\title{
Novel Cationic Sulfur Reagents and their Application in ELECTROPHILIC GROUP-TRANSFER REACTIONS
}

\author{
Dissertation \\ zur Erlangung des mathematisch-naturwissenschaftlichen Doktorgrades \\ "Doctor rerum naturalium" \\ der Georg-August-Universität Göttingen \\ im Promotionsprogramm: Chemie \\ der Georg-August-University School of Science (GAUSS) \\ vorgelegt von \\ Kai Florian Gustav Averesch \\ aus Essen, Deutschland
}

Göttingen, 2019 
Betreuungsausschuss

Prof. Dr. Manuel Alcarazo (Institut für Organische und Biomolekulare Chemie, Tammannstr. 2, 37077 Göttingen)

Prof. Dr. Franc Meyer (Institut für Anorganische Chemie, Tammannstr. 4, 37077 Göttingen)

Mitglieder der Prüfungskommission

Referent: Prof. Dr. Manuel Alcarazo (Institut für Organische und Biomolekulare Chemie, Tammannstr. 2, 37077 Göttingen)

Korreferent: Prof. Dr. Franc Meyer (Institut für Anorganische Chemie, Tammannstr. 4, 37077 Göttingen)

Weitere Mitglieder der Prüfungskommission:

Prof. Dr. Claudia Steinem (Institut für Organische und Biomolekulare Chemie, Tammannstr. 2, 37077 Göttingen)

Prof. Dr. Konrad Koszinowski (Institut für Organische und Biomolekulare Chemie, Tammannstr. 2, 37077 Göttingen)

Prof. Dr. Dietmar Stalke (Institut für Anorganische Chemie, Tammannstr. 4, 37077 Göttingen)

Dr. Christian Sindlinger (Institut für Anorganische Chemie, Tammannstr. 4, 37077 Göttingen)

Tag der mündlichen Prüfung: 18. Dezember 2019 
I hereby declare, that this dissertation has been written independently and with no sources or aids other than those quoted. The parts performed by project collaborators have been clearly indicated.

Kai F. G. Averesch 


\section{Abbreviations}

\begin{tabular}{|c|c|}
\hline${ }^{\circ} \mathrm{C}$ & degree Celsius \\
\hline $3 c-4 e$ & three-center four-electron \\
\hline 9-BBN & 9-Borabicyclo[3.3.1]nonane \\
\hline 4CZIPN & $\begin{array}{l}\text { 2,4,5,6-Tetra }(9 H \text {-carbazol-9- } \\
\text { yl)isophthalonitrile }\end{array}$ \\
\hline $4 P O B N$ & $\alpha$-(4-Pyridyl $N$-oxide)- $N$-tert-butylnitrone \\
\hline$A^{-}$ & generic Anion \\
\hline$A B X$ & Azido-benziodoxol(on)e \\
\hline Ac & acetyl \\
\hline acac & acetylacetonate anion \\
\hline $\mathrm{Ad}$ & Adamantyl \\
\hline Anthr & Anthranyl \\
\hline aq. & aqueous media \\
\hline $\mathrm{Am}$ & Amyl \\
\hline AMLA & ambiphilic metal ligand activation \\
\hline atm & Atmosphere (unit) \\
\hline ATR & Attenuated total reflectance \\
\hline$\AA$ & Ångström (unit) \\
\hline B & generic base \\
\hline$B-I-9-B B N$ & 9-lodo-9-borabicyclo[3.3.1]nonane \\
\hline BDMAE & bis(2-dimethylaminoethyl) ether \\
\hline BIES & $\begin{array}{l}\text { base-assisted internal electrophilic(-type) } \\
\text { substitution }\end{array}$ \\
\hline Boc & tert-Butyloxycarbonyl \\
\hline bs & broad NMR singlet \\
\hline $\mathrm{Bu}$ & butyl \\
\hline $\mathrm{Bn}$ & benzyl \\
\hline BXs & Benziodoxol(on)es \\
\hline $\mathrm{Bz}$ & benzoyl \\
\hline ca. & circa \\
\hline calcd. & calculated \\
\hline cat. & catalytic amount \\
\hline CBX & Cyanobenziodoxol(on)e \\
\hline CCSD & coupled cluster single double \\
\hline CDBX & Cyano-dimethyl-benziodoxol(on)e \\
\hline $\mathrm{cm}$ & centimetres \\
\hline CMD & concerted metalation-deprotonation \\
\hline CTA & Chain-transfer agent \\
\hline CUAAC & copper-catalyzed azide-alkyne cycloaddition \\
\hline CuTC & Copper(I) thiophene-2-carboxylate \\
\hline Cy & cyclohexyl \\
\hline$\delta$ & chemical shift \\
\hline d & doublet (NMR) \\
\hline DBU & 1,8-Diazabicyclo[5.4.0]undec-7-ene \\
\hline
\end{tabular}




\begin{tabular}{|c|c|}
\hline DCE & dichloroethane \\
\hline DCM & dichloromethane \\
\hline dd & doublet of doublets (NMR) \\
\hline ddd & doublet of doublets of doublets (NMR) \\
\hline ddt & doublet of doublet of triplets (NMR) \\
\hline dddt & $\begin{array}{l}\text { doublet of doublet of doublet of triplets } \\
\text { (NMR) }\end{array}$ \\
\hline deg & Degree (Angle) \\
\hline DG & directing group \\
\hline DIPEA & $N, N$-Diisopropylethylamine \\
\hline DMA & $N, N$-dimethylacetamide \\
\hline DMAP & 4-Dimethylaminopyridine \\
\hline DMTA & $\mathrm{N}, \mathrm{N}$-dimethylthioacetamide \\
\hline dme & Dimethoxyethane \\
\hline DMF & $\mathrm{N}, \mathrm{N}$-Dimethylformamide \\
\hline DMTF & $\mathrm{N}, \mathrm{N}$-dimethylthioformamide \\
\hline DMSO & dimethyl sulfoxide \\
\hline dppen & 1,2-cis(diphenylphosphino)ethylene \\
\hline $\mathrm{dt}$ & doublet of triplets (NMR) \\
\hline$e^{-}$ & electron \\
\hline e.r. & enantiomeric ratio \\
\hline EBX & Ethynylbenziodoxol(on)e \\
\hline EDG & electron-donating group \\
\hline$e e$ & enantiomeric excess \\
\hline $\mathrm{EI}$ & Electron Ionization \\
\hline $\mathrm{El}$ & Generic Electrophile \\
\hline EN & Electronegativity \\
\hline equiv & equivalents \\
\hline ESI & Electrospray lonization \\
\hline Et & Ethyl \\
\hline et al. & et alii \\
\hline $\mathrm{eV}$ & electronvolt \\
\hline EWG & electron-withdrawing group \\
\hline g & gram \\
\hline GC & Gas Chromatography \\
\hline GP & General Procedure \\
\hline h & hour \\
\hline hept & heptet (NMR) \\
\hline $\mathrm{HMBC}$ & Heteronuclear Multiple Bond Correlation \\
\hline HOMO & Highest Occupied Molecular Orbital \\
\hline HRMS & High Resolution Mass Spectroscopy \\
\hline HSQC & Heteronuclear Single Quantum Coherence \\
\hline $\mathrm{Hz}$ & Herz \\
\hline i.e. & id est \\
\hline$i$ & iso- \\
\hline IES & internal electrophilic substitution \\
\hline
\end{tabular}




\begin{tabular}{|c|c|}
\hline IR & infrared spectroscopy \\
\hline J & coupling constant \\
\hline K & Kelvin \\
\hline kcal & kilocalorie \\
\hline KIE & kinetic isotope effect \\
\hline kJ & kilojoule \\
\hline L & generic ligand \\
\hline $\mathrm{L}$ & litre \\
\hline LA & Lewis acid \\
\hline LB & Lewis base \\
\hline LC & Liquid chromatography \\
\hline LDA & Lithium diisopropylamide \\
\hline LED & Light-emitting diode \\
\hline LiHMDS & Lithium bis(trimethylsilyl)amide \\
\hline LR & Lawesson's Reagent \\
\hline$m$ & meta- \\
\hline $\mathrm{m}$ & multiplet (NMR) \\
\hline $\mathrm{m} / \mathrm{z}$ & mass-to-charge ratio \\
\hline $\mathrm{M}$ & molar \\
\hline $\mathrm{Me}$ & methyl \\
\hline $\min$ & minute \\
\hline MS & Mass Spectrometry \\
\hline NCTS & $N$-Cyano- $N$-phenyl- $p$-toluenesulfonamide \\
\hline NFSI & $N$-Fluorobenzenesulfonimide \\
\hline NFOBS & $\mathrm{N}$-fluoro-o-benzenedisulfonimide \\
\hline NMR & Nuclear Magnetic Resonance \\
\hline NOE & Nuclear Overhauser Effect \\
\hline $\mathrm{Nu}$ & generic nucleophile \\
\hline NXS & $\mathrm{N}-\mathrm{X}$-succinimide \\
\hline 0 & ortho- \\
\hline oct & octet (NMR) \\
\hline$p$ & pentet (quintet) \\
\hline p.t. & proton transfer \\
\hline$p$ & para- \\
\hline $\mathrm{Ph}$ & phenyl \\
\hline Piv & Pivaloyl \\
\hline ppm & parts per million \\
\hline $\mathrm{Pr}$ & propyl \\
\hline pyr & pyridine \\
\hline$q$ & quartet (NMR) \\
\hline $\mathrm{R}$ & generic substituent \\
\hline RAFT & $\begin{array}{l}\text { Reversible addition-fragmentation chain } \\
\text { transfer }\end{array}$ \\
\hline $\mathrm{rt}$ & room temperature \\
\hline $\mathrm{s}$ & second \\
\hline $\mathrm{s}$ & singlet (NMR) \\
\hline
\end{tabular}




\begin{tabular}{|c|c|}
\hline 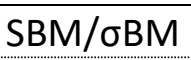 & sigma/ $\sigma$ bond metatesis \\
\hline sept & septet (NMR) \\
\hline SET & Single Electron Transfer \\
\hline sext & sextet (NMR) \\
\hline Sir & Saturation-Inversion-Recovery \\
\hline $\mathrm{S}_{\mathrm{N}} \mathrm{Ar}$ & Nucleophilic aromatic substitution \\
\hline t & time \\
\hline $\mathrm{t}$ & triplet (NMR) \\
\hline $\mathrm{T}$ & Temperature \\
\hline$t$ & tert- \\
\hline TBAF & Tetra- $n$-butylammonium fluoride \\
\hline TBPB & tert-Butyl peroxybenzoate \\
\hline td & triplet of doublets (NMR) \\
\hline Tf & Triflyl (trifluoromethanesulfonyl) \\
\hline THF & tetrahydrofurane \\
\hline TIPS & triisopropylsilyl \\
\hline TLC & Thin Layer Chromatography \\
\hline TM & Transition Metal \\
\hline TMEDA & Tetramethylethylenediamine \\
\hline TMG & 1,1,3,3-Tetramethylguanidine \\
\hline TMS & trimethylsilyl \\
\hline Tol & Tolyl \\
\hline Ts & Tosyl (toluenesulfonyl) \\
\hline $\mathrm{tt}$ & triplet of triplets (NMR) \\
\hline UV & Ultraviolet \\
\hline vs & versus \\
\hline $\mathrm{X}$ & generic halogen \\
\hline Z & generic substituent \\
\hline
\end{tabular}




\section{ACKNOWLEDGEMENTS}

Zuallererst gilt mein Dank Prof. Dr. Manuel Alcarazo, dafür mich in seine Arbeitsgruppe aufgenommen und mir die Gelegenheit gegeben zu haben, meine Promotion durchzuführen. Ich danke Ihm dafür, mir von Anfang an die Möglichkeit gegeben zu haben, Forschung nach meiner eigenen Neugierde zu betreiben und mich dabei konsequent zu unterstützen. Weiterhin danke ich Ihm für seine Unermüdlichkeit darin, trotz wechselnder Umgebung, die bestmöglichen Arbeitsbedingungen für seine Mitarbeiter geschaffen zu haben. Schlussendlich bin ich $\mathrm{lhm}$ dankbar für jedweden wissenschaflichen als auch nicht-wissenschaftlichen Wissensgewinn während meiner Promotionszeit, der ohne Ihn nicht möglich gewesen wäre.

Mein Dank gilt außerdem Prof. Dr. Franc Meyer, für die gewissenhafte Übernahme des Zweitgutachtens.

Ich danke den Mitarbeitern des Max-Planck Instituts für Kohlenforschung, besonders Sigrid Lutz und Gerlinde Mehler dafür, die Anfangsphase der Promotion, so leicht wie möglich gemacht zu haben. Ebenfalls bin ich den Mitarbeitern der Georg-August Universität Göttingen dankbar, zuerst für die Unterstützung während der Umzugsphase nach Göttingen und weiterhin die stetige Hilfsbereitschaft in formellen Angelegenheiten sowie die freundliche Atmosphäre am Arbeitsplatz. Hier gilt mein Dank besonders, Martina Pretor, Sabine Schacht, Dr. Christopher Golz, Dr. Sergei I. Kozhushkov und Martin Simon.

Ich danke den Serviceabteilungen des MPI, sowie der Universität Göttingen, den NMR-, Massenspektrometrie- und Röntgenkristallstruktur-Abteilungen, für ihre gewissenhafte Arbeit, die Messung zahlreicher analytischer Proben.

Ein großer Dank gilt allen momentanen und ehemaligen Arbeitskollegen der Forschungsgruppe, die meine Promotion zu einer einzigartigen Erfahrung gemacht haben: Dr. Elisa González Fernández, Dr. Hendrik Tinnermann, Dr. Lianghu Gu, Dr. Isaac Alonso, Dr. Jonathan Dube, Dr. Leo Nicholls, Dr. Alejandro García Barrado, Dr. Yin Zhang, Dr. Javier Peña, Dr. Garazi Talavera, Lukas Schaaf, Menno Hinrich, Maximilian Marx, Dr. Bernd Waldecker, Dr. Samuel Suarez Pantiga, Dr. Vijaykumar Gonela, Dr. Xiangdong Li, Dr. Jianwei Zhang, Marvin Böhm, Tim Johannsen, Thierry Hartung, Pablo Redero Garcia, Morwenna Mögel, Christian Rugen, Kristin Sprenger, Kevin Kafuta, Sebastian Kolle, Adam Zielinski, Steve Karreman und Henner Pesch.

Ich bin dankbar für all die Freundschaften die geschlossen wurden, auf dass sie lange währen.

Für das Korrekturlesen dieser Arbeit und die hilfreichen Anmerkungen danke ich noch einmal Adaś Zielinski, Dr. Bernd Waldecker und Dr. Sergei I. Kozhushkov.

Ich danke meiner Familie und meiner Freundin für die unentwegte Unterstützung, die mich bis hierhin gebracht hat. Danke Mama, danke Nils, danke Kim. 
„....and I don't care how tough you are, life will beat you to your knees and keep you there permanently if you let it. You, me, or nobody is gonna hit as hard as life. But it ain't about how hard you hit. It's about how hard you can get hit and keep moving forward; how much you can take and keep moving forward."

-Rocky Balboa 


\section{TABLE OF CONTENTS}

Novel Cationic SUlfur Reagents and their Application in EleCtrophilic Group-Transfer Reactions ..........1

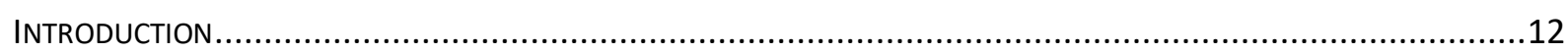

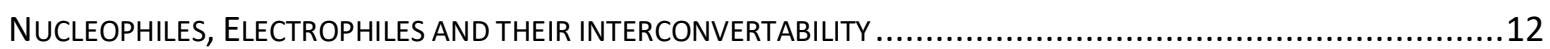

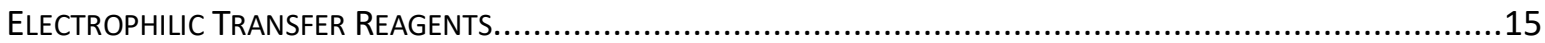

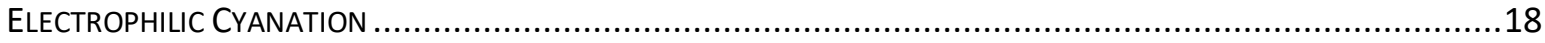

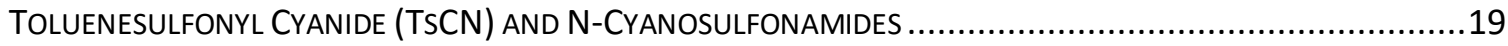

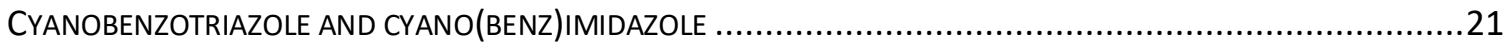

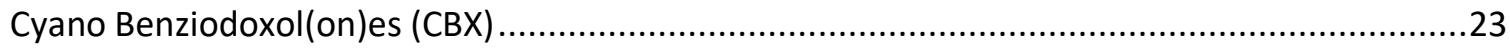

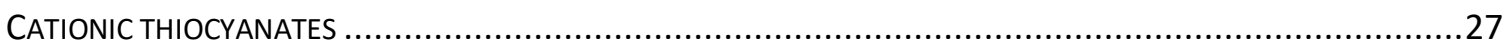

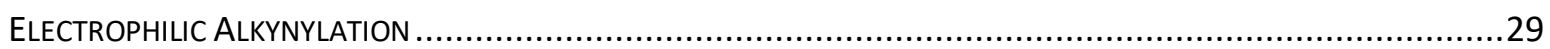

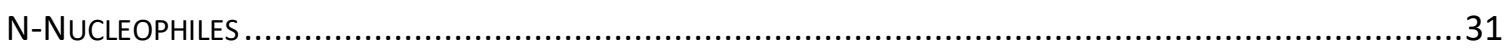

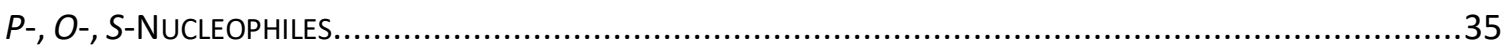

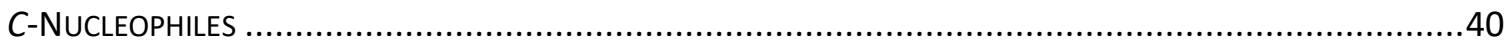

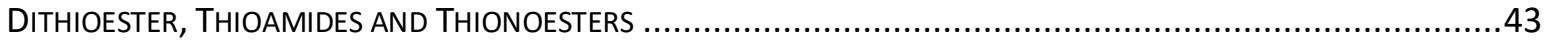

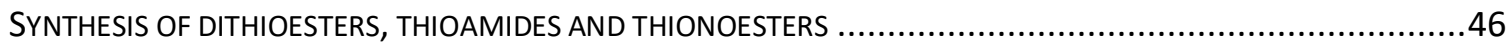

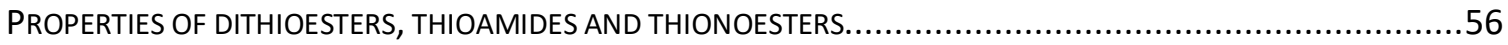

APPLICATION AND REACTIVITY OF DITHIOESTERS, THIOAMIDES AND THIONOESTERS ...................................58

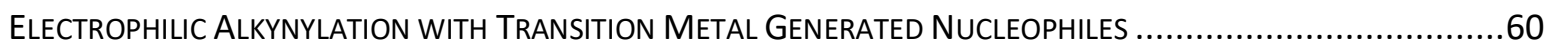

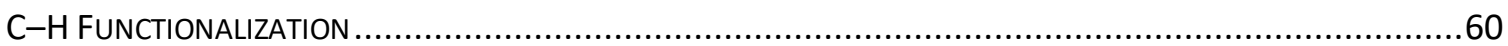

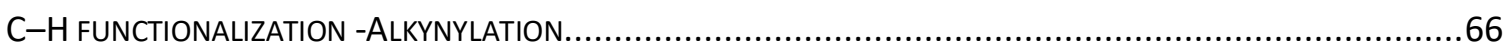

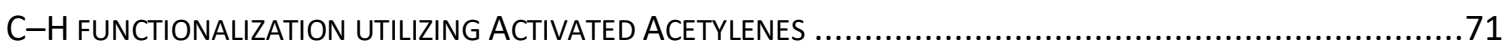

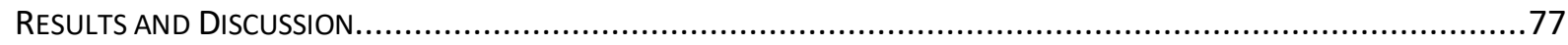

SYNTHESIS AND EVALUATION OF NOVEL PYrIdINIUM BASEd EleCtRoPHILIC TRANSFER REAGENTS .....................77

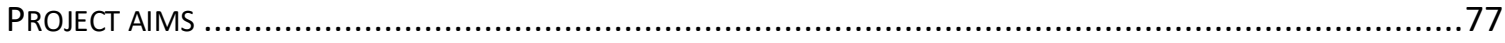

SYNTHESIS, Characteristic Properties and Application of Novel PyridinIUM-BASEd EleCtrophiliC

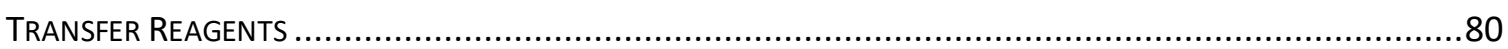

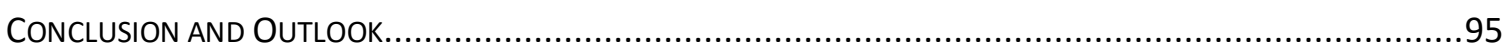

C-H FUNCTIONALIZATION UtILIZING THIOIMIDAZOLONE-BASEd ELECTROPHILIC TRANSFER REAGENTS ................97

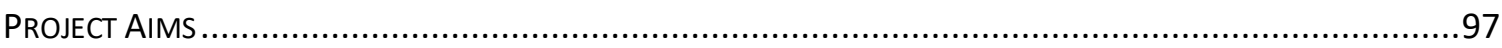

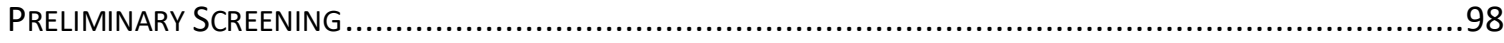

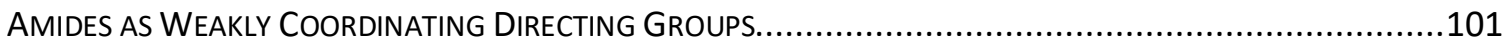

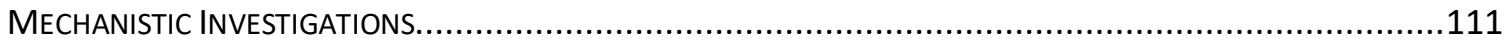

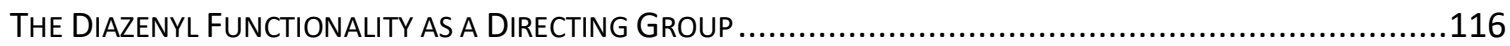

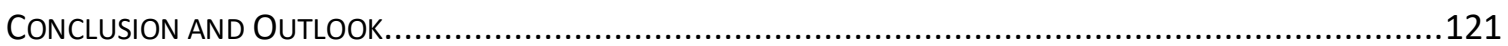


Topic:" Synthesis and Evaluation of Novel Pyridinium-BaSed Electrophilic Transfer Reagents" ......131

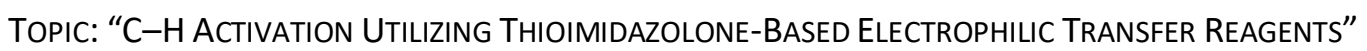
152 NON- AND LOW REACTIVE SUBSTRATES .152

APPENDIX....... .174

X-RAY StRUCtURES . 175

SPECTRA. .234 


\section{INTRODUCTION}

\section{NUCLEOPHILES, ELECTROPHILES AND THEIR INTERCONVERTABILITY}

One of the most fundamental concepts of organic chemistry is the classification of organic molecules or fragments thereof into electrophiles (Greek: "electron loving") and nucleophiles (Greek: "nucleus loving"). Nucleophiles are electron rich; they possess a filled lone pair or $\pi$-orbital resulting in a partial of fully developed negative charge, while electrophiles are electron deficient and are characterized by a partial or fully developed positive charge or an incomplete octet. They react with each other by donation of the electron pair from the occupied orbital of the nucleophile into the vacant orbital of the electrophile, forming a new molecular bond (Scheme 1).

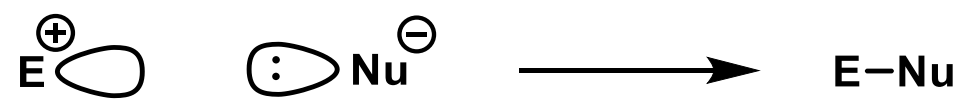

Scheme 1: Reaction of a generic electrophile with a generic nucleophile in an elementary step.

The successful identification of nucleophiles and electrophiles enables the chemist to predict the most probable outcome of an elementary step of a reaction.

In organic chemistry functional groups also can be categorized into nucleophilic and electrophilic, or ones that hold electrophilic and nucleophilic positions. Arguably, the most commonly used example is the carbonyl moiety, where a partially positive charged carbon (electrophilic) is bonded to a partially negative charged oxygen (nucleophilic) by the means of a $\sigma$ - and $\pi$-bond. Similar polarization of a carbon bound to a heteroatom is found in nitriles, however, additionally, their bonding situation is resemblant to that of alkynes, since they both possess a $\sigma$ - and two $\pi$-bonds (Scheme 2$)^{[1]}$
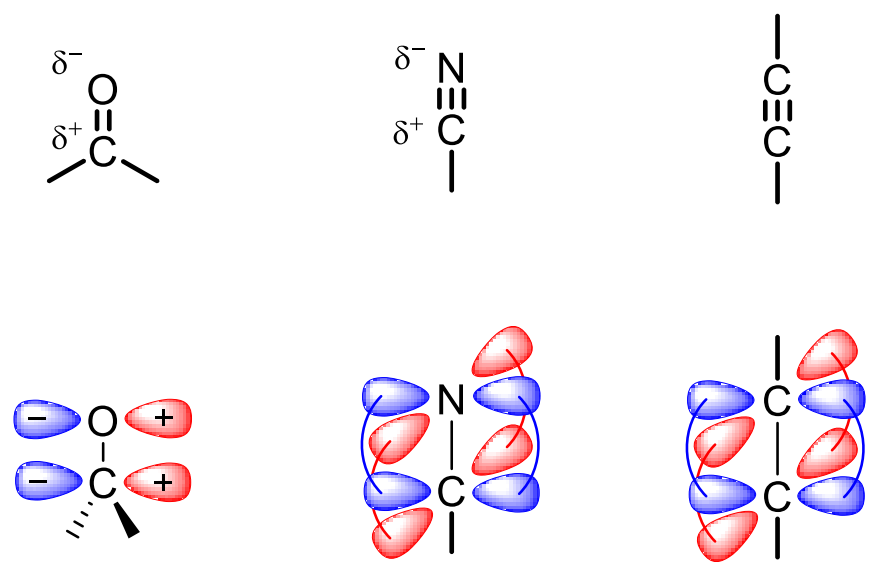

Scheme 2: Partial polarization and selected orbitals of carbonyl, nitrile, and alkyne functionalities. 
The fact, that the electrophilicity of the carbon in carbonyl and nitrile groups is a direct result of its electron withdrawing neighboring atom $(\mathrm{O}-, \mathrm{N}-)$, can be abstracted and used as a methodology to also tune the electronic properties of other functional groups such as the aforementioned alkynes. By employing an electron withdrawing atom or electron withdrawing group (EWG) adjacent to the functional group, a partial positive charge can be induced or enhanced. In the same manner a partial negative polarization can be introduced or magnified by electron donating atoms or groups (EDG). Furthermore by employing, for example the right EWG adjacent to a partially negative charged atom, this atom can be rendered so electron poor that its reactivity is inverted, and the former nucleophile becomes an electrophile. The conversion of nucleophiles into electrophiles and vice versa is known as "Umpolung" or polarity inversion. The concept was originally introduced by SEEBACH and COREY. ${ }^{[2]}$ The canonical Umpolung reagent is the cyanide ion, which, for example, finds application in the benzoin condensation, first observed by WÖHLER and LIEBIG. ${ }^{[3]}$ Here, the cyanide anion facilitates the reversion of polarity on the carbonyl croup of benzaldehyde 1 to generate nucleopile $\mathbf{3}$, which can then undergo condensation with another equivalent of benzaldehyde, which functions as electrophile giving benzoin $\mathbf{5}$.

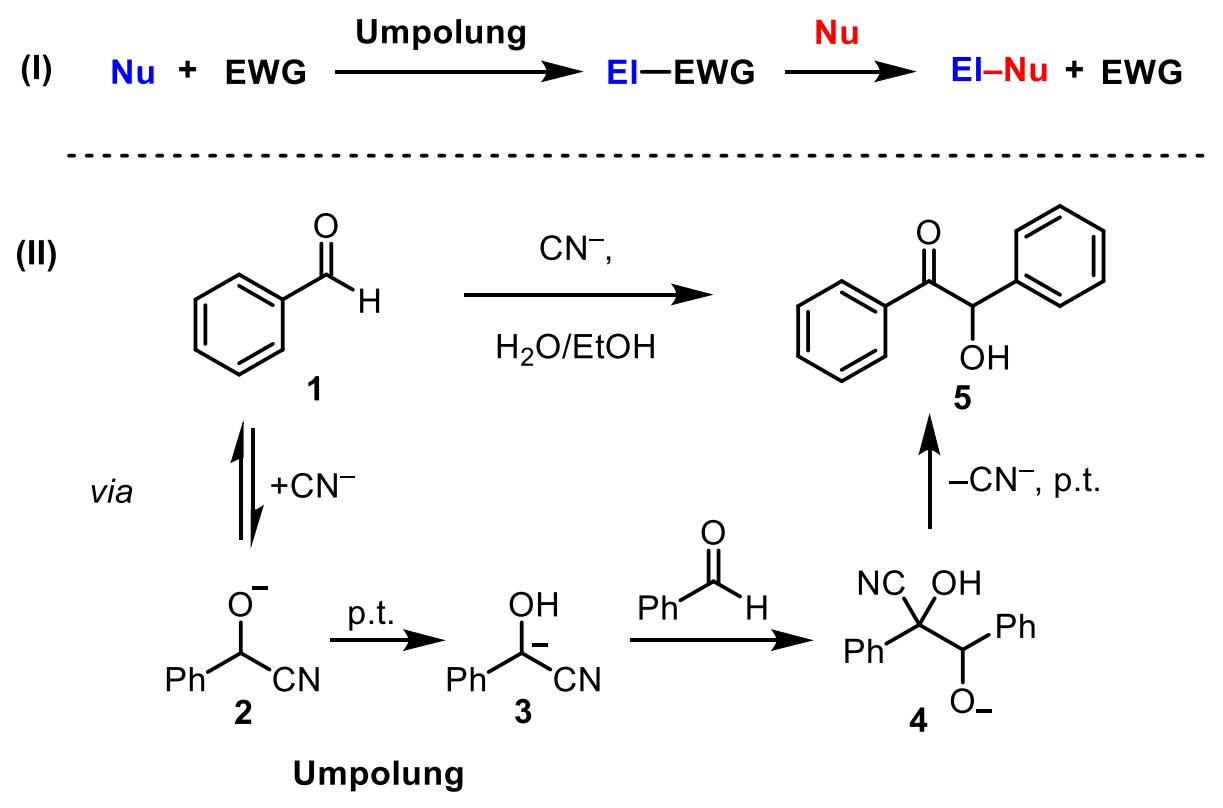

Scheme 3: Depiction of a generic initial Umpolung (I) of a nucleophile and a subsequent secondary reaction, and the benzoin reaction (II) as an example for the polarity inversion of a carbonyl group. (p.t. = proton transfer)

All functional groups possess an intrinsic polarization which is reflected in their most common reactivity. For example, electron rich groups like the cyano group or acetylides classically react as nucleophiles with electrophiles. As a direct result they find broad application in nucleophilic cyanation and alkynylation reactions, respectively. A general approach to broaden the scope of this type of reaction is to invert the polarization in Umpolung, as described above, generating an electrophilic fragment which then, in turn, can react with another nucleophile (Scheme 3-I). 
To realize an Umpolung from nucleophile to an electrophile, various groups can be attached to the moiety that needs to be transferred. Generally, they all have electron-withdrawing properties. It is logical that atoms with high electronegativity, such as halides or well-known EWGs like tosyl or triflate, are prominent examples for the realization of Umpolung reactions. The next Chapters will cover the methods that have been developed to facilitate this kind of transformations and cover the reagents that have proven to be most successful in applications. 


\section{ELECTROPHILIC TRANSFER REAGENTS}

A plethora of different electrophilic transfer reagents, among these several patented and commercially available, are known and their applications have been reported in literature. $^{[4,5]}$ In theory, any desired organic fragment can be polarized to undergo an Umpolung and later be transferred, however selected groups are of more significance than others (Scheme 4).

Halogenation:

$\mathrm{I}_{2}, \mathrm{Br}_{2}$<smiles>[X]N1C(=O)CCC1=O</smiles>

NXS

$\mathrm{X}=\mathrm{Br}, \mathrm{Cl}, \mathrm{I}$<smiles>O=S1(=O)c2ccccc2S(=O)(=O)N1F</smiles>

NFOBS<smiles>O=S(=O)(c1ccccc1)N(F)S(=O)(=O)c1ccccc1</smiles>

NFSI<smiles>FC(F)(F)[N+]12CC[N+](CCl)(CC1)CC2</smiles>

Selectfluor

Alkylation, Thiolation:<smiles>[R][R]1ccc2c(c1)[Y7]([Z])c1cc[R1]([H])cc12</smiles>

Umemoto reagent and derivatives

$\mathrm{Y}=\mathrm{S}, \mathrm{O}, \mathrm{Se}, \mathrm{Te}$

$\mathrm{Z}=\mathrm{CF}_{3}, \mathrm{SCF}_{3}, \mathrm{CN}$, Alkyne,

$\mathrm{R}^{1}, \mathrm{R}^{2}=\mathrm{F}, \mathrm{NO}_{2}, \mathrm{SO}_{3}, \mathrm{H}$, $\mathrm{Me}$,<smiles>C[N+](C)(C(F)(F)Br)S(=O)(=O)c1ccccc1</smiles>

Shibata reagent<smiles>Cc1cc(C)c([S+](c2c(C)cc(C)cc2C)C(F)(F)F)c(C)c1</smiles>

Mes-Umemoto<smiles>[Z][IH]1OC([R])([Z])c2ccccc21</smiles>

Togni reagent, EBX, CBX, $A B X$ and derivatives $\mathrm{R}^{3}=\mathrm{H}, \mathrm{R}^{4}=\mathrm{O}$ or $\mathrm{R}^{3}, \mathrm{R}^{4}=\mathrm{Me}$ $\mathrm{Z}=\mathrm{CF}_{3}, \mathrm{CN}, \mathrm{N}_{3}$, Alkyne Reagents developed in our group:<smiles>[Z][As]c1n([R8])c(C)c(C)[n+]1[B]</smiles><smiles>[Z][S-]([3H])c1c(C)cc(C)c[n+]1[3H]</smiles>

$\mathrm{R}^{5}=\mathrm{Me}, \mathrm{iPr}$ $\mathrm{R}^{6}=\mathrm{Me}, \mathrm{Et}$ $\mathrm{Z}=\mathrm{Cl}, \mathrm{Br}, \mathrm{CN}$, Alkyne, $\mathrm{Ar}$

Scheme 4: Selected electrophilic transfer reagents (NXS $=N$-X-succinimide, NFOBS $=N$-fluoro- $O$ benzenedisulfonimide, NFSI = N-fluorobenzenesulfonimide, $\mathrm{EBX}=$ ethynyl-1,2-benziodoxol-3(1H)-one, $\mathrm{CBX}=1$ cyano-1,2-benziodoxol-3-(1H)-one, $A B X=1$-azido-1,2-benziodoxol-3(1H)-one).

As can be seen from Scheme 4, the most prevalent transferred groups are (pseudo)halogens and selected alkyl, perfluoroalkyl, thiol and alkynyl groups. Introduction of halides allows for subsequent functionalization applying classical cross-coupling reactions like the Heck 
reaction, the Stille, Suzuki-Miaura, Negishi or Sonogashira-Hagihara coupling. ${ }^{[6-15]}$ Other groups like the trifluoromethyl moiety change the stability or solubility of the resulting product. Introduction of a $\mathrm{CF}_{3}$ group plays a significant role in drug development to increase the stability of compounds towards metabolization and to increase the acceptor properties of hydrogen bonds. This group is normally introduced into a key structure by manipulation of trifluoracetic acid, for example by condensation. However, the Umemoto reagent (Scheme 4) opens up new possibilities to introduce such a moitey. ${ }^{[16]}$ Other functional groups such as nitriles and alkynes, which effectively are $C_{1}$ - and $C_{2}$-synthons, but can also be modified in various other ways, are additional important motifs.

Some of the reagents presented herein possess the properties commonly described as hypervalent. It is duely noted at this point, that the term hypervalent itself and the concept of hypervalency is still a controverse topic. ${ }^{[17]}$

Nevertheless it is necessary to discuss the unique structure and properties of hypervalent compounds due to their relevance for this work. The iodonium salts discussed below, described for the first time by BERINGER in $1965,{ }^{[18]}$ have a unique structure owing to their hypervalency, which is partly reflected in their reactivity (Scheme 5). They adopt a T-shaped geometry with an almost linear $\mathbf{X}-\mathbf{I}-\mathbf{Y}$ triad. Stabilization is gained by electron withdrawing axial ligands, which stabilize the molecule due to the 3-center-4-electron bond $(3 c-4 e)$ in which more electron density resides on the ligands at the ends ( $X \& \mathbf{Y})$ than at the center (visualized by the node for the nonbonding orbital of the central atom). The three-centrefour electron bond, has a lower Bond Order than a normal sigma bond and is thus weaker. Attached organic groups $\mathbf{Y}$ are consequently prone to nucleophilic attack. This allows transfer of organic groups to small molecules and also advanced intermediates.

Furthermore, trans influences effecting their stability have been reported. ${ }^{[19]}$ Manifestation of trans influences in general can be observed by trends in NMR, IR and solid state structures. ${ }^{[20]}$ With the recent surge in the use of hypervalent iodine compounds, availability of solid state structures has also increased. Investigations of these towards trans influences of the ligands $\mathbf{X}$ and $\mathbf{Y}$ lead to the conclusion that combinations of ligands $\mathbf{X}$ and $\mathbf{Y}$ where one shows a strong and the other a weak trans influence or the scenario in which both ligands have a moderate trans influence seem to be favoured. Other scenarios where both ligands $\mathbf{X}$ and $\mathbf{Y}$ have strong or weak trans influences both result in labile compounds.

How the trans influence manifests itself for hypervalent iiodine compounds can be explained as follows: In a linear X-I-Y hypervalent bonding situation the central iodine retains its $5 s^{2}$ lone pair and an exclusively inductive trans influence by the $\mathbf{X}$ ligand is transmitted through the $5 p_{\sigma}$ orbital of the iodine atom. With increasing donor ability of $\mathbf{X}$, the $\mathbf{X}-\mathbf{I}$ bond strength increases while the $\mathbf{I}-\mathbf{Y}$ bond trans to the $\mathbf{X}-\mathbf{I}$ bond is weakened proportionately, thus making a potential nucleophilic attack towards $Y$ easier. ${ }^{[21]}$ 


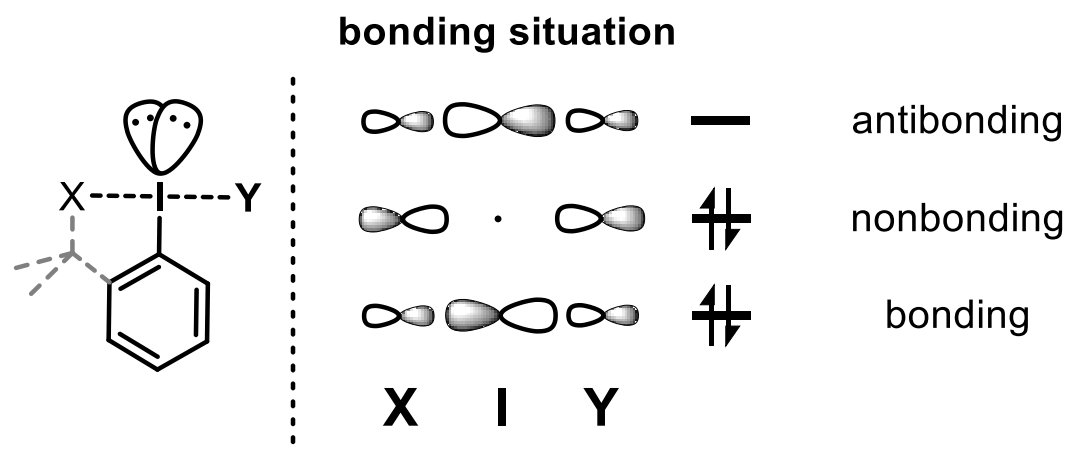

Scheme 5: The Rundle-Pimentel orbital model for 3-center-4-electron bonds applied to hypervalent iodonium salts. ${ }^{[22]}$

The unique properties, which enable hypervalent iodonium salts to reverse the polarity of certain organic groups in Umpolung reactions, have been unveiled, and their applicability in different research fields is documented in numerous publications. Modification of the structure from acyclic to cyclic, by introduction of an oxy-tether, gives these compounds an increased stability (Scheme $\mathbf{5}, \mathbf{X}=0$ ). Nevertheless, thermal decomposition or decomposition in the presence of Lewis acids, bases or transition metal catalysts remains an issues for hypervalent iodonium salts. ${ }^{[23]}$

Another example for a structure exploited due to its unique properties are (perfluoroalkyl)chalcogen salts, the most prominent member of which is the Umemoto reagent. It was designed after its acyclic predecessor $\mathrm{Ar}_{2} \mathrm{~S}^{+} \mathrm{CF}_{3} \mathrm{SbF}_{6}{ }^{-}$, originally discovered by YAGUPOLSKII. ${ }^{[5,24,25]}$ At first developed for electrophilic $\mathrm{CF}_{3}{ }^{+}$transfer, this motif has now found a significantly broader range of applications. 


\section{Electrophilic Cyanation}

To open up new reaction pathways and enable the synthesis of novel CN-containing compounds, numerous electrophilic cyanating reagents have been developed, commercialized and employed. While the very early reagents like cyanogen chloride, bromide, or dicyan are simple in constitution, their high toxicity in combination with their low boiling points and high vapor pressure are major drawbacks in handling these chemicals even on laboratory scale. A logic improvement consists of attaching a larger, electron withdrawing substituent to the nitrile group giving the resulting compounds a higher boiling point and thus, making them easier to handle. Ideally, with the choice of the correct polarizing group, highly stable solid compounds, which only release the desired electrophilic $\mathrm{CN}$-synthon upon choice of the correct reaction conditions, can be designed.

One of the first steps towards such electrophilic cyanation reagent was taken by KURZER with the synthesis of $\mathrm{N}$-cyanosulfonamides in 1949 (Scheme 7-I). ${ }^{[26]} 32$ years later the first successful electrophilic cyanation employing toluenesulfonyl cyanide (TsCN) 6 was reported. ${ }^{[27]}$ Over the past 25 years the field experienced a renaissance, and up to today a significant amount of additional electrophilic transfer reagents were developed, underlining the demand to access and broaden this mode of reactivity (Scheme 6). ${ }^{[28,29-42]}$
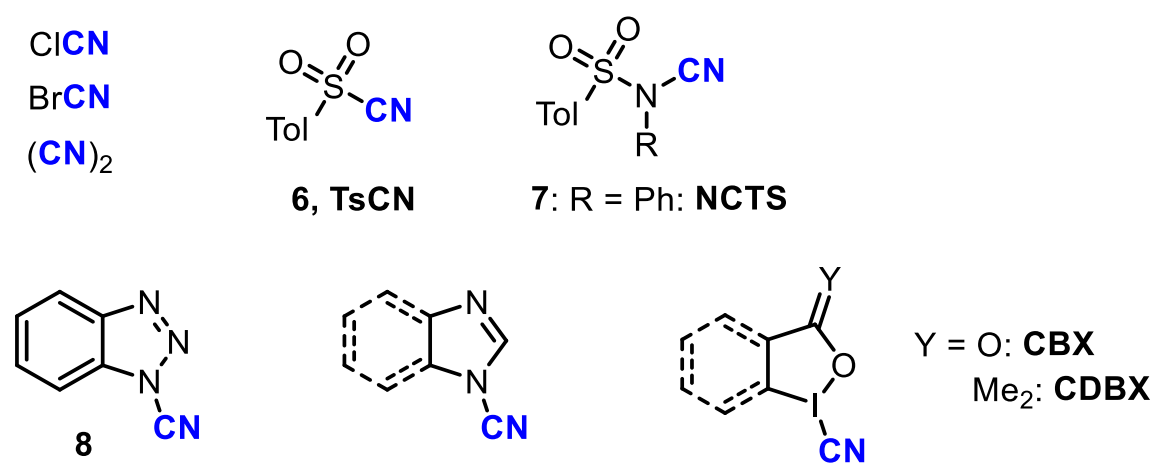<smiles>[Y]Sc1n(CCC)c(C)c(C)[n+]1C([Y])[I-]</smiles>

9a, $\mathrm{X}=\mathrm{Br}$

$9 b, X=\mathrm{SbF}_{6}$<smiles>N#C[s+]1c2ccccc2c2ccccc21</smiles>

10

Scheme 6: A selection of electrophilic cyanide transfer reagents (Tol = tolyl, NCTS $=N$-cyano- $N$-phenyl- $p$ methylbenzenesulfonamide, $C B X=$ cyanobenziodoxol(on)e, $C D B X=$ cyano dimethylbenziodoxol(on)e.

The following Sections will cover the most relevant applications of transfer reagents shown above, by highlighting selected reactions. 


\section{TOLUENESUlfonyl CyANIDE (TsCN) AND N-CyANOSUlfonAmides}

(I) Kurzer (1949)

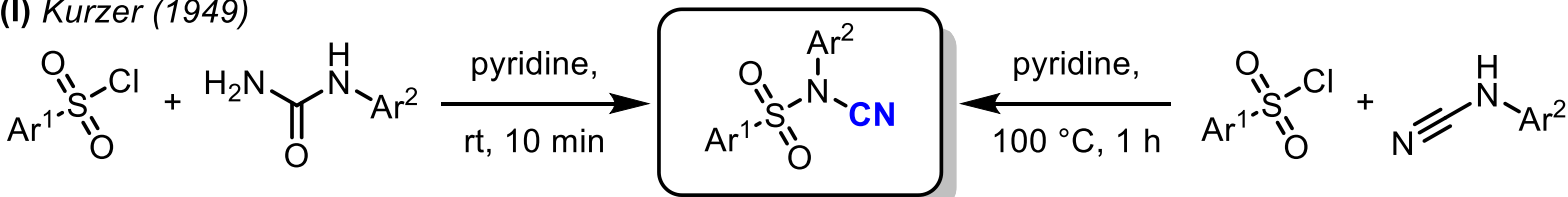

(II) Cox \& Ghosh (1969)

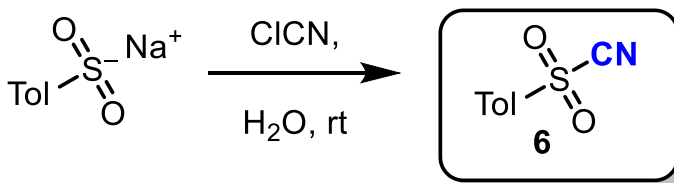

(III) Kahne \& Collum (1981)<smiles>[R]C1CCCCC1=O</smiles>

11

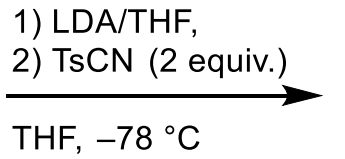
$80 \%$<smiles>[R]C1CCCC(C#N)C1=O</smiles>

12

selected examples:

(IV) Beller (2011)<smiles>[R]c1ccc(NC(C)(Cl)Cl)cc1</smiles>

13

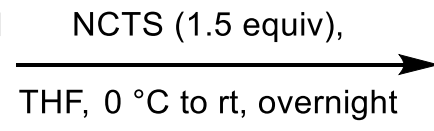
up to $93 \%$<smiles></smiles>

14<smiles>N#CC1CCc2ccccc2C1=O</smiles>

(V) Wang (2011)<smiles></smiles>

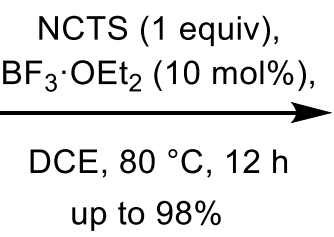<smiles>[R]c1c(C#N)c2c(n1[R])=CC#[Y14]=2</smiles>

$\mathrm{R}^{1}=\mathrm{Me}, \mathrm{NO}_{2}, \mathrm{OMe}$

$\mathrm{R}^{2}=\mathrm{Me}, \mathrm{H}, \mathrm{Bn}$, anisyl

$\mathrm{R}^{3}=\mathrm{Me}, \mathrm{H}, \mathrm{Ph}$, anisyl, etc.

$\mathrm{Cl}, \mathrm{F}$ dioxolane $\mathrm{NC}, \mathrm{CONiPr}$ etc.

\section{further examples:}<smiles>CC1(C)CC(=O)C(C#N)=C(O)C1</smiles><smiles>N#CC1C(=O)c2ccccc2C1O</smiles>

(VII) Minakata \& Kiyokawa (2016)

$$
\begin{aligned}
& \text { TsCN ( } 1.5 \text { equiv), } \\
& \mathrm{K}_{2} \mathrm{CO}_{3} \text { ( } 1.5 \text { equiv), } \\
& \stackrel{\begin{array}{c}
\mathrm{THF} / \mathrm{H}_{2} \mathrm{O}(9: 1), \mathrm{rt} \\
\text { up to } 95 \%
\end{array}}{ }
\end{aligned}
$$

NCTS (1 equiv), 9-BBN (1.05 equiv),

THF, $40{ }^{\circ} \mathrm{C}, 12 \mathrm{~h}$ up to $94 \%$<smiles>[R]C(=O)C(C#N)C([R])=O</smiles>
$\mathrm{R}^{1}, \mathrm{R}^{2}=\mathrm{Me}, \mathrm{Ph}$<smiles>[R]C=C([R])C([R])=O</smiles>

18<smiles>[124IH]</smiles><smiles>CCC12CCCC(CC1)[Pb]2I</smiles><smiles>[R]CC([R])([R])C([R])=O</smiles>
$\mathrm{R}^{1}=\mathrm{Ph}, \mathrm{Me}, i \mathrm{Pr}, \mathrm{Et}, t \mathrm{Bu}$, etc. $\mathrm{R}^{2}=\mathrm{H}, \mathrm{Me}$, etc. $\mathrm{R}^{3}=\mathrm{Bn}, \mathrm{Et}, \mathrm{iBu}$, etc.

\section{(1.1 equiv)}

selected examples:<smiles>[R]C(=O)C([R])[R]</smiles>

19
$i-\mathrm{Pr}_{2} \mathrm{NEt}$ ( 1.1 equiv), $\mathrm{Et}_{2} \mathrm{O}, 0{ }^{\circ} \mathrm{C}$ to $\mathrm{rt}, 1 \mathrm{~h}$

2) NCTS (1.2 equiv), rt up to $94 \%$<smiles>N#CC1CCCCCC1C(=O)C1(c2ccccc2)CC1</smiles>

Scheme 7: Electrophilic cyanation utilizing $N$-cyanosulfonamides.

A rather early example for the utilization of $\operatorname{TsCN}(6)$ as an electrophilic cyanating agent is given by KAHNE and ColLUM (Scheme 7-III). ${ }^{[27]}$ Within the context of a natural product 
synthesis study, they required a protocol to effectively cyanate a ketone moiety in compounds 11, without compromising the integrity of an asymmetric center. After successful identification of a protocol utilizing TsCN to obtain $\beta$-ketonitriles 12 via lithium enolates, they could show the procedure to be general for five additional examples. ${ }^{[27]}$

30 years later, BELLER and coworkers (Scheme 7-IV) ${ }^{[33]}$ realized the first electrophilic cyanation of aryl and heteroaryl Grignard reagents 13 by utilization of $N$-cyano- $N$-phenyl- $p$ methyl-benzenesulfonamide (NCTS, 7). For selected examples they could also realize monocyanation of dibromoarenes. The relevancy for the developed procedure was further underlined by the exemplary synthesis of three different key building blocks for pharmacological and agrochemical intermediates. ${ }^{[33]}$

Using the same electrophilic cyanating reagent 7 but employing $\mathrm{BF}_{3} \bullet \mathrm{OEt}_{2}$ as a strong Lewis acid, WANG (Scheme $7-\mathrm{V}$ ) could show that both $\mathrm{N}$-unsubstituted, $\mathrm{N}$-aryl and $\mathrm{N}$-alkyl indoles $\mathbf{1 5 a}$ as well as pyrroles $15 \mathbf{b}$ gave the corresponding heteroarylnitriles $\mathbf{1 6} \mathbf{a}, \mathbf{b}$. The authors claim the use of an appropriate Lewis acid activates the cyanating agent; however, they do not indicate how. ${ }^{[34]}$

A significantly milder approach compared to that of KAHNE and COLLUM with regard to the use of a base was reported in 2013 by IBRAHIM et al. (Scheme 7-VI). With use of the mild base $\mathrm{K}_{2} \mathrm{CO}_{3}$ instead of strong lithiumorganyls, they were able to cyanate both acyclic and cyclic 1,3-dicarbonyl compounds 17. Modification of the standard reaction conditions further enabled them to develop an efficient one-pot cyanation/pyrazole formation sequence to 4cyano pyrazoles. ${ }^{[31]}$

KIYOKAWA and MINAKATA (Scheme 7-VII) could extend the scope of modifiable substrates to $\alpha, \beta$-unsaturated ketones 18 by using 9-BBN as a Lewis acid to generate boron enolates via 1,4-hydroboration. Choice of the appropriate reaction temperature and solvent was crucial to facilitate complete hydroboration in this case. By switching from 9-BBN to $B-I-9-B B N 20$ and addition of a base, a protocol for saturated ketones 19 was also elaborated. The latter is remarkably orthogonal to the first system, enabling cyanation in the presence of conjugated alkene moieties with their conservation. During their studies, both TSCN and NCTS appeared to be versatile sources for electrophilic cyanation. A preliminary result with a chiral boron enolate was also reported. The cyanated product was obtained with $94 \% e e$, making the reaction to be one of the few efficient examples for enantioselective electrophilic cyanation. ${ }^{[35]}$

Advantages of both TsCN and NCTS are their mild reaction conditions and high reactivity towards C-nucleophiles. However, as in all nitrogen-based transfer reagents, polarization is relatively weak, and strong nucleophiles are necessary to achieve satisfying results. 
(I) Cava (1998)

\section{LDA}

2.<smiles>c1ccc2[nH]nnc2c1</smiles><smiles>N#CCc1ccccc1</smiles>

21

(II) $W u(2000)$

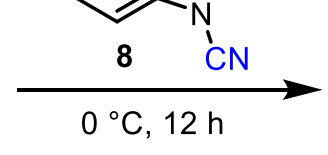

$0^{\circ} \mathrm{C}, 12 \mathrm{~h}$<smiles>N#CC(C#N)c1ccccc1</smiles>

22
R-CN

$\mathrm{RN}-\mathrm{CN}$

$\mathrm{RS}-\mathrm{CN}$
$\mathrm{RLi}$, $\mathrm{RMgBr}$,

$\mathrm{RNH}_{2}$, $\mathrm{RSH}$,

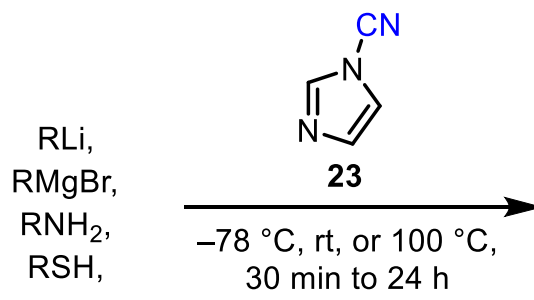

(III) Katritzky (2007)

Selected examples:

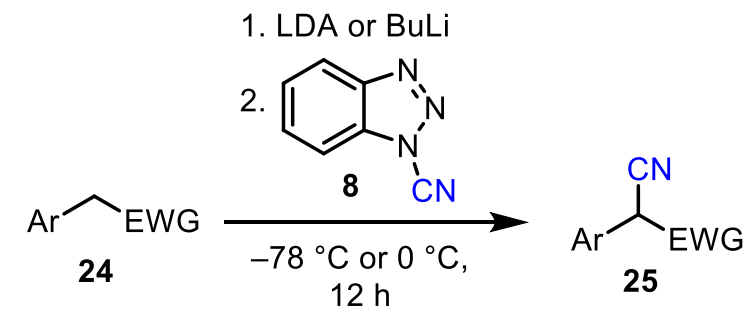

(IV) Beller (2010)<smiles>COc1ccc(SC(C)(C)C)cc1OC</smiles>

Selected examples:<smiles>C=Cc1ccc(C(C)(C)C)cc1</smiles>

Scheme 8: Electrophilic cyanation utilizing cyanobenzotriazole or cyano(benz)imidazole.

Before CAVA et al. (Scheme 8-I) published a two-step way to access 1-cyanobenzotriazole (8), only syntheses which resided on the use of either cyanogen chloride or cyanogen bromide were known. In fact, the authors also postulated in situ formation of $\mathrm{ClCN}$ for their procedure, which is then consumed upon the product formation. Their synthesis, however, is significantly safer since it resides on the use of $5 \%$ sodium hypochlorite solution for the chlorination, followed by treatment with sodium cyanide to give 1-cyanobenzotriazole (8) after sublimation. They also found the first examples of the use of cyanating agents to obtain arylmalononitriles $\mathbf{2 2}$ from arylacetonitriles $\mathbf{2 1}$ with employment of LDA as a base. ${ }^{[29]}$ 
1-Cyanoimidazole (23), which was originally used as a coupling partner in polynucleotide synthesis, was found by Wu et al. ${ }^{[43]}$ to be a potent ${ }^{+} \mathrm{CN}$-synthon for reactions with $\mathrm{N}$-, $\mathrm{C}$ - and $S$-nucleophiles (Scheme 8-II). Notably, no additional base was necessary. During their experiments with thiols as nucleophiles they observed concomitant disulfide formation, which was caused by further addition of thiol to the already formed thiocyanate product. A major drawback of this reagent is the use of cyanogen bromide for the synthesis.

KATRITZKY (Scheme 8-III) expanded the chemistry reported by CAVA and showed that not only compounds $\mathbf{2 4}$ decorated with EWGs such as ketones, esters, sulfoxides, but in addition also substrates with pyridyl, furyl, pyrrolyl moieties without EWGs undergo electrophilic cyanation facilitated by lithiumorganyls. ${ }^{[36]}$

In analogy to the their work with NCTS, BELLER et al. (Scheme 8-IV) also reported the reaction of arylgrignards 13 with 1-cyanobenzimidazole (26). To extend the developed methodology, they also showed that a domino Grignard-coupling/cyanation to give access to 2 -cyano-1,1'biaryls is feasible. ${ }^{[32]}$

Just like TsCN and NCTS, 1-cyanobenzotriazole (8), 1-cyanoimidazole (23) and 1cyanobenzimidazole (26) are nitrogen-based reagents and thus mild cyanating agents. All of which show activity with strong $C$-nucleophilies such as Grignards or lithiumorganyls; however, compounds $\mathbf{8 , 2 3}$ and $\mathbf{2 6}$ react also with $N$ - and $S$-nucleophiles. 
Cyano Benziodoxol(on)es (CBX)

Remarkable pioneering and continued work in the field of hypervalent iodine compounds has been carried out by ZHDANKIN, OCHIAI and others. ${ }^{[44]}$

Since 2008 the field has experienced a renaissance, notably due to J. WASER and coworkers, who could clearly show that up to then, the potential of benziodoxolones (BXs) had been seriously underestimated. ${ }^{[45]}$ One of the members of the BX group of compounds is cyano benziodoxol(on)e (CBX) 27, which is used for electrophilic cyanation. ${ }^{[30]}$ 
(I) Zhdankin (1995)

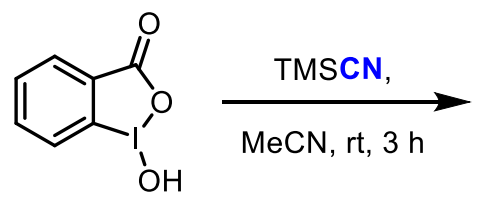<smiles>N#CI1OC(=O)c2ccccc21</smiles>

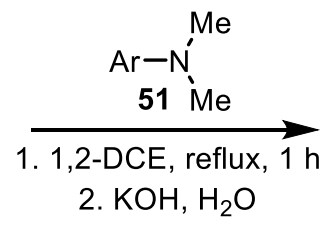<smiles>CN([Al])CC#N</smiles>

(II) Chen, Zheng, M. Waser (2015)<smiles>[Y]C(=O)C1Cc2ccccc2C1=O</smiles>

28<smiles>CC1[IH]OC(=O)c2ccccc21</smiles><smiles>[Y]C(=O)C1(C)Cc2ccccc2C1=O</smiles>

(III) J. Waser (2015)

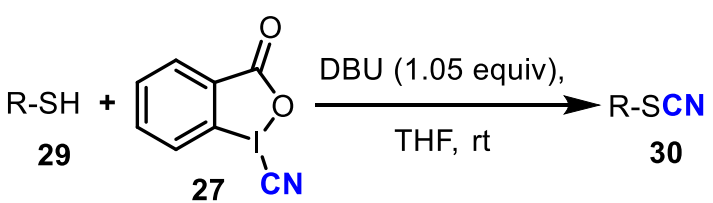
$\mathrm{R}=$ aryl, alkyl

(IV) Ma \& Zhang (2018)

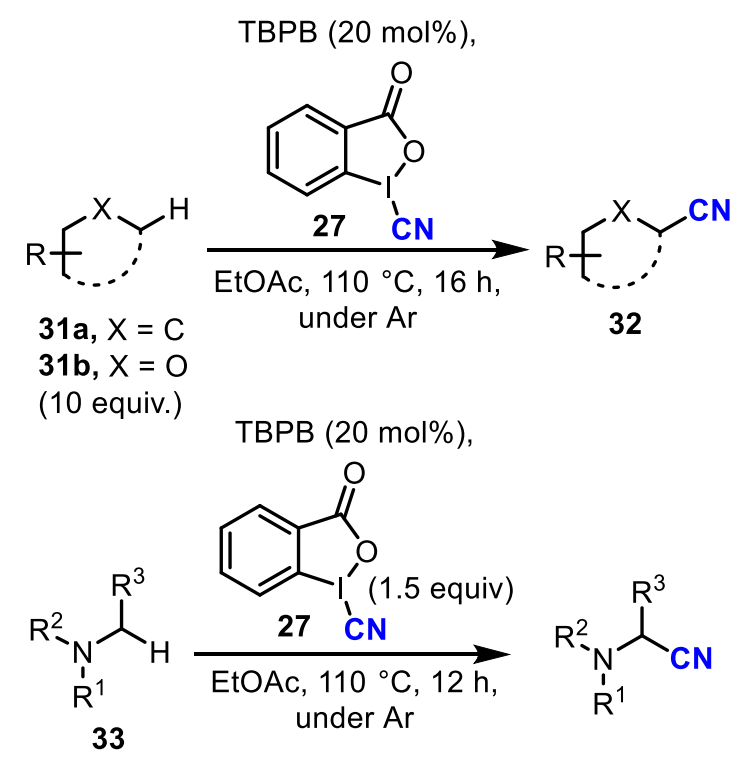

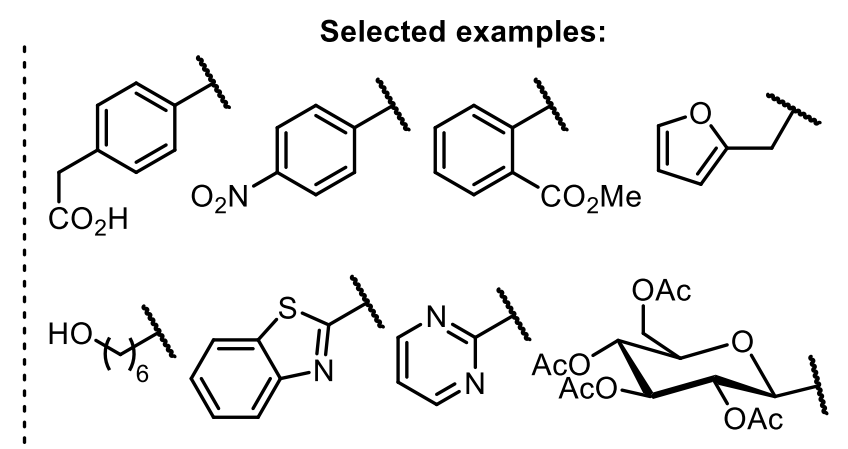

Selected examples:<smiles>CC(C)C1CCCc2ccccc2C1C(C)C</smiles><smiles>CC(C)C(C)Oc1ccccc1C(C)C(C)C</smiles><smiles>CCCCCCCCC(C)CN(C)c1ccc(C(=O)OC)cc1</smiles><smiles>CC(C)C1CCc2ccccc2N1C(=O)OC(C)(C)C</smiles>

Scheme 9: Electrophilic cyanation utilizing benziodoxol(on)es. 
The groups of ZHENG, M. WASER and also CHEN (Scheme 9-II) all described the $\alpha$-cyanation of $\beta$ keto esters 28 with CBX 27 in 2015: [37,39,40] While CHEN et al. ${ }^{[37]}$ focused on a base-free approach and also reported successful cyanation of $\beta$-keto amides, the main emphasis of $M$. WASER $^{[39]}$ and ZHENG ${ }^{[0]}$ was on developing an enantioselective synthesis. Both groups used alkaloid-based phase transfer catalysts for the stereoselective induction.

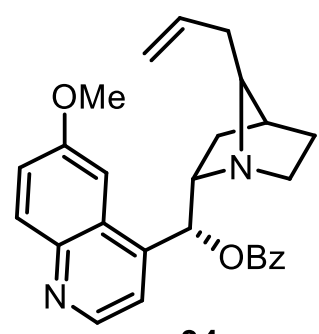

$34 a$

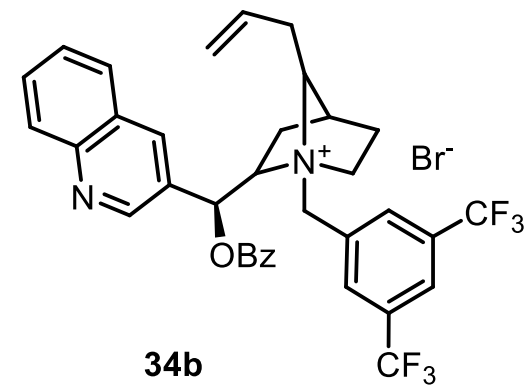

34b

Scheme 10: Phase transfer catalysts identified to be suitable for enantioselective electrophilic cyanation.

While the group of M. WASER identified alkaloid 34a to catalyze the reaction best in chloroform without addition of base with a maximum e.r. of 76:24, the group of ZHENG found catalyst 34b to be the best in a solvent mixture of THF and toluene with addition of DMAP as a base, thus affording products with the highest ee of 93\% (Scheme 10).

Few procedures have been proven to be straightforward for the cyanation of thiols. Among them is the reported by J. WASER (Scheme 9-III) in 2015 protocol for cyanation of compounds

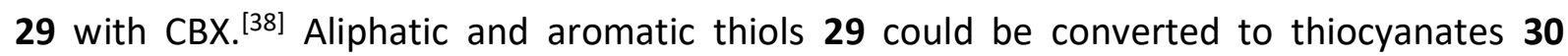
within minutes at room temperature in the presence of DBU. They have also found disulfides to be viable substrates which could be converted in up to $92 \%$ yield. Mechanistic investigations were carried out in silico and suggest either a SET process or a concerted transition state, both would be in agreement with the high reaction rate reported. Selected examples of significance for synthetic chemistry, chemical biology and materials science further underline the impact of this work

MA and ZHANG (Scheme 9-IV) made use of the fact that CBX is not only capable of electrophilic cyano transfer, but can also act as an oxidant. Normally an inconvenience causing undesirable side reactions, this property was used to develop a procedure for direct $\mathrm{C}\left(\mathrm{sp}^{3}\right)$ cyanation. The results from ZHDANKIN et al., who reported on azidation with azidobenziodoxole $(A B X)$ with similar oxidative properties, ${ }^{[46]}$ were applied to the cyanation protocol by $M A$ and ZHANG. ${ }^{[47]}$ Assuming a free radical chain mechanism proposed by ZHDANKIN, the groups of MA and ZHANG used tert-butyl-peroxybenzoate (TBPB) as the initiator. They successfully functionalized alkanes $\mathbf{3 1 a}$, ethers $\mathbf{3 1} \mathbf{b}$ and tertiary amines $\mathbf{3 3}$. To investigate the mechanism, they conducted their cyanations in the presence of $N$-tert-butyl$\alpha$-(4-pyridyl-1-oxide) nitrone (4-POBN, 35) as spin-trap reagent (Scheme 11). 


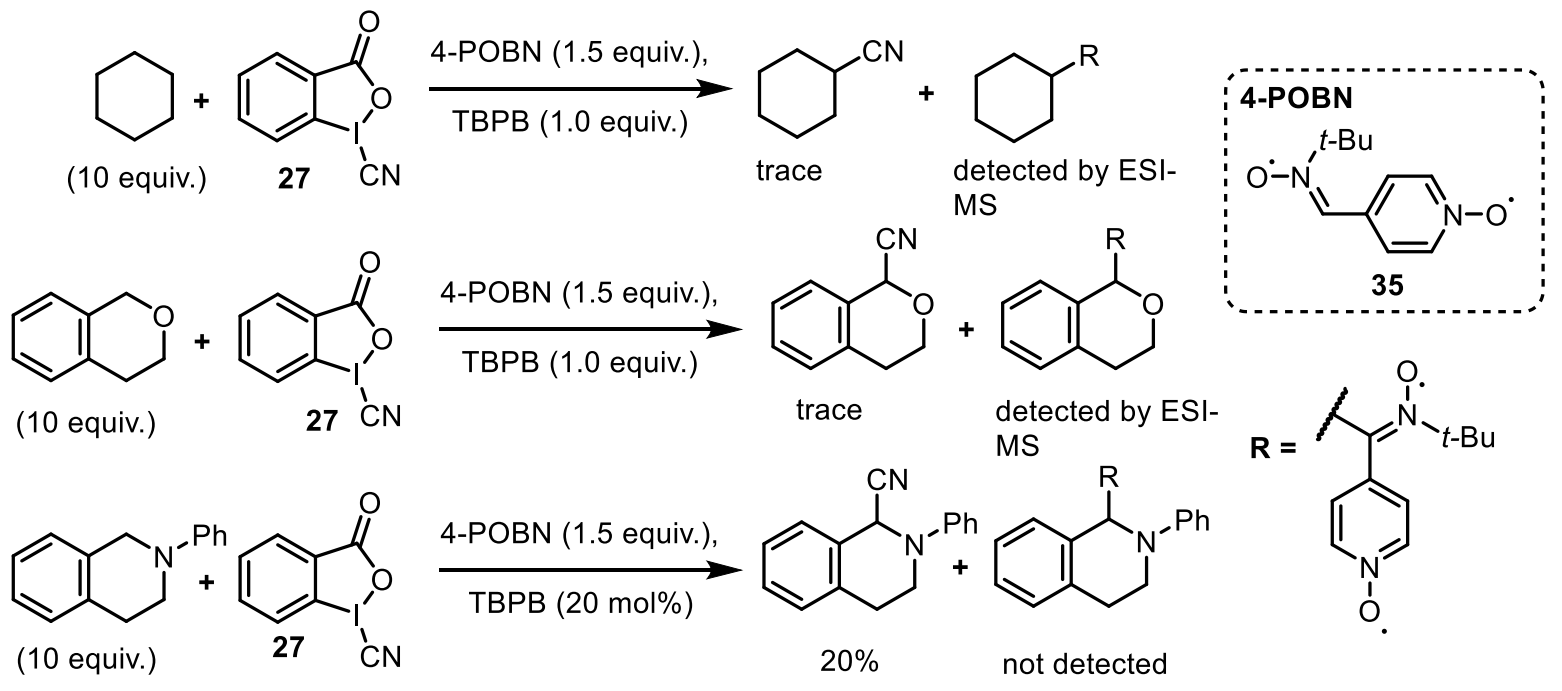

Scheme 11: Mechanistic investigations with 4-POBN as a spin trapping reagent.

While for alkanes and ethers the reaction was completely suppressed, tertiary amines still gave moderate yields. The authors assumed an oxidative reaction process, which was tested in additional competition experiments under parallel oxidative trifluoromethylation conditions in the presence of $\mathrm{TMSCF}_{3}$ and $\mathrm{CsF}$. The observation of the trifluoromethylated product verified the transient formation of an iminium cation intermediate. Based on the findings, they postulated two different mechanisms, depending on the nature of the substrate, i. e. alkanes and ethers versus tertiary amines. 


\section{Cationic thiocyanates}

(I) Alcarazo (2015)<smiles>[Y]C(C)[n+]1c(C)c(C)n([In]CC)c1SC</smiles>

a) DIPEA (1 equiv),

$$
\mathrm{CH}_{2} \mathrm{Cl}_{2}, \mathrm{rt}
$$

$\mathrm{R}-\mathrm{H}$

(II) Alcarazo (2018)

b) $\mathrm{BF}_{3} \cdot \mathrm{OEt}_{2}(20 \mathrm{~mol} \%)$

1,2-DCE, $80^{\circ} \mathrm{C}$

$\mathrm{X}=\mathrm{Br}, \mathrm{SbF}_{6}$<smiles>N#C[s+]1c2ccccc2c2ccccc21</smiles>

$\mathrm{R}-\mathrm{H} \stackrel{\mathrm{Cs}_{2} \mathrm{CO}_{3} \text { or } \mathrm{K}_{3} \mathrm{PO}_{4}(1.5 \text { equiv }),}{\mathrm{CH}_{2} \mathrm{Cl}_{2}, \mathrm{rt}} \mathrm{R}-\mathrm{CN}$
Selected examples:<smiles>COc1ccc(C(C)(C)c2cccn2-c2ccccc2)c(OC)c1</smiles>

Selected examples:

$t$-Bu<smiles>Cc1ccc(NC(C)C)cc1</smiles><smiles>CC(C)N1CCN([O+])CC1</smiles><smiles>CC(C)Sc1ccccc1Br</smiles><smiles>CC(C)CC(=O)c1ccccc1</smiles><smiles>CC(C)c1cn(-c2ccccc2)c2ccccc12</smiles><smiles>COc1cc(OC)c(C(C)C)c(OC)c1</smiles><smiles>CC(C)c1c2ccccc2cc2cc3ccccc3cc12</smiles>

cyano cyclization<smiles>[R]c1c(CCN)c2ccccc2n1[R2]</smiles><smiles>N#C[s+]1c2ccccc2c2ccccc21</smiles>

$\mathrm{MeCN}, \mathrm{rt}, 2 \mathrm{~h}$<smiles>[Y19]C1CN[C@H]2N([R])c3ccccc3[C@]12C</smiles>

$R^{1}, R^{2}=H, M e, P h, B n$

Scheme 12: Electrophilic cyanation utilizing imidazolium thiocyanates and dibenzothiophenium cyanates.

Based on the work of ARDUENGO \& BURGESS ${ }^{[48]}$ and further progressions by ROESKY, ${ }^{[49]}$ ALCARAZO and coworkers were able to exploit the hypervalent character of di(halo)imidazolium salts for the synthesis of imidazolium thiocyanates 9 (Scheme $12-1) .{ }^{[41]}$ These salts proved to be efficient transfer reagents for electrophilic cyanation, as $S_{-}, N$ - and, remarkably, also a number of $\boldsymbol{C}$-nucleophiles underwent the transformation smoothly. Advantages of these reagents are the easy and short synthesis sequence, as well as their high stability.

After successful synthesis of 5-(alkynyl)dibenzothiophenium salts by utilizing the backbone, which was already popularized by UMEMOTO, ${ }^{[5,[25]}$ LI \& ALCARAZO (Scheme 12-II) ${ }^{[42]}$ could demonstrate that the cyano derivative $\mathbf{1 0}$ is also stable and potent in cyanation reactions. While its behavior towards nucleophiles is milder, as compared to the imidazolium thiocyanate 9, its reactivity among electrophilic transfer reagents is unique. Thus, it shows unprecedented reactivity with indol and derivatives thereof. When 2-unsubstituted indoles 
were subjected to standard reaction conditions, cyanation in 3-position with subsequent nucleophilic attack of a second equivalent of indole resulted in dimerization of the indole moiety. To make use of this observation, tryptamine derivatives $\mathbf{3 6}$ were employed for a cyano cylization cascade. The methodology could even be extended to a cyanation-Povarov cascade. Noteworthy, upon cyclization of allylic indoles in which four consecutive stereocenters are generated, selective formation of only one diastereomer was observed.

In conclusion, although various available reagents can be used to implement electrophilic cyanation, the synthetic problems remain the same. Depending on the degree of polarization of the nitrile group within a transfer reagent, diverging pathways for the functionalization of nucleophiles exist. Reagents with relatively low polarization can cleanly provide cyanated products with high selectivity, yet the employed nucleophile needs to be strong enough. Reactions of reagents with a highly polarized nitrile group can lead to diminished yields of the desired product, due to side reactions, hence reduced selectivity. Compared to other fields that explore electrophilic group-transfer reagents and reactions, electrophilic cyanations still remain relatively scarce. Finally, it is worth noting that the extension to asymmetric transformations is only known for a hand full of examples. 


\section{ElECTROPHILIC AlKYNYLATION}

In the last Chapter the electrophilic transfer of the nitrile group was discussed. While from the first look, the transition to electrophilic alkynylation demonstrates definite similarity, the most obvious difference is the additional substitution on the opposite site of the acetylene functionality. The substitution pattern can play a vital role in the mode of reactivity and also allows tuning of it to a certain degree, as will be shown in this Chapter.

In general, the applied principles are the same as for Umpolung methodology transmutation from nucleophiles to electrophiles. In order to discover and provide access to new reaction pathways that expand the scope of acetylene transfer reactions, complementary to nucleophilic alkynylations, electrophilic alkynylation is used. Today, it already has become an established, growing field of research. Recently, it has experienced a resurgence due to its extended application in transition metal catalysis. The most prevalent methods for the Umpolung of alkynes are the use of halogenoalkynes 37, hypervalent alkynyliodonium salts $\mathbf{3 8}$, acetylene sulfones $\mathbf{3 9}$ and in situ oxidized terminal acetylenes $\mathbf{4 0}$ (Scheme 13-I).

(I) Halogens, hypervalent iodines, sulfones, oxidized terminal alkynes

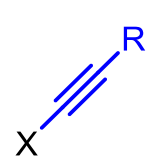

37

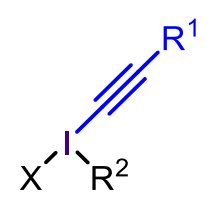

38

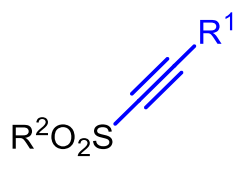

39

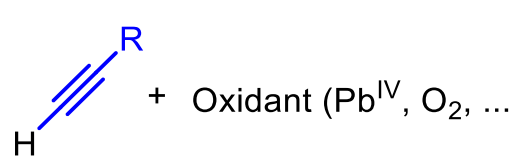

40

$\mathrm{X}=\mathrm{F}, \mathrm{Cl}, \mathrm{Br}, \mathrm{I} \quad \mathrm{X}=\mathrm{BF}_{4}, \mathrm{OTs}, \mathrm{OTf}, \ldots$

(II) Alcarazo, (2015-2019)

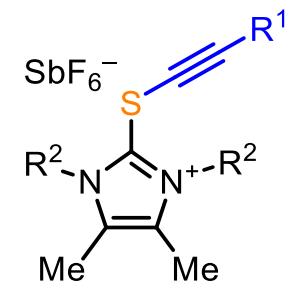

41

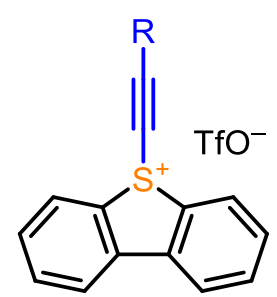

42

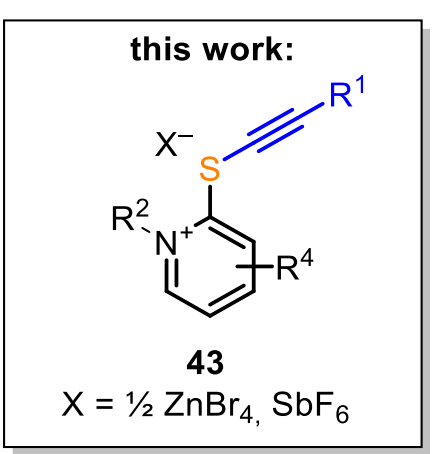

$\mathrm{X}=1 / 2 \mathrm{ZnBr}_{4}, \mathrm{SbF}_{6}$

Scheme 13: Evolution of electrophilic alkynylation reagents.

Evaluation of the different categories of reagents reveals the halogenoalkynes $\mathbf{3 7}$ to be relatively mild alkynylating reagents, when used under metal-free conditions, as the next three subchapters illustrate. In addiition, with the development of transition metal catalysis, the research field of haloalkyne functionalization in transition metal-mediated reactions has emerged as a much broader field. It enables access to novel products and alternatives to previously established reaction pathways (see Chapter "C-H FUNCTIONALIZATION UTILIZING ACTIVATED ACETYLENES"). 
On the other hand, similar to related cyano analogons, alkynyliodonium salts $\mathbf{3 8}$ are inherently more reactive than haloalkynes, due to their unusual 4-electron-3-centre bond. Their applications are covered extensively in literature, and the scope of transformations is broader than that of halogenoalkynes. ${ }^{[50,51,52]}$

Sulfone-substituted alkynes are preferably used in reactions that involve radical chemistry. Terminal alkynes can effectively be oxidized to act as electrophiles, formerly by strong oxidants such as lead(IV), and more recently by mediation with transition metal catalysts combined with external oxidants such as either dioxygen or electrochemical oxidation.

Recently the group of ALCARAZO was able to expand the range of reagents by successful synthesis and application of sulfur-based reagents. A variety of these reagents could be established as platform chemicals for electrophilic cyanation, ${ }^{[41,42,53]}$ alkynylation, ${ }^{[41,54-56]}$ thioalkynylation, ${ }^{[57]}$ alkylation and, in addition, as an aldothioketene mimetic reagent (Scheme 13-II). ${ }^{[55]}$ While the majority of the reported chemistry utilizing these reagents is metal- or at least transition metal-free, herein the expansion to transition metal catalyzed reactions is also presented (See Chapter "C-H FUnCTIONALIZATION UTILIZING THIOIMIDAZOLONE-BASED ELectrophilic TRansfer ReAgents").

In the next three subchapters examples for electrophilic alkynylation reactions of 37-42 with $\mathrm{N}_{-}, \mathrm{O}_{-}, \mathrm{S}_{-}, \mathrm{P}$ - and $\mathrm{C}$-nucleophiles will be covered. While reports for heteroatom-based nucleophiles, predominate, fewer examples are known for the still challenging C-nucleophiles.

Mechanistic proposals for the electrophilic alkynylation with hypervalent iodonium salts have been thoroughly inverstigated. They diverge, depending on a number of factors such as the kind of substrate, the class of hypervalent iodine compound (a differentiation between acyclic alkynyliodonium salts and cyclic ethynylbenziodoxol(on)es is necessary) and the nature of the R-substituent on the alkyne. As a result, different mechanisms have been proposed for the transformation of alkynyliodonium salts with $\mathrm{N}-, \mathrm{S}_{-}, \mathrm{O}$ - and $\mathrm{P}$-nucleophiles (see chapter next but one ' $P$-, $O-$, S-Nucleophiles' Scheme 17) and EBX, with either $S$ - or $C$-nucleophiles (Scheme 19 and Scheme 22 respectively). 


\section{N-NUCLEOPHILES}

Synthesis of ynamines and ynamides is feasible by utilizing alkynyl bromides in combination with copper catalysis, hypervalent iodonium salts or, very recently, sulfur-based reagents, as well as terminal alkynes and a copper salt and dioxygen as an external oxidant. 
(I) Stang (1994)<smiles>CCOC(C#CC(C)(C)C)(Oc1ccccc1)c1ccccc1</smiles>

(II) Hsung (2003)<smiles>[R]#[B-]</smiles><smiles>[R]NC([R])=O</smiles>

46

(III) Danheiser \& Dunetz (2003)

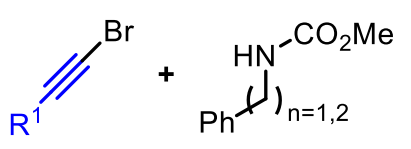

(IV) Stahl (2008)<smiles>[R][CH][CH]C#[R][H]</smiles>

$\mathrm{R}^{1}=\mathrm{Ph}$, anisyl, $n$-hexyl,TIPS, TBSO
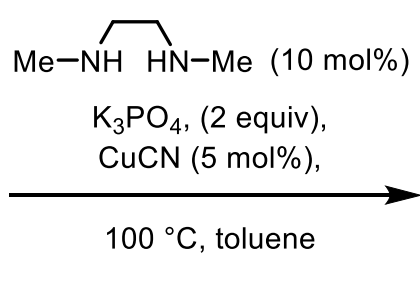<smiles>[R][X]C(=O)N([R])C#[R]</smiles>

$\mathrm{X}=\mathrm{O}, \mathrm{C}, \mathrm{N}$

$\mathrm{R}^{3}=$ aryl, alkyl, $\mathrm{SiR}_{3}$<smiles></smiles>

EWG $\frac{=}{45} N_{\mathrm{Ph}}^{P h}$

main motive

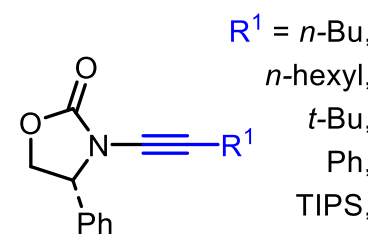

$\mathrm{R}^{1}=\mathrm{Ph}$,

TMS,

TIPS,

$n$-hexyl

$N$-nucleophiles:<smiles>[R]c1ccc(S(=O)(=O)NN(C)O[Na])cc1</smiles><smiles></smiles>

$47 \mathrm{~b}, \mathrm{R}^{1}=$ TIPS

(VI) Zhao (2018)<smiles>[R]/C(Cl)=C/Cl</smiles>

48, $\mathrm{R}^{1}=\mathrm{H}, \mathrm{Ph}$

(VII) Ghosh (2019)

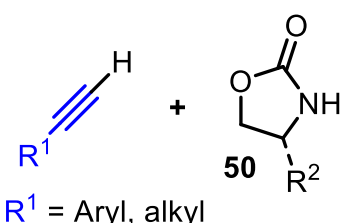

$\mathrm{R}^{1}=$ Aryl, alkyl<smiles>[R]C#CN([R])[R]</smiles>

$\vdots$<smiles>CN1CCNC1=O</smiles>

$N$-nucleophiles:

O

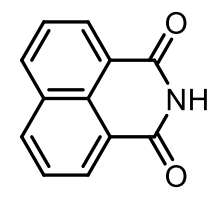

$N$-nucleophiles:

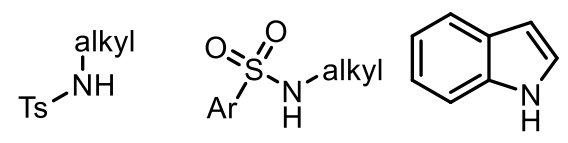<smiles>c1ccc2c(c1)[nH]c1ccccc12</smiles><smiles>O=C1NC(c2ccccc2)CO1</smiles>

$$
\mathrm{Cu}-\mathrm{MnO}(5 \mathrm{~mol} \%)
$$

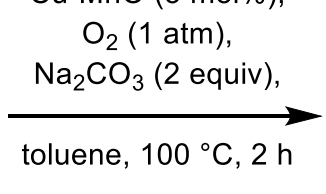<smiles>[R]C#CN1C(=O)OCC1[R]</smiles>

Scheme 14: Alkynylation of $N$-nucleophiles. ${ }^{[54,58-63]}$

Pioneering work was accomplished by STANG et al. (Scheme 14-I) by employing alkynyliodinoum salts $\mathbf{4 4}$ in the successful synthesis of push-pull ynamides $\mathbf{4 5}$; however, the 
reaction was limited to EWG-substituted alkynes and diphenylamine as a substrate. ${ }^{[58]}$ Two years later the methodology could be extended to ynamides by FELDMANN et al. ${ }^{[64]}$

FELDMAN and later WITULSKI, also proposed two mechanistic scenarios, based on migration abilities of substituents. Substitution with $\mathrm{Si}, \mathrm{H}$ or Ph gives acetylenes (Scheme 15-I), while the alkyl-substituted reagents prefer an insertion pathway (Scheme 15-II). ${ }^{[64,65]}$

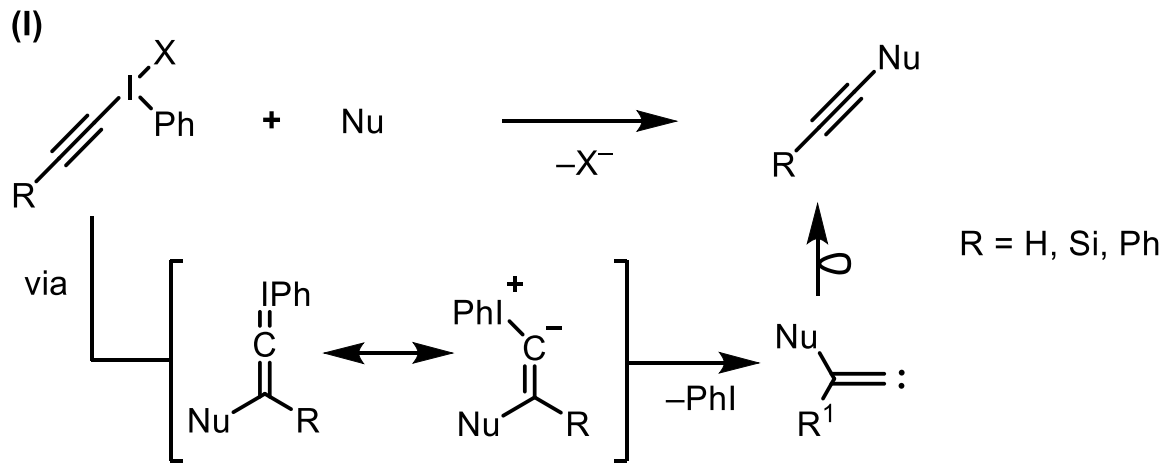

(II)

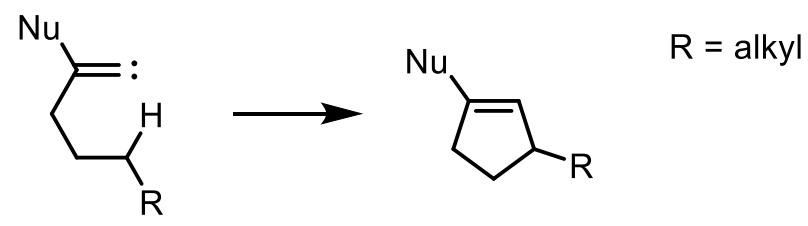

Scheme 15: Proposed mechanism for the nucleophilic attack on electrophilic alkynyl iodonium salts.

Similar observations were made for sulfonium reagents developed by WALDECKER \& ALCARAZO (Scheme 14-V). ${ }^{[54]}$

A protocol for electron-poor amines $\mathbf{4 6}$ in combination with halogenoalkynes under copper catalysis was described by HsuNG et al. (Scheme 14-II). ${ }^{[59]}$ They discovered that by replacement of iodoacetylenes with bromoacetylenes under their reaction conditions, homocoupling of the acetylene could successfully be diminished. While their work poses a breakthrough for the first successful employment of alkyl substituted acetylenes, further investigations showed the protocol to be less efficient when acyclic carbamates or sulfonamides were used.

An alternative approach to reduce the homodimerization of halogenoalkynes in their copper-mediated reaction, consisting in the initial generation of stoichiometric amounts of a copper-amine species and then adding two equivalents of bromoacetylenes, was selected by DUNETZ and DANHEISER (Scheme 14-III). ${ }^{[60]}$ In addition, this methodology allowed the reaction to proceed at room temperature.

The aforementioned direct oxidation of terminal alkynes was described by STAHL et al. (Scheme 14-IV) for the optimized catalytic system utilizing one atmosphere of dioxygen to regenerate the copper catalyst. The reaction operates well with a broad scope of amides as well as acetylenes. ${ }^{[61]}$ 
Circumventing any potential problems of homodimerization, ALCARAZO et al. (Scheme 14-V) have found that newly designed hypervalent benzothiophenium salts $47 \mathrm{a}$ and $47 \mathrm{~b}$ allow alkynylation of sulfonamides and diamides. Additional investigations towards the mode of reactivity using the reagent with a ${ }^{13} \mathrm{C}$-labeled acetylene moiety gave insight into the mechanism. ${ }^{[54]}$

Another metal-free approach was reported bei ZHAO et al. (Scheme 14-VI), who used (Z)-1,2dichloroalkenes $\mathbf{4 8}$ or alkynyl chlorides in combination with electron poor amines to elaborate an access to either terminal or internal ynamides $49 .{ }^{[62]}$

Focusing on atom economy, the group of GHOSH (Scheme 14-VII) reported on the heterogeneous $\mathrm{Cu}-\mathrm{MnO}$ catalyst for direct oxidation of terminal alkynes using air as the sole oxidant. While they observed concurrent homodimerization, kinetic studies revealed that the amount of Glaser-type coupling product could be reduced using an excess of nucleophile 50. They could also show that recycling of the catalyst was possible up to four times. ${ }^{[63]}$

Very recently, an access to highly electrophilic alkyne-pyridine conjugates, $N$ alkynylpyridinium salts $\mathbf{5 2}$, by utilizing alkynyl- $\lambda^{3}$-iodanes $\mathbf{5 1}$ has been reported by UCHIYAMA et al. (Scheme 16). ${ }^{[66]}$

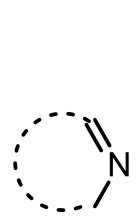

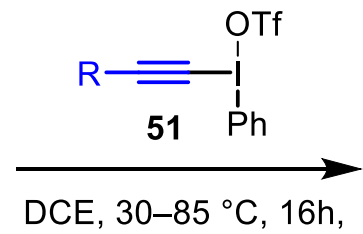

$50-99 \%$

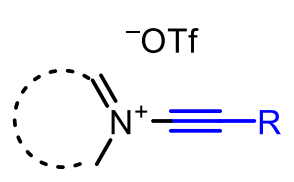

52, $\mathrm{R}=\mathrm{TMS}, \mathrm{H}$

Scheme 16: Access to $N$-alkynylpyridinium salts $\mathbf{5 2}$ by alkynylation with alkynyl- $\lambda^{3}$-iodanes $\mathbf{5 1 .}{ }^{[66]}$

The synthesized alkynylammonium salts $\mathbf{5 2}$ exhibit a high electron-accepting character with extended $\pi$-conjugation and were remarkable precursors for the synthesis of quinolizinium salts with unique optical and electrochemical properties. 
In contrast to $\mathrm{N}$-nucleophiles, reports of the transformation of $\mathrm{P}_{-}, \mathrm{O}$ - and $\mathrm{S}$-nucleophiles with electrophilic alkynes are far less prevalent.

(I) Stang (1985-1989)

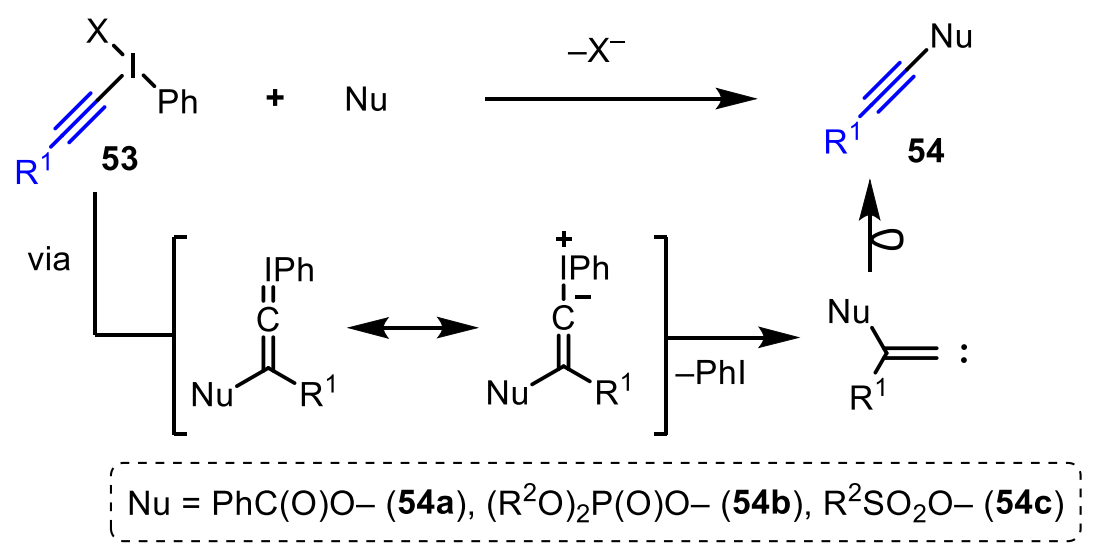

(II) a) Ochiai (1987)

b) Stang (1992)

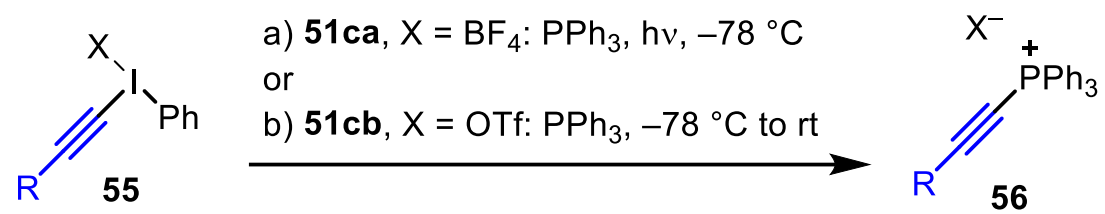

(III) Zhao, Han (2009)

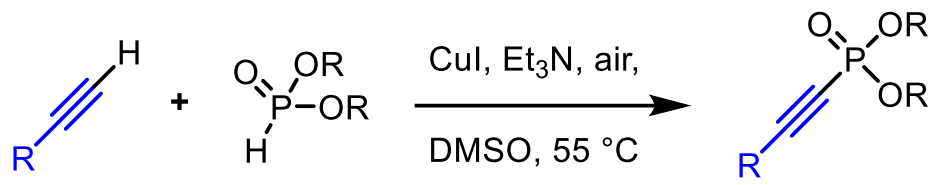

(IV) Gaumont (2011)

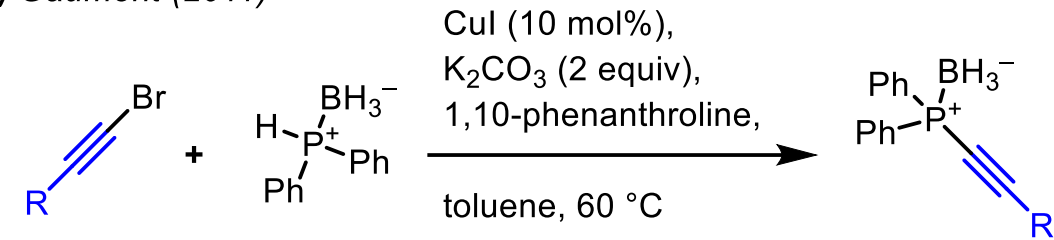

$\mathrm{R}=$ alkyl, aryl, silyl

(V) Alcarazo (2018)
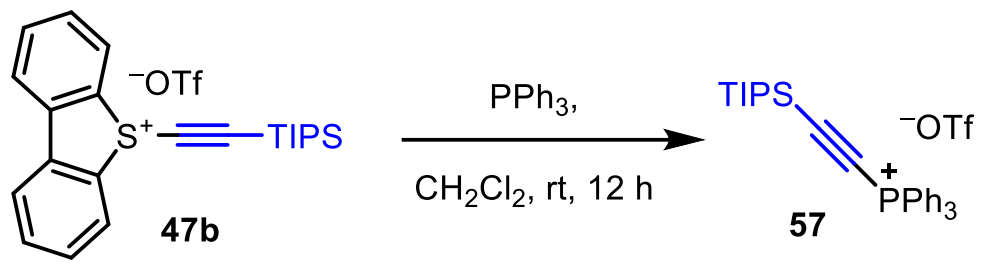

Scheme 17: Alkynylation of $O$ - and $P$-nucleophiles. 
In a number of publications, STANG (Scheme 17-I) reported on access to alkynylcarboxylates 54a, alkynyldialkylphosphates 54b and alkynylsulfonates 54c with utilization of alkynyliodonium salts $\mathbf{5 3}$ following by a Michael addition/1,2-shift sequence, albeit requiring different reaction conditions: Alkynyl sulfonates $54 \mathrm{c}$ can be obtained from alkynyl(phenyl)iodonium mesylates or tosylates under catalysis with 5-10 mol\% CuOTf or AgOTs; alkynyl carboxylates $54 \mathrm{a}$ - by either reaction of $\mathrm{Phl}\left(\mathrm{O}_{2} \mathrm{Ph}\right)_{2}$ with acetylide ions or, vice versa, of alkynyl(phenyl)iodonium triflates with $\mathrm{C}_{6} \mathrm{H}_{5} \mathrm{CO}_{2} \mathrm{Na}$. Decomposition of alkynyl(phenyl)iodinium dialkylphosphates gave alkynyl dialkylphosphate esters 54b. ${ }^{[67]}$

The mechanism proposed by STANG is in agreement with the mechanistic scenarios described for $N$-nucleophiles by FELDMANN and WITULSKI in the last Chapter.

At the same time OCHIAl et al. (Scheme 17-II) reported the synthesis of alkylethynyl triphenylphosphoniums 56, from alkynyliodonium tetrafluoroborates $\mathbf{5 5}$ and triphenylphosphines, in which the presence of light was necessary. Alternatively, the preparation of alkynyliodonium triflate $\mathbf{5 1} \mathbf{c b}$ reported by STANG et al. (Scheme 17-II) did not require light irradiation. ${ }^{[68]}$

In 2009 ZHAO, HAN et al. (Scheme 17-III) published a protocol for the oxidative coupling of terminal acetylenes and $\mathrm{H}$-phosphonates in analogy to STANG'S protocol, featuring a broad scope and large functional group tolerance. ${ }^{[69]}$

Representing the only publication on reactivity of halogenoalkynes whithin this area, GAUMONT et al. (Scheme 17-IV) utilized phosphine-boranes to couple with bromoalkynes to give access to alkynylphosphines. The use of phosphine-boranes was found to be necessary to circumvent catalyst poisoning by free phosphine. ${ }^{[70]}$

AlCARAZO et al. (Scheme 17-V) have demonstrated their novel dibenzothiophene-based electrophilic alkynylation reagent $\mathbf{4 7 b}$ to be able to convert triphenylphosphine into the corresponding alkynylphosphonium salt $\mathbf{5 7}$ under unprecedented mild conditions, cleanly and in excellent yield. ${ }^{[54]}$ 


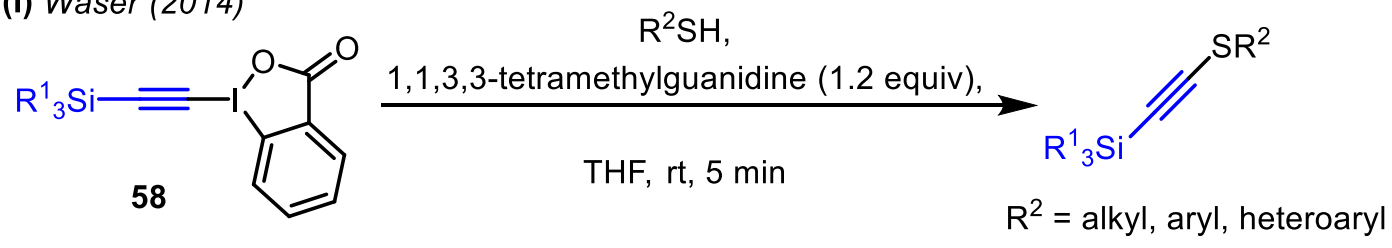

(II) Alcarazo (2015)

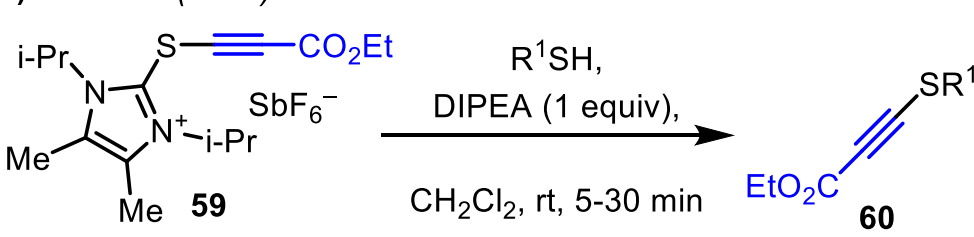

(III) Witt (2016)<smiles>[R]#C[AlH2]</smiles>
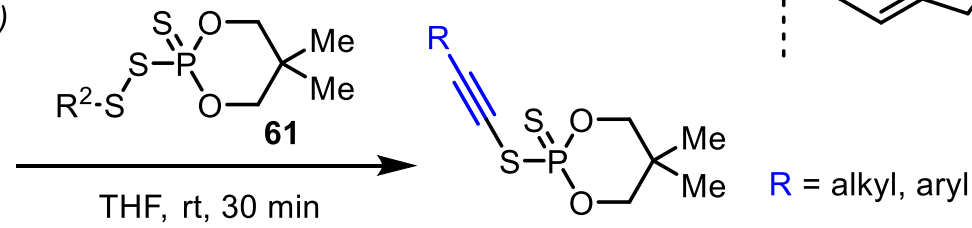

(IV) Alcarazo (2017)

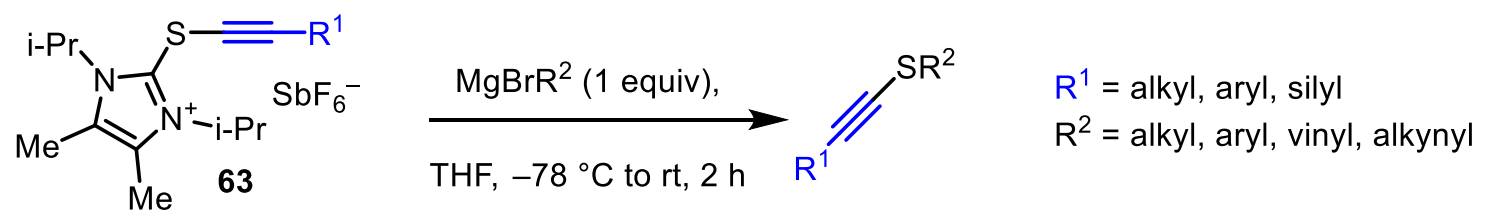

(V) Collins (2017)

\section{CzIPN (2 mol\%),}

$$
\mathrm{NiCl}_{2} \bullet \text { dme }(10 \mathrm{~mol} \%) \text {, }
$$

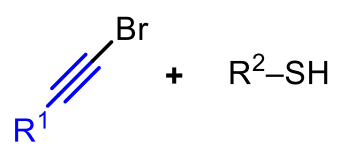

$$
\text { pyridine ( } 2 \text { equiv) }
$$

MeCN/DMF (23:)(0.05 M), $\quad R^{1}$<smiles>[R]C#[R]</smiles>

$\mathrm{R}^{1}=$ alkyl, aryl,

$\mathrm{R}^{2}=$ aryl

$t_{r}=30 \mathrm{~min}(433 \mu \mathrm{L} / \mathrm{min})$,

(VI) Zhao (2018)

$$
\text { rt, blue LED }
$$

\section{selected examples for $R^{1}$ :}

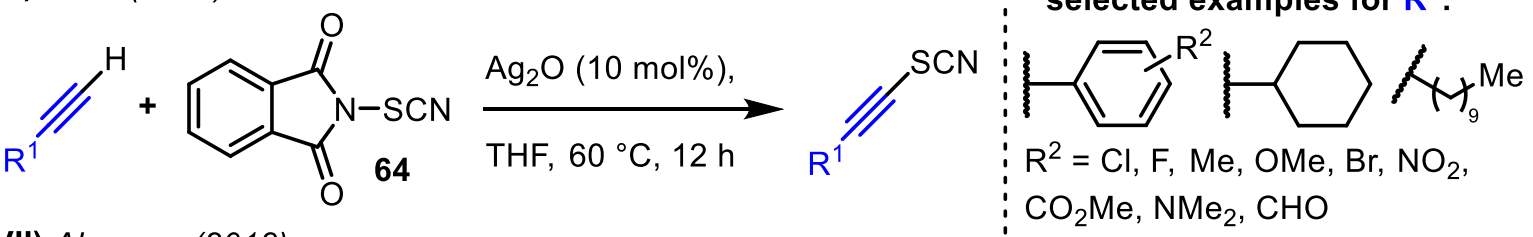

(VII) Alcarazo (2018)

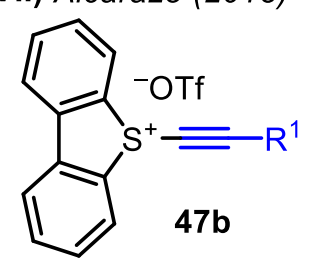

(VIII) Hosoya (2019)

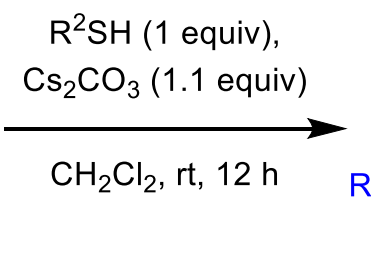

Cul $(5 \mathrm{~mol} \%)$,<smiles>[R]C#C[Hg]S[As]</smiles>

Xantphos (6 mol\%),

$\mathrm{K}_{2} \mathrm{CO}_{3}$ (1.5 equiv)<smiles>[R]C#SC</smiles>

$\mathrm{R}=$ alkyl, aryl

DMSO, rt, $24 \mathrm{~h}$

Scheme 18: Synthesis of alkynyl sulfides.

In $2014 \mathrm{~J}$. WASER and RETO (Scheme 18-I) published an unprecedented alkynylation of thiols utilizing [(triisopropylsilyl)-ethynyl] $\underline{b}$ enziodoxolone (TIPS-EBX, 58) and its other silyl 
derivatives, the latter being hypervalent iodine-based alkyne transfer reagents. ${ }^{[50]}$ The conversion of thiols takes place within five minutes at room temperature in an open flask using commercially available reagents and could be scaled up to gram levels. They further underlined the use of the thiol-alkynylation by postsynthetic modification of a cysteinecontaining peptide via a standard CUAAC reaction introducing a dansyl fluorophore tag. ${ }^{[71]}$

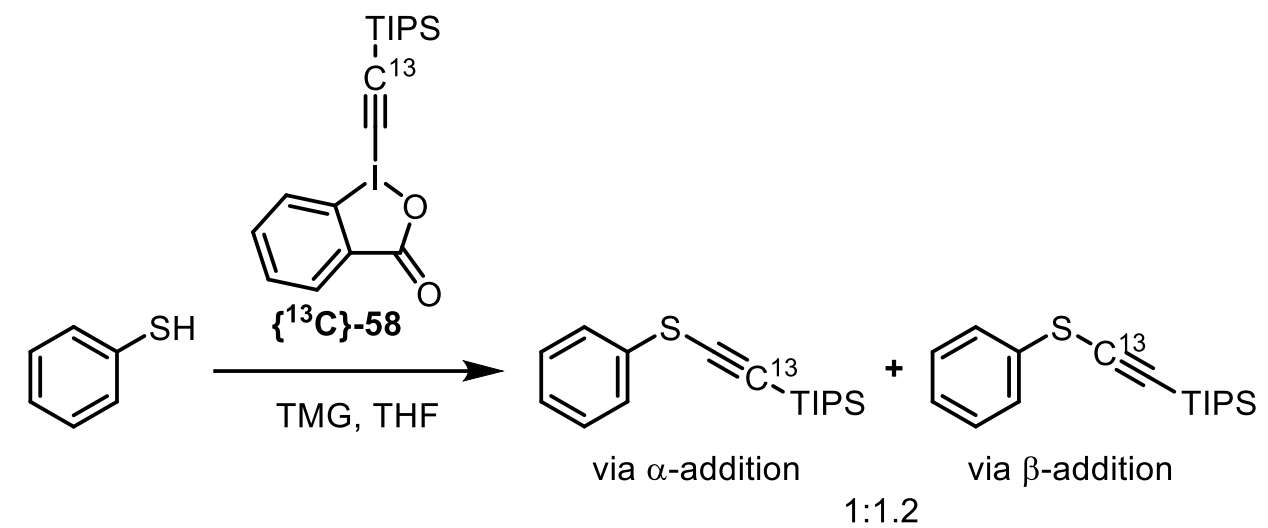

(more favored for EBX-reagents with EWG) (more favored for alkyl EBX-reagents)<smiles>O=C1O[IH](=C2[In]NC2=S)c2ccccc21</smiles>

$10.1 \mathrm{kcal} / \mathrm{mol}$ M. Wodrich (2014)

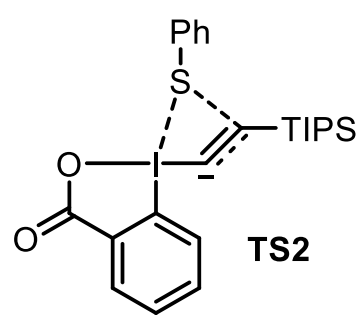

$9.3 \mathrm{kcal} / \mathrm{mol}$ M. Wodrich (2015)

refined transition states

Scheme 19: Mechanistic investigations by J. WASER et al. ${ }^{[72,73]}$

Mechanistic investigations of J. WASER in cooperation with WODRICH revealed, that two scenarios, a three atom concerted transition state TS1, in which C-S bond formation takes place on the $\alpha$-carbon versus a four atom concerted transition state TS2, in which attack of the sulfur takes place on the $\beta$-carbon, are within a very small energy range for silyl-EBX reagents. This was in agreement with experiments conducted with ${ }^{13} \mathrm{C}$-labeled TIPS-EBX $\left\{{ }^{13} \mathrm{C}\right\}$-58. Furthermore calculations showed that for EBX-reagents with EWGs, TS1 is favoured, while for alkyl substituted EBX reagents, TS2 is preferred (Scheme 19). ${ }^{[2,73]}$

In 2015, Alcarazo et al. revealed that their EWG-substituted thioimidazolioum-based acetylene transfer reagent $\mathbf{5 9}$ was able to react with thiols in a straightforward fashion to give thioacetylenes 60 (Scheme 18-II). ${ }^{[41]}$

Development of a thiation agent $\mathbf{6 1}$, structurally reminiscent of Lawesson's reagent, was reported by WITT et al. (Scheme 18-III) in 2016. [74] Functionalized unsymmetrical alkynyl sulfides 62 including hydroxy-, carboxy- or amino-substituted derivatives were obtained after reaction of the reagent $\mathbf{6 1}$ with lithium acetylides. 
Two years after their initial pulication about sulfur based electrophilic transfer reagents, ALCARAZO et al. could expand the scope of thioacetylenes reported in previously published work. $^{[41,75]}$ Synthesis of a wide range of electrophilic acetylenes $\mathbf{6 3}$ based on thioimidazolium was reported, and their successful transformation with alkyl-, aryl, vinyl- or alkynylmagnesium Grignards as well as with zinc organyls were described (Scheme 18-IV). ${ }^{\text {75] }}$ Notably, analogous reactivity was observed when imidazolium selenoalkynes were employed in the transformation.

Distinguished by its practical application, the photochemical dual-catalytic cross-coupling to form alkynyl sulfides via C(sp)-S bond formation was described by ColuINS (Scheme 18-V) in 2017. Combining a soluble organic carbazole-based photocatalyst with continuous flow techniques, the synthesis of alkynyl sulfides bearing electronically or sterically diverse aromatic alkynes and thiols could be achieved. In addition, the continuous flow setup also allows for short reaction times $\left(30 \mathrm{~min}\right.$ ) and reproducibility on gram scale. ${ }^{[76]}$

A one-pot protocol for thiocyanation of terminal alkynes with $\mathrm{N}$-thiocyanophthalimide (64) and employing silver(I) oxide as an oxidant was developed by ZHAO et al. (Scheme 18-VI). ${ }^{[77]}$ Noteworthy, when a silver/gold relay catalysis was used, access to $\alpha$-thiocyanoketones was also possible. Derivatization into a variety of valuable sulfur-containing heterocycles and sulfides could be demonstrated as well.

S-(Alkynyl)dibenzothiophenium triflate $47 \mathrm{~b}$ developed by ALCARAzo et al. also proved to be able to transform a number of $S$-nucleophiles, including aromatic and aliphatic thiols, into the corresponding thioacetylenes (Scheme 18-VII). ${ }^{[54]}$ Functional groups such as esters, carbonyls and nitro moieties were well tolerated.

Another example for the oxidation of terminal alkynes with utilization of copper catalysis and thiosulfonates as the source of sulfur was presented by Hosora (Scheme 18-VIII). In addition, subsequent iodocyclization could be used to access benzofuranes, benzothiophenes and indols. ${ }^{[78]}$ 


\section{C-NUCLEOPHILES}

(I) Beringer (1965)

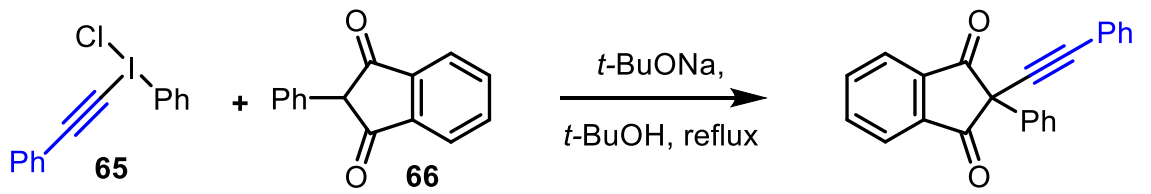

(II) Kende (1984)

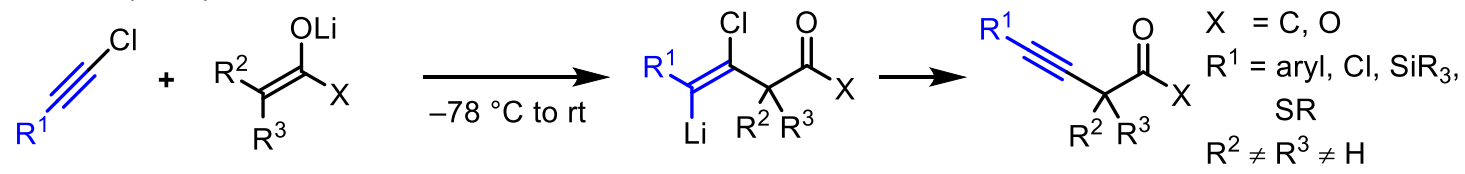

(III) Ochiai (1986)

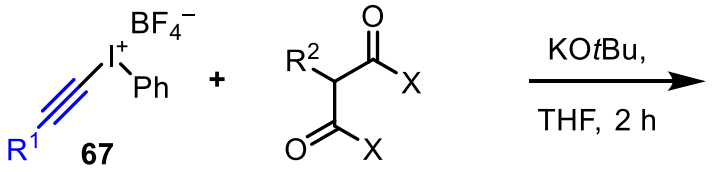<smiles>[R]C#CC([R])(C([R])=O)C([X])([R])C#[R]</smiles><smiles>[X]C(=O)C([R])(C([X])=O)C1=CC(C)CC1</smiles>

(IV) Stang (1991)

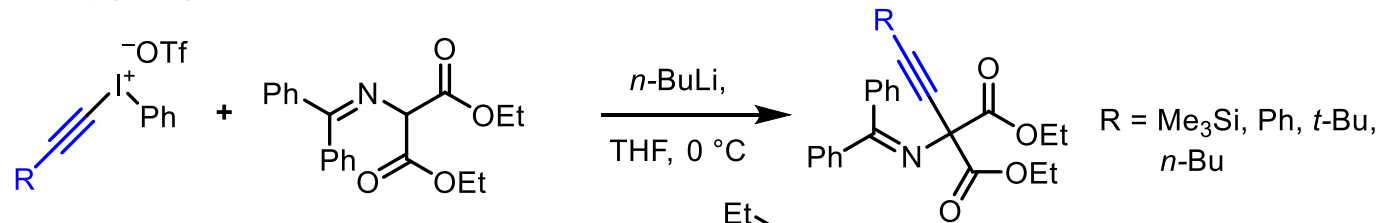

(V) Jørgensen (2007)<smiles>[X]C#COC#CCC(C)(C)OC1CCC(=O)CC1</smiles>

(VI) Waser (2010)

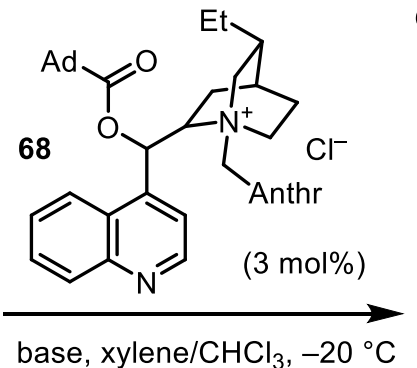<smiles></smiles>

up to $98 \%$ ee

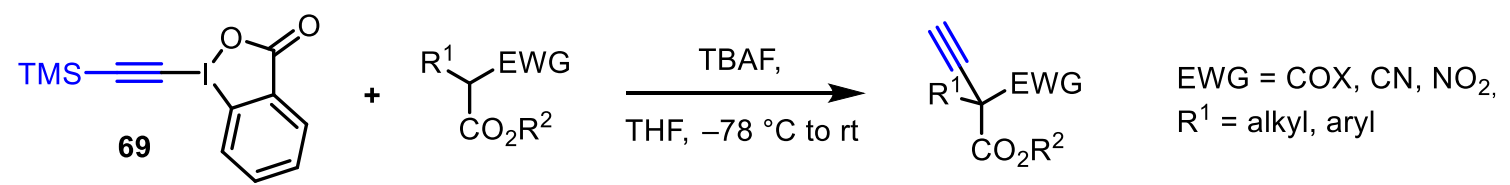

(VII) Alcarazo (2015, 2018)<smiles>CC#CSc1n(C(C)C)c(C)c(C)[n+]1C(C)C</smiles>

$+$<smiles>CCOC(=O)C1Cc2ccccc2C1=O</smiles>
DIPEA (1 equiv), 70 $\mathrm{CH}_{2} \mathrm{Cl}_{2}$, rt, $3 \mathrm{~h}$<smiles>[R]C#[S-]1c2ccccc2-c2ccccc21</smiles>

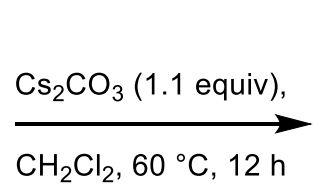<smiles>CCOC(=O)C#CC1(C(=O)OCC)Cc2ccccc2C1=O</smiles><smiles>[R]C#CC1(C(=O)OC)Cc2ccccc2C1=O</smiles>

Scheme 20: Alkynylation of C-Nucleophiles.

Laying the foundation for all research towards hypervalent iodonium salts was accomplished by BERINGER Scheme 20-I) in 1965. Successful synthesis of the acyclic chloride 65 form and 
investigation of its reactivity towards indandione 66 was performed. ${ }^{[18]}$ Even then, weak enolates were identified as good candidates for $C$-nucleophiles.

It took almost 20 years until KENDE et al. in 1984 reported the reaction of chloroalkynes, with lithiumenolates, generated by addition of LDA, from ketones or esters (Scheme 20-II). ${ }^{[79]}$ The reaction proved to tolerate chloro-, silicon-, sulfur- and aryl-substituted alkynes, but the methodology reached its limits when aliphatic alkynes were used. An explanation is given by the mechanism: The addition-elimination mechanism involves a transient $\beta$-carbanion which experiences mild stabilization, if the adjacent substituent is of aromatic nature. This mild stabilization is absent in the case of aliphatic substituents. This rationale could be further consolidated, by showing that the use of 1-bromo-1-hexyne results exclusively in the bromination, without formation of the desired product. Furthermore, only tertiary enolates underwent the reaction thus forming quarternary centres. Primary and secondary enolates gave unidentified products with only one exception: Reaction of diethylmalonate $\mathbf{7 2}$ with dichloroacetylene $\mathbf{7 3}$ in dry ether gave compound 75, which was characterized by the authors as "Ott diadduct". Most probably, product 75, resulted from the second Michael addition of another equivalent of diethylmalonate onto the chloroallene intermediate $\mathbf{7 4}$ (Scheme 21). ${ }^{[79]}$

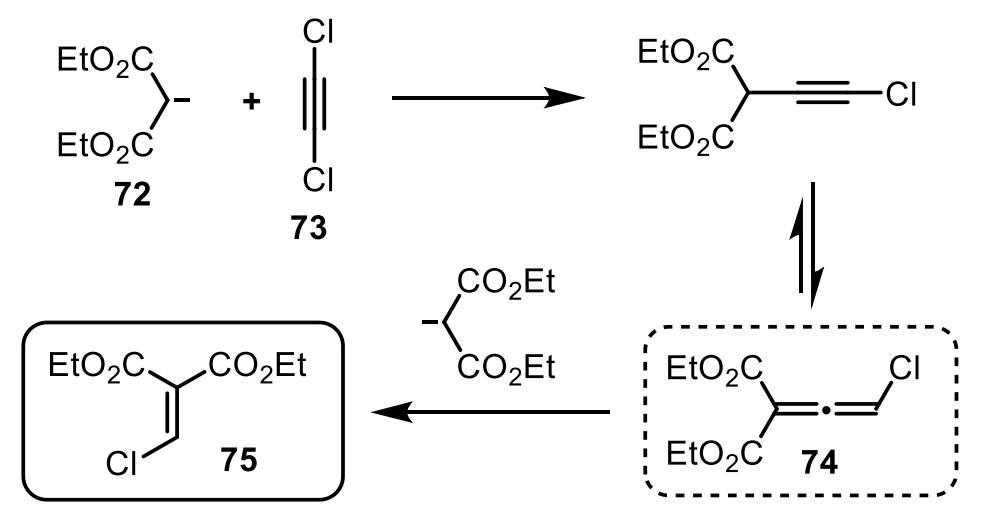

Scheme 21: Formation of „Ott diadduct“ 75 via a chloroallene intermediate 74.

Two years later OCHIAl et al. synthesized an ethynyl(pheny)iodonium salt 67 and used the weakly coordinating $\mathrm{BF}_{4}^{-}$as anion. This enabled them to subsequently modify acyclic diketones and realize tandem cyclopenten annulations (Scheme 20-III). ${ }^{[8]}$

A noteworthy variant of malonate functionalization is the alkynylation of diethyl 2aminomalonates which gives access to propargylic amines and in addition constitutes one of the few examples for which alkylsubstituted acetylenes could be employed (Scheme 20IV). ${ }^{[81]}$

While successfully applied in several additional publications, the methodology of alkynylations of activated enolates with hypervalent iodonium salts could not be further extended in the following 20 years. It was in 2007 when JØRGENSEN et al. reported the first and unique enantioselective procedure with ee values above $90 \%$. They used chloro- and 
bromoalkynes, which had to be additionally activated by electron-withdrawing groups such as ketones, esters, sulfones and amides to increase the electrophilicity of the triple bond. By employing the dihydrocinchonine-containing phase transfer catalyst $\mathbf{6 8}$, they were able to achieve an ee of up to $98 \%$ (Scheme $20-\mathbf{V}) .{ }^{[82]}$

Contributions of WASER et al. from 2010 showed that with TMS-EBX 69 it is also possible to alkynylate both cyclic and acyclic activated carbonyl compounds (Scheme 20-VI). ${ }^{[50]}$ Synthesis of a $\mathrm{C}^{13}$-labeled TIPS-EBX 76 enabled the authors to gain insight into the mechanism. Of the two possible pathways, on one hand conjugate addition to the alkyne, followed by reductive elimination and 1,2-shift leading to product $\mathbf{7 7}$ (pathway a) and, on the other hand, addition-elimination on the iodine atom (pathway $\mathbf{b}$ ), the former could be indentified as occurring (Scheme 22).

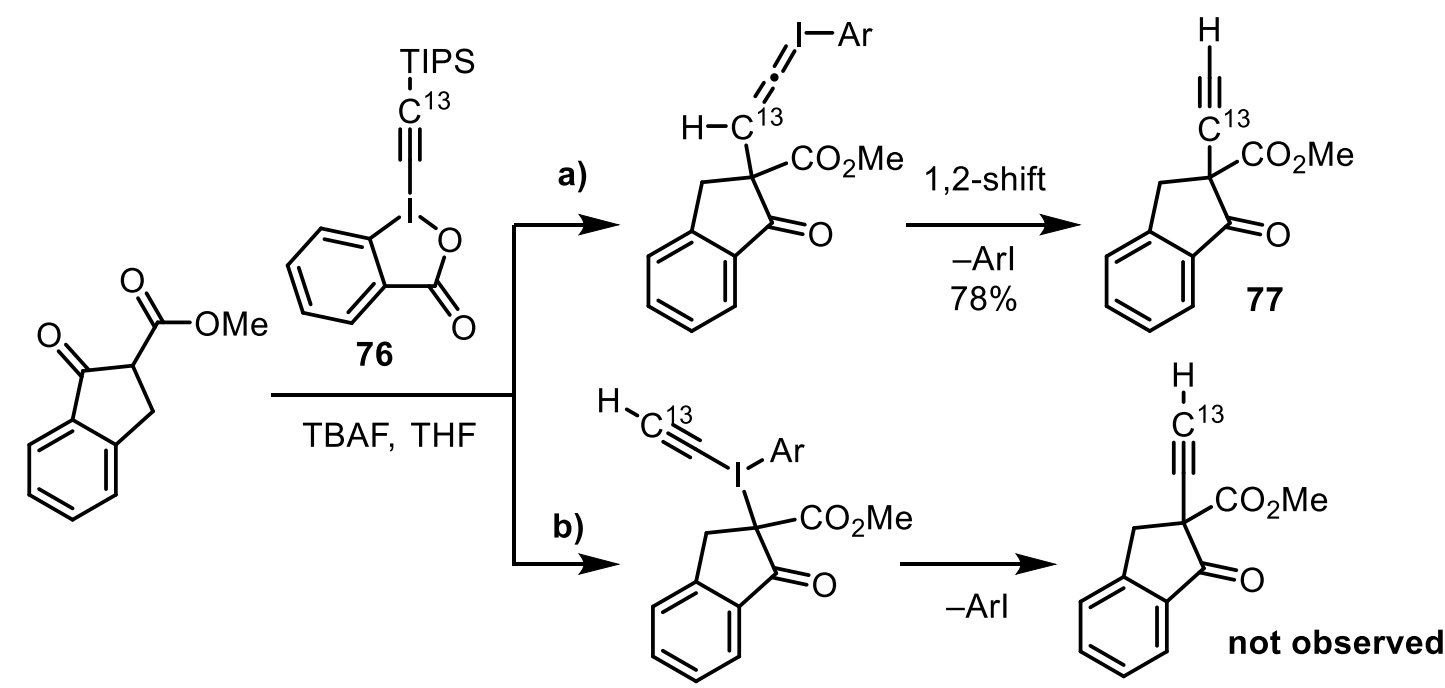

Scheme 22: Experiment with $\mathrm{C}^{13}$-labeled reagent 76 by J. WASER et al. to investigate possible mechanistic pathways.

Application of conditions similar to the JøRGENSEN protocol gave them an ee of up to $40 \%{ }^{\text {[50] }}$

The activation of acetylenes with electron-withdrawing groups was also exploited by AlCARAZO et al. for the synthesis of imidazolium thioalkyne 59. $\beta$-Ketoester 70 as well as activated amides could be alkynylated (Scheme 20-VII, top). ${ }^{[41]}$ Significant extension of the reaction scope could be achieved by replacing the imidazolium backbone with the dibenzothiophene one in $\mathbf{4 7}$. This enabled substitution of the reagents with aromatic and silyl groups on the acetylene moiety (Scheme 20-VII, bottom). ${ }^{[54]}$

As of today, the extension of electrophilic alkynylations for non-activated carbonyl compounds still poses a serious challenge to be overcome. Other frontiers are the successful application of aliphatic acetylenes: the latter, as indicated above, have only found limited application so far. In addition, the modification of non-tertiary propargylic positions also still requires further intensive studies. 


\section{DithIOESTER, THIOAMIDES AND THIONOESTERS}

The last Chapter covered the synthesis and reactivity of heteroatom substituted alkynes by nucleophilic attack of a heteroatom nucleophile on an electrophilic alkyne. This is a reasonable approach to access a multitude of products. In theory, an alternative approach would be the use of an ynol such as $\mathbf{7 8}$ or thioynol $\mathbf{8 1}$ (Scheme 23), as a nucleophile and react it with an electrophile. However, this "inverted case" is not feasible due to the fact, that these compounds (protonated or deprotonated) are not stable and equilibrate with their tautomeric form - ketenes 79 and thioketenes 82, respectively. Moreover, the equilibrium is completely shifted to the side of the carbonyl species. ${ }^{[67]}$

(l)

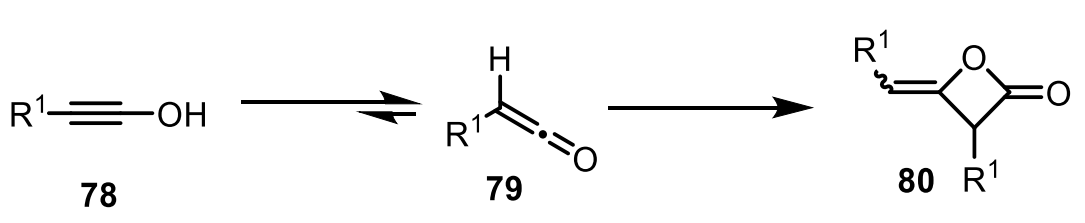

(II)

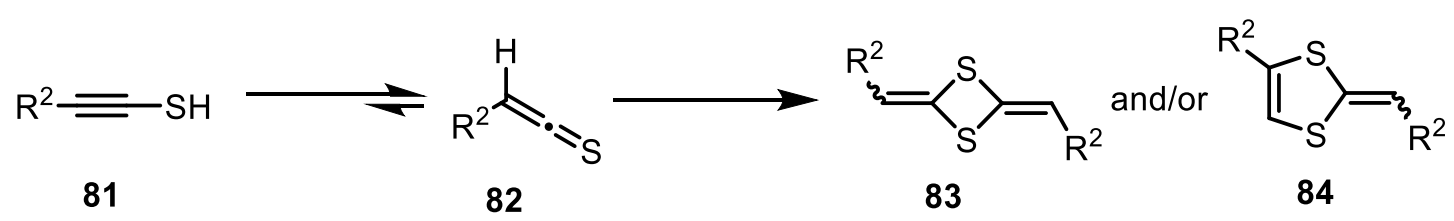

Scheme 23: Ynol-ketene equilibrium and dimerization to diketene 164 (I) and thioynol-thioketene equilibrium and dimerization to dithioketene $\mathbf{1 6 7}$ or dithiafulvenes 168 (II).

Fatefully, both aldoketenes and aldothioketenes are notoriously unstable themselves and dimerize to their corresponding diketene $\mathbf{8 0}$ /dithioketene $\mathbf{8 3}$ or to dithiafulvenes $\mathbf{8 4}$. On the other hand, for disubstituted (thio)ketenes several stable derivatives exist: RAASCH reported on the successful synthesis of bis(trifluoromethyl)thioketene 87 in 1966 and applications thereof between 1970 and 1977 (Scheme 24). ${ }^{[83,84]}$ Alongside diverse cycloadditions, he could show that thioketenes react with alcohols, thiols and amines to form thionoesters, dithioesters and thioamides respectively (see below, Scheme 29). ${ }^{[85]}$<smiles>CCOC(=O)CC(=O)OCC</smiles>

85

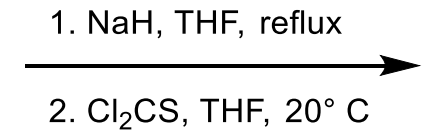

2. $\mathrm{Cl}_{2} \mathrm{CS}, \mathrm{THF}, 20^{\circ} \mathrm{C}$<smiles>CCOCC(COCC)=c1sc(=C(COCC)C(=O)OCC)s1</smiles>

$\mathrm{CO}_{2} \mathrm{Et}$

$\mathrm{HF}$ (3.3 equiv.), $\mathrm{SF}_{4}\left(7.5\right.$ equiv), $125-200^{\circ} \mathrm{C}, 4 \mathrm{~h}$

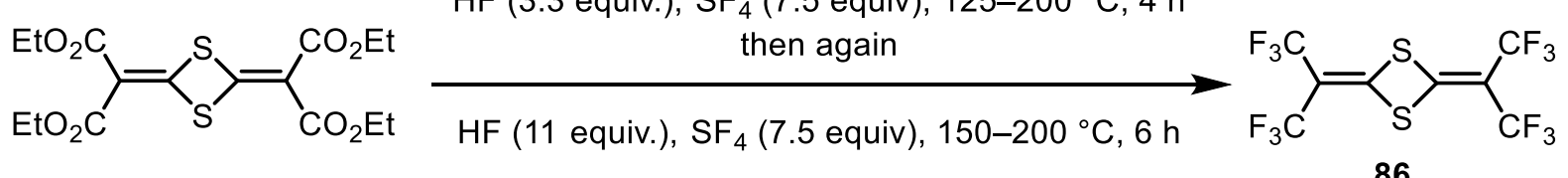

pyrolysis

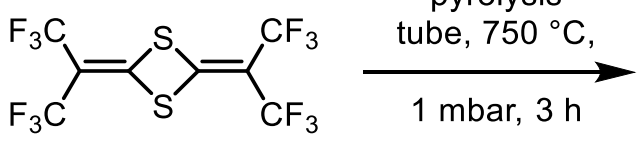

86<smiles>FC(F)(F)C(=C=S)C(F)(F)F</smiles>

87

Scheme 24: Synthesis of bis(trifluoromethyl)thioketene 87 by RAASCH. ${ }^{[83]}$ 
The tedious preparation of monomeric bis(trifluoromethyl)thioketene $\mathbf{8 7}$ requires cracking of its dimer $\mathbf{8 6}$, which is synthesized from diethylmalonate $\mathbf{8 5}$, thiophosgene and sulfur tetrafluoride (Scheme 24). After pyrolysis at $650-750{ }^{\circ} \mathrm{C}$, the monomer can be isolated as a reddish orange liquid, which is then surprisingly stable at $25^{\circ} \mathrm{C}$ in glass equipment for several months with only little dimerization.

Around the same time BRANNOCK et al. ${ }^{[86]}$ successfully synthesized di-tert-butylthioketene 89 (Scheme 24) in analogy to the synthetic route, that provides access to di-tert-butylketene $\mathbf{8 8}$, as reported by NEWMAN et al. ${ }^{[87]}$

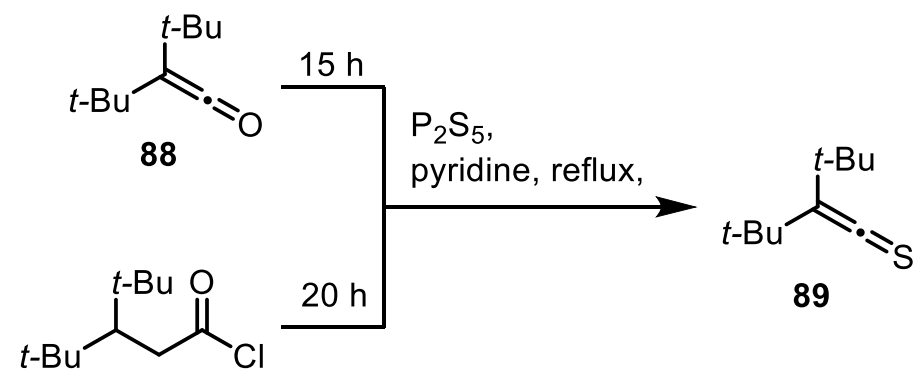

Scheme 25: Synthesis of di-tert-butylthioketene 89 by BRANNOCK et al.. ${ }^{[86]}$

While the sterical shielding of the tert-butyl groups, on the one hand, makes the compound stable, on the other hand, its reactivity also decreases for the same reason. Nevertheless, reactions with small nucleophiles were documented and are covered in the following Section. Overall the number of stable thioketenes remains scarce even today.

A viable alternative approach to facilitate transformations with thioketenes is to use thioketene equivalents, from which the reactive species are formed in situ. One of the most prominent examples which in fact exploits the aforementioned equilibrium in the described above way (Scheme 23-II), is presented by SCHAUMANN, who utilized of alkynyl silyl sulfides as thioketene equivalents. ${ }^{[88]}$

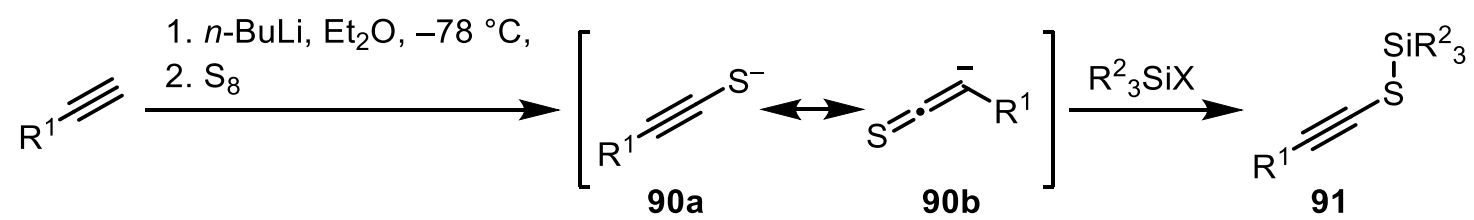

Scheme 26: Synthesis of silylated alkynylsulfides 181 by Schaumann et al.. ${ }^{[88]}$

Starting from terminal alkynes, deprotonation with an alkali metal base was carried out. The formed acetylide was also capable of breaking up the $S_{8}$ ring structure, thus affording the intermediate alkynethiolates 90. Trapping of the latter with the corresponding organosilylhalide via S-silylation gives alkynyl silyl sulfides $\mathbf{9 1}$. These represent a more stable analogon of ynethiols with regard to their reactivity. 


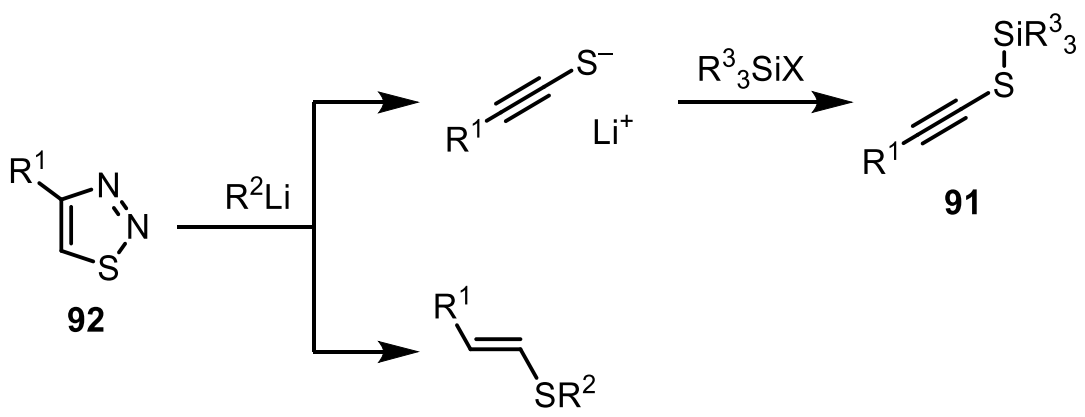

Scheme 27: Base-induced cycloreversion of 5-unsubstituted 1,2,3-thiadiazoles 92.

SCHAUMANN et al. also compared the procedure they developed for the synthesis of silylated alkynylsulfides with the known protocol that utilizes 1,2,3-thiadiazoles 92 (Scheme 27). ${ }^{[89]}$ In fact, these compounds are also used as thioketene equivalents. When 1,2,3-thiadiazoles 93 are activated either by heat or light, they give, what can best be described as thioacyl carbene, 1.3-dipole, or 1,3-diradical. Eventually either of these species rearranges to form thioketenes 94 (Scheme 28).

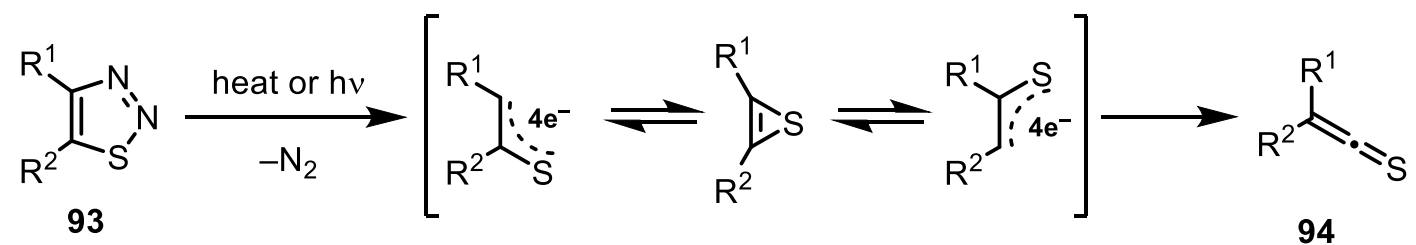

Scheme 28: Formation of thioketene 94 starting from 1,2,3-thiadiazoles 93. 
The in the Section above described thioketenes and thioketene equivalents can serve as materials for the synthesis of thiocarbonyl compounds:

(I) Brannock (1968)

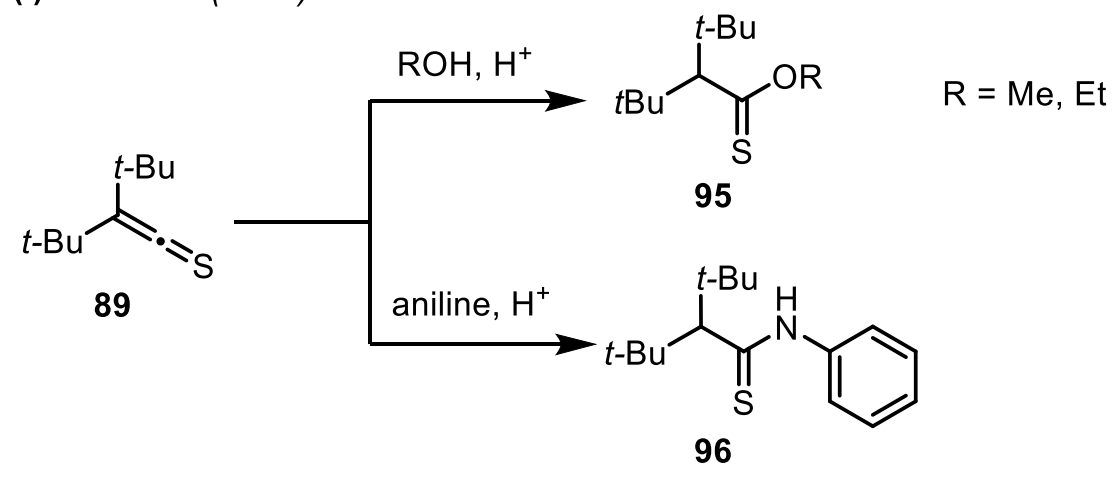

(II) Raasch (1972)

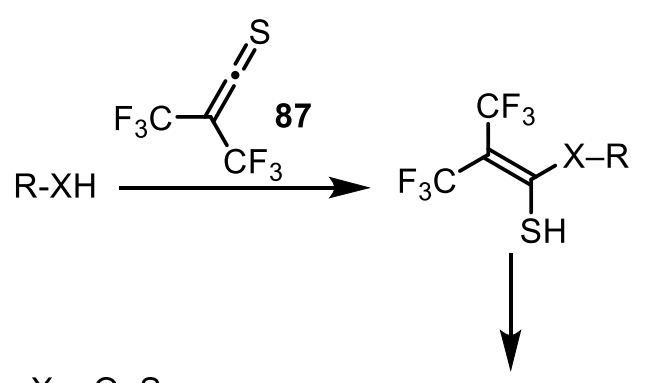

$\mathrm{X}=\mathrm{O}, \mathrm{S}$,

$\mathrm{N}, \mathrm{Se}$

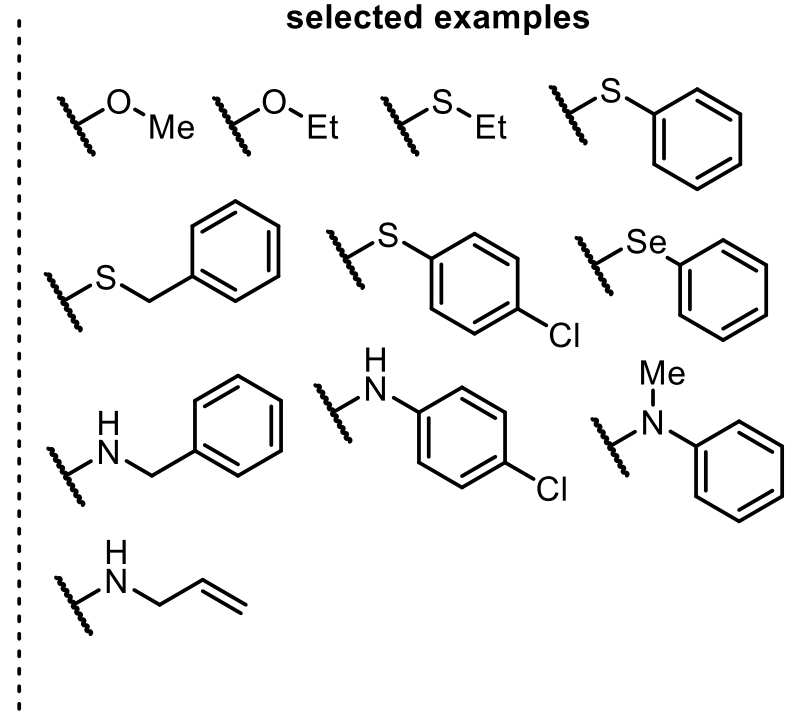

(III) Schaumann (2014)

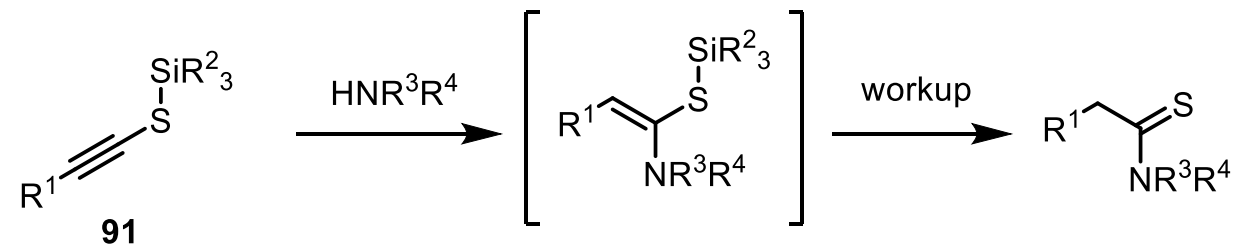

Scheme 29: Access to thioacylation products by utilization of stable thioketenes or thioketene equivalents.

After successful synthesis of the stable di-tert-butylthioketene 89, BRANNOCK et al. investigated its behavior towards small nucleophiles. Thioacylation took place with methanol and ethanol, giving rise to thionoesters 95 , and reaction with aniline gave the corresponding thioamide 96 (Scheme 29-I). No further reactions were reported, as the authors stated that, similar to the ketene analogon $\mathbf{8 8}$, the reactivity of $\mathbf{8 9}$ is severely hindered by the steric shielding, which also increases its stability. ${ }^{[86]}$

Reaction of bis(trifluoromethyl)thioketene 87 with $\mathrm{N}$ - $S$ - and $O$-nucleophiles gives the corresponding thioacylation products 97, as reported by RAASCH (Scheme 29-II). ${ }^{[85]}$ The 
obvious limitation for this procedure, as well as of the aforementioned, is the predefined substitution pattern of either bis(trifluoromethyl)- or di-tert-butyl-substitution in $\alpha$-position to the thiocarbonyl functionality.

The thioacylation protocol reported by SCHAUMANN which uses silylated alkynylsulfides $\mathbf{9 1}$, is also limited (Scheme 29-III). ${ }^{[88]}$ The oxophilic nature of silicon does not permit convenient thioacylation of alcohols, and reports with other simple $N$ - or $S$-nucleophiles are also scarce. Its advantages lie in its ability to undergo cyclization reactions with azomethines to give $\beta$ thiolactams and, furthermore, cyclizations with (thio)imidates and ynamides.

An alternative for the synthesis of thioamides, which is not relying on the use of thioketene or thioketene-mimetic chemistry, is the Willgerodt-Kindler reaction. Originally discovered as Willgerodt Carbonyl Transposition (Scheme 30-I) in 1887, ${ }^{\text {[90] }}$ the reaction was further developed by KINDLER (II) in 1923 (Scheme 30-II). ${ }^{\text {[1] }}$

(I) Willgerodt Carbonyl Transposition (1887)

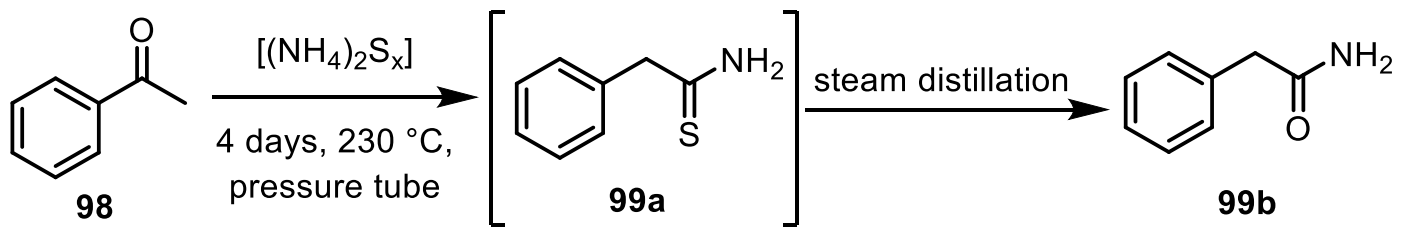

(II) Willgerodt-Kindler modification (1923)<smiles>CC(=O)c1ccccc1</smiles>

98
$H N R^{1} R^{2}$ $\mathrm{R}^{1}=\mathrm{H}$, alkyl $\mathrm{R}^{2}=$ alkyl

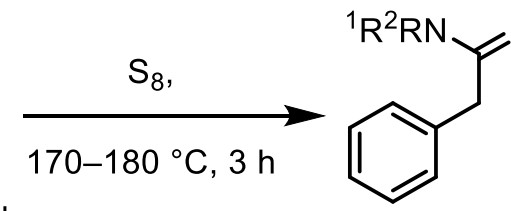

$100 \mathrm{a}$<smiles>[R20]NC(=O)Cc1cccc(C=CC)c1</smiles>

$100 \mathrm{~b}$

Scheme 30: The original Willgerodt reaction and its Willgerodt-Kindler modification.

The original protocol was used to synthesize amides $99 \mathrm{~b}$ from arylketones 98; intermediate thioamides 99a were in situ hydrolyzed to amides. The Kindler modification features much milder reaction conditions and easier to handle compounds. This procedure consists in heating of an aryl ketone $\mathbf{9 8}$ in the presence of sulfur and an amine and allows to isolate the thioamides 100a. Subjecting them to hydrolysis conditions again, the corresponding amides $100 \mathrm{~b}$ are obtained. While the reaction is historically well utilized, it is limited to aryl ketones, and yields above $60 \%$ are mostly only achieved when methyl ketones are employed.

Contemporary literature with resembing conditions of the Willgerodt-Kindler reaction is provided by ZHOU et al. and NGUYEN et al. ${ }^{\text {[92,93] }}$ 
(I) Zhou (2013)

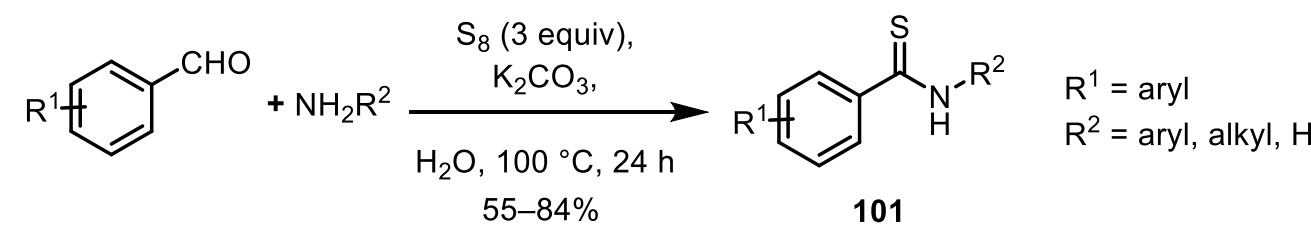

$55-84 \%$

selected examples

(II) Nguyen (2014)
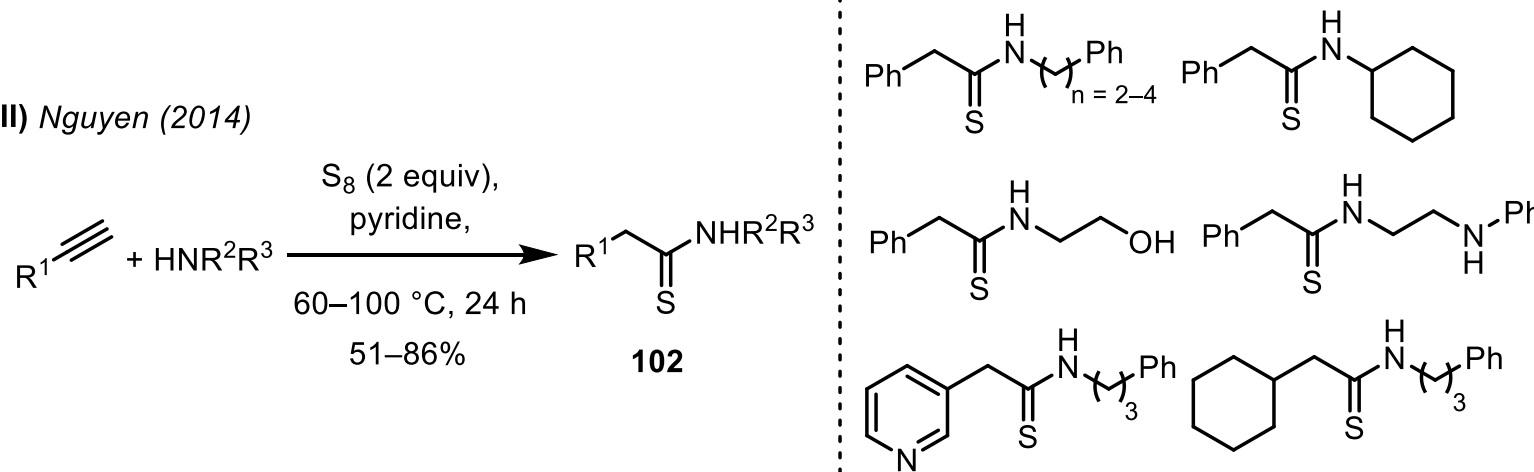

Scheme 31: Recent literature on thioamide synthesis, related to Willgerodt-Kindler conditions.

ZHOU et al. proposed that formation of the thioamide 101 takes place either by WillgerodtKindler reaction or by thionation. In a model reaction, thionation of $\mathrm{N}$-phenylbenzamide under their standard conditions gave thioamide 101 in $12 \%$ yield, whereas the reaction of $\mathrm{N}$ benzylideneaniline in the presence of sulfur, resembling Willgerodt-Kindler reaction conditions, gave the corresponding thioamide in $82 \%$ yield, thus strongly suggesting this mode of reaction to be the actual one. The advantage of the reported methodology clearly is the use of water as a solvent, in which employment of $\mathrm{K}_{2} \mathrm{CO}_{3}$ as a base prevents subsequent hydrolysis to the amide during the reaction (Scheme 31-I). ${ }^{[2]}$

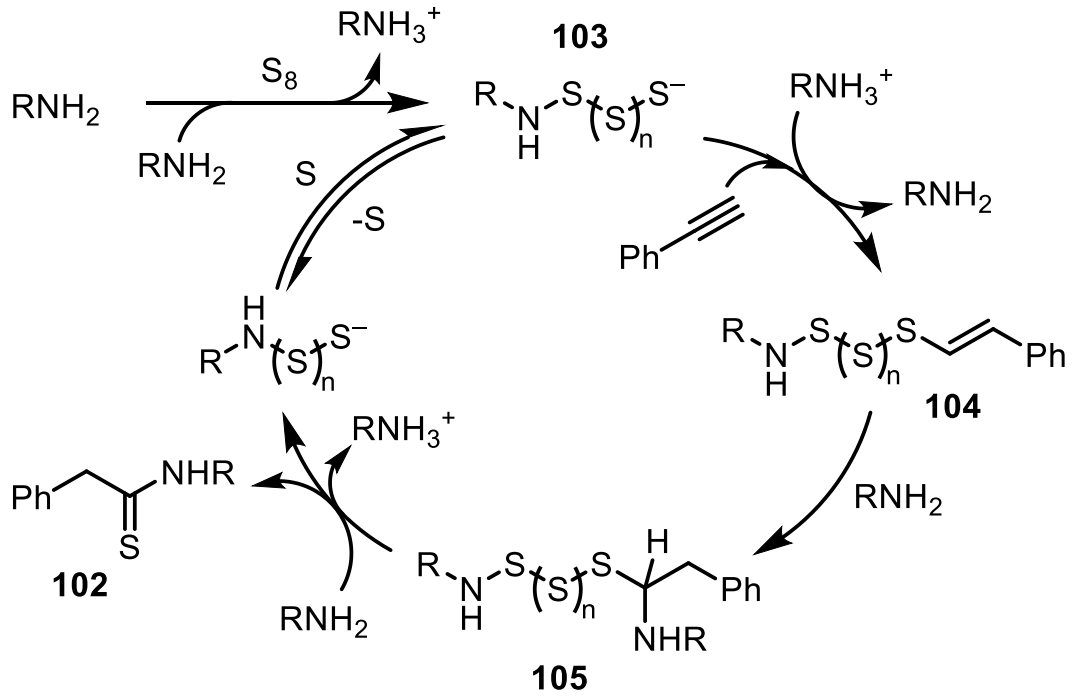

Scheme 32: Mechanism proposed by NGUYEN et al.

Instead of carbonyl compounds also terminal alkynes can be utilized to give access to thioamides 102, as reported by NGUYEN et al. (Scheme 31-II). The authors propose a mechanism in which the $S_{8}$ ring structure is cleaved by the amine, which gives the 
polysulfidic species 103 . The alkyne subsequently adds to the latter, thus resulting in a vinyl polysulfide 104. The polarized double bond in $\mathbf{1 0 4}$ is then attacked by the amine to give a thioaminal 105, which experiences stabilization by elimination to the thioamide 102 as final product (Scheme 32). ${ }^{[93]}$

On the other hand, a mechanism more similar to that proposed by SCHAUMANN et al., ${ }^{[88]}$ in which a transiently formed thioynolate ammonium salt $\mathbf{1 0 6}$ is an intermediate of the transformation, might be a viable alternative (Scheme 33).

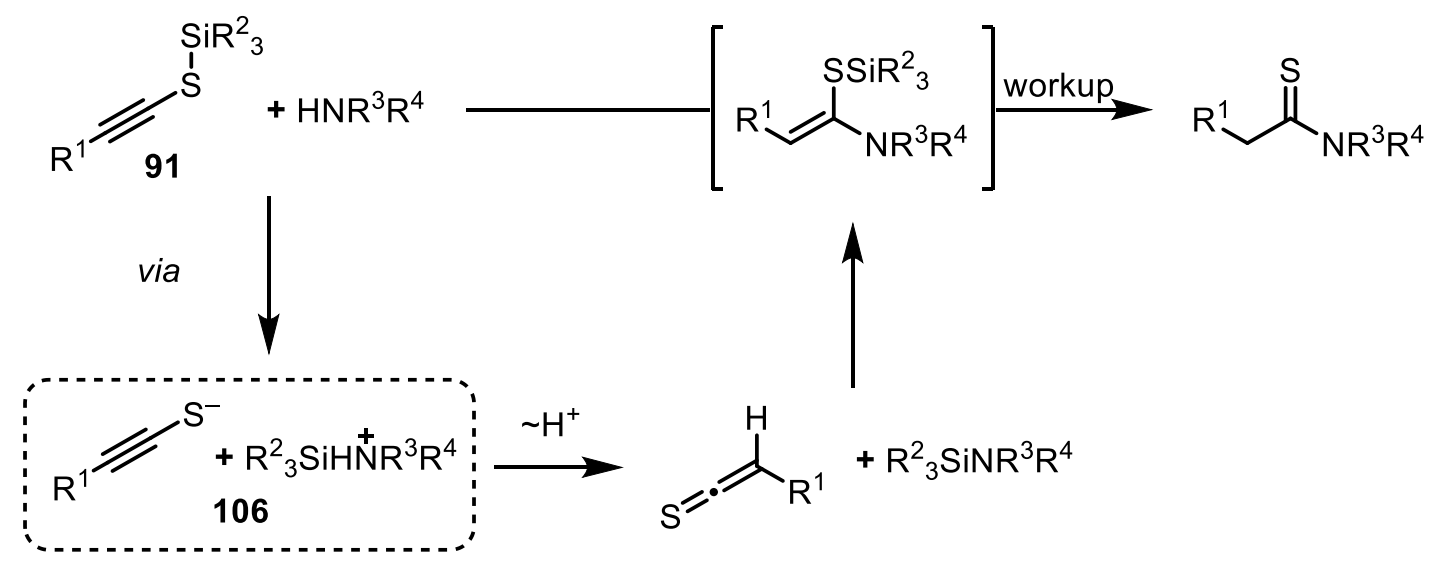

Scheme 33: Proposed mechanism by SCHAUMANN for the formation of thioamides utilizing alkynyl silyl sulfides. ${ }^{[88]}$

NGUYEN et al. pointed out that product formation could be observed neither for anilines nor for alcoholates, thus demonstrating the limitations of the developed procedure. Transformation with (triisopropylsilyl)acetylene gave the desilylated product under the reported reaction conditions. In summary, the protocol is advantageous in terms of the atom-economy, ${ }^{[94]}$ given that this is a three component reaction; however, functional group tolerance is limited. In addition, and similar to the majority of literature procedures that give access to thioamides, reaction temperatures are relatively high.

Other more conventional methods to access thioamides are the straightforward thionation of amides with either Lawesson's Reagent (LR) 125 (Scheme 34) or phosphorus pentasulfide $\left(\mathrm{P}_{4} \mathrm{~S}_{10}\right) 107$ (Scheme 38). ${ }^{[95]}$

Phosphorus pentasulfide can be obtained by reacting liquid white phosphorus $\left(\mathrm{P}_{4}\right)$ with sulfur at temperatures above $300{ }^{\circ} \mathrm{C}$. The reaction, however, can be quite violent, which is why alternative routes were developed. In one of them white phosphorus was substited with red one, another employs either elemental sulfur or pyrite $\left(\mathrm{FeS}_{2}\right)$ to react with ferrophosphorous.

Besides direct thionation, $\mathrm{P}_{4} \mathrm{~S}_{10}$ is also commonly used for the synthesis of heterocycles such as thiazines, thiazoles, thiophenes, pyrimidines, imides, dithiins, dithiazoles, thiazolines, imidazolines and thiadiazoles. General reaction conditions are refluxing $\mathrm{P}_{4} \mathrm{~S}_{10}$ and the desired 
substrate in solvents such as toluene, xylene, THF, acetonitrile or $\mathrm{CS}_{2}$, with addition of bases like pyridine, $\mathrm{Et}_{3} \mathrm{~N}, \mathrm{Na}_{2} \mathrm{CO}_{3}, \mathrm{NaHCO}_{3}$ or $\mathrm{Na}_{2} \mathrm{SO}_{3}$.

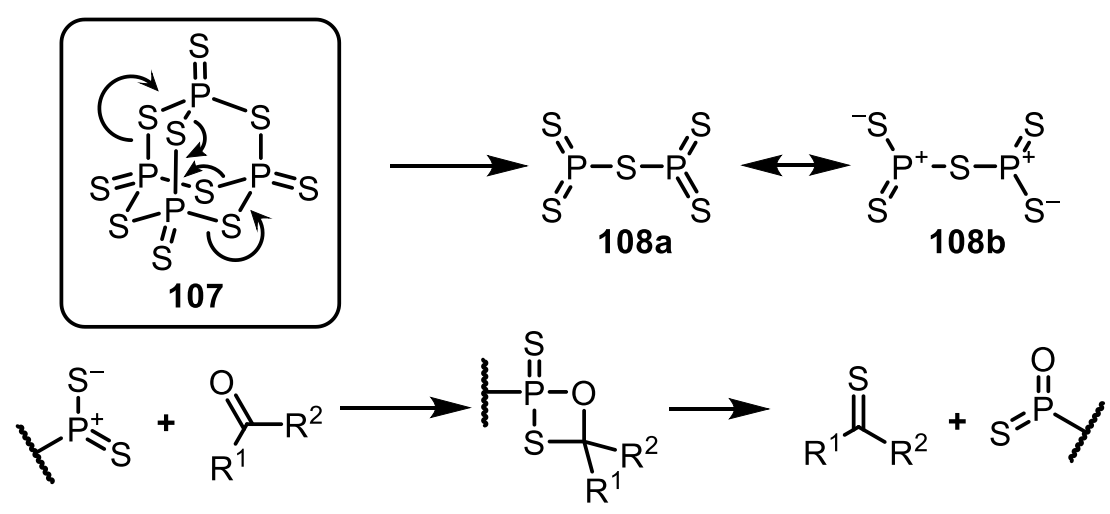

Scheme 34: Dissociation mechanism of $\mathrm{P}_{4} \mathrm{~S}_{10}$ and proposed mechanism for the thionation with $\mathrm{P}_{4} \mathrm{~S}_{10}$.

While the detailed mechanism of thionation is not fully elucidated, the general rationale is that dissociation of $\mathrm{P}_{4} \mathrm{~S}_{10}$ to $\mathrm{P}_{2} \mathrm{~S}_{5} 108$ plays a crucial role (Scheme 34). This hypothesis is generally accepted, as isolation of $\mathrm{P}_{2} \mathrm{~S}_{5}$ from reaction mixtures and its characterization by $\mathrm{X}$ ray diffractometry furnishes the evidence. 
(I) Davis (1966)

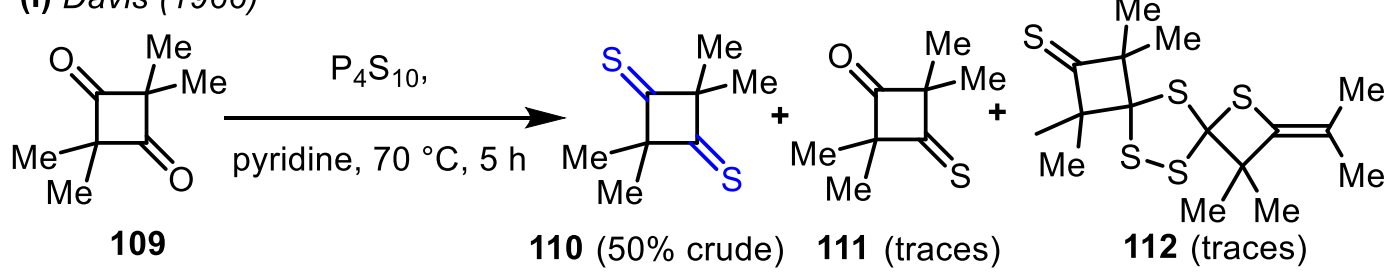

(II) Brillon (1990)

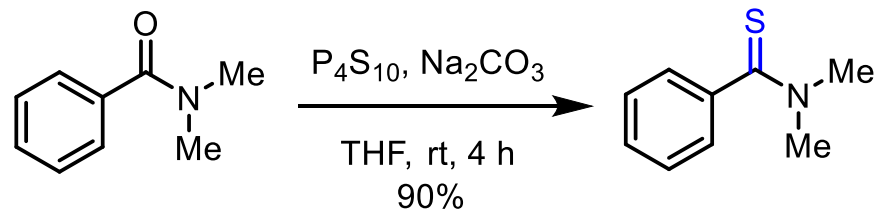

(III) Bildstein (1993)

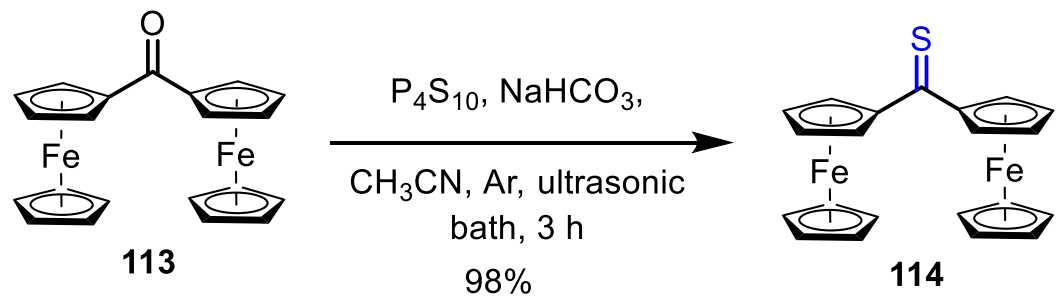

(IV) Mloston (2002)
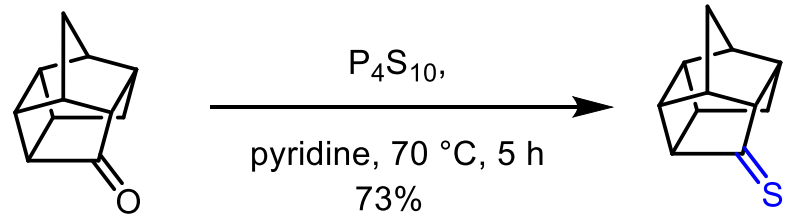

115

(V) Gnanou (2004)<smiles>O=C(O)c1ccc2cc(C(=O)O)ccc2c1</smiles>

(VI) Ibrahim (2006)

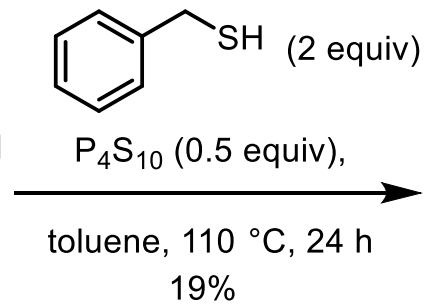<smiles>S=C(SCc1ccccc1)c1ccc2cc(C(=S)SCc3ccccc3)ccc2c1</smiles><smiles>O=c1ccc2ccc(O)cc2o1</smiles>

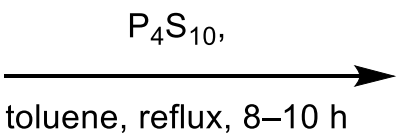<smiles>Oc1ccc2ccc(=S)oc2c1</smiles>

Scheme 35: Selected examples of thionations with $\mathrm{P}_{4} \mathrm{~S}_{10 .} .{ }^{[96-101]}$

In an attempt to find new ways to access monomeric thioketenes, DAVIS and ELAM thionated diketone dimer 109 on large scale $(2.1 \mathrm{~mol})$ and analyzed the isolable products (Scheme 35I). Depending on the amount of starting material, they were able to favour either dithionation to thioketene-dimer $\mathbf{1 1 0}$ or monothionation to obtain tetramethyl-3-thio-1,3cyclobutanedione (111). Pioneering in this field, they reported NMR data of the formerly unknown compounds. Although the authors were unsuccessful in obtaining monomeric thioketene $\mathbf{1 2 0}$ by pyrolysis, characteristic formation of thiethanethione $\mathbf{1 2 1}$ and dithiethane 
122 was observed, thus indicating the monomeric form to be a short-lived species (Scheme 36). ${ }^{[96]}$

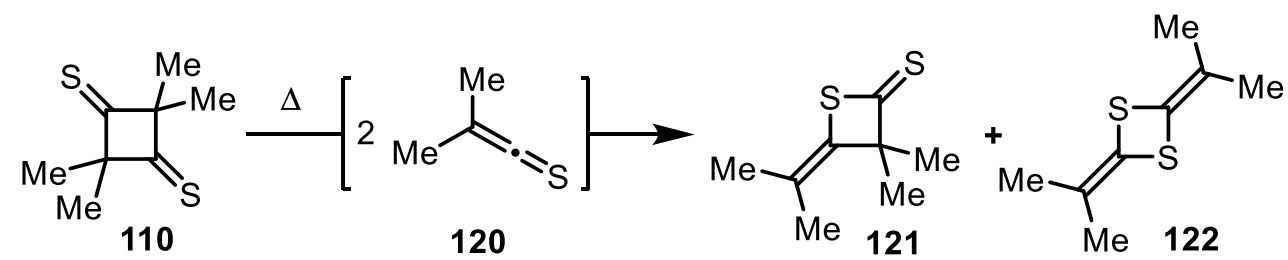

Scheme 36: Pyrolysis of thioketene-dimer.

Investigations towards enhancing the reactivity of $\mathrm{P}_{4} \mathrm{~S}_{10}$ were conducted by BRILLON as early as 1990 (Scheme 35-II). ${ }^{[97]}$ Pretreatment of $\mathrm{P}_{4} \mathrm{~S}_{10}$ with $\mathrm{Na}_{2} \mathrm{CO}_{3}$ proved to be beneficial when compared to former literature reports. He proposed the compound with the empirical formula $\left(\mathrm{P}_{4} \mathrm{~S}_{10} \mathrm{O}\right)^{2-} \mathrm{Na}_{2}{ }^{2+}$ to be the active agent, with ambiphilic properties. ${ }^{1}$ Applying this protocol, the in situ preformation of a species that is more reactive than $\mathrm{P}_{4} \mathrm{~S}_{10}$ itself, a number of thionations at room temperature could be performed.

The same concept enabled BILDSTEIN and DENIFL to transform diferrocenylketone (113) to diferrocenylthioketone (114) (Scheme 35-III). ). A combination of $\mathrm{P}_{4} \mathrm{~S}_{10}$ and $\mathrm{NaHCO}_{3}$, gave access to the corresponding thiocarbonyl compound 114, which then enabled them to study this compound's ability to coordinate different metal carbonyl complexes with the general formula $[\mathrm{THF}][\mathrm{M}(\mathrm{CO})],(\mathrm{M}=\mathrm{Cr}, \mathrm{Mo}, \mathrm{W}) .{ }^{[98]}$

For MŁOSTOŃ and ROMAŃSKI, $\mathrm{P}_{4} \mathrm{~S}_{10}$ was the thionating reagent of choice for the first synthesis of thiocarbonyl derivatives of cage ketones $\mathbf{1 1 5}$ (Scheme 35-IV). During their investigations they also employed a thionation protocol with a combination of $\mathrm{H}_{2} \mathrm{~S} / \mathrm{HCl}$ (gas) which, upon longer reaction times, gave unexpected novel cyclization products 123 and $\mathbf{1 2 4}$ (Scheme 37). ${ }^{[99]}$
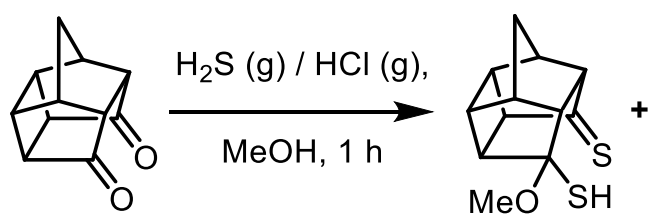

Scheme 37: Cyclization of cage thioketones.

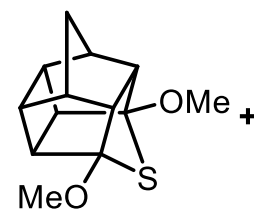

123

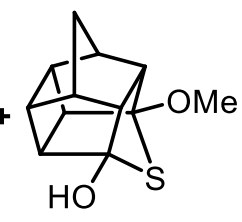

124

One of the major applications for dithioesters is as chain transfer reagents (CTA) for reversible addition-fragmentation chain transfer (RAFT) polymerization. The group of GNANOU synthesized RAFT agent 117 starting from 1,4-naphthalenedicarboxylic acid 116, which enabled them to control the molar masses and polydispersities of the formed polymers (Scheme 35-V). Thus, they were able to obtain linear poly(tert-butylacrylate- $b$ -

\footnotetext{
${ }^{1}$ According to the autor, reaction of $\mathrm{P}_{4} \mathrm{~S}_{10}$ with $\mathrm{NaHCO}_{3}$ involves phosphorus-sulfide bond breaking and formation of ionic thiophosphate groups.
} 
styrene-b-tert-butylacrylate) triblock copolymers as well as three-arm polystyrene stars following an "arm-first" approach. ${ }^{[100]}$

Investigations of the behavior of sulfur reagents towards coumarins were reported by IBRAHIM. Hydroxycoumarin 118 was converted to thionolactones 119 when $\mathrm{P}_{4} \mathrm{~S}_{10}$ was used, without touching the hydroxyl functionality (Scheme 35-VI). Different sulfur reagents were compared, and it could be shown that LR, too gave the same product for the depicted transformation. ${ }^{[101]}$

A plethora of other reactions resulting from the increased reactivity of thioanaloga compared to their oxygen parent compounds have been reported, most of which being condensation or cyclization reactions as also implied in selected examples discussed above (Scheme 35, Scheme 37).

Lawesson's reagent 125 can be synthesized in laboratory scale by heating $\mathrm{P}_{4} \mathrm{~S}_{10}$ in the presence of anisole until hydrogen sulfide evolution ceases. Alternatively it can be prepared from elemental sulfur, red phosphorous and anisole. It is commonly purified by recrystallization from xylene or toluene. It decomposes slowly at temperatures above $110^{\circ} \mathrm{C}$ in solution.

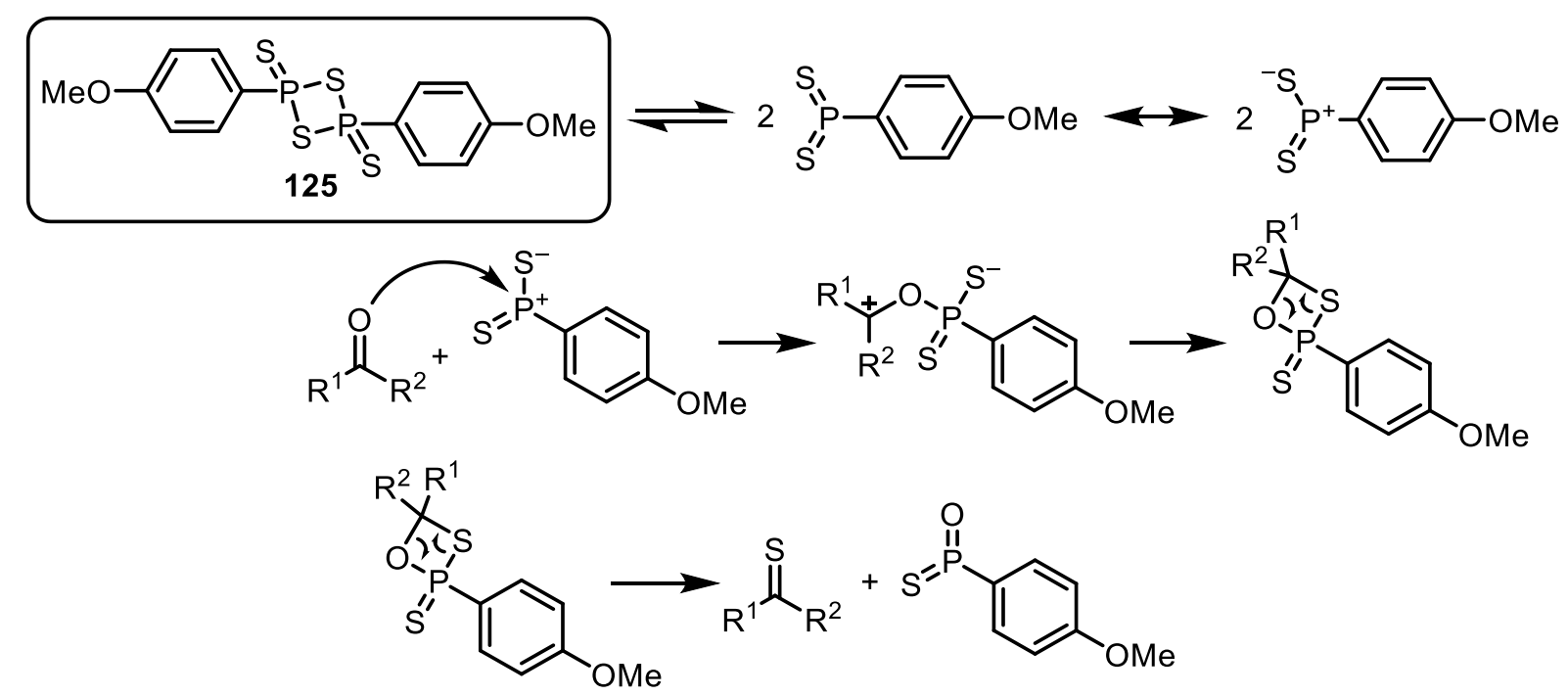

Scheme 38: Lawesson's reagent (LR) 231 and mechanism of the thionation with LR.

Usual thionations with LR are performed on carbonyl compounds, enones, esters, lactones, amides, lactams, and quinones (Scheme 38). Its diverse application possibilities are highlighted in Scheme 39. They range from modifications of complex structures, such as nucleosides (Scheme 39-II) over peptides (Scheme 39-III) to steroids (Scheme 39-IV). 
(I) Strehlow (1991)

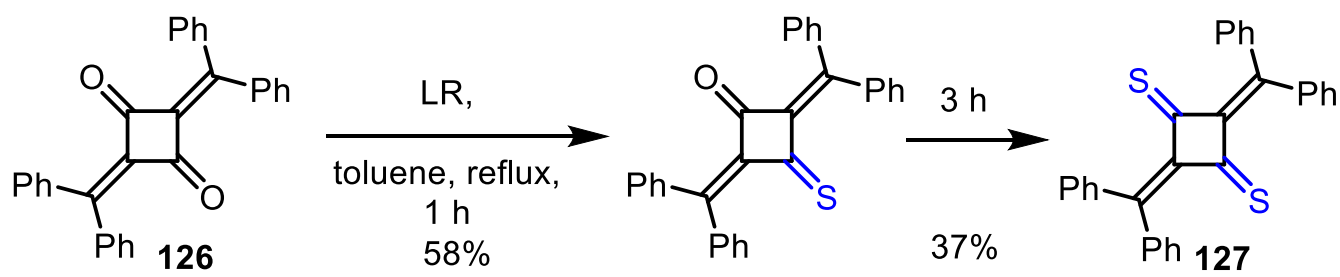

(II) Robba (1993)

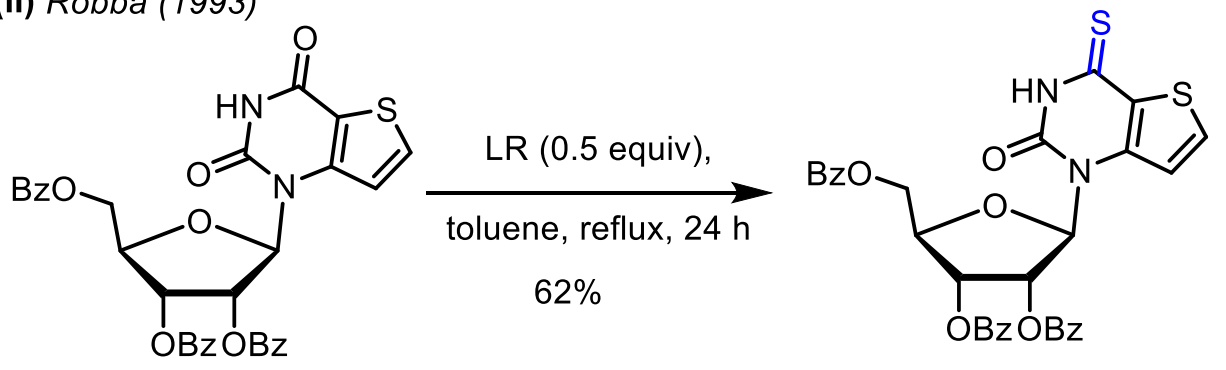

(III) Itokawa (1994)

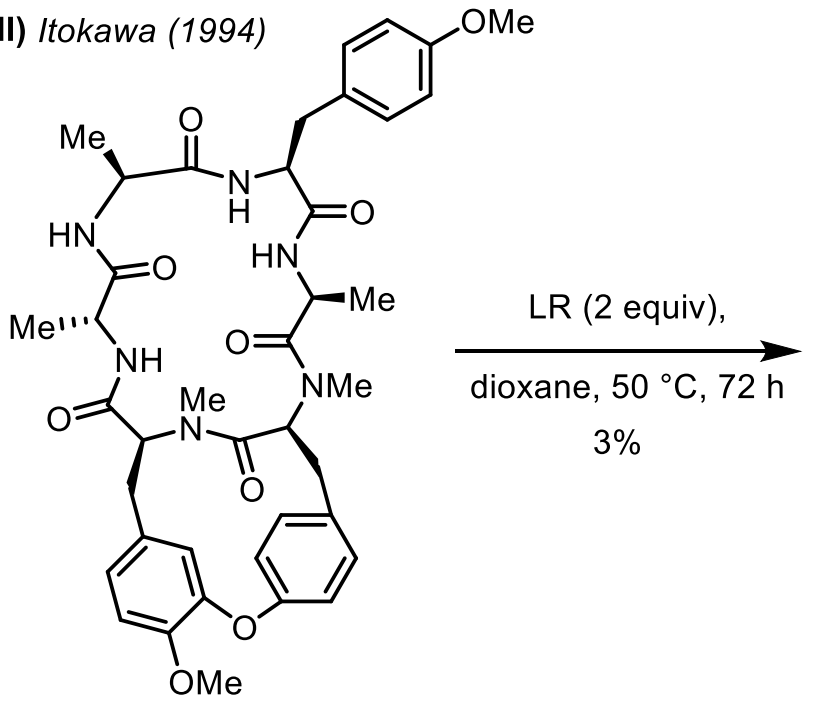

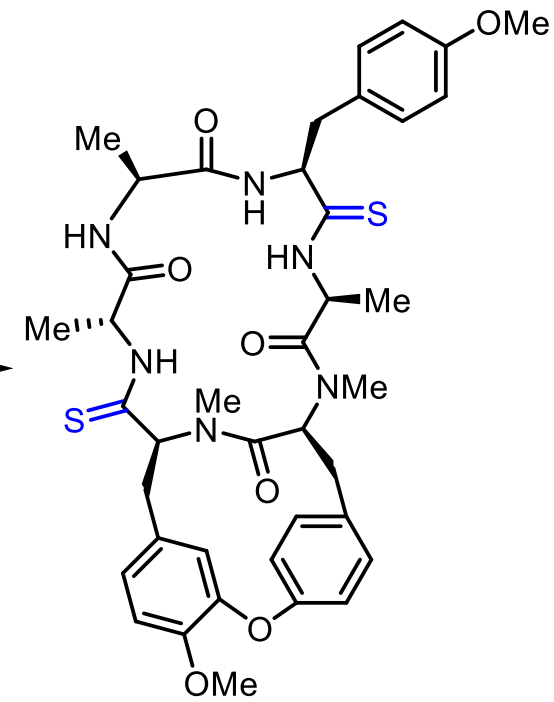

(IV) Ferraboschi (1997)

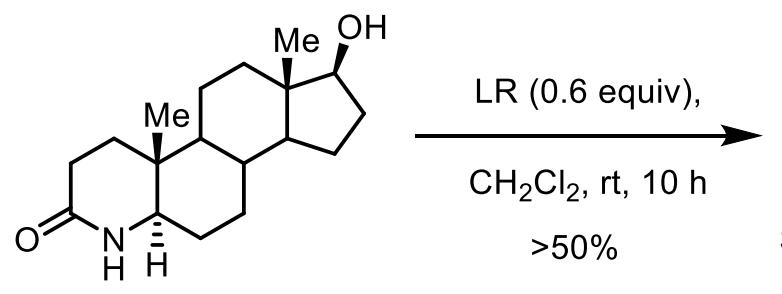<smiles>CC12CCC(=S)NC1CCC1C(CC2)C2CCC3C(C)(C)C(O)CCC3(C)C12</smiles>

Scheme 39: Highlighted products obtained by thionation with LR. ${ }^{[102]}$

In order to synthesize novel diradical dianions and to determine their electronic properties, STREHLOW et al. (I) transformed cyclobutandione 126 into their respective thioketone analogue 127. Similar to DAVIS work with $\mathrm{P}_{4} \mathrm{~S}_{10}$ (Scheme 35-I), reaction time determined either the onefold or twofold thionation products to occur as the major product.

While LR obviously is able to be applied in diverse fields, it reaches its limitations for compounds with ether-containing ester functionalities. For example, crown ether $\mathbf{1 2 8}$ with two ester moieties (Scheme 40) could not be thionated by LR. 


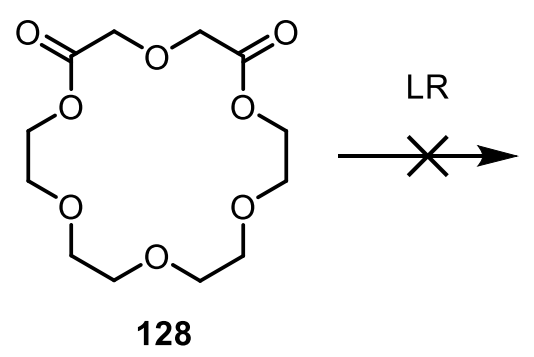

Scheme 40: Limitations of LR. ${ }^{[103]}$

While phosphorus pentasulfide, mostly due to the fact that it is one of the historically oldest thionating agents, has found countless applications as well as LR, which is still the most used chemical for thionations, their obvious drawbacks will always remain the same: Harsh reaction conditions, long reaction times, tedious chromatographic purifications, high toxicity of both the reagent, the formation of the by-products and unpleasant odour are major drawbacks of both reagents. This requires the development of novel methodologies and reagents for thionation and discovery of other reaction pathways to access thioamides, dithioesters and thionoesters.

Another possibility to obtain thionoesters is in the context of the Pinner reaction. Pinner salts 129 when reaced with $\mathrm{H}_{2} \mathrm{~S}$, give the corresponding thionoesters 130 (Scheme 41). In the same fashion, when thiols are used instead of normal alcohols, access to dithioesters becomes available.

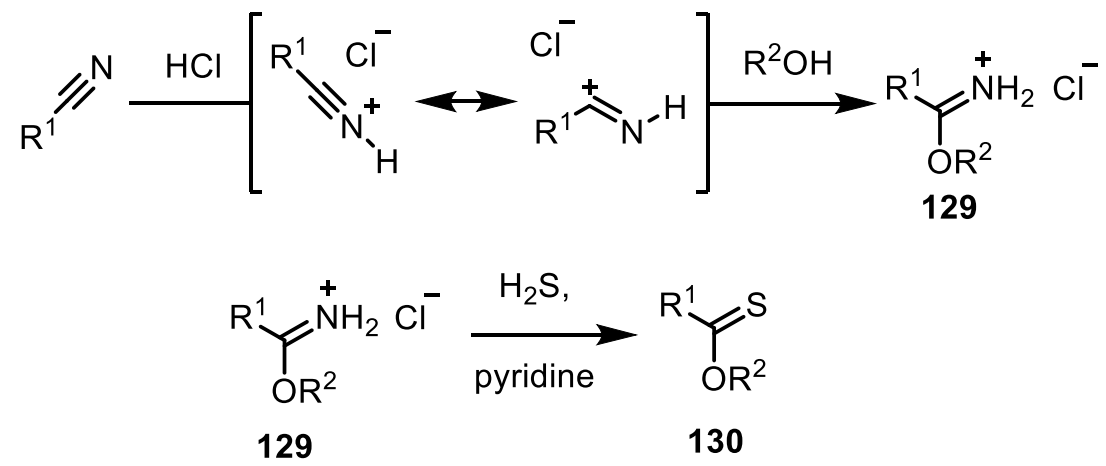

Scheme 41: Pinner salt formation and synthesis of thionoesters 130 utilizing the former.

Numerous further examples from literature, which extensively cover the topic of thioamides and dithioesters in depth, are available. ${ }^{[104]}$ 
Comparison of dithioesters, thioamides and thionoesters with their corresponding oxyanaloga shows that all three of the former generally exhibit an increased reactivity towards both electrophiles and nucleophiles.

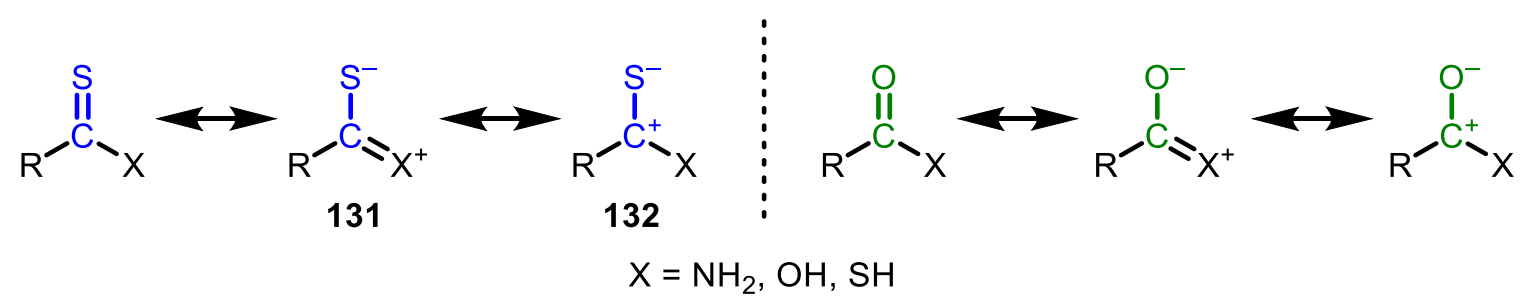

Scheme 42: Comparison of the importance of resonance structures in carbonyl and thiocarbonyl compounds.

A qualitative explanation can be drawn from evaluating the importance of resonance structures for carbonyl and thiocarbonyl compounds. For thiocarbonyls the mesoionic resonance structure 131, in which $\pi$-electron donation into the $X=C$ bond takes place and a negative charge resides on the sulfur (Scheme 42 ), is more important. ${ }^{[105]}$

Determination of rotational barriers for the $\mathrm{C}-\mathrm{N}$ bonds in thioamides gives quantitative evidence for the increased importance of the $C=X$ resonance structure and thus the actual electron density distribution. An example is given by the comparison of $\mathrm{N}, \mathrm{N}$ dimethylformamide (DMF) and $\mathrm{N}, \mathrm{N}$-dimethylacetamide (DMA) with $\mathrm{N}, \mathrm{N}$ dimethylthioformamide (DMTF) and $N, N$-dimethylthioacetamide (DMTA) (Table 1). ${ }^{[105,106]}$

Table 1: Comparison of rotational barriers (in $\mathrm{kcal} \cdot \mathrm{mol}^{-1}$ ) of DMF, DMTF, DMA and DMTA. ${ }^{[105,106]]}$

\begin{tabular}{c|ccccc}
\hline Solvent & $\boldsymbol{\epsilon}$ & DMF & DMTF & DMA & DMTA \\
\hline Gas phase & 1 & 19.25 & 22.0 & 15.33 & 17.7 \\
Cyclohexane & $2.02(1.98)$ & 19.73 & 23.5 & 16.36 & 19.6 \\
Dichloromethane & 9.08 & - & - & 17.95 & 21.9 \\
\hline
\end{tabular}

As shown in Table 1, rotational barriers for thiocarbonyl compounds are approximately $3 \mathrm{kcal} \cdot \mathrm{mol}^{-1}$ higher than for their related carbonyl compounds. This fact that can also be visualized by NMR spectroscopy: While for DMA one signal for both methyl groups is observed, for DMTA, two separate signals for each $\mathrm{CH}_{3}$ groups are detected. The rates of rotation could be obtained by selective inversion-recovery (Sir) NMR experiments. ${ }^{[107]}$

The described $\pi$-electron donation into the $\mathrm{X}=\mathrm{C}$ bond also results in weaker $\mathrm{H}$-bond acceptor and higher $\mathrm{H}$-bond donor abilities for thioamides. LEE, PARK et al. quantitatively proved this statement with a comparative study on amides and thioamides by using near IR spectroscopy. ${ }^{[108]}$ 
An additional consequence is that protons on the heteroatom are more acidic (in case of unsubstituted thiocarbonyls this accounts for $\alpha$-protons): Taken for example that the pKa values of thiobenzoic acid and thioacetic acid are 3.33 and 2.48, respectively, values for benzoic acid and acetic acid are 4.20 and 4.76. This also means that thioacids undergo easier deprotonation by the usual array of inorganic and amine bases. ${ }^{[109]}$

Yet, this does not explain the observed increased electrophilicity of thiocarbonyl carbons. The dipolar thiocarbonyl resonance structure $\mathbf{1 3 2}$ provides an answer for both, the heightened C-electrophilic-, and S-nucleophilic character of thiocarbonyl compounds. Quantitatively it can be explained by comparing the covalent radii for oxygen and sulfur, which determine their respective $\mathrm{S}-\mathrm{C} / \mathrm{O}-\mathrm{C}$ bond lengths. ${ }^{[110]}$ The longer $\mathrm{S}-\mathrm{C}$ bond in this case, results in a decrease of electron density directed from the sulfur towards the carbonyl carbon.

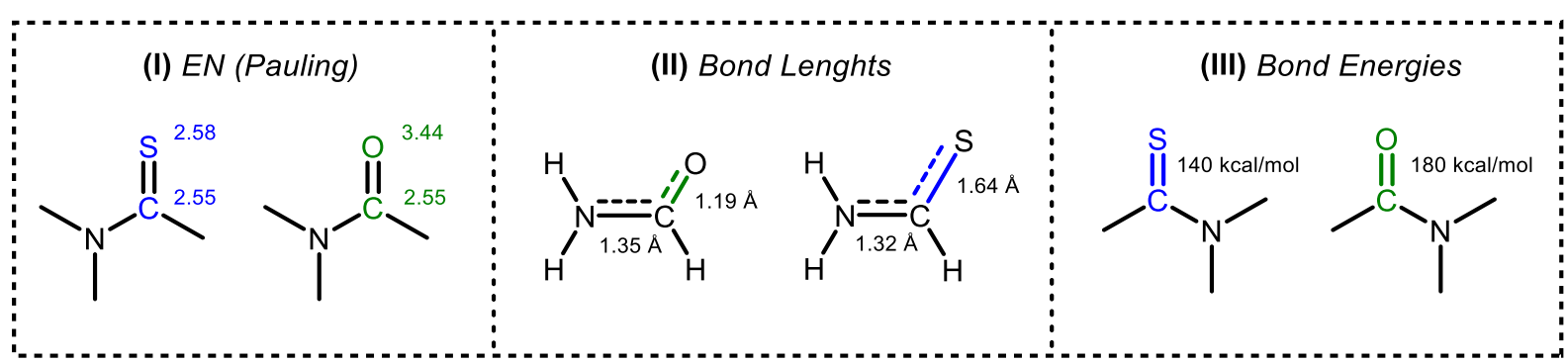

Scheme 43: Exemplary comparison of electronegativity, bond lenghts and bond energies between amides and thioamides. ${ }^{[110,111]}$

Finally, an explanation for the increased reactivity of thiocarbonyl compounds compared to their oxygen analoga can be given by examining their bond dissociation energies (Scheme 43-III and Table 2). ${ }^{[112]}$

Table 2: Bond dissociation energies $\left(D_{298}\right)$ in $\mathrm{kcal} \cdot \mathrm{mol}^{-1}{ }^{[112]}$

\begin{tabular}{c|cc}
\hline Compound & Observed & Calculated (CCSD) \\
\hline $\mathrm{H}_{2} \mathrm{C}=\mathrm{O}$ & 179 & 175 \\
$\mathrm{H}_{2} \mathrm{C}=\mathrm{S}$ & 137 & 1273 \\
$\pi \mathrm{C}=\mathrm{O}$ & $(87)$ & - \\
$\pi \mathrm{C}=\mathrm{S}$ & $(52)$ & - \\
\hline
\end{tabular}

Bond dissociation energy for thiocarbonyl compounds is lower by approximately 40 $\mathrm{kcal} \cdot \mathrm{mol}^{-1}$, meaning that the former bond is significanlty easier to break. 
As described in the previous Chapter, two properties of the thiocarbonyl group stand out. One is the electrophilic character of the carbonyl carbon, which favors condensation reactions of the thiocarbonyl unit. This also results in thiocarbonyl compounds being more prone to reduction. For example, if reduction of an amide to the amine is not possible in a straightforward manner, transformation to the thioamide and successive reduction to amines is used as a devious route. The second major difference is the increased nucleophilicity on sulfur, which, for example, results in selective $S$-alkylation for enethiolates, as compared to enolates. Along with that, as mentioned above, $\alpha$-protons show increased acidity.

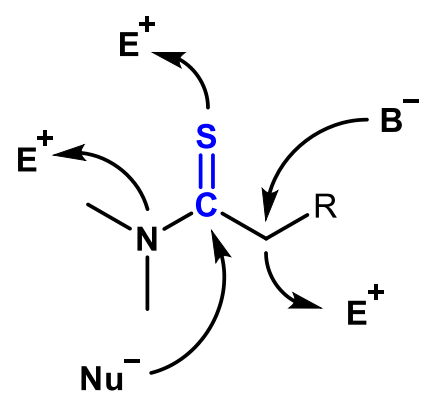

Scheme 44: Representative reactive positions of thioamides.

The described reactivity of thioamides, dithioesters and thionoesters (Scheme $\mathbf{4 4}$ and Scheme 45) is used mainly for the synthesis of heterocycles. ${ }^{[113]}$
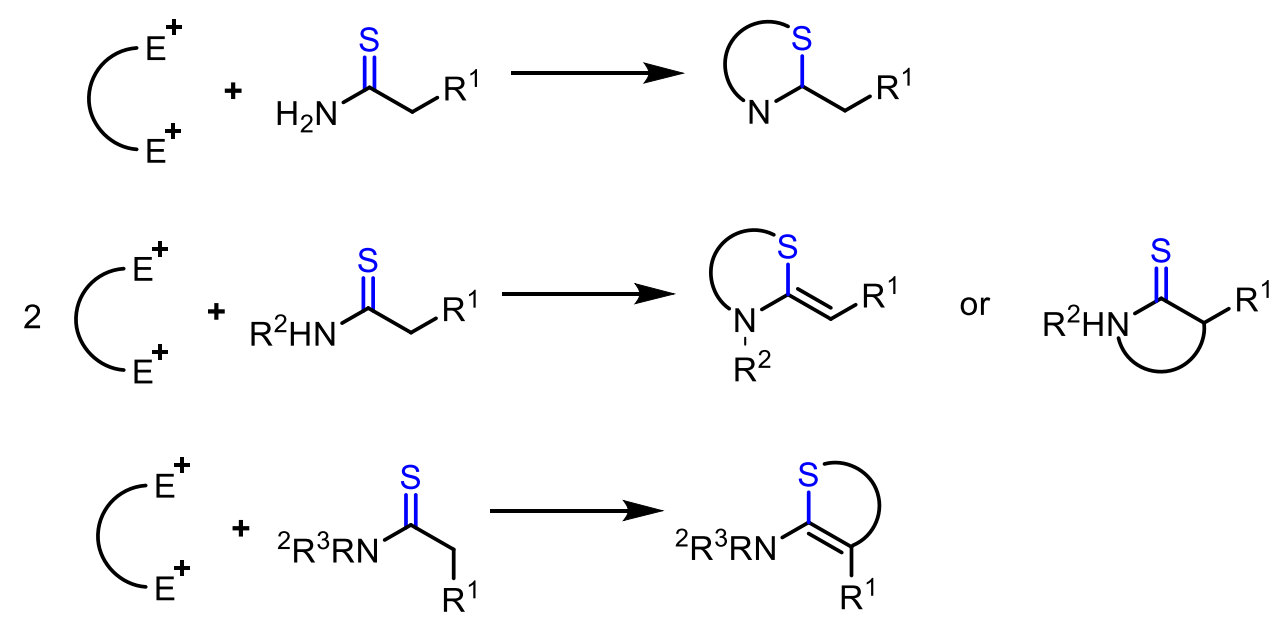

Scheme 45: Reactivity of primary, secondary or tertiary thioamides.

Other applications are thiopeptide antibiotics synthesis, for thioamides and RAFT polymerization for dithioesters respectively, as they can be used as chain transfer agents (CTA) to control the polymerization process. ${ }^{[114]}$

The Liebeskind-Srogl coupling is one of the few transition metal mediated cross couplings which utilizes sulfur containing compounds for the construction of carbon-carbon bonds 
(Scheme 46-I). ${ }^{[115]}$ A number of structures have proven to be applicable in the cross coupling, such as thiol esters, diarylsulfides, thiocyanates, (hetero)aryl thioethers, thioalkynes and cyclic thioamides.

(I) Liebeskind-Srogl coupling (2000)

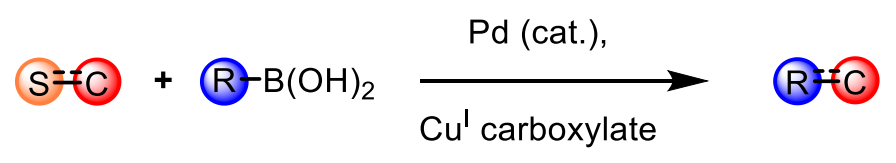

(II) Lengar \& Kappe (2004)

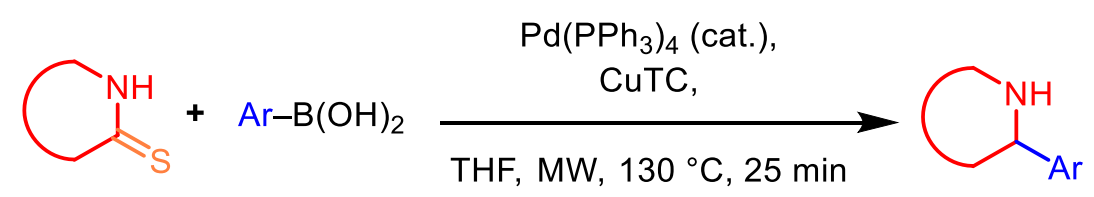

Scheme 46: Schematic Liebeskind-Srogl coupling ${ }^{[115]}$ and exemplary transformation of cyclic thioamides by LENGAR and KAPPE (CUTC $=\mathrm{Cu}(\mathrm{I})$ thiophene-2-carboxylate).$^{[116]}$

LENGAR and KAPPE reported a microwave-assisted boronic acid-thioamide Liebeskind-Srogl coupling protocol, which gave them valuable diaryls (Scheme 46-II). By addition of phenanthroline, they could also favour the selectivity of the reaction towards carbon-sulfur coupling, which gave them diarylthioethers.

The difference in $n \rightarrow \pi^{*}$ transition for thiocarbonyl and carbonyl compounds can be used for applications in protein chemistry: By implementation of the thioamide functionality as fluorescence quenching probes, PETERSSON et al were able to track protein folding and their stability. ${ }^{[117]}$ 


\section{Electrophilic Alkynylation with Transition Metal Generated Nucleophiles}

\section{C-H FUnCTIONALIZATION}

Functionalization of otherwise inert carbon hydrogen bonds ( $\mathrm{C}-\mathrm{H}$ functionalization) has been one of the last major fontiers in organic chemistry. With its high dissociation energy (110 $\mathrm{kcal} \cdot \mathrm{mol}^{-1}$ for benzene and $105 \mathrm{kcal} \cdot \mathrm{mol}^{-1}$ for methane) mediation for the bond breaking step by a metal is often required. ${ }^{[118]}$ Roughly 20 years ago the field of transition metal mediated $\mathrm{C}-\mathrm{H}$ activation, functionalization, reemerged. Since then it has significantly enriched the chemists repertoire of possible $\mathrm{C}-\mathrm{C}$ bonding reactions and has matured to a viable alternative to classic cross coupling reactions such as, Heck-, Suzuki-Miaura, Stille-, Sonogashira-Hagihara reactions and others, for many cases. ${ }^{[6-12,14,15]}$

(I) cross coupling reaction

$$
R^{1}-X+M-R^{2} \stackrel{\text { cat. }[T M]}{\underset{-M X}{\longrightarrow}} R^{1}-R^{2}
$$

(II) $\mathrm{C}-H$ functionalization

$$
\mathrm{R}^{1}-\mathrm{X}+\mathrm{H}-\mathrm{R}^{2} \stackrel{\text { cat. [TM] }}{\underset{-\mathrm{HX}}{\longrightarrow}} \mathrm{R}^{1}-\mathrm{R}^{2}
$$

(III) cross-dehydrogenative coupling

$$
\mathrm{R}^{1}-\mathrm{H}+\mathrm{H}-\mathrm{R}^{2} \stackrel{\text { cat. [TM] }}{\underset{-\mathrm{H}_{2}}{\longrightarrow}} \mathrm{R}^{1}-\mathrm{R}^{2}
$$

Scheme 47: Comparison of concepts for C-C bond formation.

As can be seen from Scheme 47, the major difference between cross-coupling reactions and $\mathrm{C}-\mathrm{H}$ functionalization is the breaking of either a $\mathrm{C}-\mathrm{X}$ or a $\mathrm{C}-\mathrm{H}$ bond and concomitant formation of either a MX or $\mathrm{HX}$ species. This in return requires prefunctionalization of the tobe-modified carbon center in an additional foregoing step. The consequence is additional necessity of reagents, solvent, energy and time. In addition, halide containing byproducts are inevitably obtained.

For the key $\mathrm{C}-\mathrm{H}$ bond breaking step of $\mathrm{C}-\mathrm{H}$ functionalization, four major pathways have been proposed, depending on the transition metal, its oxidation state and partaking ligands (Scheme 48). 
(I) oxidative addition

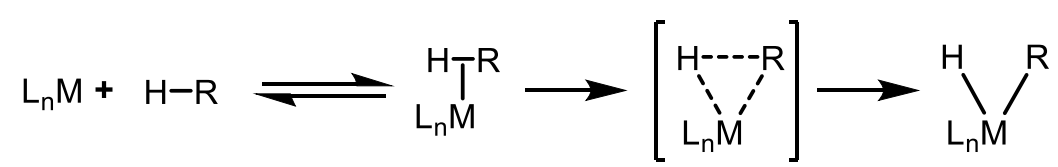

(II) $\sigma$-bond metathesis

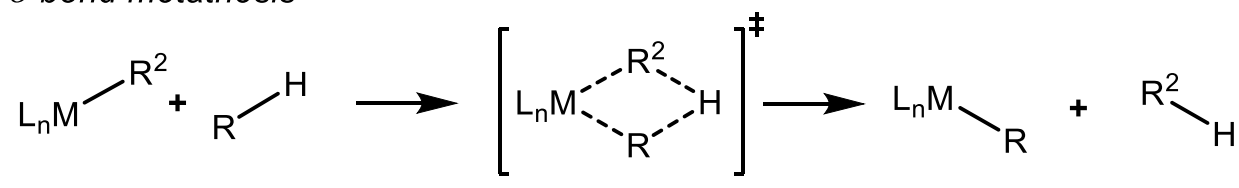

(III) electrophilic substitution

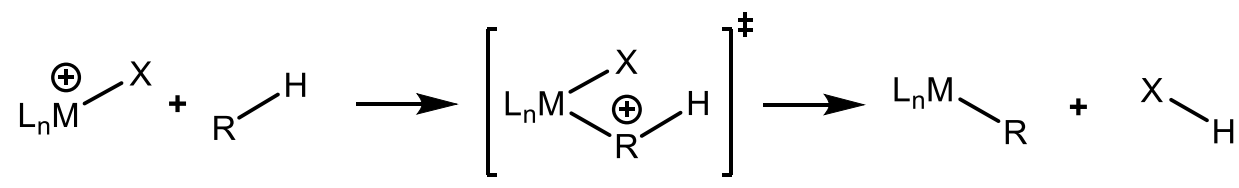

(IV) base-assisted metalation

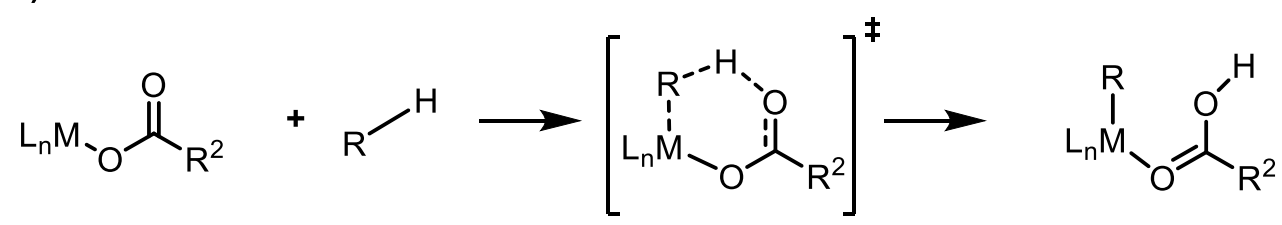

Scheme 48: Mechanisms of $\mathrm{C}-\mathrm{H}$ bond metallation.

Oxidative addition (Scheme 47-I) is the operating mechanism for late, electron rich transition metals in low oxidation states, for example rhodium(0), palladium(0), ruthenium(0) and low valent cobalt systems. Early transition metals in high oxidation states are unable to partake in oxidative addition. They favourably react via $\sigma$-bond metathesis (SBM or $\sigma B M$ ) (Scheme 47-II). For late transition metals in higher oxidation states, electrophilic substitution (Scheme 47-III) becomes favoured. In this case the Lewis acidity of the catalyst increases and dictates the mode of reaction. The historically most recently discovered (and arguably most investigated) pathway, is the base-assisted metalation (Scheme 47-IV) in which an added base for one ligates the metal centre. Secondly, it facilitates the proximal proton abstraction with an additional Lewis-basic heteroatom it posesses. The most prominent group of bases used therefor are carboxylates. ${ }^{[119,120]}$ For this fourth mechanistic pathway, additional investigations have been carried out. Four feasible transition states are discussed here. 
concerted metalation deprotonation (CMD)

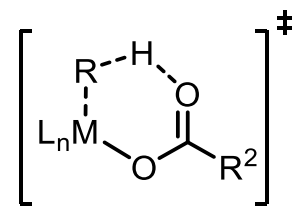

intermolecular electrophilic substitution (IES)<smiles></smiles>

ambiphilic metal ligand activation

(AMLA)

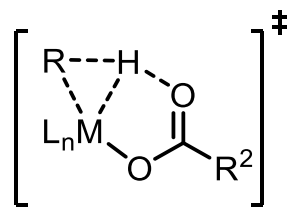

base-assisted intermolecular substitution (BIES)

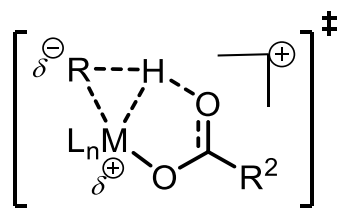

Scheme 49: Transition states for base-assisted metalation. ${ }^{[121-126]}$

Independently described by FAGNOU and DAVIES \& MACGREGOR are the concerted metalation-deprotonation (CMD) pathway and the ambiphilic metal ligand activation (AMLA) respectively, although the denomination $\mathrm{CMD}$ has prevailed. They both describe either a 4or 6-membered transition state, in which intramolecular deprotonation and metalation occur simultaneously. For CMD mechanisms the $\mathrm{C}-\mathrm{H}$ bond cleavage step is kinetically significant (Scheme 49). ${ }^{[121-123]}$

A third scenario, an 4-membered transition state, characteristic in case of either alkoxy bases or hydroxide as intramolecular base, is termed internal electrophilic substitution (IES) by OxGAARD. ${ }^{[124]}$ Adding on these results, ACKERMANN could extend the concept to carboxylates, coining the term base-assisted internal electrophilic substitution (BIES). BIES mechanisms can be identified by their dependence on the electronic nature of the substrate: The more electron-rich the arene, the lower is the activation energy for the $\mathrm{C}-\mathrm{H}$ activation step. $^{[125,126]}$

$\mathrm{C}-\mathrm{H}$ functionalization with guided selectivity requires certain considerations before using it for the modification of a molecule, regarding the choice of the directing group. If introduction, utilization for $\mathrm{C}-\mathrm{H}$ activation, functionalization and removal of the required directing group takes place in a single reaction it is considered a transient directing group. When only a preceding introduction is necessary and removal of the directing group takes place in the same reaction, the directing group is denoted traceless. When all three steps, introduction of the directing group, reaction and removal of the directing group take place separately, it is called a removable directing group. In any of these cases, evaluation of the feasibility of a $\mathrm{C}-\mathrm{H}$ functionalization protocol, or classic cross coupling is recommended. ${ }^{[127]}$ In general, any functional group with a free electron pair (lone pair) can be considered and by now, has been investigated, as a directing group. ${ }^{[128]}$ 
Choice of the right directing group is essential, since it must fulfill several roles during the reaction. First it needs to be able to coordinate the active catalyst species and selectively "direct" it to the reaction site, the $\mathrm{C}-\mathrm{H}$ bond that should be functionalized. It has to further stabilize the transition state during $\mathrm{C}-\mathrm{H}$ activation as well as partake in the metallacycle. Afterwards de-coordination is necessary to liberate the catalyst, so it can undergo the next catalytic cycle. The alternative, too strong coordination, leads to inactive substrate-catalyst species. One possibility to determine the coordination strength of directing groups is to evaluate their Lewis basicity by measuring their affinity towards Lewis acids such as $\mathrm{SbCl}_{5}$ or $\mathrm{BF}_{3 .}{ }^{[129]}$ Similar rules apply for the choice of transition metal catalyst which can be considered Lewis acids.

When discussing $\mathrm{C}-\mathrm{H}$ functionalization, precise definition of which area is neccessary, since it has become such a large field of research. Categorization is possible, for example, by employed metal, agent of oxidation, directing group or hybridization of the functionalized $\mathrm{C}-\mathrm{H}$ bond.

The first precedent for the functionalization of aromatic $\mathrm{sp}^{2}$-carbon hydrogen bonds $(\mathrm{C}-\mathrm{H}$ functionalization) arguably was given by OTTO DIMROTH in 1902, when he reported the reaction of mercury(II) acetate with benzene (Scheme $50-11),{ }^{[130]}$ predecessed only by the Butlerov-Eltekov-Lermontova-reaction for the functionalization of acyclic olefinic carbons from 1874 (Scheme 50-I). ${ }^{[131]}$ However, when compared to more contemporary literature on $\mathrm{C}-\mathrm{H}$ functionalization, these examples lack one crucial component, a directing group (DG), which is able to coordinate a metal center in order to direct the $\mathrm{C}-\mathrm{H}$ activation step to a particular proximal carbon-hydrogen bond.

Consequently $\mathrm{C}-\mathrm{H}$ functionalization with innate selectivity (no influence of directing forces) and guided selectivity (employment of a DG) is differentiated.

The first reported $\mathrm{C}-\mathrm{H}$ functionalization with guided selectivity was published by SHUNSUKE MURAHASHI in 1955 , where $\mathrm{CO}_{2}(\mathrm{CO})_{8}$ was used as a catalyst to transform $(E)-\mathrm{N}, 1-$ diphenylmethanimine (133) into 2-phenylisoindolin-1-one (134) under an atmosphere of CO (100-200 atm) at $220-230{ }^{\circ} \mathrm{C}$ (Scheme 50-II). ${ }^{[132]}$ 


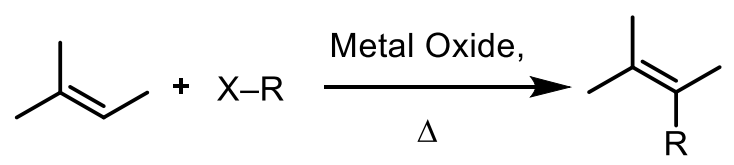

(II) Dimroth (1902)

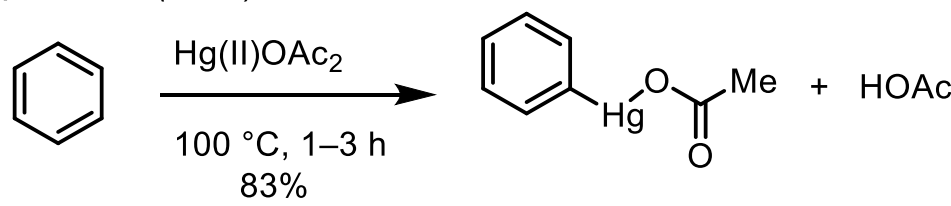

(III) Murahashi (1955)
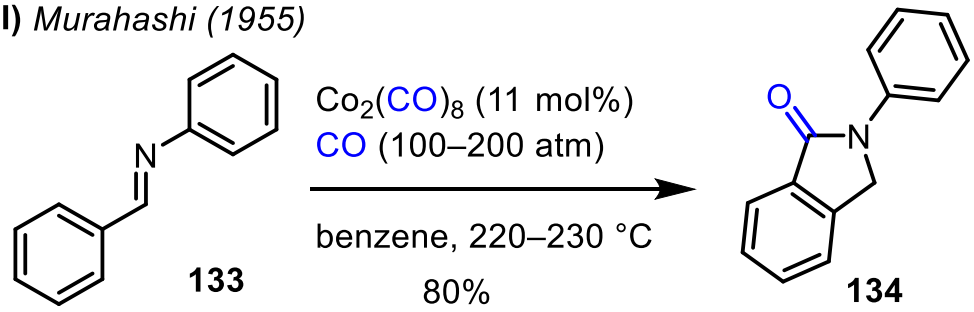

Scheme 50: Butlerov-Eltekov-Lermontova-reaction, $\mathrm{C}-\mathrm{H}$ activation of benzene with mercury(II) acetate by DIMROTH (I) as an example for innate selectivity and synthesis of indolinones by MURAHASHI by utilization of the imine functionality as a DG using guided selectivity (II). ${ }^{[130,132,133]}$

Another milestone in $\mathrm{C}-\mathrm{H}$ functionalization worth mentioning is MURAIs' work using weakly coordinating aromatic ketones with alkenes employing a ruthenium species as catalyst, which gave rise to a plethora of subsequent publications on the topic of $C\left(s p^{2}\right)-H$ functionalization. ${ }^{[134,135]}$

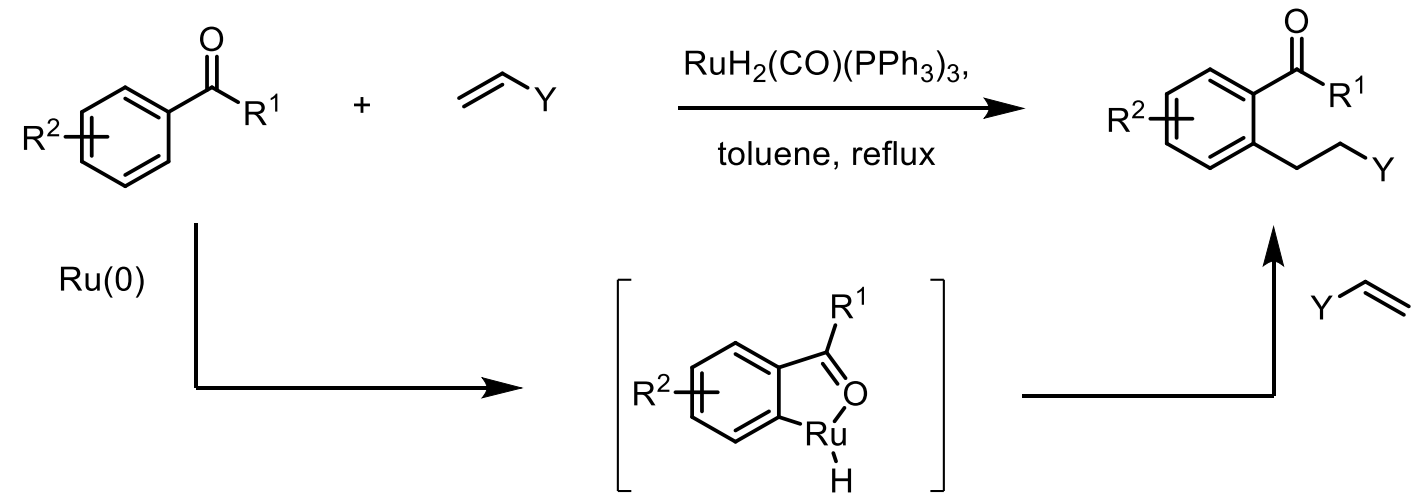

Scheme 51: MURAI's milestone in Ru-catalyzed C-H functionalization with weakly coordinating aromatic ketones.

$\mathrm{C}-\mathrm{H}$ activation has become such an immense field, that covering all literature has become simply impossible. Nevertheless, several reviews and books which thoroughly cover important aspects of the field have been written and give an excellent overview. $^{[[119,128,135,136,137,138-140]}$

While 'classical' $\mathrm{C}-\mathrm{H}$ functionalization chemistry with precious transition metals as of now seems to slowly plateau, the acquired knowledge enables today's chemists to implement- or 
combine it with other fields, such as material science, natural product synthesis, polymer science, electrochemistry, photochemistry or electrophilic transfer reagents. ${ }^{[141]}$ 
The largest part of $\mathrm{C}-\mathrm{H}$ activation alkynylation research has undoubtedly been done by employing internal alkynes. Upon these, diphenylacetylene (tolan) is commonly used as model substrate and extensively covered in the literature. ${ }^{[136,139]}$

(I)

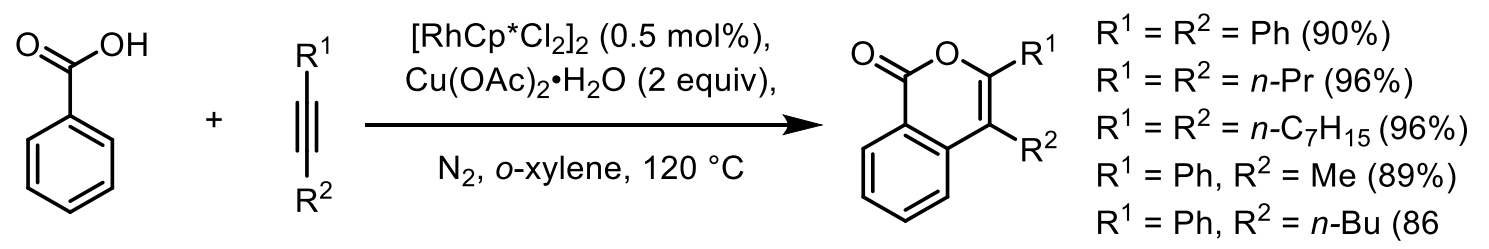

(II)

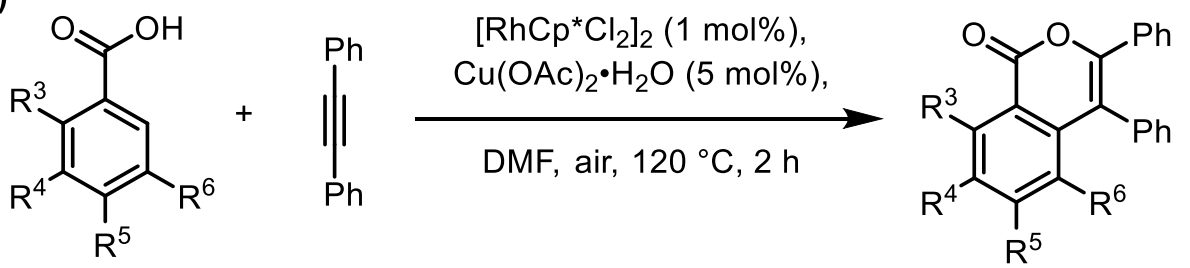

Phh<smiles>Cc1ccc2c(-c3ccccc3)c(-c3ccccc3)oc(=O)c2c1</smiles>

$97 \%$<smiles>COc1cc(OC)c2c(-c3ccccc3)c(-c3ccccc3)oc(=O)c2c1</smiles>

$99 \%$<smiles>Cc1cccc2c(-c3ccccc3)c(-c3ccccc3)oc(=O)c12</smiles>

$67 \%$<smiles>COc1cccc2c(-c3ccccc3)c(-c3ccccc3)oc(=O)c12</smiles>

$93 \%$

$\mathrm{R}^{5}=\mathrm{Me}(92 \%), \mathrm{OH}(63 \%), \mathrm{Cl}(82 \%), \mathrm{CF}_{3}(73 \%)$

Scheme 52: Coupling of benzoic acids with internal alkynes. ${ }^{[142,143]}$

In 2007 SATOH and MIURA could show that transformation of 1:1 mixture of benzoic acid derivatives and internal alkynes to 3,4-substituted isocoumarins with utilization of $\left[\mathrm{RhCp} * \mathrm{Cl}_{2}\right]_{2}(0.5 \mathrm{~mol} \%)$ and $\mathrm{Cu}(\mathrm{OAc})_{2} \bullet \mathrm{H}_{2} \mathrm{O}$ in $o$-xylene under $\mathrm{N}_{2}$ at $120{ }^{\circ} \mathrm{C}$ could be achieved (Scheme 52-I). ${ }^{[142,143]}$ Later, reaction conditions were further optimized requiring only catalytic amounts of $\mathrm{Cu}(\mathrm{OAc})_{2}$, which could be regenerated by oxygen from air (Scheme $\mathbf{5 2}$ II).

A discovered competitive reaction was the decarboxylation with concomitant insertion of the second alkyne equivalent. This is negligible when using $\left[\mathrm{RhCp}^{*} \mathrm{Cl}_{2}\right]_{2}$, but could selectively be favoured by replacing the catalyst with $\left[\mathrm{IrCp}^{*} \mathrm{Cl}_{2}\right]_{2}$ and the oxidant $\mathrm{Cu}(\mathrm{OAC})_{2} \bullet \mathrm{H}_{2} \mathrm{O}$ by $\mathrm{Ag}_{2} \mathrm{CO}_{3}$ at $160{ }^{\circ} \mathrm{C}$ (Scheme 53). 


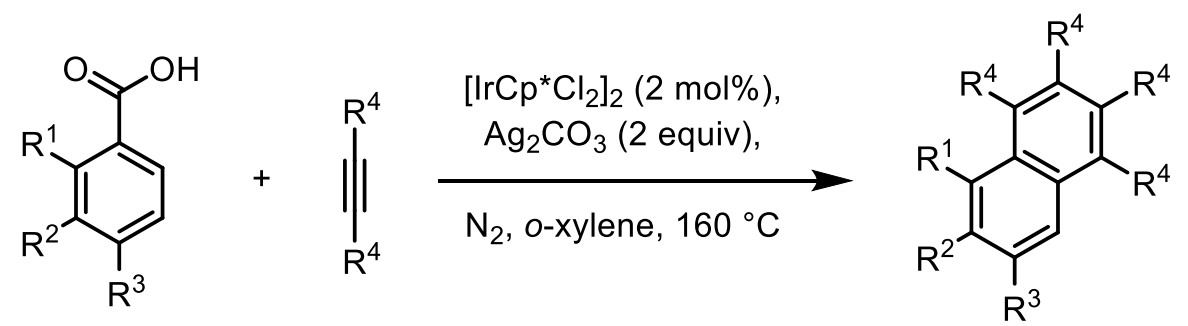<smiles>[R]c1c([R])c([R])c2ccccc2c1[R]</smiles><smiles>CC1=Cc2cc(c(-c3ccccc3)c(-c3ccccc3)c2-c2ccccc2)C=C1</smiles>

$95 \%$<smiles>Cc1cc(C)c2c(-c3ccccc3)c(-c3ccccc3)c(-c3ccccc3)c(-c3ccccc3)c2c1</smiles>

$93 \%$

$$
4-\mathrm{ClC}_{6} \mathrm{H}_{4}(91 \%)
$$

\section{Plausible Mechanism:}

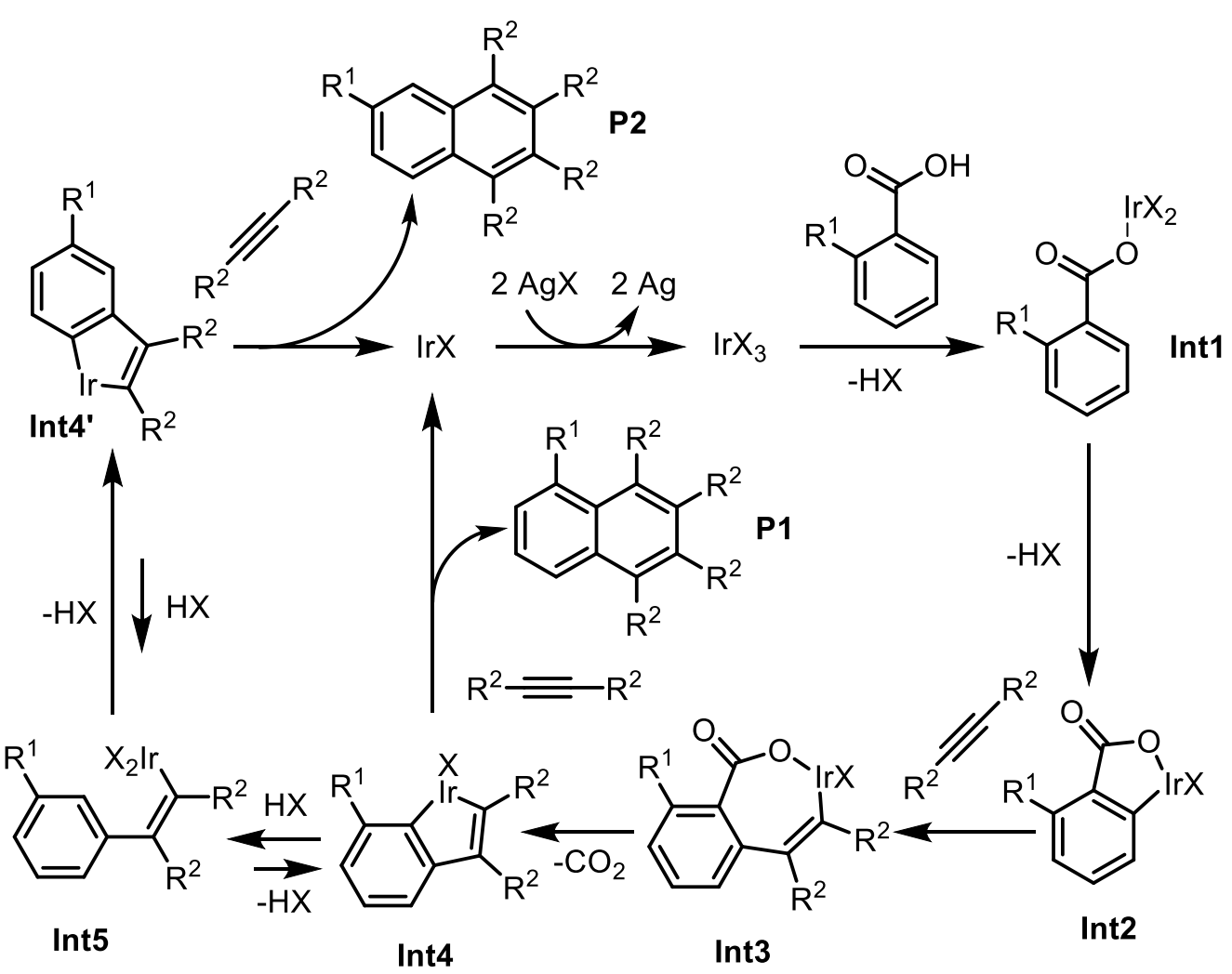

Scheme 53: Decarboxylative 1:2 coupling of benzoic acids with internal alkynes. ${ }^{[143]}$

A plausible mechanistic cycle for the decarboxylative coupling starts with coordination of the substrate to an Ir'II-species by ligand exchange, to give Int1. Ortho-iridation gives five membered iridium metallacycle Int2, which, after insertion of the internal alkyne, results in a seven membered iridium metallacycle Int3. This species then favourably undergoes decarboxylation rather than reductive elimination as observed for the aforementioned rhodium catalyzed procedure (Scheme 52), to again, give a five membered iridium 
metallacycle Int4. The subsequent second alkyne insertion then gives the naphthalene product $\mathbf{P 1}$, and the catalyst species is regenerated in the presence of a silver salt. For 2substituted benzoic acids, the key intermediate Int4 can undergo rearrangement, due to steric hindrance, passing through Int5, to give an isomeric iridacycle Int4'. This pathway explains the observed isomeric product formation of $\mathbf{P} \mathbf{2}$.

A multitude of reports followed. In order to keep this chapter in an acceptable extent, only highlights relevant for the results discussed later on, are covered (the up to now most recent comprehensive review and references to foregoing reviews therein are highly recommended). ${ }^{[140]}$ 
(I) Miura \& Satoh (2008)<smiles>CC(C)(C)[Pb]C#CPc1ccccc1-c1nc2ccccc2[nH]1</smiles>

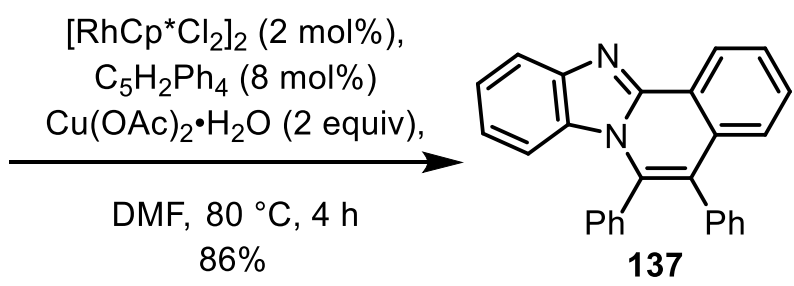

(II) Fagnou (2008)<smiles>CC#C[C+](C)C(C)=O</smiles>

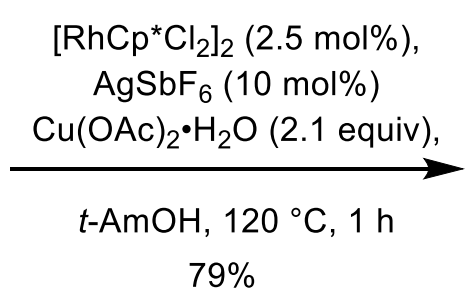<smiles>CC(=O)n1c(-c2ccccc2)c(C)c2ccccc21</smiles>

(III) Miura \& Satoh (2009)<smiles>N=C(c1ccccc1)c1ccccc1</smiles>

$\left[\mathrm{RhCp}{ }^{*} \mathrm{Cl}_{2}\right]_{2}$ (2 mol\%), $\mathrm{Cu}(\mathrm{OAc})_{2} \cdot \mathrm{H}_{2} \mathrm{O}$ (2 equiv),

DMF, $80^{\circ} \mathrm{C}, 2 \mathrm{~h}$ $90 \%$<smiles>c1ccc(-c2nc(-c3ccccc3)c3ccccc3c2-c2ccccc2)cc1</smiles>

(IV) Miura \& Satoh (2010)<smiles></smiles>

136

$$
\begin{aligned}
& {\left[\mathrm{RhCp}^{*} \mathrm{Cl}_{2}\right]_{2}(2 \mathrm{~mol} \%) \text {, }} \\
& \mathrm{Na}_{2} \mathrm{CO}_{3} \text { (2 equiv) } \\
& \mathrm{Cu}(\mathrm{OAc})_{2} \cdot \mathrm{H}_{2} \mathrm{O}(10 \mathrm{~mol} \%) \text {, } \\
& \text { o-xylene, } \mathrm{N}_{2} / \mathrm{O}_{2}, 100^{\circ} \mathrm{C} .6 \mathrm{~h} \\
& \text { 93\% }
\end{aligned}
$$<smiles>Clc1ccc2c(c1)cc1c3ccccc3c(-c3ccccc3)c(-c3ccccc3)n21</smiles>

(V) Rovis (2010)<smiles>CNC(=O)c1ccccc1</smiles>

$\left[\mathrm{RhCp}{ }^{*} \mathrm{Cl}_{2}\right]_{2}(2.5 \mathrm{~mol} \%)$, $\mathrm{Cu}(\mathrm{OAc})_{2} \cdot \mathrm{H}_{2} \mathrm{O}$ (2.1 equiv),

$t-\mathrm{AmOH}, 120^{\circ} \mathrm{C}, 16 \mathrm{~h}$ $82 \%$<smiles>Cn1c(-c2ccccc2)c(-c2ccccc2)c2ccccc2c1=O</smiles>

(VI) Fagnou (2010)<smiles>CONC(=O)c1ccccc1</smiles>

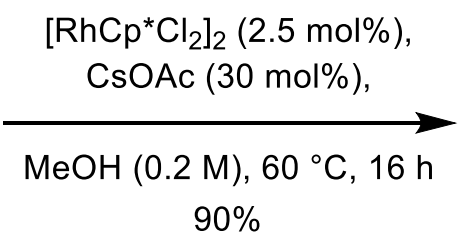<smiles>COn1c(-c2ccccc2)c(-c2ccccc2)c2ccccc2c1=O</smiles>

revised structure

Scheme 54: Progress in C-H activation-alkynylation.

In addition to their work on annulations of benzoic acids with internal alkynes, SATOH and MIURA could also prove the utility of their methodology by modifying phenylazoles 135 to obtain naphthyl- and anthrylazoles 137 (Scheme 54-I), which exhibit intense fluorescence in solid state. ${ }^{[144]}$ 
FAGNOU et al. revised their originally postulated structure for the compound $\mathbf{1 4 1}$ (Scheme 54VI) after findings by HUANG et al. This group reported a Pd-catalyzed reaction starting from the same substrate to access isoquinolines which structure was confirmed by X-ray crystallography. Controversially, no corrigendum could be found by RovIs et al. (Scheme 54V). ${ }^{[145]}$ It is possible that the reaction protocol developed by Rovis et al. in fact forms cyclic imidates, taking into account their observations of competitive isocoumarin formation, which can be formed by hydrolysis of this cyclic imidates. A rhodium-catalyzed alkynylation/cyclization of $N$-unsubstituted and $N$-monosubstituted benzamides by MIURA AND SATOH from 2010 also described $N$-cyclization. ${ }^{[146]}$

The regioselectivity for rhodium catalysts is summarized by You et al. and selectivity is also discussed within $\mathrm{YU}$ et al.'s review on $\mathrm{C}-\mathrm{H}$ functionalizations of $\mathrm{N}$-methoxy amides with an emphasis on palladium catalysis. ${ }^{[138,140]}$ Additionally, numerous ruthenium-catalyzed examples can be found in literature. ${ }^{[147]}$ 
C-H functionalization utilizing Activated Acetylenes

Along with $\mathrm{C}-\mathrm{H}$ functionalizations employing internal alkynes significant effort to employ terminal alkynes has been made. 
(I) Chatani (2012)<smiles>Cc1ccccc1-c1ccccn1</smiles>
other DGs:

(II) $L i$ (2015)<smiles>O=C(Nc1cccc2cccnc12)c1ccccc1</smiles><smiles></smiles>

(III) Balaraman (2016)

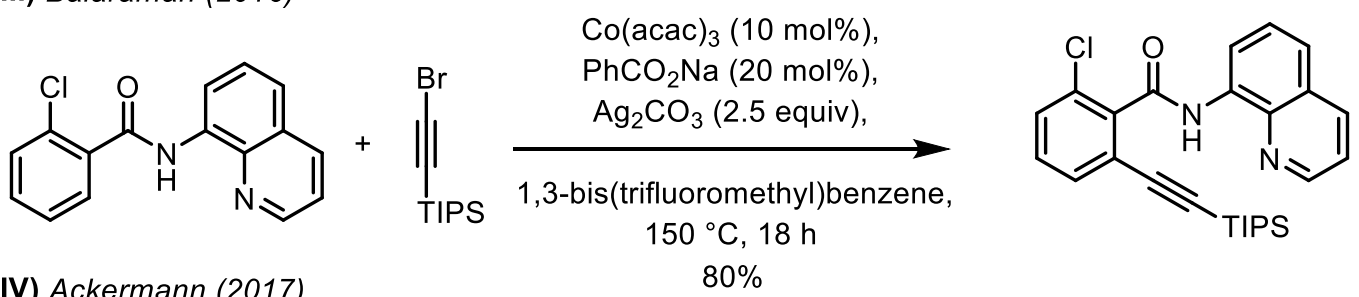

(IV) Ackermann (2017)<smiles>FC(F)([In])C#CS</smiles>

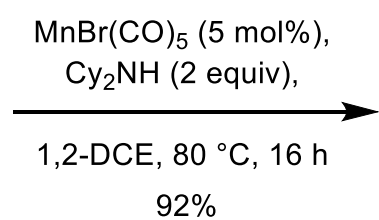<smiles>S[InH]C#Cc1cc2ccccc2n1-c1ccccn1</smiles>

(V) Ackermann (2017)<smiles>COc1cc(OC)c(C(=O)O)cc1OC</smiles>

$\left[\mathrm{Ru}\left(\mathrm{MesCO}_{2}\right)(p\right.$-cymene) (10 mol\%),

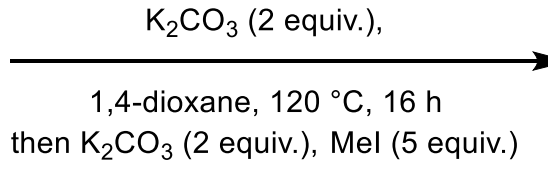

$88 \%$

(VI) Ackermann (2017)<smiles>CC(C)(NC(=O)c1ccccc1)c1cn(Cc2ccccc2)nn1</smiles><smiles>S[In]C#CBr</smiles>

$$
\mathrm{Fe}(\mathrm{acac})_{3}(10 \mathrm{~mol} \%) \text {, }
$$
dppen (15 mol\%),

$\mathrm{PhMgBr}$ (3 equiv.), $\mathrm{ZnCl}_{2} \cdot$ TMEDA (2 equiv.), THF, $65^{\circ} \mathrm{C}, 1 \mathrm{~h}$

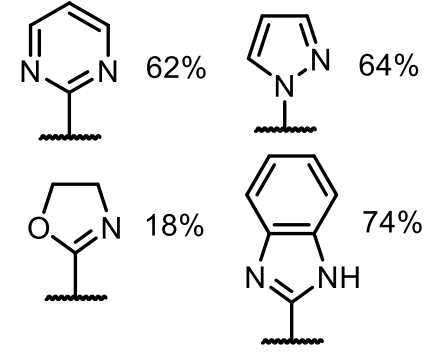


electrophile has been realized in different ways. In general, attachment of an electronwithdrawing substituent to the alkyne is the solution of a problem. The most prevalent examples are employment of halides, sulfones or hypervalent iodonium reagents.

As not only expensive transition metals such as rhodium or palladium but the roughly tenfold cheaper ruthenium as catalysts is able to provide in $\mathrm{C}-\mathrm{H}$ activation, in 2012 CHATANI et al. were able to show that by utilization of weakly polarizing bromoalkyne, arenes $\mathbf{1 4 1}$ could be directly alkynylated with the chelation assistance of nitrogen-containing heterocycles such as pyridine, pyrimidine, pyrazole, and imidazole (Scheme 55-I). ${ }^{[148]}$ Further development was performed by ACKERMANN et al. (Scheme 55-V). ${ }^{[149]}$ Extending the list of directing groups and employing one of the most exploited DGs recently, LI et al. reported on a catalytic nickel system for the direct ortho $\mathrm{C}-\mathrm{H}$ alkynylation of amides with the directing assistance of bidentate 8-aminoquinoline 142 as an auxiliary (Scheme 55-II). ${ }^{[150]}$

BALARAMAN et al. successfully demonstrated that cobalt-catalyzed selective bis-alkynylation of amides via double $\mathrm{C}-\mathrm{H}$ bond activation is possible upon the directing assistance of 8-aminoquinoline as well, and proved the feasibility of this DGs by exemplary subsequent removal of it (Scheme 55-III). ${ }^{[151]}$

Extending the range of viable metals to manganese for $\mathrm{C}-\mathrm{H}$ alkynylation of indol derivatives 143 carrying a pyridine directing group with bromoalkynes, ACKERMANN et al. could successfully introduce silyl-, aryl-, alkenyl- and alkylalkyne moieties (Scheme 55-IV). ${ }^{[152]}$ Furthermore, in the same year they could also show for the first time, that iron-catalyzed C$\mathrm{H}$ activation is possible by using a rather complex reaction protocol and involving another popular directing group, the triazole moiety. Nevertheless, a remarkable extension of the versatility of $\mathrm{C}-\mathrm{H}$ activation protocols, alkynylation of arenes, heteroarenes and even acyclic alkenes was possible (Scheme $\mathbf{5 5 - V I ) .}{ }^{[153]}$

One of the most prominent examples was given by ECHAVARREN et al. (Scheme 55-VII) in 2018: They reported alkynylation with mostly silylsubstituted bromoalkynes and a multitude of different directing groups. In addition to common DGs such as esters, ketones, and ethers also less exploited DGs like thioethers, sulfoxides, sulfones, amines, phenol esters and carbamates proved to be suitable for their protocol. Mechanistic investigations, supported by DFT calculations, suggest an BIES mechanism (Scheme 56). ${ }^{[154]}$ 


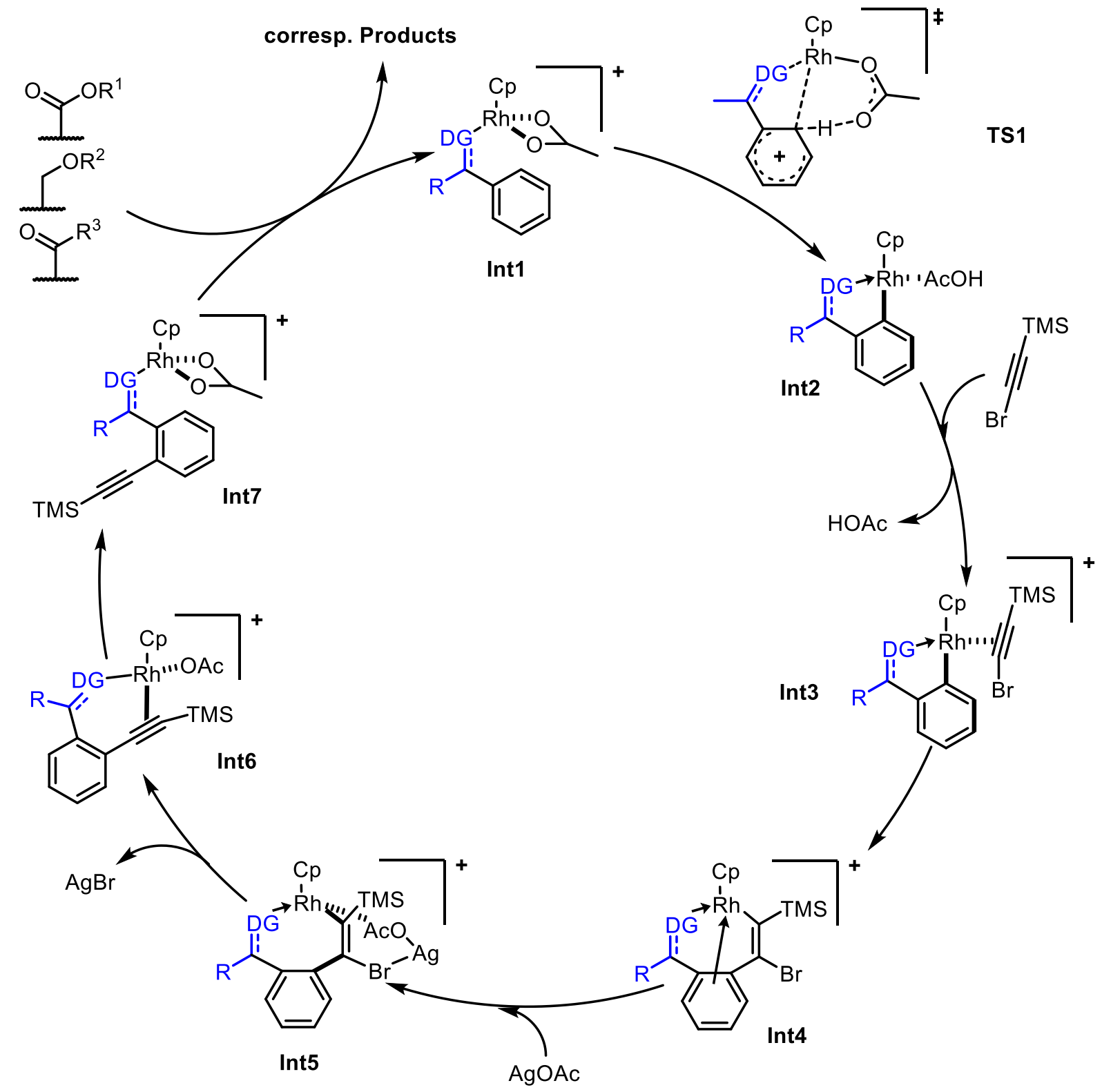

Scheme 56: Proposed mechanism by ECHAVARREN et al., based on DFT calculations.

Calculations for methyl benzoate as substrate suggest, that starting with Int1, C-H functionalization is intramolecularly assisted by the acetate ligand, undergoing the cyclic six-membered transition state TS1. Calculations for alternative transition states, such as a cyclic four membered scenario or intermolecular acetate assisted $\mathrm{C}-\mathrm{H}$ activation, gave much higher energy barriers and could thus be ruled out. The resulting Int2 then undergoes dissociative ligand exchange, to give ( $\eta^{2}$-alkyne)rhodium complex Int3. Alkyne insertion to form Int4, is followed by AgOAc-assisted bromide elimination, passing through Int5, to give Int6 and afterwards Int7. Finally, ligand exchange liberates the alkynylated product and regenerates Int1. 
(I) Loh (2014)

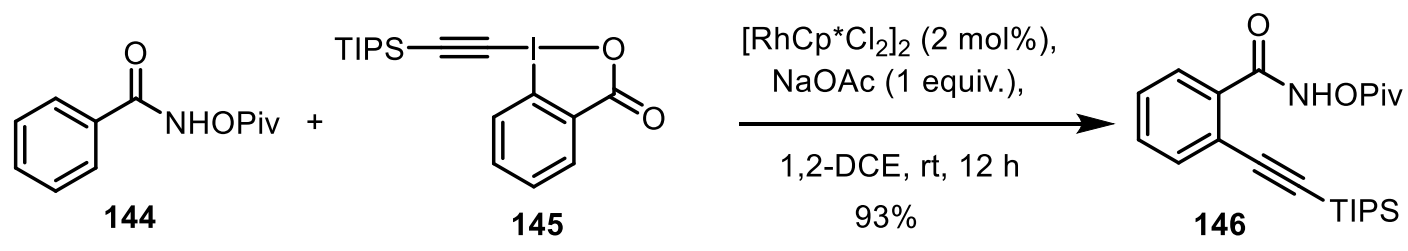

(II) $\operatorname{Li}(2014)$

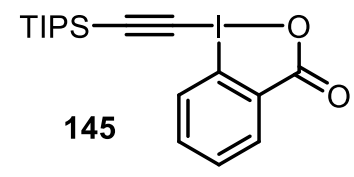<smiles>Cc1ccccc1-c1ccccn1</smiles>

141
$\left[\mathrm{RhCp}^{*} \mathrm{Cl}_{2}\right]_{2}(2 \mathrm{~mol} \%)$, ZnOTf $(10 \mathrm{~mol} \%)$,

1,2-DCE, rt or $80^{\circ} \mathrm{C}, 16 \mathrm{~h}$ $73 \%$

(III) Loh (2014)<smiles>C=C(NC)C(=O)c1ccccc1</smiles>

147<smiles>O=C1OI(C#CS[In])c2ccccc21</smiles>

145<smiles>Cc1cccc(C#C[In])c1-c1ccccn1</smiles><smiles>CC(C)(C)N=Nc1ccccc1</smiles>

(IV) Cheng (2016)<smiles>CONC(=O)c1ccccc1</smiles><smiles>CC(C)SC#CI1COC(=O)c2ccccc21</smiles>

(V) You (2017)

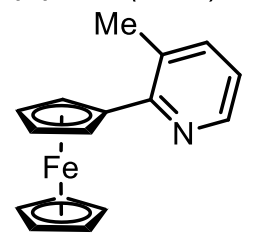<smiles>C[As](C)(=S)C#CI1OC(=O)c2ccccc21</smiles>

$\left[\mathrm{RhCp}{ }^{*} \mathrm{Cl}_{2}\right]_{2}(2.5 \mathrm{~mol} \%)$, $\mathrm{AgSbF}_{6}(20 \mathrm{~mol} \%)$,

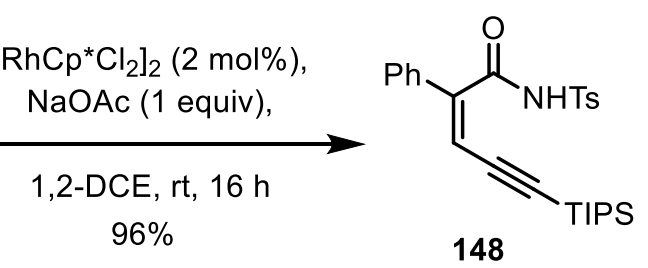

$\left[\mathrm{RuCl}_{2}(\mathrm{p} \text {-cymene })\right]_{2}(2.5 \mathrm{~mol} \%)$<smiles></smiles>

$\left[\mathrm{RhCp} \mathrm{Cl}_{2}\right]_{2}(2 \mathrm{~mol} \%)$,

$96 \%$

Scheme 57: $\mathrm{C}-\mathrm{H}$ functionalization: alkynylation utilizing hypervalent iodine-alkyne reagents.

In early 2014, the group of LOH (I) published a rhodium(III) catalyzed C-H alkynylation procedure of $N$-pivaloyloxyl amides $\mathbf{1 4 4}$ under remarkably mild reaction conditions (room temperature) and with high selectivity towards monoalkynylation products 146 (Scheme 57I). ${ }^{[155]}$

Only one month later, an extensive report on rhodium(III) catalyzed $\mathrm{C}-\mathrm{H}$ alkynylation utilizing hypervaltent iodonium salts 145 was presented by $\mathrm{LI}$ et al. (Scheme 57-II). They could prove that iodonium compounds of the BX family are compatible with a number of heterocyclic as well as non-cyclic directing groups such as $O$-methyl oximes, $N$-tert-butyl- $\alpha$ phenylnitrones, azomethine imines, $\mathrm{N}$-nitrosoanilines, azobenzene, azoxybenzene and acetanilines. Even more: a scope of rhodium(III)-catalyzed $\mathrm{C}-\mathrm{H}$ alkynylation of $\mathrm{N}$ - 
pivaloyloxybenzamides and iridium(III) catalyzed $\mathrm{C}-\mathrm{H}$ alkynylation of $\mathrm{N}$ methoxycarboxamides was reported, both utilizing TIPS-EBX as alkynylating agent. Mechanistic insights were gained by isolation of an intermediate rhodium vinyl complex formed from substrate, catalyst and reagent, which was characterized by X-ray diffractometry, and through study of kinetic isotope effects (KIE). ${ }^{[156]}$

One of the few examples in $\mathrm{C}-\mathrm{H}$ alkynylation, which makes use of acyclic substrates, was contributed by the group of LOH. They could show, that the use of TIPS-EBX $\mathbf{1 4 5}$ and a weakly-coordinating tosylimide as a DG were the best combination for the functionalization of acrylamides 147. A number of $\beta$-unsubstituted acrylamides could be transformed into the corresponding 1,3-enynes 148. By switching solvent from 1,2-DCE to $\mathrm{MeOH}$ also $\alpha, \beta-$ disubstituted acrylamides could be modified (Scheme $57-$ III). ${ }^{[157]}$

$\mathrm{N}$-methoxyamides 149 are popular substrates in $\mathrm{C}-\mathrm{H}$ activation due to their $\mathrm{N}-\mathrm{O}$ redox active bond, which is exploited as an internal oxidant in a number of transformations. ${ }^{[138]}$ However, in the reaction reported by CHEN et al. (Scheme 57-IV) the directing group remains intact. The authors postulate a redox neutral mechanism following an ES pathway. Whereas they were unable to find conditions where dialkynylation was completely excluded, after optimization, formation of the corresponding dialkynylated products were in the range of only $0-7 \%$.

You et al. (Scheme 57-VI) could functionalize rather exotic subtrates, namely ferrocene derivatives, with EBX as an alkynylating agent. They were successful in controlling mono- and dialkynylation by choice of the directing group (pyridine or isoquinoline) and the amount of EBX.

More extensive literature covering the topic of transition metal catalyzed $\mathrm{C}-\mathrm{H}$ alkynylation with hypervalent iodonium compounds is given by J. WASER in two reviews. ${ }^{[52,158]}$

It could be shown that, depending on reaction conditions, catalyst or electrophilic alkynylation agent, a plethora of either annulation or alkynylation products can be accessed. A large number of directing groups can be used, giving a wide span of possibilities for application. As already mentioned classic $\mathrm{C}-\mathrm{H}$ activation now already is a mature research area. Amalgamation with other research fields such as material science, for example highly fluorescent polyaromatic compounds, or peptide synthesis proves the relevancy of the established chemistry. 


\section{RESULTS AND DISCUSSION}

\section{Synthesis and Evaluation of Novel Pyridinium BaSed Electrophilic Transfer REAGENTS}

\section{Project Aims}

The objective of this project is to create alternative platforms for electrophilic group-transfer reactions in order to expand the field of electrophilic group-transfer reagents.

In this context utilization of scaffolds which are isolobal to hypervalent iodonium salts, yet not based on iodine, our group envisioned sulfur-based reagents isolobal to the former. Such sulfur-based reagents show similar properties as iodine compounds: In contrast to light $p$ block elements which commonly form interatomic $\pi$-bonds, a three-center-four-electron bond $(3 \mathrm{c}-4 \mathrm{e})$ is observed for both compound classes (Scheme 58). The 3c-4e bond is a popular example for a hypervalent bond. It is a weak and highly polarized bond, intrinsic to the reactivity pattern observed for these compounds.

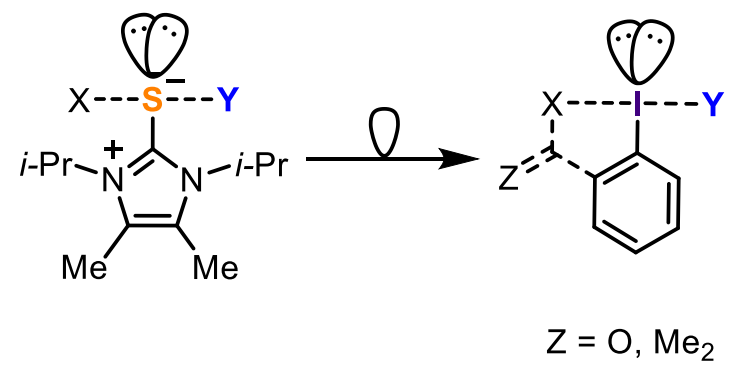

Scheme 58: Isolobality of sulfur- and iodine-based transfer reagents.

While the research field of hypervalent iodonium salts has has been a topic of constantly increasing interest for the last 20 years, their stability has been an issue since their discovery. Even significantly more stable, cyclic hypervalent iodonium salts are showing thermal instability in some cases.

The first sulfur containing transfer reagents, comprising an imidazolioum moiety, for the electrophilic transfer of nitriles as well as alkynes were successfully synthesized in 2015 by our group. ${ }^{[41]}$ After characterization and evaluation of their reactivity towards different nucleophiles, we contemplated, how the reactivity of sulfur based reagents could be further enhanced.

In this regard in-group knowledge on the research of electron poor cationic phosphines gave the decisive motivation, since the tuning of the phosphine ligands follows a similar rationale: 
By exchanging imidazolium to pyridinium in the backbone of the phosphine ligands, which are used for $\pi$-acid catalysis, these are rendered more electron deficient. ${ }^{[159]}$

Logically, by exchanging the imidazolium backbone to an even more electron withdrawing moiety, the pyridinium, an electrophilic sulfur based transfer reagent with extended reactivity will be accessible.

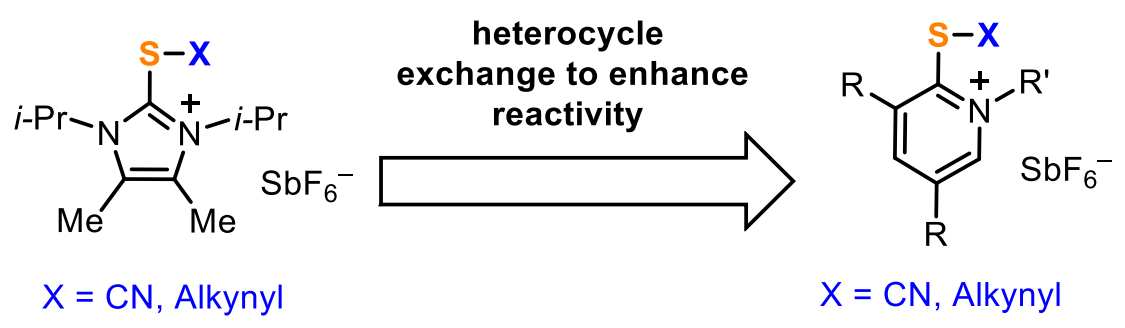

Scheme 59: Extension of reactivity by heterocycle exchange.

Thus the aims of this project can be formulated as follows:

1. A synthesis sequence to access novel sulfur based electrophilic transfer reagents, which contain the pyridinium moiety as key motif, will be developed.

2. Hypervalent dihalo(pyridinium) species $\mathbf{1 5 4}$ will be used as a key intermediates en route to electrophilic transfer reagents. They are expected to posess similar properties compared to those of known dihalo(imidazolioumsulfuranes) and will be subjected to structural investigations in order to evaluate their electronic nature.

3. From 154, pyridinium thiocyanates will be synthesized and their behavior towards nucleophiles will be tested, aiming to extend the scope of electrophilic cyanation reactions.

4. Analogously the synthesis of alkynylthiopyridinium salts from $\mathbf{1 5 4}$ will be developed and their reactivity in the presence of nucleophiles will be investigated. 
With the established knowledge for the synthesis of the original imidazolium based electrophilic cyanating reagent in hand (Scheme 60-I), a synthetic route for pyridinium based transfer reagents is envisioned (Scheme 60-II).

(I)

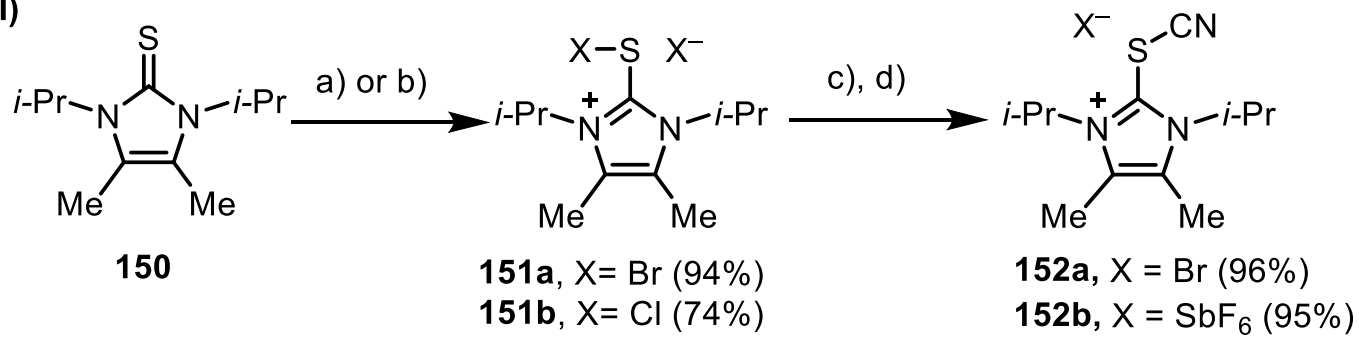

(II)<smiles>[R]c1cc([R])c(=S)n([R1])c1</smiles><smiles>[Y][Y16]1sc2[R]c([R])cc([R])c12</smiles>

c), d)<smiles>[R][R]1cc([R])c[n+]([R])c1SC#N</smiles>

153a, $R^{1}=M e, R^{2}=H$

154a, $R^{1}=M e, R^{2}=H, X=B r$

155a, $R^{1}=E t, R^{2}=M e, X=B r$

153b, $R^{1}=E t, R^{2}=H$

154ba, $R^{1}=E t, R^{2}=H, X=B r$

155b, $R^{1}=E t, R^{2}=M e, X=S b F_{6}$

153c, $R^{1}=E t, R^{2}=M e$

154bb, $R^{1}=E t, R^{2}=H, C=C l$

154c, $R^{1}=E t, R^{2}=M e, X=B r$

Scheme 60: (I) Sequence developed by the ALCARAZO group for the synthesis of imidazolium-substituted sulfuranes 152 and (II) tentative analogous synthetic route to access pyridinium thiocyanates 155 . Reaction conditions: a) $\mathrm{SO}_{2} \mathrm{Cl}_{2}, \mathrm{CH}_{2} \mathrm{Cl}_{2}, \mathrm{RT}$; b) $\mathrm{Br}_{2}, \mathrm{CH}_{2} \mathrm{Cl}_{2}, 0^{\circ} \mathrm{C} \rightarrow \mathrm{rt}$; c) TMSCN, $\mathrm{CH}_{2} \mathrm{Cl}_{2}, \mathrm{rt}$, d) aq. $\mathrm{NaSbF}_{6}$. 


\section{Synthesis, Characteristic Properties and Application of Novel Pyridinium-Based Electrophilic TRANSFER REAGENTS}

Setting out from 2-chloropyridine, $\mathrm{N}$-alkylation was achieved in high yields employing trialkyloxonium tetrafluoroborates as the alkylating reagents. Afterwards introduction of the sulfur atom was achieved by using sodium hydrogen sulfide giving the thiolactams 153a and 153b in 90 and $82 \%$ yields, respectively (Scheme 61).

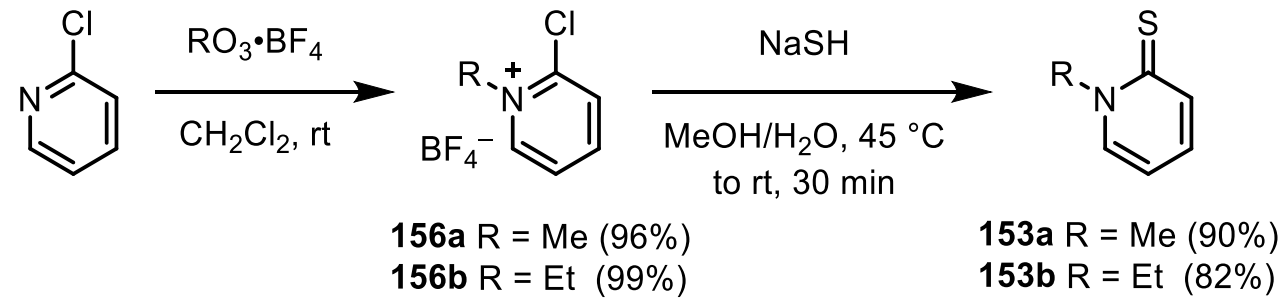

Scheme 61: Synthesis sequence towards the thiolactam backbones 153 starting from 2-chloropyridine.

With 153b in hand, the synthesis of the hypervalent dihalo(pyridinium)sulfuranes was attempted by chlorination with sulfuryl chloride (Scheme 62). ${ }^{1} \mathrm{H}$ NMR analysis of the formed product showed a deep field shifted doublet for the proton in $\alpha$-position to the cationic nitrogen $(\delta=10.71 \mathrm{ppm}$, initial shift $7.67 \mathrm{ppm})$, which is characteristic for highly electrondeficient pyridinium species. However, poor solubility and instability of the product towards air, moisture and protic solvents made a full characterization by ${ }^{13} \mathrm{C} N M R$ spectroscopy as well as by high resolution mass spectroscopy impossible. Nevertheless the target mass could be observed in traces, thus encouraging further investigations of this synthetic route. After several crystallization attempts a crystal suitable for analysis by X-Ray diffractometry was obtained and revealed $\mathrm{S}_{8}$ as the major product of decomposition of this extremely unstable compound (Scheme 62).

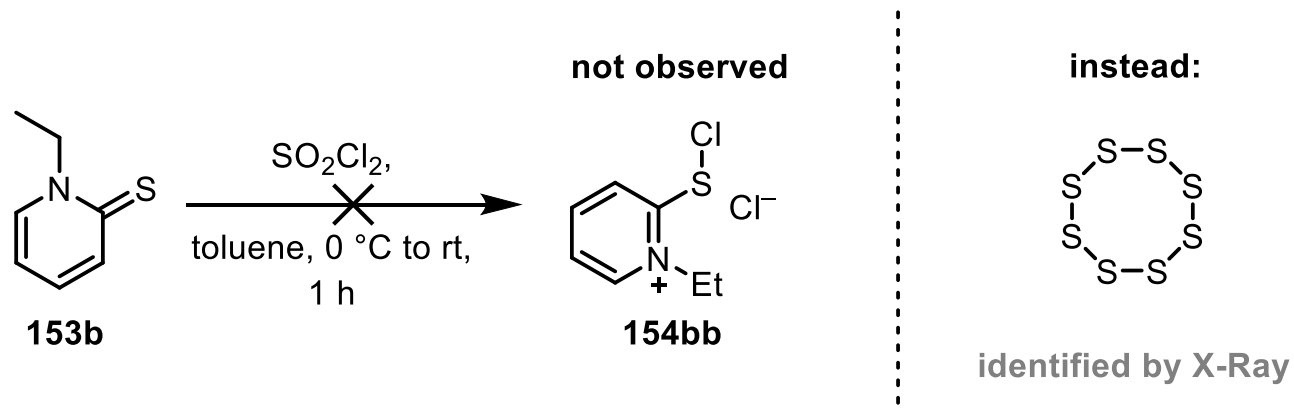

Scheme 62: Attempted synthesis of dichloro(pyridinium)sulfurane 154bb.

In an attempt to generate the dichloro(pyridinium)sulfurane 154bb in situ and directly utilize it in the nucleophilic substitution with TMSCN, more crystals were obtained, comprising another part of the decomposition pathway (Scheme 63 (I)). The analyzed structure gave important insights into the electronic properties and thus the preferential reactivity of this hypervalent pyridinium species. It is assumed that during the reaction a pyridinium species 
partly becomes so electron depleted, that the chloride anion is nucleophilic enough to attack the carbon atom in $\alpha$-position to the sulfur and thus cleaving out the sulfur. Formally the employed reaction sequence is hence reversed giving, 2-chloro-1-ethyl-pyridinium. By taking the electronegativity of a nitrile group into account, which can be calculated and scaled to be compatible with the Pauling scale, a nitrile group possesses an electronegativity (EN) of 3.3, compared to chlorine with 3.16 and bromine with 2.96. ${ }^{[160]}$ Thus the potential electrophilic cyanating reagent $\mathbf{1 5 5}$ is the most electron depleted species of these three compounds formed in the reaction and most prone to undergo nucleophilic aromatic substitution.

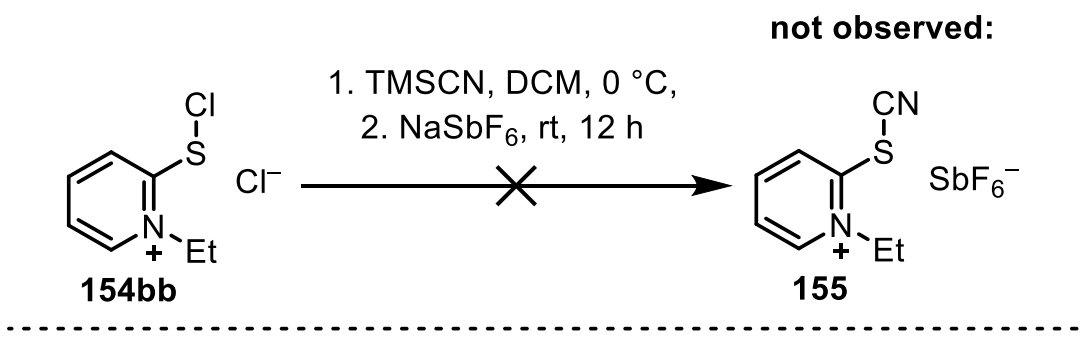

identified by X-Ray instead:

(l)<smiles>CC[n+]1ccccc1Cl</smiles>

$156 d$
(II) $2 \mathrm{SbF}_{6}^{-} \quad \mathrm{Et}$<smiles></smiles>

157

Scheme 63: Attempted syntheses of pyridinium thiocyanates starting from dichloro(pyridinium)sulfurane $154 \mathrm{bb}$.

Crystals obtained in another attempt to synthesize the electrophilic cyanating reagent 155 revealed to be dicationic species 157 (Scheme 63 (II)). This further underlines that an aromatic substitution at the pyridine is favoured and that the pyridinium moiety preferably acts as a leaving group. Additional attempts to access nitrile transfer reagents, were unsuccessful, neither anion exchange with an aqueous $\mathrm{NaSbF}_{6}$ solution nor the exchange in acetonitrile gave the desired compound.

It can be concluded, that $\mathbf{1 5 4 b b}$ is indeed formed in the reaction sequence, since subsequent reactions gave products which are a direct result of the formation of this highly reactive intermediate. However its instability under the conditions elaborated, prevented any further analysis.

Having identified, that the relatively high electronegativity of the chloride anion is one of the driving factors, which promotes a nucleophilic attack in $\alpha$-position to the sulfur, and not in the desired position, the most logical alternative was to attempt bromination instead. The resulting product, a dibromo(pyridinium) sulfurane 154ba, then holds the less electron withdrawing bromide.

The synthesis of $\mathrm{N}$-ethyldibromo(pyridinium) sulfurane (154ba) proved to be significantly more gratifying, giving instant precipitation of the desired product at $0{ }^{\circ} \mathrm{C}$. The target 
compound could be successfully characterized by NMR spectroscopy, and two additional derivatives, 154a and 154c, were synthesized (

Scheme 64). For the latter compound, a concise synthesis starting from 3,5-lutidine was designed (

Scheme 64, (II)).

(l)

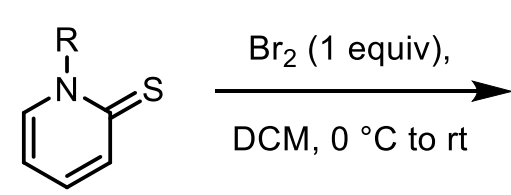<smiles></smiles>

153a: $R=M e$

154a: $R=M e(99 \%)$

153b: $R=E t$

154ba: $R=E t(99 \%)$

(II)<smiles>CCN1C=C(C)C=C(C)C1</smiles>

$156 \mathrm{c}$

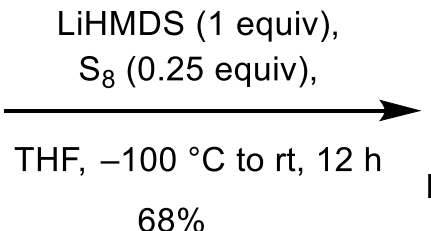

$68 \%$<smiles>CCn1cc(C)cc(C)c1=S</smiles>

$153 c$<smiles></smiles>

$154 c$

Scheme 64: Synthesis of dibromo(pyridinium) sulfuranes 154.

Deprotonation of the salt $156 \mathrm{c}$ in $\alpha$-position to the nitrogen with lithium hexamethyldisilazide followed by treatment with sulfur afforded thiolactam 153c in $68 \%$ yield. Bromination of $153 \mathrm{c}$ proceeded quantitatively at $0{ }^{\circ} \mathrm{C}$ to give the hypervalent dibromo(thiolutidinium) salt 154c.

We hypothetized that due to the higher sterical encumberment and the +1 stabilizing effect of the methyl groups, disfavouring the aforementioned decomposition pathway, this derivative will possess an increased stability as well as better solubility in organic solvents. Indeed the strategy proved to be successful and crystals of the dibromo(lutidinium) sulfurane (Martin-Arduengo N-X-L designation: $\left.{ }^{[161]} 10-S-3\right)$ 154c were grown and analyzed by X-ray crystallography (Figure 1). 


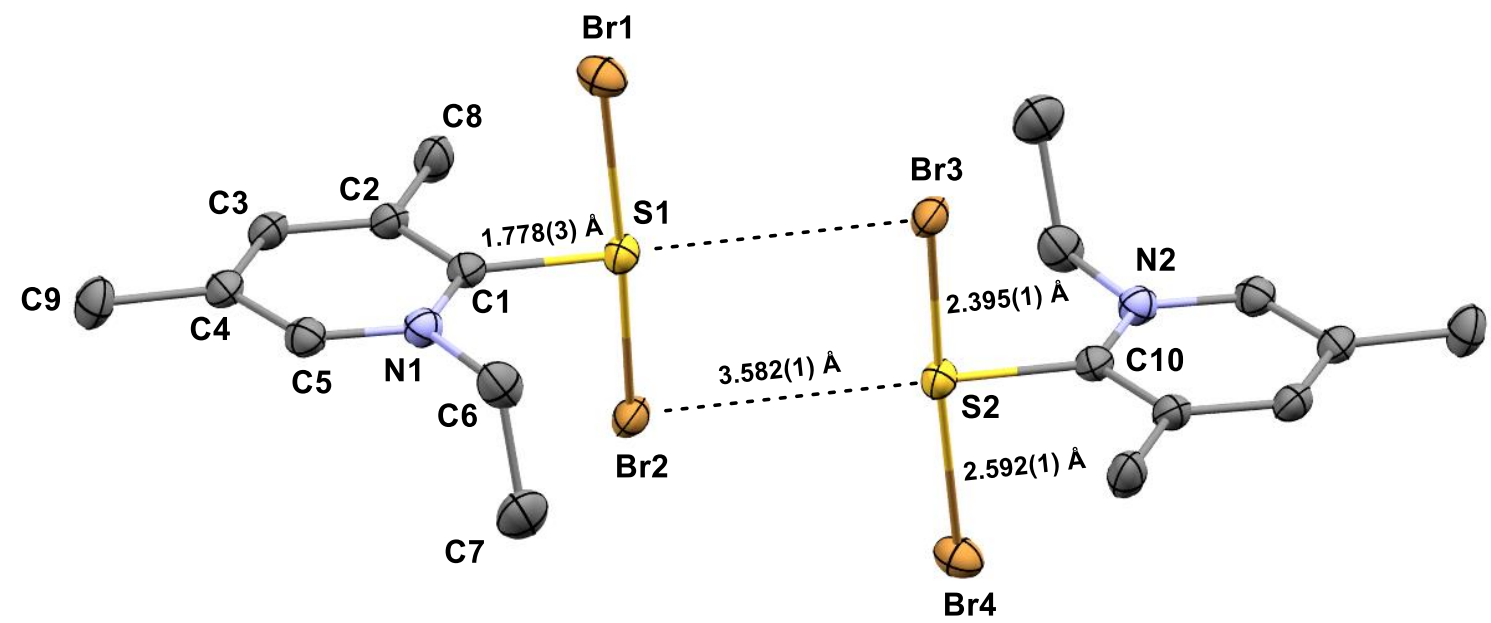

Figure 1: Structure of compound 154c in the crystal. Anisotropic displacement parameter shown at 50\% probability level, hydrogen atoms omitted for clarity. Selected bond lengths [Å] and angles [deg]: S1-Br1, 2.592(1); S1-Br2, 2.395(1); S1-C1, 1.778(3); Br1-S1-Br2, 177.50(3).

The obtained structure showed the typical T-shaped geometry similar to that in hypervalent dihalo(imidazolium) and iodonium salts (Scheme 58). Minor deviation from the ideal $180^{\circ}$ does take place $\left[177.5^{\circ}(3)\right]$ for $\mathrm{Br} 1-\mathrm{S} 1-\mathrm{Br} 2$ angle. However, of much higher interest is the

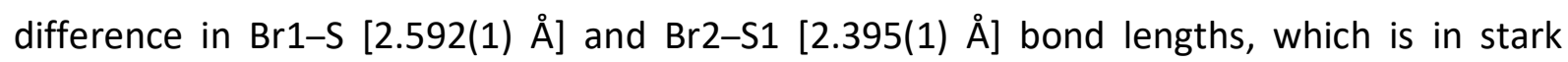
contrast to the even distances for $\mathrm{Br} 1-\mathrm{S} 1-\mathrm{Br} 2$ in a dibromoimidazolium sulfurane. Upon closer investigation and identification of the most proximal contacts, we found that the compound organizes itself as a chalcogen bonding (ChB) complex: ${ }^{[162]}$ An angle of $175.7^{\circ}$ for $\mathrm{C} 1-\mathrm{S} 1-\mathrm{Br} 3$ (and $\mathrm{Br} 2-\mathrm{S} 2-\mathrm{C} 10$, respectively) is observed, and strong positive polarization of the sulfur atom is facilitated by the pyridinium ring. The neighboring bromine atoms serve as Lewis Base (LB), interacting with the sulfur through partial donation of their electron density. This results in a shorter bond length towards the covalent bound sulfur, while for the remaining two bromine atoms elongated bonds to the sulfur centerpiece are observed.

While no further investigations towards the potential existence of charge-shift bonds in this compound have been undertaken, it shares significant similarities with compounds described to possess such. ${ }^{[163]}$

Successive synthesis of the lutidinium thiocyanate was achieved. In a first step the pyridinium thiocyanate 155a bearing a bromide anion was synthesized by nucleophilic substitution with TMSCN in $96 \%$ yield. Anion exchange with $\mathrm{SbF}_{6}{ }^{-}$then gave $155 \mathrm{~b}$ in a yield of $84 \%$. Unfortunately, this result was not reproducible in several attempts. 
(I)

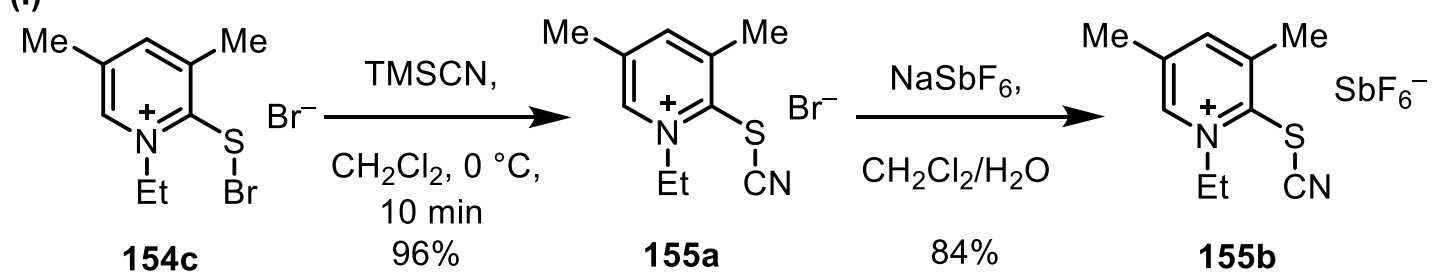

(II)

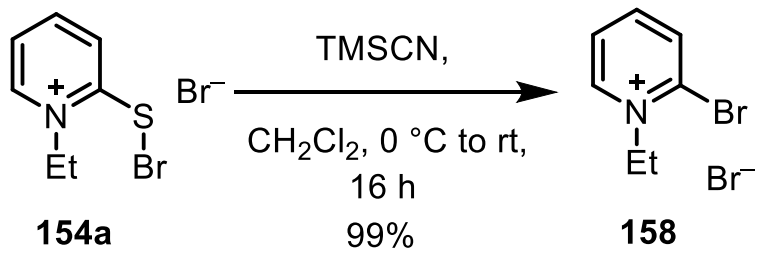

Scheme 65: Synthesis of lutidinium thiocyanate $\mathbf{1 5 5}$ and attempted synthesis of pyridinium thiocyanate.

During the unsuccesful attempts, at least three different pyridine species were formed during reaction progress, as could be observed in ${ }^{1} \mathrm{H}$ NMR spectra. Purification was possible neither applying various solvent combinations for crystallization nor by column chromatography. We suspected that all of the formed compounds are salts, due to their behavior on thin layer chromatography. This could partly be verified by stirring a solution of 154a in the presence of TMSCN for $16 \mathrm{~h}$. Full conversion to 2-bromo-1-ethylpyridinium bromide (158) was observed, indicating that the reaction time is a crucial parameter (Scheme 65 (II)). Nevertheless, compound 155b was successfully crystallized. Notably crystals could also be obtained from less successful reactions in which byproducts had formed, albeit isolation of the pure substance in significant amounts in these cases was possible neither by crystallization nor by column chromatography.

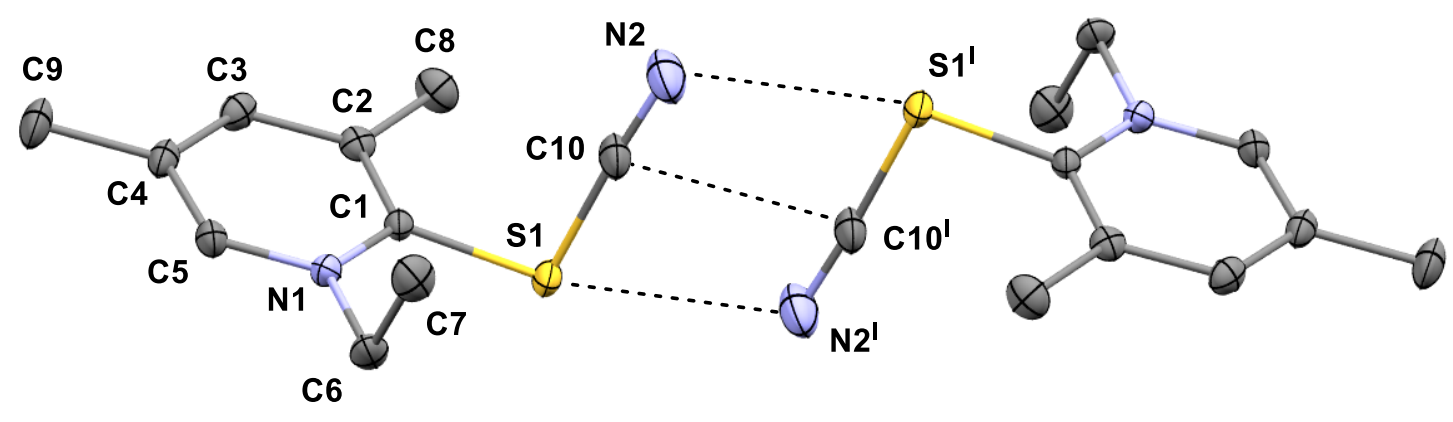

Figure 2: Crystal structure of 155b, Anisotropic displacement parameter shown at $50 \%$ probability level, hydrogen atoms and counterions omitted for clarity (Symcode: $I=-x, 1-y, 1-z)$.

The reactivity of the reagents $\mathbf{1 5 5}$ towards different nucleophiles was tested as well. Reaction conditions similar to those optimized for the analogue imidazolium thiocyanate $\mathbf{1 5 2}$ were chosen. It was found that the reagent 155a is active in the cyanation of $\mathrm{N}$ - 
isopropylbenzylamine, however, giving product 159 in lower yield of 60\%, when compared to the reaction with imidazolium thiocyanate reagent 152a (92\% after 20 min.) (Scheme 66). This is presumably due to the lower stability of the pyridinium thiocyanate. In additional experiments it was demonstrated that compound 155b decomposes in solution at temperatures above $50{ }^{\circ} \mathrm{C}$. This result was further confirmed by a control experiment in which the reagent was heated in $d_{3}$-acetonitrile to $80^{\circ} \mathrm{C}$ to recreate reaction conditions and lead to decomposition of the reagent (Scheme 66).

(l)<smiles>CC(C)NCc1ccccc1</smiles>

(II)<smiles>COc1cccc(OC)c1</smiles>

a)<smiles>CC(C)N(C)Cc1ccccc1</smiles>

a) or b)<smiles>CCC(C)(C)C</smiles><smiles>COc1ccc(C#N)c(OC)c1</smiles>

Scheme 66: Cyanation of $\mathrm{N}$-benzyl- $\mathrm{N}$-isopropylamine and attempted reactions with dimethoxybenzene. Reagents and conditions: a) lutidinium thiocyanate bromide 155a (1.2 equiv), DIPEA (1.1 equiv), $\mathrm{CH}_{2} \mathrm{Cl}_{2}, \mathrm{rt}, 20$ min; b) lutidinium thiocyanate $155 \mathrm{~b}$ (1.2 equiv), $\mathrm{BF}_{3} \cdot \mathrm{Et}_{2} \mathrm{O}$ (0.2 eq.), 1,2-DCE, $80{ }^{\circ} \mathrm{C}, 16 \mathrm{~h}$.

Due to these results, further investigations were focused on potential alkynylating reagents with a pyridinium backbone with the purpose to obtain more stable compounds. The electronegativity of a nonsubstituted acetylene group is calculated to be $3.3, i$. e. the same as of a nitrile group. This means that theoretically any transfer reagent with an unprotected acetylene moiety should be equally unstable. On the other hand, we expected to decrease this value by substituting the triple bond with electron-rich groups and to obtain more practically useful compounds. ${ }^{[160]}$ With this rationale in mind, phenylacetylene and (triisopropylsilyl)acetylene were chosen for the synthesis of the corresponding reagents. Applying a modified strategy adapted from the thioimidazolium-based reagent, gratifiyingly several promising thiopyridinium-based acetylene transfer reagents were synthesized (Scheme 67). 


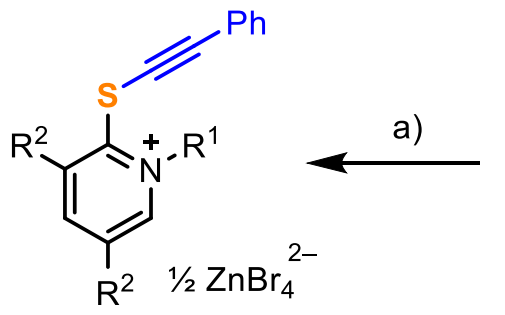

$160 a ; R^{1}=M e, R^{2}=H(39 \%)$

$160 b ; R^{1}=E t, R^{2}=H(87 \%)$

$160 c ; R^{1}=E t, R^{2}=\operatorname{Me}(71 \%)$<smiles>[R]c1cc([R])c(SBr)[n+]([R])c1</smiles>

154

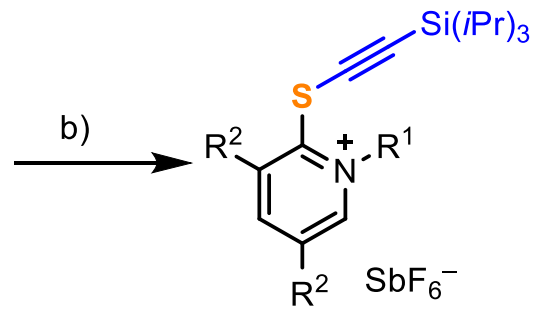

$160 d ; R^{1}=M e, R^{2}=H(65 \%)$

Scheme 67: Synthesis of alkynylthiopyridinium salts 160. Reaction conditions: a) phenylacetylene (1.1 equiv), $n$ BuLi (1.1 equiv), THF, $-78^{\circ} \mathrm{C}$ to rt, then $\mathrm{ZnBr}_{2}$ (1.1 equiv); b) TIPS-C $\mathrm{C} \equiv \mathrm{CH}$ (1 equiv), $n$-BuLi (1 equiv), THF,$-78^{\circ} \mathrm{C}$ to $\mathrm{rt}$, then $\mathrm{ZnBr}_{2}$ and finally aq. $\mathrm{NaSbF}_{6}$. TIPS = triisopropylsilyl.

Thus, compounds $160 \mathrm{a}-\mathrm{c}$ were isolated in moderate to good yields of 39,87 and $71 \%$, respectively. Attempted replacing of the counterion $\mathrm{ZnBr}_{4}{ }^{2-}$ with the non-coordinating $\mathrm{SbF}_{6}{ }^{-}$ one was unsuccessful for phenylacetylene-substituted reagents $160 \mathrm{a}-\mathrm{c}$, whereas counterion exchange underwent smoothly for the (triisopropylsilyl)acetylene-substituted derivative affording compound $160 \mathrm{~d}$ (Scheme 67). The TIPS-reagent $160 \mathrm{~d}$ could be synthesized in an overall yield of $65 \%$ over two steps.

A possible explanation for the unsuccessful counterion exchange might be the difference in lattice energy, which correlates with the ion size and thus should be smaller for $160 \mathrm{~d}$ than for $160 \mathrm{a}-\mathrm{c}$, making the anion exchange easier to proceed.

Crystallization of the alkynyl pyridinium salts unambiguously verified their connectivities (Figure 3). 

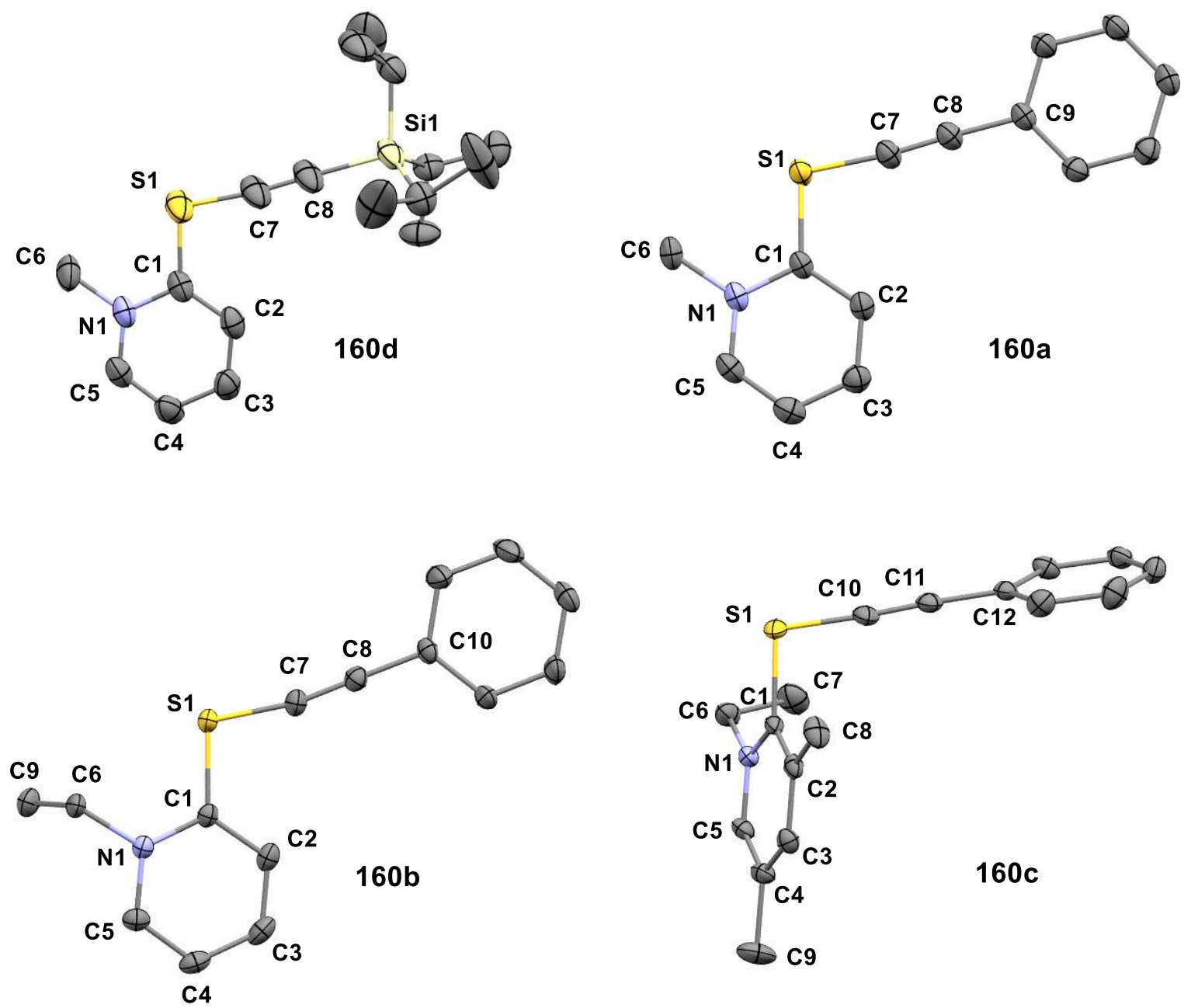

Figure 3: Structures of the salts $160 a-d$ in the crystal. Anisotropic displacement parameter shown at $50 \%$ probability level, hydrogen atoms and anions omitted for clarity.

Once characterization of the alkynylthiopyridinium salts was complete, their chemical behavior towards different kinds of nucleophiles was examined.

Already preliminary reactions between reagent $160 \mathrm{~b}$ and enamines gave intriguing results.

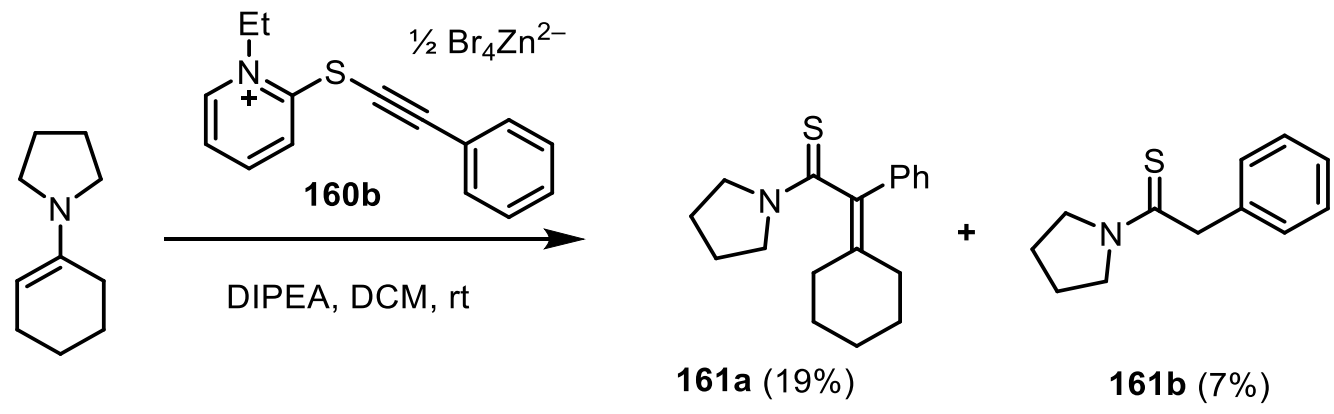

Scheme 68: Reaction of alkynylthiopyridinium salt 160b with 1-(cyclohex-1-en-1-yl)pyrrolidine.

We presume that upon slow hydrolysis of the substrate during the quenching process after the reaction, cyclohexanone and pyrrolidine formed and then subsequently reacted. This 
also explains the low yields, as hydrolysis of the starting material only sluggishly provides the reactants.

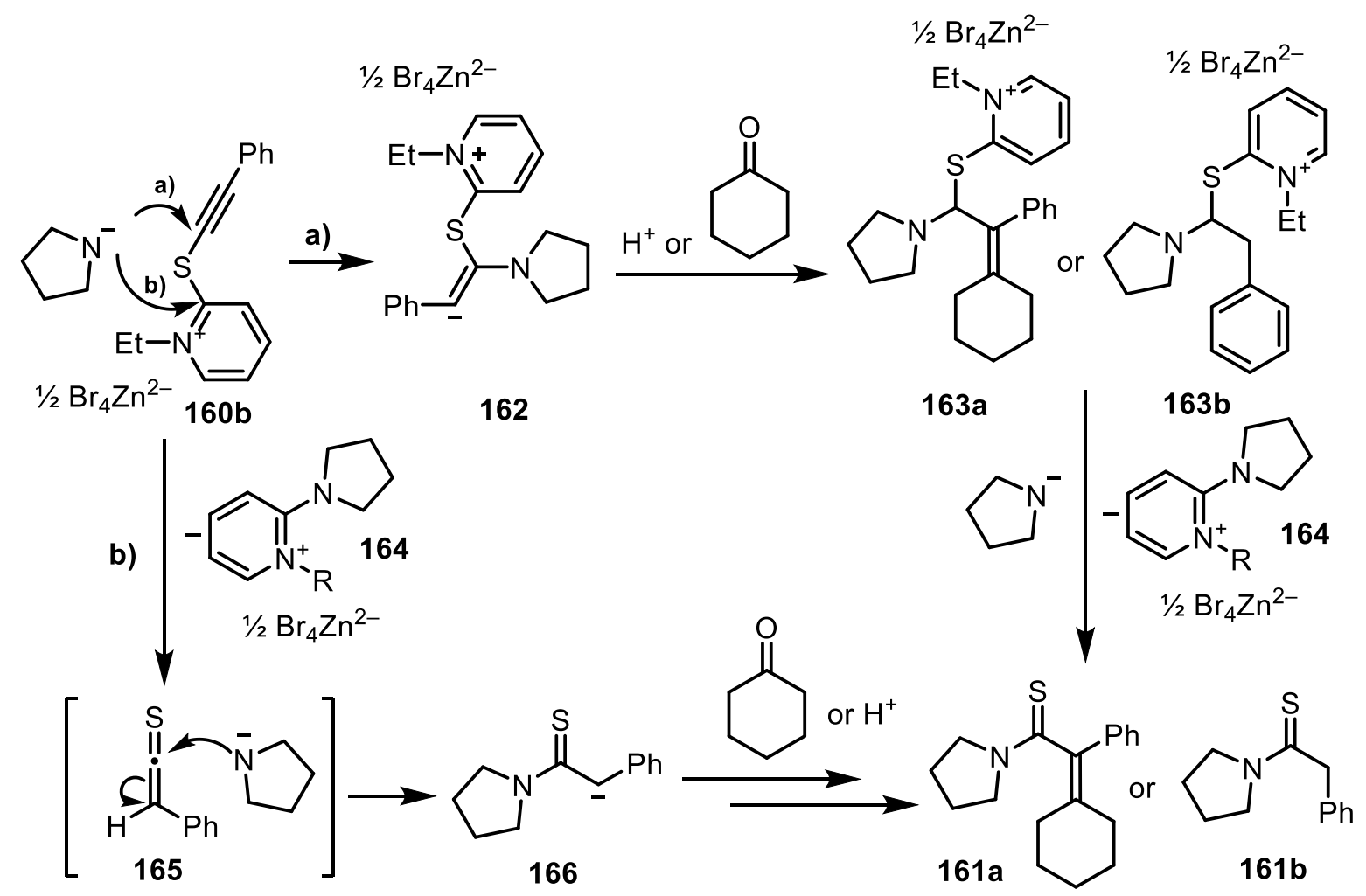

Scheme 69: Feasible mechanistic considerations.

Therefore two possible mechanistic pathways can be considered: After initial deprotonation of the pyrrolidine by Hünig's base, nucleophilic attack of the formed anion to either $\alpha$ positioned carbon atoms with respect to the sulfur can take place.

a) If the pyrrolidide attacks the acetylenic $\alpha$-position first, intermediate $\mathbf{1 6 2}$ is formed. The latter then reacts as nucleophile, either with a proton source or cyclohexanone to give product $163 \mathrm{a}$ or $163 \mathrm{~b}$ after elimination of a pyridine moiety 164 by reaction with a second equivalent of pyrrolidide.

b) If the nucleophilic attack takes place at the aromatic ring, thioketene $\mathbf{1 6 5}$ is formed. Subsequently, an additional equivalent of pyrrolidide then attacks this species to give intermediate 166, which, in turn, reacts with either cyclohexanone or a proton source to produce product $161 \mathrm{a}$ or $161 \mathrm{~b}$, respectively.

The results of the reaction illustrate that not only protons but also other compounds can serve as electrophiles in this transformation.

While these results were already quite informative from the viewpoint of the agents reactivity, more information about the behavior of the alkynylthiopyridinium salts $\mathbf{1 6 0}$ could be gathered when thiols were investigated as nucleophiles. Thus, with one equivalent of deprotonated 4-(methoxyphenyl)methanethiol (167), the successive reaction of the reagent $160 \mathrm{~d}$ gave a 1:1 mixture of dithioester $168 \mathrm{a}$ and pyridinium salt $168 \mathrm{~b}$ which presumably is the result of a $S_{N} A r$ reaction of the substrate with the carbon of the pyridinium ring in $\alpha$ - 
position to sulfur. We also observed that half an equivalent of the salt $160 \mathrm{~d}$ was still intact. For a complete conversion two equivalents of thiol were necessary, as optimization reactions revealed.<smiles>COc1ccc(CSc2cccc[n+]2C)cc1</smiles>

Scheme 70: Reaction of reagent 160d with thiol 167.

This reactivity could be proven to be general for aliphatic and aromatic thiols 168a-175 (

Scheme 71). Furthermore, the reaction scope could be extended to primary and secondary aliphatic amines 176-181. It is worth mentioning that no additional base is required to start the reaction with amines. However in order to drive the reaction to completion, equimolar amounts of DIPEA were necessary.

Double functionalization of dithiols and of substrates with the amine and thiol functionalities was possible as well. Noteworthy, in the product $\mathbf{1 8 1}$ obtained from cysteine ethyl ester, the original stereochemistry of the latter remained unchanged. It could also be shown that the reaction tolerates carboxylic acids, nitro groups and ethers. Valuable building blocks for prodrugs like morpholine and tryptamine underwent the reaction smoothly as well.

While small alcohols like methanol or tert-butanol gave no product, the phenolic constituent of $17 \alpha$-ethynylestradiol entered the transformation without observation of competing reactivity on the tertiary alcohol or on the alkyne moieties, despite the low yield of $\mathbf{1 8 2}$. However, for a more extended aromatic system the reaction went smoothly: The softer alcoholate generated from 2-naphthol gave the corresponding thionoester $\mathbf{1 8 3}$ in good yield of $59 \%$. When aromatic amines were investigated as nucleophiles, rapid characteristic thioamide formation could be observed by TLC. However, due to the presence of two equivalents of aniline in the reaction mixture, subsequent condensation of the second nucleophile takes place and gives the corresponding acetamidine $184 .^{2}$ In addition it should be pointed out, that slow hydrolysis to the amide takes place in the presence of water and base.

\footnotetext{
${ }^{2}$ To isolate the product on the thioamide stage, reaction progress needs to be monitored accurately and the reaction has to be quenched at the appropriate time.
} 


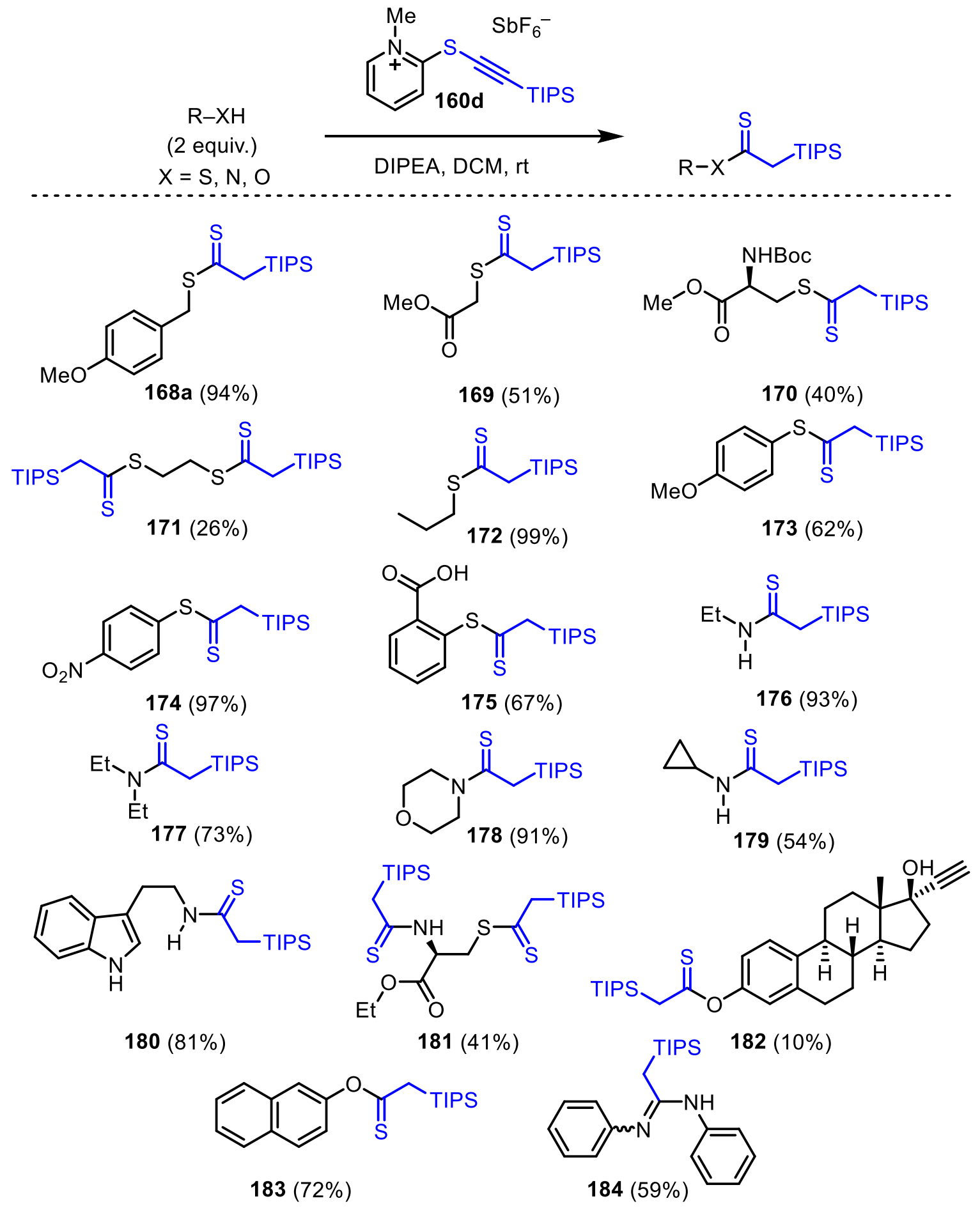

Scheme 71: Scope of $\mathrm{S}_{-}, \mathrm{N}$ - and $\mathrm{O}$-nucleophiles (isolated yields with respect to the reagent, if not otherwise mentioned ${ }^{3}$ ).

During the investigation of the general reactivity of the reagent $160 \mathrm{~d}$, a number of unusual results were obtained. When thiobenzoic acid was employed as a substrate, the alkynylation product 185 was isolated exclusively (Scheme $\mathbf{7 2}$ ). We presume that due to the more delocalized negative charge, the preferred target of attack is the softer $\alpha$-position of the

\footnotetext{
${ }^{3}$ See experimental part Topic:" Synthesis and Evaluation of Novel Pyridinium-BASEd Electrophilic Transfer REAGENTS" for details.
} 
alkyne, which gives product $\mathbf{1 8 5}$ after elimination of the thiopyridone. Similar selectivity was observed with tritylthiol, in which the sterical encumberment plays the decisive role for the formation of alkyne product 186 (Scheme 72).

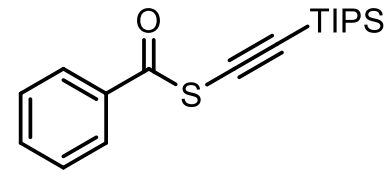

$185(100 \%)$

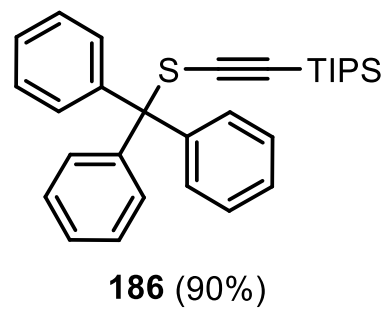

$186(90 \%)$

Scheme 72: S-Alkynylation of thiobenzoic acid and tritylthiol.

As the reagent showed reactivity reminiscent to that of thioketenes, further reactions potentially verifying this parallelism were investigated. Thioketenes and their surrogates are known to undergo cycloadditions with various substrates. In order to test the reagents behavior in this regard, a number of potential candidates for cycloaddition were tested. From examined unpolarized dienes, 2,3-dimethylbutadiene, norbornadiene and 1,3-dipole benzylazide no reactivity was observed. Surprisingly, when benzo[d]thiazole-2-thiol was employed as a nucleophile, the zwitterionic product 187 was obtained in $22 \%$ yield instead of the expected formation of a dithioester (Scheme 72-I).

(I)

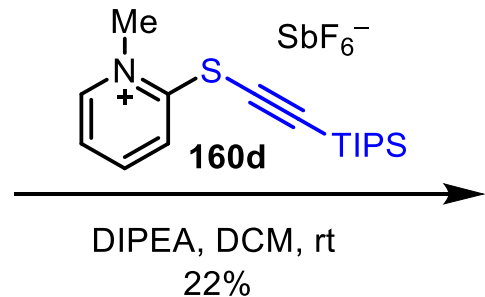

(II)<smiles>Sc1ncccn1</smiles>

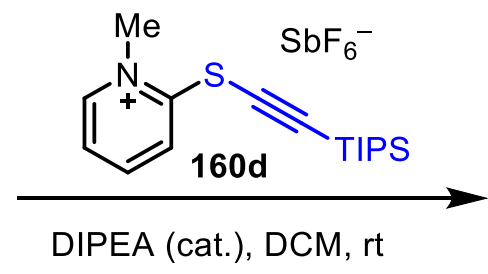

$3 \%$<smiles>[S-]c1c([I-])sc2sc3ccccc3[n+]12</smiles>

187<smiles>C[C@@H](S)/C=C(\SC(=S)C[In])Sc1ncccn1</smiles>

Scheme 73: Unexpected cycloaddition and twofold functionalization with catalytic amounts of base.

In order to validate if this reactivity is general, pyrimidine-2-thiol was employed as substrate. However, after an instant colour change of the solution was observed upon addition of the first drop of a base, further addition was stopped, and it was decided to monitor the progress of the reaction in the presence of only catalytic amounts of a base. Surprisingly, only product $\mathbf{1 8 8}$ was isolated in a low yield of $3 \%$. It is feasible to assume that in this case the intermediate aldothioketene is so highly reactive, that a direct subsequent reaction step, which leads to formation of the observed product, takes place. When the same experiment (Scheme 73-II) was conducted with stochiometric amounts of base, no cycloaddition 
products related to $\mathbf{1 8 7}$ were observed. The structure of $\mathbf{1 8 8}$ could be unambiguously confirmed by X-ray diffractometry (Figure 4).

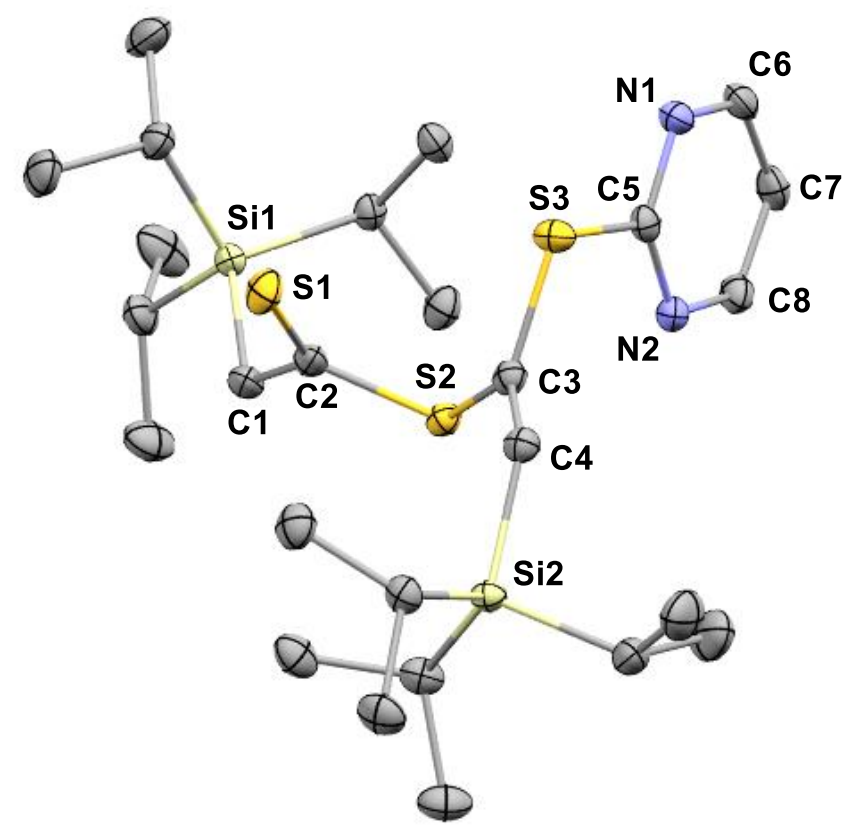

Figure 4: Crystal structure of 188, Anisotropic displacement parameter shown at 50\% probability level, hydrogen atoms omitted for clarity.

With 2,4,6-trimethylbenzoic acid as a nucleophile, an inseparable mixture of two compounds was obtained (Scheme 74). One of which could be crystallized to obtain its solid state structure, which revealed to be the anhydride $\mathbf{1 8 9}$ of the starting material. We assume that, once thiopyridone is eliminated during the reaction progress, a nucleophilic attack on the carbonyl atom by sulfur takes place. An activated intermediate is formed, which then undergoes acylation by another equivalent of substrate (mercaptopyridines are known for their application as acylating agents). ${ }^{[164]}$ In a similar fashion thioanhydrides can be synthesized starting from thioesters and 2-chloroalkylpyridinium salts. ${ }^{[109,165]}$

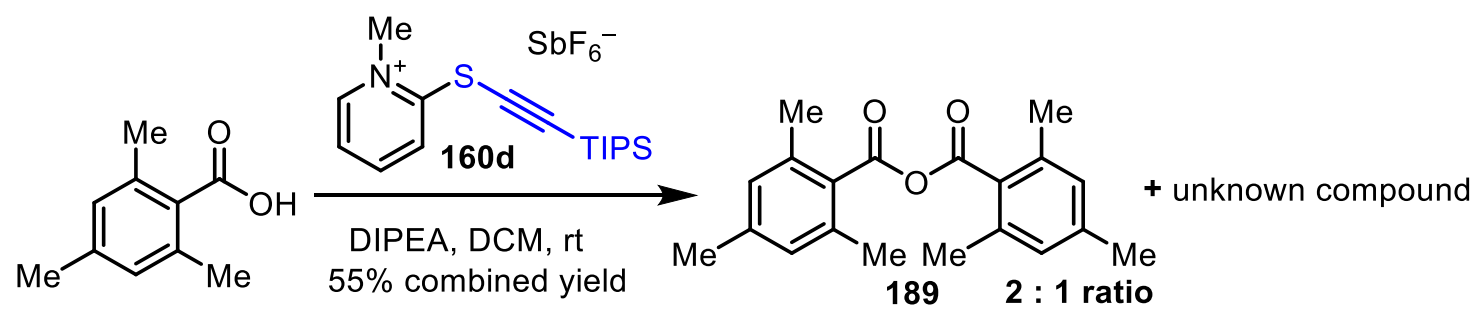

Scheme 74: Changed reactivity for sterically demanding benzoic acid derivative.

It can be concluded that an aldothioketene or a surrogate thereof is an intermediate in the major product formation pathway of alkynylthiopyridinium salts. Further proof for this hypothesis gave the reaction with a cyclic $\beta$-ketoester, in which an adduct of the substrate onto the reagent formed. This "stuck-intermediate" 190 which we assume is too sterically demanding to undergo elimination of the pyridinium moiety, appeared to be quite stable in 
our hands, as it even survived purification by column chromatography. Its absolute configuration could be determined via NOE and HSQC-NMR experiments.<smiles>[R]OC(=O)C1Cc2ccccc2C1=O</smiles>

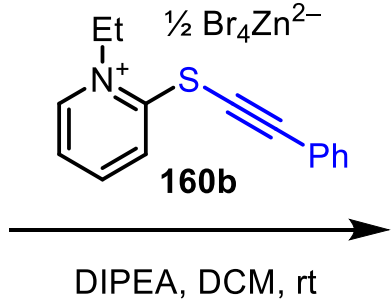

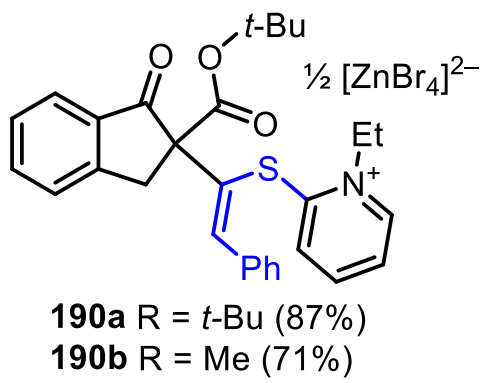

Scheme 75: Product of addition of a cyclic $\beta$-ketoesters onto the pyridinium reagent $160 \mathbf{b}$.

In order to gather an additional mechanistic evidence, we hypothetized that a relatively weak and small nucleophile such as the fluoride anion in $(\mathrm{Bu})_{4} \mathrm{NF}$ should be strong enough to

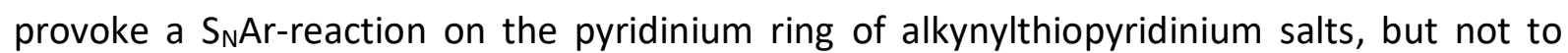
participate in the reaction with the formed thioketene. Therefore, we made use of the phenylacetylene thiopyridinium salt $160 \mathrm{~b}$, which is not subject to decomposition by a fluoride source (in contrast to $\mathbf{1 6 0 d}$ ). Indeed, we were able to observe the formation of an isomeric mixture of cis- and trans-dithiafulvenes 191, which supports our hypothesis of aldothioketene formation (Scheme 76).

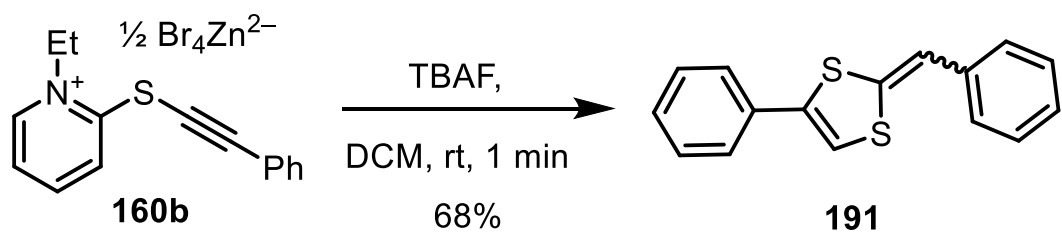

Scheme 76: Formation of dithiafulvenes 191 upon reaction of $160 \mathrm{~b}$ with TBAF.

An experiment with the deuterated substrate could prove that in 192 the protons in $\beta$ position to the sulfur originate from the nucleophile (Scheme 77).<smiles>COc1ccc([Se])cc1</smiles>

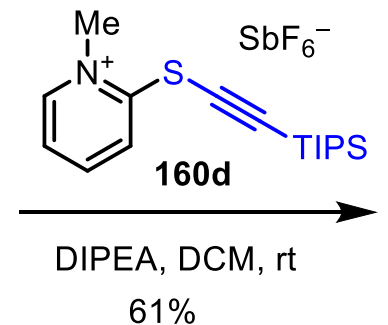

$61 \%$<smiles>[2H]C([2H])([18F])C(=S)Sc1ccc(OC)cc1</smiles>

Scheme 77: Experiment with deuterated $p$-methoxythiophenol.

Based on the formerly presented investigations, two plausible mechanistic rationalizations for the reaction are considered (Scheme 78). Initial deprotonation by a base is followed by the nucleophilic attack of the nucleophile. 
a) When nucleophilic attack takes place at the acetylenic $\alpha$-position of sulfur, an olefinic intermediate $\mathbf{1 9 3}$ is formed. Successive attack of another nucleophile then gives product 194 after elimination of pyridinium species 195.

b) If the nucleophile attacks the pyridinium carbon in $\alpha$-position of sulfur first, an aldotioketene is generated as intermediate 196. This intermediate is then attacked by a second equivalent of nucleophile to give product 194 .
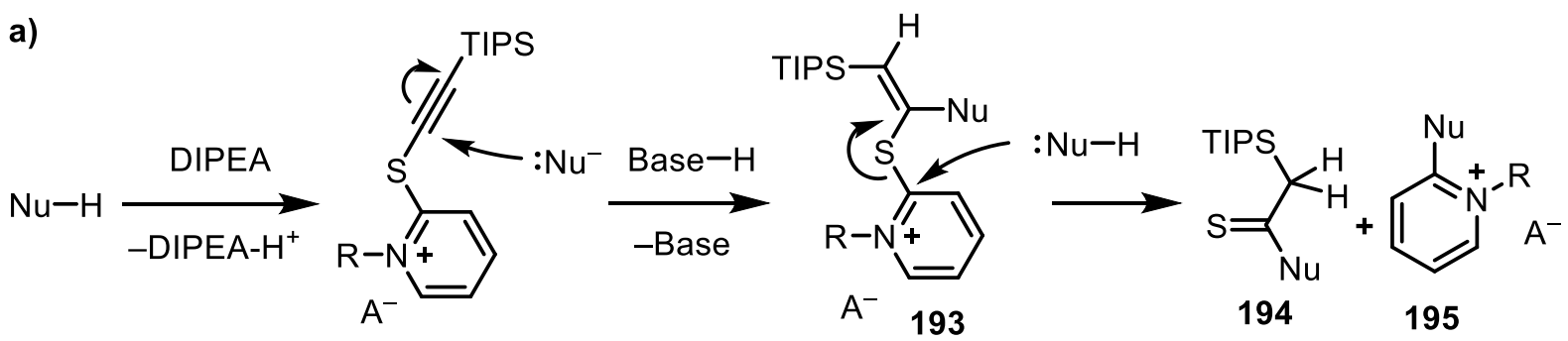

b)
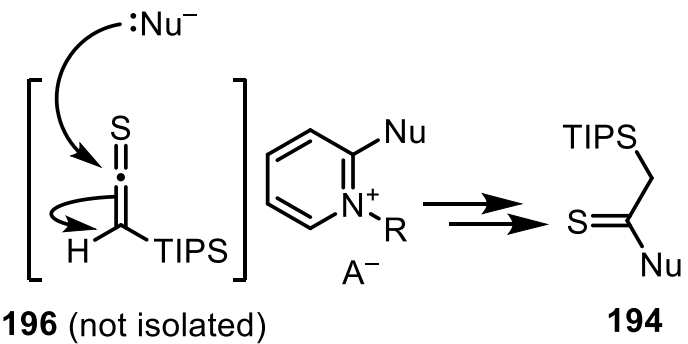

Scheme 78: Plausible mechanistic rationalizations involving an aldothioketene(-mimetic) species.

Both mechanisms lead to the same product, however whereas the behavior of cyclic $\beta$ ketoester 190 is in line with pathway a), the observations made in the experiment of reagent 160b with TBAF as a nucleophilic source are pointing towards pathway b) to be preferred. Furthermore, taking the reactivity of thiobenzoic acid and tritylthiol into account, it can be concluded, that site preference for the nucleophilic attack in either $\alpha$-position to sulfur solely depends on the nature of the nucleophile. 


\section{CONCLUSION AND OUTLOOK}

A concise synthesis of novel sulfur-based electrophilic transfer reagents was realized (Scheme 79). The initial phase of the project was focused on electrophilic cyanation reagents 155. Surprisingly, the extreme electron deficiency of the employed pyridinium backbone led to formation of highly unstable intermediates. Isolation of different decomposition products gave insight into the favored pathway thereof. Alternatively, more stable reagents 160, originally designed for electrophilic alkynylation, were then synthesized. Unexpectedly, the dominating reactivity for the majority of investigated nucleophiles was thioacylation. This gave access to dithioesters, thioamides and thionoesters, which are otherwise accessible only by employing environmentally unfriendly thionating reagents such as Lawesson's reagent or $\mathrm{P}_{4} \mathrm{~S}_{10}$. The disadvantages of the latter are toxicity of not only the reagents themselves, but also of byproducts, harsh reaction conditions and difficulties of product isolations. ${ }^{4}$ On the other hand, the use of thioketenes is limited to few available examples with defined substitution patterns.
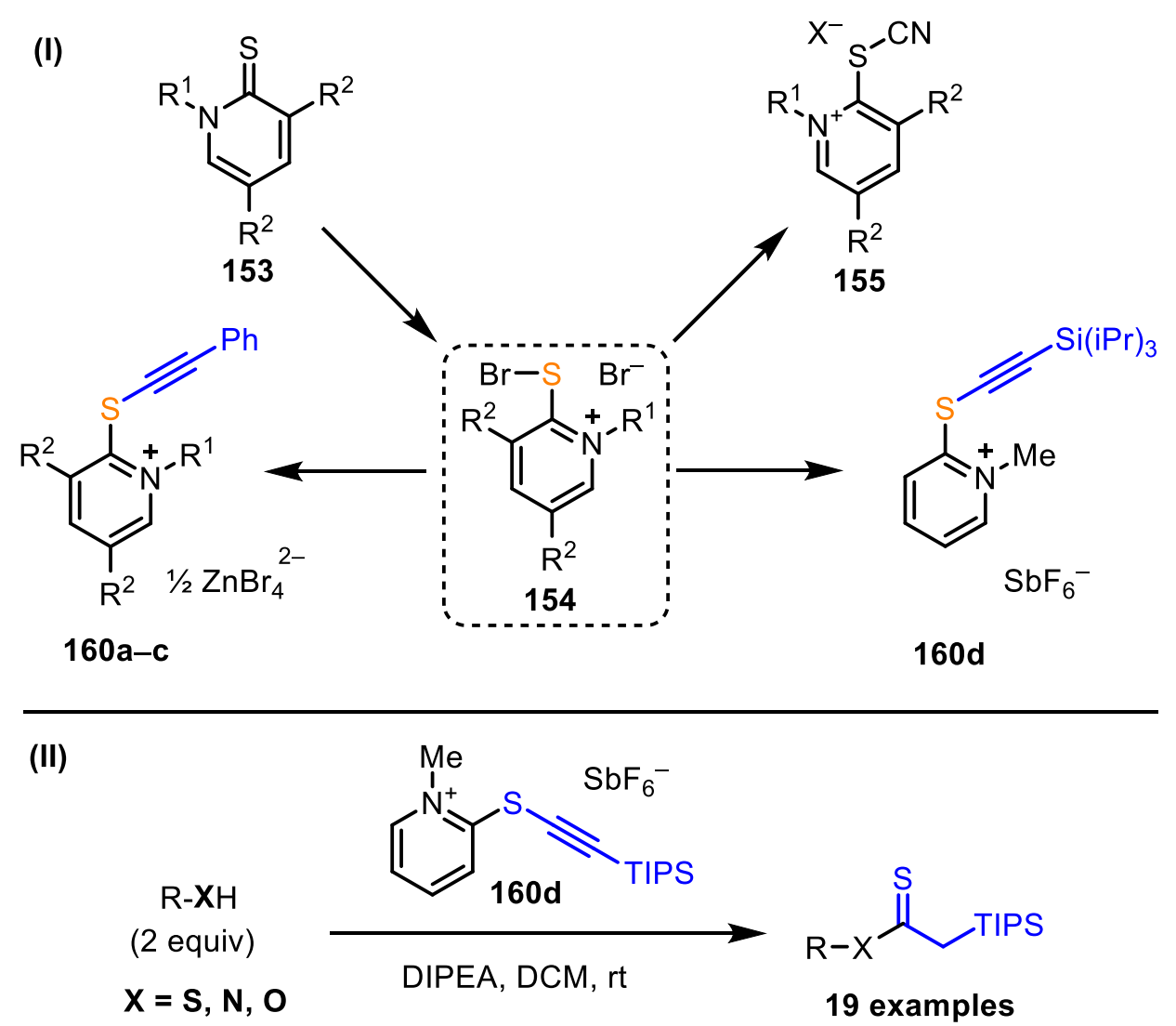

Scheme 79: (I) Synthesis of dibromo(pyridinium)sulfuranes 154, pyridinium thiocyanates 155 and pyridinium thioalkynes 160 . (II) General reactivity of pyridinium thioalkynes $160 \mathrm{~d}$ towards $S-, N$-, $\mathrm{O}$-nucleophiles.

The higher reactivity of the obtained products containing a thione group, as compared to their carbonyl analogs, makes them attractive intermediates for further functionalizations such as the modification of prodrugs in medicinal chemistry or agrochemicals, as was

\footnotetext{
${ }^{4}$ See Chapter "SYNTHESIS OF DITHIOESTERS, THIOAMIDES AND THIONOESTERS"
} 
demonstrated for selected cases. In addition, several new reactivites were discovered and illustrate that continued research on the project is justified. Exploitation of the reagent as an aldothioketene equivalent has yet to be fully extended to examples in which thioketenes show unique reactivity. These are namely a number of cyclization reactions and most relevant for medicinal chemistry, the synthesis of $\beta$-thiolactams as core units for novel antibiotics and their derivatization. Furthermore, the possibility to modify the pyridinium backbone should enable fine tuning, to regulate the reagents reactivity. Only one exemplary approach would be the introduction of an oxy-tether in analogy to hypervalent iodonium salts and the prominent example of EBX. ${ }^{5}$ 


\section{C-H functionalization Utilizing Thioimidazolone-Based Electrophilic Transfer REAGENTS}

\section{Project Aims}

The aim of this project will be to extend the scope of the in group developed imidazolium thioalkynes towards their application in transition metal catalysis. More particular this Chapter will cover the development of a catalytic system in which the mentioned imidazolioum thioalkynes will function as terminal electrophilic alkynylation synthons, which are difficult to be developed by other approaches. $\mathrm{C}-\mathrm{H}$ activation by a transition metal will be used to facilitate the generation of a C-nucleophile from a suitable substrate (Scheme 80). In this context, the choice of an appropriate directing group will be one of the focal points. Once a suitable system is found, investigation of the scope and limitations as well as elucidation of the mechanism will be conducted.

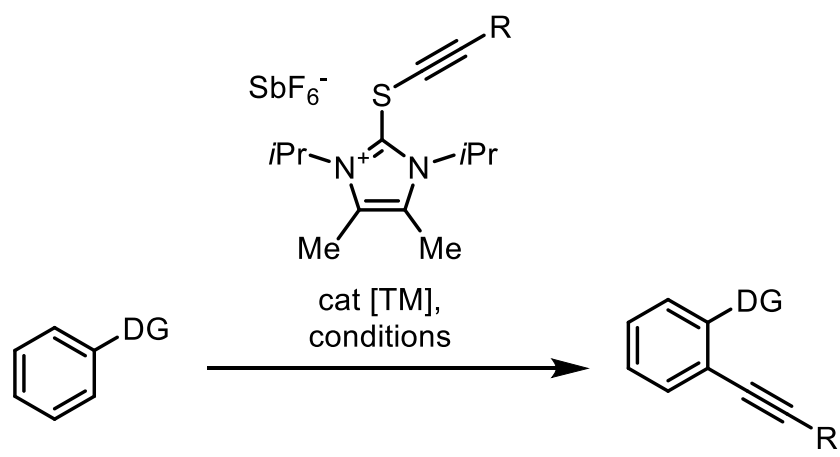

Scheme 80: Proposed C-H activation protocol for the electophilic akynylation with imidazolium thioalkynes. 


\section{Preliminary SCREening}

To commence the research in this project, high throughput screenings with a plethora of different reaction parameters were run, after which a selection of promising conditions were further investigated and optimized. Core of these screenings was the variation of transition metals, oxidants, bases, substrates with varying directing groups, solvents and a range of thioimidazolium based electrophilic transfer reagents (Scheme 81). ${ }^{6}$

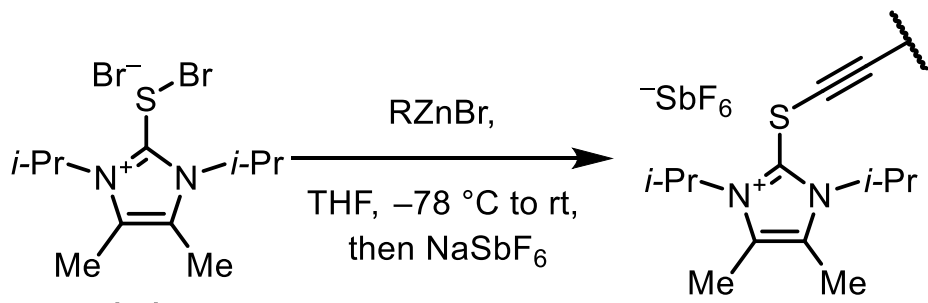

$151 \mathrm{a}$

197-202
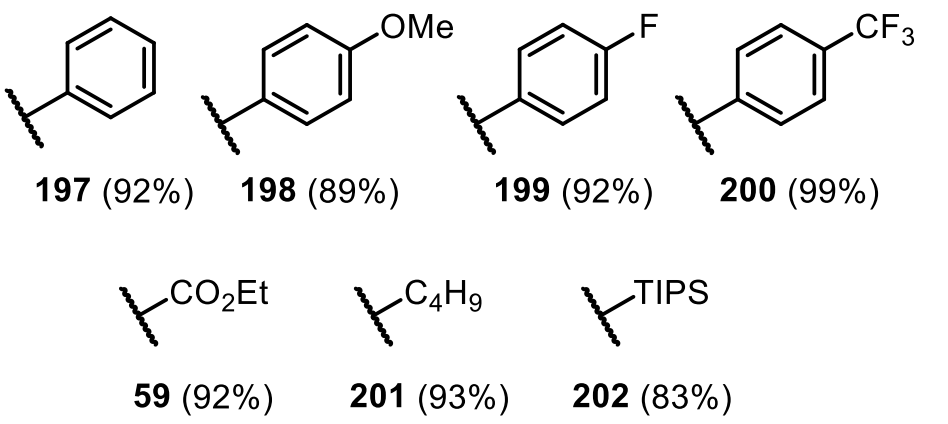

Scheme 81: Alkynylthioimidazolium salts employed during the investigation of $\mathrm{C}-\mathrm{H}$ functionalization reactions.

1-Phenylpyrazole as a substrate gave one of the most promising initial hits obtained from high throughput screening. The reaction took place in the presence of pentamethylcyclopentadienylrhodium(III) chloride dimer (5 mol\%), 0.5 equivalents of copper(II) acetate, silver hexafluoroantimonate(V) and thioimidazolium salt 197. However, instead of the expected alkynylated product, compound 203 was isolated in $50 \%$ yield (Scheme 82). Salt 203, which structure was also verified by X-ray diffractometry (Figure 5), is most probably the result of a 6-endo-dig cyclization after successful $\mathrm{C}-\mathrm{H}$ functionalization.

\footnotetext{
${ }^{6}$ High throughput screenings were conducted in cooperation with Bayer, a NDA was signed.
} 
197 (1.2 equiv),<smiles>c1ccc(-n2cccn2)cc1</smiles>
$\left[\mathrm{RhCp}^{*} \mathrm{Cl}_{2}\right]_{2}(5 \mathrm{~mol} \%)$, $\mathrm{Cu}(\mathrm{OAc})_{2}$ (0.5 equiv), $\mathrm{AgSbF}_{6}$ (1.2 equiv)

1,2-DCE, $80^{\circ} \mathrm{C}, 20 \mathrm{~h}$<smiles></smiles>
not observed:

$203,50 \%$

Scheme 82: Obtained product for the $\mathrm{C}-\mathrm{H}$ functionalization of 1-phenylpyrazole with electrophilic thioimidazolium acetylene transfer reagent 197.

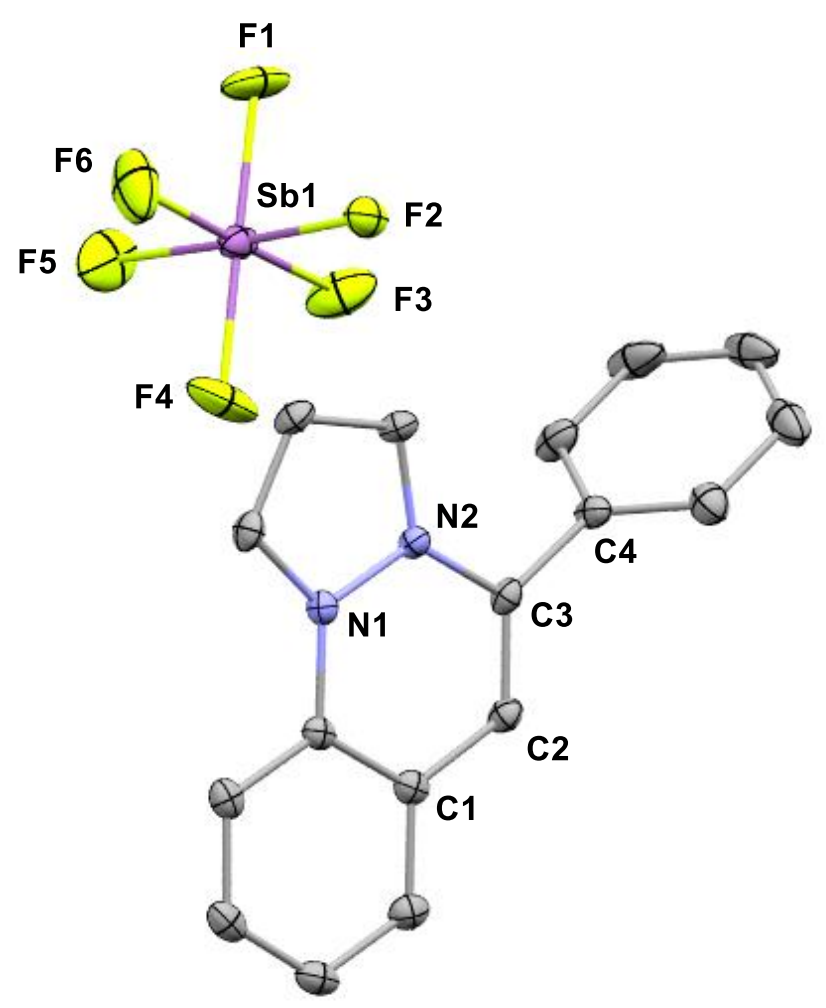

Figure 5: Structure of compound 203 in the crystal. Anisotropic displacement parameter shown at 50\% probability level, hydrogen atoms are omitted for clarity.

Other preliminary results utilizing benzophenone, pivalophenone and acetophenone showed consumption of substrate and reagent as well. While several attempts to isolate a defined product for experiments with benzophenone failed, in one case isolation of a benzophenone derivative was possible. After structural elucidation the latter proved to be the diketone $\mathbf{2 0 4}$ (Scheme 83). The structure implies, that after successful $\mathrm{C}-\mathrm{H}$ alkynylation, hydration of the alkyne functionality takes place, either still during the reaction process or during purification by LC-MS. In addition, mass analysis suggested the formation of an adduct of the transfer reagent and the substrate for benzophenone, pivalophenone and acetophenone. ${ }^{7}$ Nevertheless, from these preliminary reactions with ketones, it could be concluded, that

\footnotetext{
${ }^{7}$ Recently isolation of such an adduct starting from azobenzene as a substrate was successful as well (see Chapter: "The Diazenyl Functionality as a Directing Group").
} 
weakly coordinating DGs are promising candidates for $\mathrm{C}-\mathrm{H}$ functionalization reactions with the designed alkynylthioimidazolium salts.

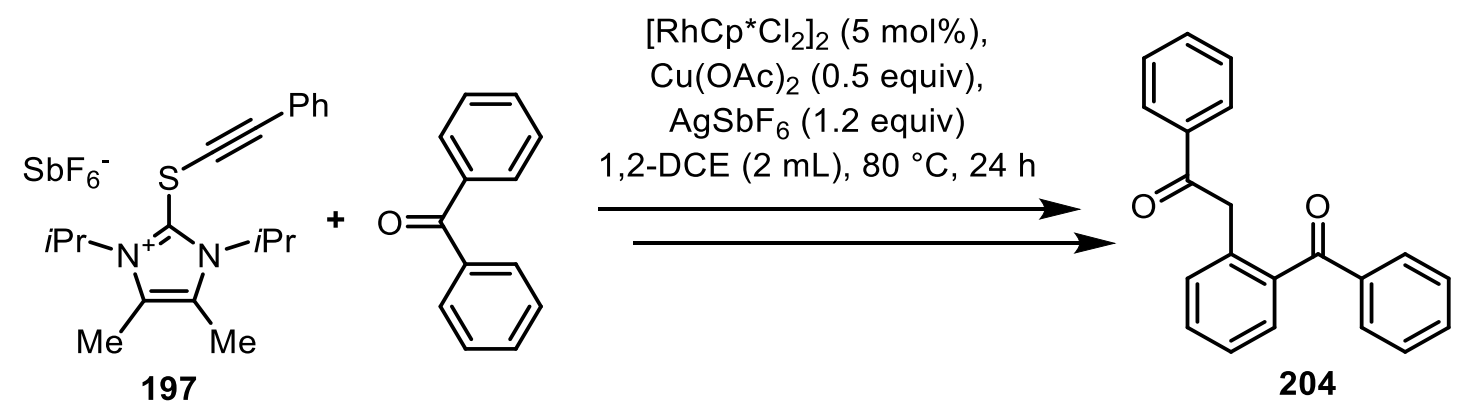

Scheme 83: Diketone 204 isolated after applying C-H alkynylation protocol with benzophenone as a substrate. 


\section{Amides as Weakly Coordinating Directing Groups}

The results obtained with ketones as directing groups motivated us to investigate other weakly coordinating directing groups, such as benzamides. When $\mathrm{N}, \mathrm{N}$-diisopropylbenzamide was employed as a substrate, TLC monitoring of the transfer reaction with alkynylating agent 197 under the elaborated conditions showed the formation of a mixture of salts (Scheme 84). Purification gave a crystalline solid in a crude yield of $84 \%$ with respect to the alkynylation product. NMR analysis revealed that a mixture of two isomers 205a and 205b had formed.

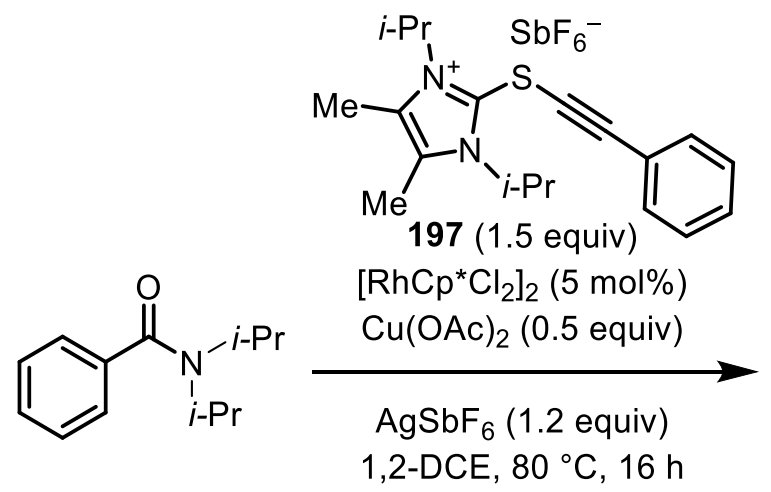

$47 \%$<smiles>CCC[N+](CC)=C1OC(=Cc2ccccc2)c2ccccc21</smiles><smiles>CCC[N+](CCC)=c1oc(-c2ccccc2)cc2ccccc12</smiles>

205a
$2: 1$ ratio $205 b$

Scheme 84: $\mathrm{C}-\mathrm{H}$ functionalization of $\mathrm{N}, \mathrm{N}$-diisopropylbenzamide with alkynylthioimidazolium salt 197.

Crystals suitable for X-ray analysis were grown by recrystallization from warm acetone on air. X-ray diffractometry unambiguously verified the structure of one of the isomers as the salt 205a (Figure 6). Based on the obtained product structure we suggest that $O$-cyclization occurs after $\mathrm{C}-\mathrm{H}$ activation and alkynylation.

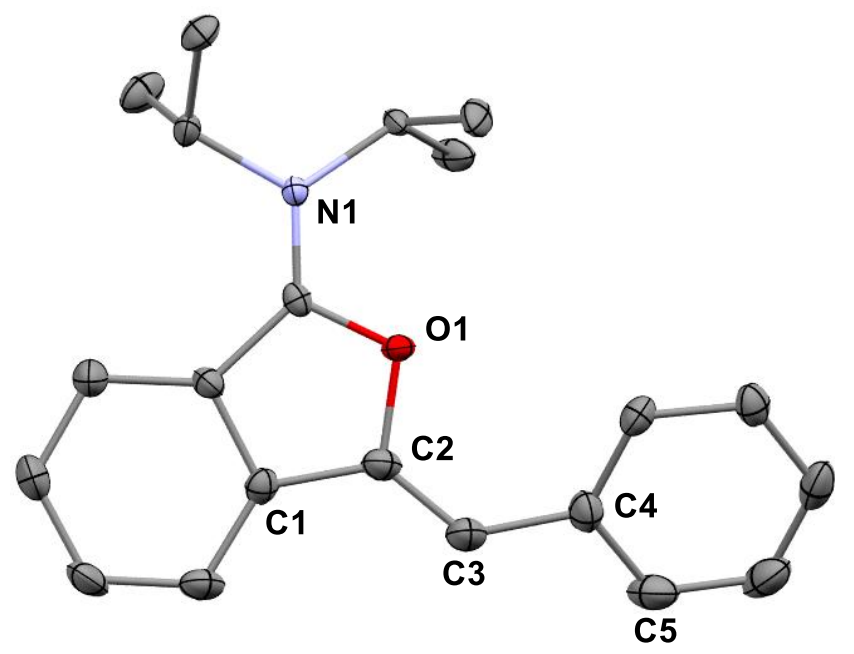

Figure 6: Structure of compound 205a in the crystal. Anisotropic displacement parameter shown at 50\% probability level, hydrogen atoms and anion are omitted for clarity. 
Taking this results into account, a final yield of $47 \%$ was determined. In contrast to the reactivity of 1-phenylpyrazole (Scheme $\mathbf{8 2}$ ), an isomeric mixture of 5-exo- and 6-endo-dig cyclization products is formed, giving a phthalanylideneammonium salt 205a and the corresponding isochromene iminium salt 205b. Attempts to separate these salts failed due to high similarity in their physical properties. It is worth mentioning, that synthesis of this particular class of compounds, in which the nitrogen is a quaternary all-carbon substituted center, and further reactions thereof are described in only two papers by BoyD. ${ }^{[166,167]}$

Much to our delight, it was possible to conduct the hydrolysis of the iminium salts (Scheme 85). The resulting stable products could be purified by column chromatography. ${ }^{8}$ These results are in accordance with the methods formerly described by BoyD. ${ }^{[167]}$<smiles></smiles>
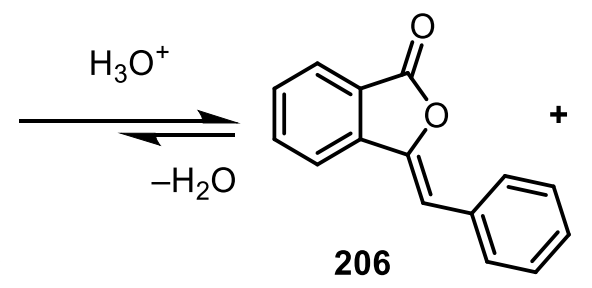

$$
(i-\mathrm{Pr})_{2} \mathrm{NH}_{2}{ }^{+} \mathrm{SbF}_{6}^{-}
$$

Scheme 85: Equilibrium of phthalanylideneammonium salt and phthalide.

Optimizational studies were conducted in order to improve overall yield, as well as identify parameters, which influence the selectivity towards either of the constitutional isomers (Table 3).

Table 3: Screening of catalyst, base and temperature.

\begin{tabular}{|c|c|c|c|c|c|}
\hline Entry & Catalyst & Base & $\mathrm{mmol}$ & Temperature $\left({ }^{\circ} \mathrm{C}\right)$ & Yield of 205 (\%) \\
\hline 1 & {$\left[\mathrm{RhCp} * \mathrm{Cl}_{2}\right]_{2}$} & $\mathrm{Cu}(\mathrm{OAc})_{2}$ & 0.15 & 80 & 47 \\
\hline 2 & {$\left[\mathrm{RhCp} * \mathrm{Cl}_{2}\right]_{2}$} & $\mathrm{Cu}(\mathrm{OAc})_{2}$ & 0.15 & $\mathrm{rt}$ & 51 \\
\hline 3 & {$\left[\mathrm{RhCp} * \mathrm{Cl}_{2}\right]_{2}$} & $\mathrm{Na}_{2} \mathrm{CO}_{3}$ & 0.3 & 80 & - \\
\hline 4 & {$\left[\mathrm{RhCp} * \mathrm{Cl}_{2}\right]_{2}$} & $\mathrm{~K}_{2} \mathrm{CO}_{3}$ & 0.3 & $\mathrm{rt}$ & - \\
\hline 5 & {$\left[\mathrm{RhCp} * \mathrm{Cl}_{2}\right]_{2}$} & $\mathrm{NaOAc}$ & 0.3 & 80 & 47 \\
\hline 6 & {$\left[\mathrm{IrCp} * \mathrm{Cl}_{2}\right]_{2}$} & $\mathrm{NaOAc}$ & 0.3 & 80 & - \\
\hline
\end{tabular}

When $\mathrm{N}, \mathrm{N}$-diisopropylbenzamide was employed as a substrate at $80{ }^{\circ} \mathrm{C}$ and $\mathrm{Cu}(\mathrm{OAc})_{2}$ as a base, an initial yield of $47 \%$ was determined (Table 3 , Entry 1 ). Under the same conditions at room temperature, the yield slightly increased to $51 \%$ (Table 3, Entry 2). Substitution of $\mathrm{Cu}(\mathrm{OAc})_{2}$ with $\mathrm{NaOAc}$ as the base, gave the same yield of $47 \%$ (Table 3, Entry 5 ). When

\footnotetext{
${ }^{8}$ While isolation of the neutral imines obtained as products starting from $N$-methylbenzamides is possible, by quenching the reaction with aqueous $\mathrm{K}_{2} \mathrm{CO}_{3}$ solution and subsequent column chromatography, yields are either equal, but more often lower than yields for the hydrolysis products.
} 
carbonates were used as a base or iridium catalyst was used instead of rhodium one, no reaction was observed (Table 3, Entries 3, 4, 6).

With suitable conditions in hand, the selectivity of isomers was still an issue to be adressed. In fact the difference in selectivity for 1-phenylpyrazole (exclusively 6-endo). and $\mathrm{N}$ substituted benzamide (competitive 5-exo and 6-endo cyclization), motivated us to investigate the importance of steric bulk of the $N$-substituents of the benzamide, reasoning that 6-endo-dig cyclization becomes more favoured with less hindrance. Thus the starting material was changed to $\mathrm{N}, \mathrm{N}$-dimethylbenzamide (Table 3 ).

Table 4: Screenings with N,N-dimethylbenzamide.

\begin{tabular}{ccccc}
\hline Entry & Solvent & Temperature $\left({ }^{\circ} \mathrm{C}\right)$ & Yield (\%) & Isomeric ratio (6-endo:5-exo) \\
\hline 1 & $\mathrm{DCE}$ & 80 & 48 & $1.4: 1$ \\
2 & $\mathrm{DCE}$ & 80 & traces $^{\mathrm{a})}$ & - \\
3 & $\mathrm{DCE}{ }^{\mathrm{b})}$ & 100 & 51 & $1.5: 1$ \\
4 & $\mathrm{CH}_{3} \mathrm{CN}$ & 80 & - & - \\
5 & $\mathrm{CH}_{2} \mathrm{Cl}_{2}$ & 60 & 37 & $3.6: 1$ \\
6 & $\mathrm{CH}_{2} \mathrm{Cl}_{2}$ & $\mathrm{rt}$ & - & - \\
$7 \mathrm{c})$ & $\mathrm{DCE}$ & $\mathrm{rt}$ & 60 & $16: 1$ \\
\hline
\end{tabular}

Reaction conditions: $\left[\mathrm{RhCp}^{*} \mathrm{Cl}_{2}\right]_{2}(9.3 \mathrm{mg}, 15 \mu \mathrm{mol}, 5 \mathrm{~mol} \%), \mathrm{AgSbF}_{6}(125.5 \mathrm{mg}, 0.37 \mathrm{mmol}), 197$ (247.2 mg, 0.45 mmol), $\mathrm{N}, \mathrm{N}$-dimethylbenzamide ( $44.8 \mathrm{mg}, 0.3 \mathrm{mmol}$ ), NaOAc $(24.6 \mathrm{mg}, 0.3 \mathrm{mmol}), 16 \mathrm{~h}, \mathrm{~N}_{2}$; a) $0.06 \mathrm{mmol}$ $\mathrm{AgSbF}_{6}$, b) $5 \mathrm{~mL}$, c) $2.5 \mathrm{~mol} \%\left[\mathrm{RhCp}^{*} \mathrm{Cl}_{2}\right]_{2}, \mathrm{~N}$-methylbenzamide was used instead of $\mathrm{N}, \mathrm{N}$-dimethylbenzamide.

The experiments conducted with $\mathrm{N}, \mathrm{N}$-dimethylbenzamide verified, that decrease of steric bulk on the imine moiety favors 6-endo cyclization, while overall yields remained similar (Table 4, Entry 1). When substociometric amounts of silver-salt were used, only traces of product formation were observed (Table 4, Entry 2). Increasing the temperature to $100{ }^{\circ} \mathrm{C}$ only marginally increased the overall yield and exerts only little influence on the selectvity (Table 4, Entry 3). Switching to the more polar acetonitrile as a solvent completely supressed product formation (Table 4, Entry 4). Using dichloromethane as a solvent resulted in a decrease of the yield. On the other hand, an increase in the proportion of 6-endo product was observed, which can either be attributed to the nature of solvent or to the decrease in temperature (Table 4, Entry 5). However, no product was formed under essentially the same conditions, but at room temperature (Table 4, Entry 6). Finally, switching to $N$-methylbenzamide as a substrate as further decreasing the size of $\mathrm{N}$-substituents, not only gave an 6-endo:5-exo isomeric mixture in ratio $16: 1$, but also increased the combined yield to $60 \%$. In addition, lowering the catalyst loading to $2.5 \mathrm{~mol} \%$ did not give any drop in the yield (Table 4, Entry 7).

Summarizing these results, on going from $\mathrm{N}, \mathrm{N}$-diisopropylbenzamide to $\mathrm{N}$-methylbenzamide and applying the $\mathrm{C}-\mathrm{H}$ functionalization/hydrolysis sequence, access to isocoumarins was elaborated (Scheme 86). At last, switching from anhydrous 1,2-dichloroethane to technical 
grade solvent increased the efficacy again, affording homalicine 207 from $N$ methylbenzamide in $70 \%$ yield (Scheme 87 ).

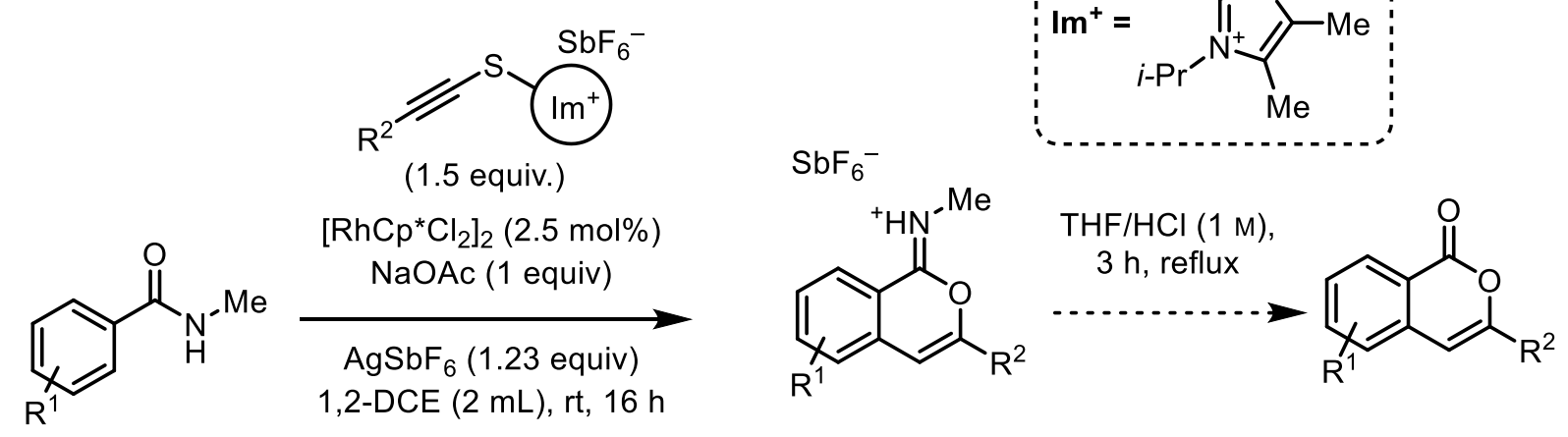

Scheme 86: Developed catalytic system for the synthesis of 3-substituted isocoumarins from $\mathrm{N}$ methylbenzamides and alkynylthioimidazolium salts.

After identification of a suitable directing group, the influence of electron withdrawing as well as of electron donating subtituents in the backbone of the starting material was explored (Scheme 87). During this process, the influence on chemoselectivity by sterically demanding substituents was recognized.

It is worth mentioning, that, since annulation also incorporates the directing group, overfunctionalization through a second $\mathrm{C}-\mathrm{H}$-activation step is circumvented. 
<smiles>[R]c1ccc2c(=O)oc(-c3ccccc3)cc2c1</smiles>

$207 \mathrm{R}=\mathrm{H}(70 \%)$

$208 \mathrm{R}=\mathrm{Me}(68 \%)$

$209 \mathrm{R}=\mathrm{MeO}(58 \%)$

$210 R=F(66 \%)$

$211 \mathrm{R}=\mathrm{Cl}(41 \%)$

$212 \mathrm{R}=\mathrm{Br}(52 \%)$

$213 \mathrm{R}=\mathrm{I}(29 \%)$

$214 \mathrm{R}=\mathrm{CF}_{3}\left(41 \%, 80{ }^{\circ} \mathrm{C}\right)$

$215 \mathrm{R}=\mathrm{CO}_{2} \mathrm{Me}(35 \%)$<smiles>[M]Oc1cc2c3c(ccc2oc1=O)OCO3</smiles>

$216(53 \%)$<smiles>Cc1ccc2cc(-c3ccccc3)oc(=O)c2c1</smiles>

$217(64 \%)$<smiles>COc1ccc2cc(-c3ccccc3)oc(=O)c2c1</smiles><smiles>Cc1cccc2c1C(=O)O/C2=C\c1ccccc1</smiles>

$219 a+219 b(12 \%, 1: 1.2)$<smiles>O=c1oc(-c2ccccc2)cc2cc3ccccc3cc12</smiles>

$221 a+221 b(72 \%, 5: 1)$<smiles>COc1ccc(-c2cc3ccccc3c(=O)o2)cc1</smiles>

$222(73 \%)$<smiles>O=c1oc(-c2ccc(F)cc2)cc2cc(F)ccc12</smiles>

$226(65 \%)$<smiles>O=c1oc(-c2ccccc2)cc2cc(-c3ccccc3)ccc12</smiles>

223 (45\%)<smiles>CNC(=O)c1ccc2cc(-c3ccccc3)oc(=O)c2c1</smiles>

$227(74 \%)$<smiles>O=c1oc(-c2ccccc2)cc2cccc(F)c12</smiles>

224 (50\%)<smiles>[R6][AsH3]</smiles>

$225(9 \%)$<smiles>O=c1oc(-c2ccccc2)cc2ccc(C(F)(F)F)cc12</smiles>

Scheme 87: Scope of $N$-methylbenzamides to synthesize 3-substituted isocoumarins.

To investigate the effects of electron donating groups, the tolyl derivative with the methyl group in para-position, featuring an additional +1 effect, was tested in the reaction. Yet, the observed yield of $68 \%$ for $\mathbf{2 0 8}$ was similar to that of 207. Assuming, that employing an EDG with the stronger $+\mathrm{M}$ effect in a starting material would increase the nucleophilicity of the in situ formed metallacycle (see below, Scheme 93-II) and thus enhance the yield, $p$-methoxy$\mathrm{N}$-methylbenzamide was used as a subtrate. Selective transformation to the corresponding isocoumarin 209, however, gave the product in a yield of $58 \%$. Furthermore, when the reactivity of an electron richer substrate containing the dioxolane moiety was tested, the 
yield remained similar with $53 \%$ for 216 . Surprisingly, product analysis revealed that only one of two possible isomers was formed. The structure of the product suggested that a lone pair-containing meta substituent might play a decisive mechanistic role in the reaction. To verify this hypothesis, $m$-methoxy- $N$-methyl benzamide was employed in the reaction, giving two regioisomers $\mathbf{2 1 8 a}$ and $\mathbf{2 1 8 b}$ in an $1: 2$ ratio and overall yield of $61 \%$. Formation of the latter might be explained by a coordinated chelating effect of the amido group and a secondary chelating effect exerted by the methoxy substituents. This resulted in the initial $\mathrm{C}-\mathrm{H}$-activation in ortho-position to both the directing and the methoxy groups. Such a secondary stabilizing effect by interaction of the adjacent $O$-containig group with the catalyst during the $\mathrm{C}-\mathrm{H}$-activation step can be considered as a general explanation (Scheme 88). Formation of a chelate with the active rhodium species would also explain the selectivity for the aforementioned dioxolane compound 216.
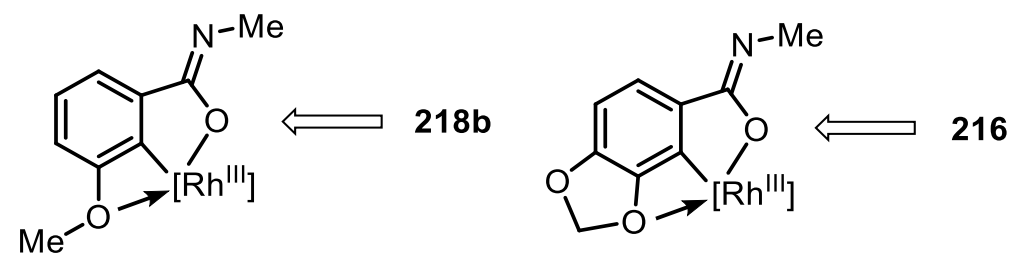

Scheme 88: Directed selectivity observed for O-containing meta substituents.

To conclude that this hypothesis is true, the reactivity of $N$-methyl- $m$-toluamide was investigated. But in case of aliphatic substituents in meta position, no secondary effects play any role, as isochromene $\mathbf{2 1 7}$ was isolated in $64 \%$ yield as a single isomer.

For ortho-substituted substrates the reaction strongly depends on sterical factors. Thus, from $\mathrm{N}$-methyl-o-tolyamide, an isomeric mixture of isocoumarin 219a and phthalide $219 \mathrm{~b}$ with a respective ratio of $1: 1.2$ was isolated in $12 \%$ yield. We suggest that the initially formed five-membered rhodacycle underwent an insertion of the alkyne moiety affording a seven-membered metallacycle (Scheme 89, right, and intermediate III in Scheme 93). ${ }^{[168,169]}$ For this scenario, 0 -methyl substitution creates a disfavoured transition state due to sterical repulsion of the methyl group and the iminium group (Scheme 89 ).

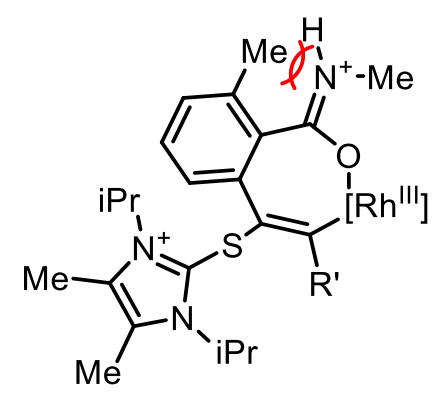

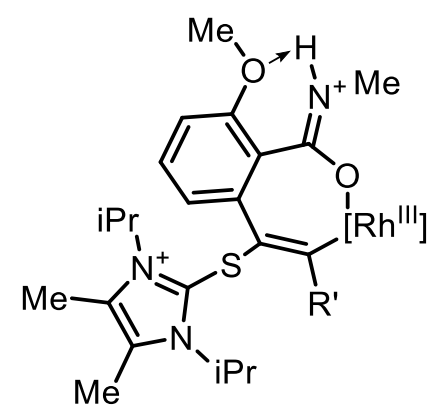

Scheme 89: Sterical clash created by $o$-alkyl-substituents (left) and H-bonding by the o-methoxy group (right).

For the comparison, when o-methoxybenzamide was employed as a substrate, an isomeric mixture of isocoumarin $\mathbf{2 2 0 a}$ and phthalide $\mathbf{2 2 0 b}$ in an overall $64 \%$ yield with a ratio of 1:1.4 
was isolated. This is an additional evidence for the postulated mechanism: In this case the methoxy group in ortho position stabilizes the transition state through hydrogen bonding.

Noteworthy, while potentially competing $N$-cyclization has been reported in literature, ${ }^{[146]}$ it was not observed in any reaction conducted within this project. Even more: based on our findings, we suggest that nothing but the oxygen of the amide functionality is exclusively responsible for the coordination of the catalytically active rhodium species during the formation of the metallacycle in the $\mathrm{C}-\mathrm{H}$ activation step and subsequently for the nucleophilic attack in the ring closing step. Consequently, the nitrogen faces away from the reaction site.

On the other hand, hydrogen bonding (Scheme 89, right) is not the sole factor determining the spatial orientation of $\mathrm{N}$-methyl groups, as sterical reasons are of prime importance as well (analogously to Scheme $\mathbf{8 9}$, left). Thus, only one singlet for the $N$-methyl group was observed in crude NMR spectra of compounds 229-233 (Scheme 90)..$^{9}$<smiles>CN=c1oc(-c2ccccc2)cc2cc(OC)ccc12</smiles><smiles>CN=c1oc(-c2ccccc2)cc2ccccc12</smiles>

232

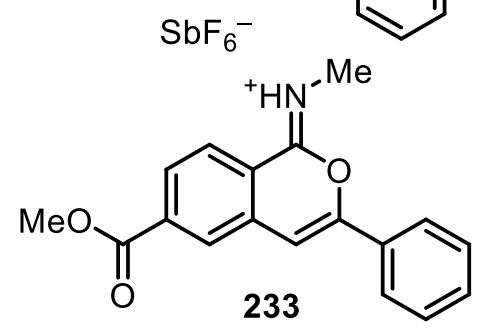<smiles></smiles><smiles>[R]c1cc2ccccc2c(=[N+](C)[O-])o1</smiles>

Scheme 90: Structures of compounds 229-233 picked as crystals from reaction crudes and identified by X-ray diffractometry.

According to their crystal structures (Figure 7), the methyl substituents on the nitrogen were found to be anti oriented towards the parent aryl ring from the substrate in all cases. This cannot be explained by the packing effects and thus indicates that such an orientation is very probably favored and determined during the $\mathrm{C}-\mathrm{H}$ activation and insertion steps (See next Chapter, Scheme 93).

\footnotetext{
${ }^{9}$ All crystals shown in Figure 7 were taken from reaction crudes before hydrolysis to the final isocoumarins presented in Scheme 87.
} 

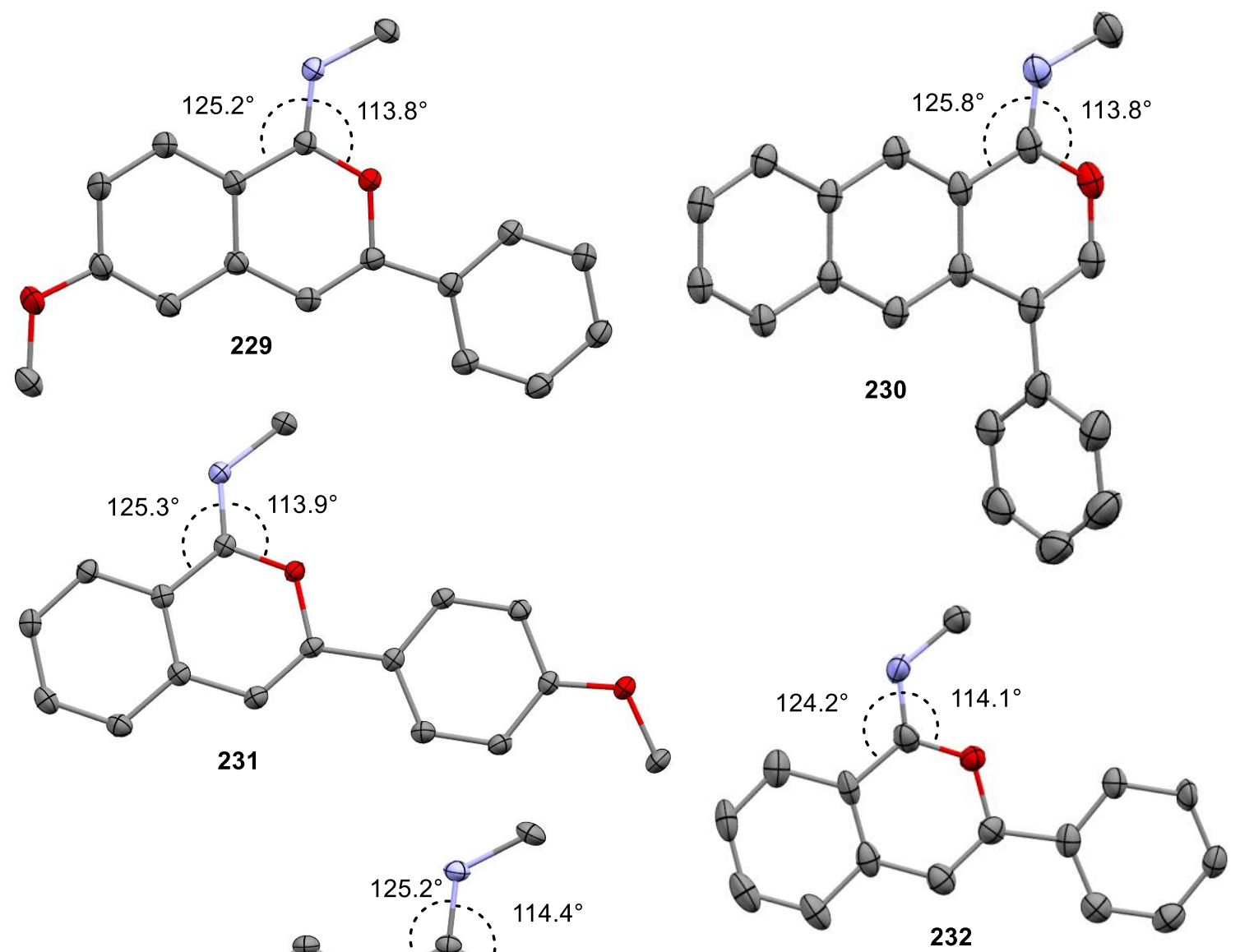

Figure 7: Structures of 229-233 in the crystals. Anisotropic displacement parameter shown at 50\% probability level, hydrogen atoms and anions are omitted for clarity. ${ }^{10}$

The deviation from an ideal $120^{\circ}$ angle depicted for compounds $\mathbf{2 2 9 - 2 3 3}$ is a result of the shorter $\mathrm{C}-\mathrm{O}$ bond. Similar angles are also observerd for the corresponding isocoumarins, which means that the bending does not originate from steric repulsion.

Finally, the observed conformational preference is in line with the results of $a b$ initio calculations to compare relative energies of $E$ - and $Z$-isomers for model substrates (Scheme 91).

${ }^{10}$ The unusual regioselectivity for compound $\mathbf{2 3 0}$ is discussed in the next Chapter (Figure 13). 

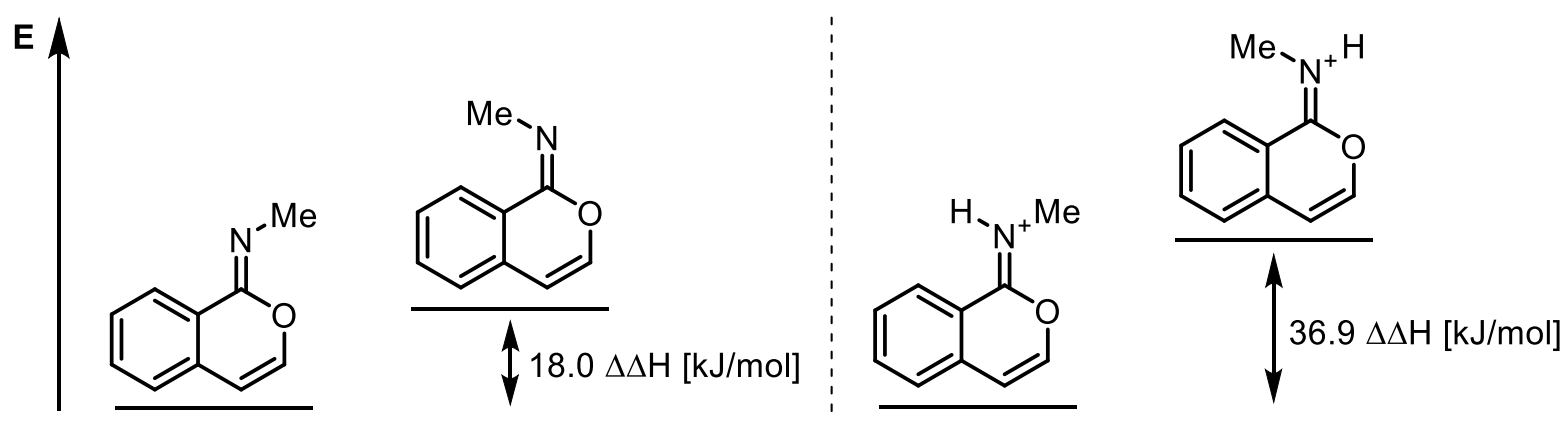

Scheme 91: Calculated energy difference for isochromene imine and iminium isomers as model substrates (B3LYP/6-31G(d) Opt Freq).

Nevertheless it can not be excluded that the observed orientation of the methyl group is the result of the thermodynamically favoured isomer.

It quickly became clear, that electron withdrawing groups in para-position to the directing group reduce the product formation. The explanation is the overall reduced electron density: this makes the generated nucleophile weaker after the $\mathrm{C}-\mathrm{H}$ activation step. Thus, $p$ trifluoromethyl and $p$-methylcarboxylate-substituted substrates afforded the corresponding products $\mathbf{2 1 4}$ and $\mathbf{2 1 5}$ in only moderate yields (41\% and 35\%, respectively). As expected, in line with their higher electronegativity, halogen substituents $p$-chloro- and $p$-bromo- slightly decrease the yields of the corresponding isochromenes 211 (41\%) 212 (52\%), when compared with the non-substituted compound 207. Due to its lowest electronegativity among the other halogens, $p$-iodo- $N$-methylbenzamide was expected to give product $\mathbf{2 1 3}$ in better yield, than compound 212 . Surprisingly, the product yield of $29 \%$ was the lowest from all obtained with the halogen-substituted substrates. $p$-Fluorine substitution did not decrease the efficacy essentially, as compared to the model substrate, giving the desired product in $66 \%$ yield. In contrast, $p$-nitro- $N$-metylbenzamide as a substrate furnished only traces of product, observed in crude ${ }^{1} \mathrm{H}$ NMR spectrum and by TLC. Besides the electron poor character of the compound, in this case we presume the low solubility of the substrate under the reaction conditions to play a major role.

Wondering if the employment of the directing group twice in the same molecule would provoke a twofold transformation and would give an increase in yield, $N^{1}, N^{3}$ dimethylisophthalamide was synthetized and used as a substrate. Inspite of the fact that this substrate can be considered as bearing two ortho-DGs, the single $\mathrm{C}-\mathrm{H}$ activation occurred in $o, p$-position. While purification proved to be difficult, the desired mono-cyclized product 227 was isolated in a good yield of $74 \%$. To further investigate whether the predominating effect for the increase in yield was due to the two DGs or the ortho-EWG, o- $\mathrm{CF}_{3}-\mathrm{N}$ methylbenzamide was used as a substrate. The product was isolated in excellent $89 \%$ yield, thus indicating that an 0 -EWG substitution was indeed benefiting product formation substantially. When a phenyl ring in the substrate was replaced by a heterocyclic moiety, such as thiophene or furane, no product could be obtained under different reaction conditions. 
Nevertheless, characteristic strong UV-activity on TLC suggested product formation, at least in traces. Treatment of the benzofurane derivative under standard reaction conditions lead to isolation of the desired product; however, only in $9 \%$ yield, thus discouraging any further investigations towards other heterocyclic substrates.

Low reaction temperatures proved to be detrimental for product formation. While $p$ (dimethylamino)- $N$-methylbenzamide only showed traces of product formation under standard conditions, the same reaction at $80{ }^{\circ} \mathrm{C}$ resulted in the complete removal of the directing group. These findings supplement the reports by GLORIUS et al. ${ }^{[169,170]}$ who observed dehydration and dehydrocarbonylation as common side reactions in the $\mathrm{C}-\mathrm{H}$ functionalization of aryl ketones and aldehydes, respectively. 


\section{MeCHANISTIC INVESTIGATIONS}

To gain insight into the mechanism of the cyclization step, model substrate 234 was synthesized from phenylacetylene and ortho-iodo- $N$-methylbenzamide via SonogashiraHagihara coupling following the modified literature procedure (Scheme 92). Fortunately, a small amount of palladium-coordinated cyclized intermediate 235 was isolated and crystallized during the purification process of 234 . Investigation by X-Ray diffractometry revealed its structure (Figure $\mathbf{8}$ ), hinting at the fact that the target cyclization of $\mathbf{2 3 4}$ can also take place under various catalytic conditions.<smiles>C[NH2+]C(=O)c1ccccc1I</smiles><smiles>C#Cc1ccccc1</smiles>
$\left(\mathrm{Ph}_{3} \mathrm{P}\right)_{2} \mathrm{PdCl}_{2}(2 \mathrm{~mol} \%)$ Cul (1 mol\%), $\mathrm{Et}_{3} \mathrm{~N}, 55^{\circ} \mathrm{C}, 1 \mathrm{~h}$<smiles>CNC(=O)c1ccccc1C#Cc1ccccc1</smiles>

$234,89 \%$ Scheme 92: Synthesis of cyclization-model substrate $\mathbf{2 3 4}$ via Sonogashira-Hagihara coupling. 


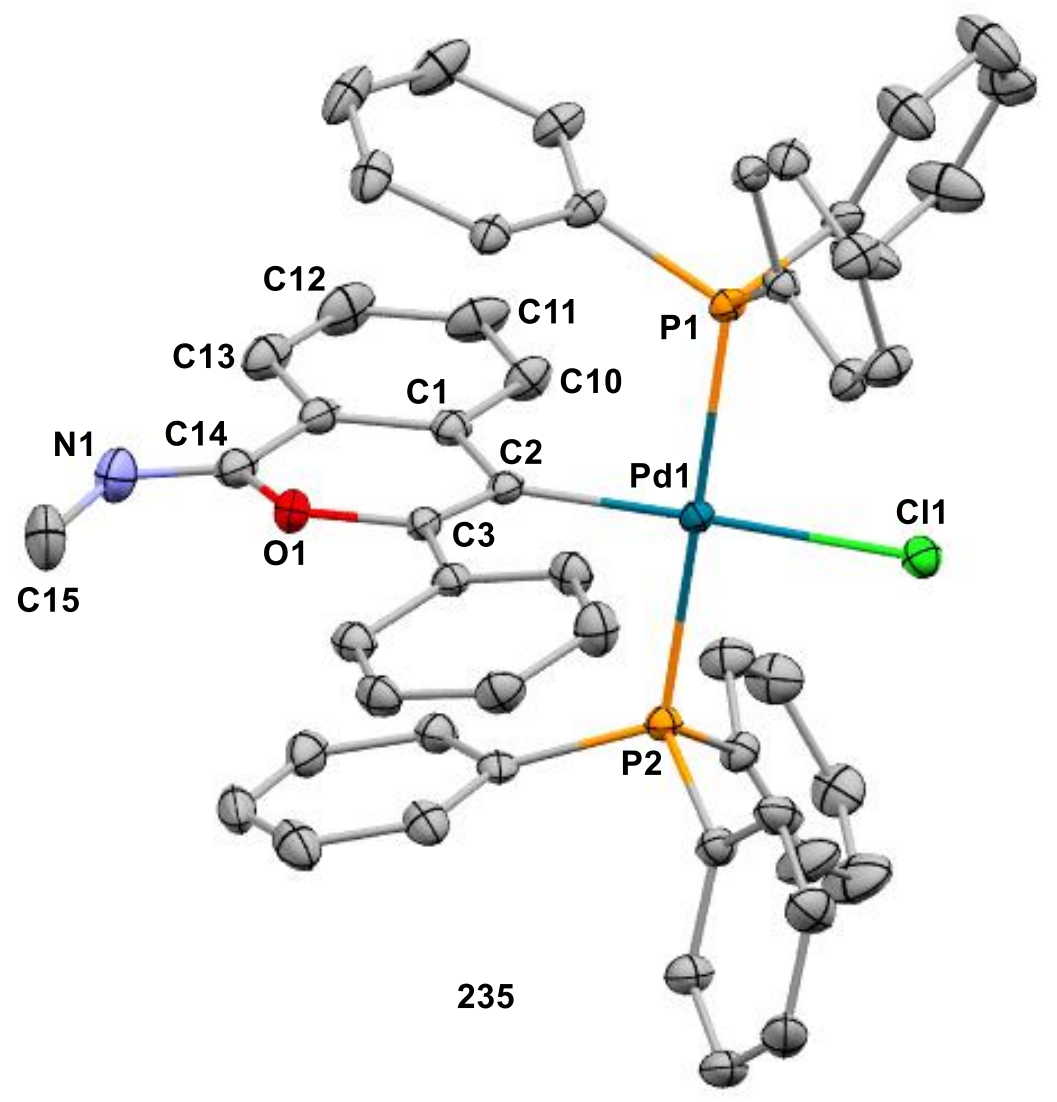

Figure 8: Structure of deactivated Pd-substrate complex 235 in the crystal with the substrate still attached. Anisotropic displacement parameter shown at $50 \%$ probability level, hydrogen atoms and anions, minor disorder part (11\%) of isochromene imine, are omitted for clarity. The chlorine position is partially occupied with an iodine atom (refined to $22 \%$ occupancy).

With model substrate $\mathbf{2 3 4}$ in hand, a set of experiments under different reaction conditions was carried out (Table 5):

Table 5: Investigation of the annulation step.

\begin{tabular}{cccc}
\hline Entry & Rh-precatalyst & AgSbF $_{6}$ (quantity) & Conversion (\%) \\
\hline 1 & {$\left[\mathrm{RhCp}^{*} \mathrm{Cl}_{2}\right]_{2}(5 \mathrm{~mol} \%)$} & - & - \\
2 & - & 1.5 equiv & 100 \\
3 & {$\left[\mathrm{RhCp}^{*} \mathrm{Cl}_{2}\right]_{2}(5 \mathrm{~mol} \%)$} & 1.5 equiv & 100 \\
4 & {$\left[\mathrm{RhCp}^{*} \mathrm{Cl}_{2}\right]_{2}(5 \mathrm{~mol} \%)$} & $5 \mathrm{~mol} \%$ & traces \\
5 & $\mathrm{RhCp} *\left(\mathrm{MeCN}_{3}\left(\mathrm{SbF}_{6}\right)_{2}(2.5 \mathrm{~mol} \%)\right)$ & - & 86 \\
\hline
\end{tabular}

After analysis of the experiments summarized in Table 5, it was concluded, that both - the catalytically active rhodium species as well as the silver salt - are responsible for the annulation process. Actually, this type of annulation has been reported to proceed with benzamides and similar substrates under catalysis with a variety of Lewis and Brønstedt acids. ${ }^{[171]}$ Interestingly, from the attempted cyclization with $\mathrm{RhCp} *(\mathrm{MeCN})_{3}\left(\mathrm{SbF}_{6}\right)_{2}$ (Table 5, Entry 5) deep red crystals were collected. The latter revealed to be the deactivated catalyst 
species 236a (Figure 9), in which one equivalent of thiourea is coordinated to the rhodium center, hinting at one of the catalysts deactivation pathways.

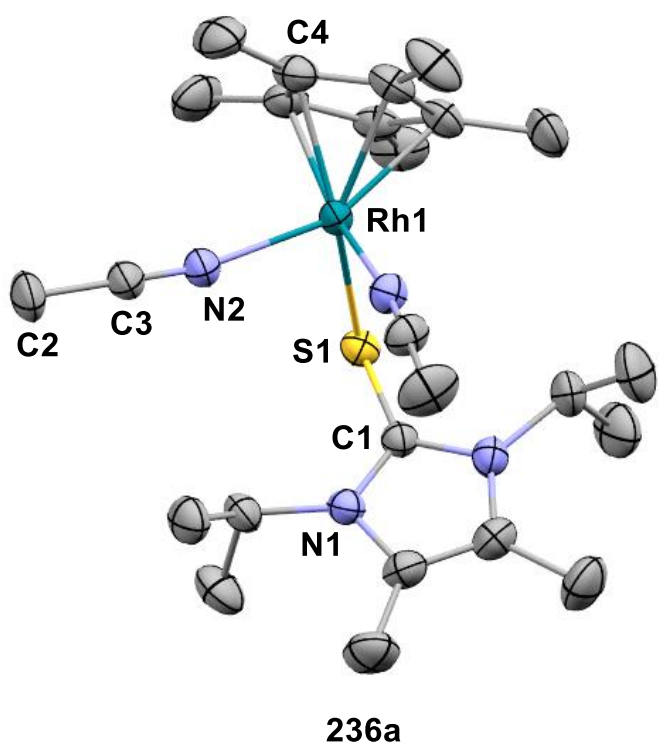

Figure 9: Structure of isolated deactivated rhodium species 236a in the crystal. Anisotropic displacement parameter shown at 50\% probability level, hydrogen atoms and anions are omitted for clarity.

Analyzing the informations gained during the study of the reaction scope with respect to the influence of different substitution patterns, as well as the investigations towards the cyclization step, a tentative catalytic cycle can be postulated (Scheme 93). 


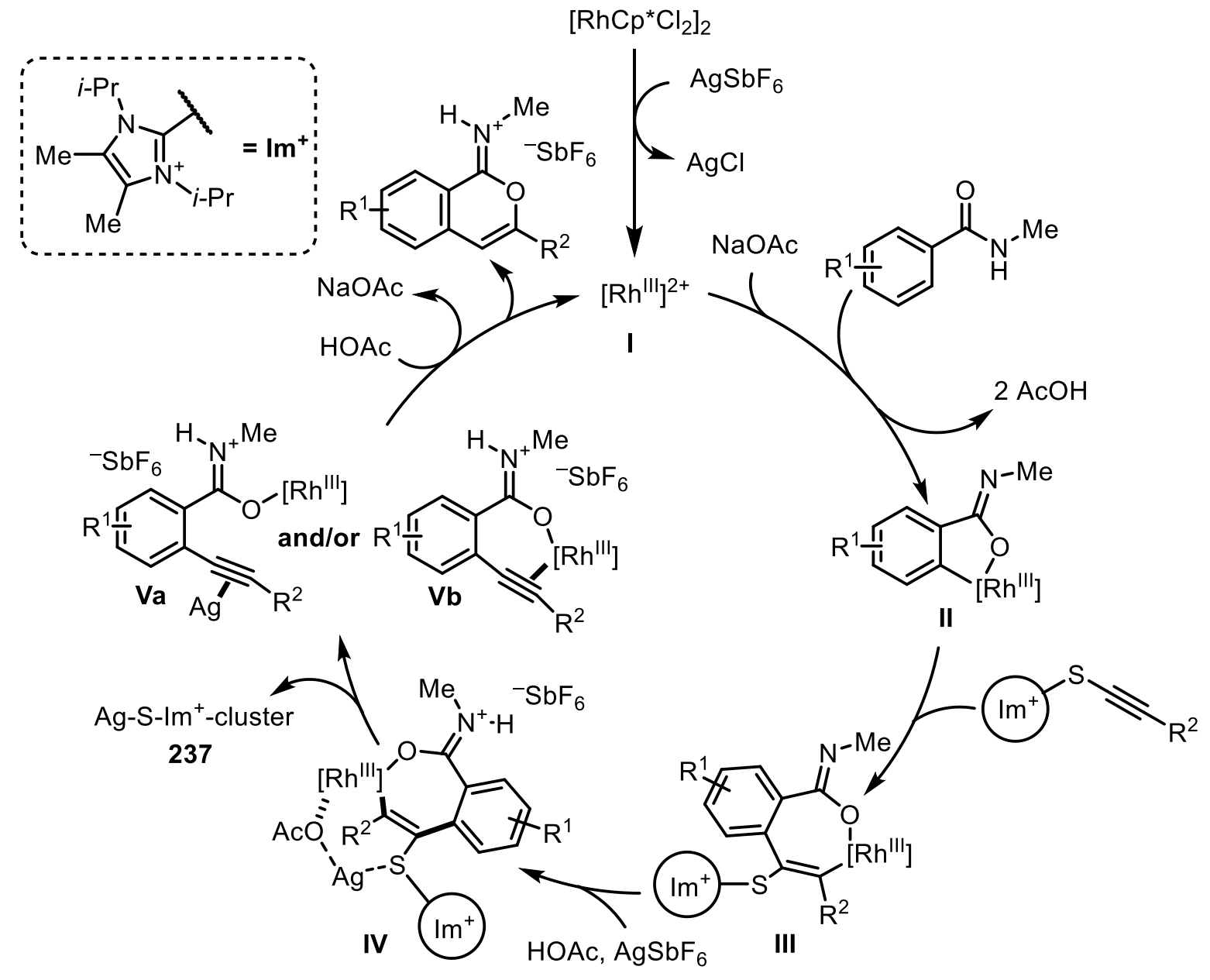

Scheme 93: Postulated catalytic cycle for the $\mathrm{C}-\mathrm{H}$ functionalization of $\mathrm{N}$-methylbenzamides with alkynylthioimidazolium salts.

Generation of the active catalyst species (I) is facilitated by abstraction of the chloride ligands by $\mathrm{AgSbF}_{6}$. Then coordination of the amide oxygen to the catalyst followed by acetate-assisted deprotonation affords the five-membered metallacycle (II). After alkyne insertion and formation of a seven-membered metallacycle (III), silver assisted sulfur elimination gives compound $\mathbf{V a}$ or $\mathbf{V b}$ and $\mathbf{2 3 7}$ (Figure 10). Subsequent annulation gives the isochromene iminium salt as a pre-product and the free catalyst. 


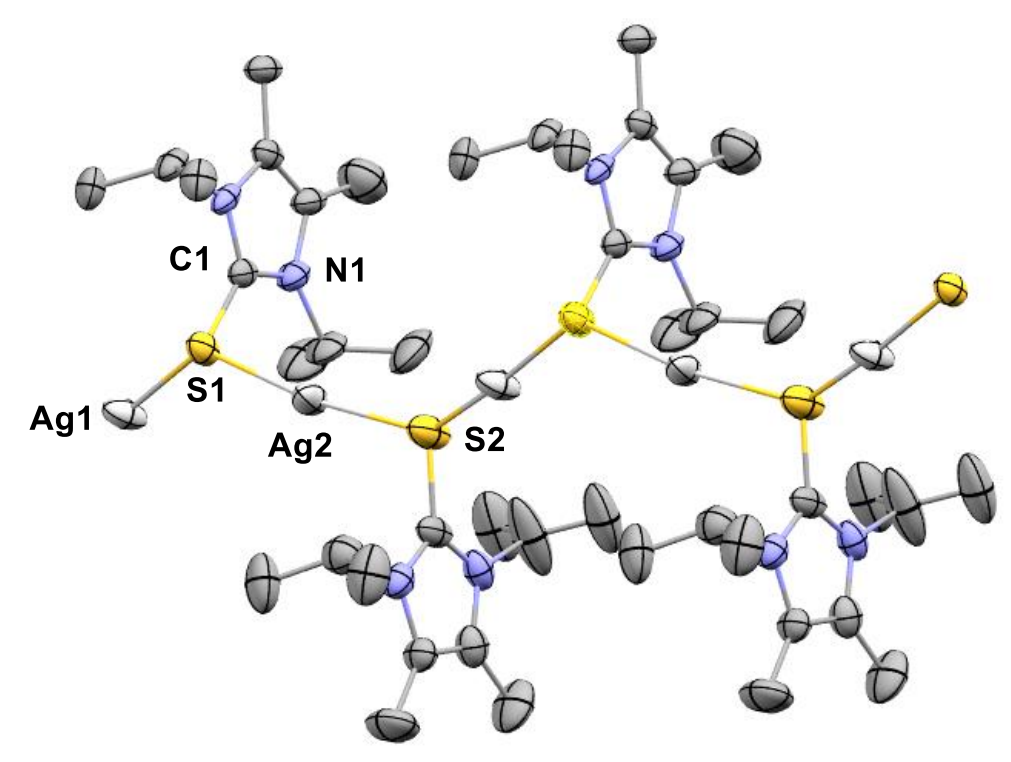

Figure 10: Structure of Ag-S-Im+-cluster 237 in the crystal. Anisotropic displacement parameter shown at $50 \%$ probability level, hydrogen atoms, solvent and anions are omitted for clarity.

Another compound suggesting a deactivation pathway, which is considered to be in equilibrium with species $\mathbf{2 3 7}$, is $\mathbf{2 3 6}$ b, justifying the amount of silver-salt used (Figure $\mathbf{1 1}$ ).

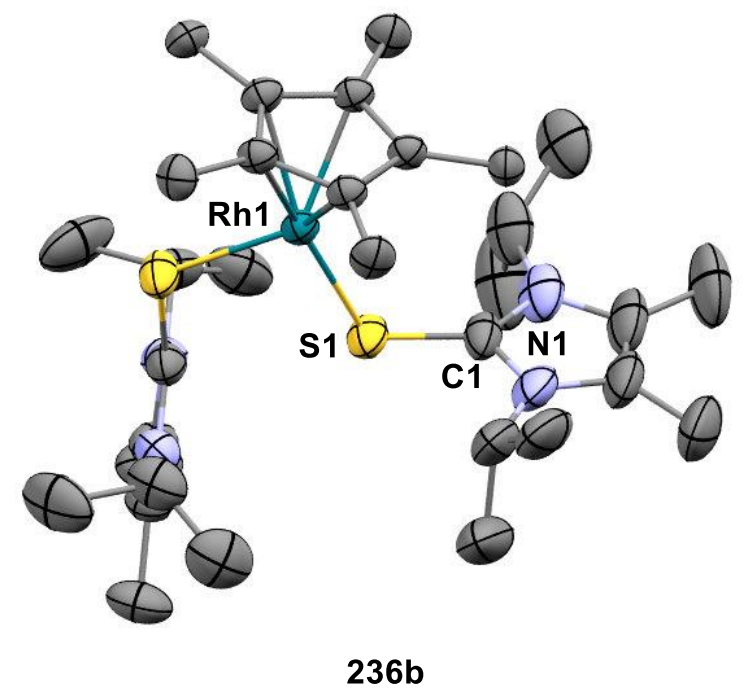

Figure 11: Structure of deactivated catalyst species 236b in the crystal. Anisotropic displacement parameter shown at $50 \%$ probability level, hydrogen atoms, solvent and anions are omitted for clarity. 


\section{The Diazenyl Functionality as a Directing Group}

After the mode of reactivity of 1-phenylpyrazole was resolved, the structurally similar compounds phenylhydrazine and azobenzene were investigated as substrates. Whereas phenylhydrazine showed no reactivity at all, when employing azobenze, full consumption of the starting material was observed under slightly modified reactions condition.

The reaction was worked up as described by CHENG et al. ${ }^{[172]} \mathrm{X}$-ray crystal structure analysis revealed that an annulation of the former triple bond took place in a similar manner as observed before. However, inverted regioselectivity with respect to the alkyne was observed this time. In addition, the thiourea backbone was still attached (Scheme 94 and Figure 12).
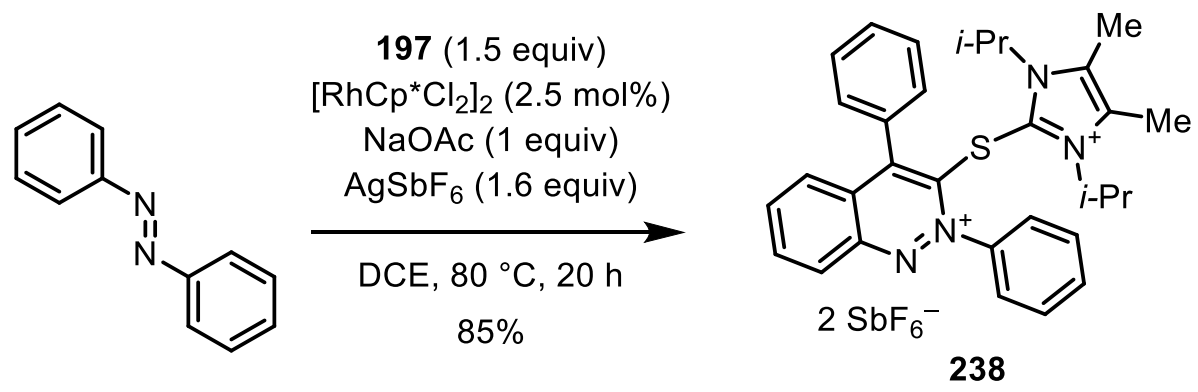

Scheme 94: Reactivity observed for the C-H activation-initiated functionalization of azobenzene with 197.

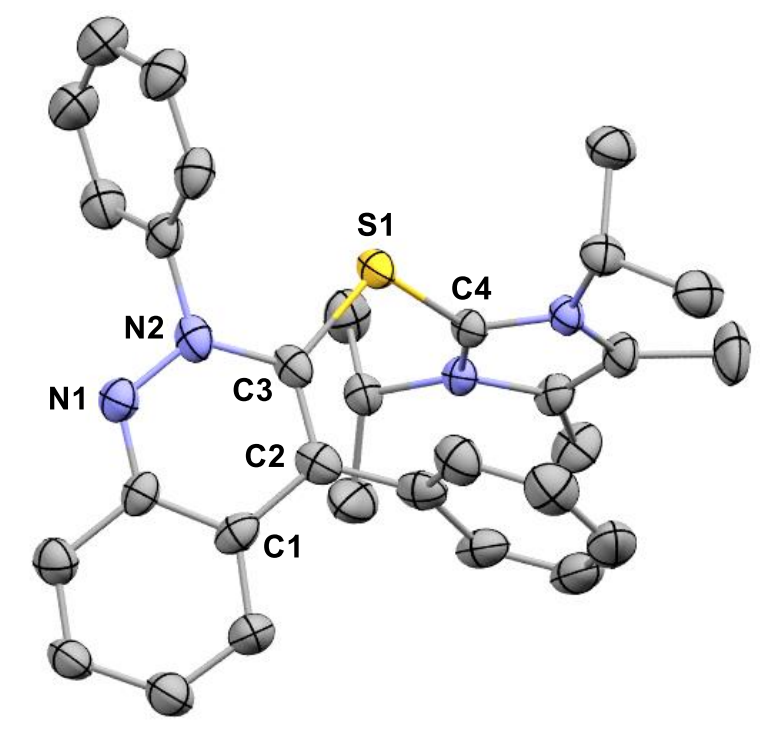

Figure 12: Structure of the adduct obtained after $\mathrm{C}-\mathrm{H}$ activation of azobenzene followed by cyclization with 197 in the crystal. Anisotropic displacement parameter shown at $50 \%$ probability level, hydrogen atoms and anions are omitted for clarity.

This unexpected result along with isochromene iminium 230 (Figure 7, Figure 13), which was obtained in a single case from a crude reaction mixture upon the synthesis of compound $221^{11}$, motivated us to further investigate the mode of cyclization by experiments of both $\mathrm{N}$ methylbenzamide and azobenzene as substrates with ${ }^{13} \mathrm{C}$-labeled reagent $241 .{ }^{[54]}$

${ }^{11}$ The reaction was run a total of three times. 

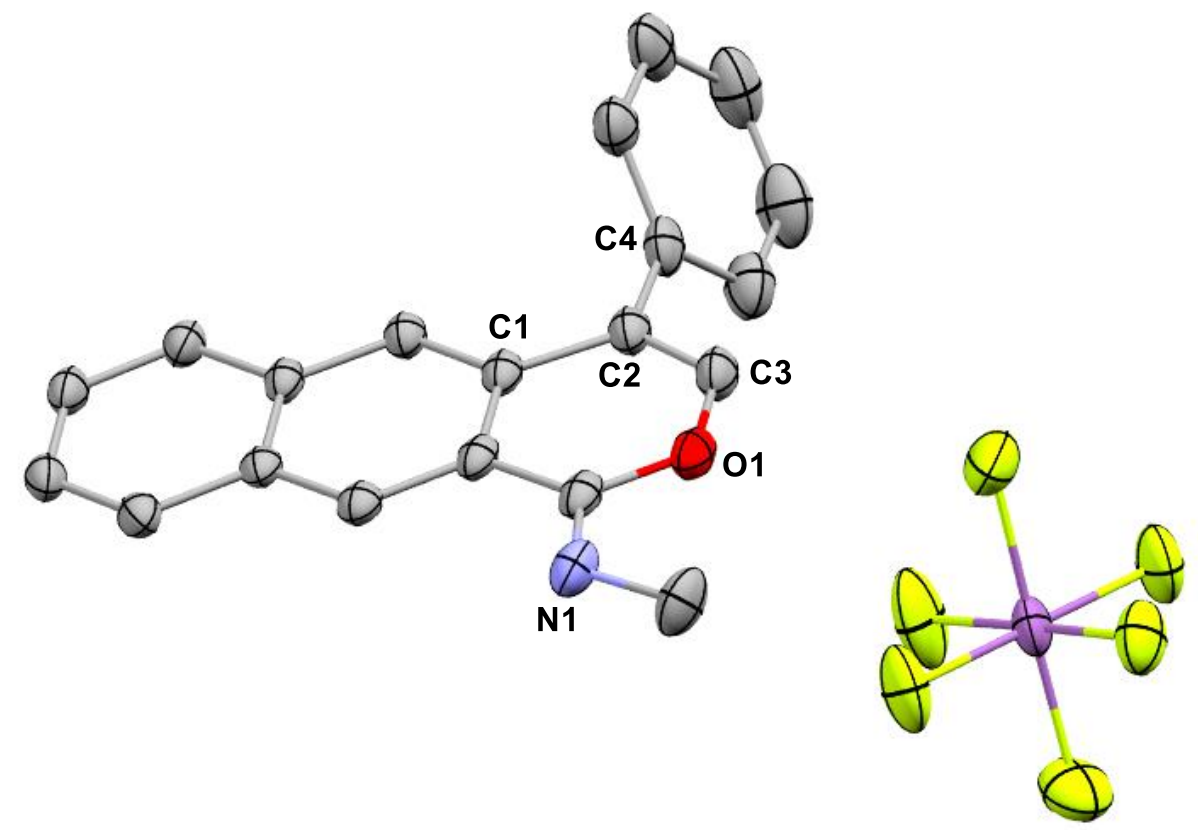

Figure 13: Molecular structure of isochromene iminium 230, obtained in a single case from a crude reaction mixture upon the synthesis of compound 221, in the crystal. Anisotropic displacement parameter shown at $50 \%$ probability level, hydrogen atoms are omitted for clarity.

Carbon scrambling observed for reactions involving (alkynyl)dibenzothiophenium reagents, as developed by ALCARAZO and WALDECKER, ${ }^{[54]}$ gave reason to investigate the observed regioselectivity of the aforementioned reactions.

Thus synthesis of a ${ }^{13} \mathrm{C}$-enriched imidazolium thioalkyne reagent 241 was conducted as presented in Scheme 93.

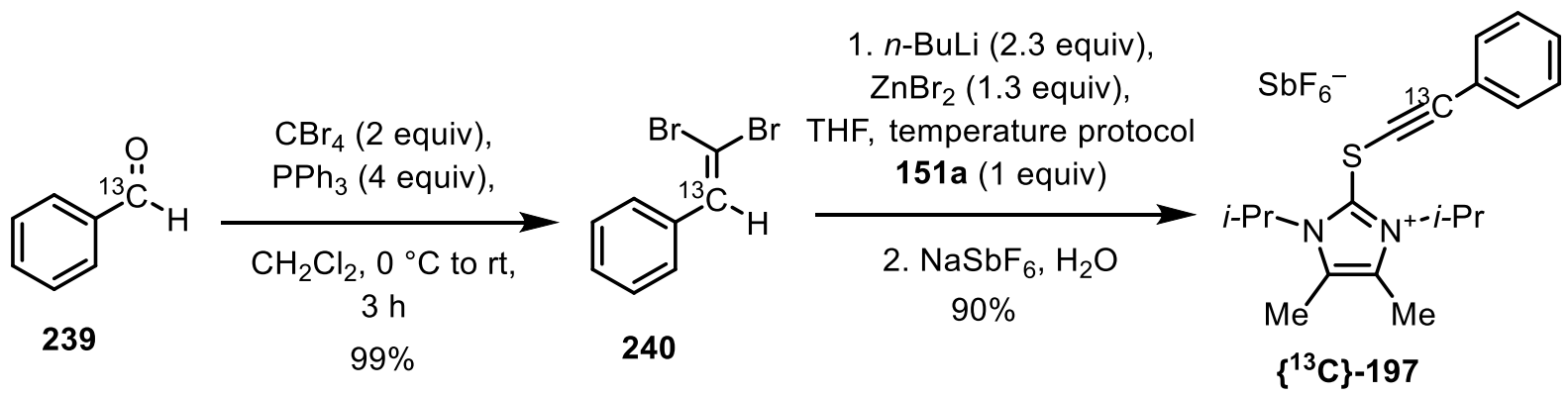

Scheme 95: Synthesis of the ${ }^{13} \mathrm{C}$-enriched alkynylthioimidazolium salt. ${ }^{12}$

In the first step, ${ }^{13} \mathrm{C}$-labeled benzaldehyde 239 was quantitatively transformed to dibromoalkene $\mathbf{2 4 0}$ employing a Corey-Fuchs reaction. Treatment of $\mathbf{2 4 0}$ with $n$-butyllithium caused the Fritsch-Buttenberg-Wiechell rearrangement followed by intermediately formation of alkynyllithium, which was directly transmetalated with a zinc salt. In situ reaction of the latter with dibromo(imidazolium)sulfurane $151 \mathrm{a}$ gave the ${ }^{13} \mathrm{C}$-labeled alkynylating agent $\left\{{ }^{13} \mathrm{C}\right\}-197$ in $90 \%$ yield.

${ }^{12}$ GP C was applied for the last step. 

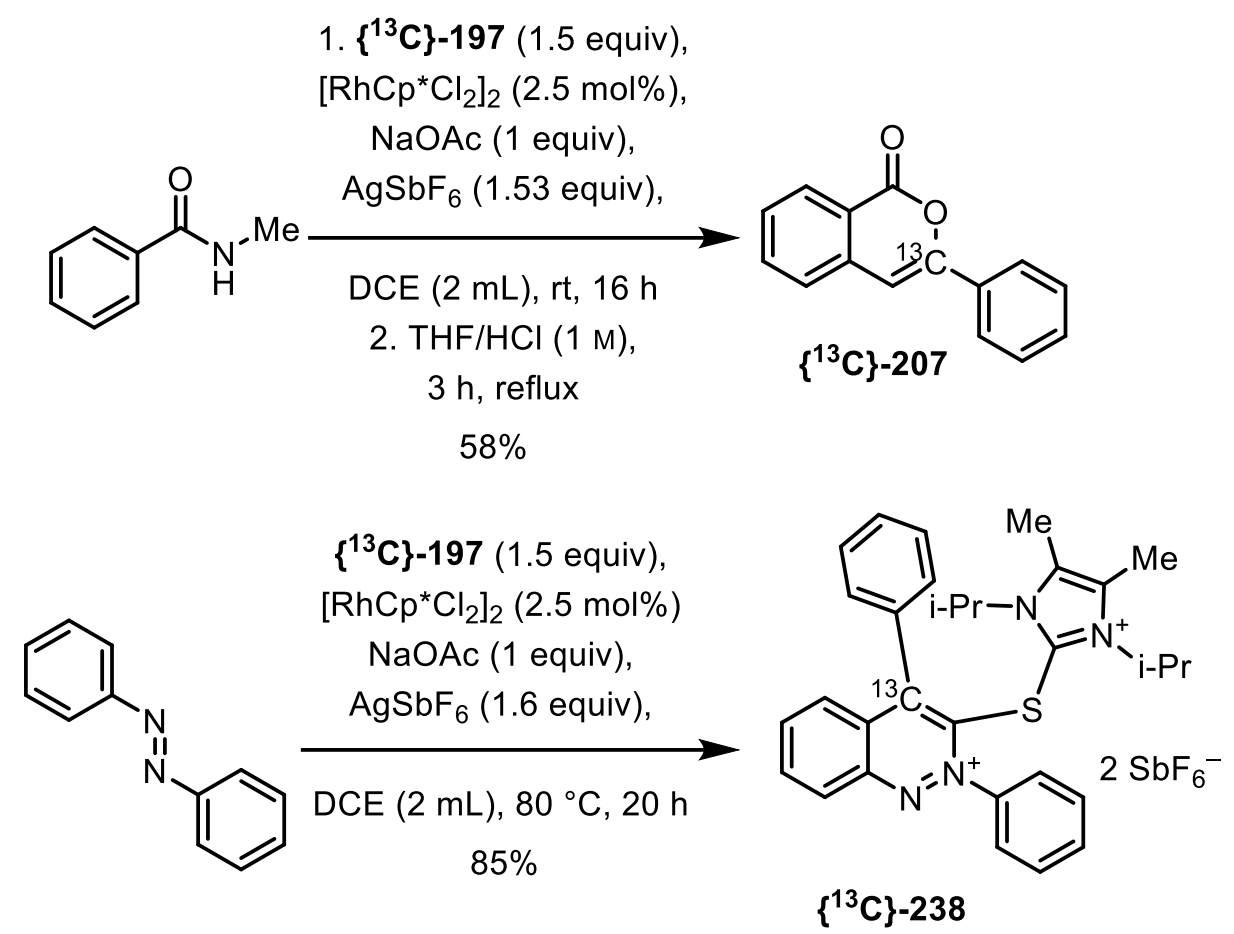

Scheme 96: Reaction of ${ }^{13} \mathrm{C}$-enriched alkynylthioimidazolium salt 241 with $N$-methylbenzamide and azobenzene.

While the aforementioned results indicated, that scrambling of the phenyl group during the reagent synthesis or the catalytic reaction itself might occur, ${ }^{[54]}$ our experiments suggest that scrambling in the products 242 and 243 (Scheme 96) does not proceed at all in both cases. Accordingly, the regioselectivity of the nucleophilic attack on the alkyne is predefined by the properties of the nucleophile.

Additional information was obtained from experiments carried out with dication 238 . Exposure to air and moisture led to the formation of an apolar species, as was observed by TLC. Successful isolation, crystallization and structural analysis revealed the product to possess the structure of cinnolinone 244 (Scheme 97 and Figure 14). ${ }^{13}$<smiles></smiles>

Scheme 97: Cinnolinone $\mathbf{2 4 1}$ obtained after prolonged exposure to ambient conditions.

\footnotetext{
${ }^{13}$ Sufficient amounts for characterization were isolated, however no final yield was determined.
} 


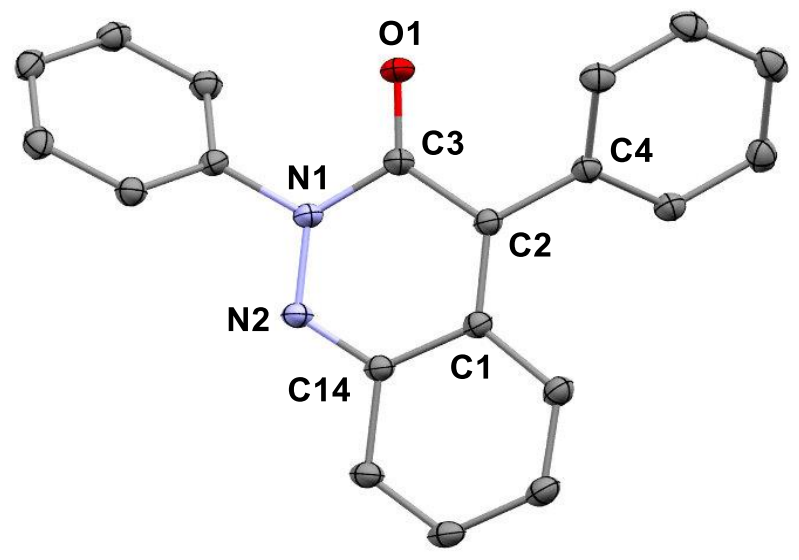

Figure 14: Molecular structure of compound 241 in the crystal. Anisotropic displacement parameter shown at $50 \%$ probability level, hydrogen atoms and anions are omitted for clarity.

When cinnolinium salt $\mathbf{2 3 8}$ was heated in presence of a strong base such as $\mathrm{NaOH}$, ringcontracted product $\mathbf{2 4 5}$ was obtained. Presumably, initial nucleophilic attack of hydroxide furnished an acyclic carboxylic acid, followed by ring closure and successive decarboxylation and air oxidation afforded the indazole framework (Scheme 98 and Figure 15). ${ }^{14[174]}$

On the other hand, participation of the thiourea to facilitate either decarbonylation or decarboxylation by activation of the carbonyl group in a similar fashion as observed for thiolactam 153a in Scheme 74, can not be excluded. ${ }^{[173]}$

\footnotetext{
${ }^{14}$ Sufficient amounts for characterization were isolated, however no final yield was determined.
} 
<smiles>CCCn1c(C)c(C)[n+](CC)c1Sc1c(-c2ccccc2)c2ccccc2n[n+]1-c1ccccc1</smiles>

1. $\mathrm{NaOH}, i-\mathrm{PrOH}, \Delta$<smiles>[R4]c1ccccc1-c1c2ccccc2nn1-c1ccccc1</smiles>

2. $\mathrm{O}_{2}$

242<smiles>Cc1c(C)[n+]([PH2+])c(S/C(O)=C(\c2ccccc2)c2ccccc2/N=N/c2ccccc2)n1[In]</smiles><smiles>COC(=O)C1CC1C</smiles><smiles>O=C(O)C1(c2ccccc2)c2ccccc2NN1c1ccccc1</smiles>

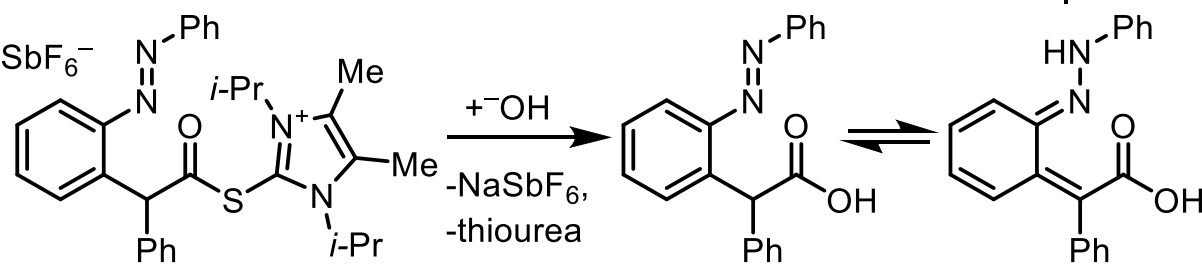

Scheme 98: Reactivity of cinnolinium salt $\mathbf{2 3 8}$ under basic, oxidative conditions to give arylated indazole 242.

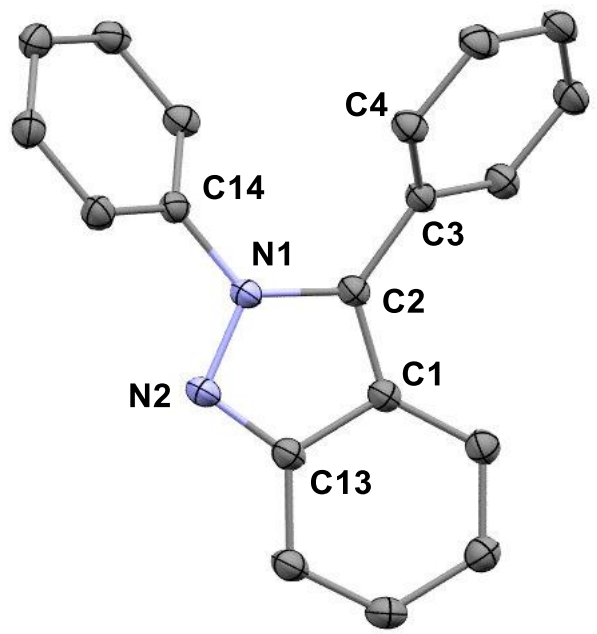

Figure 15: Molecular structure of $\mathbf{2 4 2}$ in the crystal. Anisotropic displacement parameter shown at 50\% probability level, hydrogen atoms and anions are omitted for clarity. 


\section{CONCLUSION AND OUTLOOK}

The task to elaborate an appropriate catalytic system to prove the compatibility of cationic sulfur reagents transferring electrophilic groups in a transition metal-mediated $\mathrm{C}-\mathrm{H}$ activation/functionalization reaction was successfully accomplished. After identification of an appropriate directing group and careful optimization of the reaction conditions, a sequence consisting in electrophilic alkynylation of $\mathrm{N}$-methylbenzamides followed by annulation and final hydrolysis gave isocoumarins in moderate to good yields. The substitution pattern on the aromatic framework of the benzamides revealed to be decisive for the selectivity of product formation. The observed selectivities during the establishment of the scope (Scheme $\mathbf{8 8}$, Scheme $\mathbf{8 9}$ ), the interpretation of the crystallographic evidence from structures 229-233, 237 and $\mathbf{2 3 6}$ as well as the ${ }^{13} \mathrm{C}$-labeling experiments enabled us to formulate a tentative mechanism (Scheme 93). It can be concluded that the substitution pattern on the nitrogen of the amide functionality plays a vital role, but also depending on the decoration of the benzyl ring of the benzamide, secondary effects can favor selectivity or suppress product formation.
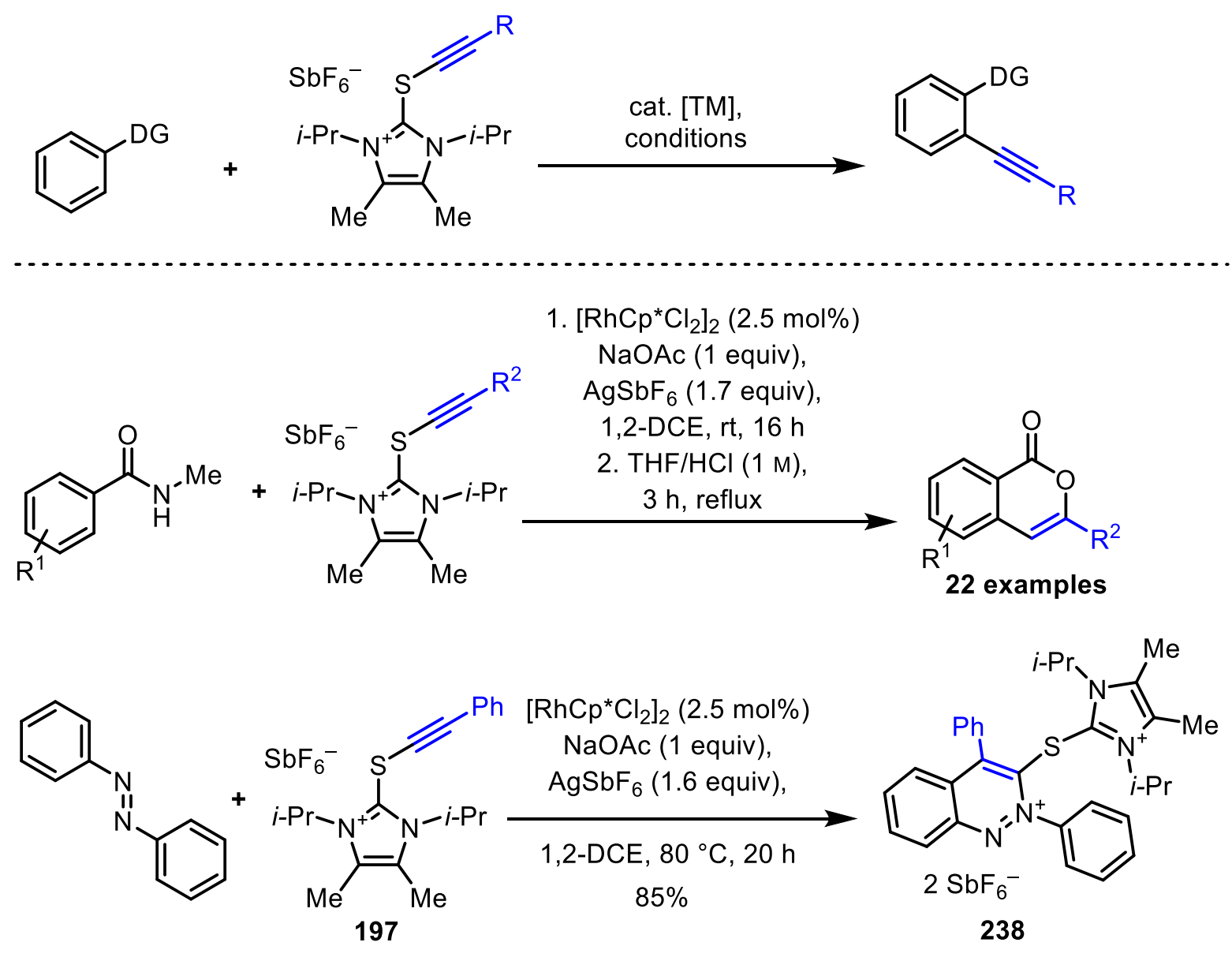

Scheme 99: Established catalytic systems utilizing imidazolium thioalkynes.

Furthermore, numerous other directing groups were screened and showed promising compatibility. For azobenzene, inverted selectivity with respect to the nucleophilic attack on 
the alkyne was observed. In addition, structure of the product suggests, that elimination of the transfer reagent is also substrate-dependent, as similar behavior was observed in initial experiments with benzophenone and other ketones. ${ }^{15}$ The dicationic product 238 was easily purified and proved to open up new exciting opportunities for diversification in preliminiary experiments.<smiles>O=C(O)c1ccc2nn(-c3ccccc3)c(=O)c(-c3ccccc3)c2c1</smiles>

241<smiles>Cc1c(C)[n+](C(C)C)c(Sc2c(-c3ccccc3)c3ccccc3n[n+]2-c2ccccc2)n1C(F)(F)F</smiles>

238

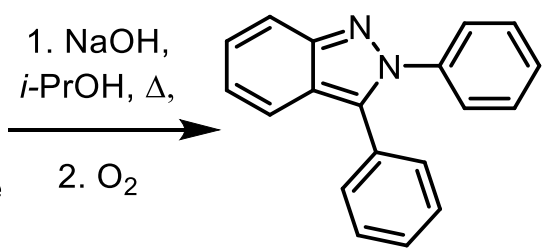

242

Scheme 100: Diversification of dicationic cinnolinium salt 238.

15 The mass of the corresponding adduct was observed in ESI-MS for numerous examples with the keto functionality. 


\section{BIBLIOGRAPHY}

[1] J. McMurry, Organic chemistry, Cengage Learning, Boston, MA, USA, 2015.

[2] a) E. J. Corey, D. Seebach, Angew. Chem. 1965, 77, 1134; b) D. Seebach, Angew. Chem. Int. Ed. Engl. 1979, 18, 239.

[3] Wöhler, Liebig, Ann. Pharm. 1832, 3, 249. Catalytic version: b) N. Zinin, Ann. Pharm. 1839, 31, 329.

[4] a) C. Djerassi, Chem. Rev. 1948, 43, 271; b) N. Shibata, A. Matsnev, D. Cahard, Beilstein J. Org. Chem. 2010, 6.

[5] T. Umemoto, Chem. Rev. 1996, 96, 1757.

[6] R. F. Heck, J. P. Nolley, J. Org. Chem. 1972, 37, 2320.

[7] T. Mizoroki, K. Mori, A. Ozaki, Bull. Chem. Soc. Jpn. 1971, 44, 581.

[8] D. Milstein, J. K. Stille, J. Am. Chem. Soc. 1978, 100, 3636.

[9] D. Milstein, J. K. Stille, J. Am. Chem. Soc. 1979, 101, 4992.

[10]J. K. Stille, Angew. Chem. Int. Ed. Engl. 1986, 25, 508.

[11] M. Kosugi, Y. Shimizu, T. Migita, Chem. Lett. 1977, 6, 1423.

[12] N. Miyaura, K. Yamada, A. Suzuki, Tetrahedron Lett. 1979, 20, 3437.

[13] N. Miyaura, A. Suzuki, J. Chem. Soc., Chem. Commun. 1979, 866.

[14]N. Miyaura, A. Suzuki, Chem. Rev. 1995, 95, 2457.

[15] K. Sonogashira, Y. Tohda, N. Hagihara, Tetrahedron Lett. 1975, 16, 4467.

[16]C. Zhang, Org. Biomol. Chem. 2014, 12, 6580.

[17]W. B. Jensen, J. Chem. Educ. 2006, 83, 1751.

[18]F. M. Beringer, S. A. Galton, J. Org. Chem. 1965, 30, 1930.

[19]M. Ochiai, T. Sueda, K. Miyamoto, P. Kiprof, V. V. Zhdankin, Angew. Chem. Int. Ed. 2006, 45, 8203.

[20]a) T. G. Appleton, H. C. Clark, L. E. Manzer, Coord. Chem. Rev. 1973, 10, 335; b) A. Pidcock, R. E. Richards, L. M. Venanzi, J. Chem. Soc. A 1966, 1707.

[21] a) E. M. Shustorovich, Y. A. Buslaev, Inorg. Chem. 1976, 76, 1142; b) E. Shustorovich, J. Am. Chem. Soc. 1978, 100, 7513; c) E. M. Shustorovich, Y. A. Buslaev, Koord. Khim. 1975, 1, 1020.

[22]a) R. J. Hach, R. E. Rundle, J. Am. Chem. Soc. 1951, 73, 4321; b) G. C. Pimentel, J. Chem. Phys. 1951, 19, 446.

[23]a) V. Zhdankin, Curr. Org. Synth. 2005, 2, 121; b) T.-Y. Sun, X. Wang, H. Geng, Y. Xie, Y.-D. Wu, X. Zhang, H. F. Schaefer, Chem. Commun. 2016, 52, 5371.

[24]a) U. Teruo, I. Sumi, Tetrahedron Lett. 1990, 31, 3579; b) H. Li, Synlett 2012, 23, 2289; c)

L. M., Yagupolskii, N. V. Kondratenko, G. N. Timofeeva, J. Org. Chem. USSR 1984, 20, 103.

[25]T. Umemoto, S. Ishihara, J. Am. Chem. Soc. 1993, 115, 2156.

[26]F. Kurzer, J. Chem. Soc. 1949, 1034.

[27]D. Kahne, D. B. Collum, Tetrahedron Lett. 1981, 22, 5011.

[28] a) S. Thambidurai, K. Jeyasubramanian, S. K. Ramalingam, Polyhedron 1996, 15, 4011; b)

S. Kim, C. J. Lim, Angew. Chem. Int. Ed. 2002, 41, 3265; c) Y. Kita, S. Akai, T. Okuno, M. Egi, T. Takada, H. Tohma, Heterocycles 1996, 42, 47; d) M. Kasthuri, H. Babu, K. Kumar, C. 
Sudhakar, P. Kumar, Synlett 2015, 26, 897; e) S. V. Bhat, D. Robinson, J. E. Moses, P. Sharma, Org. Lett. 2016, 18, 1100; f) A.-P. Schaffner, V. Darmency, P. Renaud, Angew. Chem. Int. Ed. 2006, 45, 5847; g) G. L. Adams, P. J. Carroll, A. B. Smith, J. Am. Chem. Soc. 2012, 134, 4037; h) O. Loreau, N. Camus, F. Taran, D. Audisio, Synlett 2016, 27, 1798; i) M. V. Vita, P. Caramenti, J. Waser, Org. Lett. 2015, 17, 5832.

[29]T. V. Hughes, S. D. Hammond, M. P. Cava, J. Org. Chem. 1998, 63, 401.

[30] V. V. Zhdankin, C. J. Kuehl, A. P. Krasutsky, J. T. Bolz, B. Mishmash, J. K. Woodward, A. J. Simonsen, Tetrahedron Lett. 1995.

[31]R. Akula, Y. Xiong, H. Ibrahim, RSC Adv. 2013, 3, 10731.

[32]P. Anbarasan, H. Neumann, M. Beller, Chem. Eur. J. 2010, 16, 4725.

[33]P. Anbarasan, H. Neumann, M. Beller, Chem. Eur. J. 2011, 17, 4217.

[34]Y. Yang, Y. Zhang, J. Wang, Org. Lett. 2011, 13, 5608.

[35] K. Kiyokawa, T. Nagata, S. Minakata, Angew. Chem. Int. Ed. 2016, 55, 10458.

[36]Alan R. Katritzky, Rufine Akue-Gedu, Anatoliy V. Vakulenko, Arkivoc 2006, 2007, 5.

[37]Y.-F. Wang, J. Qiu, D. Kong, Y. Gao, F. Lu, P. G. Karmaker, F.-X. Chen, Org. Biomol. Chem. 2015, 13, 365.

[38]R. Frei, T. Courant, M. D. Wodrich, J. Waser, Chem. Eur. J. 2015, 21, 2662.

[39]R. Chowdhury, J. Schörgenhumer, J. Novacek, M. Waser, Tetrahedron Lett. 2015, 56, 1911.

[40]M. Chen, Z.-T. Huang, Q.-Y. Zheng, Org. Biomol. Chem. 2015, 13, 8812.

[41]G. Talavera, J. Peña, M. Alcarazo, J. Am. Chem. Soc. 2015, 137, 8704.

[42]X. Li, C. Golz, M. Alcarazo, Angew. Chem. Int. Ed. 2019, 58, 9496.

[43]Y.-q. Wu, D. C. Limburg, D. E. Wilkinson, G. S. Hamilton, Org. Lett. 2000, 2, 795.

[44]a) A. Yoshimura, V. V. Zhdankin, Chem. Rev. 2016, 116, 3328; b) M. Ochiai, Y. Masaki, M. Shiro, J. Org. Chem. 1991, 56, 5511. Essentially, the first hypervalent iodine compound, $\mathrm{PhICl}_{2}$, was prepared in 1886: c) Willgerodt, J. Prakt. Chem. 1886, 33, 185; however, a renaissance in the chemistry of polyvalent iodine has occured only in the past 30 years: d) V. V. Zhdankin, Hypervalent lodine Chemistry: Preparation, Structure, and Synthetic Applications of Polyvalent lodine Compounds, Wiley, Chichester, 2013; e) Special Issue Hypervalent lodine Chemistry, T. Wirth (Ed.), Top. Curr. Chem. 2016, 373, 316 pp.

[45]D. P. Hari, P. Caramenti, J. Waser, Acc. Chem. Res. 2018, 51, 3212.

[46] V. V. Zhdankin, A. P. Krasutsky, C. J. Kuehl, A. J. Simonsen, J. K. Woodward, B. Mismash, J. T. Bolz, J. Am. Chem. Soc. 1996, 118, 5192.

[47]M.-X. Sun, Y.-F. Wang, B.-H. Xu, X.-Q. Ma, S.-J. Zhang, Org. Biomol. Chem. 2018, 16, 1971. [48]A. J. Arduengo, E. M. Burgess, J. Am. Chem. Soc. 1977, 99, 2376.

[49]H. W. Roesky, U. N. Nehete, S. Singh, H.-G. Schmidt, Y. G. Shermolovich, Main Group Chem. 2005, 4, 11.

[50]a) D. Fernández González, J. P. Brand, J. Waser, Chem. Eur. J. 2010, 16, 9457. b) J. P. Brand, J. Charpentier, J.; Waser, Angew. Chem. Int. Ed. 2009, 48, 9346.

[51] a) J. P. Brand, J. Waser, Chem. Soc. Rev. 2012, 41, 4165; b) J. Waser, Johnson Matthey Technol. Rev. 2015, 59, 284.

[52]J. Waser, Synlett 2016, 27, 2761. 
[53]A. G. Barrado, A. Zieliński, R. Goddard, M. Alcarazo, Angew. Chem. Int. Ed. 2017, 56, 13401.

[54]B. Waldecker, F. Kraft, C. Golz, M. Alcarazo, Angew. Chem. Int. Ed. 2018, 57, 12538.

[55] K. F. G. Averesch, H. Pesch, C. Golz, M. Alcarazo, Chem. Eur. J. 2019, 25, 10472.

[56]J. Peña, G. Talavera, B. Waldecker, M. Alcarazo Chem. Eur. J. 2017, 23, 75.

[57]M. J. Böhm, C. Golz, I. Rüter, M. Alcarazo, Chem. Eur. J. 2018, 24, 15026.

[58] P. Murch, B. L. Williamson, P. J. Stang, Synthesis 1994, 1994, 1255.

[59] M. O. Frederick, J. A. Mulder, M. R. Tracey, R. P. Hsung, J. Huang, K. C. M. Kurtz, L. Shen, C. J. Douglas, J. Am. Chem. Soc. 2003, 125, 2368.

[60]J. R. Dunetz, R. L. Danheiser, Org. Lett. 2003, 5, 4011.

[61]T. Hamada, X. Ye, S. S. Stahl, J. Am. Chem. Soc. 2008, 130, 833.

[62]Y. Tu, X. Zeng, H. Wang, J. Zhao, Org. Lett. 2018, 20, 280.

[63]H. Singh, T. Sahoo, C. Sen, S. M. Galani, S. C. Ghosh, Catal. Sci. Technol. 2019, 9, 1691.

[64]K. S. Feldman, M. M. Bruendl, K. Schildknegt, A. C. Bohnstedt, J. Org. Chem. 1996, 61, 5440.

[65]B. Witulski, T. Stengel, Angew. Chem. Int. Ed. 1998, 37, 489.

[66] N. Toriumi, N. Asano, K. Miyamoto, A. Muranaka, M. Uchiyama, J. Am. Chem. Soc. 2018, $140,3858$.

[67]P. J. Stang, Acc. Chem. Res. 1991, 91, 304.

[68]a) M. Ochiai, M. Kunishima, Y. Nagao, K. Fuji, E. Fujita, J. Chem. Soc., Chem. Commun. 1987, 1708; b) P. J. Stang, C. M. Crittell, J. Org. Chem. 1992, 57, 4305.

[69]Y. Gao, G. Wang, L. Chen, P. Xu, Y. Zhao, Y. Zhou, L.-B. Han, J. Am. Chem. Soc. 2009, 131, 7956.

[70]E. Bernoud, C. Alayrac, O. Delacroix, A.-C. Gaumont, Chem. Commun. 2011, 47, 3239.

[71]R. Frei, J. Waser, J. Am. Chem. Soc. 2013, 135, 9620.

[72] M. D. Wodrich, P. Caramenti, J. Waser, Org. Lett. 2016, 18, 60.

[73]R. Frei, M. D. Wodrich, D. P. Hari, P.-A. Borin, C. Chauvier, J. Waser, J. Am. Chem. Soc. 2014, 136, 16563.

[74]J. Doroszuk, M. Musiejuk, S. Demkowicz, J. Rachon, D. Witt, RSC Adv. 2016, 6, 105449.

[75]J. Peña, G. Talavera, B. Waldecker, M. Alcarazo, Chem. Eur. J. 2017, 23, 75.

[76]J. Santandrea, C. Minozzi, C. Cruché, S. K. Collins, Angew. Chem. Int. Ed. 2017, 56, 12255.

[77]J. Y. See, Y. Zhao, Org. Lett. 2018, 20, 7433.

[78] K. Kanemoto, S. Yoshida, T. Hosoya, Org. Lett. 2019, 21, 3172.

[79]A. S. Kende, P. Fludzinski, J. H. Hill, J. Am. Chem. Soc. 1984, 106, 3551.

[80]a) M. Ochiai, M. Kunishima, Y. Nagao, K. Fuji, M. Shiro, E. Fujita, J. Am. Chem. Soc. 1986, 108,$8281 ;$ b) M. Ochiai, T. Ito, Y. Takaoka, Y. Masaki, M. Kunishima, S. Tani, Y. Nagao, J. Chem. Soc., Chem. Commun. 1990, 118.

[81] M. D. Bachi, N. Bar-Ner, C. M. Crittell, P. J. Stang, B. L. Williamson, J. Org. Chem. 1991, 56, 3912.

[82]T. B. Poulsen, L. Bernardi, J. Aleman, J. Overgaard, K. A. Jørgensen, J. Am. Chem. Soc. 2007, 129, 441.

[83]M. S. Raasch, Chem. Commun. (London) 1966, 577. 
[84] M. S. Raasch, J. Org. Chem. 1970, 35, 3470.

[85]M. S. Raasch, J. Org. Chem. 1972, 37, 1347.

[86]E. U. Elam, F. H. Rash, J. T. Dougherty, V. W. Goodlett, K. C. Brannock, J. Org. Chem. 1968, 33, 2738.

[87]M. S. Newman, A. Arkell, T. Fukunaga, J. Am. Chem. Soc. 1960, 82, 2498.

[88] C. Spanka, E. Schaumann, Synlett 2014, 25, 2415.

[89]a) U. Timm, U. Merkle, H. Meier, Chem. Ber. 1980, 113, 2519; b) E. Schaumann, Tetrahedron 1988, 44, 1827.

[90]C. Willgerodt, Ber. Dtsch. Chem. Ges. 1887, 20, 2467.

[91] K. Kindler, Justus Liebigs Ann. Chem. 1923, 431, 187.

[92] H. Xu, H. Deng, Z. Li, H. Xiang, X. Zhou, Eur. J. Org. Chem. 2013, 2013, 7054.

[93]T. B. Nguyen, M. Q. Tran, L. Ermolenko, A. Al-Mourabit, Org. Lett. 2014, 16, 310.

[94] For the principles of atom- and step-economy, see a) B. M. Trost, Acc. Chem. Res. 2002, 35, 695; b) P. A. Wender, V. A. Verma, T. J. Paxton, T. H. Pillow, Acc. Chem Res. 2008, 41, 40.

[95]a) M. Jesberger, T. P. Davis, L. Barner, Synthesis 2003, 1929; b) T. Ozturk, E. Ertas, O. Mert, Chem. Rev. 2010, 110, 3419.

[96]E. U. Elam, H. E. Davis, J. Org. Chem. 1967, 32, 1562.

[97]D. Brillon, Synth. Commun. 1990, 20, 3085.

[98] P. Denifl, B. Bildstein, J. Org. Chem. 1993, 453, 53.

[99]J. Romański, G. Mlostoń, Synthesis 2002, 1355.

[100] A. Duréault, D. Taton, M. Destarac, F. Leising, Y. Gnanou, Macromolecules 2004, 37, 5513.

[101] N. M. Ibrahim, Phosphorus, Sulfur, Silicon Relat. Elem. 2006, 181, 1773.

[102] a) T. Strehlow, J. Voß, R. Spohnholz, G. Adiwidjaja, Chem. Ber. 1991, 124, 1397; b) Y. Hitotsuyanagi, J. Suzuki, Y. Matsumoto, K. Takeya, H. Itokawa, J. Chem. Soc., Perkin Trans. 1 1994, 1887; c) P. Grisenti, A. Magni, A. Manzocchi, P. Ferraboschi, Steroids 1997, 62, 504; d) C. Fossey, H. Landelle, D. Ladureey, M. Robba, Nucleosides Nucleotides 1993, $12,973$.

[103] S. L. Baxter, J. S. Bradshaw, J. Org. Chem. 1981, 46, 831.

[104] a) K. A. Petrov, L. N. Andreev, Russ. Chem. Rev. 1969, 38, 21; b) Brown, Ellis, V., Synthesis 1975, 1975, 358; c) A. B. Charette, M. Grenon, J. Org. Chem. 2003, 68, 5792; d) J. Houben, Ber. Dtsch. Chem. Ges. 1906, 39, 3219; e) J. Houben, K. M. L. Schultze, Ber. Dtsch. Chem. Ges. 1910, 43, 2481; f) E. J. Hedgley, H. G. Fletcher, J. Org. Chem. 1965, 30, 1282; g) P. J. W. Schuijl, L. Brandsma, J. F. Arens, Recl. Trav. Chim. Pays-Bas 1966, 85, 889; h) J. Meijer, P. Vermeer, L. Brandsma, Recl. Trav. Chim. Pays-Bas 1973, 92, 601; i) H. Westmijze, H. Kleijn, J. Meijer, P. Vermeer, Synthesis 1979, 1979, 432; j) R. Hoffmann, K. Hartke, Chem. Ber. 1980, 113, 919; k) A. C. Worth, C. E. Needham, D. B. Franklin, A. J. Lampkins, Synth. Commun. 2012, 42, 2694.

[105] K. B. Wiberg, D. J. Rush, J. Am. Chem. Soc. 2001, 123, 2038.

[106] K. B. Wiberg, P. R. Rablen, D. J. Rush, T. A. Keith, J. Am. Chem. Soc. 1995, 117, 4261.

[107] T. J. Williams, A. D. Kershaw, V. Li, X. Wu, J. Chem. Educ. 2011, 88, 665. 
[108] B. K. Min, H.-J. Lee, Y. S. Choi, J. Park, C.-J. Yoon, J.-A. Yu, J. Mol. Struct. 1998, 471, 283.

[109] S. J. Collier, Science of Synthesis 2007.

[110] K. B. Wiberg, Acc. Chem. Res. 1999, 32, 922.

[111] L. Pauling, The Nature of the Chemical Bond, Cornell University Press, Ithaca, U.S.A., 1960.

[112] K. B. Wiberg, Y. Wang, Arkivoc 2011, 2011, 45.

[113] T. S. Jagodziński, Chem. Rev. 2003, 103, 197.

[114] a) M. C. Bagley, J. W. Dale, E. A. Merritt, X. Xiong, Chem. Rev. 2005, 105, 685; b) T. Sifferlen, M. Rueping, K. Gademann, B. Jaun, D. Seebach, Helv. Chim. Acta 1999, 82, 2067; c) G. Moad, E. Rizzardo, S. H. Thang, Aust. J. Chem. 2012, 65, 985.

[115] H.-G. Cheng, H. Chen, Y. Liu, Q. Zhou, Asian J. Org. Chem. 2018, 7, 490.

[116] A. Lengar, C. O. Kappe, Org. Lett. 2004, 6, 771.

[117] E. J. Petersson, J. M. Goldberg, R. F. Wissner, Phys. Chem. Chem. Phys. 2014, 16, 6827.

[118] a) D. R. Lide (Ed.) CRC handbook of chemistry and physics. A ready-reference book of chemical and physical data, CRC Press, Boca Raton, Fla., 1990; b) V. V. Grushin, H. Alper, Chem. Rev. 1994, 94, 1047; c) J. D. Cox, G. Pilcher, Thermochemistry of organic and organometallic compounds, Acad. Press, London, 1970.

[119] L. Ackermann, Chem. Rev. 2011, 111, 1315.

[120] a) L. Ackermann (Ed.) Modern arylation methods, Wiley-VCH, Weinheim, 2009; b) N. Chatani, L. Ackermann (Eds.) Topics in organometallic chemistry, Vol. 24, Springer, Berlin, Heidelberg, 2007.

[121] D. Lapointe, K. Fagnou, Chem. Lett. 2010, 39, 1118.

[122] Y. Boutadla, D. L. Davies, S. A. Macgregor, A. I. Poblador-Bahamonde, Dalton Trans. 2009, 5887.

[123] Y. Boutadla, D. L. Davies, S. A. Macgregor, A. I. Poblador-Bahamonde, Dalton Trans. 2009, 5820.

[124] J. Oxgaard, W. J. Tenn, R. J. Nielsen, R. A. Periana, W. A. Goddard, Organometallics 2007, 26, 1565.

[125] W. Ma, R. Mei, G. Tenti, L. Ackermann, Chem. Eur. J. 2014, 20, 15248.

[126] D. Zell, M. Bursch, V. Müller, S. Grimme, L. Ackermann, Angew. Chem. Int. Ed. 2017, $56,10378$.

[127] F. Zhang, D. R. Spring, Chem. Soc. Rev. 2014, 43, 6906.

[128] Z. Chen, B. Wang, J. Zhang, W. Yu, Z. Liu, Y. Zhang, Org. Chem. Front. 2015, 2, 1107.

[129] C. Laurence, J.-F.. Gal, Lewis basicity and affinity scales. Data and measurement, Wiley, Chichester, 2010.

[130] O. Dimroth, Ber. Dtsch. Chem. Ges. 1898, 31, 2154.

[131] L. Y., Zh. Russ. Phys-Khim. Obshch. 1876, 8, 281.

[132] S. Murahashi, J. Am. Chem. Soc. 1955, 77, 6403.

[133] W. C. Baird, R. L. Hartgerink, J. H. Surridge, J. Org. Chem. 1985, 50, 4601. 
[134] a) S. Murai, F. Kakiuchi, S. Sekine, Y. Tanaka, A. Kamatani, M. Sonoda, N. Chatani, Nature 1993, 366, 529; b) D. Alberico, M. E. Scott, M. Lautens, Chem. Rev. 2007, 107, 174; c) E. M. Beccalli, G. Broggini, M. Martinelli, S. Sottocornola, Chem. Rev. 2007, 107, 5318; d) D. A. Colby, R. G. Bergman, J. A. Ellman, Chem. Rev. 2010, 110, 624; e) G. P. McGlacken, L. M. Bateman, Chem. Soc. Rev. 2009, 38, 2447.

[135] X. Chen, K. M. Engle, D.-H. Wang, J.-Q. Yu, Angew. Chem. Int. Ed. 2009, 48, 5094.

[136] T. Satoh, M. Miura, Chem. Eur. J. 2010, 16, 11212.

[137] a) G. Song, F. Wang, X. Li, Chem. Soc. Rev. 2012, 41, 3651; b) K. M. Engle, T.-S. Mei, M. Wasa, J.-Q. Yu, Acc. Chem. Res. 2012, 45, 788; c) R. Shang, L. Ilies, E. Nakamura, Chem. Rev. 2017, 117, 9086; d) M. Moselage, J. Li, L. Ackermann, ACS Catal. 2016, 6, 498; e) R. Das, G. S. Kumar, M. Kapur, Eur. J. Org. Chem. 2017, 2017, 5439; f) A. Peneau, C. Guillou, L. Chabaud, Eur. J. Org. Chem. 2018, 2018, 5777; g) P. G. Chirila, C. J. Whiteoak, Dalton Trans. 2017, 46, 9721; h) L. Ackermann, Acc. Chem. Res. 2014, 47, 281; i) H. M. L. Davies, D. Morton, J. Org. Chem. 2016, 81, 343; j) X.-F. Wu (Ed.) Transition metal-catalyzed heterocycle synthesis via $\mathrm{C}-\mathrm{H}$ activation, Wiley-VCH, Weinheim, 2016; k) J.-Q. Yu, L. Ackermann (Eds.), Top. Curr. Chem., Vol. 292, Springer, Berlin, 2010; I) F. W. Patureau, J. Wencel-Delord, F. Glorius, Aldrichimica Acta 2012, 45, 31.

[138] R.-Y. Zhu, M. E. Farmer, Y.-Q. Chen, J.-Q. Yu, Angew. Chem. Int. Ed. 2016, 55, 10578.

[139] Z. Huang, H. N. Lim, F. Mo, M. C. Young, G. Dong, Chem. Soc. Rev. 2015, 44, 7764.

[140] Y. Yang, K. Li, Y. Cheng, D. Wan, M. Li, J. You, Chem. Commun. 2016, 52, 2872.

[141] D. J. Abrams, P. A. Provencher, E. J. Sorensen, Chem. Soc. Rev. 2018, 47, 8925.

[142] K. Ueura, T. Satoh, M. Miura, Org. Lett. 2007, 9, 1407.

[143] K. Ueura, T. Satoh, M. Miura, J. Org. Chem. 2007, 72, 5362.

[144] N. Umeda, H. Tsurugi, T. Satoh, M. Miura, Angew. Chem. Int. Ed. 2008, 47, 4019.

[145] a) T. K. Hyster, T. Rovis, J. Am. Chem. Soc. 2010, 132, 10565; b) H. Zhong, D. Yang, S. Wang, J. Huang, Chem. Commun. 2012, 48, 3236.

[146] S. Mochida, N. Umeda, K. Hirano, T. Satoh, M. Miura, Chem. Lett. 2010, 39, 744.

[147] M. Deponti, S. I. Kozhushkov, D. S. Yufit, L. Ackermann, Org. Biomol. Chem. 2013, 11, 142.

[148] Y. Ano, M. Tobisu, N. Chatani, Synlett 2012, 23, 2763.

[149] R. Mei, S.-K. Zhang, L. Ackermann, Org. Lett. 2017, 19, 3171.

[150] J. Yi, L. Yang, C. Xia, F. Li, J. Org. Chem. 2015, 80, 6213.

[151] V. G. Landge, G. Jaiswal, E. Balaraman, Org. Lett. 2016, 18, 812.

[152] Z. Ruan, N. Sauermann, E. Manoni, L. Ackermann, Angew. Chem. Int. Ed. 2017, 56, 3172.

[153] G. Cera, T. Haven, L. Ackermann, Chem. Eur. J. 2017, 23, 3577.

[154] E. Tan, O. Quinonero, M. Elena de Orbe, A. M. Echavarren, ACS Catal. 2018, 8, 2166.

[155] C. Feng, T.-P. Loh, Angew. Chem. Int. Ed. 2014, 53, 2722.

[156] F. Xie, Z. Qi, S. Yu, X. Li, J. Am. Chem. Soc. 2014, 136, 4780.

[157] C. Feng, D. Feng, Y. Luo, T.-P. Loh, Org. Lett. 2014, 16, 5956.

[158] J. Waser, Top. Curr. Chem. 2016, 373, 187.

[159] M. Alcarazo, Acc. Chem. Res. 2016, 49, 1797. 
[160] A. Streitwieser, R. W. Taft, Progress in Physical Organic Chemistry, John Wiley \& Sons, Inc, Hoboken, NJ, USA, 1968.

[161] a) C. W. Perkins, J. C. Martin, A. J. Arduengo, W. Lau, A. Alegria, J. K. Kochi, J. Am. Chem. Soc. 1980, 102, 7753; b) P. J. Stang, V. V. Zhdankin, Chem. Rev. 1996, 96, 1123.

[162] L. Vogel, P. Wonner, S. M. Huber, Angew. Chem. Int. Ed. 2019, 58, 1880, and references cited therein.

[163] a) S. Shaik, D. Danovich, W. Wu, P. C. Hiberty, Nat. Chem. 2009, 1, 443; b) B. Braïda, P. C. Hiberty, Nat. Chem. 2013, 5, 417.

[164] E. J. Adams, T. Skrydstrup, K. B. Lindsay in Encyclopedia of Reagents for Organic Synthesis, John Wiley \& Sons, Ltd, Chichester, 2001.

[165] H. Masumoto, H. Tsutumi, T. Kanda, M. Komada, T. Murai, S. Kato, Sulfur Lett. 1989, 10, 103.

[166] G. V. Boyd, Tetrahedron Lett. 1972, 13, 2711.

[167] G. V. Boyd, J. Chem. Soc., Perkin Trans. 1 1973, 1731.

[168] K. Muralirajan, K. Parthasarathy, C.-H. Cheng, Angew. Chem. Int. Ed. 2011, 50, 4169.

[169] F. W. Patureau, T. Besset, N. Kuhl, F. Glorius, J. Am. Chem. Soc. 2011, 133, 2154.

[170] S. H. Park, J. Y. Kim, S. Chang, Org. Lett. 2011, 13, 2372.

[171] a) G. Liu, Y. Zhou, D. Ye, D. Zhang, X. Ding, H. Jiang, H. Liu, Adv. Synth. Catal. 2009, 351, 2605; b) M. Bian, W. Yao, H. Ding, C. Ma, J. Org. Chem. 2010, 75, 269; c) H. Sashida, Synthesis 1999, 1145; d) J. D. Tovar, T. M. Swager, J. Org. Chem. 1999, 64, 6499; e) T. Wakamatsu, Y. Ogawa, M. Maruno, Heterocycles 1995, 41, 2587; f) P. Zhao, D. Chen, G. Song, K. Han, X. Li, J. Org. Chem. 2012, 77, 1579; g) H. Sun, L. Xiao, W. Li, Q. Xie, L. Shao, Synthesis 2017, 49, 4845; h) X. Zhang, B. Liu, X. Shu, Y. Gao, H. Lv, J. Zhu, J. Org. Chem. 2012, 77, 501.

[172] K. Muralirajan, C.-H. Cheng, Chem. Eur. J. 2013, 19, 6198.

[173] K. Inomata, H. Kinoshita, H. Fukuda, K. Tanabe, H. Kotake, BCSJ 1978, 51, 1866.

[174] J. Chi, C. Hang, Y. Zhu, H. Katayama, Synth. Commun. 2010, 40, 1123. 


\section{EXPERIMENTAL}

General: If not otherwise specified, all reactions were carried out in oven-dried glassware under $\mathrm{N}_{2}$. All solvents were purified by distillation over the appropiate drying agents and were transferred under $\mathrm{N}_{2}$. Alternatively, dry solvents were obtained using an MBraun MBSPS-800 solvent purification system (tetrahydrofuran, diethyl ether, toluene, pentane, dichloromethane, acetonitrile). IR: Nicolet FT-7199 spectrometer, JASCO FT-4100 spectrometer, wavenumbers in $\mathrm{cm}^{-1}$. MS (EI): Finnigan MAT 8200 (70 eV), ESIMS: Finnigan MAT 95, accurate mass determinations: Bruker APEX III FT-MS (7 T magnet). NMR: Spectra were recorded on a Bruker AV 600, AV 500, AV 400 or DPX 300; ${ }^{1} \mathrm{H}$ and ${ }^{13} \mathrm{C}$ chemical shifts $(\delta)$ are given in ppm relative to TMS, coupling constants $(J)$ in $\mathrm{Hz}$. Residual solvent signals were used as references and the chemical shifts converted to the TMS scale. X-ray-diffraction: Single crystal structure determination was performed by Dr. Christopher Golz at the University of Göttingen or by the department of chemical crystallography at the Max-PlanckInstitut für Kohlenforschung. Experimental setup in Göttingen: Data collection was done on a Bruker D8 Venture four-circle-diffractometer from Bruker AXS GmbH; detector: Photon II from Bruker AXS GmbH; X-ray sources: microfocus I $\mu \mathrm{S} \mathrm{Cu} / \mathrm{Mo}$ from Incoatec $\mathrm{GmbH}$ with mirror optics HELIOS and single-hole collimator from Bruker AXS GmbH. Used programs: APEX3 Suite (v2017.3-0) and therein integrated programs SAINT (Integration) und SADABS (Absorption correction) from Bruker AXS GmbH; structure solution was done with SHELXT, refinement with SHELXS (Both: G.M. Sheldrick, Acta Cryst. 2008, A64, 112-122.); OLEX² was used for data finalization (O.V. Dolomanov, L.J. Bourhis, R.J Gildea, J.A.K. Howard, H. Puschmann, J. Appl. Cryst. 2009, 42, 339-341.). Special Utilities: SMZ1270 stereomicroscope from Nikon Metrology $\mathrm{GmbH}$ was used for sample preparation; crystals were mounted on MicroMounts or MicroLoops from MiTeGen; for sensitive samples the X-TEMP 2 System was used for picking of crystals (T. Kottke, D. Stalke, J. Appl. Cryst. 1993, 26, 615-619.); crystals were cooled to given temperature with Cryostream 800 from Oxford Cryosystems. Experimental set up at the Max-Planck-Institut für Kohlenforschung: Data collection was done on a Bruker AXS Proteum X8, Bruker AXS KappaCCD and/or Bruker AXS Apex II diffractometers. The crystal structures were solved by direct methods using SHELXS97 and refined with SHELXL-2014. Column chromatography was performed on Merck 60 silica gel (40-63 $\mu \mathrm{m})$, and for thin-layer chromatography (TLC) analysis, Merck silica gel 60 F254 TLC plates or polygram SIL G/UV254 from Macherey Nagel were used and visualized by UV irradiation and/or phosphomolybdic acid or $\mathrm{KMnO}_{4}$ dip. All commercially available compounds (ABCR, Acros Organics, Alfa Aesar, Chempur GmbH, J and K Scientific, Sigma Aldrich, Thermo Fisher Scientific, Tokyo Chemical Industry) were used as received. Triethyloxonium tetrafluoroborate ${ }^{[16]}, \quad \mathrm{N}$-methylbenzamides ${ }^{17}\left[\mathrm{RhCp}^{*} \mathrm{Cl}_{2}\right]_{2}{ }^{18}$,

\footnotetext{
${ }^{16}$ H. Meerwein, Org. Synth. 1966, 46, $113 \mathrm{ff}$.

${ }^{17}$ L. Ackermann, A. V. Lygin, N. Hofmann, Angew. Chem. Int. Ed. 2011, 50, 6379-6382.

18 J.W. Kang, K. Moseley, P. M. Maitlis, J. Am. Chem. Soc. 1969, 91, 5970-5977.
} 
$\mathrm{RhCp} *(\mathrm{MeCN})_{3}\left[\mathrm{SbF}_{6}\right]_{2}{ }^{19}$, were prepared according to literature procedures. X-ray structures of 230-233 were obtained by picking suitable crystals from the reaction crude. If not mentioned otherwise, no further characterization for these compounds was conducted.

\section{Topic:" Synthesis and Evaluation of Novel Pyridinium-Based Electrophilic Transfer REAGENTS"}

\section{2-Chloro-1-methylpyridin-1-ium tetrafluoroborate (156a):}<smiles>C[n+]1ccccc1Cl</smiles>

Following the literature procedure, ${ }^{[20]}$ the salt $156 a$ was prepared by alkylation of 2-chloropyridine (2.2 g, $19.38 \mathrm{mmol}, 1$ equiv.) with trimethyloxonium tetrafluoroborate $(3.0 \mathrm{~g}, 20.3 \mathrm{mmol}, 1.05$ equiv.) in dry $\mathrm{CH}_{2} \mathrm{Cl}_{2}(15 \mathrm{~mL})$ at $0{ }^{\circ} \mathrm{C}$. The solution was stirred overnight, the $\mathrm{CH}_{2} \mathrm{Cl}_{2}$ was filtered off with a filter stick and the residue was washed two more times with $\mathrm{CH}_{2} \mathrm{Cl}_{2}$. Compound 156a was obtained as a white solid after drying in vacuo (4.0 g, $18.57 \mathrm{mmol}$, 96\%). ${ }^{[21]}$

${ }^{1} \mathrm{H}$ NMR: $\left(300 \mathrm{MHz}, \mathrm{CD}_{3} \mathrm{CN}\right) \delta=8.74(\mathrm{~d}, J=6.0 \mathrm{~Hz}, 1 \mathrm{H}), 8.46(\mathrm{td}, J=8.1,1.7 \mathrm{~Hz}, 1 \mathrm{H}), 8.19-$ $8.06(\mathrm{~m}, 1 \mathrm{H}), 7.94(\mathrm{t}, J=6.8 \mathrm{~Hz}, 1 \mathrm{H}), 4.29(\mathrm{~s}, 3 \mathrm{H})$ ppm. ${ }^{19} \mathrm{~F}$ NMR: $\left(282 \mathrm{MHz}, \mathrm{CD}_{3} \mathrm{CN}\right) \delta=-$ $151.77 \mathrm{ppm}$.

\section{2-Chloro-1-ethylpyridin-1-ium tetrafluoroborate (156b):}<smiles>CC[n+]1ccccc1Cl</smiles>

Salt 156b was prepared by alkylation of 2-chloropyridine (1 equiv., $1.135 \mathrm{~g}$, $10 \mathrm{mmol}$ ) with triethyloxonium tetrafluoroborate (1 equiv., $1.9 \mathrm{~g}, 10 \mathrm{mmol}$ ) in dry $\mathrm{CH}_{2} \mathrm{Cl}_{2}(40 \mathrm{~mL})$ at $0{ }^{\circ} \mathrm{C}$. The solution was stirred overnight, the $\mathrm{CH}_{2} \mathrm{Cl}_{2}$ was removed in vacuo and the residue was washed with $\mathrm{Et}_{2} \mathrm{O}(3 \times 20 \mathrm{~mL})$ to afford compound $156 \mathrm{~b}$ as a white solid (2.3 g, $10 \mathrm{mmol}, 99 \%){ }^{[22]}$

${ }^{1}$ H NMR: $\left(300 \mathrm{MHz}, \mathrm{CD}_{3} \mathrm{CN}\right) \delta=8.77(\mathrm{dd}, J=6.3,1.7 \mathrm{~Hz}, 1 \mathrm{H}), 8.46(\mathrm{td}, J=8.0,1.8 \mathrm{~Hz}, 1 \mathrm{H})$, $8.11(\mathrm{dd}, J=8.3,1.4 \mathrm{~Hz}, 1 \mathrm{H}), 8.04-7.90(\mathrm{~m}, 1 \mathrm{H}), 4.72(\mathrm{q}, J=7.3 \mathrm{~Hz}, 2 \mathrm{H}), 1.58(\mathrm{t}, J=7.3 \mathrm{~Hz}$, 3H) ppm. ${ }^{13} \mathrm{C}$ NMR: $\left(101 \mathrm{MHz}, \mathrm{CD}_{3} \mathrm{CN}\right) \delta=148.2,147.7,131.4,127.8,57.3,14.8 \mathrm{ppm}$.

\footnotetext{
${ }^{19}$ Y. Du, T. K. Hyster, T. Rovis, Chem. Commun 2011, 47, 12074-12076

${ }^{20}$ H. Tinnermann, C. Wille, M. Alcarazo, Angew. Chem. Int. Ed. 2014, 53, 8732-8736.

${ }^{21}$ Analytical data are in agreement with the previously published ones: H. Tinnermann, C. Wille, M. Alcarazo., Angew. Chem. Int. Ed. 2014, 53, 8732-8736, Compound 6.

${ }^{22}$ Analytical data are in agreement with the previously reported ones: $\mathrm{H}$. Tinnermann, PhD Thesis, University of Göttingen (Germany), 2017, Compound 129c.
} 


\section{1-Ethyl-3,5-dimethylpyridin-1-ium tetrafluoroborate (156c):}<smiles>CC[n+]1cc(C)cc(C)c1</smiles>

Salt 156c was prepared by alkylation of 3,5-lutidine ( 1 equiv., $5.7 \mathrm{~g}, 53.2$ $\mathrm{mmol}$ ) with triethyloxonium tetrafluoroborate (0.99 equiv., $10.0 \mathrm{~g}, 52.6$ mmol) in dry $\mathrm{CH}_{2} \mathrm{Cl}_{2}(50 \mathrm{~mL})$ at $0{ }^{\circ} \mathrm{C}$. The solution was stirred overnight, then the solvent was removed in vacuo and the residue was washed with

$\mathrm{Et}_{2} \mathrm{O}(3 \times 50 \mathrm{~mL})$. Salt $156 \mathrm{c}$ was obtained as white solid after drying in vacuo $(10.6 \mathrm{~g}, 47.53$ $\mathrm{mmol}, 90 \%)$.

${ }^{1}$ H NMR: $\left(400 \mathrm{MHz}, \mathrm{CD}_{3} \mathrm{CN}\right) \delta=8.39(\mathrm{~s}, 2 \mathrm{H}), 8.13(\mathrm{~s}, 1 \mathrm{H}), 4.46(\mathrm{q}, J=7.4 \mathrm{~Hz}, 3 \mathrm{H}), 2.47(\mathrm{~s}, 3 \mathrm{H})$, $2.46(\mathrm{~s}, 3 \mathrm{H}), 1.56(\mathrm{t}, J=7.4 \mathrm{~Hz}, 3 \mathrm{H})$ ppm. ${ }^{13} \mathrm{C}$ NMR: $\left(101 \mathrm{MHz}, \mathrm{CD}_{3} \mathrm{CN}\right) \delta=147.3,141.9(\mathrm{t}, J=$ $8.8 \mathrm{~Hz}), 139.9,57.8(\mathrm{t}, J=3.8 \mathrm{~Hz}), 18.2,16.5 \mathrm{ppm} .{ }^{19} \mathrm{~F}$ NMR: $\left(377 \mathrm{MHz}, \mathrm{CD}_{3} \mathrm{CN}\right) \delta=-151.70$ ppm. IR: (ATR, cm ${ }^{-1}$ ) 3082, 2360, 1497, 1458, 1391, 1304, 1189, 1093, 1047, 1033, 887, 811, 691, 521. HRMS: calcd. for $\mathrm{C}_{9} \mathrm{H}_{14} \mathrm{~N}^{+}\left[\mathrm{M}-\mathrm{BF}_{4}^{-}\right]$: 136.112074 ; found (ESI): 136.112230.

\section{1-Methylpyridine-2(1H)-thione (153a):}<smiles>Cn1ccccc1=S</smiles>

Compound 156a (4.0 g, $18.57 \mathrm{mmol})$ was dissolved in methanol (70 mL). A solution of sodium hydrosulfide hydrate (2 equiv, $2.8 \mathrm{~g}, 37.8 \mathrm{mmol}$ ) in water (140 $\mathrm{mL}$ ) was prepared and subsequently added to the former dropwise at $45^{\circ} \mathrm{C}$. After stirring for $30 \mathrm{~min}$, the reaction mixture was poured into $100 \mathrm{~mL}$ of water, the solution extracted with $\mathrm{CH}_{2} \mathrm{Cl}_{2}(3 \times 70 \mathrm{~mL})$, and the organic phase was dried over $\mathrm{Na}_{2} \mathrm{SO}_{4}$. Removal of all solvents in vacuo afforded crude 153a, which was purified by column chromatography (EtOAc) and obtained as a pale yellow crystalline solid $(2.1 \mathrm{~g}, 16.8 \mathrm{mmol}$, 90\%).

Alternatively compound AVK-AA-429 was prepared following the same procedure but starting from commercially available 2-chloro-1-methylpyridin-1-ium iodide $(30.0 \mathrm{~g}, 117.4$ $\mathrm{mmol})$, in methanol $(60 \mathrm{~mL})$, sodium hydrosulfide hydrate $(1.7$ equiv, $14.3 \mathrm{~g}, 193 \mathrm{mmol}$ ) in water $(30 \mathrm{~mL}$ ) after column chromatography (EtOAc) as a pale yellow crystalline solid (13.4 g, $107 \mathrm{mmol}, 91 \%)$.

${ }^{1} \mathrm{H}$ NMR: $\left(300 \mathrm{MHz}, \mathrm{CD}_{3} \mathrm{CN}\right) \delta=7.82(\mathrm{dd}, J=6.6,1.3 \mathrm{~Hz}, 1 \mathrm{H}), 7.51(\mathrm{~d}, J=8.6 \mathrm{~Hz}, 1 \mathrm{H}), 7.24$ (ddd, $J=8.6,6.9,1.6 \mathrm{~Hz}, 1 \mathrm{H}), 6.68(\mathrm{td}, J=6.8,1.4 \mathrm{~Hz}, 1 \mathrm{H}), 3.87(\mathrm{~s}, 3 \mathrm{H}) \mathrm{ppm} .{ }^{1} \mathrm{H}$ NMR: (400 $\left.\mathrm{MHz}, \mathrm{C}_{2} \mathrm{D}_{2} \mathrm{Cl}_{4}\right) \delta=7.58(\mathrm{dd}, J=6.6,1.3 \mathrm{~Hz}, 1 \mathrm{H}$ ), 7.55 (ddd, $J=8.7,1.5,0.7 \mathrm{~Hz}, 1 \mathrm{H}$ ), 7.13 (ddd, $J$ $=8.6,7.0,1.6 \mathrm{~Hz}, 1 \mathrm{H}), 6.57(\mathrm{td}, J=6.8,1.5 \mathrm{~Hz}, 1 \mathrm{H}), 3.85(\mathrm{~s}, 3 \mathrm{H})$ ppm. ${ }^{13} \mathrm{C}$ NMR: $(101 \mathrm{MHz}$, $\left.\mathrm{C}_{2} \mathrm{D}_{2} \mathrm{Cl}_{4}\right) \delta=180.1,141.3,136.0,134.7,113.9,46.4$ ppm. IR: (ATR, $\mathrm{cm}^{-1}$ ) 3006, 1617, 1536, $1474,1408,1310,1187,1142,1108,1054,1022,802,752,707$. HRMS: calcd. for $\mathrm{C}_{6} \mathrm{H}_{8} \mathrm{NS}^{+}$ $[\mathrm{M}+\mathrm{H}]^{+}:$126.0372; found (ESI): 126.0372 . 


\section{1-Ethylpyridine-2(1H)-thione (153b):}

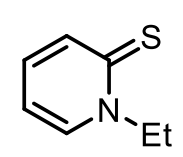

Salt 156b (7.6 g, $33.1 \mathrm{mmol})$ was dissolved in methanol $(120 \mathrm{~mL})$. A solution of sodium hydrosulfide hydrate (2 equiv., $3.7 \mathrm{~g}, 66.3 \mathrm{mmol}$ ) in water $(240 \mathrm{~mL}$ ) was prepared and subsequently added dropwise to the former one at $45{ }^{\circ} \mathrm{C}$. After stirring for $30 \mathrm{~min}$, the reaction mixture was poured into $100 \mathrm{~mL}$ of water, the solution extracted with $\mathrm{CH}_{2} \mathrm{Cl}_{2}(3 \times 70 \mathrm{~mL})$ and the combined organic phases dried over $\mathrm{Na}_{2} \mathrm{SO}_{4}$. Removal of the solvents in vacuo afforded $\mathbf{1 5 3 b}$ as a yellow crystalline material $(3.8 \mathrm{~g}, 27.3$ $\mathrm{mmol}, 82 \%)$.

${ }^{1} \mathrm{H}$ NMR: $\left(300 \mathrm{MHz}, \mathrm{CDCl}_{3}\right) \delta=7.67$ (ddd, $J=8.7,1.5,0.7 \mathrm{~Hz}, 1 \mathrm{H}$ ), 7.61 (ddd, $J=6.6,1.7,0.7$ $\mathrm{Hz}, 1 \mathrm{H}$ ), 7.14 (ddd, $J=8.6,6.8,1.7 \mathrm{~Hz}, 1 \mathrm{H}), 6.64(\mathrm{td}, J=6.7,1.5 \mathrm{~Hz}, 1 \mathrm{H}), 4.58(\mathrm{q}, J=7.2 \mathrm{~Hz}$, $2 \mathrm{H}), 1.46$ (t, $J=7.2 \mathrm{~Hz}, 3 \mathrm{H}$ ) ppm. ${ }^{1} \mathrm{H}$ NMR: $\left(300 \mathrm{MHz}, \mathrm{CD}_{3} \mathrm{CN}\right) \delta=7.80$ (ddd, $J=6.7,1.7,0.8$ $\mathrm{Hz}, 1 \mathrm{H}$ ), 7.50 (ddd, $J=8.7,1.5,0.7 \mathrm{~Hz}, 1 \mathrm{H}), 7.21$ (ddd, $J=8.6,6.9,1.7 \mathrm{~Hz}, 1 \mathrm{H}), 6.71(\mathrm{td}, J=$ $6.8,1.5 \mathrm{~Hz}, 1 \mathrm{H}$ ), $4.53(\mathrm{q}, J=7.1 \mathrm{~Hz}, 2 \mathrm{H}), 1.36$ (t, $J=7.1 \mathrm{~Hz}, 3 \mathrm{H})$ ppm. ${ }^{1} \mathrm{H}$ NMR: $(400 \mathrm{MHz}$, DMSO-d6) $\delta=8.14$ (ddd, $J=6.6,1.7,0.8 \mathrm{~Hz}, 1 \mathrm{H}$ ), 7.45 (ddd, $J=8.6,1.6,0.7 \mathrm{~Hz}, 1 \mathrm{H}$ ), 7.31 (ddd, $J=8.6,6.9,1.7 \mathrm{~Hz}, 1 \mathrm{H}), 6.81(\mathrm{td}, J=6.7,1.6 \mathrm{~Hz}, 1 \mathrm{H}), 4.51(\mathrm{q}, J=7.1 \mathrm{~Hz}, 2 \mathrm{H}), 1.30(\mathrm{t}, J=$ $7.1 \mathrm{~Hz}, 3 \mathrm{H})$ ppm. ${ }^{13} \mathrm{C}$ NMR: $\left(101 \mathrm{MHz}, \mathrm{CDCl}_{3}\right) \delta=179.6,139.6,136.7,133.8,113.9,52.2,14.0$ ppm. ${ }^{13} \mathrm{C}$ NMR: (101 MHz, DMSO-d6) $\delta=178.0,141.5,134.8,134.6,113.8,50.8,13.7$ ppm. IR: $\left(A T R, \mathrm{~cm}^{-1}\right)$ 3060, 3019, 2967, 2927, 1615, 1531, 1471, 1415, 1374, 1260, 1185, 1137, 1106, 1080, 961, 770, 699, 487, 458. HRMS: calcd. for $\mathrm{C}_{7} \mathrm{H}_{9} \mathrm{NNaS}^{+}[\mathrm{M}+\mathrm{Na}]^{+}:$162.034791; found: 162.034770 .

\section{1-Ethyl-3,5-dimethylpyridine-2(1H)-thione (153c):}<smiles>CCn1cc(C)cc(C)c1=S</smiles>

To a stirred suspension of $156 \mathrm{c}$ ( 1 equiv, $8.0 \mathrm{~g}, 35.9 \mathrm{mmol}$ ) and $\mathrm{S}_{8}(1 / 4$ equiv, $2.3 \mathrm{~g}, 72 \mathrm{mmol})$ in THF ( $10 \mathrm{~mL} / \mathrm{mmol})$, a solution of LiHMDS (1 equiv, $5 \mathrm{~mL} / \mathrm{mmol}$ of a solution in THF) was added at $-100{ }^{\circ} \mathrm{C}$. The resulting mixture was stirred overnight, while allowing it to slowly warm up to room temperature. After removal of all solvents, the crude mixture was purified by column chromatography (pentane/EtOAc, 1:1) and dried in vacuo to afford thione 153c as yellow crystals (4.1 g, $24.5 \mathrm{mmol}, 68 \%)$.

${ }^{1} \mathrm{H}$ NMR: $\left(400 \mathrm{MHz}, \mathrm{CDCl}_{3}\right) \delta=7.45(\mathrm{~s}, 1 \mathrm{H}), 7.19(\mathrm{~s}, 1 \mathrm{H}), 4.66(\mathrm{q}, \mathrm{J}=7.2 \mathrm{~Hz}, 2 \mathrm{H}), 2.45(\mathrm{~s}, 3 \mathrm{H})$, $2.15(\mathrm{~s}, 3 \mathrm{H}), 1.47(\mathrm{t}, \mathrm{J}=7.2 \mathrm{~Hz}, 3 \mathrm{H})$ ppm. ${ }^{13} \mathrm{C}$ NMR: $\left(101 \mathrm{MHz}, \mathrm{CDCl}_{3}\right) \delta=176.6,142.3,136.3$, 136.0, 122.5, 53.1, 23.9, 17.6, 14.1 ppm. IR: (ATR, cm ${ }^{-1}$ ) 1634, 1544, 1455, 1402, 1375, 1279, $1240,1180,1120,1083,1019,980,861,717,658,539$. HRMS: calcd. for $\mathrm{C}_{9} \mathrm{H}_{14} \mathrm{NNaS}^{+}$ $[\mathrm{M}+\mathrm{Na}]^{+}:$190.066090; found (ESI): 190.066110 . 


\section{1-Ethyl-5-(trifluoromethyl)pyridine-2(1H)-thione (153d):}

2-Chloro-1-ethyl-5-(trifluoromethyl)pyridin-1-ium tetrafluoroborate (537 mg, $1.8 \mathrm{mmol}$ ) was dissolved in methanol $(10 \mathrm{~mL})$. A solution of sodium $\mathrm{F}_{3} \mathrm{C}$ 'Et hydrosulfide hydrate $(202 \mathrm{mg}, 3.6 \mathrm{mmol})$ in water $(20 \mathrm{~mL})$ was prepared and added to the former dropwise at $45{ }^{\circ} \mathrm{C}$. After stirring for $30 \mathrm{~min}$, the reaction mixture was poured into $50 \mathrm{~mL}$ of water, the solution extracted with $\mathrm{CH}_{2} \mathrm{Cl}_{2}(3 \times 70 \mathrm{~mL})$, and the organic phase was dried over $\mathrm{Na}_{2} \mathrm{SO}_{4}$. Removal of all solvents in vacuo afforded compound 153d as yellow crystals (292 mg, $1.4 \mathrm{mmol}, 78 \%$ ).

${ }^{1} \mathrm{H}$ NMR: $\left(300 \mathrm{MHz}, \mathrm{CDCl}_{3}\right) \delta=7.87(\mathrm{td}, J=2.0,1.0 \mathrm{~Hz}, 1 \mathrm{H}), 7.68(\mathrm{dt}, J=9.2,0.9 \mathrm{~Hz}, 1 \mathrm{H}), 7.20$ $(\mathrm{dd}, J=9.1,2.2 \mathrm{~Hz}, 1 \mathrm{H}), 4.57(\mathrm{q}, J=7.2 \mathrm{~Hz}, 2 \mathrm{H}), 1.49(\mathrm{t}, J=7.2 \mathrm{~Hz}, 3 \mathrm{H}) \mathrm{ppm}$.

\section{2-(Chlorothio)-1-ethylpyridin-1-ium chloride (154bb):}<smiles>CC[n+]1ccccc1SCl</smiles>

Sulfuryl chloride (1.35 equiv, $158 \mu \mathrm{L}, 264 \mathrm{mg}, 1.95 \mathrm{mmol}$ ) was added to a solution of thiolactam 153a (1 equiv, $200 \mathrm{mg}, 1.44 \mathrm{mmol}$ ) in toluene (10 mL) at $0{ }^{\circ} \mathrm{C}$. The reaction mixture was stirred at $0{ }^{\circ} \mathrm{C}$ for $15 \mathrm{~min}$ and then allowed to warm up to room temperature over $1 \mathrm{~h}$. The supernatant was filtered off, the residue was washed with cold toluene $(10 \mathrm{~mL})$ and dried in vacuo to afford $154 \mathrm{bb}$ as a bright yellow powder in $78 \%$ yield.

${ }^{1} \mathrm{H}$ NMR: $\left(300 \mathrm{MHz}, \mathrm{CDCl}_{3}\right) \delta=10.71(\mathrm{dd}, J=6.2,1.6 \mathrm{~Hz}, 1 \mathrm{H}), 8.54(\mathrm{td}, J=7.9,1.5 \mathrm{~Hz}, 1 \mathrm{H})$, $8.22(\mathrm{t}, J=6.7 \mathrm{~Hz}, 1 \mathrm{H}), 8.03(\mathrm{dd}, J=8.2,1.3 \mathrm{~Hz}, 1 \mathrm{H}), 5.30(\mathrm{q}, J=7.2 \mathrm{~Hz}, 2 \mathrm{H}), 1.73(\mathrm{t}, J=7.2 \mathrm{~Hz}$, 3H) $\mathrm{ppm}$.

\section{2-(Bromothio)-1-methylpyridin-1-ium bromide (154a):}<smiles>[N+]#[N+]c1ccccc1Br</smiles>

To an oven-dried schlenk flask charged with 153a (893 mg, $7.1 \mathrm{mmol}$ ); dry $\mathrm{CH}_{2} \mathrm{Cl}_{2}(20 \mathrm{~mL})$ was added, and the solution was cooled to $0{ }^{\circ} \mathrm{C}$. Bromine (1 equiv, $365 \mu \mathrm{L}, 1.133 \mathrm{~g}, 7.1 \mathrm{mmol}$ ) was added slowly at $0{ }^{\circ} \mathrm{C}$ under stirring, and the reaction mixture was then slowly warmed up to room temperature over 2 h. After removal of all volatiles in vacuo, 154a was obtained as a bright orange solid $(2.0 \mathrm{~g}$, $7.0 \mathrm{mmol}, 99 \%)$.

${ }^{1} \mathrm{H}$ NMR: $\left(500 \mathrm{MHz}, \mathrm{C}_{2} \mathrm{D}_{2} \mathrm{Cl}_{4}\right) \delta=8.39(\mathrm{~d}, J=7.9 \mathrm{~Hz}, 1 \mathrm{H}), 8.28(\mathrm{~d}, J=6.2 \mathrm{~Hz}, 1 \mathrm{H}), 8.17(\mathrm{t}, J=7.8$ $\mathrm{Hz}, 1 \mathrm{H}), 7.69$ (ddd, $J=7.7,6.2,1.6 \mathrm{~Hz}, 1 \mathrm{H}), 4.44(\mathrm{~s}, 3 \mathrm{H})$ ppm. ${ }^{13} \mathrm{C}$ NMR: $\left(126 \mathrm{MHz}, \mathrm{C}_{2} \mathrm{D}_{2} \mathrm{Cl}_{4}\right) \delta=$ 163.5, 145.5, 144.6, 137.5, 125.8, 48.9 ppm. HRMS: calcd. for $\mathrm{C}_{6} \mathrm{H}_{7} \mathrm{BrNS}^{+}[\mathrm{M}-\mathrm{Br}]^{+}$: 203.9477; found (ESI): 203.9475. 


\section{2-(Bromothio)-1-ethylpyridin-1-ium bromide (154ba):}

$\overbrace{}^{\mathrm{Et}} \mathrm{Br}^{-}$

To an oven-dried schlenk flask charged with 153b (1.5 g, $10.8 \mathrm{mmol})$; dry $\mathrm{CH}_{2} \mathrm{Cl}_{2}(50 \mathrm{~mL})$ was added, and the solution was cooled to $0{ }^{\circ} \mathrm{C}$. Bromine $(1$ equiv., $554 \mu \mathrm{L}, 1.719 \mathrm{~g}, 10.8 \mathrm{mmol}$ ) was added slowly at $0{ }^{\circ} \mathrm{C}$ under stirring, and the reaction mixture was then slowly warmed up to room temperature over $2 \mathrm{~h}$. After removal of all volatiles in vacuo, 154ba was obtained as a bright orange solid (3.2 g, $10.7 \mathrm{mmol}, 99 \%)$.

${ }^{1} \mathrm{H}$ NMR $\left(400 \mathrm{MHz}, \mathrm{CD}_{2} \mathrm{Cl}_{2}\right) \delta=8.46(\mathrm{~d}, J=8.0 \mathrm{~Hz}, 1 \mathrm{H}), 8.39(\mathrm{~d}, J=5.7 \mathrm{~Hz}, 1 \mathrm{H}), 8.27-8.18(\mathrm{~m}$, 1H), $7.85-7.77(\mathrm{~m}, 1 \mathrm{H}), 5.04(\mathrm{q}, J=7.4 \mathrm{~Hz}, 2 \mathrm{H}), 1.84(\mathrm{t}, J=7.4 \mathrm{~Hz}, 3 \mathrm{H})$ ppm ${ }^{13} \mathrm{C}$ NMR: (101 $\mathrm{MHz}, \mathrm{CD}_{2} \mathrm{Cl}_{2}$ ) $\delta=164.2$ (only visible by $\mathrm{HMBC}$ ), 145.3, 143.7, 138.4, 126.4, 57.1, $15.2 \mathrm{ppm}$.

HRMS measurement of methanol solutions of 154 a revealed the methanol-adduct $\mathrm{C}_{7} \mathrm{H}_{9} \mathrm{NS} \bullet \mathrm{CH}_{3} \mathrm{O}$ to be the major compound present, in addition the decomposition product $\mathrm{C}_{7} \mathrm{H}_{9} \mathrm{NBr}^{+}$was detected.

HRMS: calcd. for $\mathrm{C}_{8} \mathrm{H}_{12} \mathrm{NSO}$ [M-Br${ }_{2}+\mathrm{MeOH}$ ]: 170.0630; found (ESI): 170.0634. HRMS: calcd. for $\mathrm{C}_{7} \mathrm{H}_{9} \mathrm{NBr}^{+}[\mathrm{M}-\mathrm{SBr}]^{+}:$185.9913, found (ESI): 186.0577

\section{2-(Bromothio)-1-ethyl-3,5-dimethylpyridin-1-ium bromide (154c):}<smiles>CC[n+]1cc(C)cc(C)c1SBr</smiles>

To an oven-dried schlenk flask charged with $153 \mathrm{c}(2.82 \mathrm{~g}, 16.9 \mathrm{mmol})$; dry $\mathrm{CH}_{2} \mathrm{Cl}_{2}(20 \mathrm{~mL})$ was added, and the solution was cooled to $0{ }^{\circ} \mathrm{C}$. Bromine ( 1 equiv, $870 \mu \mathrm{L}, 2.700 \mathrm{~g}, 16.9 \mathrm{mmol}$ ) was added slowly at $0{ }^{\circ} \mathrm{C}$, and the reaction mixture was then slowly warmed up to room temperature over $2 \mathrm{~h}$. After removal of all volatiles under vacuum, the residue was washed with dry $\mathrm{Et}_{2} \mathrm{O}(3 \times 20 \mathrm{~mL})$. Compound $154 \mathrm{c}$ was obtained as a bright orange solid $(5.1 \mathrm{~g}, 15.6$ $\mathrm{mmol}, 92 \%)$.

${ }^{1} \mathrm{H}$ NMR: $\left(300 \mathrm{MHz}, \mathrm{CD}_{3} \mathrm{CN}\right) \delta=8.46(\mathrm{~s}, 1 \mathrm{H}), 8.15-8.07(\mathrm{~m}, 1 \mathrm{H}), 4.98(\mathrm{q}, \mathrm{J}=6.6 \mathrm{~Hz}, 2 \mathrm{H}), 2.66$ $(\mathrm{s}, 3 \mathrm{H}), 2.41(\mathrm{~s}, 3 \mathrm{H}), 1.77(\mathrm{t}, \mathrm{J}=7.3 \mathrm{~Hz}, 3 \mathrm{H}) \mathrm{ppm} .{ }^{1} \mathrm{H}$ NMR: $\left(400 \mathrm{MHz}, \mathrm{CD}_{2} \mathrm{Cl}_{2}\right) \delta=8.14(\mathrm{~s}, 1 \mathrm{H})$, $7.97(\mathrm{~s}, 1 \mathrm{H}), 5.06(\mathrm{q}, J=7.4 \mathrm{~Hz}, 2 \mathrm{H}), 2.78(\mathrm{~s}, 3 \mathrm{H}), 2.44(\mathrm{~s}, 3 \mathrm{H}), 1.86(\mathrm{t}, J=7.4 \mathrm{~Hz}, 3 \mathrm{H}) \mathrm{ppm}$. ${ }^{13} \mathrm{C}$ NMR: $\left(101 \mathrm{MHz}, \mathrm{CD}_{2} \mathrm{Cl}_{2}\right) \delta=159.9,147.4,145.0,141.5,137.6,57.6,22.4,18.8,15.5$ ppm. HRMS: calcd. for $\mathrm{C}_{9} \mathrm{H}_{13} \mathrm{BrNS}^{+}[\mathrm{M}-\mathrm{Br}]^{+}:$245.9947; found: 245.9944.

\section{1-Ethyl-3,5-dimethyl-2-thiocyanatopyridin-1-ium bromide (155a):}<smiles>CC[n+]1cc(C)cc(C)c1SCBr</smiles>

Under stirring, to a solution of compound 154c (1 equiv, $2.5 \mathrm{~g}, 7.6 \mathrm{mmol}$ ) in dry $\mathrm{CH}_{2} \mathrm{Cl}_{2}(40 \mathrm{~mL}$ ) TMSCN (1.18 equiv, $1.13 \mathrm{~mL}, 893 \mathrm{mg}, 9.0 \mathrm{mmol}$ ) was slowly added at $0{ }^{\circ} \mathrm{C}$. After addition, the ice bath was removed, and the mixture was stirred for another 10 minutes, before removing all 
volatiles in vacuo. The residue was washed with dry $\mathrm{Et}_{2} \mathrm{O}(3 \times 20 \mathrm{~mL})$. Compound 155a was isolated as a pale yellow-orange solid $(2.0 \mathrm{~g}, 7.3 \mathrm{mmol}, 96 \%)$.

${ }^{1} \mathrm{H}$ NMR: $\left(400 \mathrm{MHz}, \mathrm{CD}_{3} \mathrm{CN}\right) \delta=8.85(\mathrm{~s}, 1 \mathrm{H}), 8.31(\mathrm{~s}, 1 \mathrm{H}), 4.96(\mathrm{q}, \mathrm{J}=7.3 \mathrm{~Hz}, 2 \mathrm{H}), 2.75(\mathrm{~s}, 3 \mathrm{H})$, $2.51(\mathrm{~s}, 3 \mathrm{H}), 1.68(\mathrm{t}, \mathrm{J}=7.3 \mathrm{~Hz}, 3 \mathrm{H}) \mathrm{ppm}$.

\section{1-Ethyl-3,5-dimethyl-2-thiocyanatopyridin-1-ium hexafluoroantimonate (155b):}<smiles></smiles>

Compound 155a (1 equiv, $700 \mathrm{mg}, 2.56 \mathrm{mmol}$ ) was dissolved in $\mathrm{CH}_{2} \mathrm{Cl}_{2}$ $\left(70 \mathrm{~mL}\right.$ ), and an aqueous solution of $\mathrm{NaSbF}_{6}$ (2.7 equiv, $1.8 \mathrm{~g}, 6.96$ $\mathrm{mmol}$ in $70 \mathrm{~mL} \mathrm{H}_{2} \mathrm{O}$ ) was added. The mixture was stirred for $5 \mathrm{~min}$ at room temperature, transferred to a seperation funnel, and the aqueous phase was extracted with $\mathrm{CH}_{2} \mathrm{Cl}_{2}(3 \times 20 \mathrm{~mL})$. The combined organic phases were dried over $\mathrm{Na}_{2} \mathrm{SO}_{4}$, filtered, and all volatiles were removed in vacuo. The product $155 \mathrm{~b}$ (927.4 mg, $2.16 \mathrm{mmol}, 84 \%$ ) was isolated as colourless crystals. It is highly recommended to store this salt under inert atmosphere.

${ }^{1} \mathrm{H}$ NMR: $\left(400 \mathrm{MHz}, \mathrm{CD}_{3} \mathrm{CN}\right) \delta=8.71(\mathrm{~d}, J=1.5 \mathrm{~Hz}, 1 \mathrm{H}), 8.34(\mathrm{~s}, 1 \mathrm{H}), 4.86(\mathrm{q}, J=7.3 \mathrm{~Hz}, 2 \mathrm{H})$, $2.76(\mathrm{~s}, 3 \mathrm{H}), 2.53(\mathrm{~s}, 3 \mathrm{H}), 1.63(\mathrm{t}, J=7.3 \mathrm{~Hz}, 3 \mathrm{H}) \mathrm{ppm} .{ }^{13} \mathrm{C}$ NMR: $\left(101 \mathrm{MHz}, \mathrm{CD}_{3} \mathrm{CN}\right) \delta=149.7$, 148.2, 147.1, 142.9, 106.9, 59.4, 21.6, 18.4, 16.2 ppm. ${ }^{23}{ }^{19} \mathrm{~F}$ NMR: $\left(282 \mathrm{MHz}, \mathrm{CD}_{3} \mathrm{CN}\right) \delta=-$ 123.98 (sext, $J_{\mathrm{F}-121 \mathrm{Sb}}=1938.3 \mathrm{~Hz}$ ), -123.98 (oct, $\left.J_{\mathrm{F}-123 \mathrm{Sb}}=1052.1 \mathrm{~Hz}\right) \mathrm{ppm}$. IR: $\left(\right.$ ATR, $\mathrm{cm}^{-1}$ ) 2359, 2342, 1602, 1508, 1457, 1253, 1174, 1109, 1031, 657, 630, 540. HRMS: calcd. for $\mathrm{C}_{10} \mathrm{H}_{13} \mathrm{~N}_{2} \mathrm{~S}^{+}\left[\mathrm{M}-\mathrm{SbF}_{6}{ }^{-}\right]$: 193.079395; found (ESI): 193.079640.

\section{1-Ethyl-2-thiocyanatopyridin-1-ium tetrafluoroborate (155c):}<smiles>CC[n+]1ccccc1SC#N</smiles>

Compound 154bb was dissolved in cold DCM (10 mL) and stirred in an ice bath. TMSCN (1.0 equiv, ) was added slowly to the solution and after addition, the mixture was stirred for 20 more minutes. Dry $\mathrm{NaBF}_{4}$ was added, and the reaction mixture was stirred overnight. The supernatant was filtered off, and the solvent was removed under vacuum. $\mathrm{Et}_{2} \mathrm{O}(10 \mathrm{~mL})$ was added, and the reaction mixture was ultrasonicated for $5 \mathrm{~min}$. The ether was then filtered off, and the residue was dried in vacuo.

${ }^{1}$ H NMR: $\left(300 \mathrm{MHz}, \mathrm{CD}_{3} \mathrm{CN}\right) \delta=8.96(\mathrm{dd}, J=6.2,1.6 \mathrm{~Hz}, 1 \mathrm{H}), 8.45(\mathrm{td}, J=8.0,1.6 \mathrm{~Hz}, 1 \mathrm{H})$, 8.10 (ddd, $J=7.7,6.2,1.4 \mathrm{~Hz}, 1 \mathrm{H}$ ), $7.92(\mathrm{dd}, J=8.3,1.4 \mathrm{~Hz}, 1 \mathrm{H}$ ), $4.80(\mathrm{q}, J=7.3 \mathrm{~Hz}, 2 \mathrm{H}$ ), 1.65 (t, $J=7.3 \mathrm{~Hz}, 3 \mathrm{H}) \mathrm{ppm}$.

${ }^{19}$ F NMR: $\left(282 \mathrm{MHz}, \mathrm{CD}_{3} \mathrm{CN}\right) \delta=-152.98,-153.03 \mathrm{ppm}$.

\footnotetext{
${ }^{23}$ The quarternary carbon in $\alpha$-position to sulfur was not visible in a routine ${ }^{13} \mathrm{C}$ measurement, similar behaviour is observed for derivatives $154 a-c$.
} 


\section{2,2'-Thiobis(1-ethylpyridin-1-ium) bishexafluoroantimonate (157):}<smiles>CC[n+]1ccccc1Sc1cccc[n+]1CC</smiles>

$2 \mathrm{SbF}_{6}^{-}$

Compound 154bb (255.4 mg, $1.22 \mathrm{mmol}$ ) was dissolved in cold DCM (20 $\mathrm{mL}$ ) and stirred in an ice bath. TMSCN (1.0 equiv, $151 \mu \mathrm{L}, 119 \mathrm{mg}, 1.22$ $\mathrm{mmol}$ ) was added slowly to the solution. The mixture was stirred for 10 minutes before $\mathrm{NaSbF}_{6}$ ( 1 equiv, $314.4 \mathrm{mg} 1.22 \mathrm{mmol}$ ) was added. The reaction mixture was stirred overnight, and the supernatant was filtered off. and the solvent was removed in vacuo. $\mathrm{Et}_{2} \mathrm{O}(10 \mathrm{~mL})$ was added, and the reaction mixture was ultrasonicated for $5 \mathrm{~min}$. The ether was then filtered off. After filtration, the residue was taken of with $\mathrm{CH}_{2} \mathrm{Cl}_{2}$, forming colorless crystals of 157 .

${ }^{1} \mathrm{H}$ NMR: $\left(400 \mathrm{MHz}, \mathrm{DMSO}-\mathrm{d}_{6}\right) \delta=9.34(\mathrm{dd}, J=6.2,1.5 \mathrm{~Hz}, 1 \mathrm{H}), 8.55(\mathrm{td}, J=8.0,1.6 \mathrm{~Hz}, 1 \mathrm{H})$, 8.23 (ddd, $J=7.7,6.1,1.4 \mathrm{~Hz}, 1 \mathrm{H}$ ), $8.16(\mathrm{dd}, J=8.2,1.4 \mathrm{~Hz}, 1 \mathrm{H}$ ), $4.83(\mathrm{q}, J=7.3 \mathrm{~Hz}, 2 \mathrm{H}$ ), 1.60 $(\mathrm{t}, J=7.3 \mathrm{~Hz}, 3 \mathrm{H}) .{ }^{13} \mathrm{C}$ NMR: $\left(101 \mathrm{MHz}, \mathrm{DMSO}-\mathrm{d}_{6}\right) \delta=149.25,148.23,146.23,133.06,128.21$, 55.97, 15.23 ppm. ${ }^{19} \mathrm{~F}$ NMR: $\left(282 \mathrm{MHz}, \mathrm{DMSO}^{-\mathrm{d}_{6}}\right) \delta=-102.28$ to -137.00 (m) ppm. IR: (ATR, $\mathrm{cm}^{-1}$ ) 3141, 3108, 1605, 1567, 1489, 1467, 1434, 1284, 1225, 1554, 778, 651. HRMS: calcd. for $\mathrm{C}_{14} \mathrm{H}_{18} \mathrm{~F}_{6} \mathrm{~N}_{2} \mathrm{SSb}\left[\mathrm{M}-\mathrm{SbF}_{6}{ }^{-}\right.$]: 481.012689; found: 481.012570.

\section{General Procedure for the synthesis of alkynylthiopyridinium salts (GP A):}

$n$-BuLi (1.6 M/2.5 $\mathrm{M}$ in hexanes, 1.1 equiv.) was added dropwise to a solution of the corresponding acetylene (1.1 equiv.) in THF $(10 \mathrm{~mL})$ at $-78{ }^{\circ} \mathrm{C}$. The reaction mixture was allowed to warm up to room temperature over $2 \mathrm{~h}$, then cooled down to $-78^{\circ} \mathrm{C}$ again. Then a solution of $\mathrm{ZnBr}_{2}$ (1.1 equiv.) in THF (1 M) was added dropwise, to the former one and the reaction mixture was stirred for an additional $1 \mathrm{~h}$ before allowing it to reach $\mathrm{rt}$. The solution thus obtained was then added dropwise to a solution of 154 (1 equiv.) in THF ( $0.5 \mathrm{M}$ ) at -78 ${ }^{\circ} \mathrm{C}$. Once the addition finished, the reaction mixture was removed from the cooling bath and allowed to warm to rt. After $\mathbf{1 5 4}$ was completely consumed, the solvents were removed in vacuum and the solid obtained washed three times with $\mathrm{Et}_{2} \mathrm{O}$ (ultrasonication for 5 minutes each time) and dried.

\section{1-Methyl-2-[(phenylethynyl)thio]pyridin-1-ium tetrabromozincate (160a):}<smiles>C[n+]1ccccc1SC#CC#CC(C)(C)Br</smiles>

Following GP A, 160a was prepared from 154a (1 equiv, $1.3 \mathrm{~g}, 4.56$ $\mathrm{mmol}$ ), phenylacetylene (1.1 equiv, $549 \mu \mathrm{L}, 511 \mathrm{mg}, 5.0 \mathrm{mmol}$ ), $n$-BuLi (1.1 equiv, $2.5 \mathrm{M}, 2.0 \mathrm{~mL}, 5.0 \mathrm{mmol}$ ) and $\mathrm{ZnBr}_{2}$ (1.16 equiv, $1.2 \mathrm{~g}, 5.3$ $\mathrm{mmol}$ ) in THF as a pale yellow powder (747 mg $1.78 \mathrm{mmol}, 39 \%)$.

${ }^{1} \mathrm{H}$ NMR: $\left(400 \mathrm{MHz}, \mathrm{CD}_{3} \mathrm{CN}\right) \delta=8.68-8.64(\mathrm{~m}, 1 \mathrm{H}), 8.47(\mathrm{dd}, \mathrm{J}=8.4,1.3 \mathrm{~Hz}, 1 \mathrm{H}), 8.44-8.39$ (m, 1H), 7.81 (ddd, J = 7.7, 6.3, $1.5 \mathrm{~Hz}, 1 \mathrm{H}), 7.73-7.67(\mathrm{~m}, 2 \mathrm{H}), 7.59-7.53(\mathrm{~m}, 1 \mathrm{H}), 7.52-$ $7.46(\mathrm{~m}, 2 \mathrm{H}), 4.17(\mathrm{~s}, 3 \mathrm{H}) \mathrm{ppm} .{ }^{13} \mathrm{C}$ NMR: $\left(101 \mathrm{MHz}, \mathrm{CD}_{3} \mathrm{CN}\right) \delta=157.4,148.4,145.9,133.4$, 
131.8, 129.9, 127.5, 125.5, 121.3, 105.4, 67.2, 47.1 ppm. IR: (ATR, $\mathrm{cm}^{-1}$ ) 1614, 1563, 1481, 1440, 1281, 1187, 1161, 1108, 759, 693, 563, 520. HRMS: calcd. for $\mathrm{C}_{14} \mathrm{H}_{12} \mathrm{NS}^{+}\left[\mathrm{M}-1 / 2 \mathrm{ZnBr}_{4}\right]$ : 226.0685; found (ESI): 226.0693

\section{1-Ethyl-2-[(phenylethynyl)thio]pyridin-1-ium tetrabromozincate (160b):}

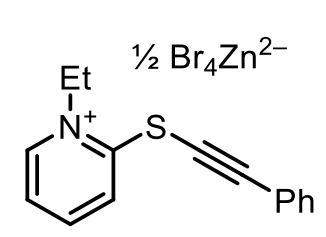

Following the modified GP A, 160b $(2.5 \mathrm{~g}, 5.8 \mathrm{mmol}, 87 \%)$ was prepared as a pale, off white solid from 154ba (1 equiv., $2.0 \mathrm{~g}, 6.7 \mathrm{mmol}$ ), phenylacetylene (1.1 equiv., $813 \mu \mathrm{L}, 756 \mathrm{mg}, 7.4 \mathrm{mmol}), n$-BuLi (1.6 M, $4.6 \mathrm{~mL}, 7.4 \mathrm{mmol}, 1.1$ equiv.) and $\mathrm{ZnBr}_{2}$ (1.1 equiv, $1.67 \mathrm{~g}, 7.4 \mathrm{mmol}$ ) in THF. However, in order to obtain pure material, crude $160 \mathrm{~b}$ was washed with THF $(2 \times 10$ $\mathrm{mL})$.

${ }^{1} \mathrm{H}$ NMR: $\left(400 \mathrm{MHz}, \mathrm{CD}_{3} \mathrm{CN}\right) \delta=8.79(\mathrm{dd}, J=6.3,1.7 \mathrm{~Hz}, 1 \mathrm{H}), 8.49(\mathrm{ddd}, J=8.5,1.6,0.6 \mathrm{~Hz}$, $1 \mathrm{H}), 8.42$ (ddd, $J=8.6,7.5,1.5 \mathrm{~Hz}, 1 \mathrm{H}$ ), 7.89 (ddd, $J=7.5,6.2,1.4 \mathrm{~Hz}, 1 \mathrm{H}), 7.74-7.64(\mathrm{~m}$, $2 \mathrm{H}), 7.59-7.44(\mathrm{~m}, 3 \mathrm{H}), 4.56(\mathrm{q}, J=7.3 \mathrm{~Hz}, 3 \mathrm{H}), 1.59(\mathrm{t}, J=7.3 \mathrm{~Hz}, 3 \mathrm{H})$ ppm. ${ }^{13} \mathrm{C}$ NMR: (101 $\left.\mathrm{MHz}, \mathrm{CD}_{3} \mathrm{CN}\right) \delta=156.1,147.3,145.9,133.3,131.7,129.8,128.0,126.2,121.3,105.77,55.9$, 14.6 ppm. IR: (ATR, cm ${ }^{-1}$ ) 3052, 2159, 2007, 1607, 1564, 1488, 1461, 1431, 1325, 1177, 1148, 1113, 771, 753, 716, 689, 569, 516. HRMS: calcd. for $\mathrm{C}_{15} \mathrm{H}_{14} \mathrm{NS}^{+}\left[\mathrm{M}-1 / 2 \mathrm{ZnBr}_{4}\right]: 240.084146$, found (ESI): 240.084210 .

\section{1-Ethyl-3,5-dimethyl-2-[(phenylethynyl)thio]pyridin-1-ium tetrabromozincate (160c):}<smiles>CC[n+]1cc(C)cc(C)c1SC#Cc1ccccc1</smiles>

Following the modified GP A, 160c (689 mg, $1.50 \mathrm{mmol}, 71 \%$ ) was prepared as a pale, off white solid from 154c (1 equiv, $684 \mathrm{mg}, 2.1$ mmol), phenylacetylene (1.1 equiv, $252 \mu \mathrm{L}, 234 \mathrm{mg}, 2.3 \mathrm{mmol}), n$ BuLi (1.1 equiv, $2.5 \mathrm{M}, 920 \mu \mathrm{L}, 2.3 \mathrm{mmol}$ ), $\mathrm{ZnBr}_{2}$ (1.1 equiv, $528 \mathrm{mg}$, $2.34 \mathrm{mmol}$ ) in THF. However, in order to obtain pure material, crude 160c was washed with THF $(2 \times 5 \mathrm{~mL})$.

${ }^{1} \mathrm{H}$ NMR: $\left(400 \mathrm{MHz}, \mathrm{CDCl}_{3}\right) \delta=9.58(\mathrm{~d}, J=2.0 \mathrm{~Hz}, 1 \mathrm{H}), 8.04(\mathrm{~s}, 1 \mathrm{H}), 7.47-7.29(\mathrm{~m}, 5 \mathrm{H}), 5.33$ $(\mathrm{q}, J=7.2 \mathrm{~Hz}, 2 \mathrm{H}), 2.83(\mathrm{~s}, 3 \mathrm{H}), 2.71(\mathrm{~s}, 3 \mathrm{H}), 1.79(\mathrm{t}, J=7.3 \mathrm{~Hz}, 3 \mathrm{H})$ ppm. ${ }^{1} \mathrm{H}$ NMR: $(400 \mathrm{MHz}$, $\left.\mathrm{CD}_{3} \mathrm{CN}\right) \delta=8.88(\mathrm{~d}, J=5.0 \mathrm{~Hz}, 1 \mathrm{H}), 8.32-8.25(\mathrm{~m}, 1 \mathrm{H}), 7.46(\mathrm{~d}, J=1.3 \mathrm{~Hz}, 3 \mathrm{H}), 7.44-7.35$ $(\mathrm{m}, 6 \mathrm{H}), 5.06-4.97(\mathrm{~m}, 4 \mathrm{H}), 2.79(\mathrm{~s}, 3 \mathrm{H}), 2.53(\mathrm{~d}, J=0.9 \mathrm{~Hz}, 4 \mathrm{H}), 1.67(\mathrm{t}, J=7.2 \mathrm{~Hz}, 4 \mathrm{H}) \mathrm{ppm}$. ${ }^{13} \mathrm{C}$ NMR: $\left(101 \mathrm{MHz}, \mathrm{CD}_{3} \mathrm{CN}\right) \delta=149.1,147.0,145.6,140.9,133.0,130.9,129.7,122.1,96.1$, 58.8, 21.5, 18.5, 16.8 ppm. IR: (ATR, cm ${ }^{-1}$ ) 2360, 2341, 1602, 1585, 1486, 1464, 1443, 1381, $1304,1250,1092,1029,876,864,766,753,720,689,539$. HRMS: calcd. for $\mathrm{C}_{17} \mathrm{H}_{18} \mathrm{NS}^{+}[\mathrm{M}-1 / 2$ $\mathrm{ZnBr}_{4}$ ]: 268.1154; found (ESI): 268.1156. 
<smiles>C[n+]1ccccc1SC#C[In]S</smiles>

Following the modified GP A, 160d was prepared from 154a (13.3 g, $46.7 \mathrm{mmol}$ ), (triisopropylsilyl)acetylene (1.1 equiv, $11.5 \mathrm{~mL}, 9.36 \mathrm{~g}$, $51.3 \mathrm{mmol}$ ), $n$-BuLi (1.1 equiv, $2.5 \mathrm{M}, 20.5 \mathrm{~mL}, 51.3 \mathrm{mmol}$ ) and $\mathrm{ZnBr}_{2}$ (1.1 equiv, $11.55 \mathrm{~g}, 51.3 \mathrm{mmol}$ ). Instead of removing the solvents after consumption of 154a, an aqueous solution of $\mathrm{NaSbF}_{6}$ (3.0 equiv., $36.2 \mathrm{~g}, 139.9 \mathrm{mmol}$ ) was added while stirring vigorously, and successively the reaction mixture was transferred to a separatory funnel. The aqueous phase was extracted with EtOAc $(3 \times 100 \mathrm{~mL})$, the combined organic phases were dried with $\mathrm{Na}_{2} \mathrm{SO}_{4}$, filtered, and the solvent was removed in vacuo to afford $160 \mathrm{~d}$ as a beige solid $(16.3 \mathrm{~g}, 30.0 \mathrm{mmol}, 64 \%)$.

${ }^{1} \mathrm{H}$ NMR: $\left(300 \mathrm{MHz}, \mathrm{CDCl}_{3}\right) \delta=8.73(\mathrm{~d}, J=6.2 \mathrm{~Hz}, 1 \mathrm{H}), 8.42-8.26(\mathrm{~m}, 2 \mathrm{H}), 7.83(\mathrm{ddd}, J=7.8$, 6.3, $1.7 \mathrm{~Hz}, 1 \mathrm{H}), 4.22(\mathrm{~s}, 3 \mathrm{H}), 1.29-1.03(\mathrm{~m}, 21 \mathrm{H})$ ppm. ${ }^{1} \mathrm{H}$ NMR: $\left(300 \mathrm{MHz}, \mathrm{CD}_{3} \mathrm{CN}\right) \delta=8.64$ $-8.56(\mathrm{~m}, 1 \mathrm{H}), 8.44(\mathrm{td}, J=8.0,7.6,1.5 \mathrm{~Hz}, 1 \mathrm{H}), 8.31$ (dd, $J=8.5,1.4 \mathrm{~Hz}, 1 \mathrm{H}), 7.78$ (ddd, $J=$ 7.6, 6.2, $1.5 \mathrm{~Hz}, 1 \mathrm{H}), 4.09(\mathrm{~s}, 3 \mathrm{H}), 1.32-1.07(\mathrm{~m}, 21 \mathrm{H})$ ppm. ${ }^{13} \mathrm{C}$ NMR: $\left(75 \mathrm{MHz}, \mathrm{CD}_{3} \mathrm{CN}\right) \delta=$ 157.3, 148.4, 146.0, 127.0, 125.5, 113.6, 82.7, 46.9, 18.8, 11.9 ppm. IR: (ATR, $\left.\mathrm{cm}^{-1}\right)$ 3516, 3140, 2943, 2867, 2104, 1617, 1571, 1489, 1456, 1282, 1168, 1110, 993, 878, 851, 767, 656, 625, 595. HRMS: calcd. for $\mathrm{C}_{17} \mathrm{H}_{28} \mathrm{NSSi}^{+}[\mathrm{M}]^{+}$: 306.1706; found (ESI): 306.1707 . 
General Procedure for the synthesis of dithioesters and thioamides (GP B):

An oven dried schlenk flask was charged with solid 160 (1 equiv.), $\mathrm{CH}_{2} \mathrm{Cl}_{2}(2 \mathrm{~mL})$ and the desired substrate. Subsequently DIPEA (1-2 equiv.) was added, and the progress of reaction was monitored by TLC. Once the starting materials were consumed, the solvent was removed in vacuo, and the product purified by column chromatography.

\section{2-Cyclohexylidene-2-phenyl-1-(pyrrolidin-1-yl)ethane-1-thione (161a) and 2-phenyl-1-} (pyrrolidin-1-yl)ethane-1-thione (161b):<smiles>S=C(C(=C1CCCCC1)c1ccccc1)N1CCCC1</smiles><smiles></smiles>

Column chromatography (petrol ether/EtOAc, 10:1) of the reaction mixture obtained from 1-(cyclohex-1-en-1-yl)pyrrolidine (1 equiv, 48.4 $\mathrm{mg}, 51.5 \mu \mathrm{L}, 0.32 \mathrm{mmol}$ ) and $160 \mathrm{~b}$ (1.1 equiv, $151.3 \mathrm{mg}, 0.35 \mathrm{mmol}$ ) in $\mathrm{CH}_{2} \mathrm{Cl}_{2}\left(2 \mathrm{~mL}\right.$ ) following GP B ${ }^{24}$ afforded products 161a (17.5 mg, 61.3 $\mu \mathrm{mol}, 19 \%$ ) as a pale yellow solid and $161 \mathrm{~b}(4.6 \mathrm{mg}, 22.4 \mu \mathrm{mol}, 7 \%)$ as a crystalline off-white solid.

Compound 161a: ${ }^{1} \mathrm{H}$ NMR: $\left(500 \mathrm{MHz} \mathrm{CD}_{2} \mathrm{Cl}_{2}\right) \delta=7.47-7.43(\mathrm{~m}, 2 \mathrm{H})$, $7.35-7.30(\mathrm{~m}, 2 \mathrm{H}), 7.28-7.24(\mathrm{~m}, 1 \mathrm{H}), 3.88-3.80(\mathrm{~m}, 1 \mathrm{H}), 3.73-3.64$ $(\mathrm{m}, 2 \mathrm{H}), 3.51-3.43(\mathrm{~m}, 1 \mathrm{H}), 2.29(\mathrm{~m}, 1 \mathrm{H}), 2.21-2.09(\mathrm{~m}, 2 \mathrm{H}), 2.05-$ $1.87(\mathrm{~m}, 4 \mathrm{H}), 1.76-1.67(\mathrm{~m}, 1 \mathrm{H}), 1.64-1.53(\mathrm{~m}, 5 \mathrm{H}) .{ }^{13} \mathrm{C}$ NMR: $(126$ $\left.\mathrm{MHz}, \mathrm{CD}_{2} \mathrm{Cl}_{2}\right) \delta=197.8,137.5,137.1,136.3,130.2,128.5,127.5,52.8,52.2,33.2,31.2,28.6$, 28.0, 27.1, 26.8, 25.0 ppm. IR: (ATR, cm ${ }^{-1}$ ) 2925, 2851, 1732, 1598, 1469, 1444, 1327, 1257, 1191, 1088, 952, 851, 752, 703, 675. HRMS: calcd. for $\mathrm{C}_{18} \mathrm{H}_{24} \mathrm{NS}^{+}[\mathrm{M}+\mathrm{H}]^{+}:$: 286.1624; found: 286.1626 .

Compound 161b: ${ }^{25}{ }^{1} \mathrm{H}$ NMR: $\left(400 \mathrm{MHz}, \mathrm{CD}_{2} \mathrm{Cl}_{2}\right) \delta=7.37-7.20(\mathrm{~m}, 5 \mathrm{H}), 4.15(\mathrm{~d}, J=0.6 \mathrm{~Hz}$, $2 \mathrm{H}$ ), 3.82 (dddt, $J=7.2,6.1,1.1,0.6 \mathrm{~Hz}, 2 \mathrm{H}$ ), 3.54 (ddt, $J=8.0,6.9,1.1 \mathrm{~Hz}, 2 \mathrm{H}$ ), $2.04-1.88$ (m, 4H) ppm. ${ }^{13} \mathrm{C}$ NMR: $\left(126 \mathrm{MHz}, \mathrm{CD}_{2} \mathrm{Cl}_{2}\right) \delta=197.4,136.6,129.1,129.1,127.3,51.5,51.5$, $30.2,27.0,24.9 \mathrm{ppm}$.

\section{N,N-Diethyl-2-phenylethanethioamide (161c):}

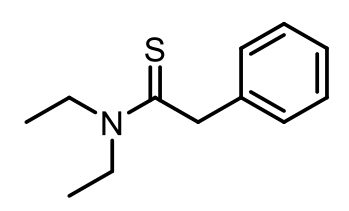

Following GP B, compound 161c was obtained from diethylamine (1 equiv, $17.4 \mu \mathrm{L}, 0.17 \mathrm{mmol}$ ) and $160 \mathrm{a}$ (1 equiv, $73.0 \mathrm{mg}, 0.17 \mathrm{mmol}$ ) in

\footnotetext{
${ }^{24}$ No additional base was added.

${ }^{25}$ Analytical data are in agreement with the previously published ones: T. Guntreddi, R. Vanjari, K. N. Singh, Org. Lett. 2014, 16, 3624-3627.
} 
$\mathrm{CH}_{2} \mathrm{Cl}_{2}(2 \mathrm{~mL}$ ) as a pale brown solid (8.4 mg, $41 \mu \mathrm{mol}, 24 \%)$ after column chromatography (petrol ether/EtOAc, 10:1). ${ }^{26}$

${ }^{1} \mathrm{H}$ NMR: $\left(600 \mathrm{MHz}, \mathrm{CD}_{2} \mathrm{Cl}_{2}\right) \delta=7.34-7.29(\mathrm{~m}, 3 \mathrm{H}), 7.26-7.22(\mathrm{~m}, 1 \mathrm{H}), 4.25(\mathrm{~s}, 2 \mathrm{H}), 3.98(\mathrm{q}$, $J=7.1 \mathrm{~Hz}, 2 \mathrm{H}), 3.48(\mathrm{q}, J=7.2 \mathrm{~Hz}, 2 \mathrm{H}), 1.26(\mathrm{t}, J=7.1 \mathrm{~Hz}, 3 \mathrm{H}), 1.11(\mathrm{t}, J=7.2 \mathrm{~Hz}, 3 \mathrm{H}) \mathrm{ppm} .{ }^{13} \mathrm{C}$ NMR: $\left(126 \mathrm{MHz}, \mathrm{CD}_{2} \mathrm{Cl}_{2}\right) \delta=199.3,137.3,129.1,128.4,127.2,50.8,48.1,47.1,13.7,11.3$ ppm.

4-Methoxybenzyl 2-(triisopropylsilyl)ethanedithioate (168a) and 2-[(4methoxybenzyl)thio]-1-methylpyridin-1-ium hexafluoroantimonate (168b):

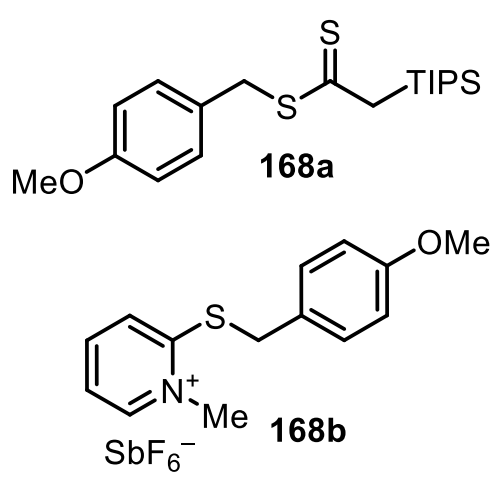

orange oil in $94 \%$ yield.
The reaction mixture obtained from (4-methoxyphenyl)methanethiol ( 2 equiv, $92.5 \mathrm{mg}, 83.6 \mu \mathrm{L}, 0.6 \mathrm{mmol}$ ), $160 \mathrm{~d}$ (1 equiv, $162.7 \mathrm{mg}, 0.3 \mathrm{mmol}$ ) and DIPEA (2 equiv, $77.6 \mathrm{mg}$, $104.5 \mu \mathrm{L}, 0.6 \mathrm{mmol})$ in $\mathrm{CH}_{2} \mathrm{Cl}_{2}(2 \mathrm{~mL})$ following $\mathrm{GP} \mathrm{B}^{27}$ was filtered. The precipitate was washed with $\mathrm{CH}_{2} \mathrm{Cl}_{2}(3 \times 2 \mathrm{~mL})$ affording a white solid, which was identified as $168 \mathrm{~b}$ (136.9 $\mathrm{mg}, 28.4 \mu \mathrm{mol}$, 95\%). The combined filtrates were concentrated and purified by column chromatography (hexanes/ethyl acetate, 20:1) to give compound 168a as an

Compound 168a: ${ }^{1} \mathrm{H}$ NMR: $\left(300 \mathrm{MHz}, \mathrm{CDCl}_{3}\right) \delta=7.25-7.20(\mathrm{~m}, 2 \mathrm{H}), 6.87-6.80(\mathrm{~m}, 2 \mathrm{H})$, $4.39(\mathrm{~s}, 2 \mathrm{H}), 3.79(\mathrm{~s}, 3 \mathrm{H}), 3.16(\mathrm{~s}, 2 \mathrm{H}), 1.32-1.17(\mathrm{~m}, 3 \mathrm{H}), 1.16-1.05(\mathrm{~m}, 18 \mathrm{H}) \mathrm{ppm} .{ }^{13} \mathrm{C}$ NMR: $\left(126 \mathrm{MHz}, \mathrm{CDCl}_{3}\right) \delta=236.1,159.2,130.5,127.5,114.2,55.4,42.5,41.8,18.7,11.6$ ppm. IR: (ATR, cm ${ }^{-1}$ ) 2940, 2864, 1610, 1510, 1462, 1301, 1248, 1174, 1102, 1036, 911, 880, 830, 658, 510. HRMS: calcd. for $\mathrm{C}_{19} \mathrm{H}_{33} \mathrm{OS}_{2} \mathrm{Si}^{+}[\mathrm{M}+\mathrm{H}]^{+}$: 369.1737; found: 369.1736 .

Compound 168b: ${ }^{1} \mathrm{H}$ NMR: $\left(600 \mathrm{MHz}, \mathrm{CD}_{3} \mathrm{CN}\right) \delta=8.50$ (ddt, $\left.J=6.3,1.3,0.6 \mathrm{~Hz}, 1 \mathrm{H}\right), 8.23$ (ddd, $J=8.9,7.5,1.6 \mathrm{~Hz}, 1 \mathrm{H}$ ), $7.89(\mathrm{dt}, J=8.5,0.8 \mathrm{~Hz}, 1 \mathrm{H}$ ), 7.61 (ddd, $J=7.7,6.3,1.4 \mathrm{~Hz}, 1 \mathrm{H}$ ), $7.44-7.40(\mathrm{~m}, 2 \mathrm{H}), 6.97-6.94(\mathrm{~m}, 2 \mathrm{H}), 4.55(\mathrm{~s}, 2 \mathrm{H}), 4.07(\mathrm{~s}, 3 \mathrm{H}), 3.79(\mathrm{~s}, 3 \mathrm{H}) \mathrm{ppm} .{ }^{13} \mathrm{C}$ NMR: $\left(126 \mathrm{MHz}, \mathrm{CD}_{3} \mathrm{CN}\right) \delta=160.9,160.8,147.5,144.3,131.6,126.4,125.6,123.4,115.5,56.1$, 47.1, 37.9 ppm. ${ }^{19}$ F NMR: $\left(282 \mathrm{MHz}, \mathrm{CD}_{3} \mathrm{CN}\right) \delta=-104.38$ to $-144.00(\mathrm{~m}) \mathrm{ppm}$. IR: (ATR, $\left.\mathrm{cm}^{-1}\right)$ 2918, 2849, 2359, 1611, 1566, 1513, 1492, 1456, 1438, 1250, 1178, 1117, 1228, 845, 766, 658, 641. HRMS: calcd. for $\mathrm{C}_{14} \mathrm{H}_{16} \mathrm{NOS}^{+}\left[\mathrm{M}-\mathrm{SbF}_{6}\right]^{+}: 246.0947$; found: 246.0947.

\footnotetext{
${ }^{26}$ Analytical data are in agreement with the previously published ones: Y. Sun, H. Jiang, W. Wu, W. Zeng, J. Li, Org. Biomol. Chem. 2014, 12, 700-707.

${ }^{27}$ Experiment was run on air.
} 
Methyl 2-\{[(2-(triisopropylsilyl)ethanethioyl]thio\}acetate (169):

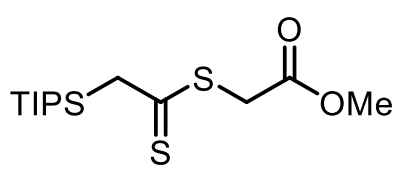

Following GP B, compound 169 (49.0 mg, $0.153 \mathrm{mmol}, 51 \%)$ was obtained as a yellow oil from methyl thioglycolate (1 equiv, 31.8 $\mathrm{mg}, 26.8 \mu \mathrm{L}, 0.3 \mathrm{mmol}$ ), 160d, (1 equiv, $162.7 \mathrm{mg}, 0.3 \mathrm{mmol}$ ) and DIPEA (1 equiv, $77.6 \mathrm{mg}, 104.5 \mu \mathrm{L}, 0.6 \mathrm{mmol}$ ) in $\mathrm{CH}_{2} \mathrm{Cl}_{2}(2 \mathrm{~mL}$ ) after column chromatography (hexanes/EtOAc, 80:1).

${ }^{1} \mathrm{H}$ NMR: $\left(300 \mathrm{MHz}, \mathrm{CDCl}_{3}\right) \delta=4.07(\mathrm{~s}, 2 \mathrm{H}), 3.72(\mathrm{~s}, 3 \mathrm{H}), 3.20(\mathrm{~s}, 2 \mathrm{H}), 1.34-1.18(\mathrm{~m}, 3 \mathrm{H}), 1.14$ - 1.07 (m, 18H) ppm. ${ }^{13} \mathrm{C}$ NMR: $\left(126 \mathrm{MHz} \mathrm{CDCl}_{3}\right) \delta=234.12,168.40,52.82,42.44,39.17$, 18.72, 11.57 ppm. IR: (ATR, cm ${ }^{-1}$ ) 2942, 2865, 1741, 1461, 1435, 1294, 1231, 1155, 1138, 1102, 999, 913, 879, 660, 510. HRMS: calcd. for $\mathrm{C}_{14} \mathrm{H}_{29} \mathrm{O}_{2} \mathrm{~S}_{2} \mathrm{Si}^{+}[\mathrm{M}+\mathrm{H}]^{+}: 321.1373$; found (ESI): 321.1375 .

\section{Methyl N-(tert-butoxycarbonyl)-S-[2-(triisopropylsilyl)ethanethioyl]-L-cysteinate (170):}<smiles></smiles>

Following GP B, compound 170 (54.6 mg, $0.12 \mathrm{mmol}$, 40\%, 59\% RSM) was obtained from $\mathrm{N}$-(tert-butoxycarbonyl)-L-cysteine methyl ester ( 1 equiv, $70.6 \mathrm{mg}, 61.8 \mu \mathrm{L}, 0.3 \mathrm{mmol}$ ), 160d, (1 equiv, $162.7 \mathrm{mg}, 0.3 \mathrm{mmol}$ ) and DIPEA (1 equiv, $77.6 \mathrm{mg}, 54 \mu \mathrm{L}$, $0.31 \mathrm{mmol}$ ) in $\mathrm{CH}_{2} \mathrm{Cl}_{2}(2 \mathrm{~mL}$ ) after column chromatography (hexanes/EtOAc, 80:1 to 5:1) as bright yellow crystals.

${ }^{1} \mathrm{H}$ NMR: $\left(300 \mathrm{MHz}, \mathrm{CDCl}_{3}\right) \delta=5.21(\mathrm{~d}, J=7.6 \mathrm{~Hz}, 1 \mathrm{H}), 4.65-4.54(\mathrm{~m}, 1 \mathrm{H}), 3.81(\mathrm{dd}, J=13.9$, $4.5 \mathrm{~Hz}, 1 \mathrm{H}), 3.76(\mathrm{~s}, 3 \mathrm{H}), 3.63(\mathrm{dd}, J=13.7,7.2 \mathrm{~Hz}, 1 \mathrm{H}), 3.18(\mathrm{~s}, 2 \mathrm{H}), 1.43(\mathrm{~s}, 9 \mathrm{H}), 1.30-1.17$ (m, 3H), 1.09 (d, $J=6.7 \mathrm{~Hz}, 18 \mathrm{H}) .{ }^{13} \mathrm{C}$ NMR: $\left(126 \mathrm{MHz}, \mathrm{CDCl}_{3}\right) \delta=235.6,171.3,155.2,80.4$, 52.9, 52.66, 43.2, 38.9, 28.4, 18.8, 11.6 ppm. IR: (ATR, cm ${ }^{-1}$ ) 3369, 2943, 2866, 1747, 1719, $1500,1366,1220,1166,1105,913,881,661$. HRMS: calcd. forC ${ }_{20} \mathrm{H}_{40} \mathrm{NO}_{4} \mathrm{~S}_{2} \mathrm{Si}^{+}[\mathrm{M}+\mathrm{H}]^{+}$: 450.2163; found (ESI): 450.2167.

\section{Ethane-1,2-diyl bis[2-(triisopropylsilyl)ethanedithioate] (171):}

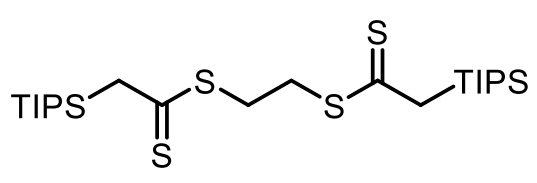

Following GP B, compound 171 (40.2 mg, $76.8 \mu \mathrm{mol}, 26 \%)$ was obtained from ethane-1,2-dithiol (1 equiv, $28.3 \mathrm{mg}$, $25.2 \mu \mathrm{L}, 0.3 \mathrm{mmol}$ ), 160d, (1 equiv, $162.7 \mathrm{mg}, 0.3 \mathrm{mmol}$ ) and DIPEA (1 equiv, $77.6 \mathrm{mg}, 54 \mu \mathrm{L}, 0.31 \mathrm{mmol}$ ) in $\mathrm{CH}_{2} \mathrm{Cl}_{2}(2$ $\mathrm{mL}$ ) after column chromatography (hexanes/EtOAc, 200:1) as bright yellow crystals.

${ }^{1} \mathrm{H}$ NMR: $\left(400 \mathrm{MHz}, \mathrm{CDCl}_{3}\right) \delta=3.49(\mathrm{~s}, 4 \mathrm{H}), 3.17(\mathrm{~s}, 4 \mathrm{H}), 1.29-1.20(\mathrm{~m}, 6 \mathrm{H}), 1.10(\mathrm{~d}, J=7.3$ $\mathrm{Hz}, 36 \mathrm{H})$ ppm. ${ }^{13} \mathrm{C}$ NMR: $\left(101 \mathrm{MHz}, \mathrm{CDCl}_{3}\right) \delta=235.9,43.0,34.6,27.1,18.8,11.6$ ppm. IR: $\left(\right.$ ATR, cm ${ }^{-1}$ ) 2941, 2875, 1463, 1388, 1226, 1103, 912, 882, 658. HRMS: calcd. for $\mathrm{C}_{24} \mathrm{H}_{51} \mathrm{~S}_{4} \mathrm{Si}_{2}{ }^{+}$ $[\mathrm{M}+\mathrm{H}]^{+}:$523.2407; found (ESI): 523.2410 
Propyl 2-(triisopropylsilyl)ethanedithioate (172):<smiles>CCCSC(=S)C[In][Sn]</smiles>

Following GP B, compound 172 (87.1 mg, $0.3 \mathrm{mmol}$, 99\%) was obtained from 1-propanethiol (2 equiv, $45.7 \mathrm{mg}, 54.4 \mu \mathrm{L}, 0.6 \mathrm{mmol}$ ), 160d, (1 equiv, $162.7 \mathrm{mg}, 0.3 \mathrm{mmol}$ ) and DIPEA (1 equiv, $77.6 \mathrm{mg}, 54$ $\mu \mathrm{L}, 0.31 \mathrm{mmol}$ ) in $\mathrm{CH}_{2} \mathrm{Cl}_{2}(2 \mathrm{~mL}$ ) after column chromatography (hexanes/EtOAc, 50:1) as a yellow solid.

${ }^{1} \mathrm{H}$ NMR: $\left(300 \mathrm{MHz}, \mathrm{CDCl}_{3}\right) \delta=3.21-3.13(\mathrm{~m}, 4 \mathrm{H}), 1.69(\mathrm{~h}, J=7.3 \mathrm{~Hz}, 2 \mathrm{H}), 1.33-1.17(\mathrm{~m}$, $3 \mathrm{H}), 1.10(\mathrm{~d}, J=6.8 \mathrm{~Hz}, 18 \mathrm{H}), 1.01(\mathrm{t}, J=7.3 \mathrm{~Hz}, 3 \mathrm{H})$ ppm. ${ }^{13} \mathrm{C} \mathrm{NMR:}\left(126 \mathrm{MHz}, \mathrm{CDCl}_{3}\right) \delta=$ 237.0, 42.9, 39.2, 21.1, 18.8, 13.8, 11.6 ppm. IR: (ATR, cm ${ }^{-1}$ ) 2961, 2941, 2866, 1715, 1634, 1551, 1462, 1224, 1109, 915, 881, 662. HRMS: calcd. for $\mathrm{C}_{14} \mathrm{H}_{31} \mathrm{~S}_{2} \mathrm{Si}^{+}[\mathrm{M}+\mathrm{H}]^{+}:$: 291.1631; found (ESI): 291.1629.

\section{4-Methoxyphenyl 2-(triisopropylsilyl)ethanedithioate (173):}<smiles>COc1ccc(SC(=S)CC(F)(F)F)cc1</smiles>

Following GP B, compound 173 (66.2 $\mathrm{mg}, 0.19 \mathrm{mmol}$, 62\%) was obtained from 4-methoxythiophenol (1 equiv, $42.1 \mathrm{mg}, 36.9 \mu \mathrm{L}$, $0.3 \mathrm{mmol}$ ), $160 \mathrm{~d}$ ( 1 equiv, $162.7 \mathrm{mg}, 0.3 \mathrm{mmol}$ ) and DIPEA (1 equiv, $77.6 \mathrm{mg}, 54 \mu \mathrm{L}, 0.31 \mathrm{mmol})$ in $\mathrm{CH}_{2} \mathrm{Cl}_{2}(2 \mathrm{~mL})$ after column chromatography (hexanes/EtOAc, 80:1 to $60: 1$ ) as a yellow oil.

${ }^{1}$ H NMR: $\left(300 \mathrm{MHz}, \mathrm{CDCl}_{3}\right) \delta=7.31-7.25(\mathrm{~m}, 2 \mathrm{H}), 7.02-6.95(\mathrm{~m}, 2 \mathrm{H}), 3.85(\mathrm{~s}, 3 \mathrm{H}), 3.24(\mathrm{~s}$, $2 \mathrm{H}), 1.43-1.21(\mathrm{~m}, 3 \mathrm{H}), 1.21-1.09(\mathrm{~m}, 18 \mathrm{H})$ ppm. ${ }^{13} \mathrm{C}$ NMR: $\left(126 \mathrm{MHz}, \mathrm{CDCl}_{3}\right) \delta=237.9$, 161.2, 136.7, 123.5, 115.1, 55.5, 41.9, 18.8, 11.6 ppm. IR: (ATR, cm ${ }^{-1}$ ) 2941, 2864, 1591, $1494,1462,1291,1250,1173,1103,1032,904,882,824,799,664,645$. HRMS: calcd. for $\mathrm{C}_{18} \mathrm{H}_{31} \mathrm{OS}_{2} \mathrm{Si}^{+}[\mathrm{M}+\mathrm{H}]^{+}:$355.1580; found: 355.1570 .

\section{Compound 174, 4-Nitrophenyl 2-(triisopropylsilyl)ethanedithioate (174)}<smiles>O=[N+]([O-])c1ccc(SC(=S)C[In]Sc2ccccc2)cc1</smiles>

Following GP B, compound 174 (108.0 mg, $0.29 \mathrm{mmol}, 97 \%)$ was obtained from 4-nitrothiophenol (2 equiv, $93.1 \mathrm{mg}, 0.6 \mathrm{mmol}$ ), 160d (1 equiv, $162.7 \mathrm{mg}, 0.3 \mathrm{mmol}$ ) and DIPEA (1 equiv, $77.6 \mathrm{mg}$, $54 \mu \mathrm{L}, 0.31 \mathrm{mmol}$ ) in $\mathrm{CH}_{2} \mathrm{Cl}_{2}(2 \mathrm{~mL})$ after column chromatography (hexanes/EtOAc, 50:1) as a bright yellow oil.

${ }^{1}$ H NMR: $\left(300 \mathrm{MHz}, \mathrm{CDCl}_{3}\right) \delta=8.29(\mathrm{~d}, J=8.9 \mathrm{~Hz}, 2 \mathrm{H}), 7.55(\mathrm{~d}, J=8.9 \mathrm{~Hz}, 2 \mathrm{H}), 3.28(\mathrm{~s}, 2 \mathrm{H})$, $1.39-1.24(\mathrm{~m}, 3 \mathrm{H}), 1.15(\mathrm{~d}, \mathrm{~J}=7.1 \mathrm{~Hz}, 18 \mathrm{H})$ ppm. ${ }^{13} \mathrm{C}$ NMR: $\left(126 \mathrm{MHz}, \mathrm{CDCl}_{3}\right) \delta=233.4$, 148.8, 139.8, 136.3, 124.4, 43.0, 18.8, 11.6 ppm. IR: (ATR, $\mathrm{cm}^{-1}$ ) 2942, 2866, 1600, 1522, 
$1463,1343,1229,1101,902,882,851,743$. HRMS: calcd. for $\mathrm{C}_{17} \mathrm{H}_{28} \mathrm{NO}_{2} \mathrm{~S}_{2} \mathrm{Si}^{+}[\mathrm{M}+\mathrm{H}]^{+}$: 370.1325; found (ESI): 370.1320 .

\section{2-\{[2-(Triisopropylsilyl)ethanethioyl]thio\}benzoic acid (175):}<smiles>O=C(O)c1ccccc1SC(=S)C[In]S</smiles>

Following GP B, compound $175(73.6 \mathrm{mg}, 0.2 \mathrm{mmol}, 67 \%)$ was obtained from thiosalicylic acid ( 2 equiv, $92.5 \mathrm{mg}, 62.1 \mu \mathrm{L}, 0.6 \mathrm{mmol}$ ), 160d (1 equiv, $162.7 \mathrm{mg}, 0.3 \mathrm{mmol}$ ) and DIPEA (1 equiv, $77.6 \mathrm{mg}, 54$ $\mu \mathrm{L}, 0.31 \mathrm{mmol})$ in $\mathrm{CH}_{2} \mathrm{Cl}_{2}(2 \mathrm{~mL})$ after column chromatography (cyclohexane/EtOAc, 10:1 to 5:1) as a yellow solid.

${ }^{1} \mathrm{H}$ NMR: $\left(400 \mathrm{MHz}, \mathrm{CDCl}_{3}\right) \delta=8.11$ (ddd $\left.J=7.6,1.7,0.4 \mathrm{~Hz}, 1 \mathrm{H}\right), 7.63(\mathrm{td}, J=7.5,1.7 \mathrm{~Hz}, 1 \mathrm{H})$, 7.57 (td, $J=7.6,1.5 \mathrm{~Hz}, 1 \mathrm{H}$ ), 7.52 (ddd, $J=7.7,1.5,0.4 \mathrm{~Hz}, 1 \mathrm{H}), 3.26(\mathrm{~s}, 2 \mathrm{H}), 1.37-1.24(\mathrm{~m}$, $3 \mathrm{H}), 1.14$ (d, $J=7.2 \mathrm{~Hz}, 18 \mathrm{H}$ ) ppm. ${ }^{13} \mathrm{C}$ NMR: $\left(101 \mathrm{MHz}, \mathrm{CDCl}_{3}\right) \delta=234.7,171.0,138.0,134.1$, 133.2, 133.06, 131.8, 130.4, 42.3, 18.9, 11.7 ppm. IR: (ATR, $\mathrm{cm}^{-1}$ ) 2942, 2866, 2363, 2342, $1698,1465,1406,1296,1272,1228,1136,1106,906,882,748,699,658$. HRMS: calcd. for $\mathrm{C}_{18} \mathrm{H}_{29} \mathrm{O}_{2} \mathrm{~S}_{2} \mathrm{Si}^{+}[\mathrm{M}+\mathrm{H}]^{+}:$369.1373; found (ESI): 369.1372 .

\section{N-Ethyl-2-(triisopropylsilyl)ethanethioamide (176):}<smiles>CCNC(=S)C[In]S</smiles>

Following the GP B, compound $176(72.7 \mathrm{mg}, 0.28 \mathrm{mmol}, 93 \%)$ was obtained from ethylamine ( $12 \mathrm{M}$ in $\mathrm{H}_{2} \mathrm{O}, 2$ equiv, $50 \mu \mathrm{L}, 0.6 \mathrm{mmol}$ ), 160d (1 equiv, $162.7 \mathrm{mg}, 0.3 \mathrm{mmol}$ ) and DIPEA (1 equiv, $77.6 \mathrm{mg}, 54 \mu \mathrm{L}, 0.31$ $\mathrm{mmol}$ ) in $\mathrm{CH}_{2} \mathrm{Cl}_{2}(2 \mathrm{~mL}$ ) after separation by column chromatography (cyclohexane/EtOAc, 50:1 to $10: 1$ ) as an off-white crystalline solid.

${ }^{1} \mathrm{H}$ NMR: $\left(300 \mathrm{MHz}, \mathrm{CDCl}_{3}\right) \delta=6.86$ (bs, $\left.1 \mathrm{H}\right), 3.65$ (qd, $\left.J=7.3,5.2 \mathrm{~Hz}, 2 \mathrm{H}\right), 2.53(\mathrm{~s}, 2 \mathrm{H}), 1.30-$ $1.15(\mathrm{~m}, 6 \mathrm{H}), 1.09$ (d, $J=6.8 \mathrm{~Hz}, 18 \mathrm{H})$ ppm. ${ }^{13} \mathrm{C} \mathrm{NMR:}\left(75 \mathrm{MHz}, \mathrm{CDCl}_{3}\right) \delta=204.1,41.1,34.2$, 18.8, 13.5, 11.5 ppm. IR: (ATR, cm ${ }^{-1}$ ) 3238, 2940, 2875, 1527, 1459, 1383, 1328, 1203, 1103, 1036, 881, 799, 735, 642, 605, 559. HRMS: calcd. for $\mathrm{C}_{13} \mathrm{H}_{30} \mathrm{NSSi}^{+}[\mathrm{M}+\mathrm{H}]^{+}:$260.1863; found (ESI): 260.1866 .

\section{N,N-Diethyl-2-(triisopropylsilyl)ethanethioamide (177):}<smiles>CCN(CC)C(=S)C[In]S</smiles>

Following the GP B, compound $177(76.0 \mathrm{mg}, 0.264 \mathrm{mmol}, 73 \%)$ was obtained from diethylamine (1 equiv, $26.3 \mathrm{mg}, 37.2 \mu \mathrm{L}, 0.36 \mathrm{mmol}$ ), 160d (1 equiv, $195.2 \mathrm{mg}, 0.36 \mathrm{mmol}$ ) and DIPEA (1 equiv, $46.5 \mathrm{mg}, 62.7 \mu \mathrm{L}$, $0.36 \mathrm{mmol})$ in $\mathrm{CH}_{2} \mathrm{Cl}_{2}(2 \mathrm{~mL})$ after column chromatography (hexanes/EtOAc, 20:1) as an off-white solid. 
${ }^{1} \mathrm{H}$ NMR: $\left(400 \mathrm{MHz}, \mathrm{CDCl}_{3}\right) \delta=4.02(\mathrm{q}, J=7.1 \mathrm{~Hz}, 2 \mathrm{H}), 3.57(\mathrm{q}, J=7.1 \mathrm{~Hz}, 2 \mathrm{H}), 2.69(\mathrm{~s}, 2 \mathrm{H})$, $1.34-1.21(\mathrm{~m}, 9 \mathrm{H}), 1.10(\mathrm{~d}, J=7.3 \mathrm{~Hz}, 18 \mathrm{H})$ ppm. ${ }^{13} \mathrm{C} \mathrm{NMR:}\left(101 \mathrm{MHz}, \mathrm{CDCl}_{3}\right) \delta=201.8,47.7$, 46.5, 30.1, 18.9, 13.2, 11.8, 11.5 ppm. IR: (ATR, cm ${ }^{-1}$ ) 3321, 2971, 2873, 2360, 1454, 1381, 1087, 1045, 879, 637. HRMS: calcd. for $\mathrm{C}_{15} \mathrm{H}_{34} \mathrm{NSSi}^{+}[\mathrm{M}+\mathrm{H}]^{+}:$: 288.2176; found (ESI): 288.2173.

\section{1-Morpholino-2-(triisopropylsilyl)ethane-1-thione (178):}<smiles>FC(F)(F)CC(=S)N1CCOCC1</smiles>

Following GP B, compound $178(82.0 \mathrm{mg}, 0.272 \mathrm{mmol}, 91 \%)$ was obtained from morpholine ( 2 equiv, $52.3 \mathrm{mg}, 51.7 \mu \mathrm{L}, 0.6 \mathrm{mmol}$ ), 160d (1 equiv, $162.7 \mathrm{mg}, 0.3 \mathrm{mmol}$ ) and DIPEA (1 equiv, $77.6 \mathrm{mg}, 54 \mu \mathrm{L}, 0.31$ $\mathrm{mmol}$ ) in $\mathrm{CH}_{2} \mathrm{Cl}_{2}(2 \mathrm{~mL}$ ) after column chromatography (hexanes/EtOAc, 20:1 to $10: 1)$ as a white crystalline solid.

${ }^{1} \mathrm{H}$ NMR: $\left(300 \mathrm{MHz}, \mathrm{CDCl}_{3}\right) \delta=4.44-4.34(\mathrm{~m}, 2 \mathrm{H}), 3.83-3.70(\mathrm{~m}, 6 \mathrm{H}), 2.79(\mathrm{~s}, 2 \mathrm{H}), 1.37-$ $1.18(\mathrm{~m}, 3 \mathrm{H}), 1.18-1.05(\mathrm{~m}, 18 \mathrm{H}) \mathrm{ppm} .{ }^{13} \mathrm{C} \mathrm{NMR:}\left(126 \mathrm{MHz}, \mathrm{CDCl}_{3}\right) \delta=203.5,66.7,66.3$, 50.6, 49.7, 30.1, 18.8, 11.8 ppm. IR: (ATR, cm ${ }^{-1}$ ) 2941, 2865, 1477, 1412, 1276, 1248, 1114, 1024, 1007, 881, 707, 658. HRMS: calcd. for $\mathrm{C}_{15} \mathrm{H}_{32} \mathrm{NOSSi}^{+}[\mathrm{M}+\mathrm{H}]^{+}:$302.1968; found (ESI): 302.1970 .

\section{N-Cyclopropyl-2-(triisopropylsilyl)ethanethioamide (179):}

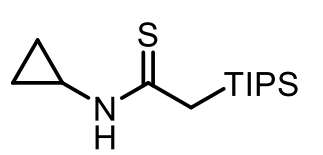

Following GP B, compound $179(43.9 \mathrm{mg}, 0.162 \mathrm{mmol}, 54 \%)$ was obtained from cyclopropanamine (2 equiv, $34.3 \mathrm{mg}, 41.6 \mu \mathrm{L}, 0.6 \mathrm{mmol}$ ), 160d (1 equiv, $162.7 \mathrm{mg}, 0.3 \mathrm{mmol}$ ) and DIPEA (1 equiv, $77.6 \mathrm{mg}, 54 \mu \mathrm{L}$, $0.31 \mathrm{mmol}$ ) in $\mathrm{CH}_{2} \mathrm{Cl}_{2}(2 \mathrm{~mL}$ ) after column chromatography (cyclohexane/EtOAc, 20:1) as an off-white crystalline solid.

${ }^{1} \mathrm{H}$ NMR: $\left(300 \mathrm{MHz}, \mathrm{CDCl}_{3}\right) \delta=6.89(\mathrm{~s}, 1 \mathrm{H}), 3.17(\mathrm{tt}, J=7.7,3.9 \mathrm{~Hz}, 1 \mathrm{H}), 2.52(\mathrm{~s}, 2 \mathrm{H}), 1.32-$ $1.12(\mathrm{~m}, 3 \mathrm{H}), 1.09(\mathrm{~d}, \mathrm{~J}=6.5 \mathrm{~Hz}, 18 \mathrm{H}), 0.97-0.86(\mathrm{~m}, 2 \mathrm{H}), 0.67-0.60(\mathrm{~m}, 2 \mathrm{H}) \mathrm{ppm} .{ }^{13} \mathrm{C} \mathrm{NMR}$ : $\left(101 \mathrm{MHz}, \mathrm{CDCl}_{3}\right) \delta=206.3,34.1,29.0,18.8,11.5,7.3$ ppm. IR: $\left(\right.$ ATR, $\left.\mathrm{cm}^{-1}\right)$ 3330, 3244, 2969, 2942, 2866, 1459, 1382, 1089, 1052, 883, 783, 732, 647, 612. HRMS: calcd. for $\mathrm{C}_{14} \mathrm{H}_{30} \mathrm{NSSi}^{+}$ $[\mathrm{M}+\mathrm{H}]^{+}: 272.1863$ found (ESI): 272.1864 .

\section{$N$-[2-(1H-Indol-3-yl)ethyl]-2-(triisopropylsilyl)ethanethioamide (180):}<smiles>FC(F)(F)CC(=S)NCCc1c[nH]c2ccccc12</smiles>

Following GP B, 180 (90.6 mg, $0.242 \mathrm{mmol}, 81 \%$ ) was obtained from tryptamine ( 2 equiv, $96.1 \mathrm{mg}, 0.6 \mathrm{mmol}$ ), 160d (1 equiv, $162.7 \mathrm{mg}, 0.3 \mathrm{mmol}$ ) and DIPEA (1 equiv, $77.6 \mathrm{mg}, 54 \mu \mathrm{L}, 0.31$ mmol) in $\mathrm{CH}_{2} \mathrm{Cl}_{2}(2 \mathrm{~mL})$ after column chromatography 
(cyclohexane/EtOAc, 10:1) as a colorless oil.

${ }^{1}$ H NMR: $\left(500 \mathrm{MHz}, \mathrm{CDCl}_{3}\right) \delta=8.12(\mathrm{~s}, 1 \mathrm{H}), 7.63(\mathrm{dd}, J=7.9,1.0 \mathrm{~Hz}, 1 \mathrm{H}), 7.39(\mathrm{dt}, J=8.2,0.9$ $\mathrm{Hz}, 1 \mathrm{H}$ ), 7.23 (ddd, $J=8.2,7.0,1.2 \mathrm{~Hz}, 1 \mathrm{H}$ ), 7.14 (ddd, $J=8.0,7.0,1.0 \mathrm{~Hz}, 1 \mathrm{H}$ ), 7.07 (d, $J=2.4$ $\mathrm{Hz}, 1 \mathrm{H}), 6.89(\mathrm{~s}, 1 \mathrm{H}), 3.98(\mathrm{td}, J=6.7,5.0 \mathrm{~Hz}, 2 \mathrm{H}), 3.12(\mathrm{td}, J=6.6,0.9 \mathrm{~Hz}, 2 \mathrm{H}), 2.45(\mathrm{~s}, 2 \mathrm{H})$, $1.08-0.99(\mathrm{~m}, 3 \mathrm{H}), 0.96(\mathrm{~d}, J=6.5 \mathrm{~Hz}, 17 \mathrm{H})$ ppm. ${ }^{13} \mathrm{C}$ NMR: $\left(126 \mathrm{MHz}, \mathrm{CDCl}_{3}\right) \delta=204.2$, 136.7, 127.2, 122.7, 122.2, 119.9, 118.8, 112.4, 111.4, 46.2, 34.0, 23.9, 18.6, 11.3 ppm. IR: $\left(\right.$ ATR, cm ${ }^{-1}$ ) 3408, 3353, 3262, 2939, 2864, 1512, 1456, 1390, 1338, 1228, 1128, 1104, 1011, 999, 880, 739, 647. HRMS: calcd. for $\mathrm{C}_{21} \mathrm{H}_{35} \mathrm{~N}_{2} \mathrm{SSi}^{+}[\mathrm{M}+\mathrm{H}]^{+}: 375.2285$; found (ESI): 375.2271.

Ethyl N,S-bis[2-(triisopropylsilyl)ethanethioyl]-L-cysteinate (181):<smiles></smiles>

Following GP B, compound 181 (72 $\mathrm{mg}, 0.13 \mathrm{mmol}$, 43\%) was prepared from L-cysteine ethyl ester hydrochloride (1 equiv, $55.7 \mathrm{mg}, 0.3 \mathrm{mmol}$ ), 160d (1 equiv, $162.7 \mathrm{mg}, 0.3 \mathrm{mmol}$ ) and DIPEA (2 equiv, $78.8 \mathrm{mg}, 106.3 \mu \mathrm{L}, 0.61 \mathrm{mmol}$ ) in $\mathrm{CH}_{2} \mathrm{Cl}_{2}(2 \mathrm{~mL}$ ) after column chromatography (hexanes/EtOAc, 95:5) as a

yellow oil.

${ }^{1}$ H NMR: $\left(600 \mathrm{MHz}, \mathrm{CDCl}_{3}\right) \delta=7.70(\mathrm{~d}, J=7.0 \mathrm{~Hz}, 1 \mathrm{H}), 5.43(\mathrm{td}, J=6.5,4.6 \mathrm{~Hz}, 1 \mathrm{H}), 4.28-$ $4.15(\mathrm{~m}, 2 \mathrm{H}), 4.01-3.88(\mathrm{~m}, 2 \mathrm{H}), 3.18(\mathrm{~s}, 2 \mathrm{H}), 2.67-2.46(\mathrm{~m}, 2 \mathrm{H}), 1.29(\mathrm{t}, J=7.2 \mathrm{~Hz}, 3 \mathrm{H})$, $1.27-1.19(\mathrm{~m}, 6 \mathrm{H}), 1.09$ (ddd, $J=7.3,4.8,2.2 \mathrm{~Hz}, 36 \mathrm{H})$ ppm. ${ }^{13} \mathrm{C} \mathrm{NMR:}\left(126 \mathrm{MHz}, \mathrm{CDCl}_{3}\right) \delta=$ 236.2, 205.6, 169.7, 62.4, 57.6, 43.4, 37.6, 34.4, 18.9, 18.8, 14.3, 11.7, 11.5 ppm. IR: (ATR, $\mathrm{cm}^{-1}$ ) 3369, 2941, 2866, 1734, 1499, 1464, 1370, 1222, 1193, 1135, 1105, 912, 881, 660. HRMS: calcd. for $\mathrm{C}_{27} \mathrm{H}_{56} \mathrm{NO}_{2} \mathrm{~S}_{3} \mathrm{Si}_{2}{ }^{+}[\mathrm{M}+\mathrm{H}]^{+}$: 578.3006; found (ESI): 578.2994.

$O-[(8 R, 9 S, 13 S, 14 S, 17 R)-17-E t h y n y l-17-h y d r o x y-13-m e t h y l-7,8,9,11,12,13,14,15,16,17-$ decahydro-6H-cyclopenta[a]phenanthren-3-yl] 2-(triisopropylsilyl)ethanethioate (182):

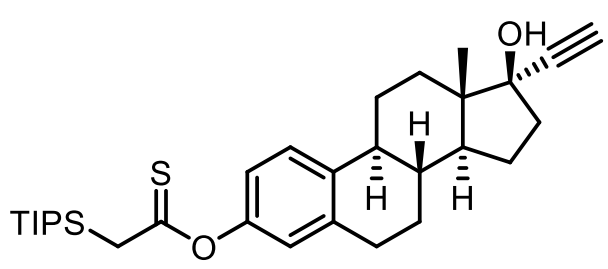

Following GP B, compound $182(15.5 \mathrm{mg}, 30.3 \mu \mathrm{mol}$, $10 \%$ ) was obtained from $17 \alpha$-ethynylestradiol (2 equiv, $177.8 \mathrm{mg}, 0.06 \mathrm{mmol}$ ), 160d (1 equiv, $162.7 \mathrm{mg}, 0.3$ mmol) and DIPEA ( 1 equiv, $77.6 \mathrm{mg}, 54 \mu \mathrm{L}, 0.31 \mathrm{mmol}$ ) in $\mathrm{CH}_{2} \mathrm{Cl}_{2}(2 \mathrm{~mL})$ after column chromatography

(cyclohexane/EtOAc, 20:1) as a colorless oil.

${ }^{1}$ H NMR: $\left(300 \mathrm{MHz}, \mathrm{CDCl}_{3}\right) \delta=7.33(\mathrm{~d}, J=8.3 \mathrm{~Hz}, 1 \mathrm{H}), 6.81(\mathrm{dd}, J=8.5,2.6 \mathrm{~Hz}, 1 \mathrm{H}), 6.73(\mathrm{~d}, J$ $=2.4 \mathrm{~Hz}, 1 \mathrm{H}), 3.00(\mathrm{~s}, 2 \mathrm{H}), 2.90-2.87(\mathrm{~m}, 2 \mathrm{H}), 2.61(\mathrm{~s}, 1 \mathrm{H}), 2.36(\mathrm{~m}, 2 \mathrm{H}), 2.28(\mathrm{~m}, 1 \mathrm{H}), 2.03$ (ddd, J = 13.6, 11.9, 3.9 Hz, $1 \mathrm{H}$ ), $1.95-1.87(\mathrm{~m}, 3 \mathrm{H}), 1.80$ (dddd, J = 12.0, 9.6, 7.6, $4.0 \mathrm{~Hz}, 1 \mathrm{H}$ ), $1.76-1.68(\mathrm{~m}, 2 \mathrm{H}), 1.58-1.37(\mathrm{~m}, 4 \mathrm{H}), 1.31-1.25(\mathrm{~m}, 3 \mathrm{H}), 1.17(\mathrm{~d}, J=7.3 \mathrm{~Hz}, 18 \mathrm{H}), 0.89(\mathrm{~d}$, $J=0.7 \mathrm{~Hz}, 3 \mathrm{H})$ ppm. ${ }^{13} \mathrm{C}$ NMR: $\left(126 \mathrm{MHz}, \mathrm{CDCl}_{3}\right) \delta=223.3,152.5,138.4,138.3,126.5,121.9$, 
119.2, 87.6, 80.0, 74.3, 49.7, 47.3, 43.9, 39.2, 39.2, 36.3, 32.9, 29.8, 27.2, 26.4, 23.0, 18.8, 12.8, 11.7 ppm. IR: (ATR, cm $\left.{ }^{-1}\right)$ 3305, 2936, 2865, 1493, 1459, 1268, 1192, 1120, 1058, 1048, 880, 651. HRMS: calcd. for $\mathrm{C}_{31} \mathrm{H}_{47} \mathrm{O}_{2} \mathrm{SSi}^{+}[\mathrm{M}+\mathrm{H}]^{+}$: 511.3061; found (ESI): 511.3053 .

\section{O-(Naphthalen-2-yl) 2-(triisopropylsilyl)ethanethioate (183):}

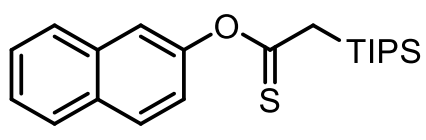

Following GP B, compound 183 (64.0 $\mathrm{mg}, 0.18 \mathrm{mmol}$, 60\%) was obtained from 2-naphthol (2 equiv, $86.5 \mathrm{mg}, 0.6 \mathrm{mmol}$ ), 160d (1 equiv, $162.7 \mathrm{mg}, 0.3 \mathrm{mmol}$ ) and DIPEA (1 equiv, $77.6 \mathrm{mg}, 54 \mu \mathrm{L}$, $0.31 \mathrm{mmol}$ ) in $\mathrm{CH}_{2} \mathrm{Cl}_{2}(2 \mathrm{~mL}$ ) after column chromatography (cyclohexane/EtOAc, 50/1) as a brown crystalline solid.

${ }^{1} \mathrm{H}$ NMR: $\left(300 \mathrm{MHz}, \mathrm{CDCl}_{3}\right) \delta=7.93-7.79(\mathrm{~m}, 3 \mathrm{H}), 7.55-7.43(\mathrm{~m}, 3 \mathrm{H}), 7.21(\mathrm{dd}, J=8.9,2.3$ $\mathrm{Hz}, 1 \mathrm{H}), 3.08(\mathrm{~s}, 2 \mathrm{H}), 1.46-1.26(\mathrm{~m}, 3 \mathrm{H}), 1.21(\mathrm{~d}, J=6.6 \mathrm{~Hz}, 18 \mathrm{H})$ ppm. ${ }^{13} \mathrm{C}$ NMR: $(126 \mathrm{MHz}$, $\left.\mathrm{CDCl}_{3}\right) \delta=223.4,152.4,133.9,131.8,129.4,128.0,127.9,126.7,126.0,121.9,119.1,36.3$, 18.8, 11.7 ppm. IR: (ATR, cm ${ }^{-1}$ ) 3369, 2943, 2866, 1747, 1719, 1500, 1366, 1220, 1166, 1105, 913, 881,661. HRMS: calcd. for $\mathrm{C}_{21} \mathrm{H}_{31} \mathrm{OSSi}^{+}[\mathrm{M}+\mathrm{H}]^{+}$: 359.1859; found (ESI): 359.1863 .

\section{N,N'-Diphenyl-2-(triisopropylsilyl)acetimidamide (184):}<smiles>FC(F)(S)CC(=Nc1ccccc1)Nc1ccccc1</smiles>

Following GP B, compound $184(79.6 \mathrm{mg}, 0.217 \mathrm{mmol}, 72 \%)$ was obtained from anilline ( 2 equiv, $55.9 \mathrm{mg}, 54.8 \mu \mathrm{L}, 0.6 \mathrm{mmol}$ ), 160d (1 equiv, $162.7 \mathrm{mg}, 0.3 \mathrm{mmol}$ ) and DIPEA (1 equiv, $77.6 \mathrm{mg}, 54 \mu \mathrm{L}, 0.31$ $\mathrm{mmol}$ ) in $\mathrm{CH}_{2} \mathrm{Cl}_{2}(2 \mathrm{~mL}$ ) after column chromatography (hexanes/EtOAc, 10:1 to pure EtOAc) as brownish crystals.

${ }^{1} \mathrm{H}$ NMR: $\left(400 \mathrm{MHz}, \mathrm{CDCl}_{3}\right) \delta=7.38-6.94(\mathrm{~m}, 11 \mathrm{H}), 1.99(\mathrm{~s}, 2 \mathrm{H}), 1.20-1.05(\mathrm{~m}, 5 \mathrm{H}), 1.00(\mathrm{~d}$, $J=6.9 \mathrm{~Hz}, 16 \mathrm{H}) .{ }^{13} \mathrm{C}$ NMR: $\left(101 \mathrm{MHz}, \mathrm{CDCl}_{3}\right) \delta=155.4,129.0,122.4,121.8-120.3$ (s, br), 18.6, 14.4, 11.6 ppm. IR: (ATR, $\mathrm{cm}^{-1}$ ) 3452, 2940, 2865, 1640, 1589, 1521, 1497, 1486, 1435, $1318,1207,1155,881,750,698,653,639$. 505. HRMS: calcd. for $\mathrm{C}_{23} \mathrm{H}_{35} \mathrm{~N}_{2} \mathrm{Si}[\mathrm{M}+\mathrm{H}]^{+}$: 367.2564; found: 367.2566 .

\section{S-(Triisopropylsilyl)ethynyl benzothioate (185):}

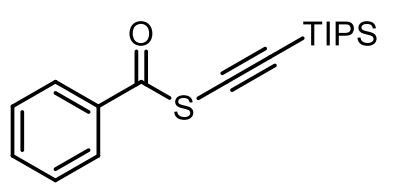

Following GP B, compound $185(95.7 \mathrm{mg}, 0.3 \mathrm{mmol}, 100 \%)$ was obtained from thiobenzoic acid (1 equiv, $41.5 \mathrm{mg}, 35.2 \mu \mathrm{L}, 0.3$ $\mathrm{mmol}$ ), 160d (1 equiv, $162.7 \mathrm{mg}, 0.3 \mathrm{mmol}$ ) and DIPEA (1 equiv, 77.6 $\mathrm{mg}, 54 \mu \mathrm{L}, 0.31 \mathrm{mmol})$ in $\mathrm{CH}_{2} \mathrm{Cl}_{2}(2 \mathrm{~mL})$ after column chromatography (hexanes/EtOAc, 95:5) as a yellow oil. 
${ }^{1} \mathbf{H}$ NMR: $\left(400 \mathrm{MHz}, \mathrm{CDCl}_{3}\right) \delta=7.88(\mathrm{dd}, J=8.5,1.3 \mathrm{~Hz}, 2 \mathrm{H}), 7.67-7.57(\mathrm{~m}, 1 \mathrm{H}), 7.51-7.45$ (m, 2H), 1.15 (d, $J=1.8 \mathrm{~Hz}, 21 \mathrm{H})$ ppm. ${ }^{13} \mathrm{C} \mathrm{NMR:}\left(101 \mathrm{MHz}, \mathrm{CDCl}_{3}\right) \delta=187.6,135.6,134.4$, 129.1, 127.6, 109.6, 86.0, 18.7, 11.5 ppm. IR: (ATR, cm ${ }^{-1}$ ) 2942, 2834, 2105, 1703, 1462, $1448,1257,1202,1177,1072,998,881,857,769,675,637,597,516$. HRMS: calcd. for $\mathrm{C}_{18} \mathrm{H}_{27} \mathrm{OSSi}^{+}[\mathrm{M}+\mathrm{H}]^{+}:$319.1546; found (ESI): 319.1545.

Triisopropyl[(tritylthio)ethynyl]silane (186):<smiles>FC(F)(F)C#CSC(c1ccccc1)(c1ccccc1)c1ccccc1</smiles>

Following GP B, compound 186 (123.7 mg, $0.271 \mathrm{mmol}$, 90\%) was obtained from triphenylmethanethiol (2 equiv, $165.8 \mathrm{mg}, 0.6$ mmol), 160d (1 equiv, $162.7 \mathrm{mg}, 0.3 \mathrm{mmol}$ ) and DIPEA (1 equiv, 77.6 $\mathrm{mg}, 54 \mu \mathrm{L}, 0.31 \mathrm{mmol})$ in $\mathrm{CH}_{2} \mathrm{Cl}_{2}(2 \mathrm{~mL})$. Isolated as colorless crystals after column chromatography (cyclohexane/EtOAc, 50:1).

${ }^{1} \mathbf{H}$ NMR $\left(300 \mathrm{MHz}, \mathrm{CD}_{2} \mathrm{Cl}_{2}\right) \delta=7.37-7.23(\mathrm{~m}, 15 \mathrm{H}), 0.91(\mathrm{~d}, J=1.5 \mathrm{~Hz}, 21 \mathrm{H}) \mathrm{ppm} .{ }^{13} \mathrm{C}$ NMR: $\left(126 \mathrm{MHz}, \mathrm{CD}_{2} \mathrm{Cl}_{2}\right) \delta=144.4,130.3,128.3,127.8,102.8,97.0,71.8,18.9,11.9$ ppm. IR: (ATR, $\mathrm{cm}^{-1}$ ) 3059, 2940, 2863, 2083, 1596, 1492, 1461, 1443, 995, 881, 854, 755, 735, 695, 671, 659, 631, 590. HRMS: calcd. for $\mathrm{C}_{30} \mathrm{H}_{36} \mathrm{NaSSi}^{+}[\mathrm{M}+\mathrm{Na}]^{+}$: 479.2199 ; found (ESI): 479.2198.

\section{2-(Triisopropylsilyl)benzo[d]thiazolo[2,3-b]thiazol-4-ium-3-thiolate (187):}<smiles>[S-]c1c([I-])sc2sc3ccccc3[n+]12</smiles>

(hexanes/EtOAc, 20:1) as colorless crystals.

${ }^{1}$ H NMR: $\left(600 \mathrm{MHz}, \mathrm{CDCl}_{3}\right) \delta=10.99(\mathrm{dd}, J=8.6,1.3 \mathrm{~Hz}, 1 \mathrm{H}), 7.82-7.78(\mathrm{~m}, 1 \mathrm{H}), 7.62$ (ddd, $J$ $=8.5,7.3,1.6 \mathrm{~Hz}, 1 \mathrm{H}), 7.61-7.56(\mathrm{~m}, 1 \mathrm{H}), 1.82(\mathrm{~h}, J=7.5 \mathrm{~Hz}, 3 \mathrm{H}), 1.23(\mathrm{~d}, J=7.5 \mathrm{~Hz}, 18 \mathrm{H})$ ppm. ${ }^{13} \mathrm{C}$ NMR: $\left(126 \mathrm{MHz}, \mathrm{CDCl}_{3}\right) \delta=166.5,160.3,138.4,133.0,127.5,126.5,122.5,119.8$ (d, $J=15.8 \mathrm{~Hz}$ ), 115.7, 19.3, 12.7 ppm. IR: (ATR, cm${ }^{-1}$ ) 3076, 2944, 2865, 1676, 1568, 1462, 1426, 1310, 1234, 1187, 1043, 999, 853, 757, 660, 574. HRMS: calcd. for $\mathrm{C}_{18} \mathrm{H}_{26} \mathrm{NS}_{3} \mathrm{Si}^{+}$ $[\mathrm{M}+\mathrm{H}]^{+}:$: 380.0991; found: 380.0995 . 
<smiles>FC(F)(F)NC(=S)/C=C(\SC(=S)C[In]S)Sc1ncccn1</smiles>

Following the GP B ${ }^{28}$, compound $188(4.6 \mathrm{mg}, 8.5 \mu \mathrm{mol}$, 3\%) was obtained from pyrimidine-2-thiol (1 equiv, $33.65 \mathrm{mg}, 0.3 \mathrm{mmol}$ ), 160d (1 equiv, $162.7 \mathrm{mg}, 0.3 \mathrm{mmol}$ ) and DIPEA (0.095 equiv, $3.6 \mathrm{mg}, 4.9 \mu \mathrm{L}$, $28 \mu \mathrm{mol})$ in $\mathrm{CH}_{2} \mathrm{Cl}_{2}(2 \mathrm{~mL})$ after column chromatography (cyclohexane/EtOAc, 20:1) as brown crystals.

${ }^{1}$ H NMR: $\left(600 \mathrm{MHz} \mathrm{CDCl}_{3}\right) \delta=8.47(\mathrm{~d}, J=4.8 \mathrm{~Hz}, 2 \mathrm{H}), 7.13(\mathrm{~s}, 1 \mathrm{H}), 6.93(\mathrm{t}, J=4.8 \mathrm{~Hz}, 1 \mathrm{H})$, $3.04(\mathrm{~s}, 2 \mathrm{H}), 1.34-1.24(\mathrm{~m}, 3 \mathrm{H}), 1.18-1.12(\mathrm{~m}, 4 \mathrm{H}), 1.10(\mathrm{~d}, J=7.5 \mathrm{~Hz}, 20 \mathrm{H}), 1.02$ (d, J = 7.3 $\mathrm{Hz}, 18 \mathrm{H})$ ppm. ${ }^{13} \mathrm{C}$ NMR: $\left(126 \mathrm{MHz} \mathrm{CDCl}_{3}\right) \delta=232.4,173.6,159.6,157.3,139.1,116.7,41.4$, 19.0, 18.8, 12.5, 11.6 ppm. IR: (ATR, $\mathrm{cm}^{-1}$ ) 2942, 2865, 1561, 1548, 1463, 1380, 1188, 912, 882, 671. HRMS: calcd. for $\mathrm{C}_{26} \mathrm{H}_{49} \mathrm{~N}_{2} \mathrm{~S}_{3} \mathrm{Si}_{2}{ }^{+}[\mathrm{M}+\mathrm{H}]^{+}$: 541.2591; found: 541.2593.

\section{2,4,6-Trimethylbenzoic anhydride (189):}<smiles>Cc1cc(C)c(C(=O)OC(=O)c2c(C)cc(C)cc2C)c(C)c1</smiles>

Following GP B, anhydride 189 was obtained in ca. $36 \%$ yield from 2,4,6-trimethylbenzoic acid (2 equiv, $98.5 \mathrm{mg}, 0.6$ mmol), 160d (1 equiv, $162.7 \mathrm{mg}, 0.3 \mathrm{mmol}$ ) and DIPEA (2 equiv, $78.8 \mathrm{mg}, 106.3 \mu \mathrm{L}, 0.61 \mathrm{mmol})$ in $\mathrm{CH}_{2} \mathrm{Cl}_{2}(2 \mathrm{~mL})$ after column chromatography (pure cyclohexane, then cyclohexane/EtOAc, 200:1) as brown crystals.

${ }^{1} \mathrm{H} \operatorname{NMR}\left(300 \mathrm{MHz}, \mathrm{CDCl}_{3}\right) \delta=6.88(\mathrm{~s}, 4 \mathrm{H}), 2.40(\mathrm{~s}, 12 \mathrm{H}), 2.29(\mathrm{~s}, 6 \mathrm{H}) \mathrm{ppm} .{ }^{29}$

(Z)-2-[\{1-[2-(tert-Butoxycarbonyl)-1-oxo-2,3-dihydro-1H-inden-2-yl]-2-phenylvinyl\}thio]-1ethylpyridin-1-ium tetrabromozincate (190a):<smiles>CC[n+]1ccccc1S/C(=C\c1ccccc1)C1(C(=O)OC(C)(C)C)Cc2ccccc2C1=O</smiles>

To a solution of tert-butyl 1-oxo-2,3-dihydro- $1 H$-indene-2carboxylate (1 equiv, $69.7 \mathrm{mg}, 0.3 \mathrm{mmol}$ ) in dry $\mathrm{CH}_{2} \mathrm{Cl}_{2}(2 \mathrm{~mL})$, DIPEA ( 1 equiv, $77.6 \mathrm{mg}, 54 \mu \mathrm{l}, 0.31 \mathrm{mmol}$ ) was added, and the solution was stirred for $5 \mathrm{~min}$. Afterwards $160 \mathrm{~b}$ ( 0.8 equiv, $103.5 \mathrm{mg}, 239 \mu \mathrm{mol})$ was added. The reaction takes place instantly, and was quenched with sat. aqueous $\mathrm{NH}_{4} \mathrm{Cl}$ solution and extracted with $\mathrm{CH}_{2} \mathrm{Cl}_{2}$. The organic layers were dried over

\footnotetext{
${ }^{28}$ Only catalytic amounts of the base were added.

${ }^{29}$ Analytical data are in agreement with the previously published ones: F. Kazemi, H. Shargi, M. A. Nasseri, Synthesis 2004, 2, 205-207.
} 
anhydrous $\mathrm{Na}_{2} \mathrm{SO}_{4}$, filtered, and all volatiles were removed in vacuo. The residue was carefully washed with EtOAc and purified by column chromatography $\left(\mathrm{CH}_{2} \mathrm{Cl}_{2} / \mathrm{MeOH}, 95: 5\right)$. Compound 190 (137.9 mg, $207 \mu \mathrm{mol}, 87 \%$ ) was obtained as a pale beige oil. ${ }^{30}$

${ }^{1} \mathrm{H}$ NMR: $\left(400 \mathrm{MHz}, \mathrm{CD}_{2} \mathrm{Cl}_{2}\right) \delta=9.91-9.83(\mathrm{~m}, 1 \mathrm{H}), 7.99(\mathrm{t}, J=7.8 \mathrm{~Hz}, 1 \mathrm{H}), 7.90(\mathrm{dd}, J=8.5$, $1.2 \mathrm{~Hz}, 1 \mathrm{H}), 7.79(\mathrm{~d}, J=7.7 \mathrm{~Hz}, 1 \mathrm{H}), 7.75-7.65(\mathrm{~m}, 2 \mathrm{H}), 7.63(\mathrm{~s}, 1 \mathrm{H}), 7.57(\mathrm{~d}, J=7.7 \mathrm{~Hz}, 1 \mathrm{H})$, $7.48(\mathrm{t}, J=7.5 \mathrm{~Hz}, 1 \mathrm{H}), 7.41-7.37(\mathrm{~m}, 2 \mathrm{H}), 7.24(\mathrm{~d}, J=7.1 \mathrm{~Hz}, 3 \mathrm{H}), 4.89(\mathrm{q}, J=7.0 \mathrm{~Hz}, 2 \mathrm{H})$, $3.94(\mathrm{~d}, J=17.5 \mathrm{~Hz}, 1 \mathrm{H}), 3.57(\mathrm{~d}, J=17.5 \mathrm{~Hz}, 1 \mathrm{H}), 1.54(\mathrm{t}, J=7.2 \mathrm{~Hz}, 3 \mathrm{H}), 1.39(\mathrm{~s}, 9 \mathrm{H}) \mathrm{ppm} .{ }^{13} \mathrm{C}$ NMR: $\left(126 \mathrm{MHz}, \mathrm{CD}_{2} \mathrm{Cl}_{2}\right) \delta=198.6,168.9,156.7,152.1,147.9,145.8,143.3,136.7,135.2$, 134.6, 130.2, 129.2, 129.0, 129.0, 127.5, 127.0, 125.9, 125.5, 124.6, 84.7, 69.3, 55.1, 39.7, 28.0, 15.4ppm. IR: (ATR, cm ${ }^{-1}$ ) 1708, 1605, 1463, 1368, 1251, 1147, 839, 754, 695. HRMS: calcd. for $\mathrm{C}_{29} \mathrm{H}_{30} \mathrm{NO}_{3} \mathrm{~S}^{+}\left[\mathrm{M}-1 / 2 \mathrm{ZnBr}_{4}\right]^{+}: 472.1941$; found (ESI): 472.1942 .

\section{(Z)-1-Ethyl-2-[\{1-[2-(methoxycarbonyl)-1-oxo-2,3-dihydro-1H-inden-2-yl]-2-phenylvinyl\}- thio]pyridin-1-ium tetrabromozincate (190b):}

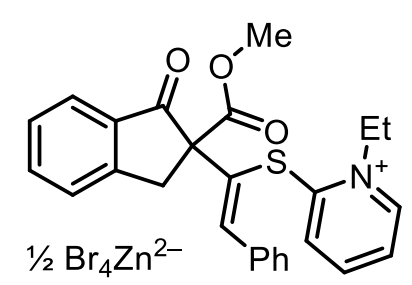

To a solution of methyl 1-oxo-2,3-dihydro- $1 \mathrm{H}$-indene-2-carboxylate (1 equiv, $65.5 \mathrm{mg}, 0.35 \mathrm{mmol}$ ) in dry $\mathrm{CH}_{2} \mathrm{Cl}_{2}(2 \mathrm{~mL}$ ) was added DIPEA (1 equiv, $44.5 \mathrm{mg}, 60 \mu \mathrm{l}, 0.35 \mathrm{mmol}$ ), and the solution was stirred for $5 \mathrm{~min}$ to assure complete deprotonation. Afterwards 160b (1.1 equiv, $167.9 \mathrm{mg}, 0.39 \mathrm{mmol})$ was added. The reaction takes place instantly, and was quenched with sat. aqueous $\mathrm{NH}_{4} \mathrm{Cl}$ solution and extracted with $\mathrm{CH}_{2} \mathrm{Cl}_{2}$. The organic layers were dried over anhydrous $\mathrm{Na}_{2} \mathrm{SO}_{4}$, filtered, and all volatiles were removed in vacuo. The residue was carefully washed with EtOAc, leaving the pure product 190b as a brown oil (155.4 mg, $0.25 \mathrm{mmol}, 71 \%){ }^{31}$

${ }^{1} \mathrm{H}$ NMR: $\left(500 \mathrm{MHz}, \mathrm{CDCl}_{3}\right) \delta=9.65(\mathrm{~d}, J=5.8 \mathrm{~Hz}, 1 \mathrm{H}), 8.00(\mathrm{t}, J=7.6 \mathrm{~Hz}, 1 \mathrm{H}), 7.84(\mathrm{~d}, J=8.2$ $\mathrm{Hz}, 2 \mathrm{H}), 7.80(\mathrm{~d}, J=7.7 \mathrm{~Hz}, 1 \mathrm{H}), 7.69(\mathrm{t}, J=7.1 \mathrm{~Hz}, 1 \mathrm{H}), 7.63(\mathrm{~s}, 1 \mathrm{H}), 7.60(\mathrm{~d}, J=7.7 \mathrm{~Hz}, 1 \mathrm{H})$, $7.45(\mathrm{t}, J=7.5 \mathrm{~Hz}, 1 \mathrm{H}), 7.38(\mathrm{~d}, J=6.6 \mathrm{~Hz}, 2 \mathrm{H}), 7.24-7.20(\mathrm{~m}, 3 \mathrm{H}), 4.88(\mathrm{q}, J=7.2 \mathrm{~Hz}, 2 \mathrm{H})$, $4.01(\mathrm{~d}, J=17.6 \mathrm{~Hz}, 1 \mathrm{H}), 3.72(\mathrm{~s}, 3 \mathrm{H}), 3.60(\mathrm{~d}, J=17.6 \mathrm{~Hz}, 1 \mathrm{H}), 1.54(\mathrm{t}, J=7.1 \mathrm{~Hz}, 3 \mathrm{H}) \mathrm{ppm} .{ }^{13} \mathrm{C}$ NMR: $\left(126 \mathrm{MHz}, \mathrm{CDCl}_{3}\right) \delta=198.1,170.1,154.9,151.9,148.5,146.0,143.1,136.8,134.4$, 133.8, 130.0, 128.9, 128.7 (2C), 127.0, 126.5, 125.4, 125.2, 124.5, 68.1, 55.2, 53.9, 39.3, 15.3 ppm. IR: (ATR, $\mathrm{cm}^{-1}$ ) 3053, 2360, 1607, 1564, 1488, 1461, 1431, 1149, 1113, 770, 753, 689, $517 \mathrm{MS}$ : calcd. for $\mathrm{C}_{26} \mathrm{H}_{24} \mathrm{NO}_{3} \mathrm{~S}^{+}\left[\mathrm{M}-1 / 2 \mathrm{ZnBr}_{4}\right]^{+} 430.1471$; found: 430.1 .

\footnotetext{
${ }^{30}$ 2D-NMR experiments gave the absolute configuration of the product.

${ }^{31}$ 2D-NMR Experiments gave the absolute configuration of the product.
} 


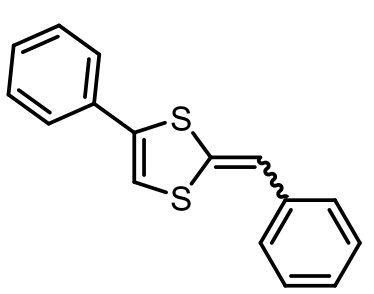

TBAF (1 equiv., $400 \mu \mathrm{L}$ of a $1 \mathrm{~N}$ solution in THF, $0.4 \mathrm{mmol}$ ) was added to a solution of $160 \mathrm{~b}$ (1 equiv., $174.0 \mathrm{mg}, 0.4 \mathrm{mmol}$ ) in $\mathrm{CH}_{2} \mathrm{Cl}_{2}(2.5$ $\mathrm{mL})$. An instant color change to orange-red was observed. After stirring for $10 \mathrm{~min}$, the reaction was quenched with saturated aqueous $\mathrm{NH}_{4} \mathrm{Cl}$ solution $(2.5 \mathrm{~mL})$, and the aqueous phase was extracted three times with $\mathrm{CH}_{2} \mathrm{Cl}_{2}$. The combined organic phases were dried with $\mathrm{Na}_{2} \mathrm{SO}_{4}$, filtered, and the solvent was removed in vacuo. After purification by column chromatography (petrol ether/EtOAc, 50:1 to 20:1), compound 191 (73 mg, 0.27 $\mathrm{mmol}, 68 \%)$ was isolated as a colorless solid, approximately 1:1 (E/Z)-isomeric mixture. ${ }^{32}$

${ }^{1}$ H NMR: $\left(300 \mathrm{MHz}, \mathrm{CDCl}_{3}\right) \delta=7.49-7.26(\mathrm{~m}, 2 \mathrm{H}), 7.21-7.13(\mathrm{~m}, 2 \mathrm{H}), 6.61(\mathrm{~d}, J=1.4 \mathrm{~Hz}$, $1 \mathrm{H}), 6.54(\mathrm{~s}, 1 \mathrm{H}), 6.52(\mathrm{~s}, 1 \mathrm{H}), 6.45(\mathrm{~d}, J=1.5 \mathrm{~Hz}, 1 \mathrm{H})$ ppm. ${ }^{13} \mathrm{C}$ NMR: $\left(126 \mathrm{MHz}, \mathrm{CDCl}_{3}\right) \delta=$ 136.8, 135.5, 135.2, 134.3/134.1, 132.7/132.2, 130.6, 128.9, 128.6, 128.5, 128.4, 126.7, 126.3, 126.3, 125.7, 125.6, 113.7/113.3, 111.9/111.8 ppm. HRMS: calcd. for $\mathrm{C}_{16} \mathrm{H}_{12} \mathrm{~S}_{2}{ }^{+}[\mathrm{M}]^{+}$: 268.0375; found (ESI): 268.0375.

\section{4-Methoxyphenyl 2,2-dideuterio-2-(triisopropylsilyl)ethanedithioate (192):}<smiles>[2H]C([2H])([InH])C(=S)Sc1ccc(OC)cc1</smiles>

To an oven dried schlenk flask the transfer reagent $160 \mathrm{~d}$ (1 equiv, $162.7 \mathrm{mg}, 0.3 \mathrm{mmol}$ ) was added, and the schlenk flask was evacuated and flushed with $\mathrm{N}_{2}$ three times. Dry acetonitrile $(2 \mathrm{~mL})$ was added followed by $S$-deuterio- $p$-methoxythiophenol ( 2 equiv, $84.7 \mathrm{mg}, 74.9 \mu \mathrm{L}, 0.6 \mathrm{mmol}$ ) under stirring. After this, DIPEA (1 equiv, $77.6 \mathrm{mg}, 54$ $\mu \mathrm{l}, 0.31 \mathrm{mmol}$ ) was added and the reaction mixture was stirred at ambient temperature under progress monitoring by TLC. After completion of the reaction, the solvent was removed in vacuo and the product $192(64.9 \mathrm{mg}, 0.182 \mathrm{mmol}, 61 \%)$ was isolated as a yellow oil after separation by column chromatography (cyclohexane/EtOAc, 100:1).

${ }^{1} \mathrm{H}$ NMR: $\left(300 \mathrm{MHz}, \mathrm{CDCl}_{3}\right) \delta=7.28(\mathrm{~d}, J=8.6 \mathrm{~Hz}, 2 \mathrm{H}), 6.98(\mathrm{~d}, J=8.7 \mathrm{~Hz}, 2 \mathrm{H}), 3.85(\mathrm{~s}, 3 \mathrm{H})$, $3.25(\mathrm{~s}, 0.1 \mathrm{H}), 3.22(\mathrm{~s}, 0.27 \mathrm{H}), 1.40-1.24(\mathrm{~m}, 3 \mathrm{H}), 1.15(\mathrm{~d}, J=7.1 \mathrm{~Hz}, 18 \mathrm{H}) \mathrm{ppm} .{ }^{13} \mathrm{C}$ NMR: $\left(101 \mathrm{MHz} \mathrm{CDCl}_{3}\right) \delta=237.9,161.2,136.7,123.4$ (d, $\left.J=2.6 \mathrm{~Hz}\right), 115.1,55.5,18.8,11.6$ (d, $J=$ $1.4 \mathrm{~Hz}$ ) ppm. IR: (ATR, cm ${ }^{-1}$ ) 2940, 2864, 1591, 1494, 1461, 1293, 1250, 1183, 1173, 1068, 1032, 964, 882, 824, 770, 670, 638, 527. HRMS: calcd. for $\mathrm{C}_{18} \mathrm{H}_{29} \mathrm{D}_{2} \mathrm{OS}_{2} \mathrm{Si}^{+}[\mathrm{M}+\mathrm{H}]^{+}:$:357.1706; found (ESI): 357.1703 .

\footnotetext{
${ }^{32}$ Analytical data are in agreement with the previously published ones: (a) U. Timm, U. Merkle, H. Meier, Chem. Ber. 1980, 113, 2519-2529; (b) K. Naka, A. Gelover-Santiago, Y. Chujo, J. Polym. Sci. A 2004, 42, 5872-5876.
} 
Topic: "C-H Activation Utilizing Thioimidazolone-Based Electrophilic Transfer REAGENTS"

\section{Non- AND Low ReActive Substrates}

Substrates containing various directing groups were investigated; the ones performing below our expectations are shown in Scheme 101 with short remarks.<smiles>[R]OCC(=O)c1ccccc1CCC(=O)NC</smiles>

Scheme 101: Investigated non-reactive substrates and substrates with low reactivity (n. R. = no reaction). 
<smiles>CCCn1c([N+](=O)[O-])c(C)c(=S)n1C(C)C</smiles>
Following literature procedure, ${ }^{33}$ acetoin $(20.89 \mathrm{~g}, 20.68 \mathrm{~mL}, 237.1 \mathrm{mmol})$ was added to a stirred solution of diisopropylthiourea $(38.00 \mathrm{~g}, 237.1$ $\mathrm{mmol})$ in 1-hexanol $(225 \mathrm{~mL})$, and the reaction mixture was stirred at 158 ${ }^{\circ} \mathrm{C}$ for $12 \mathrm{~h}$. Subsequently, the solution was cooled down to room temperature, and the solid formed was filtered off and washed with cold ethanol to afford compound 150 (37.20 g, $175.2 \mathrm{mmol}, 73 \%)$ as a white solid. ${ }^{33}$

\section{2-(Bromothio)-1,3-diisopropyl-4,5-dimethyl-1H-imidazol-3-ium bromide (151a):}<smiles>CCCn1c(C)c(C)[n+](C(C)C)c1SBr</smiles>

Following literature procedure, to a stirred solution of compound $\mathbf{1 5 0}$ (20.13 g, $94.79 \mathrm{mmol})$ in dry $\mathrm{CH}_{2} \mathrm{Cl}_{2}(100 \mathrm{~mL})$, bromine $(15.15 \mathrm{~g}, 4.9 \mathrm{~mL}$, $94.79 \mathrm{mmol}$ ) was added at $0{ }^{\circ} \mathrm{C}$, and the reaction mixture was allowed to warm up to rt over a period of $3 \mathrm{~h}$. After removal of all volatiles under vacuum, compound 151a (33.05 g, $88.8 \mathrm{mmol}, 94 \%$ ) was obtained as an orange crystalline solid. ${ }^{33}$

${ }^{1} \mathrm{H}$ NMR: $\left(400 \mathrm{MHz}, \mathrm{CDCl}_{3}\right) \delta=5.64$ (hept, $\left.J=7.1 \mathrm{~Hz}, 2 \mathrm{H}\right), 2.36(\mathrm{~s}, 6 \mathrm{H}), 1.66(\mathrm{~d}, J=7.1 \mathrm{~Hz}$, $12 \mathrm{H})$ ppm. ${ }^{13} \mathrm{C}$ NMR: $\left(101 \mathrm{MHz}, \mathrm{CDCl}_{3}\right) \delta=145.7,128.3,53.6,20.5,10.7 \mathrm{ppm}$.

\section{2-[(3-Ethoxy-3-oxoprop-1-yn-1-yl)thio]-1,3-diisopropyl-4,5-dimethyl-1H-imidazol-3-ium hexafluoroantimonate (59):}<smiles>CCCn1c(C)c(C)[n+](C(C)C)c1SC#CC(=O)OCC</smiles>

Following a literature procedure, ${ }^{33}$ compound $\mathbf{5 9}$ was prepared from dibromide 151a (1.67 g, $4.49 \mathrm{mmol})$, silver acetylide of ethyl propiolate $(0.92 \mathrm{~g}, 4.49 \mathrm{mmol})$ in dry DCM $(20 \mathrm{~mL})$ maintaining $\mathrm{rt}$ with a water bath (note: the reaction is slightly exothermic and may cause DCM to boil). After $5 \mathrm{~min}, \mathrm{NaSbF}_{6}(2.32 \mathrm{~g}, 8.97 \mathrm{mmol}$ ) was added, and the reaction mixture stirred vigorously for another $5 \mathrm{~min}$.

Silver salts were then filtered off and the solvent removed under vacuum. The remaining solid was washed twice with diethyl ether to afford compound $\mathbf{5 9}$ as a pale yellow solid $(2.25$ g, $4.13 \mathrm{mmol}, 92 \%) .^{33}$

\footnotetext{
${ }^{33}$ Analytical data were identical to the previously published ones: G. Talavera, J. Peña, M. Alcarazo, J. Am. Chem. Soc., 2015, 137, 8704-8707.
} 


\section{General Procedure C (GP C) for the synthesis of alkynylthioimidazolium transfer reagents:}

In a Schlenk flask, $n$-BuLi (1.6 M/2.5 $\mathrm{M}$ in hexanes, 1.1 equiv.) was added to a stirred solution of the corresponding terminal alkyne ( 1.1 equiv.) in THF $(10 \mathrm{~mL})$ at $-78{ }^{\circ} \mathrm{C}$. After letting the reaction mixture to warm up to room temperature over $2 \mathrm{~h}$, the formed lithium acetylide solution was re-cooled to $-78^{\circ} \mathrm{C}$ and treated with an ice-cold solution of $\mathrm{ZnBr}_{2}$ (1.1 equiv.) in THF (1 M). The reaction mixture was stirred for an additional $1 \mathrm{~h}$ at this temperature to ensure the formation of the organozinc derivative, then slowly added to a solution of 151 (1 equiv.) in THF at $-78^{\circ} \mathrm{C}$. After 30 minutes at this temperature, the reaction was allowed to warm up to room temperature by removing the cool bath. Upon this, 151 was slowly consumed. Successively an aqueous solution of $\mathrm{NaSbF}_{6}$ (3 equiv.) was added, and the reaction mixture was transferred to a separation funnel. The organic phase was separated, and the aqueous phase was extracted two more times with either EtOAc or DCM. The combined organic phases were dried, and the solvent was removed in vacuo. DCM was added, the solution was filtered through a plug of celite and washed two more times with DCM. The solvent was removed again, and the final product was obtained as a white to offwhite solid, either directly or after crystallizing with $\mathrm{Et}_{2} \mathrm{O}$. In several cases, a final washing with $n$-pentane is necessary to solidify the product. If the anion exchange did not proceed completely, as detected by ESI-MS, it has to be repeated. Further purification is possible by column chromatography $\left(\mathrm{CH}_{2} \mathrm{Cl}_{2}\right.$ to $\mathrm{CH}_{2} \mathrm{Cl}_{2}$ /acetone, 20:1); however, in this case yields dropped.

\section{1,3-Diisopropyl-4,5-dimethyl-2-[(phenylethynyl)thio]-1H-imidazol-3-ium hexafluoroanti- monate (197):}

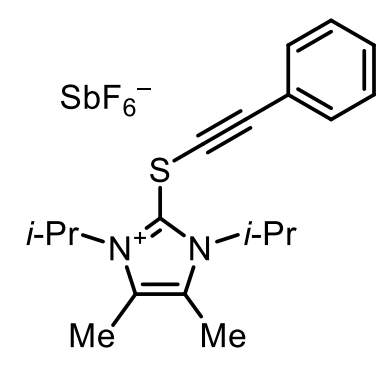

Following GP C, from phenylacetylene $(3.27 \mathrm{~g}, 3.5 \mathrm{~mL}, 32 \mathrm{mmol}), n$ BuLi (1 equiv, $2.5 \mathrm{M}, 12.8 \mathrm{~mL}, 32 \mathrm{mmol}$ ), $\mathrm{ZnBr}_{2}(7.1 \mathrm{~g}, 31.5 \mathrm{mmol}$ ), 151a (11.1 g, $30 \mathrm{mmol})$ and $\mathrm{NaSbF}_{6}(24.84 \mathrm{~g}, 96 \mathrm{mmol})$, compound 197 (15.1 g, $27.5 \mathrm{mmol}, 92 \%$ ) was obtained as a pale white solid. ${ }^{34}$

${ }^{1} \mathrm{H}$ NMR: $\left(300 \mathrm{MHz}, \mathrm{CDCl}_{3}\right) \delta=7.45-7.29(\mathrm{~m}, 5 \mathrm{H}), 5.26$ (hept, $J=6.8$ $\mathrm{Hz}, 3 \mathrm{H}), 2.40(\mathrm{~s}, 6 \mathrm{H}), 1.71(\mathrm{~d}, J=7.1 \mathrm{~Hz}, 12 \mathrm{H}) \mathrm{ppm}$.

\footnotetext{
${ }^{34}$ Analytical data are in agreement with the previously published ones: J. Peña, G. Talavera, B. Waldecker, M. Alcarazo, Chem. Eur. J. 2017, 23, 75-78
} 
1,3-Diisopropyl-2-\{[(4-methoxyphenyl)ethynyl]thio\}-4,5-dimethyl-1H-imidazol-3-ium hexafluoroantimonate (198):

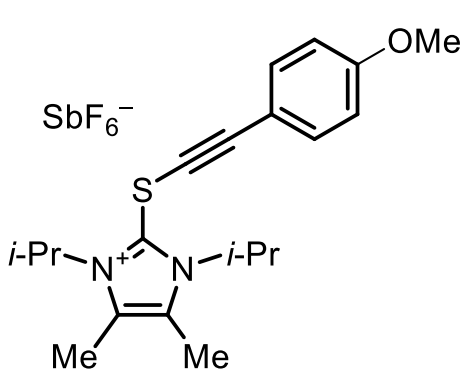

Following GP C, from 1-ethynyl-4-methoxybenzene (519 $\mu \mathrm{L}, 4$ mmol), $n$-BuLi (2.5 M, $1.7 \mathrm{~mL}, 4.2 \mathrm{mmol}$ ), $\mathrm{ZnBr}_{2}$ (945 mg, 4.2 $\mathrm{mmol}$ ), 151a (1.5 g, $4 \mathrm{mmol}$ ) and $\mathrm{NaSbF}_{6}(3.1 \mathrm{~g}, 12 \mathrm{mmol})$, compound 198 ( $2.1 \mathrm{~g}, 3.6 \mathrm{mmol}, 89 \%)$ was obtained as a pale grey powder. ${ }^{35}$

${ }^{1}$ H NMR: $\left(300 \mathrm{MHz} \mathrm{CDCl}_{3}\right) \delta=7.37(\mathrm{~d}, J=9.0 \mathrm{~Hz}, 2 \mathrm{H}), 6.85(\mathrm{~d}, J$ $=8.9 \mathrm{~Hz}, 2 \mathrm{H}$ ), 5.27 (hept, $J=7.6,7.1 \mathrm{~Hz}, 2 \mathrm{H}$ ), $3.81(\mathrm{~s}, 3 \mathrm{H}), 2.41(\mathrm{~s}, 6 \mathrm{H}), 1.72(\mathrm{~d}, J=7.0 \mathrm{~Hz}$, 12H) ppm. ${ }^{13} \mathrm{C}$ NMR: $\left(75 \mathrm{MHz}, \mathrm{CDCl}_{3}\right) \delta=161.4,134.4,131.1,114.5,112.6,96.1,68.0,55.6$, 54.0, 21.3, $10.5 \mathrm{ppm}$.

2-\{[(4-Fluorophenyl)ethynyl]thio\}-1,3-diisopropyl-4,5-dimethyl-1H-imidazol-3-ium hexafluoroantimonate (199):

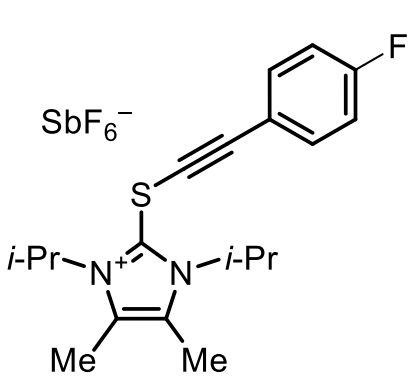

Following GP C, from 1-ethynyl-4-fluorobenzene $(956.2 \mathrm{mg}, 912.4$ $\mu \mathrm{L}, 7.96 \mathrm{mmol}), n$-BuLi (1.6 M, $5.2 \mathrm{~mL}, 8.32 \mathrm{mmol}), \mathrm{ZnBr}_{2}(1.88 \mathrm{~g}$, $8.36 \mathrm{mmol}), 151 \mathrm{a}(2.6676 \mathrm{~g}, 7.168 \mathrm{mmol})$ and $\mathrm{NaSbF}_{6}(5.5639 \mathrm{~g}$, $21.50 \mathrm{mmol}$ ), compound 199 (3.741 g, $6.60 \mathrm{mmol}, 92 \%)$ was obtained as an orange solid.

${ }^{1}$ H NMR: $\left(300 \mathrm{MHz}, \mathrm{CDCl}_{3}\right) \delta=7.42(\mathrm{dd}, J=8.9,5.2 \mathrm{~Hz}, 2 \mathrm{H}), 7.03$ $(\mathrm{t}, J=8.7 \mathrm{~Hz}, 2 \mathrm{H}), 5.37-5.18(\mathrm{~m}, 2 \mathrm{H}), 2.42(\mathrm{~s}, 6 \mathrm{H}), 1.72(\mathrm{~d}, J=7.0 \mathrm{~Hz}, 12 \mathrm{H}) \mathrm{ppm} .{ }^{1} \mathrm{H}$ NMR: $\left(300 \mathrm{MHz}, \mathrm{CD}_{3} \mathrm{CN}\right) \delta=7.51(\mathrm{dd}, J=9.0,5.4 \mathrm{~Hz}, 2 \mathrm{H}), 7.14(\mathrm{t}, J=8.9 \mathrm{~Hz}, 2 \mathrm{H}), 5.26(\mathrm{dt}, J=13.8$, $6.8 \mathrm{~Hz}, 2 \mathrm{H}), 2.37(\mathrm{~s}, 6 \mathrm{H}), 1.65(\mathrm{~d}, J=7.1 \mathrm{~Hz}, 12 \mathrm{H}) \mathrm{ppm} .{ }^{13} \mathrm{C} \mathrm{NMR:}\left(75 \mathrm{MHz}, \mathrm{CDCl}_{3}\right) \delta=165.33$, 161.98, 134.62 (d, $J=8.7 \mathrm{~Hz}$ ), 131.39, 116.92 (d, $J=3.5 \mathrm{~Hz}), 116.29$ (d, $J=22.3 \mathrm{~Hz}$ ), 94.42, 69.87, 54.04, 21.38, 10.72 ppm. ${ }^{19} \mathrm{~F}$ NMR: (300 Mhz, $\mathrm{CDCl}_{3}$ ) $\delta=-107.14$ ppm. IR: (ATR, $\mathrm{cm}^{-1}$ ) 2987, 2941, 2172, 1614, 1598, 1502, 1456, 1410, 1374, 1219, 1159, 1113, 843, 795, 639, 535. HRMS: calcd. for $\mathrm{C}_{19} \mathrm{H}_{24} \mathrm{FN}_{2} \mathrm{~S}^{+}\left[\mathrm{M}-\mathrm{SbF}_{6}\right]^{+}:=331.1636$; found: 331.1639 .

\footnotetext{
${ }^{35}$ Analytical data are in agreement with the previously published ones: J. Peña, G. Talavera, B. Waldecker, M. Alcarazo, Chem. Eur. J. 2017, 23, 75-78
} 
1,3-Diisopropyl-4,5-dimethyl-2-[\{[4-(trifluoromethyl)phenyl]ethynyl\}thio]-1H-imidazol-3ium hexafluoroantimonate (200):

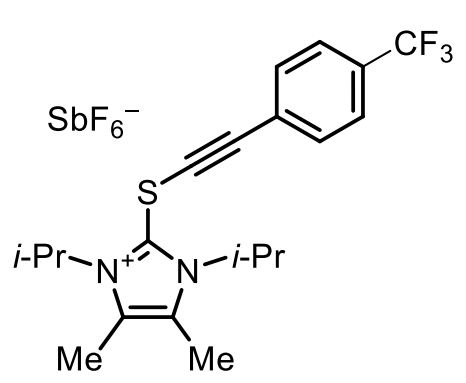

Following GP C, from 1-ethynyl-4-(trifluoromethyl)benzene (2.04 g, $1.96 \mathrm{~mL}, 12 \mathrm{mmol}), n$-BuLi $(2.5 \mathrm{M}, 4.8 \mathrm{~mL}, 12 \mathrm{mmol})$, $\mathrm{ZnBr}_{2}(2.75 \mathrm{~g}, 12 \mathrm{mmol}), 151 \mathrm{a}(3.8 \mathrm{~g}, 10 \mathrm{mmol})$ and $\mathrm{NaSbF}_{6}$ (7.06 g, $27 \mathrm{mmol})$, compound $200(6.16 \mathrm{~g}, 10 \mathrm{mmol}, 99 \%)$ was obtained as a pale orange solid. ${ }^{35}$

2-(Hex-1-yn-1-ylthio)-1,3-diisopropyl-4,5-dimethyl-1H-imidazol-3-ium hexafluoroantimonate (201):

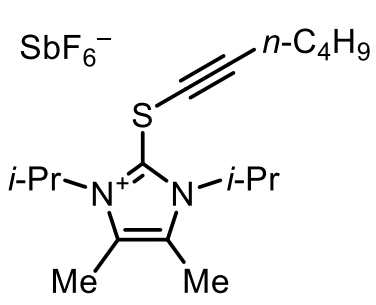

Following GP C, from hex-1-yne (1.2 equiv, $985.7 \mathrm{mg}, 1.38 \mathrm{~mL}, 12$ mmol), $n$-BuLi (1.2 equiv, $2.5 \mathrm{M}, 4.8 \mathrm{~mL}, 12 \mathrm{mmol}$ ), $\mathrm{ZnBr}_{2}$ (1.2 equiv, $2.70 \mathrm{~g}, 12 \mathrm{mmol}$ ), 151a (1 equiv, $3.72 \mathrm{~g}, 10 \mathrm{mmol}$ ) and $\mathrm{NaSbF}_{6}(3$ equiv, $7.76 \mathrm{~g}, 30 \mathrm{mmol}$ ), compound 201 (4.9 g, $9.3 \mathrm{mmol}, 93 \%$ ) was obtained as a pale, off-white solid.

${ }^{1}$ H NMR: $\left(400 \mathrm{MHz}, \mathrm{CDCl}_{3}\right) \delta=5.27-5.12(\mathrm{~m}, 2 \mathrm{H}), 2.39(\mathrm{~s}, 6 \mathrm{H}), 2.29(\mathrm{t}, J=7.1 \mathrm{~Hz}, 2 \mathrm{H}), 1.67$ (d, $J=7.1 \mathrm{~Hz}, 12 \mathrm{H}), 1.53-1.42(\mathrm{~m}, 2 \mathrm{H}), 1.41-1.30(\mathrm{~m}, 2 \mathrm{H}), 0.88(\mathrm{t}, J=7.3 \mathrm{~Hz}, 3 \mathrm{H}) \mathrm{ppm}$. ${ }^{13} \mathrm{C}$ NMR: $\left(101 \mathrm{MHz}, \mathrm{CDCl}_{3}\right) \delta=131.0,98.6,59.6,53.8,30.0,22.0,21.2,19.6,13.5,10.4 \mathrm{ppm}$. ${ }^{19}$ F NMR: $\left(376 \mathrm{MHz}, \mathrm{CDCl}_{3}\right) \delta=-106.97$ to -147.46 (m) ppm. IR: (ATR, $\mathrm{cm}^{-1}$ ) 2959, 2935, $2873,1617,1456,1380,1219,1140,1116,908,750,653$. HRMS: calcd. for $\mathrm{C}_{17} \mathrm{H}_{29} \mathrm{~N}_{2} \mathrm{~S}^{+}[\mathrm{M}-$ $\left.\mathrm{SbF}_{6}\right]^{+}:$293.2046; found: 293.2049 .

\section{1,3-Diisopropyl-4,5-dimethyl-2-\{[(triisopropylsilyl)ethynyl]thio\}-1H-imidazol-3-ium hexaflu-} oroantimonate (202):

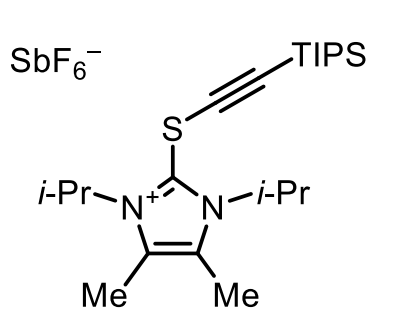

Following GP C, from ethynyltriisopropylsilane (1.2 equiv, $2.19 \mathrm{~g}, 2.7$ $\mathrm{mL}, 12 \mathrm{mmol}$ ), $n$-BuLi (1.2 equiv, $2.5 \mathrm{M}, 4.8 \mathrm{~mL}, 12 \mathrm{mmol}$ ), $\mathrm{ZnBr}_{2}$ (1.1 equiv, $2.5 \mathrm{~g}, 11.1 \mathrm{mmol}), 151 \mathrm{a}\left(3.72 \mathrm{~g}, 10 \mathrm{mmol}\right.$ ) and $\mathrm{NaSbF}_{6}(3$ equiv, $7.762 \mathrm{~g}, 30 \mathrm{mmol}$ ), compound 202 (5.212 g, $8.28 \mathrm{mmol}, 83 \%)$ was obtained as a white solid.

${ }^{1} \mathrm{H}$ NMR: $\left(500 \mathrm{MHz}, \mathrm{CDCl}_{3}\right) \delta=5.21$ (brs, 2H), $2.41(\mathrm{~s}, 6 \mathrm{H}), 1.69(\mathrm{~d}, J=7.1 \mathrm{~Hz}, 12 \mathrm{H}), 1.09-$ $0.99(\mathrm{~m}, 21 \mathrm{H})$ ppm. ${ }^{13} \mathrm{C}$ NMR: $\left(126 \mathrm{MHz}, \mathrm{CDCl}_{3}\right) \delta=131.3,130.2,102.4,84.7,77.2,54.0,21.2$, 18.5, 11.2, 10.5 ppm. IR: (ATR, cm ${ }^{-1}$ ) 2946, 2870, 1459, 1377, 1216, 1113, 881, 838, 653, 590. HRMS: calcd. for $\mathrm{C}_{22} \mathrm{H}_{41} \mathrm{~N}_{2} \mathrm{SSi}^{+}\left[\mathrm{M}-\mathrm{SbF}_{6}\right]^{+}:=393.2754$; found: 393.2750 . 


\section{5-Phenylpyrazolo[1,2-a]cinnolin-4-ium hexafluoroantimonate (203):}

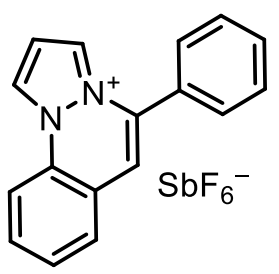

A pressure vial was charged with $\left[\mathrm{RhCp}^{*} \mathrm{Cl}_{2}\right]_{2}(5 \mathrm{~mol} \%, 6.2 \mathrm{mg}, 10 \mu \mathrm{mol})$, $\mathrm{Cu}(\mathrm{OAc})_{2}$ (0.5 equiv., $18.1 \mathrm{mg}, 0.1 \mathrm{mmol}$ ), 1-phenylpyrazol (1 equiv, 28.8 $\mathrm{mg}, 26.4 \mu \mathrm{L}, 0.2 \mathrm{mmol}$ ), 197 (1.1 equiv, $120.6 \mathrm{mg}, 219.5 \mu \mathrm{mol}$ ), and finally $\mathrm{AgSbF}_{6}$ (1.1 equiv., $75.6 \mathrm{mg}, 2.2 \mathrm{mmol}$ ). The vial was sealed and flushed with $3 \mathrm{~N}_{2}$ /Vacuum-Cycles. 1,2-Dichloroethane $(2 \mathrm{~mL})$ was added via syringe, and the reaction was stirred for $20 \mathrm{~h}$ at $80^{\circ} \mathrm{C}$. After letting the reaction mixture cool down to room temperature, the solution was diluted with acetonitrile $(6 \mathrm{~mL})$, and supernatant phase was filtered through a pad of celite. The operation was repeated twice. Subsequently the organic solvents were removed in vacuo. Separation by column chromatography $\left(\mathrm{CH}_{2} \mathrm{Cl}_{2}\right.$ /acetone, 100:1 to $\left.20: 1\right)$ gave the salt 203 as pale yellow crystals (50 $\mathrm{mg}, 103.9 \mu \mathrm{mol}, 52 \%)$.

${ }^{1} \mathrm{H}$ NMR: $\left(400 \mathrm{MHz}, \mathrm{CD}_{3} \mathrm{CN}\right) \delta=9.25(\mathrm{~d}, J=3.2 \mathrm{~Hz}, 1 \mathrm{H}), 8.43(\mathrm{~d}, J=3.2 \mathrm{~Hz}, 1 \mathrm{H}), 8.26(\mathrm{~d}, J=8.4$ $\mathrm{Hz}, 1 \mathrm{H}$ ), 7.94 (dd, $J=7.9,1.4 \mathrm{~Hz}, 1 \mathrm{H}$ ), 7.90 (ddd $J=8.7,7.4,1.5 \mathrm{~Hz}, 1 \mathrm{H}), 7.78$ (td, $J=7.6,1.1$ $\mathrm{Hz}, 1 \mathrm{H}), 7.71(\mathrm{~d}, J=2.7 \mathrm{~Hz}, 5 \mathrm{H}), 7.39-7.34(\mathrm{~m}, 2 \mathrm{H}) \mathrm{ppm} .{ }^{13} \mathrm{C}$ NMR: $(151 \mathrm{MHz}$, DMSO-d 6 ) $\delta=$ 134.0, 131.3, 131.1, 130.1, 129.5, 129.5, 129.4, 129.2, 129.0, 128.7, 128.5, 120.6, 115.6, 114.6, 110.5 ppm. IR: (ATR, cm ${ }^{-1}$ ) 3150, 2359, 1507, 1465, 1437, 1353, 1296, 1118, 860, 768, 648. HRMS: calcd. for $\mathrm{C}_{17} \mathrm{H}_{13} \mathrm{~N}_{2}{ }^{+}\left[\mathrm{M}-\mathrm{SbF}_{6}\right]^{+}:=245.1073$; found: 245.1079 .

\section{2-(2-Benzoylphenyl)-1-phenylethan-1-one (204):}<smiles>O=C(Cc1ccccc1C(=O)c1ccccc1)c1ccccc1</smiles>

diluted with acetonitrile $(6 \mathrm{~mL})$, and supernatant phase was filtered through a pad of celite. The operation was repeated twice. Subsequently the organic solvents were removed in vacuo. $^{36}$

${ }^{1}$ H NMR: $\left(400 \mathrm{MHz}, \mathrm{DMSO}-d_{6}\right) \delta=7.94(\mathrm{dd}, J=8.2,1.1 \mathrm{~Hz}, 2 \mathrm{H}), 7.69(\mathrm{dd}, J=8.2,1.3 \mathrm{~Hz}, 2 \mathrm{H})$, $7.67-7.36(\mathrm{~m}, 10 \mathrm{H}), 4.65(\mathrm{~s}, 2 \mathrm{H}) \mathrm{ppm} .{ }^{13} \mathrm{C}$ NMR: $\left(126 \mathrm{MHz}, \mathrm{DMSO}-d_{6}\right) \delta=197.4,196.8$, $137.9,137.4,136.4,134.9,133.2,133.0,132.5,130.9,129.9,129.7,128.7,128.4,128.0$, $126.3,42.8 \mathrm{ppm}$.

\footnotetext{
${ }^{36}$ Analytical data are in agreement with the previously published ones H. Yoshida, M. Watanabe, J. Ohshita, A. Kunai, Chem. Commun. 2005, 3292-3294.
} 


\section{General Procedure D (GP D) for the alkynylation, cyclization of $\boldsymbol{N}$-methylbenzamides}

A pressure vial was charged with $\left[\mathrm{RhCp}^{*} \mathrm{Cl}_{2}\right]_{2}(2.5 \mathrm{~mol} \%, 4.6 \mathrm{mg}, 7.44 \mu \mathrm{mol}), \mathrm{NaOAc}$ (1 equiv, $24.3 \mathrm{mg}, 0.3 \mathrm{mmol}$ ), substrate, (1 equiv, $0.3 \mathrm{mmol}$ ), reagent 197, 198 or 199 (1.5 equiv, 0.45 mmol) and finally $\mathrm{AgSbF}_{6}$ (1.7 equiv, $175.2 \mathrm{mg}, 0.51 \mathrm{mmol}$ ). The vial was sealed and flushed three times using $\mathrm{N}_{2} /$ Vacuum-Cycles. 1,2-Dichloroethane $(2 \mathrm{~mL}$ ) was added via syringe, and the reaction mixture was stirred at room temperature for $20 \mathrm{~h}$, unless otherwise stated.

For work-up, the reaction mixture was diluted with acetonitrile $(6 \mathrm{~mL})$, and supernatant phase was filtered through a pad of celite. The operation was repeated twice. Subsequently the organic solvents were removed in vacuo.

For hydrolysis, THF $(15 \mathrm{~mL})$ and $\mathrm{HCl}(1 \mathrm{M}, 3 \mathrm{~mL})$ were added to the crude residue, and the reaction mixture was stirred under reflux $\left(69^{\circ} \mathrm{C}\right)$ for $3 \mathrm{~h}$, unless otherwise specified.

Afterwards a solution of saturated aqueous $\mathrm{NaHCO}_{3}$ was added until the aqueous phase showed neutral $\mathrm{pH}$. The aqueous phase was then extracted with EtOAc $(3 \times 20 \mathrm{~mL})$, and the combined organic phases were dried over $\mathrm{Na}_{2} \mathrm{SO}_{4}$. The solvent was removed in vacuo and the final product was obtained after separation by column chromatography (pure DCM, unless otherwise mentioned).

\section{3-Phenyl-1H-isochromen-1-one (Homalicine, 207):}<smiles>O=c1oc(-c2ccccc2)cc2ccccc12</smiles>

Following GP D, from $N$-methylbenzamide (1 equiv, $40.6 \mathrm{mg}, 0.3 \mathrm{mmol}$ ), 197 (1.5 equiv, $247.2 \mathrm{mg}, 0.45 \mathrm{mmol})$, [ $\left[\mathrm{RhCp}^{*} \mathrm{Cl}_{2}\right]_{2}(2.5 \mathrm{~mol} \%, 4.6 \mathrm{mg}$, $7.44 \mu \mathrm{mol}$ ), NaOAc (1 equiv, $24.3 \mathrm{mg}, 0.3 \mathrm{mmol}$ ), and $\mathrm{AgSbF}_{6}$ (1.7 equiv, $175.2 \mathrm{mg}, 0.51 \mathrm{mmol})$, compound $207(46.7 \mathrm{mg}, 210 \mu \mathrm{mol}, 70 \%)$ was obtained as a white solid.

${ }^{1} \mathrm{H}$ NMR: $\left(300 \mathrm{MHz}, \mathrm{CDCl}_{3}\right) \delta=8.32(\mathrm{dd}, J=8.2,0.9 \mathrm{~Hz}, 1 \mathrm{H}), 7.90(\mathrm{dd}, J=8.0,1.7 \mathrm{~Hz}, 2 \mathrm{H}), 7.77$ - $7.69(\mathrm{~m}, 1 \mathrm{H}), 7.55-7.42(\mathrm{~m}, 6 \mathrm{H}), 6.97(\mathrm{~s}, 1 \mathrm{H})$ ppm. ${ }^{13} \mathrm{C}$ NMR: $\left(126 \mathrm{MHz}, \mathrm{CDCl}_{3}\right) \delta=162.3$, 153.7, 137.6, 134.9, 132.1, 130.0, 129.7, 128.9, 128.2, 126.0, 125.3, 120.7, 101.9 ppm. IR: $\left(\right.$ ATR, cm ${ }^{-1}$ ) 2972, 1734, 1635, 1344, 1086, 1045, 878, 769, 693. HRMS: calcd. for $\mathrm{C}_{15} \mathrm{H}_{11} \mathrm{O}_{2}{ }^{+}$ $[\mathrm{M}+\mathrm{H}]^{+}:$223.0757; found: 223.0754 .

\section{6-Methyl-3-phenyl-1H-isochromen-1-one (208):}<smiles>Cc1ccc2c(=O)oc(-c3ccccc3)cc2c1</smiles>

Following GP D, from $N$-methyl-p-toluamide (1 equiv, $44.8 \mathrm{mg}, 0.3$ $\mathrm{mmol}$ ), 197 (1.5 equiv, $247.2 \mathrm{mg}, 0.45 \mathrm{mmol}),\left[\mathrm{RhCp}^{*} \mathrm{Cl}_{2}\right]_{2}(2.5$ mol\%, $4.6 \mathrm{mg}, 0.0075 \mathrm{mmol}$ ), NaOAc (1 equiv, $24.3 \mathrm{mg}, 0.3 \mathrm{mmol}$, and $\mathrm{AgSbF}_{6}$ (1.7 equiv, $175.2 \mathrm{mg}, 0.51 \mathrm{mmol}$ ), compound 208 (48.0 
$\mathrm{mg}, 203 \mu \mathrm{mol}, 68 \%$ ) was obtained as a white crystalline solid.

${ }^{1}$ H NMR: $\left(400 \mathrm{MHz}, \mathrm{CDCl}_{3}\right) \delta=8.19(\mathrm{~d}, J=8.0 \mathrm{~Hz}, 1 \mathrm{H}), 7.87(\mathrm{~d}, J=7.3 \mathrm{~Hz}, 2 \mathrm{H}), 7.45(\mathrm{q}, J=8.9$, $7.8 \mathrm{~Hz}, 3 \mathrm{H}), 7.34-7.27(\mathrm{~m}, 2 \mathrm{H}), 6.89(\mathrm{~s}, 1 \mathrm{H}), 2.48(\mathrm{~s}, 3 \mathrm{H}) \mathrm{ppm} .{ }^{13} \mathrm{C} \mathrm{NMR:}\left(101 \mathrm{MHz}, \mathrm{CDCl}_{3}\right) \delta=$ 162.5, 153.8, 146.1, 137.8, 132.2, 130.0, 129.8, 129.7, 128.9, 126.1, 125.4, 118.3, 101.9, 22.1 ppm. IR: (ATR, $\mathrm{cm}^{-1}$ ): 2360, 2340, 1710, 1063, 1032, 897, 771, 756, 681, 641. HRMS: calcd. for $\mathrm{C}_{16} \mathrm{H}_{13} \mathrm{O}_{2}^{+}[\mathrm{M}+\mathrm{H}]^{+}:$237.0910; found: 237.0914 .

\section{6-Methoxy-3-phenyl-1H-isochromen-1-one (209):}<smiles>COc1ccc2c(=O)oc(-c3ccccc3)cc2c1</smiles>

Following GP $\mathbf{D}$, from 4-methoxy- $N$-methylbenzamide (1 equiv, $49.6 \mathrm{mg}, 0.3 \mathrm{mmol}$ ), 197 (1.5 equiv, $247.2 \mathrm{mg}, 0.45 \mathrm{mmol}$ ), $\left[\mathrm{RhCp}{ }^{*} \mathrm{Cl}_{2}\right]_{2}$ (2.5 mol\%, $\left.4.6 \mathrm{mg}, 0.0075 \mathrm{mmol}\right), \mathrm{NaOAc}$ (0.5 equiv, $12.3 \mathrm{mg}, 0.15 \mathrm{mmol}$ ), and $\mathrm{AgSbF}_{6}$ (1.7 equiv, $175.2 \mathrm{mg}, 0.51$ mmol), compound 209 (44.0 mg, $174.4 \mu \mathrm{mol}, 58 \%$ ) was obtained as a white solid.

${ }^{1} \mathrm{H}$ NMR: $\left(400 \mathrm{MHz}, \mathrm{CDCl}_{3}\right) \delta=8.24(\mathrm{~d}, J=8.8 \mathrm{~Hz}, 1 \mathrm{H}), 7.89(\mathrm{~d}, \mathrm{~J}=7.4 \mathrm{~Hz}, 2 \mathrm{H}), 7.46(\mathrm{q}, J=7.4$ $\mathrm{Hz}, 3 \mathrm{H}), 7.04(\mathrm{~d}, J=8.8 \mathrm{~Hz}, 1 \mathrm{H}), 6.89(\mathrm{~d}, J=6.5 \mathrm{~Hz}, 2 \mathrm{H}), 3.94(\mathrm{~s}, 3 \mathrm{H}) \mathrm{ppm} .{ }^{13} \mathrm{C}$ NMR: $(101 \mathrm{MHz}$, $\left.\mathrm{CDCl}_{3}\right) \delta=164.9,162.2,154.4,140.0,132.2,132.1,130.1,129.0,125.5,116.7,113.9,108.1$, 102.0, 77.2, 55.8 ppm. IR: (ATR, cm ${ }^{-1}$ ) 2926, 1717, 1601, 1489, 1260, 1029, 859, 769, 690, 611. HRMS: calcd. for $\mathrm{C}_{16} \mathrm{H}_{13} \mathrm{O}_{3}{ }^{+}[\mathrm{M}+\mathrm{H}]^{+}:$253.0859; found: 253.0859 .

\section{6-Fluoro-3-phenyl-1H-isochromen-1-one (210):}<smiles>O=c1oc(-c2ccccc2)cc2cc(F)ccc12</smiles>

Following GP D, from 4-fluoro- $N$-methylbenzamide (1 equiv, $45.9 \mathrm{mg}$, $0.3 \mathrm{mmol}$ ), 197 (1.5 equiv, $247.2 \mathrm{mg}, 0.45 \mathrm{mmol}),\left[\mathrm{RhCp}^{*} \mathrm{Cl}_{2}\right]_{2}(2.5$ mol\%, $4.6 \mathrm{mg}, 0.0075 \mathrm{mmol}$ ), NaOAc (1 equiv, $24.3 \mathrm{mg}, 0.3 \mathrm{mmol}$ ), and $\mathrm{AgSbF}_{6}$ (1.7 equiv, $175.2 \mathrm{mg}, 0.51 \mathrm{mmol}$ ), compound 210 (47.4 $\mathrm{mg}, 197 \mu \mathrm{mol}, 66 \%)$ was obtained as a white solid.

${ }^{1} \mathrm{H}$ NMR: $\left(300 \mathrm{MHz}, \mathrm{CDCl}_{3}, 25^{\circ} \mathrm{C}\right) \delta=8.33(\mathrm{dd}, J=8.7,5.6 \mathrm{~Hz}, 1 \mathrm{H}), 7.93-7.84(\mathrm{~m}, 2 \mathrm{H}), 7.54-$ $7.41(\mathrm{~m}, 3 \mathrm{H}), 7.23-7.12(\mathrm{~m}, 2 \mathrm{H}), 6.91(\mathrm{~s}, 1 \mathrm{H}) . \mathrm{ppm} .{ }^{13} \mathrm{C} \mathrm{NMR:}\left(126 \mathrm{MHz}, \mathrm{CDCl}_{3}\right) \delta=166.77$ (d, $J=256.2 \mathrm{~Hz}$ ), 161.36, 154.99, 140.29 (d, $J=10.8 \mathrm{~Hz}), 133.09$ (d, $J=10.4 \mathrm{~Hz}), 131.67,130.44$, 128.97, 125.51, 117.11 (d, $J=2.2 \mathrm{~Hz}), 116.52(\mathrm{~d}, J=23.4 \mathrm{~Hz}), 111.59(\mathrm{~d}, J=22.6 \mathrm{~Hz}), 101.31$ $(\mathrm{d}, J=2.9 \mathrm{~Hz})$ ppm. ${ }^{19} \mathrm{~F}$ NMR: $\left(376 \mathrm{MHz}, \mathrm{CD}_{3} \mathrm{Cl}, 25^{\circ} \mathrm{C}\right) \delta=-101.85(\mathrm{td}, J=8.6,5.7 \mathrm{~Hz}) \mathrm{ppm}$. IR: $\left(\right.$ ATR, cm ${ }^{-1}$ ) 2925, 1717, 1617, 1483, 1453, 1257, 1063, 765, 686. HRMS: calcd. for $\mathrm{C}_{15} \mathrm{H}_{10} \mathrm{FO}_{2}{ }^{+}$ $[\mathrm{M}+\mathrm{H}]^{+}:$241.0659; found: 241.0659 . 
<smiles>O=c1oc(-c2ccccc2)cc2cc(Cl)ccc12</smiles>

Following GP D, from 4-chloro- $N$-methylbenzamide (1 equiv, 50.9 $\mathrm{mg}, 0.3 \mathrm{mmol}$ ), 197 (1.5 equiv, $247.2 \mathrm{mg}, 0.45 \mathrm{mmol}$ ), [RhCp*Cl$]_{2}$ (2.5 mol\%, $4.6 \mathrm{mg}, 0.0075 \mathrm{mmol}$ ), NaOAc (1 equiv, $24.3 \mathrm{mg}, 0.3$ $\mathrm{mmol}$ ), and $\mathrm{AgSbF}_{6}$ (1.7 equiv, $175.2 \mathrm{mg}, 0.51 \mathrm{mmol}$ ), compound 211 (31.2 mg, $122 \mu \mathrm{mol}, 41 \%$ ) was obtained as a white solid.

${ }^{1}$ H NMR: $\left(300 \mathrm{MHz}, \mathrm{CDCl}_{3}\right) \delta=8.24(\mathrm{~d}, J=8.4 \mathrm{~Hz}, 1 \mathrm{H}), 7.90-7.85(\mathrm{~m}, 2 \mathrm{H}), 7.46(\mathrm{~m}, 5 \mathrm{H}), 6.88$ (s, $1 \mathrm{H})$ ppm. ${ }^{13} \mathrm{C}$ NMR: $\left(126 \mathrm{MHz}, \mathrm{CDCl}_{3}\right) \delta=161.5,155.0,141.6,139.0,131.7,131.4,130.5$, 129.0, 128.7, 125.5, 118.9, 100.9 ppm. IR: (ATR, cm ${ }^{-1}$ ) 3106, 3033, 2921, 1710, 1633, 1595, 1446, 1321, 1237, 1059, 893, 771, 755, 679, 641, 559, 532. HRMS: calcd. for $\mathrm{C}_{15} \mathrm{H}_{9} \mathrm{ClO}_{2}{ }^{+}[\mathrm{M}]^{+}$: 256.0291; found (EI): $=256.0293$.

\section{6-Bromo-3-phenyl-1H-isochromen-1-one (212):}<smiles>O=c1oc(-c2ccccc2)cc2cc(Br)ccc12</smiles>

Following GP D, from 4-bromo- $N$-methylbenzamide (0.97 equiv, $64.2 \mathrm{mg}, 0.29 \mathrm{mmol}), 197$ (1.5 equiv, $247.2 \mathrm{mg}, 0.45 \mathrm{mmol}$ ), $\left[\mathrm{RhCp} * \mathrm{Cl}_{2}\right]_{2}$ (2.5 mol\%, $4.6 \mathrm{mg}, 0.0075 \mathrm{mmol}$ ), NaOAc (1 equiv, 24.3 $\mathrm{mg}, 0.3 \mathrm{mmol}$ ), and $\mathrm{AgSbF}_{6}$ (1.7 equiv, $175.2 \mathrm{mg}, 0.51 \mathrm{mmol}$ ), compound 212 (45.2 mg, $150 \mu \mathrm{mol}, 52 \%)$ was obtained as a white

solid.

${ }^{1} \mathbf{H}$ NMR: $\left(400 \mathrm{MHz}, \mathrm{CDCl}_{3}\right) \delta=8.16(\mathrm{dt}, J=8.5,0.6 \mathrm{~Hz}, 1 \mathrm{H}), 7.87(\mathrm{dd}, J=7.9,1.8 \mathrm{~Hz}, 2 \mathrm{H}), 7.67$ $(\mathrm{dd}, J=1.9,0.5 \mathrm{~Hz}, 1 \mathrm{H}), 7.61(\mathrm{dd}, J=8.5,1.9 \mathrm{~Hz}, 1 \mathrm{H}), 7.52-7.44(\mathrm{~m}, 3 \mathrm{H}), 6.87(\mathrm{~d}, J=0.7 \mathrm{~Hz}$, 1H) ppm. ${ }^{13} \mathrm{C}$ NMR: (101 MHz, $\left.\mathrm{CDCl}_{3}\right) \delta=161.8,155.1,139.2,131.8,131.6,131.5,130.6$, 130.5, 129.1, 128.7, 125.6, 119.3, 100.8 ppm. IR: (ATR, cm ${ }^{-1}$ ) 2956, 1772, 1720, 1181, 1064, 1037, 955, 925, 849, 563. HRMS: calcd. for $\mathrm{C}_{15} \mathrm{H}_{9} \mathrm{BrO}_{2}^{+}[\mathrm{M}]^{+}:$299.9786; found (EI): 299.9786.

\section{6-lodo-3-phenyl-1H-isochromen-1-one (213):}<smiles>O=c1oc(-c2ccccc2)cc2cc(I)ccc12</smiles>

Following GP D, from 4-iodo- $N$-methylbenzamide (1 equiv, $78.3 \mathrm{mg}$, $0.3 \mathrm{mmol}), \mathbf{1 9 7}(1.5$ equiv, $247.2 \mathrm{mg}, 0.45 \mathrm{mmol}),\left[\mathrm{RhCp}^{*} \mathrm{Cl}_{2}\right]_{2}(2.5$ mol\%, $4.6 \mathrm{mg}, 0.0075 \mathrm{mmol}$ ), NaOAc (1 equiv, $24.3 \mathrm{mg}, 0.3 \mathrm{mmol}$ ), and $\mathrm{AgSbF}_{6}$ (1.7 equiv, $175.2 \mathrm{mg}, 0.51 \mathrm{mmol}$ ), compound 213 (29.8 $\mathrm{mg}, 85.5 \mu \mathrm{mol}, 29 \%)$ was obtained as pale yellow crystals.

${ }^{1} \mathbf{H}$ NMR: $\left(300 \mathrm{MHz}, \mathrm{CDCl}_{3}\right) \delta=7.99(\mathrm{~d}, J=8.3 \mathrm{~Hz}, 1 \mathrm{H}), 7.93-7.79(\mathrm{~m}, 4 \mathrm{H}), 7.53-7.43(\mathrm{~m}$, $3 \mathrm{H}), 6.85$ (s, 1H) ppm. ${ }^{13} \mathrm{C}$ NMR: $\left(126 \mathrm{MHz}, \mathrm{CDCl}_{3}\right) \delta=161.91,154.84,138.91,137.29,134.86$, 131.71, 130.98, 130.45, 129.00, 125.47, 119.76, 103.35, 100.48 ppm. IR: (ATR, cm ${ }^{-1}$ ) 3417, 3106, 3030, 1698, 1552, 1449, 1313, 1235, 1053, 893, 769, 755, 679, 638, 541, 511. HRMS: calcd. for $\mathrm{C}_{15} \mathrm{H}_{10} \mathrm{IO}_{2}{ }^{+}[\mathrm{M}+\mathrm{H}]^{+}$: 348.9720; found: 348.9727 . 
<smiles>O=c1oc(-c2ccccc2)cc2cc(C(F)(F)F)ccc12</smiles>

Following GP $\mathbf{D}^{37}$, from $N$-methyl-4-(trifluoromethyl)benzamide (1 equiv, $61.1 \mathrm{mg}, 0.3 \mathrm{mmol}$ ), 197 (1.5 equiv, $247.2 \mathrm{mg}, 0.45 \mathrm{mmol}$ ), $\left[\mathrm{RhCp}^{*} \mathrm{Cl}_{2}\right]_{2}(2.5 \mathrm{~mol} \%, 4.6 \mathrm{mg}, 0.0075 \mathrm{mmol})$, NaOAc (1 equiv, $24.3 \mathrm{mg}, 0.3 \mathrm{mmol}$ ), and $\mathrm{AgSbF}_{6}$ (1.7 equiv, $175.2 \mathrm{mg}, 0.51 \mathrm{mmol}$ ), compound 214 (36.1 mg, $124.3 \mu \mathrm{mol}, 41 \%$ ) was obtained as

${ }^{1}$ H NMR: $\left(300 \mathrm{MHz}, \mathrm{CDCl}_{3}\right) \delta=8.43(\mathrm{dq}, J=8.3,0.8 \mathrm{~Hz}, 1 \mathrm{H}), 7.93-7.87(\mathrm{~m}, 2 \mathrm{H}), 7.78(\mathrm{~s}, 1 \mathrm{H})$, 7.71 (ddd, $J=8.2,1.8,0.7 \mathrm{~Hz}, 1 \mathrm{H}), 7.55-7.45(\mathrm{~m}, 3 \mathrm{H}), 7.01(\mathrm{~s}, 1 \mathrm{H})$ ppm. ${ }^{13} \mathrm{C} \mathrm{NMR:}(101 \mathrm{MHz}$, $\left.\mathrm{CDCl}_{3}\right) \delta=161.3,155.3,138.0,136.5\left(\mathrm{q}, J_{C-F}=32.8 \mathrm{~Hz}\right), 131.5,130.8,130.7,129.2,125.6$, $124.4\left(q, J_{C-F}=3.4 \mathrm{~Hz}\right), 123.4\left(q, J_{C-F}=273.2 \mathrm{~Hz}\right), 123.3\left(q, J_{C-F}=3.9 \mathrm{~Hz}\right), 123.0,101.2 \mathrm{ppm} .{ }^{19} \mathrm{~F}$ NMR: $\left(282 \mathrm{MHz}, \mathrm{CDCl}_{3}\right) \delta=-63.47$ ppm. IR: $\left(\mathrm{ATR}, \mathrm{cm}^{-1}\right)$ 2954, 2925, 2853, 2162, 2020, 1714, $1341,1170,1121,1060,908,762$, 686. HRMS: calcd. for $\mathrm{C}_{16} \mathrm{H}_{10} \mathrm{~F}_{3} \mathrm{O}_{2}[\mathrm{M}+\mathrm{H}]^{+}:$:291.0627; found: 291.0627.

\section{Methyl 1-oxo-3-phenyl-1H-isochromene-6-carboxylate (215):}<smiles>CC(=O)c1ccc2c(=O)oc(-c3ccccc3)cc2c1</smiles>

Following GP D, from methyl 4-(methylcarbamoyl)benzoate (1 equiv, $58.0 \mathrm{mg}, 0.3 \mathrm{mmol}$ ), 197 (1.5 equiv, $247.2 \mathrm{mg}, 0.45$ $\mathrm{mmol}),\left[\mathrm{RhCp}^{*} \mathrm{Cl}_{2}\right]_{2}(2.5 \mathrm{~mol} \%, 4.6 \mathrm{mg}, 0.0075 \mathrm{mmol}), \mathrm{NaOAc}$ (0.5 equiv, $24.3 \mathrm{mg}, 0.3 \mathrm{mmol}$ ), and $\mathrm{AgSbF}_{6}$ (1.7 equiv, 175.2 $\mathrm{mg}, 0.51 \mathrm{mmol})$, compound 215 (29.1 mg, 103.8 umol, 35\%) was obtained as bright yellow crystals.

${ }^{1} \mathrm{H}$ NMR: $\left(400 \mathrm{MHz}, \mathrm{CDCl}_{3}\right) \delta=8.37(\mathrm{dt}, J=8.2,0.7 \mathrm{~Hz}, 1 \mathrm{H}), 8.19(\mathrm{dd}, J=1.6,0.5 \mathrm{~Hz}, 1 \mathrm{H}), 8.10$ $(\mathrm{dd}, J=8.3,1.6 \mathrm{~Hz}, 1 \mathrm{H}), 7.91-7.86(\mathrm{~m}, 2 \mathrm{H}), 7.52-7.43(\mathrm{~m}, 3 \mathrm{H}), 7.01(\mathrm{~s}, 1 \mathrm{H}), 3.99(\mathrm{~s}, 3 \mathrm{H})$ ppm. ${ }^{13} \mathrm{C}$ NMR: $\left(101 \mathrm{MHz}, \mathrm{CDCl}_{3}\right) \delta=166.0,161.7,154.6,137.7,135.9,131.8,130.5,130.1$, 129.1, 128.4, 127.7, 125.5, 123.5, 101.7, 52.9 ppm. IR: (ATR, $\mathrm{cm}^{-1}$ ) 3101, 1710, 1633, 1432, $1304,1235,1186,1064,963,905,748,677,641,552,527$. HRMS: calcd. for $\mathrm{C}_{17} \mathrm{H}_{13} \mathrm{O}_{4}{ }^{+}$ $[\mathrm{M}+\mathrm{H}]^{+}:$281.0808; found: 281.0807 .

\section{8-Phenyl-6H-[1,3]dioxolo[4,5-f]isochromen-6-one (216):}<smiles>O=c1oc(-c2ccccc2)cc2c3c(ccc12)OCO3</smiles>

Following GP $\mathbf{D}$, from $N$-Methyl-1,3-benzodioxole-5-carboxamide (1 equiv, $53.8 \mathrm{mg}, 0.3 \mathrm{mmol}$ ), 197 (1.5 equiv, $247.2 \mathrm{mg}, 0.45 \mathrm{mmol}$ ), $\left[\mathrm{RhCp}^{*} \mathrm{Cl}_{2}\right]_{2}(2.5 \mathrm{~mol} \%, 4.6 \mathrm{mg}, 0.0075 \mathrm{mmol}), \mathrm{NaOAc}$ (1 equiv, 24.3 $\mathrm{mg}, 0.3 \mathrm{mmol})$, and $\mathrm{AgSbF}_{6}(1.7$ equiv, $175.2 \mathrm{mg}, 0.51 \mathrm{mmol}$ ), compound $216(42.6 \mathrm{mg}, 160 \mu \mathrm{mol}, 53 \%)$ was obtained as a white

\footnotetext{
${ }^{37}$ Reaction was run at $80{ }^{\circ} \mathrm{C}$
} 
amorphous solid after separation by column chromatography (pure $\mathrm{CH}_{2} \mathrm{Cl}_{2}$ then $\mathrm{CH}_{2} \mathrm{Cl}_{2}$ /EtOAc, 1:1).

${ }^{1}$ H NMR: $\left(300 \mathrm{MHz}, \mathrm{CDCl}_{3}\right) \delta=7.93(\mathrm{dd}, J=8.4,0.7 \mathrm{~Hz}, 1 \mathrm{H}), 7.90-7.83(\mathrm{~m}, 2 \mathrm{H}), 7.50-7.41$ (m, 3H), $7.01-6.92(\mathrm{~m}, 2 \mathrm{H}), 6.18(\mathrm{~s}, 2 \mathrm{H})$ ppm. ${ }^{13} \mathrm{C}$ NMR: $\left(101 \mathrm{MHz}, \mathrm{CDCl}_{3}\right) \delta=161.5,154.0$, 152.3, 141.9, 132.1, 130.2, 128.9, 125.9, 125.4, 120.8, 114.8, 109.3, 102.9, 95.0 ppm. IR: $\left(\right.$ ATR, $\mathrm{cm}^{-1}$ ) 2956, 2883, 1726, 1465, 1287, 1186, 1064, 1037, 955, 928, 851. HRMS: calcd. for $\mathrm{C}_{16} \mathrm{H}_{10} \mathrm{O}_{4}[\mathrm{M}]^{+}: 266.0579$; found (EI): 266.0579 .

\section{7-Methyl-3-phenyl-1H-isochromen-1-one (217):}<smiles>Cc1ccc2cc(-c3ccccc3)oc(=O)c2c1</smiles>

Following GP D, from $N$-methyl- $m$-toluamide (1 equiv, $44.8 \mathrm{mg}, 0.3$ mmol), 197 (1.5 equiv, $247.2 \mathrm{mg}, 0.45 \mathrm{mmol}),\left[\mathrm{RhCp}^{*} \mathrm{Cl}_{2}\right]_{2}(2.5$ $\mathrm{mol} \%, 4.6 \mathrm{mg}, 0.0075 \mathrm{mmol}$ ), NaOAc (1 equiv, $24.3 \mathrm{mg}, 0.3 \mathrm{mmol}$ ), and $\mathrm{AgSbF}_{6}$ (1.7 equiv, $175.2 \mathrm{mg}, 0.51 \mathrm{mmol}$ ), compound 217 was prepared as a white crystalline solid (45.6 mg, $193 \mu \mathrm{mol}, 64 \%)$.

${ }^{1} \mathrm{H}$ NMR: $\left(300 \mathrm{MHz}, \mathrm{CDCl}_{3}\right) \delta=8.11(\mathrm{~s}, 1 \mathrm{H}), 7.86(\mathrm{dd}, J=8.0,1.6 \mathrm{~Hz}, 2 \mathrm{H}), 7.53(\mathrm{dd}, J=8.0,1.7$ $\mathrm{Hz}, 1 \mathrm{H}), 7.50-7.36(\mathrm{~m}, 4 \mathrm{H}), 6.92(\mathrm{~s}, 1 \mathrm{H}) 2.47$ (s, 3H) ppm. ${ }^{13} \mathrm{C} \mathrm{NMR:}\left(126 \mathrm{MHz}, \mathrm{CDCl}_{3}\right) \delta=$ 162.5, 152.8, 138.5, 136.2, 135.1, 132.1, 129.8, 129.4, 128.8, 126.0, 125.2, 120.5, 101.8, 21.6 ppm. IR: (ATR, cm ${ }^{-1}$ ) 2925, 1731, 1633, 1499, 1072, 767, 699, 538. HRMS: calcd. for $\mathrm{C}_{16} \mathrm{H}_{12} \mathrm{O}_{2}$ $[M]^{+}:$236.0837; found: 236.0840 .

7-Methoxy-3-phenyl-1H-isochromen-1-one isochromen-1-one (218b):<smiles>COc1ccc2cc(-c3ccccc3)oc(=O)c2c1</smiles>

(218a) and 5-methoxy-3-phenyl-1H-

Following GP D, from 3-methoxy- $N$ methylbenzamide (1 equiv, $49.6 \mathrm{mg}, 0.3$ mmol), 197 (1.5 equiv, $247.2 \mathrm{mg}, 0.45 \mathrm{mmol}$ ), $\left[\mathrm{RhCp}^{*} \mathrm{Cl}_{2}\right]_{2}(2.5 \mathrm{~mol} \%, 4.6 \mathrm{mg}, 0.0075 \mathrm{mmol})$, $\mathrm{NaOAc}$ ( 1 equiv, $24.3 \mathrm{mg}, 0.3 \mathrm{mmol}$ ), and $\mathrm{AgSbF}_{6}$ (1.7 equiv, $175.2 \mathrm{mg}, 0.51 \mathrm{mmol}$ ), compounds 218a and 218b (46.0 mg, $182.3 \mu \mathrm{mol}$, $61 \%$, ratio $218 a: 218 b=1: 2$ ) were obtained as a white amorphous solid. The isomers were further separated by preparative HPLC. Separation conditions: $4.6 \times 250 \mathrm{~mm}$ Zorbax SB-C18 $3.5 \mu \mathrm{m}, 30 \mathrm{~min}$ isocratic, $1.0 \mathrm{~mL} / \mathrm{min}, \mathrm{CH}_{3} \mathrm{CN} / \mathrm{H}_{2} \mathrm{O}=(50 / 50), 295 \mathrm{~K}^{38}$

\footnotetext{
${ }^{38}$ The analytical data correspond to the previopusly published ones: R. G. Chary, G. R. Reddy, Y. S. S. Ganesh,K. V. Prasad, S. K. P. Chandra, S. Mukherjee, M Pal, RSC Adv. 2013, 3, 9641-9644.
} 
Compound 218a: ${ }^{1} \mathrm{H}$ NMR: $\left(500 \mathrm{MHz}, \mathrm{CDCl}_{3}\right) \delta=7.90-7.85(\mathrm{~m}, 2 \mathrm{H}), 7.74(\mathrm{~d}, \mathrm{~J}=2.7 \mathrm{~Hz}, 1 \mathrm{H})$, $7.46(\mathrm{td}, J=8.5,2.0 \mathrm{~Hz}, 3 \mathrm{H}), 7.43-7.39(\mathrm{~m}, 1 \mathrm{H}), 7.33(\mathrm{dd}, J=8.6,2.7 \mathrm{~Hz}, 1 \mathrm{H}), 6.94(\mathrm{~s}, 1 \mathrm{H})$, 3.93 (s, 3H) ppm. ${ }^{13} \mathrm{C}$ NMR: $\left(126 \mathrm{MHz}, \mathrm{CDCl}_{3}\right) \delta=162.7,159.7,151.9,132.3,131.4,129.7$, 129.0, 127.7, 125.1, 125.0, 121.8, 110.2, 101.8, 56.0 ppm. IR: (ATR, cm ${ }^{-1}$ ) 2924, 1693, 1600, $1501,1450,1284,1245,1070,1028,715$. HRMS: calcd. for $\mathrm{C}_{16} \mathrm{H}_{12} \mathrm{O}_{3}{ }^{+}[\mathrm{M}]^{+}:=252.0786$; found: 252.0778 .

Compound 218b: ${ }^{1} \mathbf{H}$ NMR: $\left(500 \mathrm{MHz}, \mathrm{CDCl}_{3}\right) \delta=7.93-7.89(\mathrm{~m}, 3 \mathrm{H}), 7.47-7.40(\mathrm{~m}, 4 \mathrm{H})$, $7.36(\mathrm{~s}, 1 \mathrm{H}), 7.16(\mathrm{dd}, J=8.1,1.0 \mathrm{~Hz}, 1 \mathrm{H}), 3.98(\mathrm{~s}, 3 \mathrm{H})$ ppm. ${ }^{13} \mathrm{C}$ NMR: $\left(126 \mathrm{MHz}, \mathrm{CDCl}_{3}\right) \delta=$ 162.5, 154.5, 153.0, 132.4, 129.9, 128.9, 128.6, 128.3, 125.4, 121.5, 121.2, 114.5, 96.4, 56.1 ppm. IR: (ATR, $\mathrm{cm}^{-1}$ ) 2926, 1698, 1596, 1493, 1468, 1266, 1054, 752, 716, 689. HRMS: calcd. for $\mathrm{C}_{16} \mathrm{H}_{12} \mathrm{O}_{3}^{+}[\mathrm{M}]^{+}$: 252.0786; found: 252.0785 .

8-Methyl-3-phenyl-1H-isochromen-1-one

(219a) and (Z)-3-benzylidene-7-methylisobenzofuran-1(3H)-one (219b):

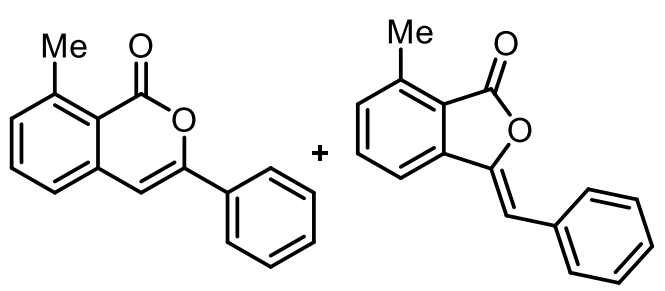

Following GP $\mathrm{D}$, from $\mathrm{N}$-methyl-o-toluamide (1 equiv, $44.8 \mathrm{mg}, 0.3 \mathrm{mmol}$ ), 197 (1.5 equiv, 247.2 $\mathrm{mg}, 0.45 \mathrm{mmol}),\left[\mathrm{RhCp}^{*} \mathrm{Cl}_{2}\right]_{2}(2.5 \mathrm{~mol} \%, 4.6 \mathrm{mg}$, $0.0075 \mathrm{mmol}$ ), $\mathrm{NaOAc}$ (1 equiv, $24.3 \mathrm{mg}, 0.3 \mathrm{mmol}$ ), and $\mathrm{AgSbF}_{6}$ (1.7 equiv, $175.2 \mathrm{mg}, 0.51 \mathrm{mmol}$ ), compounds $\mathbf{2 1 9} \mathrm{a}^{39}$ and $\mathbf{2 1 9 b ^ { 4 0 }}(8.6 \mathrm{mg}, 36.3 \mu \mathrm{mol}$, $12 \%$; isomeric mixture, $1: 1.2)$ were obtained as a white solid. No further separation was performed.

${ }^{1} \mathrm{H}$ NMR: $\left(300 \mathrm{MHz}, \mathrm{CDCl}_{3}\right) \delta=7.87(\mathrm{tt}, J=8.2,1.5 \mathrm{~Hz}, 4 \mathrm{H}), 7.62-7.27(\mathrm{~m}, 12 \mathrm{H}), 6.90(\mathrm{~s}, 1 \mathrm{H})$ (219a), $6.39(\mathrm{~s}, 1 \mathrm{H})(\mathbf{2 1 9 b}), 2.86(\mathrm{~s}, 3 \mathrm{H})$ (219a), $2.72(\mathrm{~s}, 3 \mathrm{H})$ (219b) ppm.

8-Methoxy-3-phenyl-1H-isochromen-1-one

(220a) and (Z)-3-benzylidene-7methoxyisobenzofuran-1(3H)-one (220b):<smiles>COc1cccc2c1C(=O)OC2=Cc1ccccc1</smiles>

Column chromatography $\left(\mathrm{CH}_{2} \mathrm{Cl}_{2}\right.$, then toluene:ethyl acetate, 100:0 to 20:1) of the residue obtained from 2-methoxy- $N$-methylbenzamide (1 equiv, $49.5 \mathrm{mg}$, $0.3 \mathrm{mmol}$ ), 197 (1.5 equiv, $247.2 \mathrm{mg}, 0.45 \mathrm{mmol}$ ), $\left[\mathrm{RhCp}^{*} \mathrm{Cl}_{2}\right]_{2}(2.5 \mathrm{~mol} \%, 4.6 \mathrm{mg}, 0.0075 \mathrm{mmol}), \mathrm{NaOAc}$ (1 equiv, $24.3 \mathrm{mg}, 0.3 \mathrm{mmol}$ ), and $\mathrm{AgSbF}_{6}$ (1.7 equiv, $175.2 \mathrm{mg}, 0.51 \mathrm{mmol}$ ) following GP $\mathbf{D}$,

\footnotetext{
${ }^{39}$ Analytical data are in agreement with the previously published ones: Y.-F. Liang, L. Yang, T. Rogge, L. Ackermann, Chem.Eur.J. 2018, 24, 16548-16552.

${ }^{40}$ Analytical data are in agreement with the previously published ones: Y. Liu, Y. Yang, Y. Shi, X. Wang, L. Zhang, Y. Cheng, J. You, Organometallics 2016, 35, 1350-1353
} 
afforded compounds 220a (28.1 mg, $111 \mu \mathrm{mol}, 37 \%)$ and 220b (20.0 mg, $79 \mu \mathrm{mol}, 26 \%)$ as white crystalline solids.

Compound 220a: ${ }^{1} \mathrm{H}$ NMR: $\left(600 \mathrm{MHz}, \mathrm{CDCl}_{3}\right) \delta=7.89-7.87(\mathrm{~m}, 2 \mathrm{H}), 7.62(\mathrm{dd}, J=8.3,7.7 \mathrm{~Hz}$, $1 \mathrm{H}), 7.47-7.40(\mathrm{~m}, 3 \mathrm{H}), 7.03(\mathrm{dd}, J=7.8,0.9 \mathrm{~Hz}, 1 \mathrm{H}), 6.94(\mathrm{dd}, J=8.4,1.0 \mathrm{~Hz}, 1 \mathrm{H}), 6.85(\mathrm{~s}$, $1 \mathrm{H}), 4.02(\mathrm{~s}, 3 \mathrm{H}) \mathrm{ppm} .{ }^{13} \mathrm{C}$ NMR: $\left(126 \mathrm{MHz} \mathrm{CDCl}_{3}\right) \delta=161.7,159.0,154.0,140.5,135.8$, 132.0, 130.0, 128.8, 125.4, 118.2, 110.0, 109.4, 101.9, 56.5 ppm. IR: (ATR, $\left.\mathrm{cm}^{-1}\right)$ : 2925, 1734, 1568, 1478, 1277, 1109, 996, 769, 690. HRMS: calcd. for $\mathrm{C}_{16} \mathrm{H}_{13} \mathrm{O}_{3}{ }^{+}[\mathrm{M}+\mathrm{H}]^{+}:$: 253.0859; found: 253.0854 .

Compound 220b: ${ }^{1} \mathrm{H}$ NMR: $\left(600 \mathrm{MHz}, \mathrm{CDCl}_{3}\right) \delta=7.85-7.84(\mathrm{~m}, 2 \mathrm{H}), 7.65(\mathrm{t}, J=7.9 \mathrm{~Hz}, 1 \mathrm{H})$, 7.40 (dd, $J=8.4,7.1 \mathrm{~Hz}, 2 \mathrm{H}), 7.33-7.29(\mathrm{~m}, 2 \mathrm{H}), 6.95(\mathrm{~d}, J=8.2 \mathrm{~Hz}, 1 \mathrm{H}), 6.39(\mathrm{~s}, 1 \mathrm{H}), 4.03(\mathrm{~s}$, $3 \mathrm{H}) \mathrm{ppm} .{ }^{13} \mathrm{C}$ NMR: $\left(126 \mathrm{MHz}, \mathrm{CDCl}_{3}\right) \delta=165.1,158.6,144.4,143.1,136.6,133.3,130.2$, 128.8, 128.4, 111.7, 111.3, 111.1, 107.1, 56.3 ppm. IR: (ATR, cm ${ }^{-1}$ ) 2363, 2338, 1775, 1605, 1489, 1271, 1042, 977, 805, 690. HRMS: calcd. for $\mathrm{C}_{16} \mathrm{H}_{12} \mathrm{O}_{3}{ }^{+}[\mathrm{M}+\mathrm{H}]^{+}$: 253,0859; found: 253.0859 .

3-Phenyl-1H-benzo[g]isochromen-1-one (221a) and 2-phenyl-4H-benzo[f]isochromen-4one (221b):<smiles>O=c1oc(-c2ccccc2)cc2c(=O)oc(-c3ccccc3)cc12</smiles>

The residue obtained from $\mathrm{N}$-methyl-2naphthamide, (1 equiv, $55.6 \mathrm{mg}, 0.3 \mathrm{mmol}$ ), 197 (1.5 equiv, $247.2 \mathrm{mg}, 0.45 \mathrm{mmol}$ ), $\left[\mathrm{RhCp}^{*} \mathrm{Cl}_{2}\right]_{2} \quad(2.5 \mathrm{~mol} \%, 4.6 \mathrm{mg}, 0.0075$ $\mathrm{mmol}$ ), NaOAc (1 equiv, $24.3 \mathrm{mg}, 0.3 \mathrm{mmol}$ ), and $\mathrm{AgSbF}_{6}$ (1.7 equiv, $175.2 \mathrm{mg}, 0.51 \mathrm{mmol}$ ) according to $\mathrm{GP} \mathbf{D}$, was stirred with $3 \mathrm{~mL}$ of methanol and filtered off, affording analytically pure compound 221a (44.1 mg, $162 \mu \mathrm{mol}$, $54 \%)$ as orange crystals. The mother liquor was evaporated in vacuo and purified by column chromatography $\left(\mathrm{CH}_{2} \mathrm{Cl}_{2}\right)$ furnishing enriched compounds $221 \mathrm{a}$ (4.1 $\mathrm{mg}, 5 \%$; 4.5:1 isomeric mixture of 221a:221b) and 221b (10.3 mg, $38 \mu \mathrm{mol}, 13 \% ; 1: 4.5$ isomeric mixture of 221a:221b), the latter as a white crystalline solid. ${ }^{41}$

Compound 221a: ${ }^{1} \mathrm{H}$ NMR: $\left(300 \mathrm{MHz}, \mathrm{CDCl}_{3}\right) \delta=8.93(\mathrm{~s}, 1 \mathrm{H}), 8.02(\mathrm{~d}, J=8.3 \mathrm{~Hz}, 1 \mathrm{H}), 7.91(\mathrm{~d}$, $J=5.8 \mathrm{~Hz}, 4 \mathrm{H}), 7.64(\mathrm{t}, J=7.5 \mathrm{~Hz}, 1 \mathrm{H}), 7.59-7.37(\mathrm{~m}, 4 \mathrm{H}), 7.07(\mathrm{~s}, 1 \mathrm{H}) \mathrm{ppm} .{ }^{13} \mathrm{C}$ NMR: $(126$ $\left.\mathrm{MHz}, \mathrm{CDCl}_{3}\right) \delta=162.6,152.1,136.7,132.5,132.4,132.3,132.1,129.9,129.5,128.9,127.8$, 126.8, 125.3, 124.4, 119.1, 102.1 ppm. IR: (ATR, cm ${ }^{-1}$ ) 2921, 1717, 1628, 1446, 1256, 1183, 1056, 896, 755, 736, 682, 622. HRMS: calcd. for $\mathrm{C}_{19} \mathrm{H}_{12} \mathrm{O}_{2}{ }^{+}[\mathrm{M}]^{+}:$: 272.0837; found: 272.0827.

\footnotetext{
${ }^{41}$ The analytical data were identical to the previously reported ones: Z.-Y. Ge, X.-D. Fei, T. Tang, Y.-M. Zhu, J.-K. Shen, J. Org. Chem. 2012, 77, 5736-5743.
} 
Compound 221b: ${ }^{1} \mathrm{H}$ NMR: $\left(300 \mathrm{MHz}, \mathrm{CDCl}_{3}\right) \delta=8.48-8.43(\mathrm{~m}, 1 \mathrm{H}), 8.27(\mathrm{dd}, J=8.7,0.7 \mathrm{~Hz}$, $1 \mathrm{H}), 8.05-7.93(\mathrm{~m}, 3 \mathrm{H}), 7.90(\mathrm{dq}, J=8.7,0.4 \mathrm{~Hz}, 1 \mathrm{H}), 7.79-7.68(\mathrm{~m}, 3 \mathrm{H}), 7.58-7.45(\mathrm{~m}, 3 \mathrm{H})$ ppm. ${ }^{13} \mathrm{C}$ NMR: $\left(126 \mathrm{MHz}, \mathrm{CDCl}_{3}\right) \delta=162.7,155.1,136.9,136.2,132.3,130.3,129.5,129.1$, 129.0, 128.6, 128.0, 127.4, 125.6, 124.5, 124.1, 117.8, 97.7 ppm. IR: (ATR, $\mathrm{cm}^{-1}$ ) 2925, 1732, 1632, 1217, 1059, 763, 688, 594. HRMS: calcd. for $\mathrm{C}_{19} \mathrm{H}_{12} \mathrm{O}_{3}{ }^{+}[\mathrm{M}]^{+}:$:272.0837; found: 272.0828 .

\section{3-(4-Methoxyphenyl)-1H-isochromen-1-one (222):}<smiles>COc1ccc(-c2cc3ccccc3c(=O)o2)cc1</smiles>

Following GP D, from $N$-methylbenzamide (1 equiv, $40.6 \mathrm{mg}, 0.3$ $\mathrm{mmol}), 198$ (1.5 equiv, $260.7 \mathrm{mg}, 0.45 \mathrm{mmol}),\left[\mathrm{RhCp}^{*} \mathrm{Cl}_{2}\right]_{2}(2.5$ mol\%, $4.6 \mathrm{mg}, 0.0075 \mathrm{mmol}$ ), NaOAc (0.5 equiv, $12.3 \mathrm{mg}, 0.15$ $\mathrm{mmol}$ ), and $\mathrm{AgSbF}_{6}$ (1.7 equiv, $175.2 \mathrm{mg}, 0.51 \mathrm{mmol}$ ), compound 222 (55.3 mg, $219 \mu \mathrm{mol}, 73 \%$ ) was obtained as white crystals.

${ }^{1} \mathrm{H}$ NMR: $\left(600 \mathrm{MHz}, \mathrm{CDCl}_{3}\right) \delta=8.27(\mathrm{dd}, J=8.4,1.2 \mathrm{~Hz}, 1 \mathrm{H}), 7.83-7.79(\mathrm{~m}, 2 \mathrm{H}), 7.68(\mathrm{td}, J=$ 7.5, $1.3 \mathrm{~Hz}, 1 \mathrm{H}), 7.47-7.42(\mathrm{~m}, 2 \mathrm{H}), 6.98-6.94(\mathrm{~m}, 2 \mathrm{H}), 6.81(\mathrm{~s}, 1 \mathrm{H}), 3.85(\mathrm{~s}, 3 \mathrm{H}) \mathrm{ppm} .{ }^{13} \mathrm{C}$ NMR: $\left(126 \mathrm{MHz}, \mathrm{CDCl}_{3}\right) \delta=162.4,161.1,153.7,137.9,134.8,129.6,127.7,126.9,125.7$, 124.6, 120.2, 114.3, 100.3, 55.6 ppm. IR: (ATR, cm ${ }^{-1}$ ) 2932, 1731, 1633, 1602, 1513, 1257,1178, 1029, 825, 689, 533. HRMS: calcd. for $\mathrm{C}_{16} \mathrm{H}_{13} \mathrm{O}_{3}{ }^{+},[\mathrm{M}+\mathrm{H}]^{+}:=253.0859$; found: 253.0860 .

\section{3,6-Diphenyl-1H-isochromen-1-one (223):}<smiles>O=c1oc(-c2ccccc2)cc2cc(-c3ccccc3)ccc12</smiles>

Following GP D, from $\mathrm{N}$-methyl-4-phenylbenzamide (1 equiv, $63.4 \mathrm{mg}, 0.3 \mathrm{mmol}$ ), 197 (1.5 equiv, $247.2 \mathrm{mg}, 0.45 \mathrm{mmol}$ ), $\left[\mathrm{RhCp} * \mathrm{Cl}_{2}\right]_{2}(2.5 \mathrm{~mol} \%, 4.6 \mathrm{mg}, 0.0075 \mathrm{mmol}), \mathrm{NaOAc}$ (1 equiv, $24.3 \mathrm{mg}, 0.3 \mathrm{mmol}$ ), and $\mathrm{AgSbF}_{6}(1.7$ equiv, $175.2 \mathrm{mg}, 0.51$ mmol) compound 223 (40.6 mg, $136 \mu \mathrm{mol}, 45 \%$ ) was obtained after column chromatography as bright green crystals.

${ }^{1} \mathbf{H}$ NMR: $\left(300 \mathrm{MHz}, \mathrm{CDCl}_{3}\right) \delta=8.37(\mathrm{dt}, J=8.2,0.7 \mathrm{~Hz}, 1 \mathrm{H}), 7.98-7.88(\mathrm{~m}, 2 \mathrm{H}), 7.77-7.65$ $(\mathrm{m}, 5 \mathrm{H}), 7.57-7.40(\mathrm{~m}, 7 \mathrm{H}), 7.02(\mathrm{~s}, 1 \mathrm{H})$ ppm. ${ }^{13} \mathrm{C} \mathrm{NMR:}\left(126 \mathrm{MHz}, \mathrm{CDCl}_{3}\right) \delta=162.3,154.1$, $147.8,139.5,138.1,132.1,130.4,130.1,129.2,128.9,128.8,127.5,127.3,125.4,124.2$, 119.4, 102.1 ppm. IR: (ATR, cm ${ }^{-1}$ ) 3057, 2919, 2847, 1707, 1606, 1446, 1066, 1026, 890, 752, 688, 516. HRMS: calcd. for $\mathrm{C}_{21} \mathrm{H}_{15} \mathrm{O}_{2}{ }^{+}[\mathrm{M}+\mathrm{H}]^{+}:$299.1067; found: 299.1070 . 


\section{8-Fluoro-3-phenyl-1H-isochromen-1-one (224):}<smiles>O=c1oc(-c2ccccc2)cc2cccc(F)c12</smiles>

Following GP D, from 2-fluoro- $N$-methylbenzamide (1 equiv, $45.9 \mathrm{mg}$, $0.3 \mathrm{mmol}$ ), 197 (1.5 equiv, $247.2 \mathrm{mg}, 0.45 \mathrm{mmol}),\left[\mathrm{RhCp}^{*} \mathrm{Cl}_{2}\right]_{2}(2.5$ mol\%, $4.6 \mathrm{mg}, 0.0075 \mathrm{mmol}$ ), NaOAc (1 equiv, $24.3 \mathrm{mg}, 0.3 \mathrm{mmol}$ ), and $\mathrm{AgSbF}_{6}(1.7$ equiv, $175.2 \mathrm{mg}, 0.51 \mathrm{mmol}$ ) compound 224 (36.2 mg, 151 $\mu \mathrm{mol}, 50 \%$ ) was obtained as a white solid.

${ }^{1} \mathrm{H}$ NMR: $\left(400 \mathrm{MHz}, \mathrm{CDCl}_{3}\right) \delta=7.90-7.84(\mathrm{~m}, 2 \mathrm{H}), 7.67(\mathrm{td}, J=8.1,4.9 \mathrm{~Hz}, 1 \mathrm{H}), 7.51-7.42$ (m, 3H), $7.29-7.26(\mathrm{~m}, 1 \mathrm{H}), 7.14$ (ddd, $J=10.6,8.2,1.0 \mathrm{~Hz}, 1 \mathrm{H}), 6.92(\mathrm{~d}, J=2.3 \mathrm{~Hz}, 1 \mathrm{H}$ ) ppm. ${ }^{13}$ C NMR: $\left(101 \mathrm{MHz}, \mathrm{CDCl}_{3}\right) \delta=163.0(\mathrm{~d}, J=266.9 \mathrm{~Hz}), 157.89(\mathrm{~d}, J=5.5 \mathrm{~Hz}), 154.72,140.23$, $136.32(\mathrm{~d}, J=10.1 \mathrm{~Hz}), 131.60,130.48,129.01,125.52,121.92(\mathrm{~d}, J=4.4 \mathrm{~Hz}), 115.35$ (d, $J=$ $21.2 \mathrm{~Hz}$ ), 109.50 (d, $J=7.3 \mathrm{~Hz}), 101.25$ (d, $J=3.1 \mathrm{~Hz})$ ppm. ${ }^{19} \mathrm{~F}$ NMR: $\left(376 \mathrm{MHz}, \mathrm{CDCl}_{3}\right) \delta=-$ 107.16 (ddd, $J=10.7,5.0,2.3 \mathrm{~Hz}$ ). IR: (ATR, cm ${ }^{-1}$ ) 3107, 3028, 2922, 1714, 1638, 1568, 1472, $1325,1228,1072,1053,998,904,857,799,756,683,650,549$. HRMS: calcd. for $\mathrm{C}_{15} \mathrm{H}_{10} \mathrm{FO}_{2}{ }^{+}$ $[\mathrm{M}+\mathrm{H}]^{+}:$241.0659; found: $=241.0662$.

\section{3-Phenyl-1H-pyrano[3,4-b]benzofuran-1-one (225):}

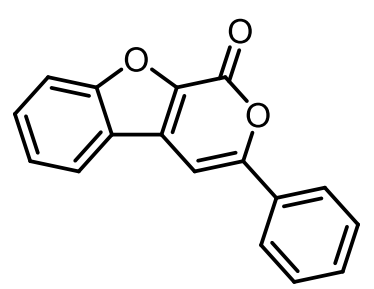

Following GP D, from $N$-methylbenzofuran-2-carboxamide (1 equiv, $52.4 \mathrm{mg}, 299.1 \mu \mathrm{mol}$ ), 197 (1.5 equiv, $247.2 \mathrm{mg}, 0.45 \mathrm{mmol}$ ), $\left[\mathrm{RhCp} * \mathrm{Cl}_{2}\right]_{2}(2.5 \mathrm{~mol} \%, 4.6 \mathrm{mg}, 0.0075 \mathrm{mmol}), \mathrm{NaOAc}$ (1 equiv, 24.3 $\mathrm{mg}, 0.3 \mathrm{mmol})$, and $\mathrm{AgSbF}_{6}(1.7$ equiv, $175.2 \mathrm{mg}, 0.51 \mathrm{mmol})$, compound $225(7.4 \mathrm{mg}, 28.2 \mu \mathrm{mol}, 9 \%)$ was obtained as off white crystals.

${ }^{1} \mathrm{H}$ NMR: $\left(300 \mathrm{MHz}, \mathrm{CDCl}_{3}\right) \delta=7.97-7.88(\mathrm{~m}, 3 \mathrm{H}), 7.71(\mathrm{dt}, J=8.5,1.0 \mathrm{~Hz}, 1 \mathrm{H}), 7.64(\mathrm{ddd}, J=$ 8.5, 7.0, $1.3 \mathrm{~Hz}, 1 \mathrm{H}), 7.56-7.43(\mathrm{~m}, 4 \mathrm{H}), 7.29(\mathrm{~s}, 1 \mathrm{H})$ ppm. ${ }^{13} \mathrm{C}$ NMR: $\left(101 \mathrm{MHz}, \mathrm{CDCl}_{3}\right) \delta=$ $157.8,157.2,154.4,138.6,132.0,131.9,130.4,130.4,129.1,125.7,124.5,122.7,122.3$, 113.4, 95.9 ppm. IR: (ATR, $\mathrm{cm}^{-1}$ ) 2926, 1795, 1744, 1682, 1617, 1462, 1448, 1291, 1231, 1069, 755, 695. HRMS: calcd. for $\mathrm{C}_{17} \mathrm{H}_{10} \mathrm{O}_{3}{ }^{+}[\mathrm{M}]^{+}:$: 262.06299; found: 262.0626 .

\section{6-Fluoro-3-(4-fluorophenyl)-1H-isochromen-1-one (226):}

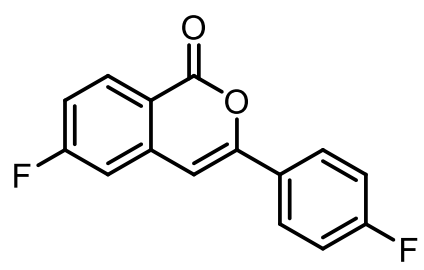

Following GP D, from 4-fluoro- $N$-methylbenzamide (1 equiv, 46.0 $\mathrm{mg}, 0.3 \mathrm{mmol}$ ), 199 (1.5 equiv, $255.3 \mathrm{mg}, 0.45 \mathrm{mmol}$ ), $\left[\mathrm{RhCp}^{*} \mathrm{Cl}_{2}\right]_{2}$ (2.5 mol\%, $4.6 \mathrm{mg}, 0.0075 \mathrm{mmol}$ ), NaOAc (1 equiv, $24.3 \mathrm{mg}, 0.3$ $\mathrm{mmol}$ ), and $\mathrm{AgSbF}_{6}$ (1.7 equiv, $175.2 \mathrm{mg}, 0.51 \mathrm{mmol}$ ), compound 226 (50.0 mg, $194 \mu \mathrm{mol}, 65 \%$ ) was obtained as a white solid.

${ }^{1} \mathrm{H}$ NMR: $\left(300 \mathrm{MHz}, \mathrm{CDCl}_{3}\right) \delta=8.32(\mathrm{ddt}, J=8.8,5.6,0.6 \mathrm{~Hz}, 1 \mathrm{H}), 7.90-7.83(\mathrm{~m}, 2 \mathrm{H}), 7.25-$ $7.08(\mathrm{~m}, 4 \mathrm{H}), 6.84(\mathrm{q}, J=0.5 \mathrm{~Hz}, 1 \mathrm{H}) \mathrm{ppm} .{ }^{13} \mathrm{C} \mathrm{NMR:}\left(126 \mathrm{MHz}, \mathrm{CDCl}_{3}\right) \delta=166.42(\mathrm{~d}, J=352.8$ 
Hz), 164.40 (d, $J=347.8 \mathrm{~Hz}), 161.2,154.1,140.2(\mathrm{~d}, J=10.8 \mathrm{~Hz}), 133.1(\mathrm{~d}, J=10.4 \mathrm{~Hz}), 127.9$ (d, $J=3.3 \mathrm{~Hz}$ ), 127.6 (d, $J=8.5 \mathrm{~Hz}$ ), 117.0 (d, J=2.2 Hz), 116.6 (d, J = $23.2 \mathrm{~Hz}$ ), 116.2 (d, $J=$ $22.1 \mathrm{~Hz}), 111.6(\mathrm{~d}, J=22.6 \mathrm{~Hz}), 101.1 \mathrm{ppm} .{ }^{19} \mathrm{~F}$ NMR: $\left(282 \mathrm{MHz}, \mathrm{CDCl}_{3}\right) \delta=-101.66$ (td, $J=$ 8.5, $5.5 \mathrm{~Hz}$ ), $-109.51(\mathrm{tt}, J=8.2,5.1 \mathrm{~Hz})$ ppm. IR: $\left(\right.$ ATR, $\mathrm{cm}^{-1}$ ) 2954, 2926, 2853, 1720, 1603, 1511, 1238, 1060, 960, 849, 772. HRMS: calcd. for $\mathrm{C}_{15} \mathrm{H}_{9} \mathrm{~F}_{2} \mathrm{O}_{2}[\mathrm{M}+\mathrm{H}]^{+}:$259.0565; found:=259.0570.

\section{N-Methyl-1-oxo-3-phenyl-1H-isochromene-7-carboxamide (227):}<smiles>CNC(=O)c1ccc2cc(-c3ccccc3)oc(=O)c2c1</smiles>

Following GP $\mathbf{D}^{42}$, from $N^{1}, N^{3}$-dimethylisophthalamide (1 equiv, $57.7 \mathrm{mg}, 0.3 \mathrm{mmol}$ ), 197 (1 equiv, $164.8 \mathrm{mg}, 0.3 \mathrm{mmol}$ ), $\left[\mathrm{RhCp} * \mathrm{Cl}_{2}\right]_{2}(2.5 \mathrm{~mol} \%, 4.6 \mathrm{mg}, 0.0075 \mathrm{mmol}), \mathrm{NaOAc}$ (1 equiv, $24.3 \mathrm{mg}, 0.3 \mathrm{mmol}$ ), and $\mathrm{AgSbF}_{6}(1.1$ equiv, $113.4 \mathrm{mg}, 0.33$ mmol), compound 227 (61.7 mg, $221 \mu \mathrm{mol}, 74 \%)$ was obtained as colorless crystals after separation by column chromatography (toluene/EtOAc, 1/1).

${ }^{1} \mathrm{H}$ NMR: $\left(300 \mathrm{MHz}, \mathrm{CDCl}_{3}\right) \delta=8.57(\mathrm{~d}, J=1.7 \mathrm{~Hz}, 1 \mathrm{H}), 8.29(\mathrm{dd}, J=8.2,1.9 \mathrm{~Hz}, 1 \mathrm{H}), 7.93-$ $7.87(\mathrm{~m}, 2 \mathrm{H}), 7.59(\mathrm{~d}, J=8.2 \mathrm{~Hz}, 1 \mathrm{H}), 7.48(\mathrm{~m}, 3 \mathrm{H}), 7.01(\mathrm{~s}, 1 \mathrm{H}), 6.40(\mathrm{br} \mathrm{s}, 1 \mathrm{H}), 3.07(\mathrm{~d}, J=4.9$ $\mathrm{Hz}, 3 \mathrm{H})$ ppm. ${ }^{13} \mathrm{C}$ NMR: $\left(126 \mathrm{MHz}, \mathrm{CDCl}_{3}\right) \delta=167.2,162.5,155.5,140.9,134.5,132.9,131.3$, 130.0, 129.5, 128.6, 127.7, 126.2, 121.3, 102.6, 26.9 ppm. IR: (ATR, cm ${ }^{-1}$ ) 3292, 1715, 1634, $1551,1497,1315,1229,1125,1065,875,761,679,642,566$. HRMS: calcd. for $\mathrm{C}_{17} \mathrm{H}_{14} \mathrm{NO}_{3}{ }^{+}$ $[\mathrm{M}+\mathrm{H}]^{+}:$280.0968; found: $=280.0970$.

\section{3-Phenyl-7-(trifluoromethyl)-1H-isochromen-1-one (228):}<smiles>O=c1oc(-c2ccccc2)cc2ccc(C(F)(F)F)cc12</smiles>

$268 \mu \mathrm{mol}, 89 \%)$.

Following GP D, from $N$-methyl-3-(trifluoromethyl)benzamide (1 equiv, $60.9 \mathrm{mg}, 0.3 \mathrm{mmol}$ ), 197 (1.5 equiv, $247.2 \mathrm{mg}, 0.45 \mathrm{mmol}$ ), $\left[\mathrm{RhCp} * \mathrm{Cl}_{2}\right]_{2}$ (2.5 mol\%, $\left.4.6 \mathrm{mg}, 0.0075 \mathrm{mmol}\right), \mathrm{NaOAc}$ (1 equiv, $24.3 \mathrm{mg}, 0.3 \mathrm{mmol}$ ), and $\mathrm{AgSbF}_{6}$ (1.7 equiv, $175.2 \mathrm{mg}, 0.51 \mathrm{mmol}$ ), compound 228 was obtained as a white amorphous solid $(77.8 \mathrm{mg}$,

${ }^{1} \mathrm{H}$ NMR: $\left(300 \mathrm{MHz}, \mathrm{CDCl}_{3}\right) \delta=8.59(\mathrm{~s}, 1 \mathrm{H}), 7.98-7.87(\mathrm{~m}, 3 \mathrm{H}), 7.63(\mathrm{~d}, J=8.3 \mathrm{~Hz}, 1 \mathrm{H}), 7.49$ (dd, $J=5.1,2.0 \mathrm{~Hz}, 3 \mathrm{H}), 7.01(\mathrm{~s}, 1 \mathrm{H}) \mathrm{ppm} .{ }^{13} \mathrm{C} \mathrm{NMR:}\left(101 \mathrm{MHz}, \mathrm{CDCl}_{3}\right) \delta=161.3,155.9,140.5$, 131.5, $131.3\left(\mathrm{q}, J_{C-F}=3.4 \mathrm{~Hz}\right), 130.9,130.3\left(\mathrm{~d}, J_{C-F}=33.6 \mathrm{~Hz}\right), 129.2,127.4\left(\mathrm{q}, J_{C-F}=4.1 \mathrm{~Hz}\right)$, 126.9, 125.7, $123.6\left(\mathrm{~d}, J_{C-F}=272.2 \mathrm{~Hz}\right), 120.7,101.0$ ppm. ${ }^{19} \mathrm{~F}$ NMR: $\left(282 \mathrm{MHz}, \mathrm{CDCl}_{3}\right) \delta=-$ 62.71 ppm. IR: (ATR, cm ${ }^{-1}$ ) 2926, 1740, 1625, 1316, 1174, 1129, 1071, 846, 765, 698. HRMS: calcd. for $\mathrm{C}_{16} \mathrm{H}_{10} \mathrm{~F}_{3} \mathrm{O}_{2}{ }^{+}[\mathrm{M}+\mathrm{H}]^{+}=291.0627$; found $=291.0625$.

\footnotetext{
421 equiv of reagent 197 was used instead of 1.5 .
} 
(Z)-N-(6-Methoxy-3-phenyl-1H-isochromen-1-ylidene)methanaminium hexafluoroantimonate (229):<smiles>CN=c1oc(-c2ccccc2)cc2cc(OC)ccc12</smiles>

A schlenk flask was charged with $\left[\mathrm{RhCp}^{*} \mathrm{Cl}_{2}\right]_{2}$ (1 equiv, $20 \mathrm{mg}, 32.3$ $\mu \mathrm{mol}$ ), NaOAc (1 equiv, $5.3 \mathrm{mg}, 64.6 \mu \mathrm{mol}$ ), 4-methoxy- $N$ methylbenzamide (1 equiv, $10.69 \mathrm{mg}, 64.7 \mu \mathrm{mol}$ ), 197 (1 equiv, $35.7 \mathrm{mg}, 65 \mu \mathrm{mol}$ ) and $\mathrm{AgSbF}_{6}$ (1 equiv, $\left.22.2 \mathrm{mg}, 64.6 \mu \mathrm{mol}\right)$. The flask was flushed three times with $\mathrm{N}_{2} /$ Vacuum-Cycles. Then 1,2dichloroethane $(2 \mathrm{~mL})$ was slowly added via syringe, and the solution was allowed to stay for $24 \mathrm{~h}$ at ambient temperature. Afterwards, dry $\mathrm{Et}_{2} \mathrm{O}(3 \mathrm{~mL})$ was carefully layered over the reaction mixture, and the solution was left for an additional $24 \mathrm{~h}$. Colorless crystals of compound $\mathbf{2 2 9}$ formed at the interphase between 1,2-DCE and $\mathrm{Et}_{2} \mathrm{O}$ and were collected for characterization.

${ }^{1} \mathrm{H}$ NMR: $\left(500 \mathrm{MHz}, \mathrm{CD}_{3} \mathrm{CN}\right) \delta=9.18(\mathrm{~s}, 1 \mathrm{H}), 8.10(\mathrm{~d}, J=9.1 \mathrm{~Hz}, 1 \mathrm{H}), 8.00-7.93(\mathrm{~m}, 2 \mathrm{H}), 7.63$ - $7.58(\mathrm{~m}, 3 \mathrm{H}), 7.57(\mathrm{~s}, 1 \mathrm{H}), 7.35(\mathrm{dd}, J=9.1,2.5 \mathrm{~Hz}, 1 \mathrm{H}), 7.29(\mathrm{~d}, J=2.5 \mathrm{~Hz}, 1 \mathrm{H}), 4.02(\mathrm{~s}, 3 \mathrm{H})$, 3.38 (s, 3H) ppm. ${ }^{13} \mathrm{C}$ NMR: $\left(126 \mathrm{MHz}, \mathrm{CD}_{3} \mathrm{CN}\right) \delta=167.9,165.1,153.2,139.9,132.3,130.8$, 130.4, 129.0, 126.4, 120.9, 110.2, 108.6, 106.0, 57.3, 29.7 ppm. ${ }^{19} \mathrm{~F}$ NMR: (471 MHz, CD ${ }_{3} \mathrm{CN}$ ) $\delta=-124.0$ (sext, $J_{\mathrm{F}-121 \mathrm{Sb}}=1932.5 \mathrm{~Hz}$ ), -124.8 (oct, $\left.J_{\mathrm{F}-123 \mathrm{Sb}}=1049.6 \mathrm{~Hz}\right) \mathrm{ppm}$. IR: $\left(\right.$ ATR, $\mathrm{cm}^{-1}$ ) 3360, 1663, 1604, 1565, 1492, 1445, 1377, 1257, 1201, 1129, 1020, 878, 824, 772, 761, 650, 623, 565. HRMS: calcd. for $\mathrm{C}_{17} \mathrm{H}_{16} \mathrm{NO}_{2}{ }^{+}[\mathrm{M}]^{+}:$: 266.1176; found: 266.1180 .

\section{N-Methyl-2-(phenylethynyl)benzamide (234):}

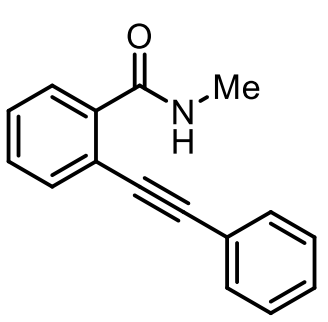

Following a modified literature procedure ${ }^{43}, \mathbf{2 3 4}$ was prepared from 2iodo- $N$-methylbenzamide (783 $\mathrm{mg}, 3 \mathrm{mmol})$, phenylacetylene $(395 \mu \mathrm{L}$, $368 \mathrm{mg}, 3.6 \mathrm{mmol})$ in $\mathrm{Et}_{3} \mathrm{~N}(12 \mathrm{~mL}), \mathrm{PdCl}_{2}\left(\mathrm{PPh}_{3}\right)_{2}(42 \mathrm{mg}, 0.06 \mathrm{mmol}, 2$ mol\%) and Cul (5.7 $\mathrm{mg}, 0.03 \mathrm{mmol}, 1 \mathrm{~mol} \%)$. The reaction mixture was stirred at $55{ }^{\circ} \mathrm{C}$, and full consumption of the starting material was observed after $30 \mathrm{~min}$ by TLC. The reaction was allowed to cool to room temperature. Water $(30 \mathrm{~mL})$ and ethyl acetate $(40 \mathrm{~mL})$ were added, the aqueous phase was extracted EtOAc $(3 \times 20 \mathrm{~mL})$, the combined organic phases were washed with brine $(50 \mathrm{~mL})$, the organic phase was dried over $\mathrm{Na}_{2} \mathrm{SO}_{4}$ and all volatiles were removed in vacuo. Separation by column chromatography (cyclohexane/ethyl acetate, 2:1 to 1:1) gave the product 234 (629 mg, $2.67 \mathrm{mmol}, 89 \%$ ) as a white crystalline solid.

${ }^{1} \mathrm{H}$ NMR: $\left(300 \mathrm{MHz}, \mathrm{CDCl}_{3}\right) \delta=8.09-8.01(\mathrm{~m}, 1 \mathrm{H}), 7.63-7.56(\mathrm{~m}, 1 \mathrm{H}), 7.55-7.49(\mathrm{~m}, 2 \mathrm{H})$, $7.47-7.36(\mathrm{~m}, 5 \mathrm{H}), 7.31$ (br s, $1 \mathrm{H}), 3.08(\mathrm{~d}, J=4.9 \mathrm{~Hz}, 3 \mathrm{H})$ ppm. ${ }^{13} \mathrm{C} \mathrm{NMR:}\left(126 \mathrm{MHz}^{\mathrm{CDCl}} \mathrm{CD}_{3} \delta\right.$ $=167.2$ (enriched), 136.0, 133.3, 131.6, 130.5, 130.0, 129.2, 129.0, 128.7, 122.3, 119.6, 95.5, $87.8,27.0 \mathrm{ppm}$.

\footnotetext{
${ }^{43}$ Analytical data are in agreement with the previously published ones: C. Schlemmer, L. Andernach, D. Schollmeyer, B. F. Straub, T. Opatz, J. Org. Chem. 2012, 77, 10118-10124.
} 
Bis(acetonitrile)\{[(1,3-diisopropyl-4,5-dimethyl-1H-imidazol-3-ium-2-yl]thio\}(pentamethylcyclopentadienyl)rhodium(I) bis(hexafluoroantimonate) (236a):<smiles>CC#[N+][Pb](Sc1n(C(C)C)c(C)c(C)[n+]1[P-]C)(C(C)C)C(C)(C)C(C)C</smiles>

A Schlenk flask was charged with $N$-methyl-p-toluamide (1 equiv, $14.9 \mathrm{mg}, 0.1 \mathrm{mmol}$ ), $\mathrm{NaOAc}$ (1 equiv, $8.2 \mathrm{mg}, 0.1$ mmol) and $\operatorname{RhCp} *\left(\mathrm{MeCN}_{3}\left(\mathrm{SbF}_{6}\right)_{2}\right.$ (1 equiv, $83.3 \mathrm{mg}, 0.1$ $\mathrm{mmol})$ in 1,2-DCE $(2 \mathrm{~mL})$, and the reaction mixture was stirred for $1 \mathrm{~h}$. Afterwards stirring was stopped and subsequently a solution of 197 (1 equiv, $54.9 \mathrm{mg}, 0.1$ $\mathrm{mmol})$ in 1,2-DCE $(1 \mathrm{~mL})$ was slowly layered over the reaction mixture. After $24 \mathrm{~h}$, dry $\mathrm{Et}_{2} \mathrm{O}(3 \mathrm{~mL})$ was carefully layered over the reaction mixture and the solution was let stand for an additional $24 \mathrm{~h}$. Red crystals of the product 236a formed and were collected for analysis. ${ }^{44}$

${ }^{1} \mathrm{H}$ NMR: $\left(500 \mathrm{MHz}, \mathrm{CD}_{3} \mathrm{CN}\right) \delta=5.29(\mathrm{p}, J=7.1 \mathrm{~Hz}, 1 \mathrm{H}), 3.81(\mathrm{~s}, 1 \mathrm{H}), 2.31(\mathrm{~s}, 3 \mathrm{H}), 1.96(\mathrm{~s}, 4 \mathrm{H})$, $1.71(\mathrm{~s}, 8 \mathrm{H}), 1.45$ (d, $J=7.2 \mathrm{~Hz}, 6 \mathrm{H}) \mathrm{ppm} .{ }^{13} \mathrm{C}$ NMR: $\left(126 \mathrm{MHz}, \mathrm{CD}_{3} \mathrm{CN}\right) \delta=147.9,128.4,101.2$, 101.1, 52.86, 45.51, 21.06, 10.77, 9.48 ppm. ${ }^{19} \mathrm{~F}$ NMR: $\left(471 \mathrm{MHz}, \mathrm{CD}_{3} \mathrm{CN}\right) \delta=-123.93\left(\mathrm{~h}, J_{F-}\right.$ $121 \mathrm{Sb}=1609.8 \mathrm{~Hz}$ ), -123.9 (oct, $J_{\mathrm{F}-\mathrm{Sb} 123}=1050.7 \mathrm{~Hz}$ ) ppm. IR: $\left(\right.$ ATR, $\mathrm{cm}^{-1}$ ) 1402, 1020, 655. HRMS: calcd. for $\mathrm{C}_{25} \mathrm{H}_{41} \mathrm{~N}_{4} \mathrm{RhS}^{2+}\left[\mathrm{M}-2 \mathrm{SbF}_{6}\right]^{2+}$ : 266.1; found: 263.2 .

Bis\{[(1,3-diisopropyl-4,5-dimethyl-1H-imidazol-3-ium-2-yl]thio\}(pentamethylcyclopentadienyl)rhodium(I) bis(hexafluoroantimonate) (236b):

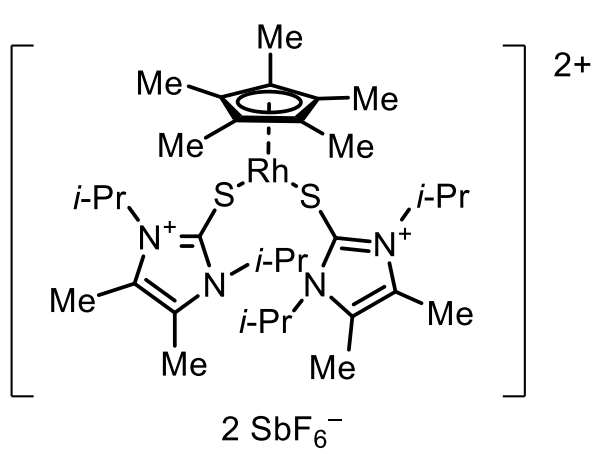

A schlenk flask was charged with $\left[\mathrm{RhCp}^{*} \mathrm{Cl}_{2}\right]_{2}$ (1 equiv, $20 \mathrm{mg}, 32.3 \mu \mathrm{mol}$ ), NaOAc (1 equiv, $5.3 \mathrm{mg}, 6.46 \mu \mathrm{mol}$ ), 4-methoxy- $N$-methylbenzamide (1 equiv, $10.69 \mathrm{mg}$, $6.47 \mu \mathrm{mol}$ ), 197 (1 equiv, $35.7 \mathrm{mg}, 6.5 \mu \mathrm{mol}$ ), and $\mathrm{AgSbF}_{6}$ (1 equiv, $22.2 \mathrm{mg}, 6.46 \mu \mathrm{mol}$ ). The flask was flushed three times with $\mathrm{N}_{2} /$ Vaccuum-Cycles. Then 1,2Dichloroethane $(2 \mathrm{~mL})$ was slowly added via syringe, and the solution was allowed to stay for $24 \mathrm{~h}$. Afterwards, dry $\mathrm{Et}_{2} \mathrm{O}(3 \mathrm{~mL})$ was carefully layered over the reaction mixture, and the solution was stayed for another $24 \mathrm{~h}$. Black crystals of $236 \mathrm{~b}$ formed at the bottom of the flask and were-collected for analysis. ${ }^{45}$

${ }^{1} \mathrm{H}$ NMR: $\left(500 \mathrm{MHz}, \mathrm{CD}_{3} \mathrm{CN}\right) \delta=2.31(\mathrm{~s}, 6 \mathrm{H}), 1.71(\mathrm{~s}, 15 \mathrm{H}), 1.45(\mathrm{~d}, J=7.2 \mathrm{~Hz}, 12 \mathrm{H}) \mathrm{ppm}$.

HRMS: calcd. for $\mathrm{C}_{32} \mathrm{H}_{55} \mathrm{~N}_{4} \mathrm{RhS}_{2}\left[\mathrm{M}-2 \mathrm{SbF}_{6}\right]^{+}: 622.2912$; found: 622.2996.

\footnotetext{
${ }^{44}$ No final yield was determined.

${ }^{45}$ No final yield was determined.
} 


\section{3-[(1,3-Diisopropyl-4,5-dimethyl-1H-imidazol-3-ium-2-yl)thio]-2,4-diphenylcinnolin-2-ium hexafluoroantimonate (238):}<smiles>CCCn1c(SCc2c(-c3ccccc3)c3ccccc3n[n+]2-c2ccccc2)c(C)c(C)[n+]1C(C)(F)F</smiles>

A pressure vial was charged with $\left[\mathrm{RhCp}^{*} \mathrm{Cl}_{2}\right]_{2}$ (2.5 mol\%, 26.4 $\mathrm{mg}, 4.27 \mu \mathrm{mmol}), \mathrm{NaOAc}(1 \mathrm{eq}, 139.9 \mathrm{mg}, 1.71 \mathrm{mmol}$ ), azobenzene ( 1 equiv, $311.6 \mathrm{mg}, 1.71 \mathrm{mmol}$ ), 197 ( 1.5 equiv, 1.4 g, $2.55 \mathrm{mmol}$ ), and finally $\mathrm{AgSbF}_{6}$ (1.6 equiv, $937.4 \mathrm{mg}, 2.73$ $\mathrm{mmol})$. The vial was sealed and flushed three times with $\mathrm{N}_{2}$ /Vacuum-Cycles. 1,2-Dichloroethane (2 $\mathrm{mL}$ ) was added via syringe, and the reaction mixture was stirred at $80^{\circ} \mathrm{C}$ for $20 \mathrm{~h}$. After cooling down to room temperature, the the reaction mixture was diluted with acetonitrile $(6 \mathrm{~mL})$, and supernatant phase was filtered through a pad of celite. The operation was repeated twice. Subsequently the organic solvents were removed in vacuo. The residue was carefully washed with $\mathrm{CHCl}_{3}(5 \times 2 \mathrm{~mL})$ to give the pure product as a dark yellow solid (1.4 $\mathrm{g}, 1.45 \mathrm{mmol}, 85 \%)$.

${ }^{1}{ }_{\mathrm{H}}$ NMR: $\left(300 \mathrm{MHz}, \mathrm{CD}_{3} \mathrm{CN}\right) \delta=8.76-8.68(\mathrm{~m}, 1 \mathrm{H}), 8.45$ (ddd, $\left.J=8.6,6.9,1.2 \mathrm{~Hz}, 1 \mathrm{H}\right), 8.36$ (ddd, $J=8.2,6.9,1.3 \mathrm{~Hz}, 1 \mathrm{H}), 7.98-7.85(\mathrm{~m}, 5 \mathrm{H}), 7.82-7.66(\mathrm{~m}, 3 \mathrm{H}), 7.62(\mathrm{~d}, J=8.7 \mathrm{~Hz}, 1 \mathrm{H})$, $7.42-7.33(\mathrm{~m}, 2 \mathrm{H}), 4.32$ (hept, $J=6.9 \mathrm{~Hz}, 2 \mathrm{H}), 2.21(\mathrm{~s}, 6 \mathrm{H}), 1.19(\mathrm{~d}, J=6.9 \mathrm{~Hz}, 12 \mathrm{H}) \mathrm{ppm}$.

${ }^{13} \mathrm{C}$ NMR: $\left(101 \mathrm{MHz}, \mathrm{CD}_{3} \mathrm{CN}\right) \delta=150.9,148.7,145.9,144.8,143.0,139.0,134.5,133.8,132.7$, 132.3, 132.1, 131.9, 131.1, 130.8, 129.6, 129.9, 129.2, 127.0, 126.4, 55.9, 21.6, 11.2 ppm. IR: $\left(\right.$ ATR, $\mathrm{cm}^{-1}$ ) 2363, 2161, 1698, 1558, 1507, 1048, 659. HRMS: calcd. for $\mathrm{C}_{31} \mathrm{H}_{34} \mathrm{~N}_{4} \mathrm{~S}^{2+}[\mathrm{M}-$ $\left.2 \mathrm{SbF}_{6}\right]^{2+}: 494.2493$; found: 494.2499 .

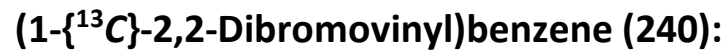

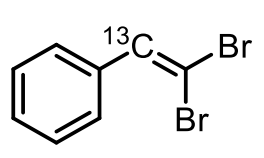

To a solution of $\mathrm{CBr}_{4}$ (2 equiv, $9.0 \mathrm{~g}, 27.1 \mathrm{mmol}$ ) and $\mathrm{PPh}_{3}$ (4 equiv, $14.2 \mathrm{~g}$, $54.3 \mathrm{mmol})$ in dry $\mathrm{CH}_{2} \mathrm{Cl}_{2}(40 \mathrm{~mL})$, benzaldehyde (20\% ${ }^{13} \mathrm{C}$-enriched) (1 equiv, $1.5 \mathrm{~mL}, 13.6 \mathrm{mmol})$ in $\mathrm{CH}_{2} \mathrm{Cl}_{2}(10 \mathrm{~mL})$ was added at $0{ }^{\circ} \mathrm{C}$. The cooling bath was removed and the reaction was stirred at $\mathrm{rt}$ for $3 \mathrm{~h}$. Afterwards the reaction solution was filtered, and the solvents removed in vacuo. Purification by column chromatography (hexanes) gave $\mathbf{2 4 0}$ as colourless oil (3.5 g, $13.4 \mathrm{mmol}, \mathbf{9 9 \%})$.

${ }^{1} \mathrm{H}$ NMR: $\left(300 \mathrm{MHz}, \mathrm{CDCl}_{3}\right) \delta=7.58-7.51(\mathrm{~m}, 2 \mathrm{H}), 7.49\left({ }^{12} \mathrm{C}\right)(\mathrm{s}, 0.8 \mathrm{H}), 7.49\left({ }^{13} \mathrm{C}\right)(\mathrm{d}, J=159.9$ $\mathrm{Hz}, 0.2 \mathrm{H}), 7.42-7.30(\mathrm{~m}, 2 \mathrm{H})$ ppm. ${ }^{13} \mathrm{C}$ NMR: (101 MHz, $\left.\mathrm{CDCl}_{3}\right) \delta=137.0\left({ }^{13} \mathrm{C}\right.$-enriched), $135.5,128.7,128.6,128.5,89.8 \mathrm{ppm}$. 
1,3-Diisopropyl-4,5-dimethyl-2-[(phenylethynyl-2- $\left.{ }^{13} \mathrm{C}\right)$ thio]-1H-imidazol-3-ium hexafluoroantimonate ( $\{13 C\}-197)$ :

Following a modification of GP C, from (2,2-dibromovinyl-1- ${ }^{13} \mathrm{C}$ ) benzene (240) (1.17 equiv, $3.8 \mathrm{~g}, 14.5 \mathrm{mmol}$ ), $n$-BuLi (12.5 M, 2.3 equiv, $11.4 \mathrm{~mL}, 28.5 \mathrm{mmol}$ ), $\mathrm{ZnBr}_{2}$ (1.26 equiv, $3.52 \mathrm{~g}$, $15.6 \mathrm{mmol}$ ), 151a ( 1 equiv, $4.6 \mathrm{~g}, 12.4 \mathrm{mmol}$ ) and $\mathrm{NaSbF}_{6}$ (3.8 equiv, $12.1 \mathrm{~g}, 46.8 \mathrm{mmol}$ ), compound 241 (6.1 g, $11.1 \mathrm{mmol}, 90 \%)$ was obtained after column chromatography $\left(\mathrm{CH}_{2} \mathrm{Cl}_{2}\right.$ /acetone, 50:1) as a white solid.

${ }^{1} \mathrm{H}$ NMR: $\left(600 \mathrm{MHz}, \mathrm{CDCl}_{3}\right) \delta=7.41(\mathrm{dt}, J=7.0,1.3 \mathrm{~Hz}, 2 \mathrm{H}), 7.40-7.37(\mathrm{~m}, 1 \mathrm{H}), 7.33(\mathrm{t}, J=$ $7.7 \mathrm{~Hz}, 2 \mathrm{H}), 2.40(\mathrm{~s}, 6 \mathrm{H}), 1.71(\mathrm{~d}, J=7.1 \mathrm{~Hz}, 12 \mathrm{H})$ ppm. ${ }^{13} \mathrm{C} \mathrm{NMR:}\left(126 \mathrm{MHz}, \mathrm{CDCl}_{3}\right) \delta=135.8$, 132.2, 131.3, 130.2, 128.7, 120.6, 95.7 ( ${ }^{13} \mathrm{C}$-enriched), 69.4, 54.1, 21.3, 10.5 ppm. IR: (ATR, $\mathrm{cm}^{-1}$ ) 2998, 2943, 1617, 1486, 1462, 1443, 1380, 1222, 1113, 756, 690, 653. HRMS: calcd. for $\mathrm{C}_{18}{ }^{13} \mathrm{CH}_{24} \mathrm{~N}_{2} \mathrm{~S}^{+}\left[\mathrm{M}-\mathrm{H}-\mathrm{SbF}_{6}\right]^{+}:=313.1688$; found: 313.1744 .

\section{3- $\left\{{ }^{13} C\right\}-3-P h e n y l-1 H$-isochromen-1-one (3- $\left\{{ }^{13} C\right\}$-Homalicine) $\left(\left\{{ }^{13} C\right\}-207\right)$ :}<smiles>O=c1oc(-c2ccccc2)cc2ccccc12</smiles>

Following GP D, from $\mathrm{N}$-methylbenzamide (1 equiv, $40.6 \mathrm{mg}, 0.3 \mathrm{mmol}$ ), 241 (1.5 equiv, $247.2 \mathrm{mg}, 0.45 \mathrm{mmol})$, $\left[\mathrm{RhCp}^{*} \mathrm{Cl}_{2}\right]_{2}(2.5 \mathrm{~mol} \%, 4.6 \mathrm{mg}$, $0.0075 \mathrm{mmol}$ ), NaOAc (1 equiv, $24.3 \mathrm{mg}, 0.3 \mathrm{mmol}$ ), and $\mathrm{AgSbF}_{6}(1.7$ equiv, $175.2 \mathrm{mg}, 0.51 \mathrm{mmol})$, compound $\left\{{ }^{13} \mathrm{C}\right\}-207$ was obtained as a white solid (38.6 mg, $173 \mu \mathrm{mol}, 58 \%)$.

${ }^{1} \mathrm{H}$ NMR: $\left(300 \mathrm{MHz}, \mathrm{CDCl}_{3}\right) \delta=8.32(\mathrm{dt}, J=8.0,0.9 \mathrm{~Hz}, 1 \mathrm{H}), 7.89(\mathrm{dd}, J=7.9,1.8 \mathrm{~Hz}, 2 \mathrm{H}), 7.76$ - $7.69(\mathrm{~m}, 1 \mathrm{H}), 7.55-7.40(\mathrm{~m}, 5 \mathrm{H}), 6.98-6.94(\mathrm{~m}, 1 \mathrm{H})$ ppm. ${ }^{13} \mathrm{C}$ NMR: $\left(75 \mathrm{MHz}, \mathrm{CDCl}_{3}\right) \delta=$ $162.5,153.8\left({ }^{13} \mathrm{C}\right.$-enriched), 137.7, 135.0, 132.1, 130.1, 129.8, 129.0, 128.3, 126.1, 125.4, 120.7, $102.0 \mathrm{ppm}$.

4-\{ $\left.{ }^{13} \mathrm{C}\right\}-3-[(1,3-D i i s o p r o p y l-4,5-d i m e t h y l-1 H$-imidazol-3-ium-2-yl)thio]-2,4-diphenylcinnolin2-ium hexafluoroantimonate $\left(\left\{{ }^{13} \mathrm{C}\right\}-238\right)$ :<smiles>CCCn1c(SCc2c(-c3ccccc3)c3ccccc3n[n+]2-c2ccccc2)c(C)c(C)[n+]1CC</smiles>

A pressure vial was charged with $\left[\mathrm{RhCp}^{*} \mathrm{Cl}_{2}\right]_{2}(2.5 \mathrm{~mol} \%, 4.6$ $\mathrm{mg}, 7.5 \mathrm{mmol}$ ), NaOAc (1 eq, $24.3 \mathrm{mg}, 0.3 \mathrm{mmol}$ ), azobenzene (1 eq, $54.7 \mathrm{mg}, 0.3 \mathrm{mmol}$ ), 241 (1.5 equiv, $247.2 \mathrm{mg}, 0.45$ $\mathrm{mmol})$, and finally $\mathrm{AgSbF}_{6}(175.2 \mathrm{mg}, 0.51 \mathrm{mmol})$. The vial was sealed and flushed three times with $\mathrm{N}_{2}$ /Vacuum-Cycles. 1,2-Dichloroethane $(2 \mathrm{~mL})$ was added via syringe, and the reaction mixture was stirred at $80{ }^{\circ} \mathrm{C}$ for $20 \mathrm{~h}$. After cooling down to room temperature, the reaction solution was diluted with acetonitrile and filtered through a pad of celite. Subsequently the organic solvents were removed in vacuo. The 
residue was carefully washed with $\mathrm{CHCl}_{3}(5 \times 2 \mathrm{~mL})$ to give the pure product 243 as a dark yellow solid (301.7 mg, $312.4 \mu \mathrm{mol}, 96 \%$ ).

${ }^{1} \mathrm{H}$ NMR: $\left(500 \mathrm{MHz}, \mathrm{CD}_{3} \mathrm{CN}\right.$ ) $\delta=8.72$ (ddd, $J=8.7,1.2,0.7 \mathrm{~Hz}, 1 \mathrm{H}$ ), 8.45 (ddd, $J=8.7,6.9,1.2$ $\mathrm{Hz}, 1 \mathrm{H}), 8.36$ (ddd, $J=8.7,6.9,1.3 \mathrm{~Hz}, 1 \mathrm{H}), 7.97-7.93(\mathrm{~m}, 1 \mathrm{H}), 7.92-7.86(\mathrm{~m}, 4 \mathrm{H}), 7.80-$ $7.75(\mathrm{~m}, 1 \mathrm{H}), 7.73-7.68(\mathrm{~m}, 2 \mathrm{H}), 7.65-7.61(\mathrm{~m}, 1 \mathrm{H}), 7.40-7.36(\mathrm{~m}, 2 \mathrm{H}), 4.33$ (hept, $J=6.9$ $\mathrm{Hz}, 2 \mathrm{H}), 2.21(\mathrm{~s}, 6 \mathrm{H}), 1.19(\mathrm{~d}, J=6.9 \mathrm{~Hz}, 12 \mathrm{H}) \mathrm{ppm} .{ }^{13} \mathrm{C}$ NMR: $\left(126 \mathrm{MHz}, \mathrm{CD}_{3} \mathrm{CN}\right) \delta=150.9$, $148.7,145.9,144.8,143.0,139.0,134.5,133.8,132.7,132.3,132.1,131.9,131.1,130.8$, 129.6, 129.1, 127.0, 126.5, 55.9, 21.6, 11.2 ppm. ${ }^{19} \mathrm{~F}$ NMR: $\left(471 \mathrm{MHz}, \mathrm{CD}_{3} \mathrm{CN}\right) \delta=-124.0$ $\left(\right.$ sext, $J_{\mathrm{F}-121 \mathrm{Sb}}=1942.5 \mathrm{~Hz}$ ), -124.0 (oct, $\left.J_{\mathrm{F}-123 \mathrm{Sb}}=1053.6 \mathrm{~Hz}\right) \mathrm{ppm}$. IR: $\left(\mathrm{ATR}, \mathrm{cm}^{-1}\right)$ 2922, 2151, 2037, 2027, 1708, 1620, 1488, 1447, 1291, 1191, 750, 697, 614, 519. HRMS: calcd. for $\mathrm{C}_{30}{ }^{13} \mathrm{CH}_{34} \mathrm{~N}_{4} \mathrm{~S}^{2+}\left[\mathrm{M}-2 \mathrm{SbF}_{6}\right]^{+}:=495.2526$; found $=495.2531$.

\section{2,4-Diphenylcinnolin-3(2H)-one (241):}<smiles>O=c1c(-c2ccccc2)c2ccccc2nn1-c1ccccc1</smiles>

Salt 238 was stored under ambient conditions for one month. Afterwards an aliquot, purified by column chromatography (pure $\mathrm{CH}_{2} \mathrm{Cl}_{2}$ to $\mathrm{CH}_{2} \mathrm{Cl}_{2}$ /EtOAc, 95:5) gave $\mathbf{2 4 4}$ as bright orange crystals. ${ }^{46}$

${ }^{1} \mathrm{H}$ NMR: $\left(300 \mathrm{MHz}, \mathrm{CDCl}_{3}\right) \delta=7.82-7.74(\mathrm{~m}, 2 \mathrm{H}), 7.67-7.33(\mathrm{~m}, 10 \mathrm{H})$, $7.24-7.12(\mathrm{~m}, 2 \mathrm{H})$ ppm. ${ }^{13} \mathrm{C}$ NMR: $\left(101 \mathrm{MHz}, \mathrm{CDCl}_{3}\right) \delta=159.56,142.74$, $142.09,134.52,132.50,131.43,130.99,130.80,129.04,128.86,128.81$, $128.53,128.36,127.63,126.00,124.6 \mathrm{ppm}$.

\section{2,3-Diphenyl-2H-indazole (242):}<smiles>c1ccc(-c2c3ccccc3nn2-c2ccccc2)cc1</smiles>

sample of $\mathbf{2 4 2}$ was obtained after separation by column chromatography (cyclohexane/EtOAc, 10/1). ${ }^{47}$

${ }^{1} \mathrm{H}$ NMR: $\left(400 \mathrm{MHz}, \mathrm{CDCl}_{3}\right) \delta=7.81(\mathrm{~d}, J=8.7 \mathrm{~Hz}, 1 \mathrm{H}), 7.72(\mathrm{~d}, J=8.5 \mathrm{~Hz}, 1 \mathrm{H}), 7.48-7.33(\mathrm{~m}$, $11 \mathrm{H}), 7.15$ (dd, $J=8.5,6.6 \mathrm{~Hz}, 1 \mathrm{H})$ ppm. ${ }^{13} \mathrm{C}$ NMR: $\left(101 \mathrm{MHz}^{\left.\mathrm{C} C \mathrm{CDC}_{3}\right)} \delta=149.2,140.4,135.6\right.$, $130.1,129.8,129.1,128.9,128.5,128.4,127.1,126.2,122.7,121.9,120.7,117.9$ ppm.

\footnotetext{
${ }^{46}$ No final yield was determined.

${ }^{47}$ No final yield was determined.
} 
N-Methyl-5-(trifluoromethyl)-2-[(triisopropylsilyl)ethynyl]benzamide (243):<smiles>CNC(=O)c1cc(C(F)(F)F)ccc1C#C[In]S</smiles>

Following a modification of GP D, from $\mathrm{N}$-methyl-3(trifluoromethyl)benzamide (1 equiv, $60.9 \mathrm{mg}, 0.3 \mathrm{mmol}$ ), $160 \mathrm{~d}$ (1.5 equiv, $244.0 \mathrm{mg}, 0.45 \mathrm{mmol}),\left[\mathrm{RhCp}^{*} \mathrm{Cl}_{2}\right]_{2}(2.5 \mathrm{~mol} \%, 4.6 \mathrm{mg}, 0.0075$ mmol), NaOAc (1 equiv, $24.3 \mathrm{mg}, 0.3 \mathrm{mmol}$ ), and $\mathrm{AgSbF}_{6}$ (1.7 equiv, $175.2 \mathrm{mg}, 0.51 \mathrm{mmol})$, compound 243 was obtained as a colorless crystalline solid (14.9 mg, $38.8 \mu \mathrm{mol}, 13 \%)$.

${ }^{1} \mathrm{H}$ NMR: $\left(400 \mathrm{MHz}, \mathrm{CDCl}_{3}\right) \delta=8.42(\mathrm{dt}, J=1.8,0.9 \mathrm{~Hz}, 1 \mathrm{H}), 7.75(\mathrm{~s}, 1 \mathrm{H}), 7.68-7.60(\mathrm{~m}, 2 \mathrm{H})$, $3.02(\mathrm{~d}, J=4.8 \mathrm{~Hz}, 3 \mathrm{H}), 1.21-1.12(\mathrm{~m}, 21 \mathrm{H}) \mathrm{ppm} .{ }^{13} \mathrm{C}$ NMR: $\left(101 \mathrm{MHz}, \mathrm{CDCl}_{3}\right) \delta=165.3$, 136.1, 134.9, $131.1(q, J=33.5 \mathrm{~Hz}), 127.3(\mathrm{dq}, J=59.3,3.7 \mathrm{~Hz}), 125.0,123.1,122.2,104.4$, 101.9, 27.0, 18.8, 11.3 ppm. IR: (ATR, $\mathrm{cm}^{-1}$ ) 3282, 2943, 2864, 1657, 1637, 1558, 1463, 1336, 1301, 1258, 1170, 1131, 882, 674, 650. HRMS: calcd. for $\mathrm{C}_{20} \mathrm{H}_{29} \mathrm{~F}_{3} \mathrm{NOSi}^{+}[\mathrm{M}+\mathrm{H}]^{+}:$384.1965, found: 384.1964 . 
APPENDIX 
2-(Bromothio)-1-ethyl-3,5-dimethylpyridin-1-ium bromide (154c):

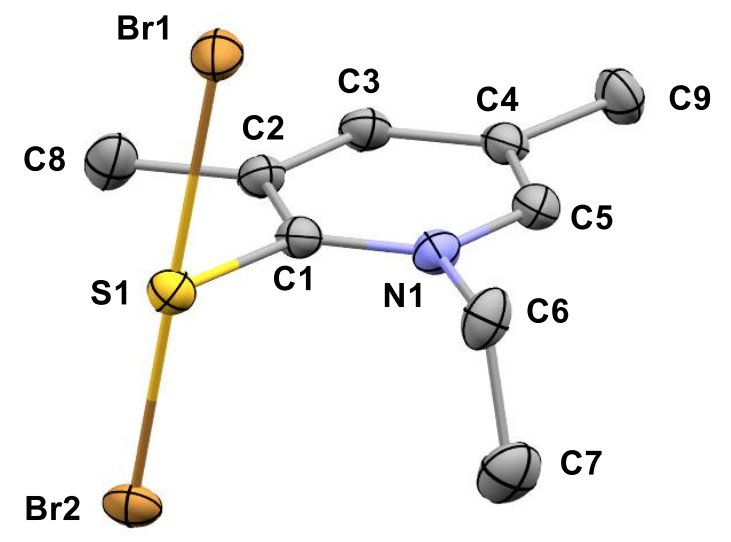

Empirical formula

Color

Formula weight

Temperature

Wavelength

Crystal system

Space group

Unit cell dimensions

Volume

Z

Density (calculated)

Absorption coefficient $\mu$

$F(000)$

Crystal size

$\theta$ range for data collection

Index ranges

Reflections collected

Independent reflections

Reflections with $\mathrm{I}>2 \sigma(\mathrm{I})$

Completeness to $\theta=65.082^{\circ}$

Absorption correction

Max. and min. transmission

Refinement method

\section{$\mathrm{C}_{9} \mathrm{H}_{13} \mathrm{Br}_{2} \mathrm{~N} \mathrm{~S}$}

orange

$327.08 \mathrm{~g} \cdot \mathrm{mol}^{-1}$

$100 \mathrm{~K}$

$1.54178 \AA$

monoclinic

C 1 2/c 1, (no. 15)

$\mathrm{a}=23.752(2) \AA \quad \alpha=90^{\circ}$.

$\mathrm{b}=7.7745(8) \AA$

$\beta=117.597(2)^{\circ}$.

$\mathrm{c}=14.5136(14) \AA$

2375.2(4) $\AA^{3}$

8

$1.829 \mathrm{Mg} \cdot \mathrm{m}^{-3}$

$9.956 \mathrm{~mm}^{-1}$

$1280 \mathrm{e}$

$0.21 \times 0.20 \times 0.08 \mathrm{~mm}^{3}$

4.200 to $65.082^{\circ}$.

$-27 \leq \mathrm{h} \leq 27,-9 \leq \mathrm{k} \leq 8,-17 \leq 1 \leq 17$

25385

$2016\left[\mathrm{R}_{\mathrm{int}}=0.0436\right]$

2003

$100.0 \%$

Gaussian

0.38153 and 0.19076

Full-matrix least-squares on $\mathrm{F}^{2}$ 
Data / restraints / parameters

Goodness-of-fit on $\mathrm{F}^{2}$

Final R indices [I>2 $\sigma(\mathrm{I})]$

$\mathrm{R}$ indices (all data)

Extinction coefficient

Largest diff. peak and hole
2016 / 0 / 121

1.162

$\begin{array}{ll}\mathrm{R}_{1}=0.0232 & \mathrm{wR}^{2}=0.0557 \\ \mathrm{R}_{1}=0.0235 & \mathrm{wR}^{2}=0.0559 \\ 0 & \\ 0.410 \text { and }-0.478 \mathrm{e} \cdot \AA^{-3} & \end{array}$


1-Ethyl-3,5-dimethyl-2-thiocyanatopyridin-1-ium hexafluoroantimonate (155b):
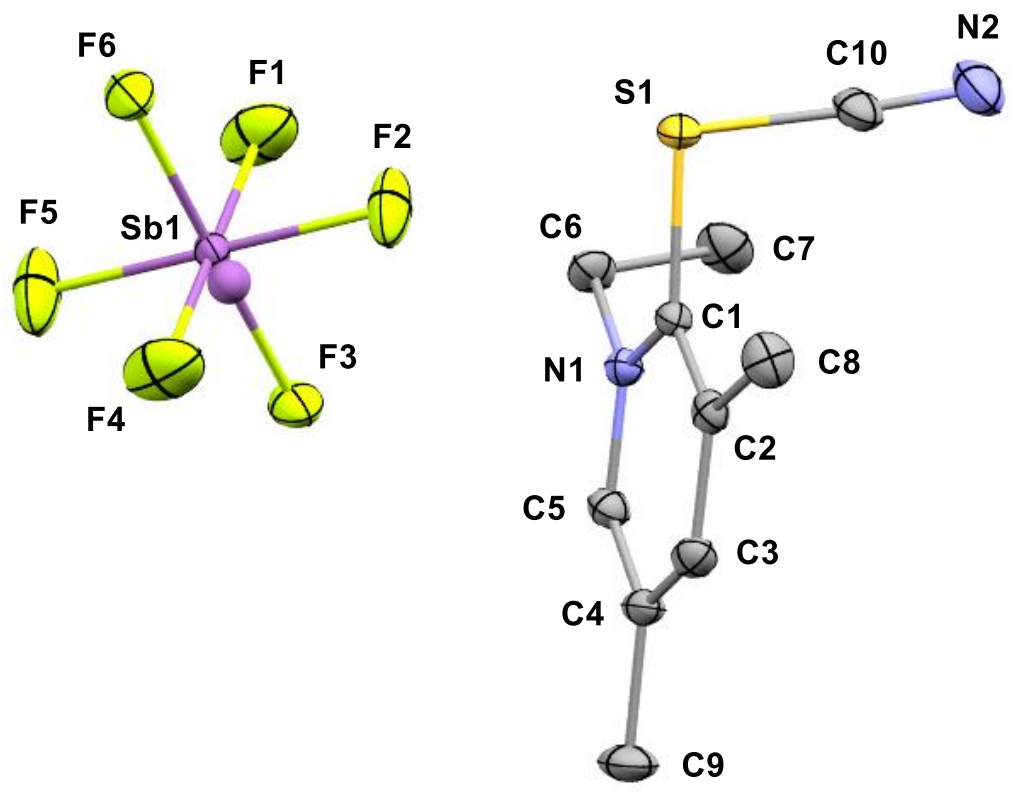

Empirical formula

Color

Formula weight

Temperature

Wavelength

Crystal system

Space group

Unit cell dimensions

Volume

$Z$

Density (calculated)

Absorption coefficient $\mu$

$F(000)$

Crystal size

$\theta$ range for data collection

Index ranges

Reflections collected

Independent reflections

Reflections with $\mathrm{I}>2 \sigma(\mathrm{I})$

Completeness to $\theta=25.242^{\circ}$

Absorption correction
$\mathrm{C}_{10} \mathrm{H}_{13} \mathrm{~F}_{6} \mathrm{~N}_{2} \mathrm{~S} \mathrm{Sb}$

colorless

$429.03 \mathrm{~g} \cdot \mathrm{mol}^{-1}$

$100 \mathrm{~K}$

$0.71073 \AA$

Monoclinic

p 21/n, (no. 14)

$\mathrm{a}=7.272(8) \AA$

$\alpha=90^{\circ}$.

$\mathrm{b}=13.130(13) \AA$

$\beta=90.92(2)^{\circ}$.

$\mathrm{c}=15.688(17) \AA$

1498(3) $\AA^{3}$

4

$1.903 \mathrm{Mg} \cdot \mathrm{m}^{-3}$

$2.036 \mathrm{~mm}^{-1}$

$832 \mathrm{e}$

$0.15 \times 0.15 \times 0.04 \mathrm{~mm}^{3}$

2.597 to $33.700^{\circ}$.

$-10 \leq \mathrm{h} \leq 11,-20 \leq \mathrm{k} \leq 19,-24 \leq 1 \leq 23$

26606

$5757\left[\mathrm{R}_{\mathrm{int}}=0.0257\right]$

5187

$100.0 \%$

Gaussian 
Max. and min. transmission

Refinement method

Data / restraints / parameters

Goodness-of-fit on $\mathrm{F}^{2}$

Final R indices [I $>2 \sigma(\mathrm{I})]$

$\mathrm{R}$ indices (all data)

Extinction coefficient

Largest diff. peak and hole
0.91378 and 0.75472

Full-matrix least-squares on $\mathrm{F}^{2}$

5757 / 0 / 188

1.045

$\mathrm{R}_{1}=0.0225 \quad \mathrm{wR}^{2}=0.0550$

$\mathrm{R}_{1}=0.0260 \quad \mathrm{wR}^{2}=0.0562$

$\mathrm{n} / \mathrm{a}$

0.838 and $-0.847 \mathrm{e} \cdot \AA^{-3}$ 
2-Chloro-1-ethylpyridin-1-ium hexafluoroantimonate (156d):

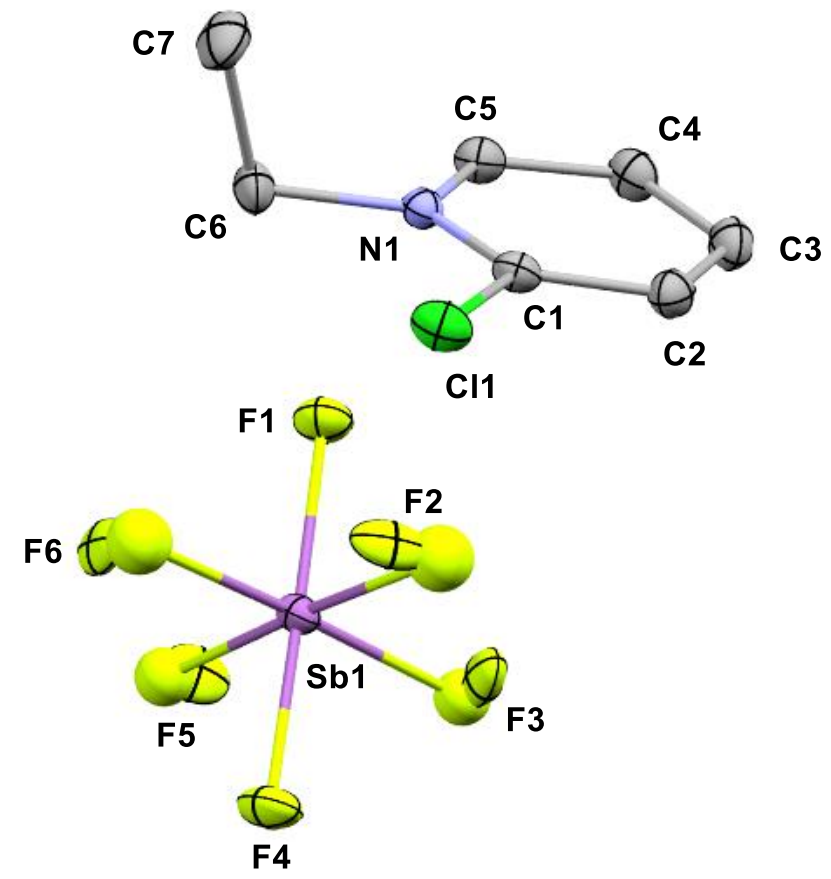

Empirical formula

$\mathrm{C}_{7} \mathrm{H}_{9} \mathrm{ClF}_{6} \mathrm{NSb}$

Color

colorless

Formula weight

$378.35 \mathrm{~g}^{\bullet} \mathrm{mol}^{-1}$

Temperature

$100 \mathrm{~K}$

Wavelength

$0.71073 \AA$

Crystal system

monoclinic

Space group

$P 21 / n$, (no. 14)

Unit cell dimensions

$\mathrm{a}=8.6285(17) \AA$

$\alpha=90^{\circ}$.

$\mathrm{b}=10.996(2) \AA$

$\beta=101.549(3)^{\circ}$.

$\mathrm{c}=12.597(3) \AA$

1171.1(4) $\AA^{3}$

Volume

4

Density (calculated)

$2.146{\mathrm{Mg} \bullet \mathrm{m}^{-3}}^{-3}$

Absorption coefficient $\mu$

$2.634 \mathrm{~mm}^{-1}$

$F(000)$

$720 \mathrm{e}$

Crystal size

$0.18 \times 0.13 \times 0.05 \mathrm{~mm}^{3}$

$\theta$ range for data collection

3.182 to $35.629^{\circ}$.

Index ranges

$-14 \leq \mathrm{h} \leq 14,-18 \leq \mathrm{k} \leq 18,-20 \leq 1 \leq 20$

Reflections collected

43856

Independent reflections

$5397\left[R_{\text {int }}=0.0278\right]$

Reflections with $I>2 \sigma(I)$

4889

Completeness to $\square=25.242^{\circ}$

$99.8 \%$ 
Absorption correction

Max. and min. transmission

Refinement method

Data / restraints / parameters

Goodness-of-fit on $\mathrm{F}^{2}$

Final $R$ indices $[I>2 \sigma(I)]$

$R$ indices (all data)

Extinction coefficient

Largest diff. peak and hole

\section{Gaussian}

0.91577 and 0.78357

Full-matrix least-squares on $\mathrm{F}^{2}$

5397 / 0 / 162

1.045

$R_{1}=0.0202 \quad \mathrm{w} R^{2}=0.0463$

$R_{1}=0.0238$

$\mathrm{w} R^{2}=0.0480$

$\mathrm{n} / \mathrm{a}$

1.518 and $-0.790 \mathrm{e} \cdot \AA^{-3}$ 
2,2'-Thiobis(1-ethylpyridin-1-ium) bishexafluoroantimonate (157):

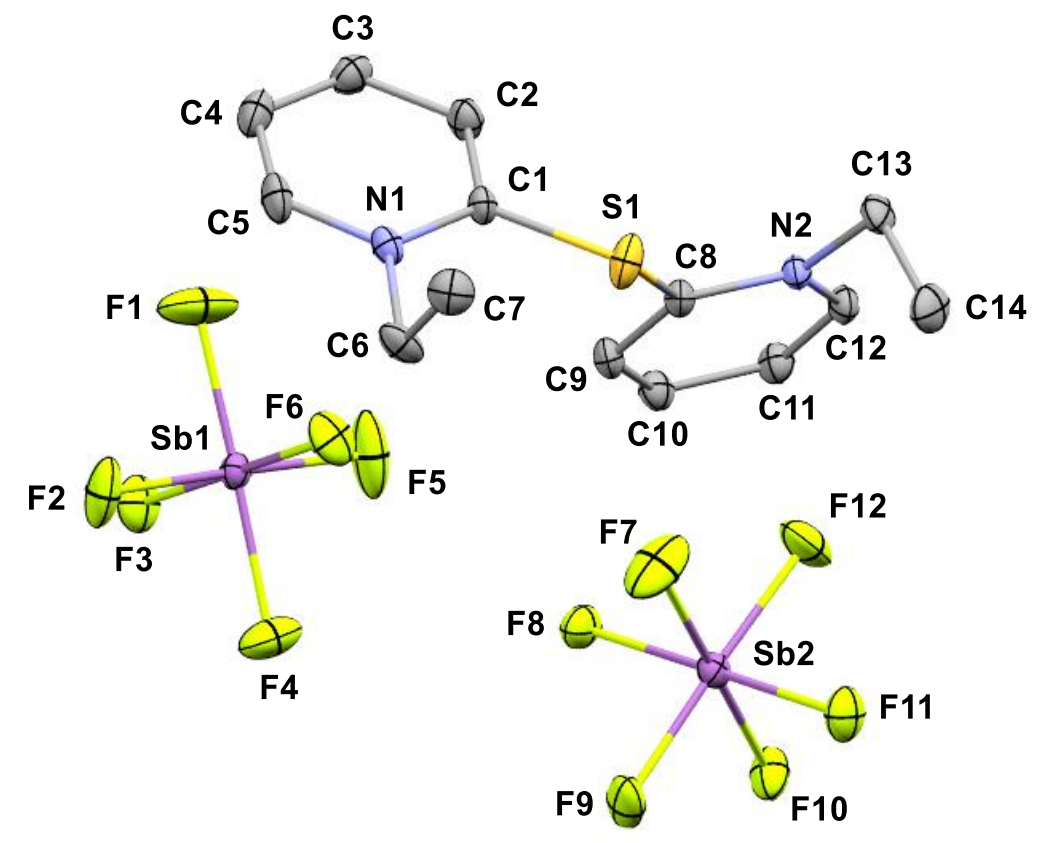

Empirical formula

$\mathrm{C}_{14} \mathrm{H}_{18} \mathrm{~F}_{12} \mathrm{~N}_{2} \mathrm{SSb}_{2}$

Color

colorless

Formula weight

$717.86 \mathrm{~g} \cdot \mathrm{mol}^{-1}$

Temperature

$100 \mathrm{~K}$

Wavelength

$0.71073 \AA$

Crystal system

MONOCLINIC

Space group

p 21/n, (no. 14)

Unit cell dimensions

$a=16.655(2) \AA$

$\alpha=90^{\circ}$.

$\mathrm{b}=23.302(3) \AA$

$\beta=101.886(2)^{\circ}$.

$\mathrm{c}=17.413$

(2) $\AA$

Volume

6613.1(14) $\AA^{3}$

$\gamma=90^{\circ}$.

$\mathrm{Z}$

12

Density (calculated)

$2.163 \mathrm{Mg} \cdot \mathrm{m}^{-3}$

Absorption coefficient

$2.650 \mathrm{~mm}^{-1}$

$\mathrm{F}(000)$

$4104 \mathrm{e}$

Crystal size

$0.24 \times 0.18 \times 0.06 \mathrm{~mm}^{3}$

$\theta$ range for data collection

1.480 to $36.319^{\circ}$.

Index ranges

$-27 \leq \mathrm{h} \leq 27,-38 \leq \mathrm{k} \leq 38,-29 \leq 1 \leq 29$

Reflections collected

258729

Independent reflections

$32018\left[\mathrm{R}_{\mathrm{int}}=0.0308\right]$

Reflections with $\mathrm{I}>2 \sigma(\mathrm{I})$

26780

Completeness to $\theta=25.242^{\circ}$

$99.9 \%$

Absorption correction

Gaussian 
Max. and min. transmission

Refinement method

Data / restraints / parameters

Goodness-of-fit on $\mathrm{F}^{2}$

Final R indices [I $>2 \sigma(\mathrm{I})]$

$\mathrm{R}$ indices (all data)

Extinction coefficient

Largest diff. peak and hole
0.7473 and 0.5745

Full-matrix least-squares on $\mathrm{F}^{2}$

32018 / 0 / 844

1.029

$\mathrm{R}_{1}=0.0208 \quad \mathrm{wR}^{2}=0.0435$

$\mathrm{R}_{1}=0.0306 \quad \mathrm{wR}^{2}=0.0473$

$\mathrm{n} / \mathrm{a}$

1.349 and $-0.746 \mathrm{e} \cdot \AA^{-3}$ 
2-Bromo-1-ethylpyridin-1-ium bromide (158):

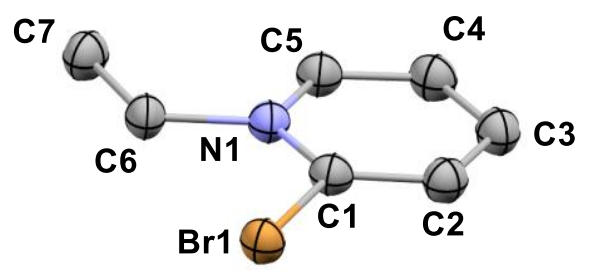

D Br2

\begin{tabular}{|c|c|}
\hline Empirical formula & $\mathrm{C}_{7} \mathrm{H}_{9} \mathrm{Br}_{2} \mathrm{~N}$ \\
\hline Formula weight & 266.97 \\
\hline Temperature/K & 100.02 \\
\hline Crystal system & orthorhombic \\
\hline Space group & Pnma \\
\hline $\mathrm{a} / \AA$ & $15.2828(6)$ \\
\hline $\mathrm{b} / \AA$ & $6.7822(2)$ \\
\hline $\mathrm{c} / \AA$ & $8.4389(4)$ \\
\hline$\alpha /^{\circ}$ & 90 \\
\hline$\beta /{ }^{\circ}$ & 90 \\
\hline$\gamma /{ }^{\circ}$ & 90 \\
\hline Volume $/ \AA^{3}$ & $874.70(6)$ \\
\hline $\mathrm{Z}$ & 4 \\
\hline$\rho_{\text {calc }} \mathrm{g} / \mathrm{cm}^{3}$ & 2.027 \\
\hline$\mu / \mathrm{mm}^{-1}$ & 9.194 \\
\hline $\mathrm{F}(000)$ & 512.0 \\
\hline Crystal size $/ \mathrm{mm}^{3}$ & $0.596 \times 0.064 \times 0.034$ \\
\hline Radiation & $\operatorname{MoK} \alpha(\lambda=0.71073)$ \\
\hline \multicolumn{2}{|c|}{$2 \Theta$ range for data collection $/{ }^{\circ} 5.332$ to 59.232} \\
\hline Index ranges & $-20 \leq \mathrm{h} \leq 21,-9 \leq \mathrm{k} \leq 9,-11 \leq 1 \leq 11$ \\
\hline Reflections collected & 13108 \\
\hline Independent reflections & $1319\left[\mathrm{R}_{\text {int }}=0.0199, \mathrm{R}_{\text {sigma }}=0.0111\right]$ \\
\hline Data/restraints/parameters & $1319 / 0 / 61$ \\
\hline Goodness-of-fit on $\mathrm{F}^{2}$ & 1.117 \\
\hline Final $R$ indexes $[\mathrm{I}>=2 \sigma(\mathrm{I})]$ & $\mathrm{R}_{1}=0.0226, \mathrm{wR}_{2}=0.0573$ \\
\hline Final $\mathrm{R}$ indexes [all data] & $\mathrm{R}_{1}=0.0232, \mathrm{wR}_{2}=0.0576$ \\
\hline Largest diff. peak/hole / e $\AA^{-3}$ & $0.60 /-0.36$ \\
\hline
\end{tabular}


1-Methyl-2-[(phenylethynyl)thio]pyridin-1-ium tetrabromozincate (160a):
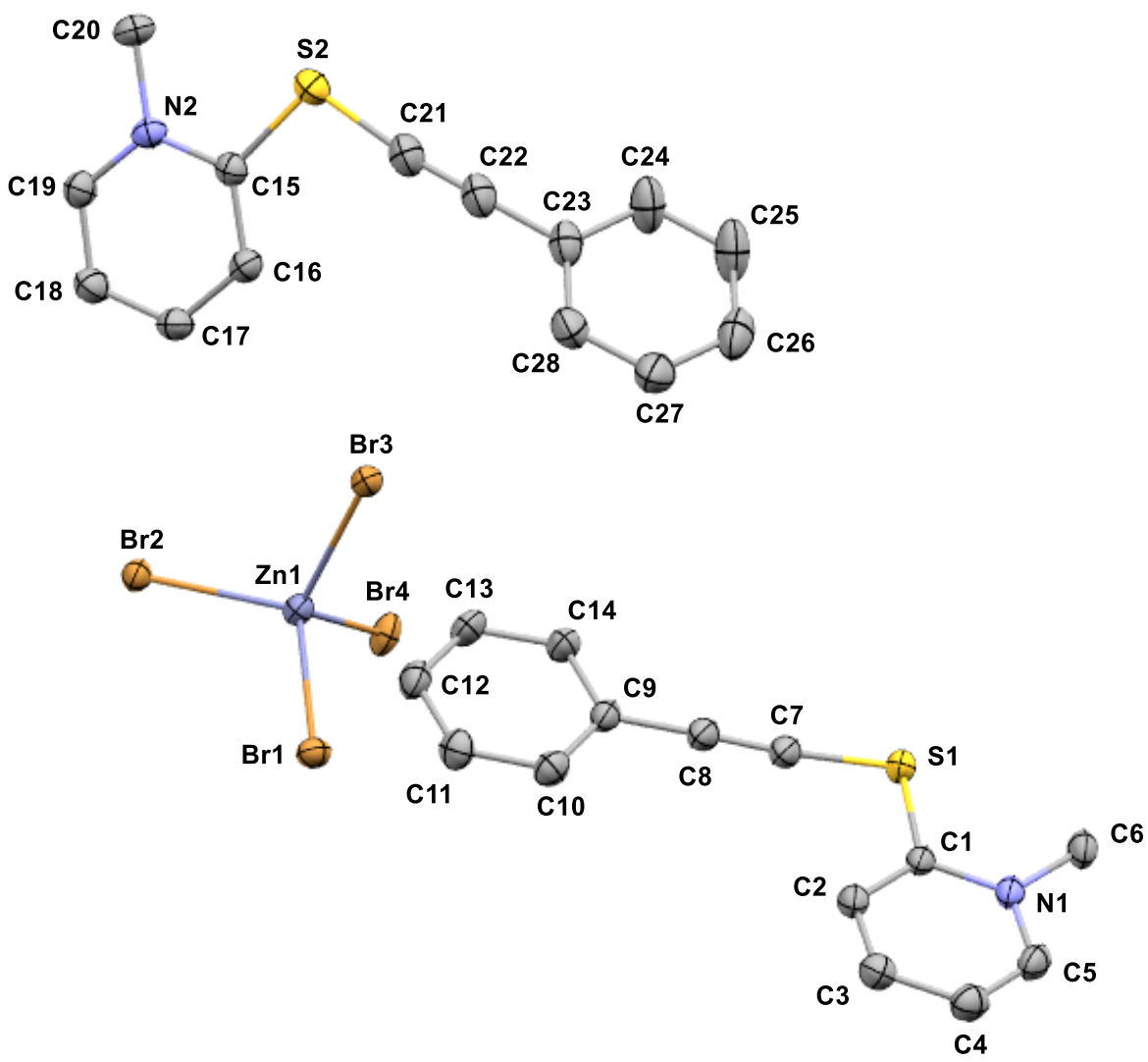

Empirical formula

Formula weight

Temperature/K

Crystal system

Space group

$\mathrm{a} / \AA$

$\mathrm{b} / \AA$

$\mathrm{c} / \AA$

$\alpha /^{\circ}$

$\beta /{ }^{\circ}$

$\gamma /{ }^{\circ}$

Volume $/ \AA^{3}$

$\mathrm{Z}$

$\rho_{\text {calc }} \mathrm{g} / \mathrm{cm}^{3}$

$\mu / \mathrm{mm}^{-1}$

$\mathrm{F}(000)$

Crystal size $/ \mathrm{mm}^{3}$

Radiation
$\mathrm{C}_{28} \mathrm{H}_{24} \mathrm{Br}_{4} \mathrm{~N}_{2} \mathrm{~S}_{2} \mathrm{Zn}$

837.62

99.99

triclinic

P-1

8.5908(4)

$11.4010(5)$

16.9074(6)

101.210(2)

102.3600(10)

$100.535(2)$

1542.58(11)

2

1.803

6.133

816.0

$0.429 \times 0.3 \times 0.122$

$\operatorname{MoK} \alpha(\lambda=0.71073)$

$2 \Theta$ range for data collection/ $/{ }^{\circ} 5.496$ to 63.134

Index ranges

Reflections collected

Independent reflections

Data/restraints/parameters
$-12 \leq \mathrm{h} \leq 12,-16 \leq \mathrm{k} \leq 16,-24 \leq 1 \leq 24$

70984

$10325\left[\mathrm{R}_{\text {int }}=0.0314, \mathrm{R}_{\text {sigma }}=0.0208\right]$

$10325 / 0 / 336$ 
Goodness-of-fit on $\mathrm{F}^{2}$

1.023

Final $R$ indexes $[\mathrm{I}>=2 \sigma(\mathrm{I})] \quad \mathrm{R}_{1}=0.0182, \mathrm{wR}_{2}=0.0453$

Final $\mathrm{R}$ indexes [all data] $\quad \mathrm{R}_{1}=0.0211, \mathrm{wR}_{2}=0.0463$

Largest diff. peak/hole / e $\AA^{-3} 0.65 /-0.38$ 
1-Ethyl-2-[(phenylethynyl)thio]pyridin-1-ium tetrabromozincate (160b):

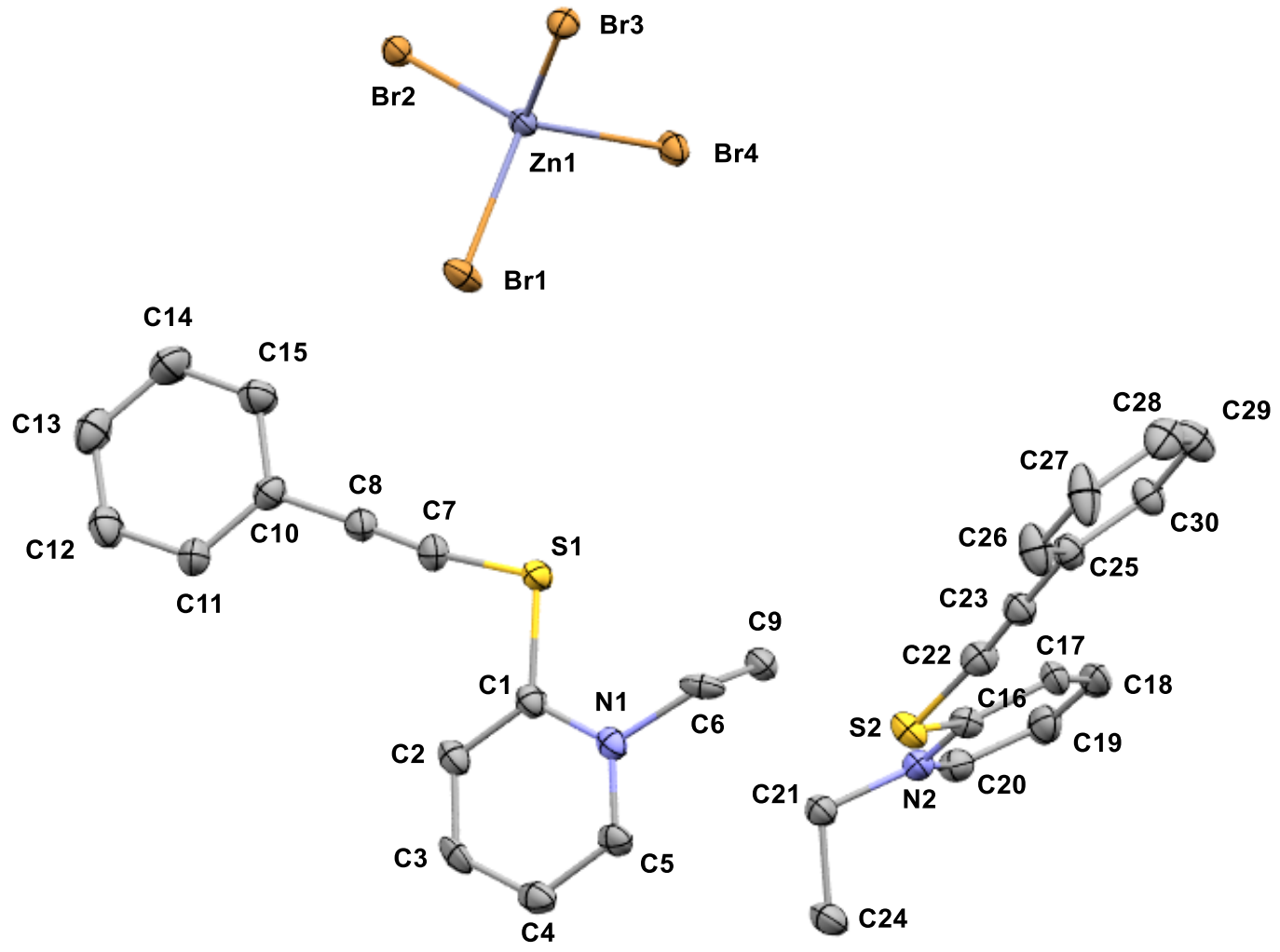

Empirical formula

Color

Formula weight

Temperature

Wavelength

Crystal system

Space group

Unit cell dimensions

Volume

$\mathrm{Z}$

Density (calculated)

Absorption coefficient

$\mathrm{F}(000)$

Crystal size

$\theta$ range for data collection

Index ranges

Reflections collected
$\mathrm{C}_{30} \mathrm{H}_{28} \mathrm{Br}_{4} \mathrm{~N}_{2} \mathrm{~S}_{2} \mathrm{Zn}$

colorless

$865.67 \mathrm{~g} \cdot \mathrm{mol}^{-1}$

$100.15 \mathrm{~K}$

$0.71073 \AA$

Triclinic

p -1, (no. 2)

$\mathrm{a}=9.3174(5) \AA \quad \alpha=104.414(5)^{\circ}$.

$\mathrm{b}=9.6047(3) \AA \quad \beta=96.515(7)^{\circ}$.

$\mathrm{c}=18.8633(18) \AA \quad \gamma=91.289(4)^{\circ}$.

1622.14(19) $\AA^{3}$

2

$1.772 \mathrm{Mg} \cdot \mathrm{m}^{-3}$

$5.835 \mathrm{~mm}^{-1}$

$848 \mathrm{e}$

$0.08 \times 0.07 \times 0.04 \mathrm{~mm}^{3}$

2.705 to $33.128^{\circ}$.

$-14 \leq \mathrm{h} \leq 14,-14 \leq \mathrm{k} \leq 14,-28 \leq 1 \leq 28$

43143 
Independent reflections

Reflections with $\mathrm{I}>2 \sigma(\mathrm{I})$

Completeness to $\theta=25.242^{\circ}$

Absorption correction

Max. and min. transmission

Refinement method

Data / restraints / parameters

Goodness-of-fit on $\mathrm{F}^{2}$

Final $\mathrm{R}$ indices [I $>2 \sigma(\mathrm{I})]$

$\mathrm{R}$ indices (all data)

Extinction coefficient

Largest diff. peak and hole
$12306\left[\mathrm{R}_{\mathrm{int}}=0.0583\right]$

10442

$99.8 \%$

Gaussian

0.72323 and 0.52922

Full-matrix least-squares on $\mathrm{F}^{2}$

$12306 / 0 / 355$

1.080

$\mathrm{R}_{1}=0.0846$

$\mathrm{wR}^{2}=0.2618$

$\mathrm{R}_{1}=0.0981$

$\mathrm{wR}^{2}=0.2750$

$\mathrm{n} / \mathrm{a}$

2.824 and $-3.220 \mathrm{e} \cdot \AA^{-3}$ 


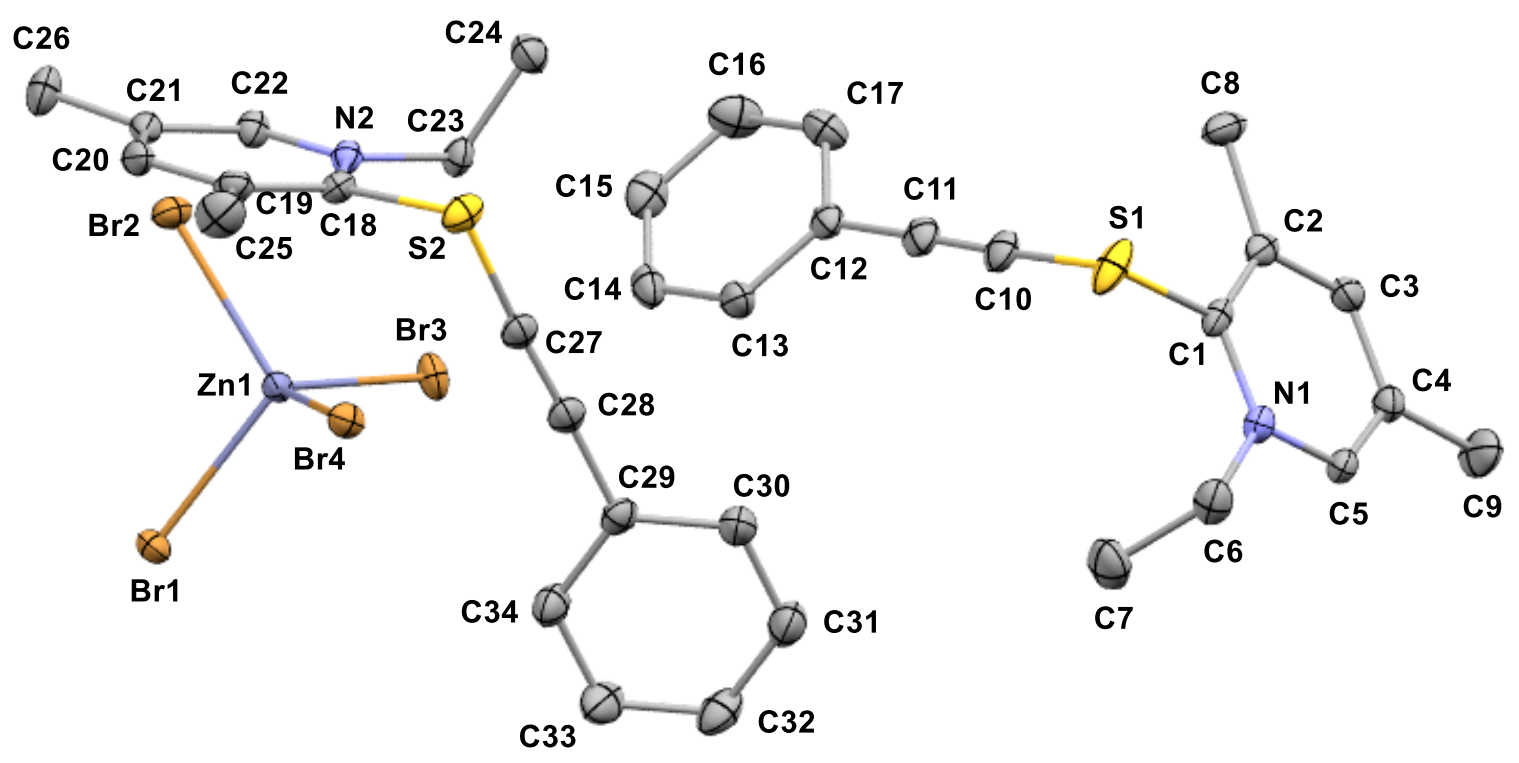

Empirical formula

Color

Formula weight

Temperature

Wavelength

Crystal system

Space group

Unit cell dimensions

Volume

Z

Density (calculated)

Absorption coefficient

$\mathrm{F}(000)$

Crystal size

$\theta$ range for data collection

Index ranges

Reflections collected
$\mathrm{C}_{34} \mathrm{H}_{36} \mathrm{Br}_{4} \mathrm{~N}_{2} \mathrm{~S}_{2} \mathrm{Zn}$

colorless

$921.78 \mathrm{~g} \cdot \mathrm{mol}^{-1}$

$100 \mathrm{~K}$

$0.71073 \AA$

MONOCLINIC

p 21/n, (no. 14)

$\mathrm{a}=18.614(2) \AA$

$\alpha=90^{\circ}$.

$\mathrm{b}=9.4805(11) \AA$

$\beta=94.396(2)^{\circ}$.

$\mathrm{c}=20.367(2) \AA$

3583.6(7) $\AA^{3}$

$\gamma=90^{\circ}$.

4

$1.709 \mathrm{Mg} \cdot \mathrm{m}^{-3}$

$5.288 \mathrm{~mm}^{-1}$

$1824 \mathrm{e}$

$0.250 \times 0.130 \times 0.080 \mathrm{~mm}^{3}$

1.429 to $32.527^{\circ}$.

$-28 \leq \mathrm{h} \leq 28,-14 \leq \mathrm{k} \leq 14,-30 \leq 1 \leq 30$

113662 
Independent reflections

Reflections with $\mathrm{I}>2 \sigma(\mathrm{I})$

Completeness to $\theta=25.242^{\circ}$

Absorption correction

Max. and min. transmission

Refinement method

Data / restraints / parameters

Goodness-of-fit on $\mathrm{F}^{2}$

Final $\mathrm{R}$ indices $[\mathrm{I}>2 \sigma(\mathrm{I})]$

$\mathrm{R}$ indices (all data)

Extinction coefficient

Largest diff. peak and hole
$12967\left[\mathrm{R}_{\mathrm{int}}=0.0420\right]$

10698

$100.0 \%$

Gaussian

0.71051 and 0.44092

Full-matrix least-squares on $\mathrm{F}^{2}$

12967 / 0 / 394

1.142

$\mathrm{R}_{1}=0.0236 \quad \mathrm{wR}^{2}=0.0601$

$\mathrm{R}_{1}=0.0366 \quad \mathrm{wR}^{2}=0.0769$

$\mathrm{n} / \mathrm{a}$

0.925 and $-0.551 \mathrm{e} \cdot \AA^{-3}$ 


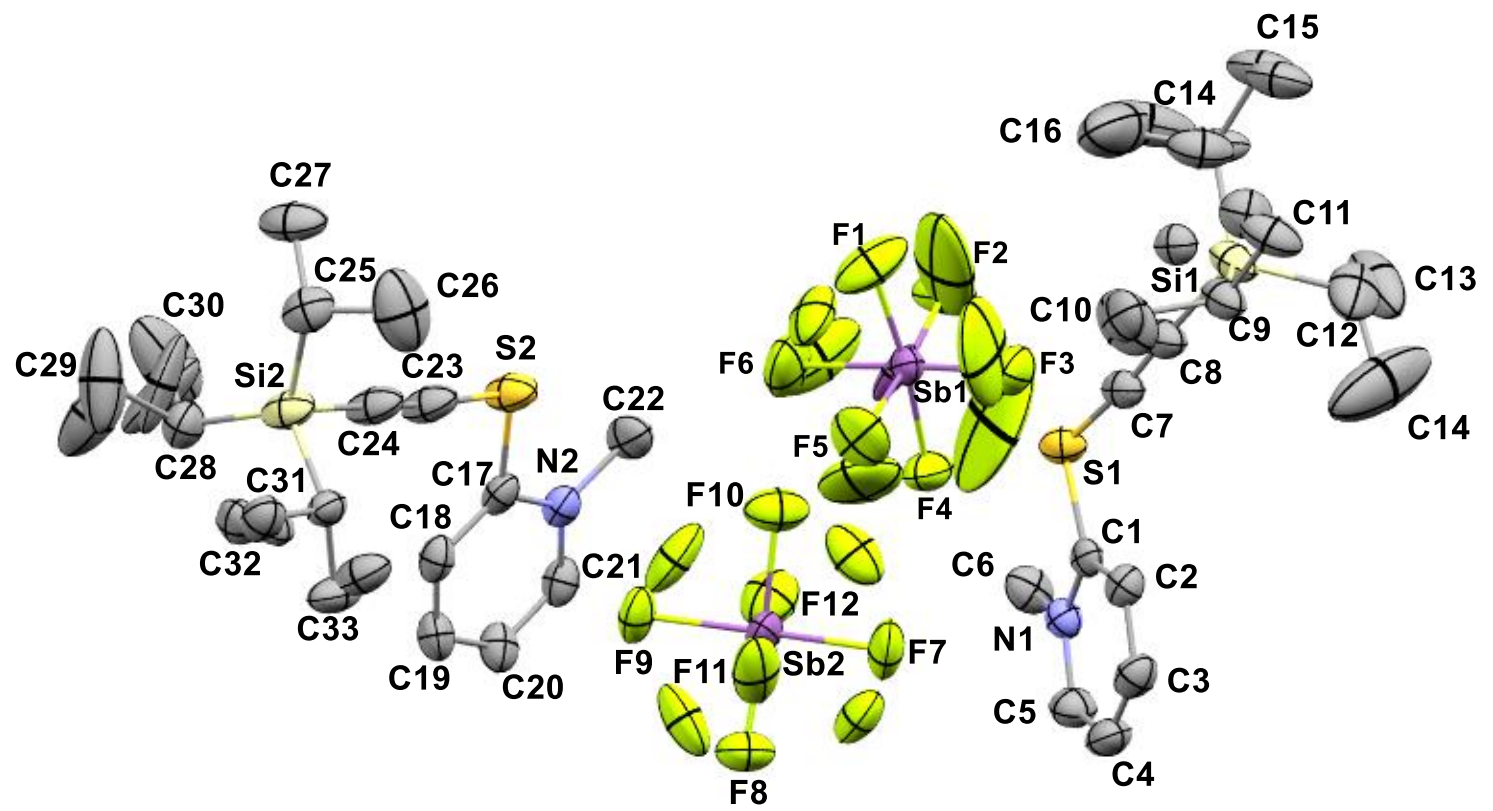

Empirical formula

Formula weight

Temperature/K

Crystal system

Space group

$\mathrm{a} / \AA$

$\mathrm{b} / \AA$

$\mathrm{c} / \AA$

$\alpha /{ }^{\circ}$

$\beta /{ }^{\circ}$

$\gamma /{ }^{\circ}$

Volume/ $/ \AA^{3}$

Z

$\rho_{\text {calc }} \mathrm{g} / \mathrm{cm}^{3}$

$\mu / \mathrm{mm}^{-1}$

$\mathrm{F}(000)$

Crystal size $/ \mathrm{mm}^{3}$

Radiation
$\mathrm{C}_{17} \mathrm{H}_{28} \mathrm{~F}_{6} \mathrm{NSSbSi}$

542.30

100.01

monoclinic

$\mathrm{P} 2 / \mathrm{c}$

22.674(2)

13.0853(8)

15.9485(10)

90

103.430(3)

90

4602.4(6)

8

1.565

1.391

2176.0

$0.361 \times 0.203 \times 0.082$

$\operatorname{MoK} \alpha(\lambda=0.71073)$

$2 \Theta$ range for data collection $/{ }^{\circ} 4.83$ to 57.498

Index ranges

Reflections collected

Independent reflections

Data/restraints/parameters

Goodness-of-fit on $\mathrm{F}^{2}$

Final $\mathrm{R}$ indexes $[\mathrm{I}>=2 \sigma(\mathrm{I})]$
$-30 \leq \mathrm{h} \leq 30,-17 \leq \mathrm{k} \leq 17,-21 \leq 1 \leq 20$

78514

$11931\left[\mathrm{R}_{\text {int }}=0.0275, \mathrm{R}_{\text {sigma }}=0.0183\right]$

$11931 / 183 / 722$

1.035

$\mathrm{R}_{1}=0.0420, \mathrm{wR}_{2}=0.0962$ 
Final $\mathrm{R}$ indexes [all data] $\quad \mathrm{R}_{1}=0.0515, \mathrm{wR}_{2}=0.1032$

Largest diff. peak/hole / e $\AA^{-3} 1.66 /-2.01$ 


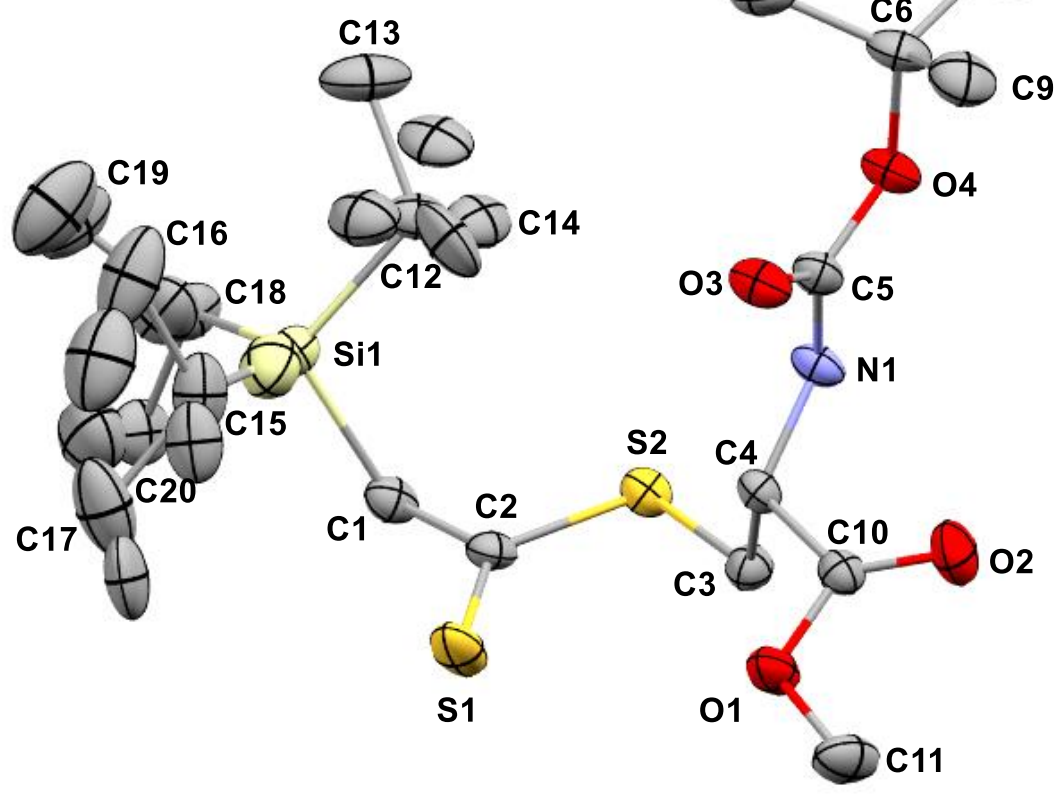

\begin{tabular}{|c|c|}
\hline Empirical formula & $\mathrm{C}_{15} \mathrm{H}_{31} \mathrm{NOSSi}$ \\
\hline Formula weight & 301.56 \\
\hline Temperature/K & 99.98 \\
\hline Crystal system & monoclinic \\
\hline Space group & $\mathrm{P} 2{ }_{1} / \mathrm{c}$ \\
\hline $\mathrm{a} / \AA$ & $15.394(2)$ \\
\hline $\mathrm{b} / \AA$ & $8.5880(10)$ \\
\hline$c / \AA$ & $14.610(2)$ \\
\hline$\alpha /^{\circ}$ & 90 \\
\hline$\beta /{ }^{\circ}$ & $115.589(4)$ \\
\hline$\gamma /{ }^{\circ}$ & 90 \\
\hline Volume $/ \AA^{3}$ & $1742.0(4)$ \\
\hline $\mathrm{Z}$ & 4 \\
\hline$\rho_{\text {calc }} \mathrm{g} / \mathrm{cm}^{3}$ & 1.150 \\
\hline$\mu / \mathrm{mm}^{-1}$ & 0.249 \\
\hline $\mathrm{F}(000)$ & 664.0 \\
\hline Crystal size $/ \mathrm{mm}^{3}$ & $0.404 \times 0.261 \times 0.038$ \\
\hline Radiation & $\operatorname{MoK} \alpha(\lambda=0.71073)$ \\
\hline \multicolumn{2}{|c|}{$2 \Theta$ range for data collection $/{ }^{\circ} 5.578$ to 57.552} \\
\hline Index ranges & $-20 \leq \mathrm{h} \leq 20,-11 \leq \mathrm{k} \leq 11,-19 \leq 1 \leq 1 \mathrm{C}$ \\
\hline Reflections collected & 29735 \\
\hline Independent reflections & $4510\left[R_{\text {int }}=0.0270, R_{\text {sigma }}=0.0190\right]$ \\
\hline Data/restraints/parameters & $4510 / 0 / 190$ \\
\hline Goodness-of-fit on $\mathrm{F}^{2}$ & 1.102 \\
\hline Final $R$ indexes $[\mathrm{I}>=2 \sigma(\mathrm{I})]$ & $\mathrm{R}_{1}=0.0298, \mathrm{w} \mathrm{R}_{2}=0.0771$ \\
\hline Final $\mathrm{R}$ indexes [all data] & $\mathrm{R}_{1}=0.0326, \mathrm{wR}_{2}=0.0790$ \\
\hline
\end{tabular}


Largest diff. peak/hole / e $\AA^{-3} 0.44 /-0.24$ 


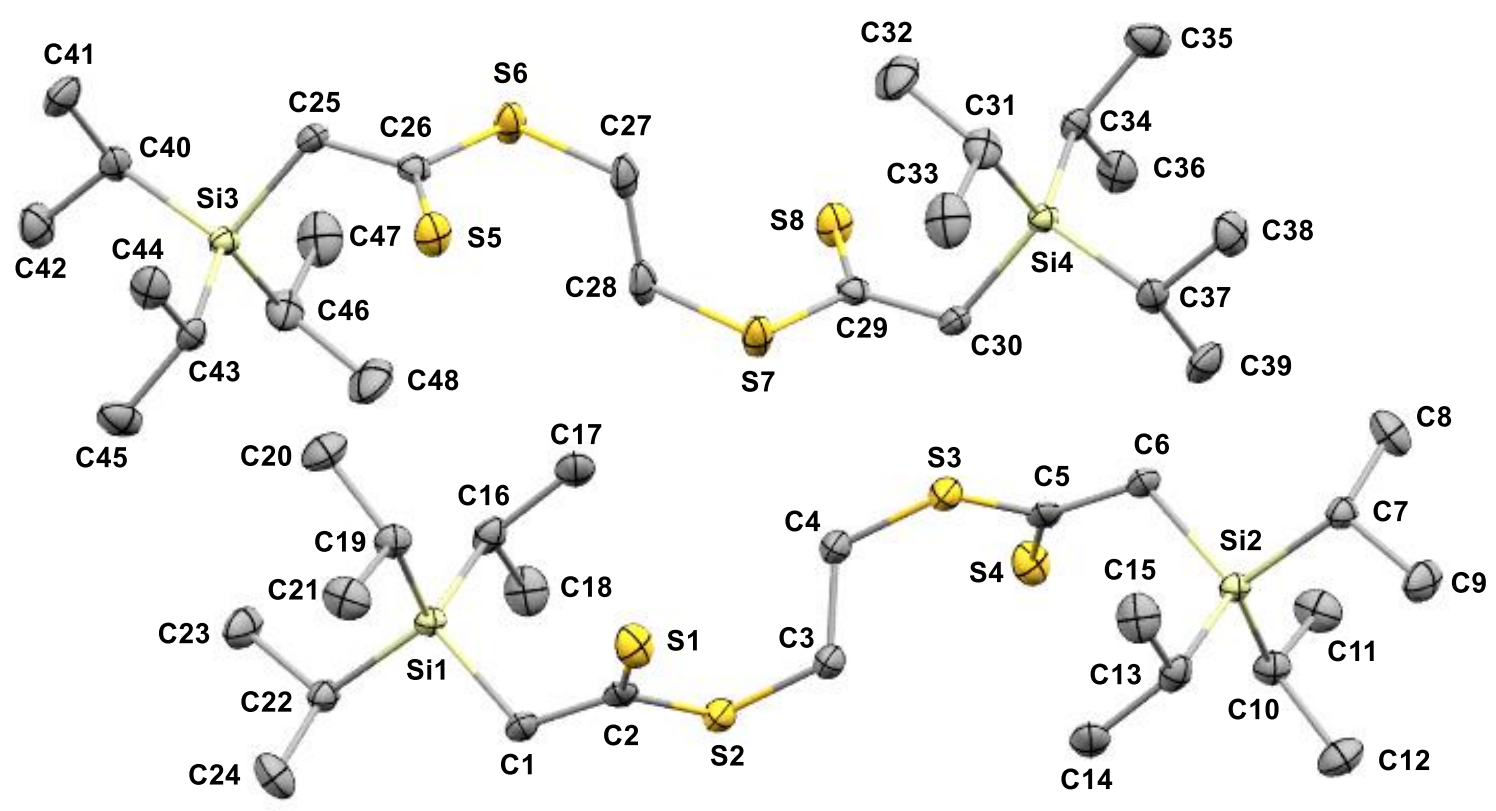

\begin{tabular}{ll} 
Empirical formula & $\mathrm{C}_{24} \mathrm{H}_{50} \mathrm{~S}_{4} \mathrm{Si}_{2}$ \\
Formula weight & 523.06 \\
Temperature/K & 100 \\
Crystal system & triclinic \\
Space group & $\mathrm{P}-1$ \\
$\mathrm{a} / \AA$ & $8.937(2)$ \\
$\mathrm{b} / \AA$ & $10.679(3)$ \\
$\mathrm{c} / \AA$ & $15.934(5)$ \\
$\alpha /{ }^{\circ}$ & $87.869(9)$ \\
$\beta /{ }^{\circ}$ & $87.665(9)$ \\
$\gamma /{ }^{\circ}$ & $89.140(7)$ \\
Volume/ $\AA^{3}$ & $1518.3(8)$ \\
$\mathrm{Z}$ & 2 \\
$\rho_{\text {calcg }} / \mathrm{cm}^{3}$ & 1.144 \\
$\mu / \mathrm{mm}^{-1}$ & 0.402 \\
$\mathrm{~F}(000)$ & 572.0 \\
Crystal size $/ \mathrm{mm}^{3}$ & $0.318 \times 0.256 \times 0.018$ \\
Radiation & $M o K \alpha(\lambda=0.71073)$ \\
\hline
\end{tabular}

$2 \Theta$ range for data collection $/{ }^{\circ} 4.518$ to 52.532

Index ranges

$-11 \leq \mathrm{h} \leq 11,-13 \leq \mathrm{k} \leq 13,0 \leq 1 \leq 19$

Reflections collected

6001

Independent reflections

$6001\left[\mathrm{R}_{\text {int }}=?, \mathrm{R}_{\text {sigma }}=0.0453\right]$ 
Data/restraints/parameters $\quad 6001 / 172 / 284$

Goodness-of-fit on $\mathrm{F}^{2} \quad 1.060$

Final $R$ indexes $[\mathrm{I}>=2 \sigma(\mathrm{I})] \quad \mathrm{R}_{1}=0.0525, \mathrm{wR}_{2}=0.1082$

Final $\mathrm{R}$ indexes [all data] $\quad \mathrm{R}_{1}=0.0634, \mathrm{wR}_{2}=0.1128$

Largest diff. peak/hole / e $\AA^{-3} 0.43 /-0.46$ 


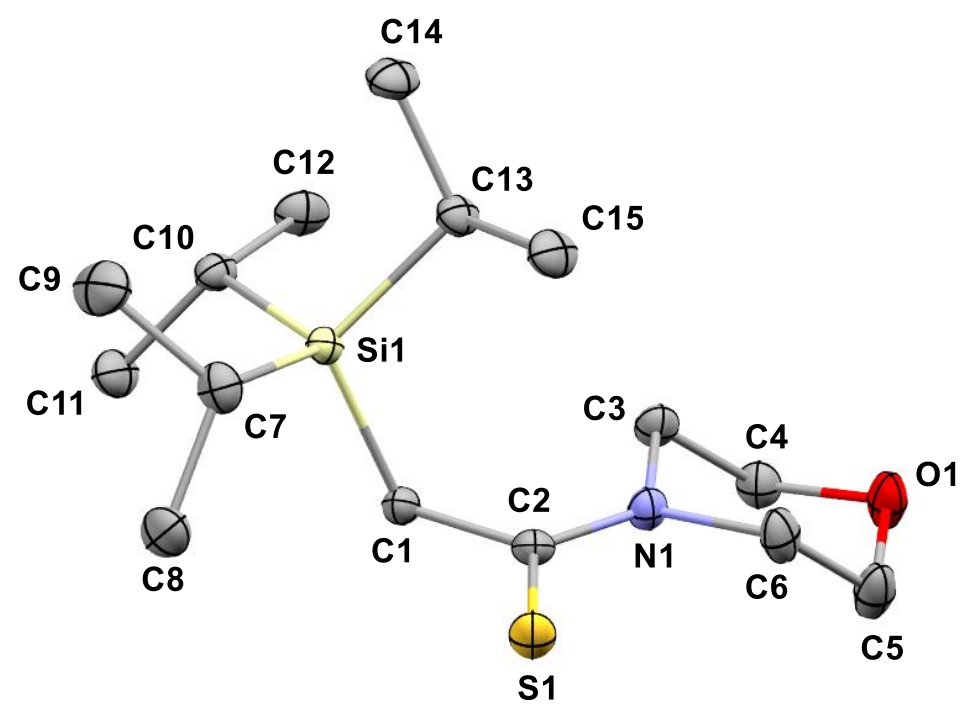

\begin{tabular}{|c|c|}
\hline Empirical formula & $\mathrm{C}_{20} \mathrm{H}_{39} \mathrm{NO}_{4} \mathrm{~S}_{2} \mathrm{Si}$ \\
\hline Formula weight & 449.73 \\
\hline Temperature/K & 99.98 \\
\hline Crystal system & orthorhombic \\
\hline Space group & $\mathrm{P} 2{ }_{1} 2_{1} 2_{1}$ \\
\hline $\mathrm{a} / \AA$ & $7.7423(8)$ \\
\hline $\mathrm{b} / \AA$ & $10.4197(8)$ \\
\hline $\mathrm{c} / \AA$ & $32.414(3)$ \\
\hline$\alpha /^{\circ}$ & 90 \\
\hline$\beta /{ }^{\circ}$ & 90 \\
\hline$\gamma /{ }^{\circ}$ & 90 \\
\hline Volume $/ \AA^{3}$ & $2614.9(4)$ \\
\hline $\mathrm{Z}$ & 4 \\
\hline$\rho_{\text {calc }} \mathrm{g} / \mathrm{cm}^{3}$ & 1.142 \\
\hline$\mu / \mathrm{mm}^{-1}$ & 0.272 \\
\hline $\mathrm{F}(000)$ & 976.0 \\
\hline Crystal size $/ \mathrm{mm}^{3}$ & $0.212 \times 0.164 \times 0.06$ \\
\hline Radiation & $\operatorname{MoK} \alpha(\lambda=0.71073)$ \\
\hline \multicolumn{2}{|c|}{$2 \Theta$ range for data collection $/{ }^{\circ} 5.026$ to 57.462} \\
\hline Index ranges & $-8 \leq \mathrm{h} \leq 10,-12 \leq \mathrm{k} \leq 14,-30 \leq 1 \leq 43$ \\
\hline Reflections collected & 21251 \\
\hline Independent reflections & $6635\left[\mathrm{R}_{\mathrm{int}}=0.0225, \mathrm{R}_{\mathrm{sigma}}=0.0250\right]$ \\
\hline Data/restraints/parameters & $6635 / 88 / 359$ \\
\hline Goodness-of-fit on $\mathrm{F}^{2}$ & 1.107 \\
\hline Final $\mathrm{R}$ indexes $[\mathrm{I}>=2 \sigma(\mathrm{I})]$ & $\mathrm{R}_{1}=0.0316, \mathrm{wR}_{2}=0.0721$ \\
\hline Final $\mathrm{R}$ indexes [all data] & $\mathrm{R}_{1}=0.0347, \mathrm{wR}_{2}=0.0738$ \\
\hline \multicolumn{2}{|c|}{ Largest diff. peak/hole / e $\AA^{-3} 0.20 /-0.24$} \\
\hline Flack parameter & $0.006(19)$ \\
\hline
\end{tabular}




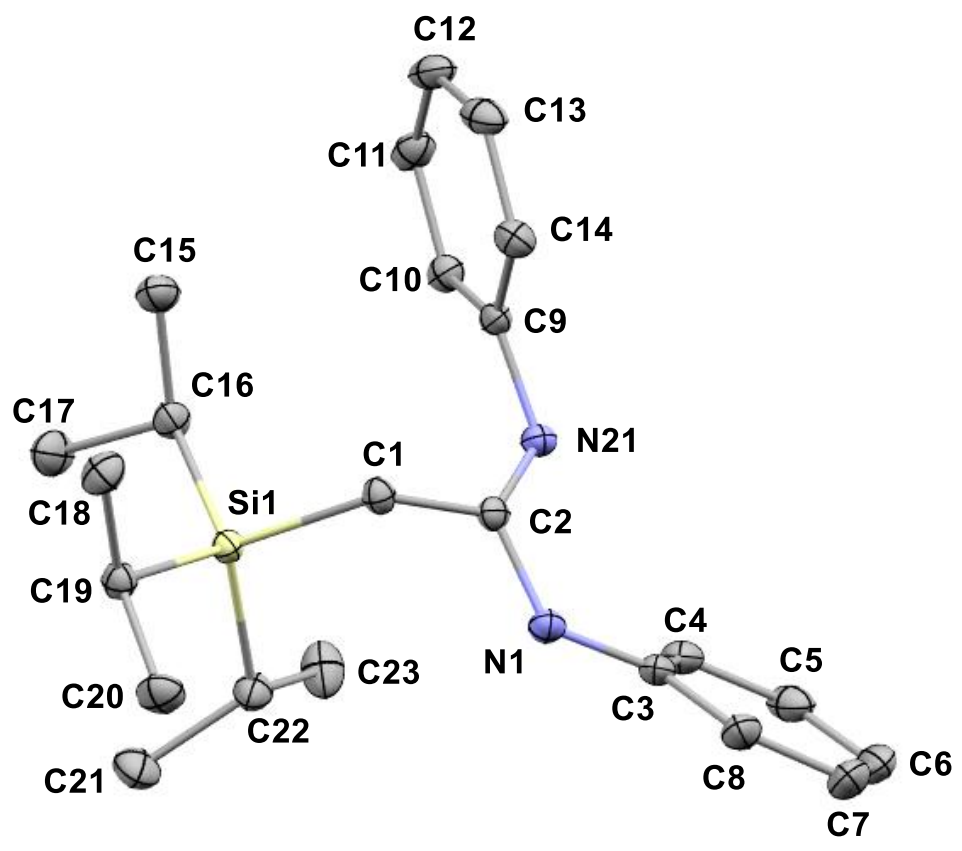

\begin{tabular}{ll} 
Empirical formula & $\mathrm{C}_{23} \mathrm{H}_{34} \mathrm{~N}_{2} \mathrm{Si}$ \\
Formula weight & 366.61 \\
Temperature/K & 99.98 \\
Crystal system & monoclinic \\
Space group & $\mathrm{P} 2{ }_{1} / \mathrm{c}$ \\
$\mathrm{a} / \AA$ & $9.7796(8)$ \\
$\mathrm{b} / \AA$ & $22.9294(18)$ \\
$\mathrm{c} / \AA$ & $9.9481(8)$ \\
$\alpha /{ }^{\circ}$ & 90 \\
$\beta /{ }^{\circ}$ & $103.240(3)$ \\
$\gamma^{\circ}$ & 90 \\
Volume/ $\AA^{3}$ & $2171.5(3)$ \\
$\mathrm{Z}$ & 4 \\
$\rho_{\text {calc }} \mathrm{g} / \mathrm{cm}^{3}$ & 1.121 \\
amidat $\mu / \mathrm{mm}^{-1}$ & 0.117 \\
$\mathrm{~F}(000)$ & 800.0 \\
Crystal size $/ \mathrm{mm}^{3}$ & $0.452 \times 0.168 \times 0.092$ \\
Radiation & $M o K \alpha(\lambda=0.71073)$ \\
\hline
\end{tabular}

$2 \Theta$ range for data collection $/{ }^{\circ} 5.562$ to 59.212

Index ranges

$-13 \leq \mathrm{h} \leq 13,-31 \leq \mathrm{k} \leq 31,-13 \leq 1 \leq 13$

Reflections collected

45001

Independent reflections

$6081\left[\mathrm{R}_{\text {int }}=0.0230, \mathrm{R}_{\text {sigma }}=0.0151\right]$

Data/restraints/parameters

$6081 / 0 / 245$

Goodness-of-fit on $\mathrm{F}^{2}$

1.072

Final $R$ indexes $[\mathrm{I}>=2 \sigma(\mathrm{I})] \quad \mathrm{R}_{1}=0.0328, \mathrm{wR}_{2}=0.0850$

Final $\mathrm{R}$ indexes [all data] $\quad \mathrm{R}_{1}=0.0346, \mathrm{wR}_{2}=0.0865$

Largest diff. peak/hole / e $\AA^{-3} 0.42 /-0.25$ 


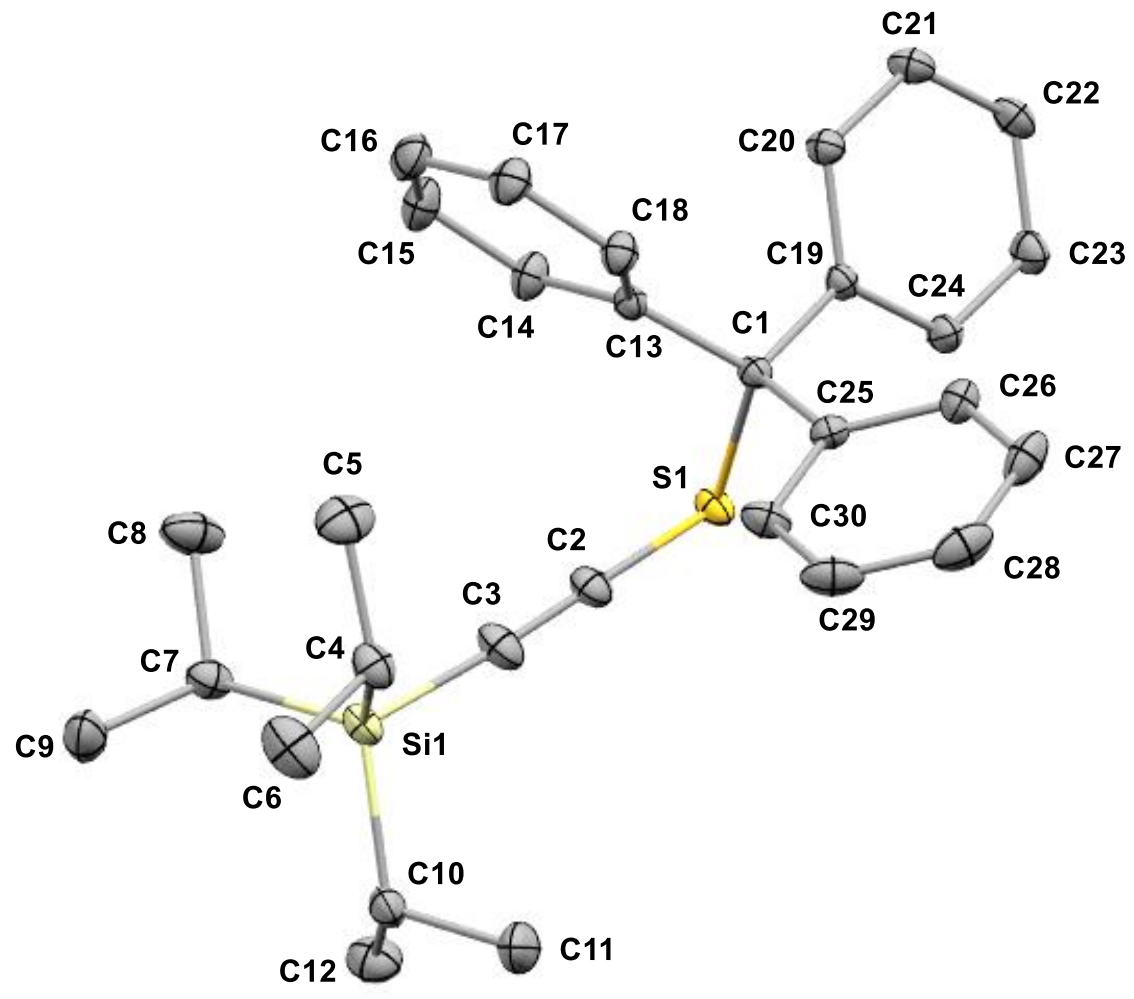

Empirical formula

Formula weight

Temperature/K

Crystal system

Space group

$\mathrm{a} / \AA$

$\mathrm{b} / \AA$

$\mathrm{c} / \AA$

$\alpha /{ }^{\circ}$

$\beta /{ }^{\circ}$

$\gamma /{ }^{\circ}$

Volume $/ \AA^{3}$

$\mathrm{Z}$

$\rho_{\text {calc }} \mathrm{g} / \mathrm{cm}^{3}$

$\mu / \mathrm{mm}^{-1}$

$\mathrm{F}(000)$

Crystal size $/ \mathrm{mm}^{3}$

Radiation
$\mathrm{C}_{30} \mathrm{H}_{36} \mathrm{SSi}$

456.74

100.02

triclinic

$\mathrm{P}-1$

9.7652(12)

$10.8607(11)$

$13.3088(15)$

98.629(4)

92.583(4)

$108.012(4)$

$1320.8(3)$

2

1.148

0.183

492.0

$0.273 \times 0.241 \times 0.198$

$\operatorname{MoK} \alpha(\lambda=0.71073)$

$2 \Theta$ range for data collection $/{ }^{\circ} 4.406$ to 61.074

Index ranges

Reflections collected

Independent reflections
$-13 \leq \mathrm{h} \leq 13,-15 \leq \mathrm{k} \leq 15,-19 \leq 1 \leq 19$

109718

$8053\left[\mathrm{R}_{\text {int }}=0.0235, \mathrm{R}_{\text {sigma }}=0.0117\right]$ 
Data/restraints/parameters $\quad 8053 / 0 / 295$

Goodness-of-fit on $\mathrm{F}^{2} \quad 1.036$

Final $R$ indexes $[\mathrm{I}>=2 \sigma(\mathrm{I})] \quad \mathrm{R}_{1}=0.0294, \mathrm{wR}_{2}=0.0809$

Final $\mathrm{R}$ indexes [all data] $\quad \mathrm{R}_{1}=0.0313, \mathrm{wR}_{2}=0.0827$

Largest diff. peak/hole / e $\AA^{-3} 0.43 /-0.27$ 

(188):

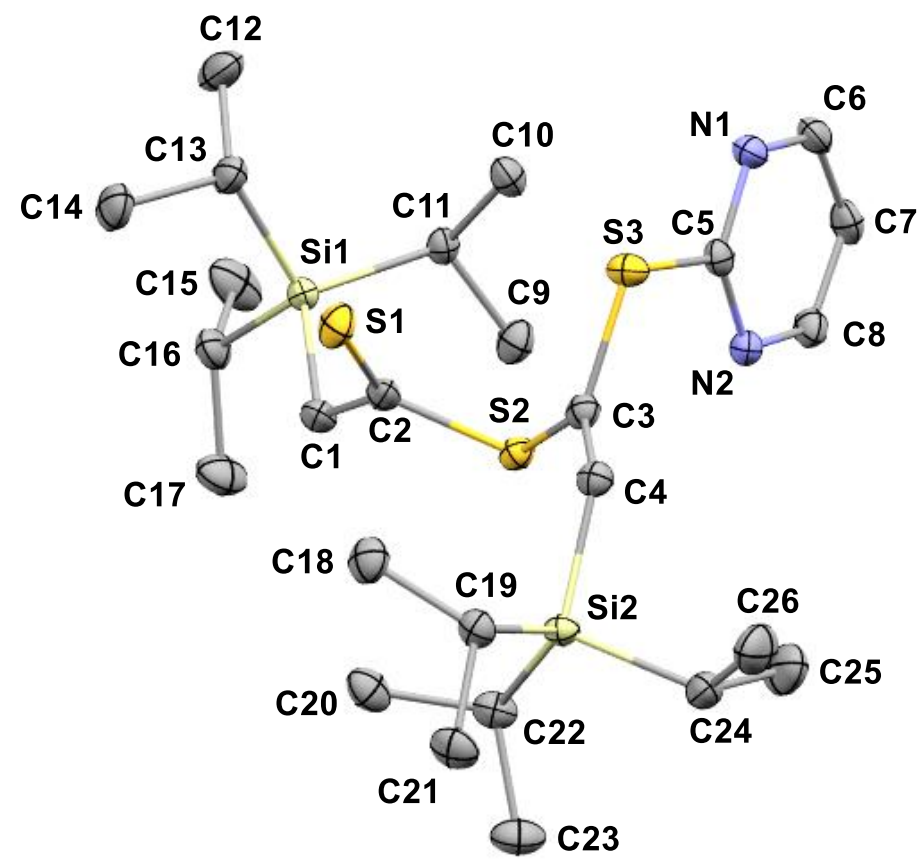

Empirical formula

$\mathrm{C}_{26} \mathrm{H}_{48} \mathrm{~N}_{2} \mathrm{~S}_{3} \mathrm{Si}_{2}$

Formula weight

541.02

Temperature/K

100.02

Crystal system

monoclinic

Space group

$\mathrm{P} 21 / \mathrm{n}$

$\mathrm{a} / \AA$

13.2581(12)

$\mathrm{b} / \AA$

$9.8280(12)$

$\mathrm{c} / \AA$

$24.350(3)$

$\alpha /^{\circ}$

90

$\beta /{ }^{\circ}$

$\gamma /{ }^{\circ}$

103.907(3)

90

Volume $/ \AA^{3}$

3079.9(6)

Z

4

$\rho_{\text {calc }} \mathrm{g} / \mathrm{cm}^{3}$

1.167

$\mu / \mathrm{mm}^{-1}$

0.336

$\mathrm{F}(000)$

1176.0

Crystal size $/ \mathrm{mm}^{3}$

$0.429 \times 0.425 \times 0.05$

Radiation

$\operatorname{MoK} \alpha(\lambda=0.71073)$

$2 \Theta$ range for data collection $/{ }^{\circ} 5.216$ to 54.262

Index ranges

Reflections collected

Independent reflections

Data/restraints/parameters

Goodness-of-fit on $\mathrm{F}^{2}$

Final $\mathrm{R}$ indexes $[\mathrm{I}>=2 \sigma(\mathrm{I})]$
$-17 \leq \mathrm{h} \leq 16,0 \leq \mathrm{k} \leq 12,0 \leq 1 \leq 31$

7023

$7023\left[\mathrm{R}_{\text {int }}=?, \mathrm{R}_{\text {sigma }}=0.0255\right]$

7023/0/311

1.052

$\mathrm{R}_{1}=0.0328, \mathrm{wR}_{2}=0.0787$ 
Final $\mathrm{R}$ indexes [all data] $\quad \mathrm{R}_{1}=0.0396, \mathrm{wR}_{2}=0.0832$

Largest diff. peak/hole / e $\AA^{-3} 0.51 /-0.21$ 


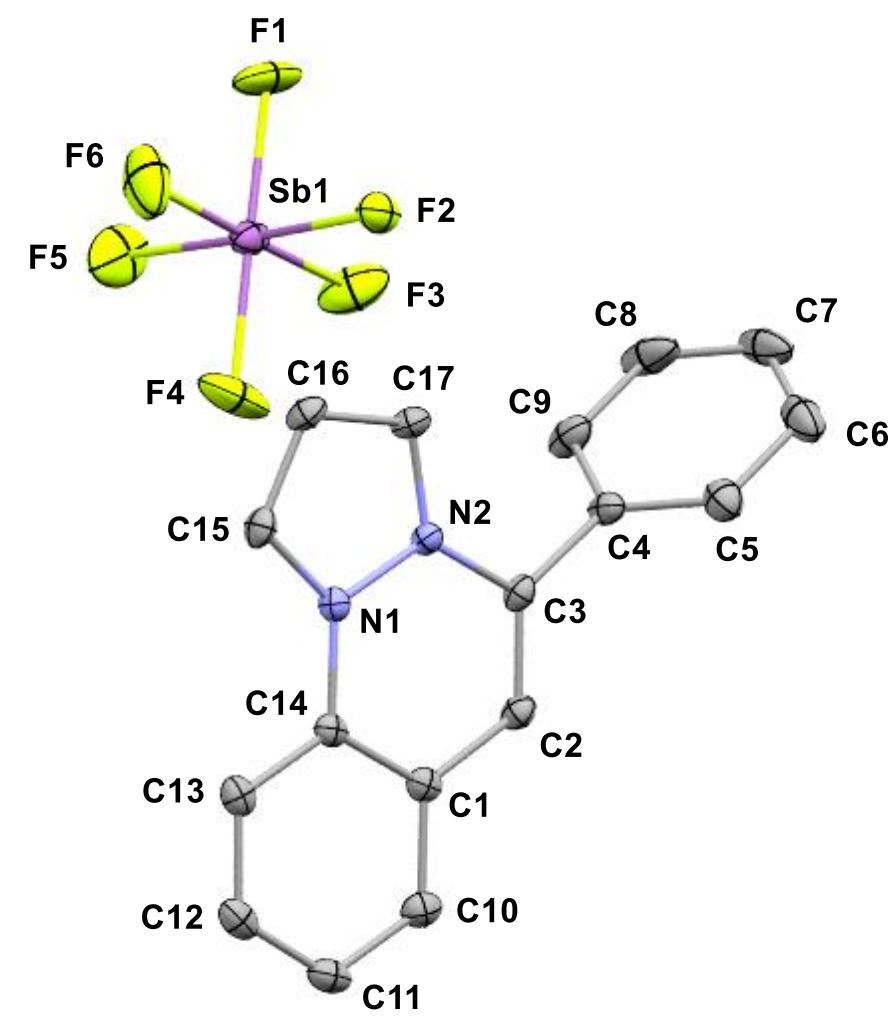

Empirical formula

Formula weight

Temperature/K

Crystal system

Space group

$\mathrm{a} / \AA$

$\mathrm{b} / \AA$

$\mathrm{c} / \AA$

$\alpha /^{\circ}$

$\beta /{ }^{\circ}$

$\gamma /{ }^{\circ}$

Volume $/ \AA^{3}$

$\mathrm{Z}$

$\rho_{\text {calc }} \mathrm{g} / \mathrm{cm}^{3}$

$\mu / \mathrm{mm}^{-1}$

$\mathrm{F}(000)$

Crystal size $/ \mathrm{mm}^{3}$

Radiation

$2 \Theta$ range for data collection/ ${ }^{\circ}$

Index ranges

Reflections collected

Independent reflections

Data/restraints/parameters

Goodness-of-fit on $\mathrm{F}^{2}$

Final $\mathrm{R}$ indexes $[\mathrm{I}>=2 \sigma(\mathrm{I})]$

Final R indexes [all data]

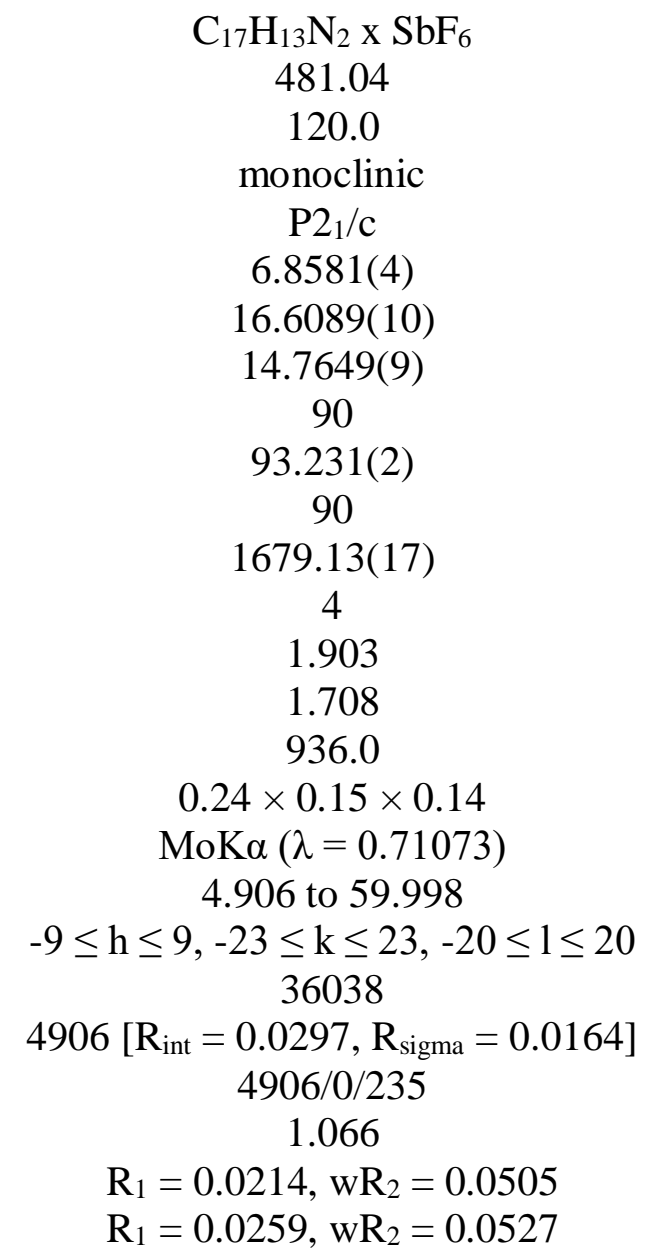


Largest diff. peak/hole / e $\AA^{-3}$

$0.67 /-0.90$ 


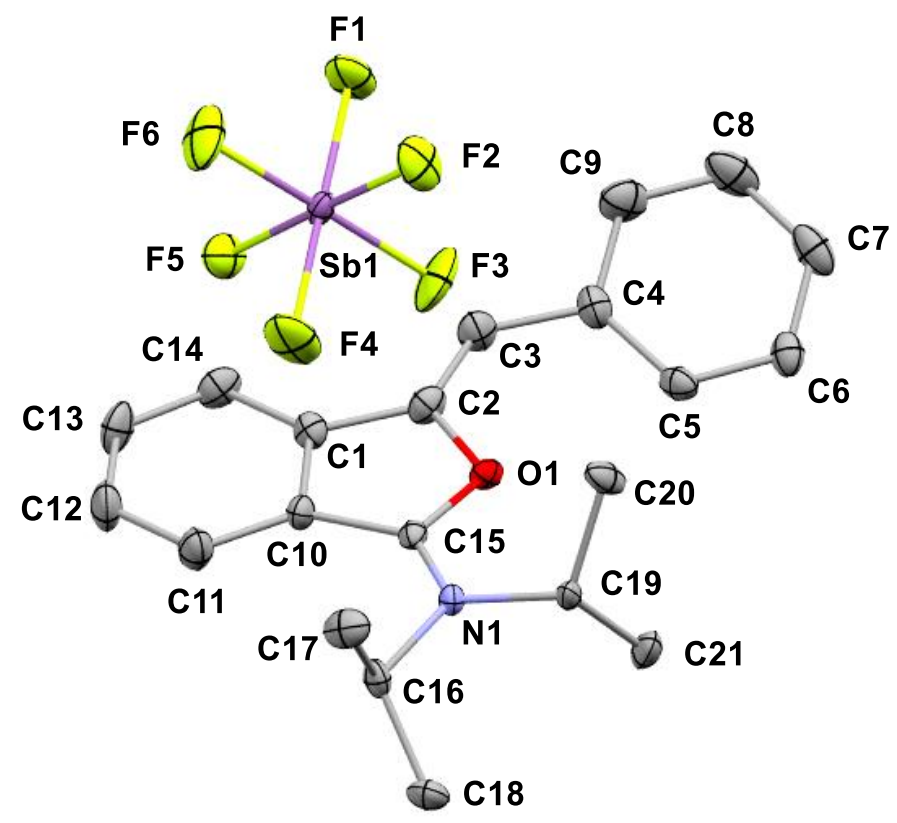

Empirical formula

Formula weight

Temperature/K

Crystal system

Space group

$\mathrm{a} / \AA$

$\mathrm{b} / \AA$

$\mathrm{c} / \AA$

$\alpha /{ }^{\circ}$

$\beta /{ }^{\circ}$

$\gamma /{ }^{\circ}$

Volume $/ \AA^{3}$

$\mathrm{Z}$

$\rho_{\text {calc }} \mathrm{g} / \mathrm{cm}^{3}$

$\mu / \mathrm{mm}^{-1}$

$\mathrm{F}(000)$

Crystal size $/ \mathrm{mm}^{3}$

Radiation
$\mathrm{C}_{21} \mathrm{H}_{24} \mathrm{~F}_{6} \mathrm{NOSb}$

542.16

100.0

monoclinic

$\mathrm{P} 2{ }_{1} / \mathrm{c}$

8.2054(3)

$17.6617(6)$

$15.2985(5)$

90

103.954(2)

90

2151.65(13)

4

1.674

1.345

1080.0

$0.177 \times 0.136 \times 0.054$

$\operatorname{MoK} \alpha(\lambda=0.71073)$

$2 \Theta$ range for data collection $/{ }^{\circ} 4.612$ to 61.166

Index ranges

Reflections collected

Independent reflections

Data/restraints/parameters

Goodness-of-fit on $\mathrm{F}^{2}$

Final $\mathrm{R}$ indexes $[\mathrm{I}>=2 \sigma(\mathrm{I})]$

Final $\mathrm{R}$ indexes [all data]
$-11 \leq \mathrm{h} \leq 11,-24 \leq \mathrm{k} \leq 24,-21 \leq 1 \leq 21$

69350

$6568\left[\mathrm{R}_{\text {int }}=0.0449, \mathrm{R}_{\text {sigma }}=0.0248\right]$

$6568 / 0 / 275$

1.034

$\mathrm{R}_{1}=0.0316, \mathrm{wR}_{2}=0.0665$

$\mathrm{R}_{1}=0.0439, \mathrm{wR}_{2}=0.0719$ 
Largest diff. peak/hole / e $\AA^{-3} 1.27 /-1.02$ 


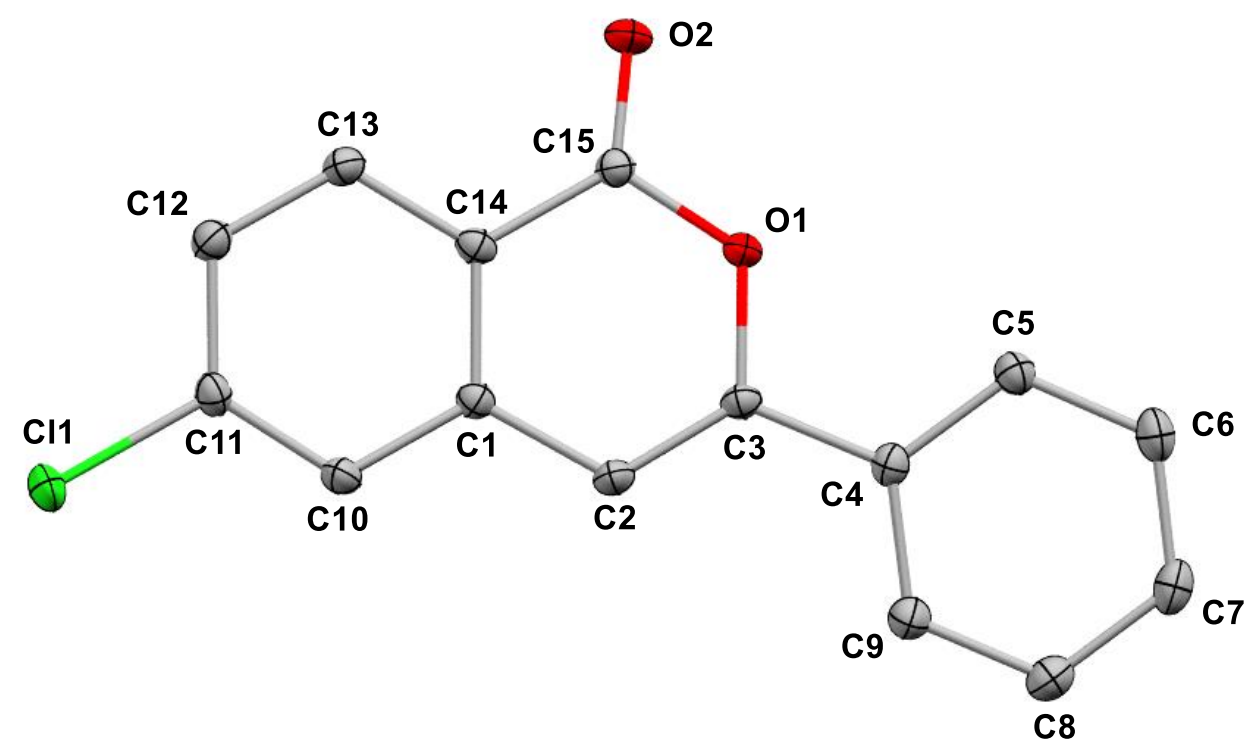

Empirical formula

Formula weight

Temperature/K

Crystal system

Space group

$\mathrm{a} / \AA$

$\mathrm{b} / \AA$

$\mathrm{c} / \AA$

$\alpha /{ }^{\circ}$

$\beta /{ }^{\circ}$

$\gamma /{ }^{\circ}$

Volume $/ \AA^{3}$

$\mathrm{Z}$

$\rho_{\text {calc }} \mathrm{g} / \mathrm{cm}^{3}$

$\mu / \mathrm{mm}^{-1}$

$\mathrm{F}(000)$

Crystal size $/ \mathrm{mm}^{3}$

Radiation
$\mathrm{C}_{15} \mathrm{H}_{9} \mathrm{ClO}_{2}$

256.67

99.98

monoclinic

$\mathrm{P} 2{ }_{1} / \mathrm{n}$

$5.9730(8)$

$3.8086(5)$

49.432(7)

90

90.944(3)

90

1124.4(3)

4

1.516

0.328

528.0

$0.971 \times 0.218 \times 0.05$

$\operatorname{MoK} \alpha(\lambda=0.71073)$

$2 \Theta$ range for data collection $/{ }^{\circ} 4.944$ to 57.44

Index ranges

Reflections collected

Independent reflections

Data/restraints/parameters

Goodness-of-fit on $\mathrm{F}^{2}$

Final $R$ indexes $[\mathrm{I}>=2 \sigma(\mathrm{I})]$

Final $R$ indexes [all data]

Largest diff. peak/hole / e $\AA^{-3} 0.51 /-0.28$
$-8 \leq \mathrm{h} \leq 8,-4 \leq \mathrm{k} \leq 5,-66 \leq 1 \leq 66$

22133

$2857\left[\mathrm{R}_{\text {int }}=0.0241, \mathrm{R}_{\text {sigma }}=0.0143\right]$

$2857 / 0 / 163$

1.172

$\mathrm{R}_{1}=0.0440, \mathrm{w} \mathrm{R}_{2}=0.1276$

$\mathrm{R}_{1}=0.0453, \mathrm{wR}_{2}=0.1286$ 


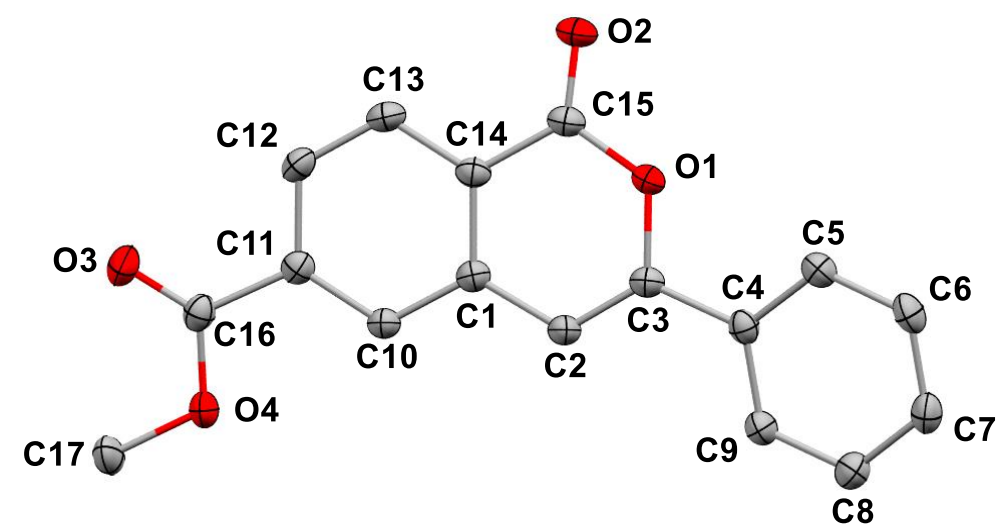

\begin{tabular}{|c|c|}
\hline Empirical formula & $\mathrm{C}_{17} \mathrm{H}_{12} \mathrm{O}_{4}$ \\
\hline Formula weight & 280.27 \\
\hline Temperature/K & 100.0 \\
\hline Crystal system & orthorhombic \\
\hline Space group & $\mathrm{P} 2{ }_{1} 2_{1} 2_{1}$ \\
\hline $\mathrm{a} / \AA$ & $3.8298(8)$ \\
\hline $\mathrm{b} / \AA$ & $6.1007(12)$ \\
\hline $\mathrm{c} / \AA$ & $54.735(11)$ \\
\hline$\alpha /^{\circ}$ & 90 \\
\hline$\beta /{ }^{\circ}$ & 90 \\
\hline$\gamma /{ }^{\circ}$ & 90 \\
\hline Volume $/ \AA^{3}$ & $1278.8(4)$ \\
\hline $\mathrm{Z}$ & 4 \\
\hline$\rho_{\text {calc }} \mathrm{g} / \mathrm{cm}^{3}$ & 1.456 \\
\hline$\mu / \mathrm{mm}^{-1}$ & 0.861 \\
\hline $\mathrm{F}(000)$ & 584.0 \\
\hline Crystal size $/ \mathrm{mm}^{3}$ & $0.322 \times 0.081 \times 0.042$ \\
\hline Radiation & $\mathrm{CuK} \alpha(\lambda=1.54178)$ \\
\hline \multicolumn{2}{|c|}{$2 \Theta$ range for data collection $/{ }^{\circ} 9.696$ to 159.788} \\
\hline Index ranges & $-4 \leq \mathrm{h} \leq 4,-7 \leq \mathrm{k} \leq 7,-69 \leq 1 \leq 49$ \\
\hline Reflections collected & 19765 \\
\hline Independent reflections & $2713\left[\mathrm{R}_{\text {int }}=0.0345, \mathrm{R}_{\text {sigma }}=0.0211\right]$ \\
\hline Data/restraints/parameters & $2713 / 0 / 191$ \\
\hline Goodness-of-fit on $\mathrm{F}^{2}$ & 1.096 \\
\hline Final $R$ indexes $[\mathrm{I}>=2 \sigma(\mathrm{I})]$ & $\mathrm{R}_{1}=0.0317, \mathrm{wR}_{2}=0.0833$ \\
\hline Final $\mathrm{R}$ indexes [all data] & $\mathrm{R}_{1}=0.0319, \mathrm{wR}_{2}=0.0834$ \\
\hline \multicolumn{2}{|c|}{ Largest diff. peak/hole / e $\AA^{-3} 0.27 /-0.17$} \\
\hline Flack parameter & $-0.09(8)$ \\
\hline
\end{tabular}




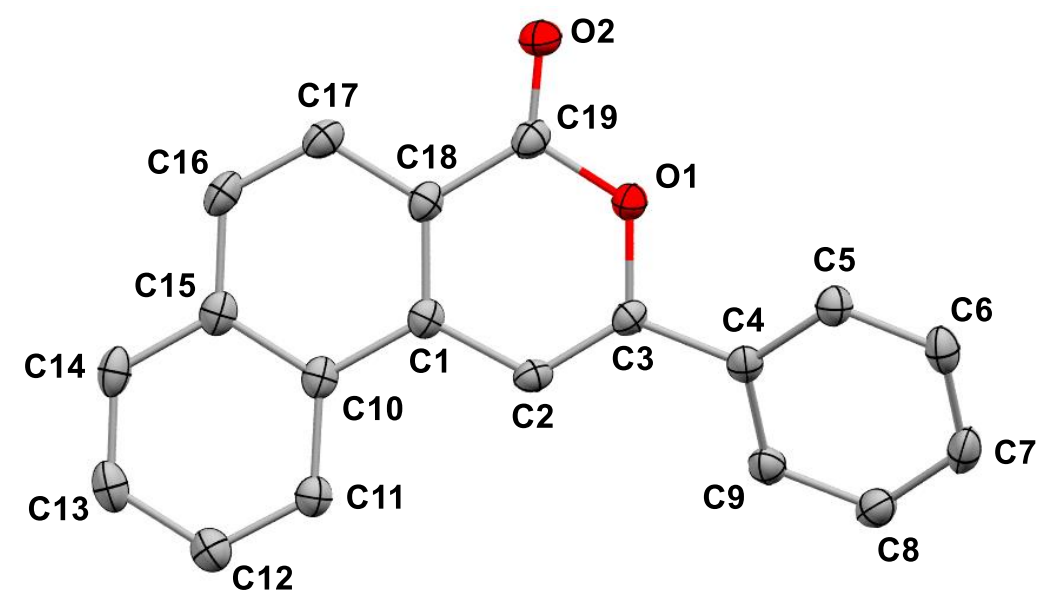

Empirical formula

Formula weight

Temperature/K

Crystal system

Space group

$\mathrm{a} / \AA$

$\mathrm{b} / \AA$

$\mathrm{c} / \AA$

$\alpha /^{\circ}$

$\beta /{ }^{\circ}$

$\gamma /{ }^{\circ}$

Volume $/ \AA^{3}$

$\mathrm{Z}$

$\rho_{\text {calc }} \mathrm{g} / \mathrm{cm}^{3}$

$\mu / \mathrm{mm}^{-1}$

$\mathrm{F}(000)$

Crystal size $/ \mathrm{mm}^{3}$

Radiation
$\mathrm{C}_{19} \mathrm{H}_{12} \mathrm{O}_{2}$

272.29

100.02

orthorhombic

$\mathrm{P} 2{ }_{1}{ }_{1} 21$

3.9872(6)

14.224(3)

22.401(4)

90

90

90

1270.4(4)

4

1.424

0.092

568.0

$0.948 \times 0.104 \times 0.038$

$\operatorname{MoK} \alpha(\lambda=0.71073)$

$2 \Theta$ range for data collection/ ${ }^{\circ} 5.728$ to 55.804

Index ranges

Reflections collected

Independent reflections

Data/restraints/parameters

Goodness-of-fit on $\mathrm{F}^{2}$

Final $\mathrm{R}$ indexes $[\mathrm{I}>=2 \sigma(\mathrm{I})]$

Final $\mathrm{R}$ indexes [all data]

Largest diff. peak/hole / e $\AA^{-3}$

Flack parameter
$-5 \leq \mathrm{h} \leq 5,-16 \leq \mathrm{k} \leq 18,-29 \leq 1 \leq 23$

12819

$3045\left[\mathrm{R}_{\text {int }}=0.0308, \mathrm{R}_{\text {sigma }}=0.0279\right]$

$3045 / 0 / 190$

1.058

$\mathrm{R}_{1}=0.0365, \mathrm{wR}_{2}=0.0843$

$\mathrm{R}_{1}=0.0431, \mathrm{wR}_{2}=0.0880$

$0.22 /-0.23$

$-0.2(5)$ 


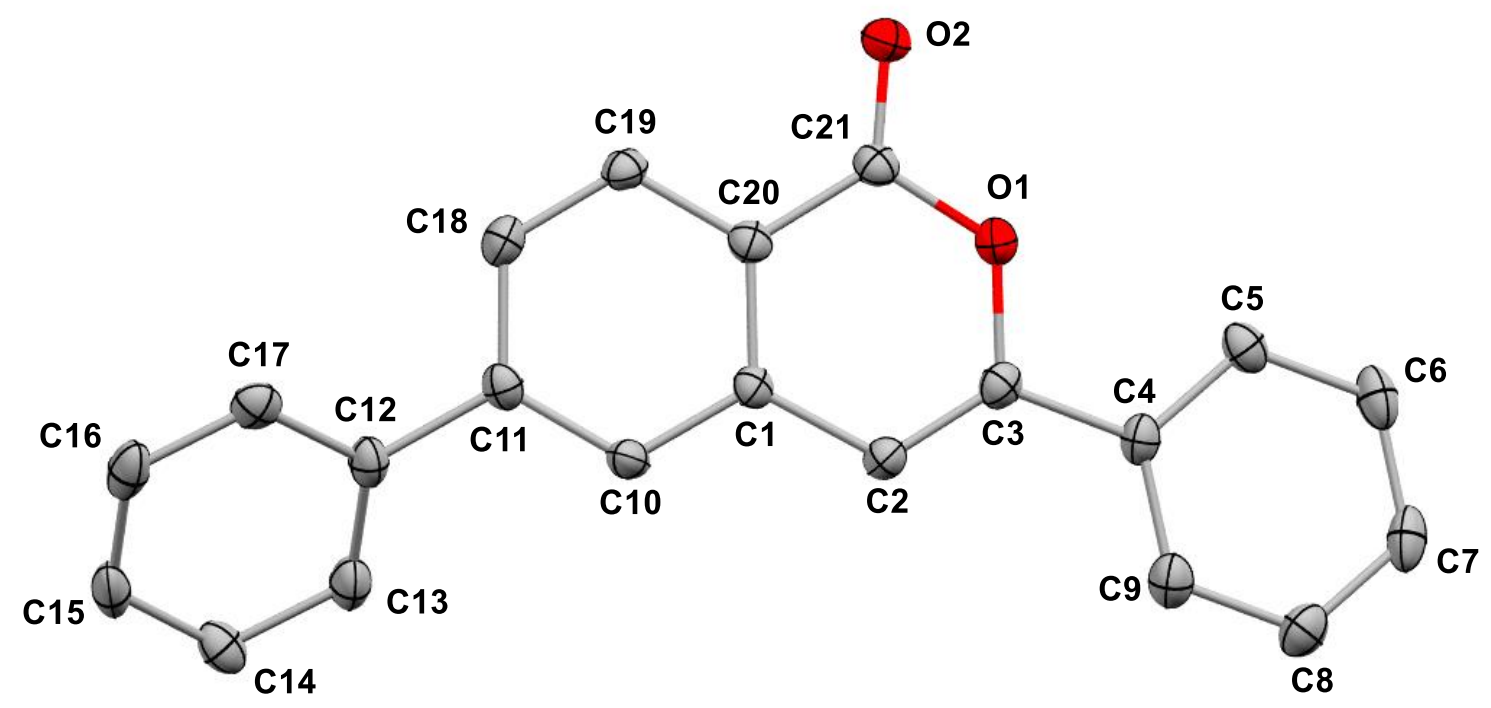

Empirical formula

$\mathrm{C}_{21} \mathrm{H}_{14} \mathrm{O}_{2}$

Formula weight

298.32

Temperature/K

100.0

Crystal system

triclinic

Space group

P-1

$\mathrm{a} / \AA$

13.7646(17)

$\mathrm{b} / \AA$

$13.8232(16)$

$\mathrm{c} / \AA$

$30.538(4)$

$\alpha /^{\circ}$

89.363(3)

$\beta /{ }^{\circ}$

$89.900(4)$

$\gamma /{ }^{\circ}$

$89.945(4)$

Volume $/ \AA^{3}$

$5810.0(12)$

$\mathrm{Z}$

16

$\rho_{\text {calc }} \mathrm{g} / \mathrm{cm}^{3}$

1.364

$\mu / \mathrm{mm}^{-1}$

0.087

$\mathrm{F}(000)$

2496.0

Crystal size $/ \mathrm{mm}^{3}$

$0.204 \times 0.187 \times 0.121$

Radiation

$\operatorname{MoK} \alpha(\lambda=0.71073)$

$2 \Theta$ range for data collection $/{ }^{\circ} 4.37$ to 57.518

Index ranges

$-18 \leq \mathrm{h} \leq 18,-18 \leq \mathrm{k} \leq 18,-41 \leq 1 \leq 41$

Reflections collected

83632

Independent reflections

$29981\left[\mathrm{R}_{\mathrm{int}}=0.0491, \mathrm{R}_{\text {sigma }}=0.0663\right]$

Data/restraints/parameters

$29981 / 0 / 1658$

Goodness-of-fit on $\mathrm{F}^{2}$

1.041

Final $\mathrm{R}$ indexes $[\mathrm{I}>=2 \sigma(\mathrm{I})]$

$\mathrm{R}_{1}=0.0580, \mathrm{wR}_{2}=0.1474$

Final $R$ indexes [all data] $\quad \mathrm{R}_{1}=0.0979, \mathrm{wR}_{2}=0.1678$

Largest diff. peak/hole / e $\AA^{-3} 0.44 /-0.30$ 


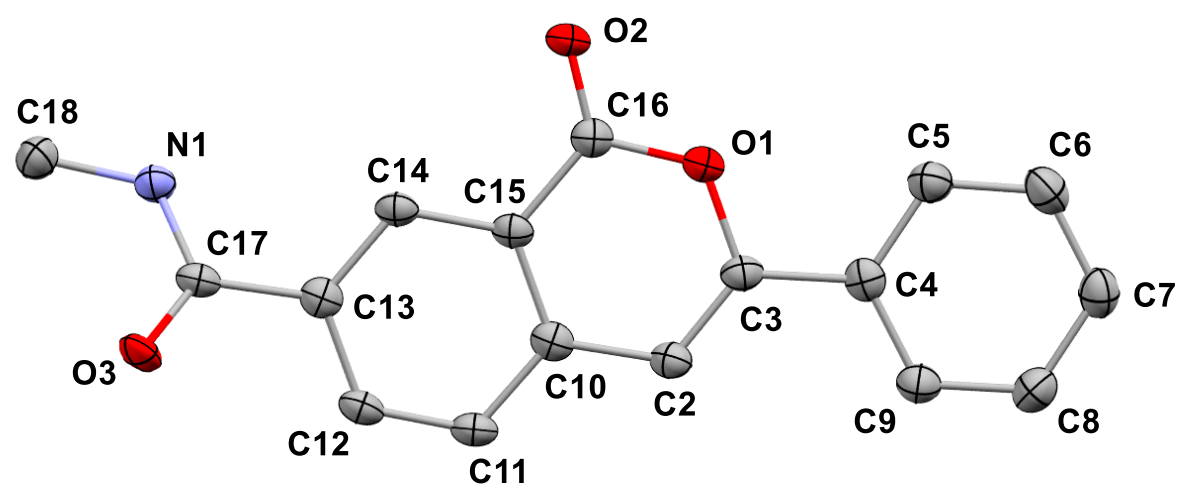

\begin{tabular}{|c|c|}
\hline Empirical formula & $\mathrm{C}_{17} \mathrm{H}_{13} \mathrm{NO}_{3}$ \\
\hline Formula weight & 279.28 \\
\hline Temperature/K & 99.98 \\
\hline Crystal system & monoclinic \\
\hline Space group & $\mathrm{P} 2_{1}$ \\
\hline $\mathrm{a} / \AA$ & $5.0291(4)$ \\
\hline $\mathrm{b} / \AA$ & $5.1512(4)$ \\
\hline $\mathrm{c} / \AA$ & $25.4093(19)$ \\
\hline$\alpha /^{\circ}$ & 90 \\
\hline$\beta /{ }^{\circ}$ & $93.484(2)$ \\
\hline$\gamma /{ }^{\circ}$ & 90 \\
\hline Volume $/ \AA^{3}$ & $657.03(9)$ \\
\hline $\mathrm{Z}$ & 2 \\
\hline$\rho_{\text {calc }} \mathrm{g} / \mathrm{cm}^{3}$ & 1.412 \\
\hline$\mu / \mathrm{mm}^{-1}$ & 0.798 \\
\hline $\mathrm{F}(000)$ & 292.0 \\
\hline Crystal size $/ \mathrm{mm}^{3}$ & $0.516 \times 0.089 \times 0.051$ \\
\hline Radiation & $\mathrm{CuK} \alpha(\lambda=1.54178)$ \\
\hline \multicolumn{2}{|c|}{$2 \Theta$ range for data collection $/{ }^{\circ} 10.464$ to 149.092} \\
\hline Index ranges & $-6 \leq \mathrm{h} \leq 6,-6 \leq \mathrm{k} \leq 6,-31 \leq 1 \leq 31$ \\
\hline Reflections collected & 2709 \\
\hline Independent reflections & $2709\left[\mathrm{R}_{\text {int }}=?, \mathrm{R}_{\text {sigma }}=0.0298\right]$ \\
\hline Data/restraints/parameters & $2709 / 1 / 195$ \\
\hline Goodness-of-fit on $\mathrm{F}^{2}$ & 1.097 \\
\hline Final $R$ indexes $[\mathrm{I}>=2 \sigma(\mathrm{I})]$ & $\mathrm{R}_{1}=0.0371, \mathrm{wR}_{2}=0.1062$ \\
\hline Final $\mathrm{R}$ indexes [all data] & $\mathrm{R}_{1}=0.0372, \mathrm{wR}_{2}=0.1068$ \\
\hline \multicolumn{2}{|c|}{ Largest diff. peak/hole / e $\AA^{-3} 0.17 /-0.18$} \\
\hline Flack parameter & $-0.23(19)$ \\
\hline
\end{tabular}


(Z)-N-(6-Methoxy-3-phenyl-1H-isochromen-1-ylidene)methanaminium hexafluoroantimonate (229):
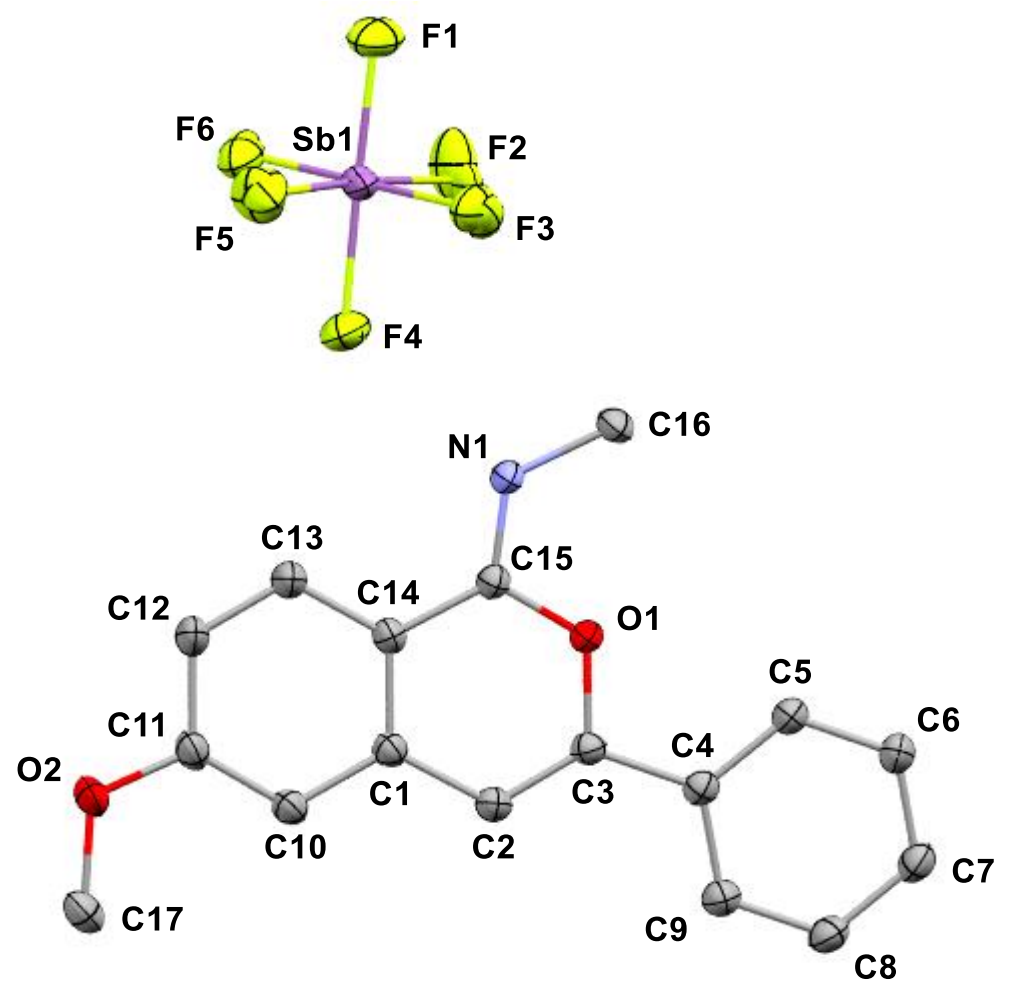

Empirical formula

Formula weight

Temperature/K

Crystal system

Space group

$\mathrm{a} / \AA$

$\mathrm{b} / \AA$

$\mathrm{c} / \AA$

$\alpha /^{\circ}$

$\beta /{ }^{\circ}$

$\gamma /{ }^{\circ}$

Volume $/ \AA^{3}$

$\mathrm{Z}$

$\rho_{\text {calc }} \mathrm{g} / \mathrm{cm}^{3}$

$\mu / \mathrm{mm}^{-1}$

$\mathrm{F}(000)$

Crystal size $/ \mathrm{mm}^{3}$

Radiation
$\mathrm{C}_{17} \mathrm{H}_{16} \mathrm{~F}_{6} \mathrm{NO}_{2} \mathrm{Sb}$

502.06

100.0

triclinic

P-1

9.1398(5)

$10.6685(7)$

$10.9432(6)$

$68.724(2)$

$68.283(2)$

$66.756(2)$

$880.98(9)$

2

1.893

1.639

492.0

$0.56 \times 0.35 \times 0.114$

$\operatorname{MoK} \alpha(\lambda=0.71073)$

$2 \Theta$ range for data collection $/{ }^{\circ} 5.276$ to 61.082

Index ranges

Reflections collected

Independent reflections

Data/restraints/parameters
$-13 \leq \mathrm{h} \leq 13,-15 \leq \mathrm{k} \leq 15,-15 \leq 1 \leq 15$

26295

$5376\left[\mathrm{R}_{\text {int }}=0.0210, \mathrm{R}_{\text {sigma }}=0.0158\right]$

5376/84/313 
Goodness-of-fit on $\mathrm{F}^{2}$

1.124

Final $R$ indexes $[\mathrm{I}>=2 \sigma(\mathrm{I})] \quad \mathrm{R}_{1}=0.0144, \mathrm{wR}_{2}=0.0384$

Final $\mathrm{R}$ indexes [all data] $\quad \mathrm{R}_{1}=0.0148, \mathrm{wR}_{2}=0.0386$

Largest diff. peak/hole / e $\AA^{-3} 0.44 /-0.27$ 


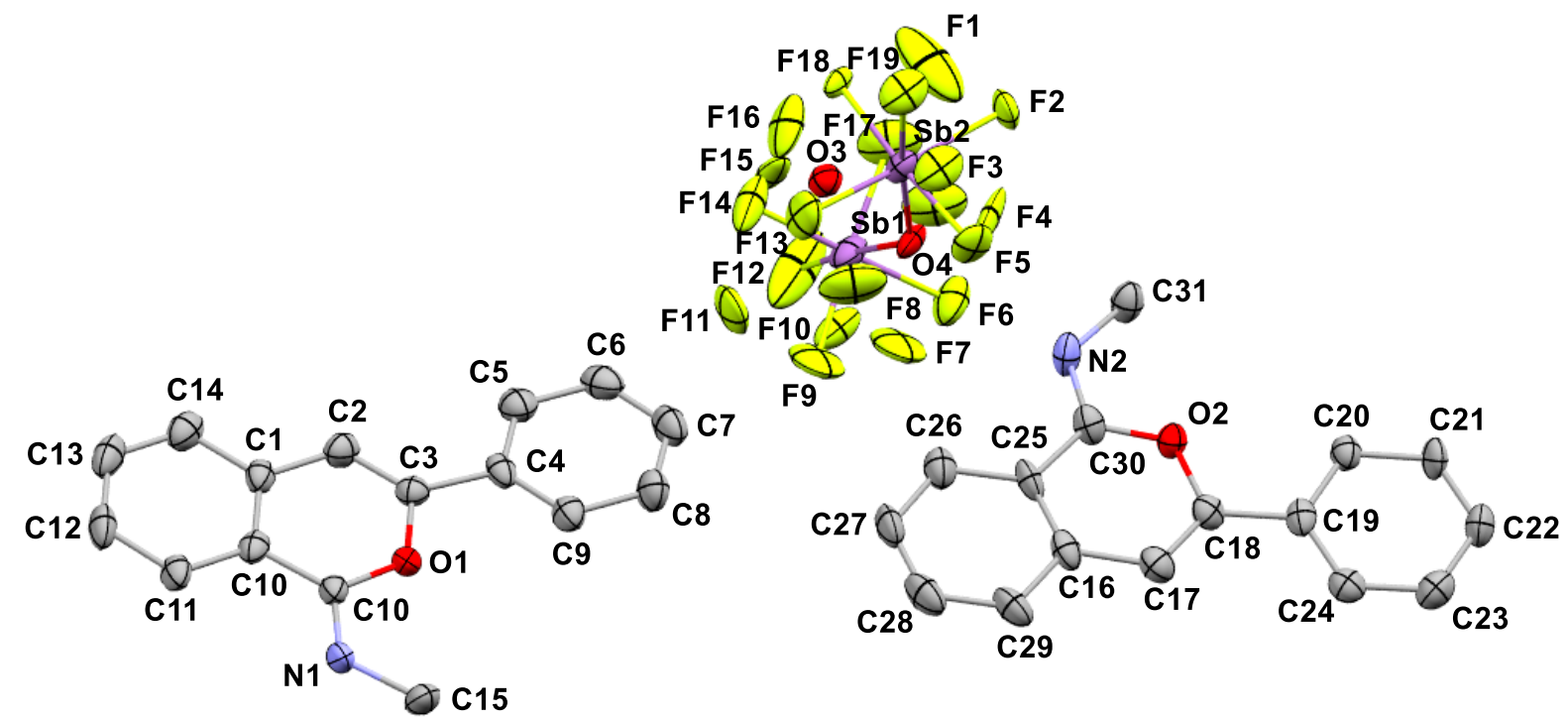



(230):

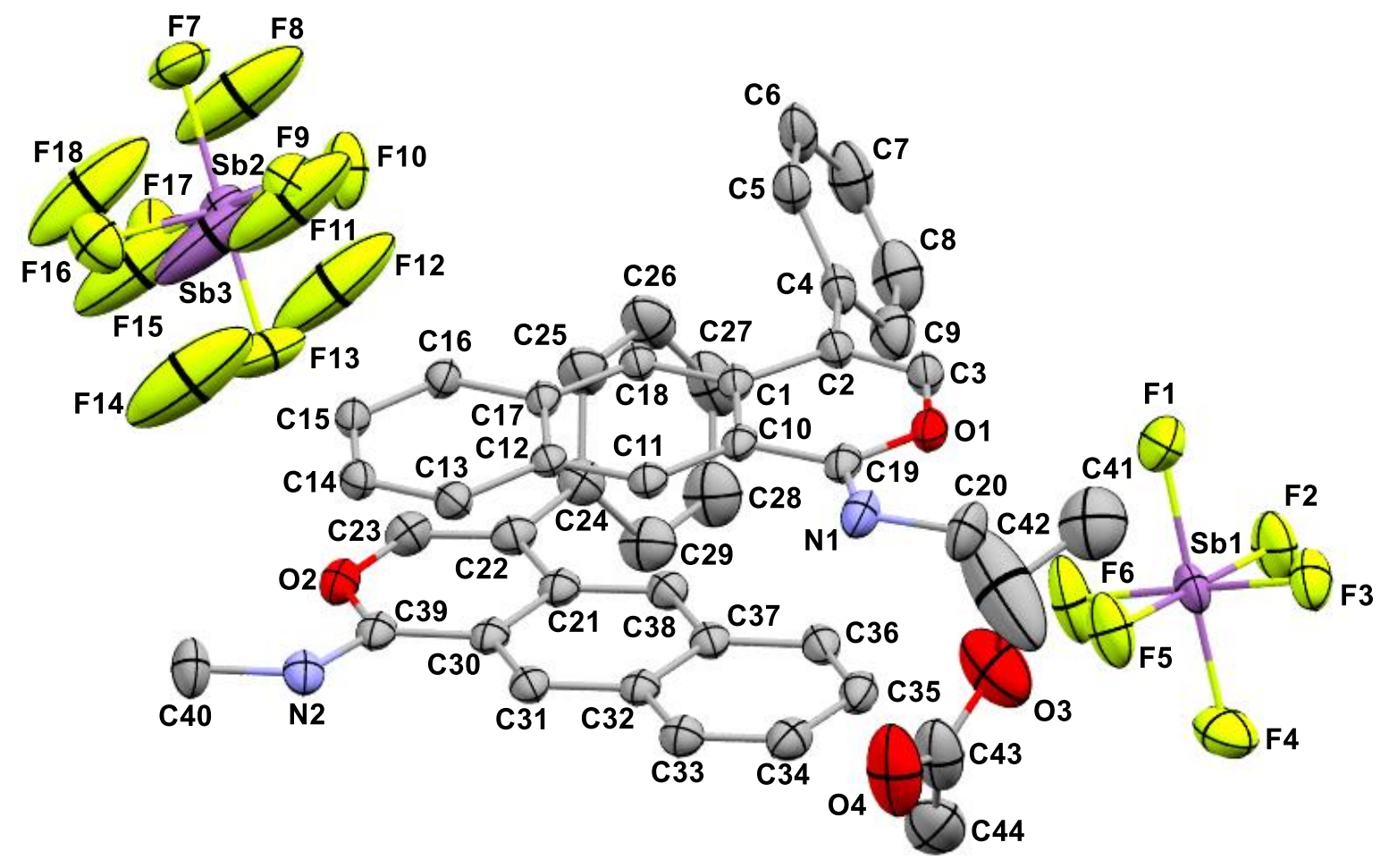

Empirical formula

Formula weight

Temperature/K

Crystal system

Space group

$\mathrm{a} / \AA$

$\mathrm{b} / \AA$

$\mathrm{c} / \AA$

$\alpha /{ }^{\circ}$

$\beta /{ }^{\circ}$

$\gamma /{ }^{\circ}$

Volume/ $\AA^{3}$

Z

$\rho_{\text {calc }} \mathrm{g} / \mathrm{cm}^{3}$

$\mu / \mathrm{mm}^{-1}$

$\mathrm{F}(000)$

Crystal size $/ \mathrm{mm}^{3}$

Radiation

$2 \Theta$ range

Index ranges

Reflections collected

Independent reflections

Data/restraints/parameters
$\mathrm{C}_{44} \mathrm{H}_{38} \mathrm{~F}_{12} \mathrm{~N}_{2} \mathrm{O}_{4} \mathrm{Sb}_{2}$

1130.26

100.22

triclinic

P-1

11.6886(15)

13.4251(17)

15.0768(18)

76.864(3)

71.293(3)

86.870(4)

2181.8(5)

2

1.720

1.334

1116.0

$0.395 \times 0.155 \times 0.022$

$\operatorname{MoK} \alpha(\lambda=0.71073)$

4.626 to 61.148

$-16 \leq \mathrm{h} \leq 16,-19 \leq \mathrm{k} \leq 19,-21 \leq 1 \leq 21$

76807

$13348\left[\mathrm{R}_{\text {int }}=0.0367, \mathrm{R}_{\text {sigma }}=0.0274\right]$

$13348 / 76 / 651$ 
Goodness-of-fit on $\mathrm{F}^{2} \quad 1.035$

Final $R$ indexes $[\mathrm{I}>=2 \sigma(\mathrm{I})] \quad \mathrm{R}_{1}=0.0376, \mathrm{wR}_{2}=0.0928$

Final $\mathrm{R}$ indexes [all data] $\quad \mathrm{R}_{1}=0.0526, \mathrm{wR}_{2}=0.1012$

Largest diff. peak/hole / e $\AA^{-3} 1.46 /-0.57$ 
(Z)- $\mathrm{N}$-[3-(p-Methoxyphenyl)-1H-isochromen-1-ylidene]methanaminium hexafluoroantimonate (231):
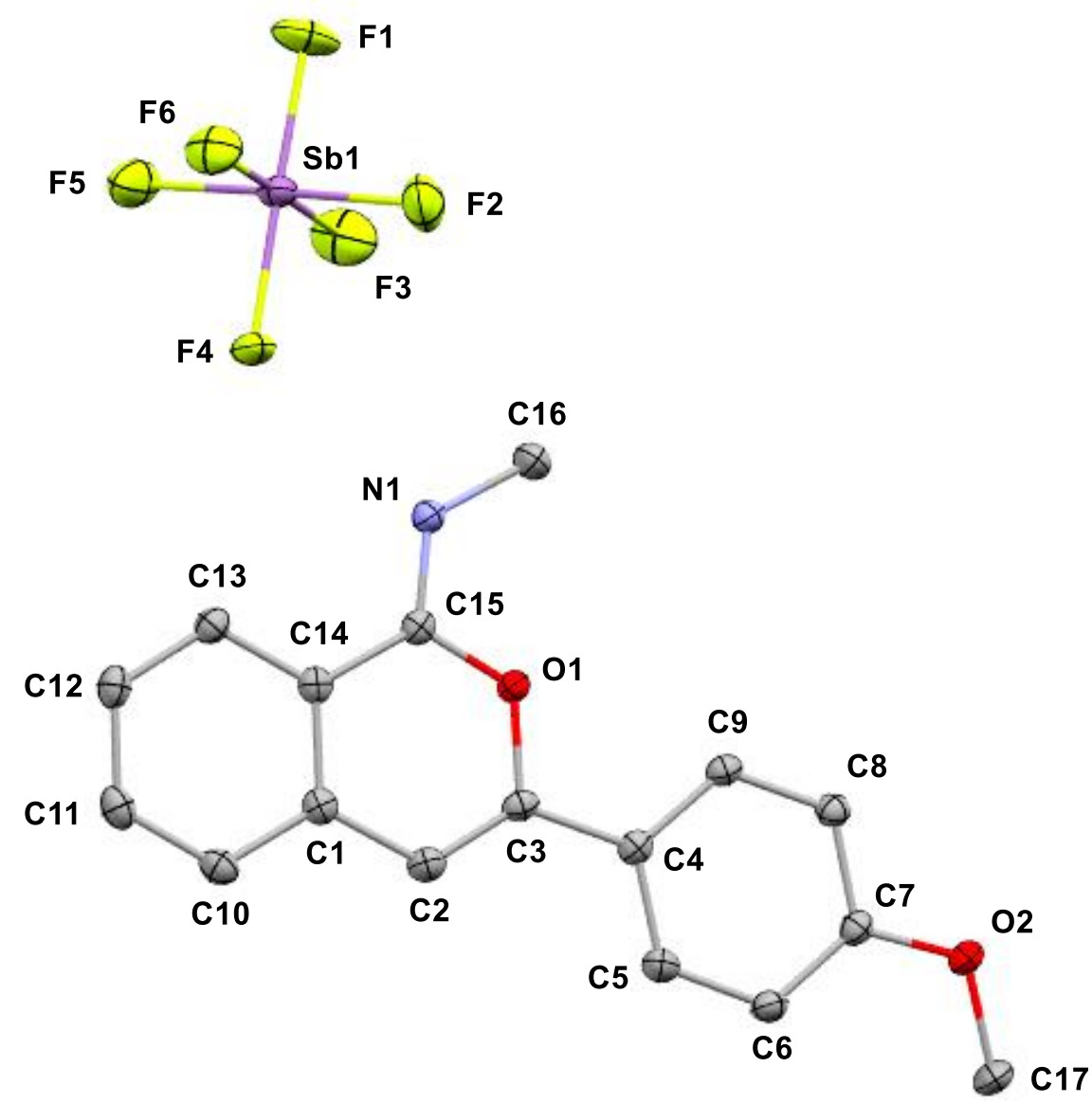

Empirical formula

$\mathrm{C}_{17} \mathrm{H}_{16} \mathrm{~F}_{6} \mathrm{NO}_{2} \mathrm{Sb}$

Formula weight

502.06

Temperature/K

100.01

Crystal system

monoclinic

Space group

$\mathrm{a} / \AA$

$\mathrm{b} / \AA$

$\mathrm{C} 2 / \mathrm{c}$

21.3857(13)

$\mathrm{c} / \AA$

7.0061(4)

$\alpha /^{\circ}$

23.513(2)

$\beta /{ }^{\circ}$

$\gamma /{ }^{\circ}$

90

92.779(4)

90

Volume $/ \AA^{3}$

$3518.8(4)$

Z

8

$\rho_{\text {calc }} \mathrm{g} / \mathrm{cm}^{3}$

1.895

$\mu / \mathrm{mm}^{-1}$

1.641

$\mathrm{F}(000)$

1968.0

Crystal size $/ \mathrm{mm}^{3}$

$0.182 \times 0.137 \times 0.043$

Radiation

$\operatorname{MoK} \alpha(\lambda=0.71073)$

$2 \Theta$ range for data collection/ ${ }^{\circ} 5.03$ to 59.998 
Index ranges

Reflections collected

Independent reflections

Data/restraints/parameters

Goodness-of-fit on $\mathrm{F}^{2}$

Final $R$ indexes $[\mathrm{I}>=2 \sigma(\mathrm{I})] \quad \mathrm{R}_{1}=0.0199, \mathrm{wR}_{2}=0.0492$

$\begin{array}{ll}\text { Final } \mathrm{R} \text { indexes [all data] } & \mathrm{R}_{1}=0.0215, \mathrm{wR}_{2}=0.0501\end{array}$

Largest diff. peak/hole / e $\AA^{-3} 0.53 /-0.45$
$-30 \leq \mathrm{h} \leq 23,-9 \leq \mathrm{k} \leq 9,-33 \leq 1 \leq 33$

27525

$5124\left[\mathrm{R}_{\mathrm{int}}=0.0199, \mathrm{R}_{\mathrm{sigma}}=0.0153\right]$

$5124 / 0 / 249$

1.093 


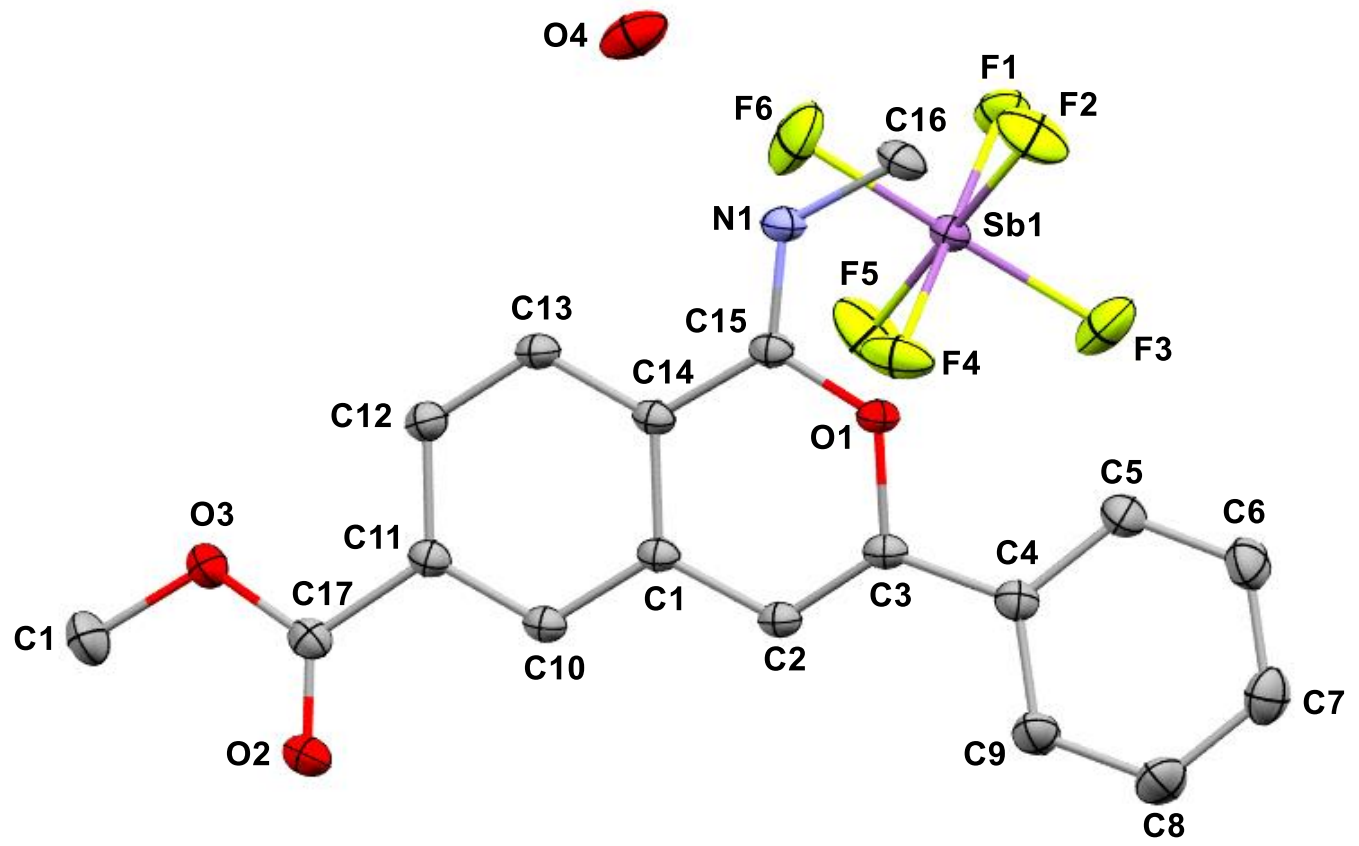

$\mathrm{C}_{18} \mathrm{H}_{18} \mathrm{~F}_{6} \mathrm{NO}_{4} \mathrm{Sb}$

548.08

100.01

monoclinic

$\mathrm{P} 2{ }_{1} / \mathrm{c}$

$11.4656(9)$

$8.2017(7)$

22.0255(14)

90

$102.279(3)$

90

2023.8(3)

4

1.799

1.442

1080.0

$0.317 \times 0.062 \times 0.037$

$\operatorname{MoK} \alpha(\lambda=0.71073)$

$2 \Theta$ range for data collection $/{ }^{\circ} 4.658$ to 61.09

Index ranges

$-16 \leq \mathrm{h} \leq 16,-11 \leq \mathrm{k} \leq 11,-31 \leq 1 \leq 30$

Reflections collected

42342

Independent reflections

Data/restraints/parameters

Goodness-of-fit on $\mathrm{F}^{2}$

$6203\left[\mathrm{R}_{\text {int }}=0.0261, \mathrm{R}_{\text {sigma }}=0.0165\right]$

$6203 / 3 / 282$

1.067

Final $R$ indexes $[\mathrm{I}>=2 \sigma(\mathrm{I})] \quad \mathrm{R}_{1}=0.0189, \mathrm{wR}_{2}=0.0462$ 
Final $\mathrm{R}$ indexes [all data] $\quad \mathrm{R}_{1}=0.0213, \mathrm{wR}_{2}=0.0476$

Largest diff. peak/hole / e $\AA^{-3} 0.68 /-0.52$ 

chloride (235):

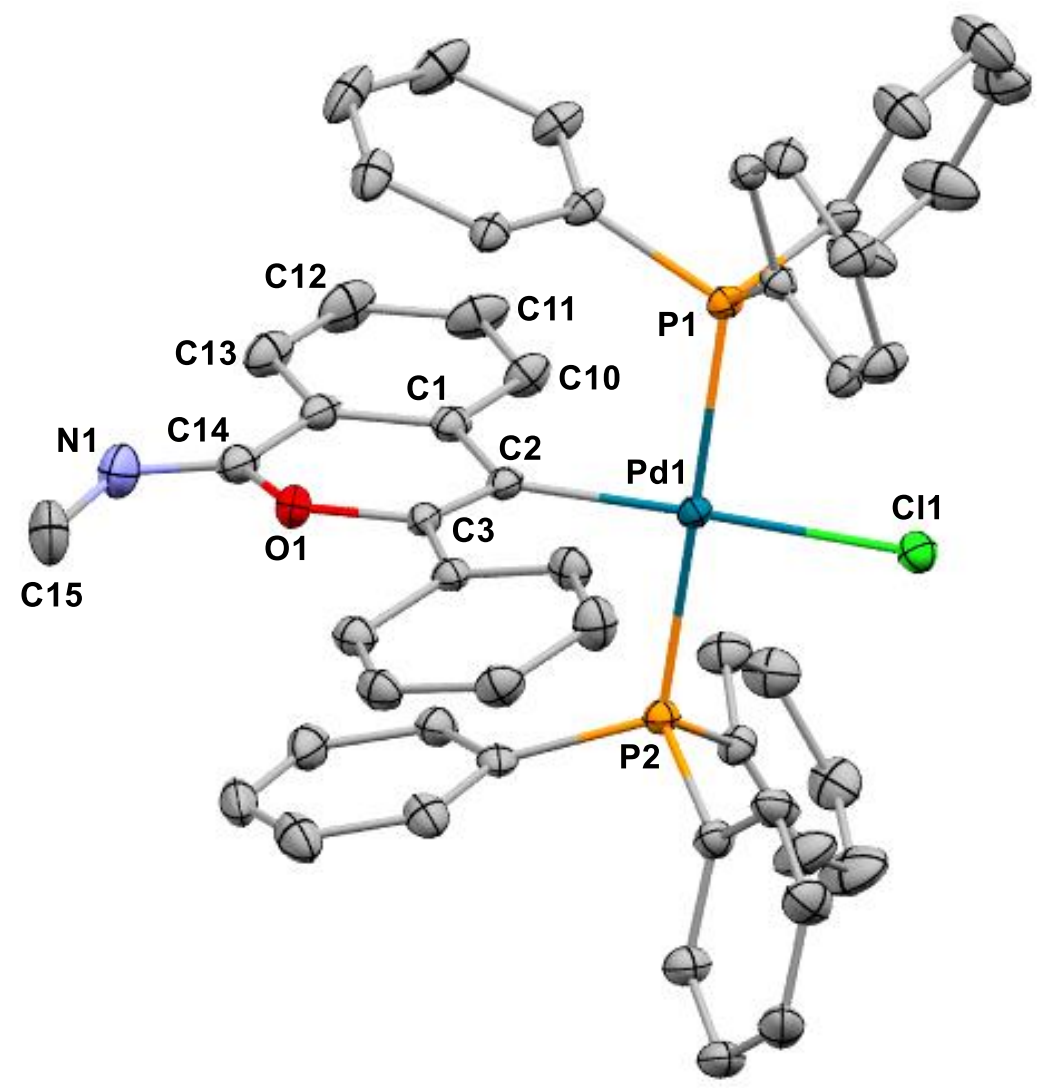

\begin{tabular}{|c|c|}
\hline Empirical formula & $\mathrm{C}_{52} \mathrm{H}_{42} \mathrm{Cl}_{0.79} \mathrm{I}_{0.22} \mathrm{NOP}_{2} \mathrm{Pd}$ \\
\hline Formula weight & 921.00 \\
\hline Temperature/K & 99.99 \\
\hline Crystal system & orthorhombic \\
\hline Space group & $\mathrm{P} 2{ }_{1} 2{ }_{1}{ }_{1}$ \\
\hline $\mathrm{a} / \AA$ & $10.9339(7)$ \\
\hline $\mathrm{b} / \AA$ & $17.1794(8)$ \\
\hline $\mathrm{c} / \AA$ & $22.2028(9)$ \\
\hline$\alpha /{ }^{\circ}$ & 90 \\
\hline$\beta /^{\circ}$ & 90 \\
\hline$\gamma /{ }^{\circ}$ & 90 \\
\hline Volume $/ \AA^{3}$ & $4170.5(4)$ \\
\hline $\mathrm{Z}$ & 4 \\
\hline$\rho_{\text {calc }} \mathrm{g} / \mathrm{cm}^{3}$ & 1.467 \\
\hline$\mu / \mathrm{mm}^{-1}$ & 0.777 \\
\hline $\mathrm{F}(000)$ & 1880.0 \\
\hline Crystal size $/ \mathrm{mm}^{3}$ & $0.282 \times 0.17 \times 0.123$ \\
\hline Radiation & $\operatorname{MoK} \alpha(\lambda=0.71073)$ \\
\hline \multicolumn{2}{|c|}{$2 \Theta$ range for data collection $/{ }^{\circ} 4.152$ to 62} \\
\hline Index ranges & $-15 \leq \mathrm{h} \leq 13,-24 \leq \mathrm{k} \leq 19,-32 \leq 1 \leq 31$ \\
\hline Reflections collected & 33108 \\
\hline
\end{tabular}


Independent reflections

Data/restraints/parameters

Goodness-of-fit on $\mathrm{F}^{2}$

Final $\mathrm{R}$ indexes $[\mathrm{I}>=2 \sigma(\mathrm{I})]$

Final $\mathrm{R}$ indexes [all data]

Largest diff. peak/hole / e $\AA^{-3}$

Flack parameter
$13250\left[\mathrm{R}_{\text {int }}=0.0356, \mathrm{R}_{\text {sigma }}=0.0469\right]$

$13250 / 14 / 571$

1.076

$\mathrm{R}_{1}=0.0335, \mathrm{wR}_{2}=0.0758$

$\mathrm{R}_{1}=0.0348, \mathrm{wR}_{2}=0.0765$

$0.091(12)$ 


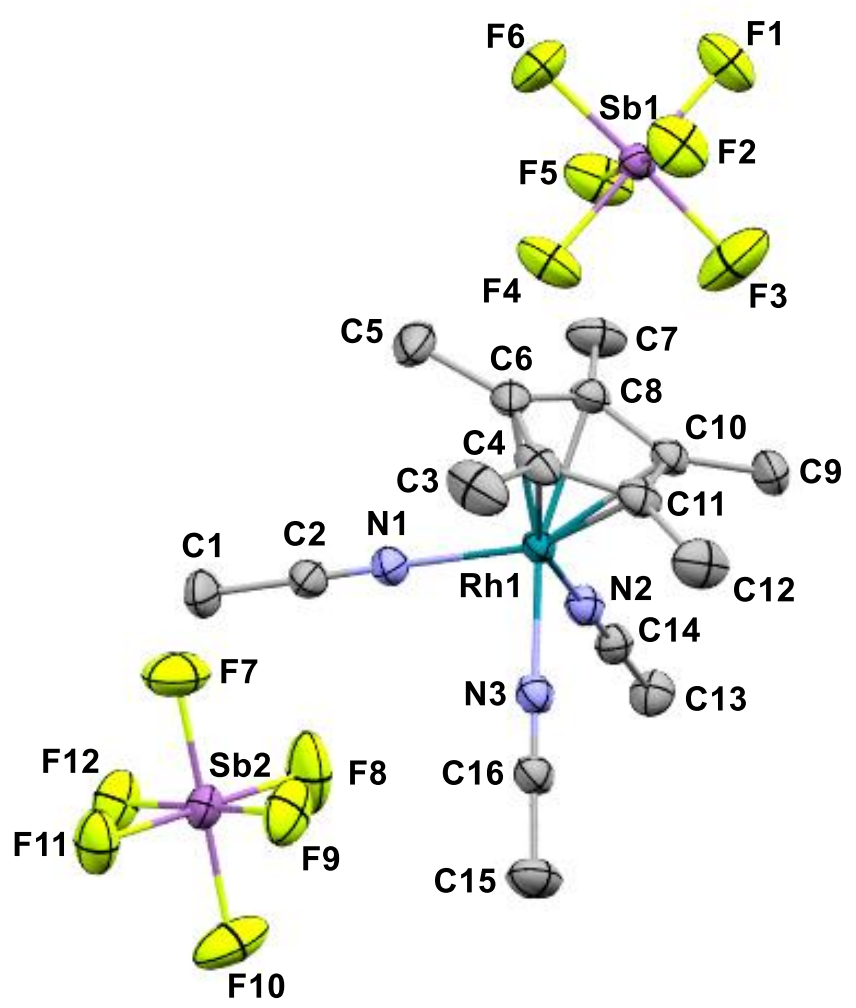

Empirical formula

Formula weight

Temperature/K

Crystal system

Space group

$\mathrm{a} / \AA$

$\mathrm{b} / \AA$

$\mathrm{c} / \AA$

$\alpha /^{\circ}$

$\beta /{ }^{\circ}$

$\gamma /{ }^{\circ}$

Volume/ $\AA^{3}$

Z

$\rho_{\text {calc }} / \mathrm{cm}^{3}$

$\mu / \mathrm{mm}^{-1}$

$\mathrm{F}(000)$

Crystal size/ $/ \mathrm{mm}^{3}$

Radiation
$\mathrm{C}_{16} \mathrm{H}_{24} \mathrm{~F}_{12} \mathrm{~N}_{3} \mathrm{RhSb}_{2}$

832.79

99.98

monoclinic

$\mathrm{P} 2{ }_{1} / \mathrm{c}$

17.2400(6)

9.7633(3)

15.8635(6)

90

90.7400(10)

90

2669.91(16)

4

2.072

2.713

1584.0

$0.337 \times 0.246 \times 0.231$

$\operatorname{MoK} \alpha(\lambda=0.71073)$

$2 \Theta$ range for data collection $/{ }^{\circ} 4.726$ to 63.068

Index ranges

$-25 \leq \mathrm{h} \leq 25,-14 \leq \mathrm{k} \leq 14,-23 \leq 1 \leq 23$

Reflections collected

55194

Independent reflections

$8901\left[\mathrm{R}_{\text {int }}=0.0194, \mathrm{R}_{\text {sigma }}=0.0130\right]$ 
Data/restraints/parameters $\quad 8901 / 0 / 315$

Goodness-of-fit on $\mathrm{F}^{2} \quad 1.116$

Final $R$ indexes $[\mathrm{I}>=2 \sigma(\mathrm{I})] \quad \mathrm{R}_{1}=0.0399, \mathrm{wR}_{2}=0.1064$

Final $\mathrm{R}$ indexes [all data] $\quad \mathrm{R}_{1}=0.0405, \mathrm{wR}_{2}=0.1068$

Largest diff. peak/hole / e $\AA^{-3} 3.86 /-2.11$ 
Bis(acetonitrile) $\{[(1,3-d i i s o p r o p y l-4,5-d i m e t h y l-1 H$-imidazol-3-ium-2-yl]thio\}(pentamethylcyclopentadienyl)rhodium(I) bis(hexafluoroantimonate) (236a):

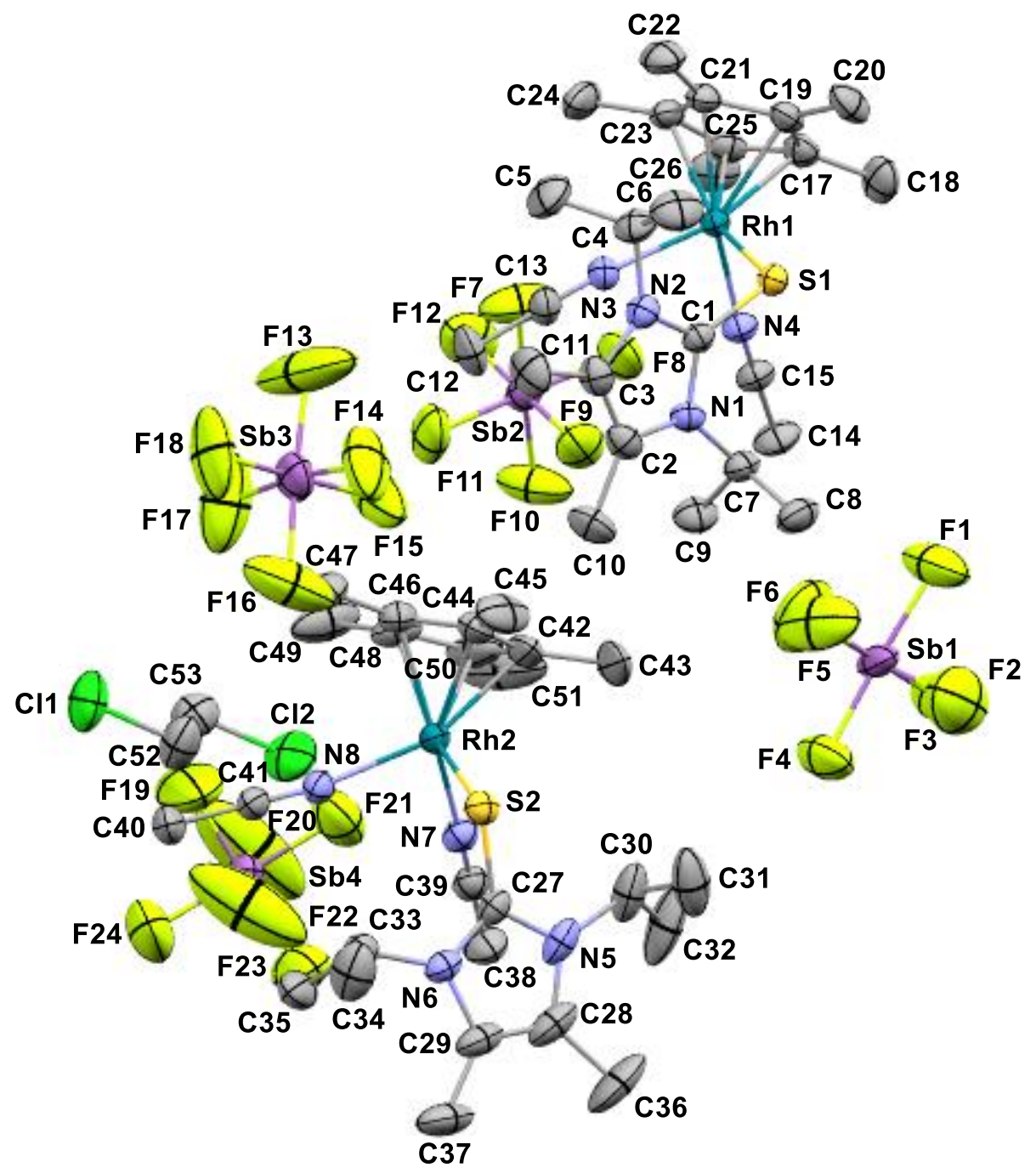

$\begin{array}{ll}\text { Empirical formula } & \mathrm{C}_{52} \mathrm{H}_{86} \mathrm{Cl}_{2} \mathrm{~F}_{24} \mathrm{~N}_{8} \mathrm{Rh}_{2} \mathrm{~S}_{2} \mathrm{Sb}_{4} \\ \text { Formula weight } & 2107.12 \\ \text { Temperature/K } & 150.01 \\ \text { Crystal system } & \text { triclinic } \\ \text { Space group } & \mathrm{P}-1 \\ \mathrm{a} / \AA & 15.3226(8) \\ \mathrm{b} / \AA & 15.8170(6) \\ \mathrm{c} / \AA & 16.9991(8) \\ \alpha /{ }^{\circ} & 106.209(2) \\ \beta /{ }^{\circ} & 92.527(2) \\ \gamma /{ }^{\circ} & 101.649(2) \\ \mathrm{Volume} / \AA^{3} & 3852.4(3) \\ \mathrm{Z} & 2 \\ \rho_{\text {calcg }} / \mathrm{cm}^{3} & 1.816 \\ \mu / \mathrm{mm}^{-1} & 2.021 \\ \mathrm{~F}(000) & 2060.0\end{array}$




$\begin{array}{ll}\text { Crystal size } / \mathrm{mm}^{3} & 0.347 \times 0.313 \times 0.172 \\ \text { Radiation } & \operatorname{MoK} \alpha(\lambda=0.71073) \\ 2 \Theta \text { range for data collection } /{ }^{\circ} 4.234 \text { to } 63.376 \\ \text { Index ranges } & -22 \leq \mathrm{h} \leq 22,-23 \leq \mathrm{k} \leq 23,-25 \leq 1 \leq 25 \\ \text { Reflections collected } & 129927 \\ \text { Independent reflections } & 25784\left[\mathrm{R}_{\text {int }}=0.0262, \mathrm{R}_{\text {sigma }}=0.0217\right] \\ \text { Data/restraints/parameters } & 25784 / 189 / 873 \\ \text { Goodness-of-fit on } \mathrm{F}^{2} & 1.020 \\ \text { Final } \mathrm{R} \text { indexes [I }>=2 \sigma(\mathrm{I})] & \mathrm{R}_{1}=0.0567, \mathrm{wR}_{2}=0.1403 \\ \text { Final } \mathrm{R} \text { indexes [all data] } & \mathrm{R}_{1}=0.0643, \mathrm{wR}_{2}=0.1466 \\ \text { Largest diff. peak/hole / e } \AA^{-3} 4.25 /-3.96\end{array}$


Bis $\{[$ (1,3-diisopropyl-4,5-dimethyl-1H-imidazol-3-ium-2-yl]thio\}(pentamethylcyclopentadienyl)rhodium(I) bis(hexafluoroantimonate) (236b):

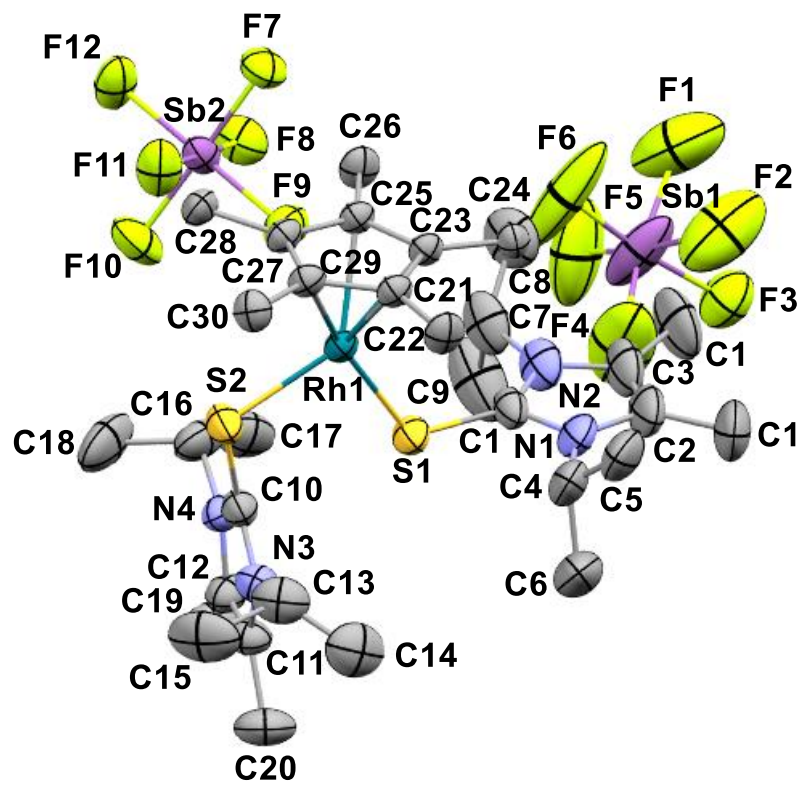


Ag-S-Im+'-cluster (237):
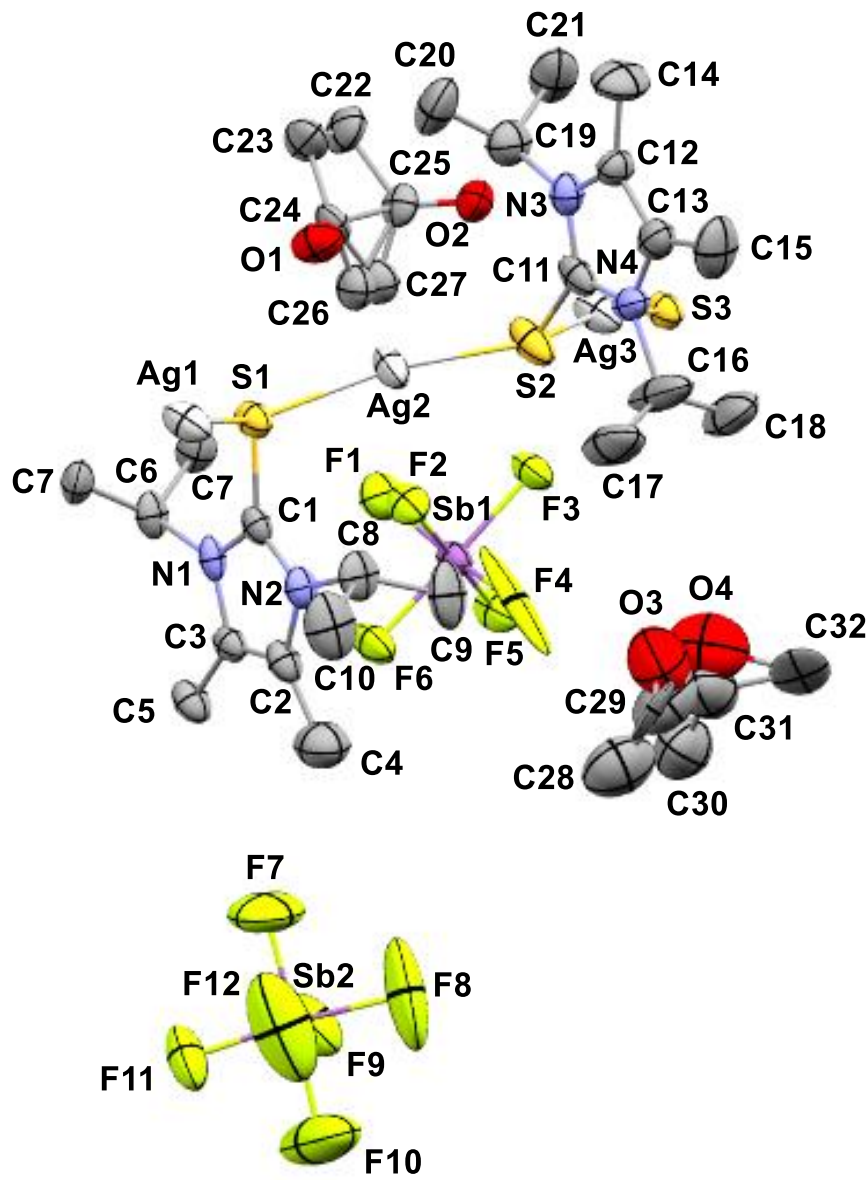

Empirical formula

$\mathrm{C}_{26.5} \mathrm{H}_{49} \mathrm{Ag}_{2} \mathrm{~F}_{12} \mathrm{~N}_{4} \mathrm{O}_{1.5} \mathrm{~S}_{2} \mathrm{Sb}_{2}$

Formula weight

1199.05

Temperature/K

99.99

Crystal system

monoclinic

Space group

$\mathrm{P} 21 / \mathrm{m}$

13.0611(6)

$\mathrm{b} / \AA$

$8.7856(4)$

$\mathrm{c} / \AA$

18.2908(9)

$\alpha /{ }^{\circ}$

90

$\beta /{ }^{\circ}$

92.823(2)

$\gamma /{ }^{\circ}$

90

Volume/ $\AA^{3}$

2096.31(17)

$\mathrm{Z}$

$\rho_{\text {calc }} \mathrm{g} / \mathrm{cm}^{3}$

2

1.900

2.376

1168.0

$\mathrm{F}(000)$

$0.254 \times 0.045 \times 0.026$

$\operatorname{MoK} \alpha(\lambda=0.71073)$

Radiation

$2 \Theta$ range for data collection/ ${ }^{\circ} 5.144$ to 55.996

Index ranges

$? \leq \mathrm{h} \leq ?, ? \leq \mathrm{k} \leq ?, ? \leq 1 \leq ?$ 


$\begin{array}{ll}\text { Reflections collected } & 5379 \\ \text { Independent reflections } & 5379\left[\mathrm{R}_{\text {int }}=?, \mathrm{R}_{\text {sigma }}=0.0205\right] \\ \text { Data/restraints/parameters } & 5379 / 80 / 307 \\ \text { Goodness-of-fit on } \mathrm{F}^{2} & 1.116 \\ \text { Final R indexes [I }>=2 \sigma(\mathrm{I})] & \mathrm{R}_{1}=0.0787, \mathrm{wR}_{2}=0.1976 \\ \text { Final R indexes [all data] } & \mathrm{R}_{1}=0.0879, \mathrm{wR}_{2}=0.2080 \\ \text { Largest diff. peak/hole / e } \AA^{-3} 4.29 /-2.88\end{array}$


3-[(1,3-Diisopropyl-4,5-dimethyl-1H-imidazol-3-ium-2-yl)thio]-2,4-diphenylcinnolin-2-ium hexafluoroantimonate (238) (AVK-AA-296):

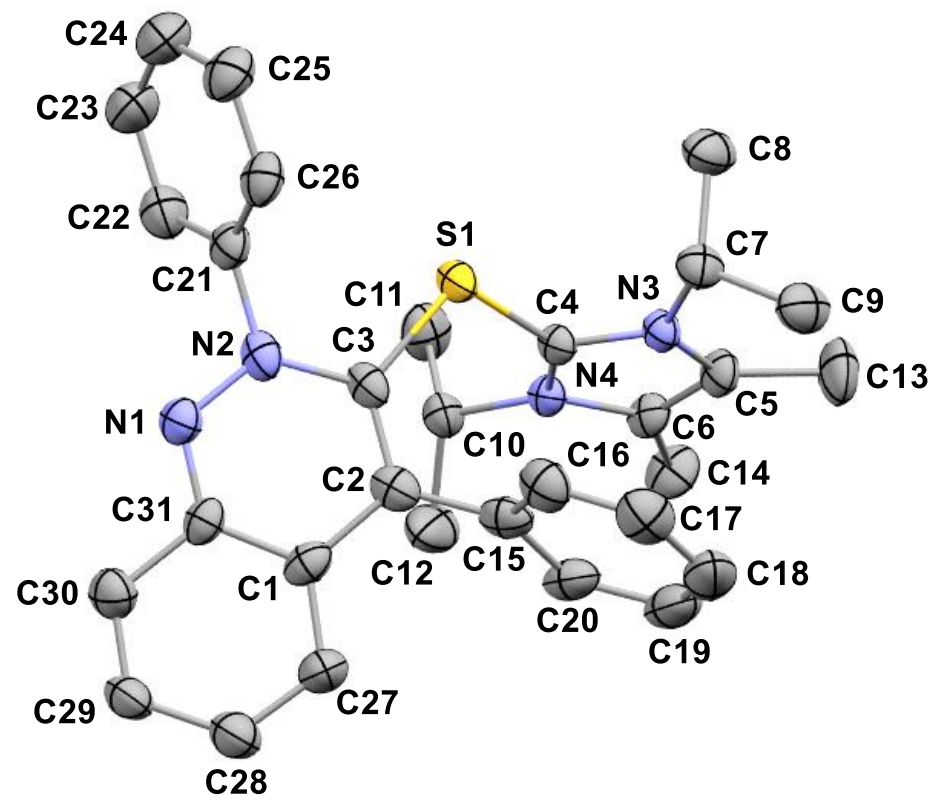

\begin{tabular}{|c|c|}
\hline Empirical formula & $\mathrm{C}_{66} \mathrm{H}_{76} \mathrm{~F}_{24} \mathrm{~N}_{8} \mathrm{OS}_{2} \mathrm{Sb}_{4}$ \\
\hline Formula weight & 2004.46 \\
\hline Temperature/K & 100.02 \\
\hline Crystal system & monoclinic \\
\hline Space group & $\mathrm{P} 2{ }_{1} / \mathrm{n}$ \\
\hline $\mathrm{a} / \AA$ & $11.3960(7)$ \\
\hline $\mathrm{b} / \AA$ & $17.4009(9)$ \\
\hline$c / \AA$ & $20.1516(12)$ \\
\hline$\alpha /^{\circ}$ & 90 \\
\hline$\beta /{ }^{\circ}$ & $105.418(2)$ \\
\hline$\gamma /{ }^{\circ}$ & 90 \\
\hline Volume $/ \AA^{3}$ & $3852.3(4)$ \\
\hline $\mathrm{Z}$ & 2 \\
\hline$\rho_{\text {calc }} \mathrm{g} / \mathrm{cm}^{3}$ & 1.728 \\
\hline$\mu / \mathrm{mm}^{-1}$ & 1.546 \\
\hline $\mathrm{F}(000)$ & 1976.0 \\
\hline Crystal size $/ \mathrm{mm}^{3}$ & $0.175 \times 0.171 \times 0.151$ \\
\hline Radiation & $\operatorname{MoK} \alpha(\lambda=0.71073)$ \\
\hline \multicolumn{2}{|c|}{$2 \Theta$ range for data collection $/{ }^{\circ} 4.384$ to 63.184} \\
\hline Index ranges & $-15 \leq \mathrm{h} \leq 16,-25 \leq \mathrm{k} \leq 20,-26 \leq 1 \leq 29$ \\
\hline Reflections collected & 35474 \\
\hline Independent reflections & $12810\left[\mathrm{R}_{\mathrm{int}}=0.0246, \mathrm{R}_{\mathrm{sigma}}=0.0316\right]$ \\
\hline Data/restraints/parameters & $12810 / 0 / 493$ \\
\hline Goodness-of-fit on $\mathrm{F}^{2}$ & 1.013 \\
\hline Final $R$ indexes $[\mathrm{I}>=2 \sigma(\mathrm{I})]$ & $\mathrm{R}_{1}=0.0553, \mathrm{wR}_{2}=0.1334$ \\
\hline
\end{tabular}


Final $\mathrm{R}$ indexes [all data] $\quad \mathrm{R}_{1}=0.0677, \mathrm{wR}_{2}=0.1412$

Largest diff. peak/hole / e $\AA^{-3} 2.36 /-1.32$ 

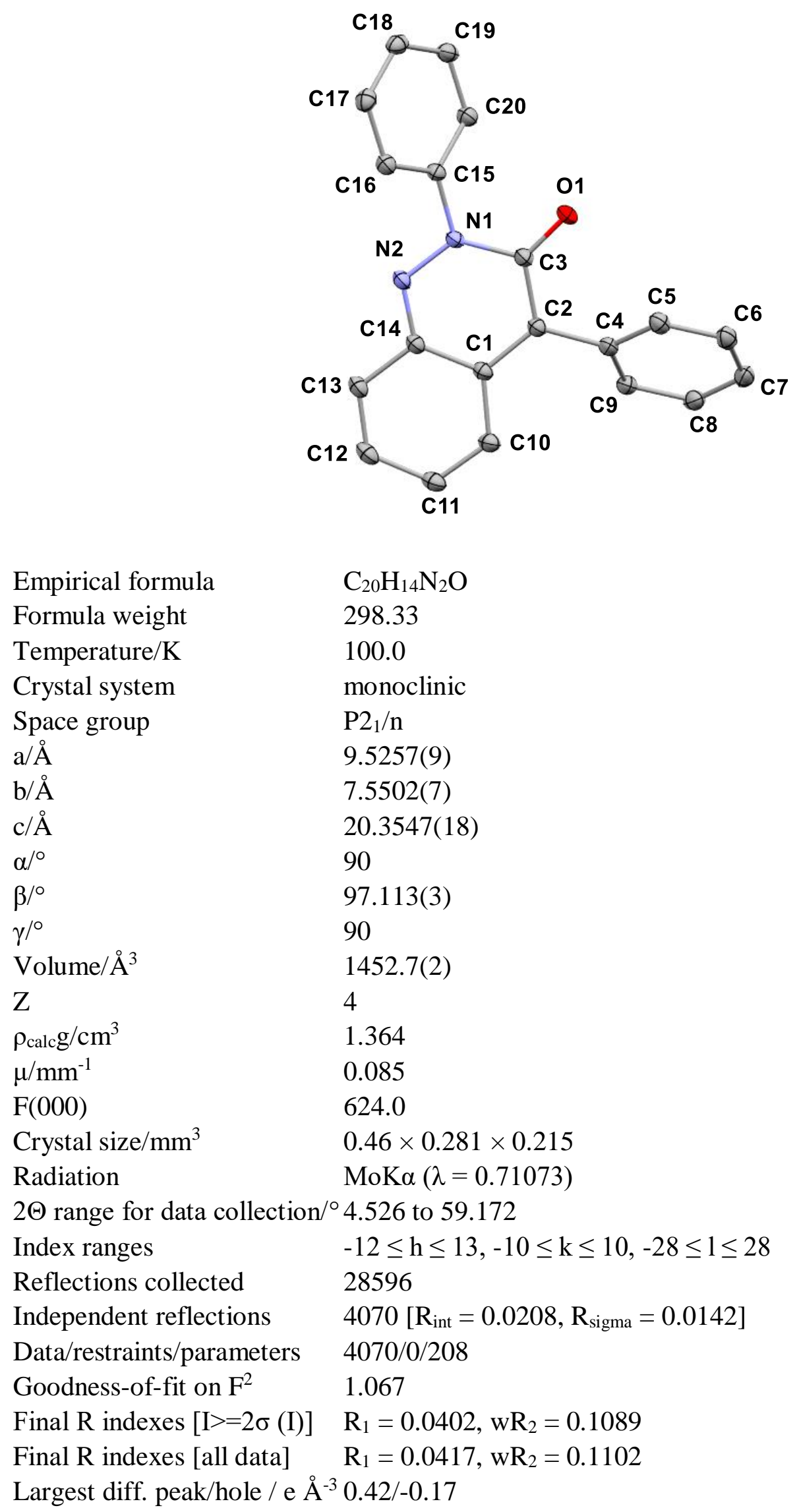


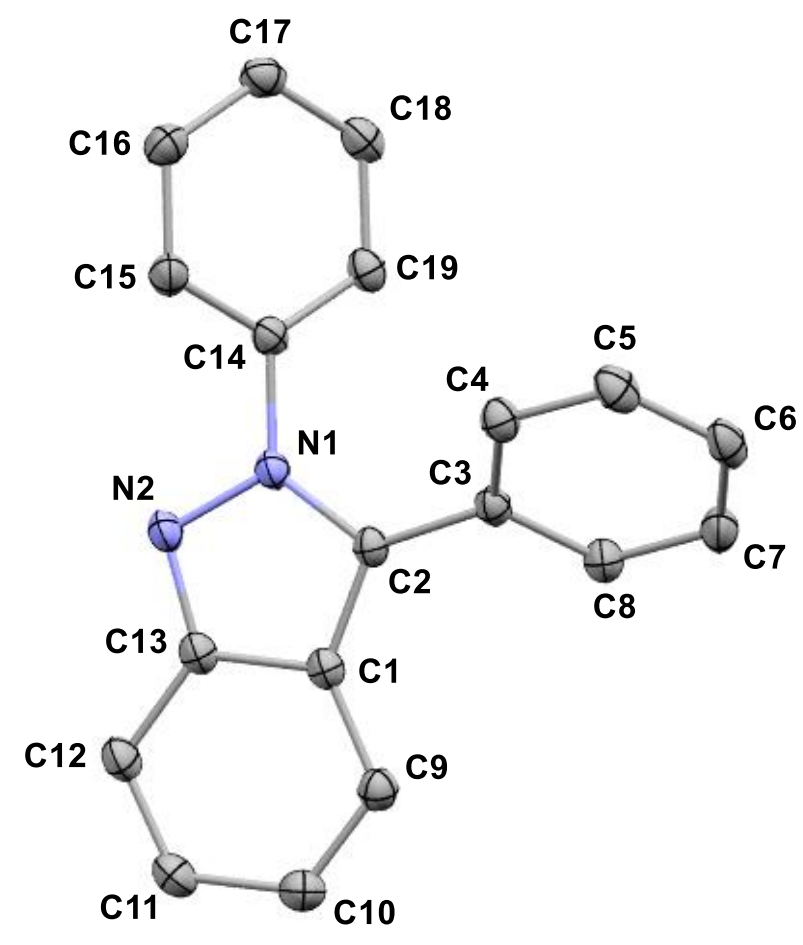

Empirical formula

$\mathrm{C}_{19} \mathrm{H}_{14} \mathrm{~N}_{2}$

Formula weight

270.32

Temperature/K

99.99

Crystal system

monoclinic

Space group

$\mathrm{C} 2 / \mathrm{c}$

$\mathrm{a} / \AA$

$10.246(3)$

$\mathrm{b} / \AA$

$16.638(4)$

$\mathrm{c} / \AA$

$17.335(6)$

$\alpha /^{\circ}$

90

$\beta /{ }^{\circ}$

105.506(14)

$\gamma /{ }^{\circ}$

90

Volume $/ \AA^{3}$

2847.5(14)

Z

8

$\rho_{\text {calc }} \mathrm{g} / \mathrm{cm}^{3}$

1.261

$\mu / \mathrm{mm}^{-1}$

0.075

$\mathrm{F}(000)$

1136.0

Crystal size $/ \mathrm{mm}^{3}$

$0.302 \times 0.174 \times 0.081$

Radiation

$\operatorname{MoK} \alpha(\lambda=0.71073)$

$2 \Theta$ range for data collection/ ${ }^{\circ} 4.798$ to 59.212

Index ranges

$-14 \leq \mathrm{h} \leq 14,-23 \leq \mathrm{k} \leq 22,-23 \leq 1 \leq 24$

Reflections collected

42375

Independent reflections

$4007\left[\mathrm{R}_{\text {int }}=0.0284, \mathrm{R}_{\text {sigma }}=0.0137\right]$

Data/restraints/parameters

Goodness-of-fit on $\mathrm{F}^{2}$

$4007 / 0 / 190$

1.051

Final $R$ indexes $[\mathrm{I}>=2 \sigma(\mathrm{I})]$

$\mathrm{R}_{1}=0.0416, \mathrm{wR}_{2}=0.1056$ 
Final $\mathrm{R}$ indexes [all data] $\quad \mathrm{R}_{1}=0.0447, \mathrm{wR}_{2}=0.1084$

Largest diff. peak/hole / e $\AA^{-3} 0.40 /-0.21$ 


\section{SPECTRA}

${ }^{1} \mathrm{H}$ NMR of compound 153a, 1-methylpyridine-2(1H)-thione, $400 \mathrm{MHz}, \mathrm{C}_{2} \mathrm{D}_{2} \mathrm{Cl}_{4}, 298 \mathrm{~K}$

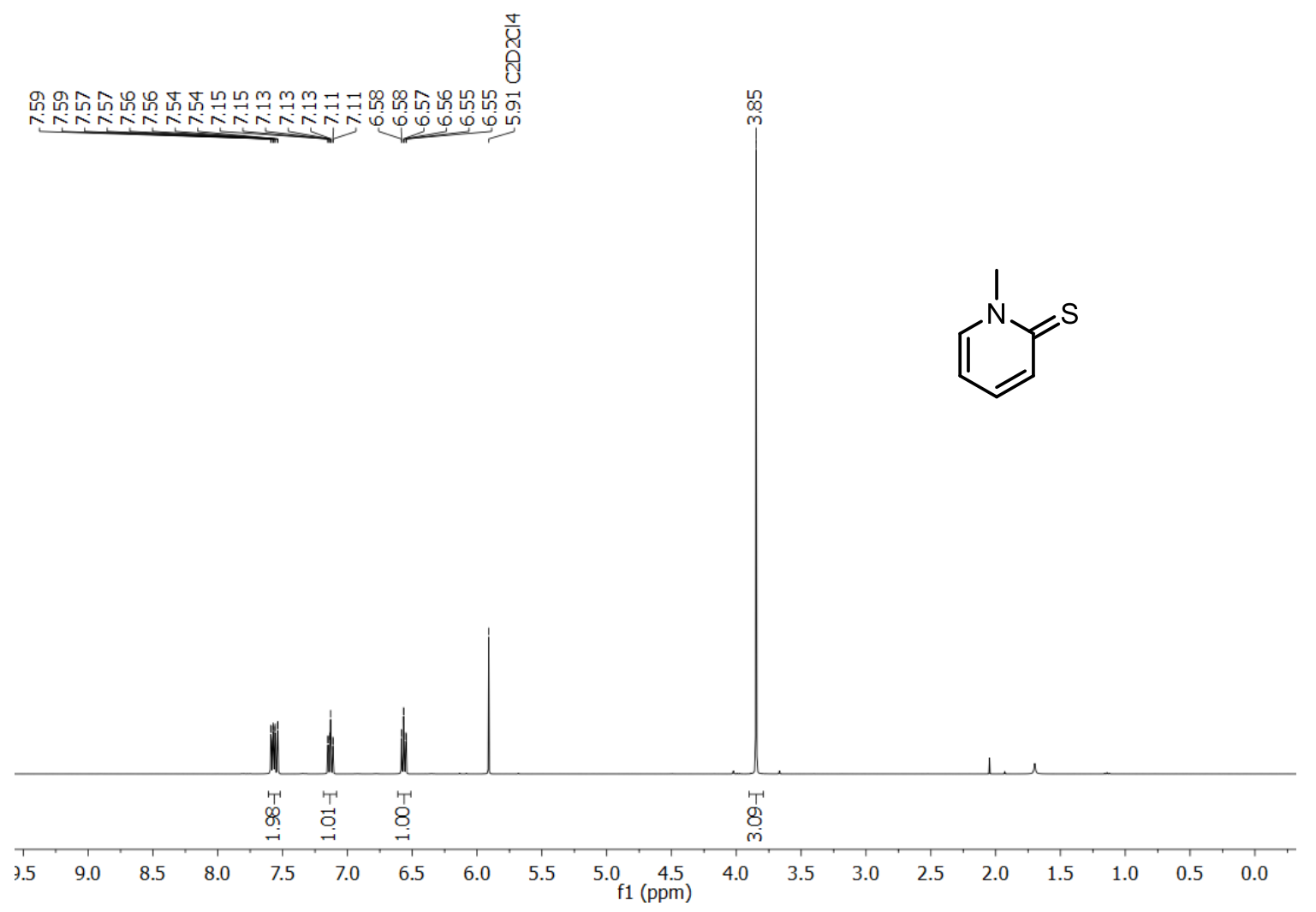

${ }^{13} \mathrm{C}$ NMR of compound 153a, 1-methylpyridine-2(1H)-thione, $101 \mathrm{MHz}, \mathrm{C}_{2} \mathrm{D}_{2} \mathrm{Cl}_{4}, 298 \mathrm{~K}$

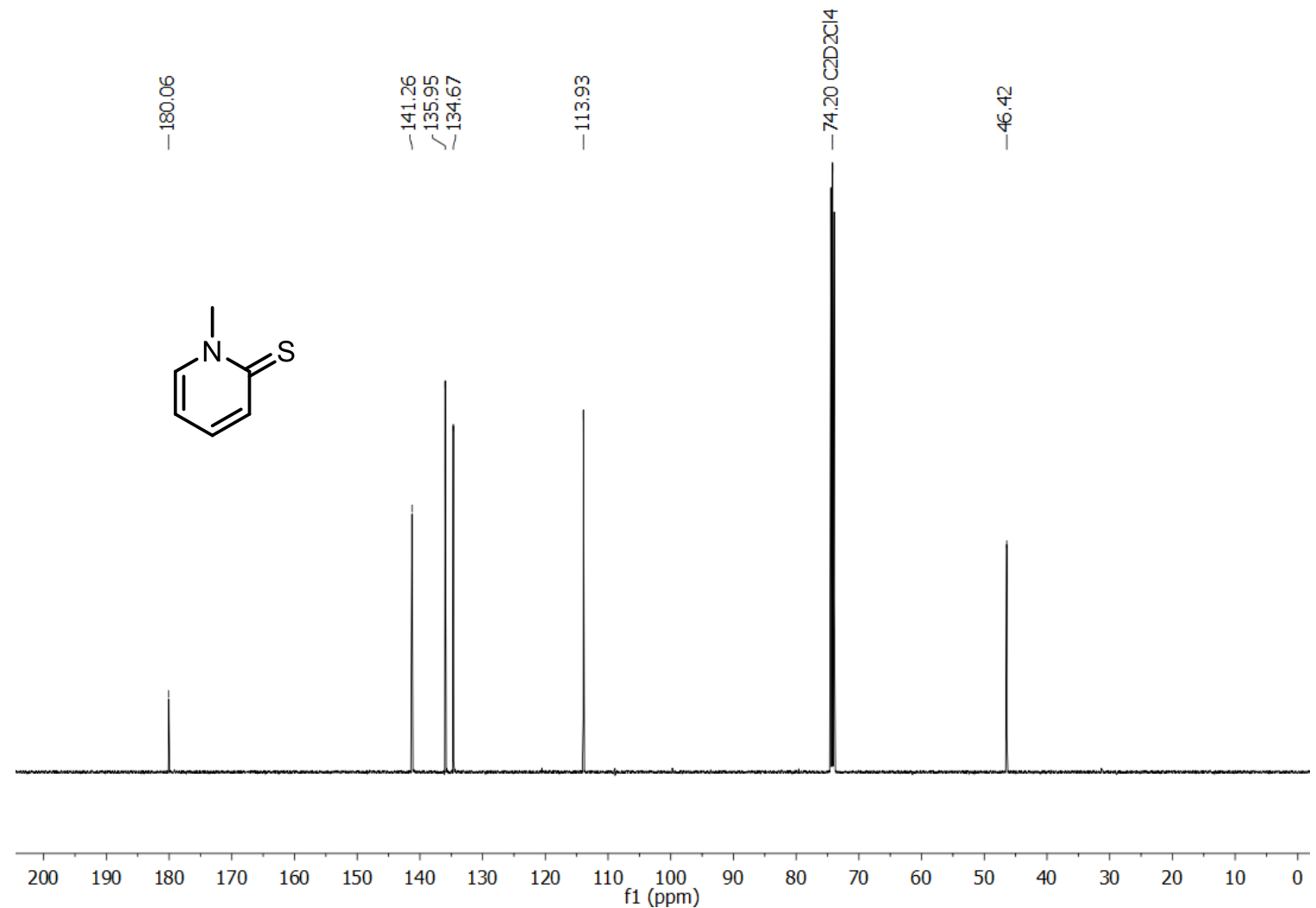


${ }^{1} \mathrm{H}$ NMR of compound 153b, 1-ethylpyridine-2(1H)-thione, $400 \mathrm{MHz}, \mathrm{CDCl}_{3}, 298 \mathrm{~K}$<smiles>CCn1ccccc1=S</smiles>

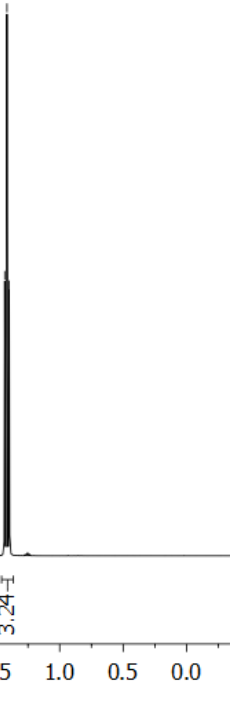

${ }^{13} \mathrm{C}$ NMR of compound 153b, 1-ethylpyridine-2(1H)-thione, $101 \mathrm{MHz}, \mathrm{CDCl}_{3}, 298 \mathrm{~K}$

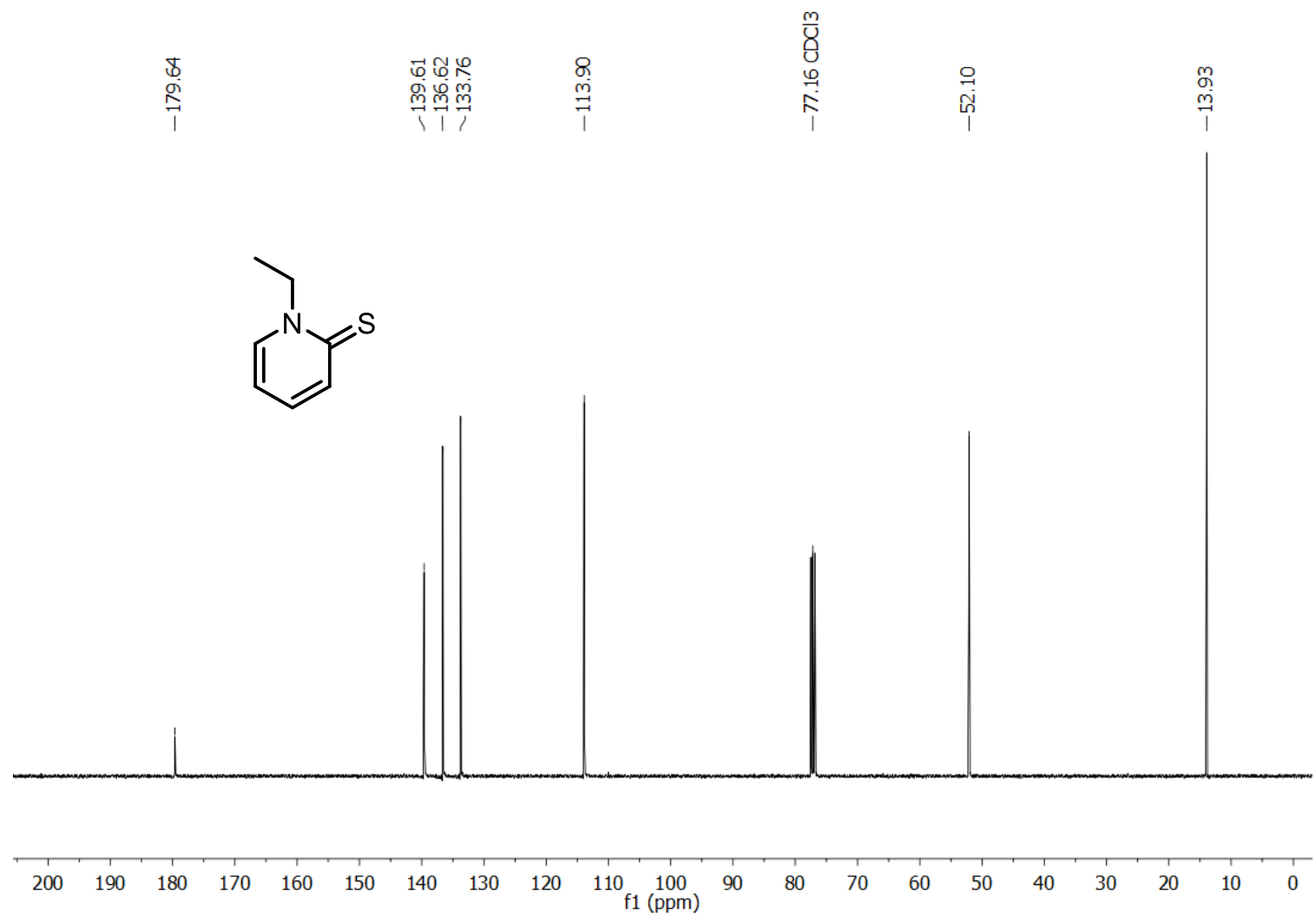


${ }^{1} \mathrm{H}$ NMR of compound 153c, 1-ethyl-3,5-dimethylpyridine-2(1H)-thione, $400 \mathrm{MHz}, \mathrm{CDCl}_{3}$, $298 \mathrm{~K}$
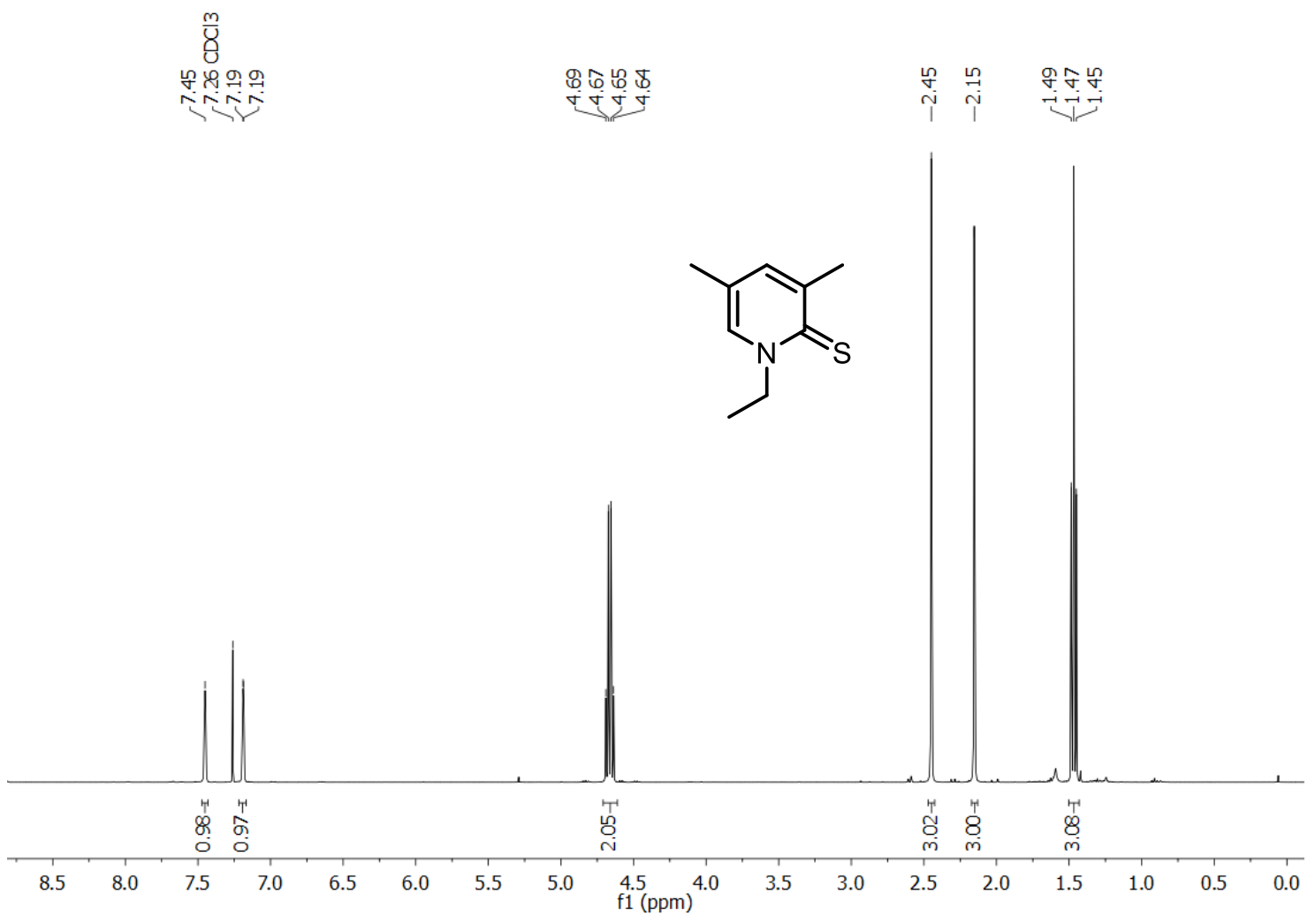

${ }^{13} \mathrm{C}$ NMR of compound 153c, 1-ethyl-3,5-dimethylpyridine-2(1H)-thione, $101 \mathrm{MHz}, \mathrm{CDCl}_{3}$, $298 \mathrm{~K}$
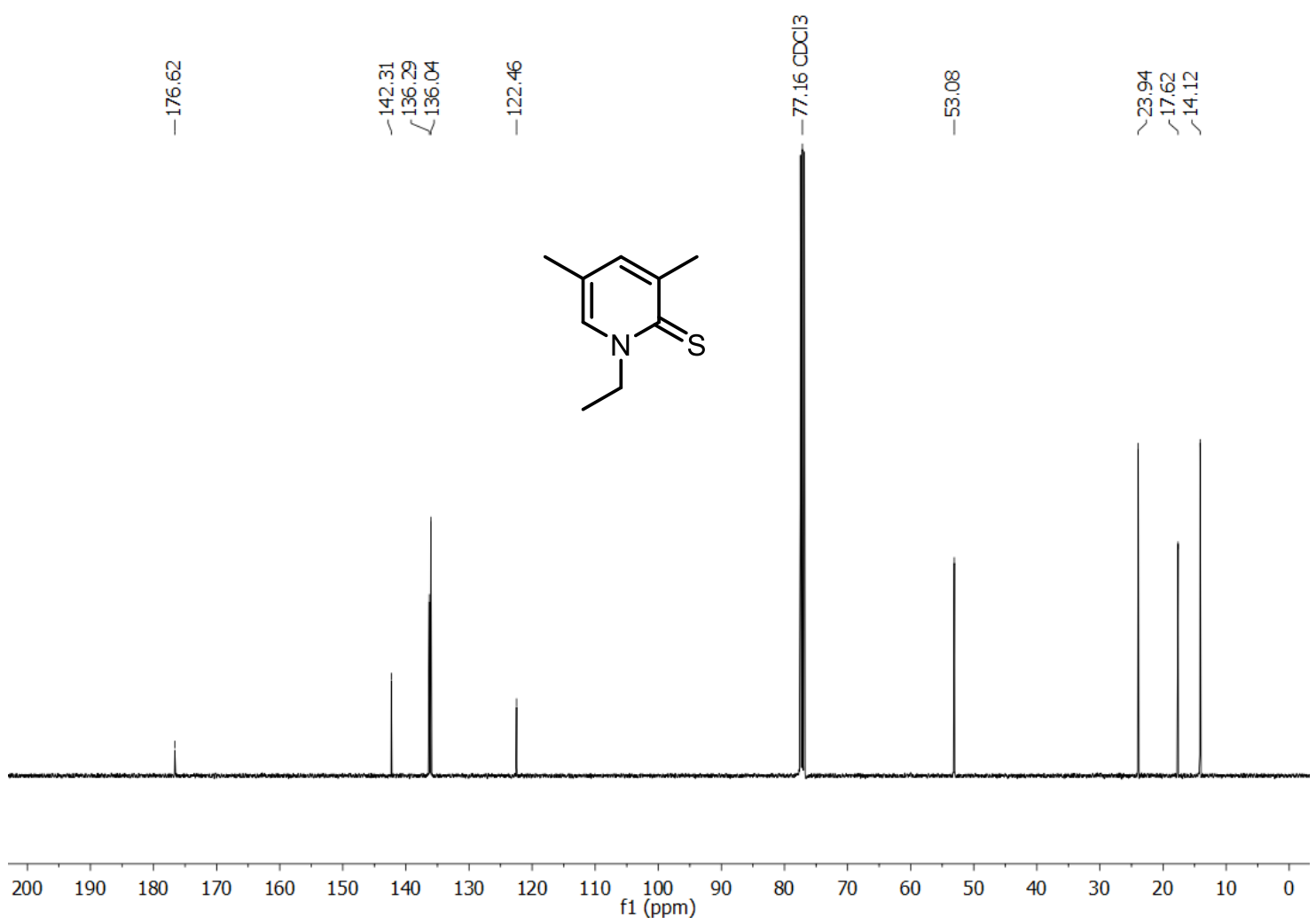

236 
${ }^{1} \mathrm{H}$ NMR of compound 154a, 2-(bromothio)-1-methylpyridin-1-ium bromide, $500 \mathrm{MHz}$, $\mathrm{C}_{2} \mathrm{D}_{2} \mathrm{Cl}_{4}, 298 \mathrm{~K}$

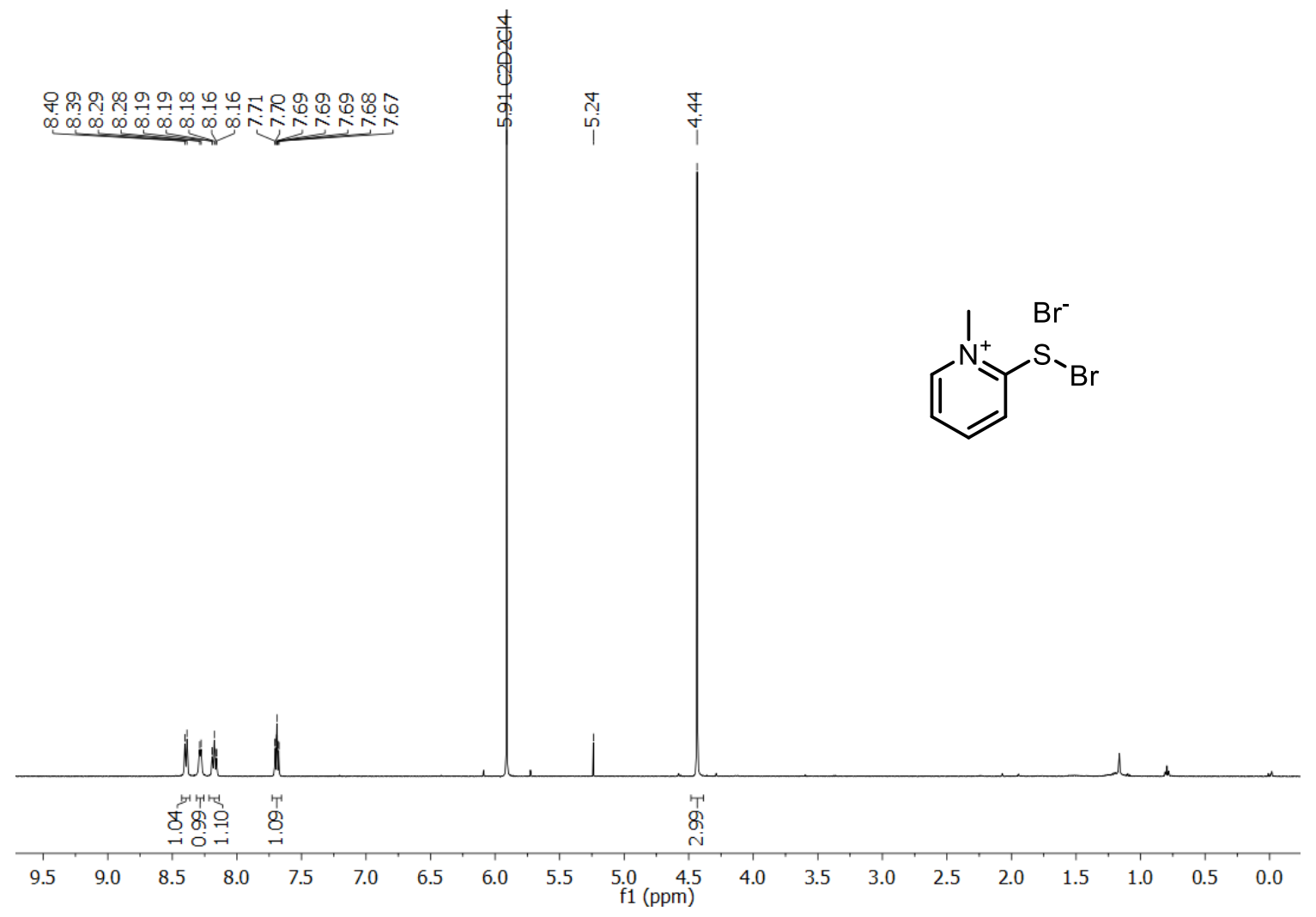

${ }^{13} \mathrm{C}$ NMR of compound 154a, 2-(bromothio)-1-methylpyridin-1-ium bromide, $126 \mathrm{MHz}$, $\mathrm{C}_{2} \mathrm{D}_{2} \mathrm{Cl}_{4}, 298 \mathrm{~K}$

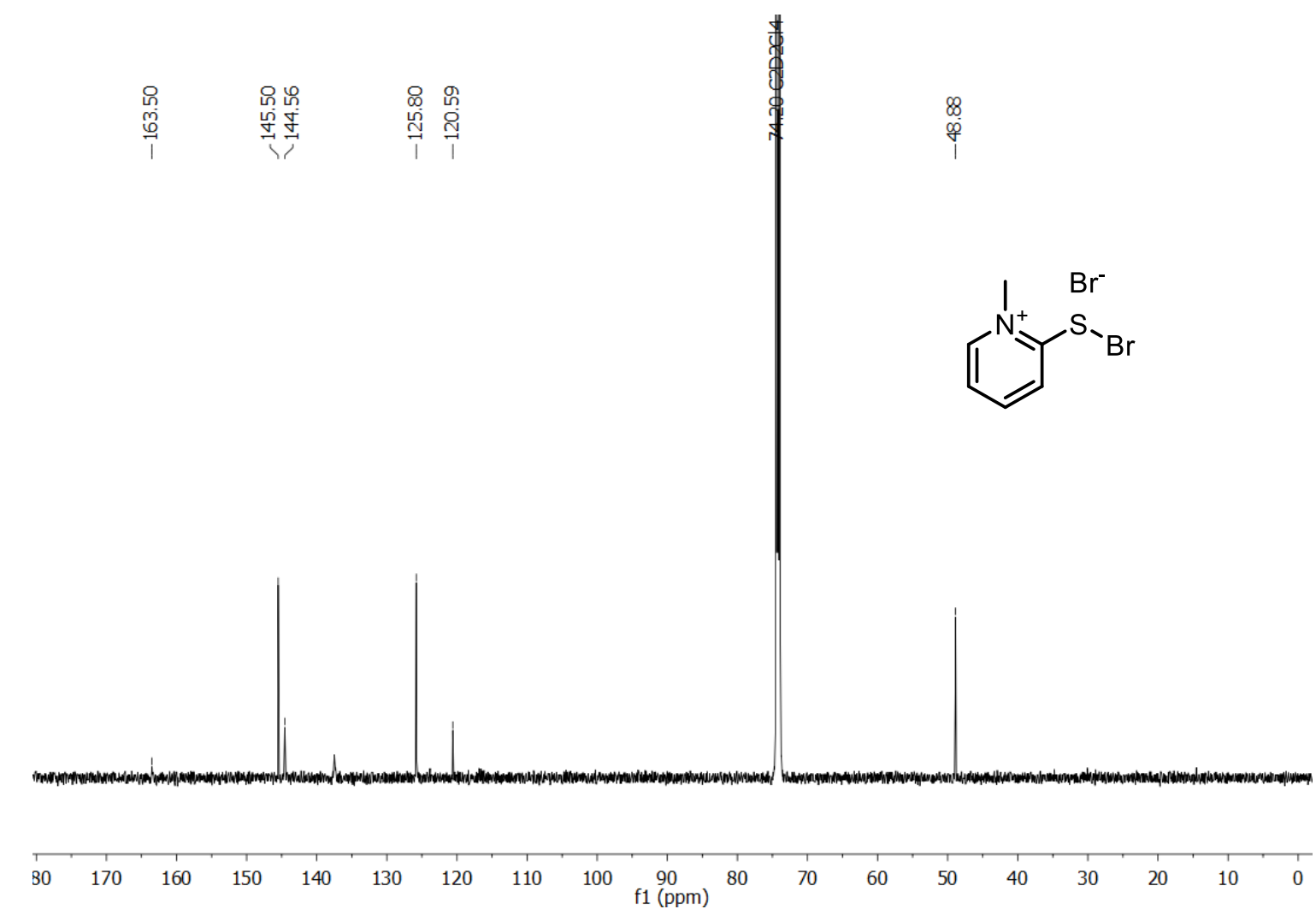


${ }^{1} \mathrm{H}$ NMR of compound 154ba, 2-(bromothio)-1-ethylpyridin-1-ium bromide, $400 \mathrm{MHz}$, $\mathrm{CD}_{2} \mathrm{Cl}_{2}, 298 \mathrm{~K}$

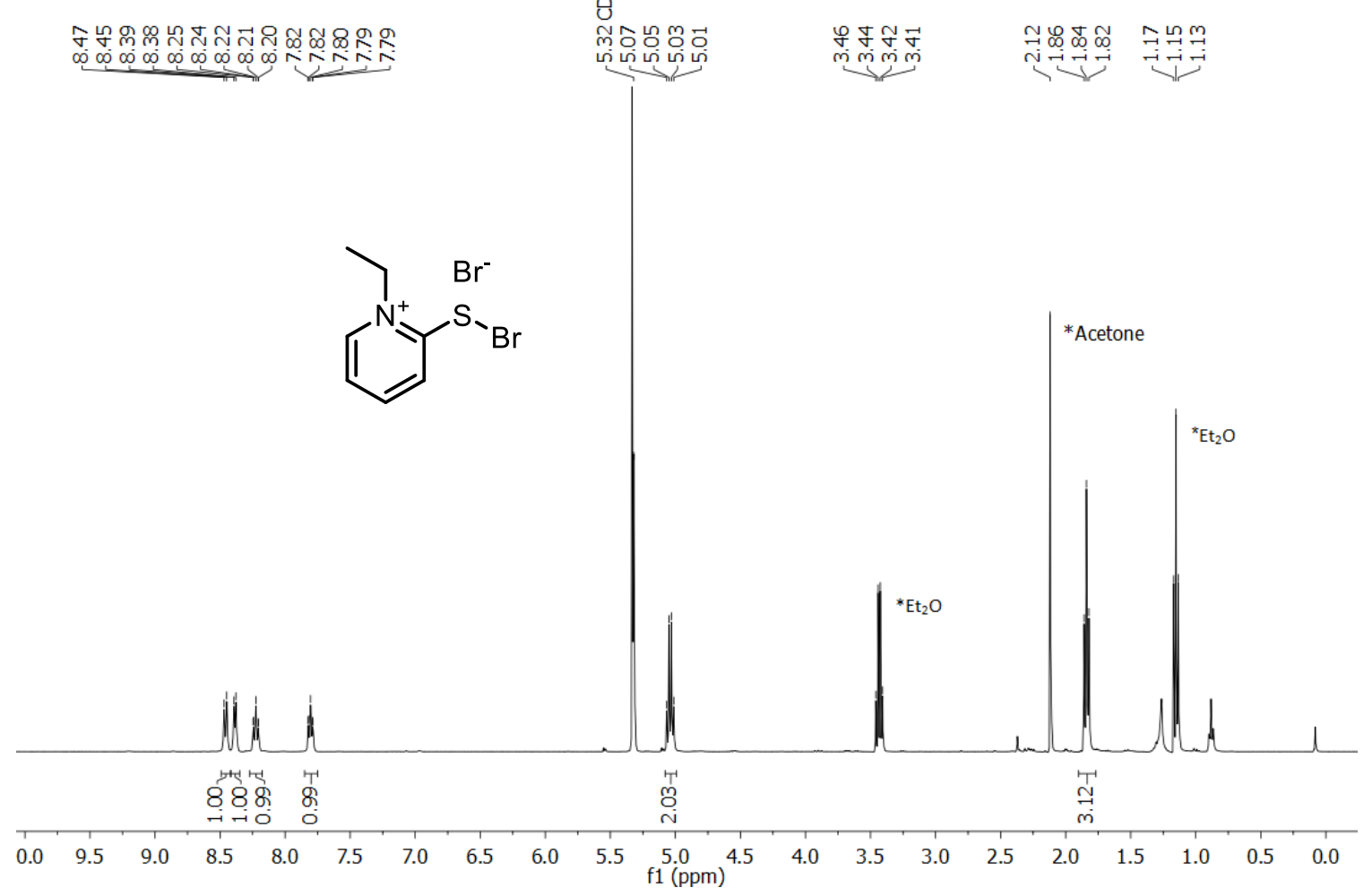

${ }^{13} \mathrm{C}$ NMR of compound 154ba, 2-(bromothio)-1-ethylpyridin-1-ium bromide, $101 \mathrm{MHz}$, $\mathrm{CD}_{2} \mathrm{Cl}_{2}, 298 \mathrm{~K}$

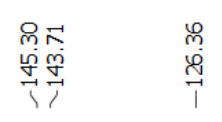

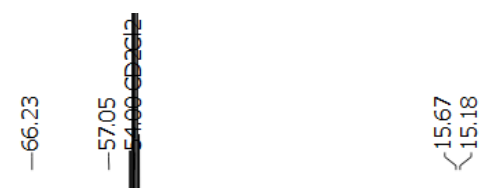<smiles>CC[n+]1ccccc1SBr</smiles>
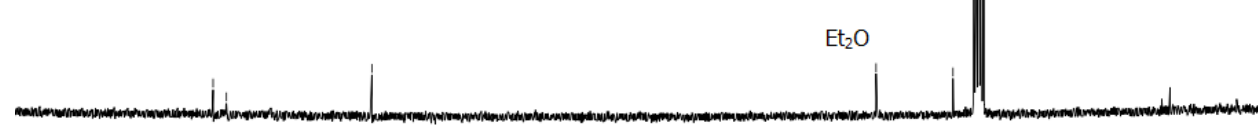

160

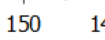

$140 \quad 130$

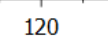

$110 \quad 100$

90

80
$1(\mathrm{ppm})$ 
Selected region of $\mathrm{HMBC}$ for compound 154ba, 2-(bromothio)-1-ethylpyridin-1-ium bromide, $400 \mathrm{MHz}$ / $101 \mathrm{MHz}, \mathrm{CD}_{2} \mathrm{Cl}_{2}, 298 \mathrm{~K}$

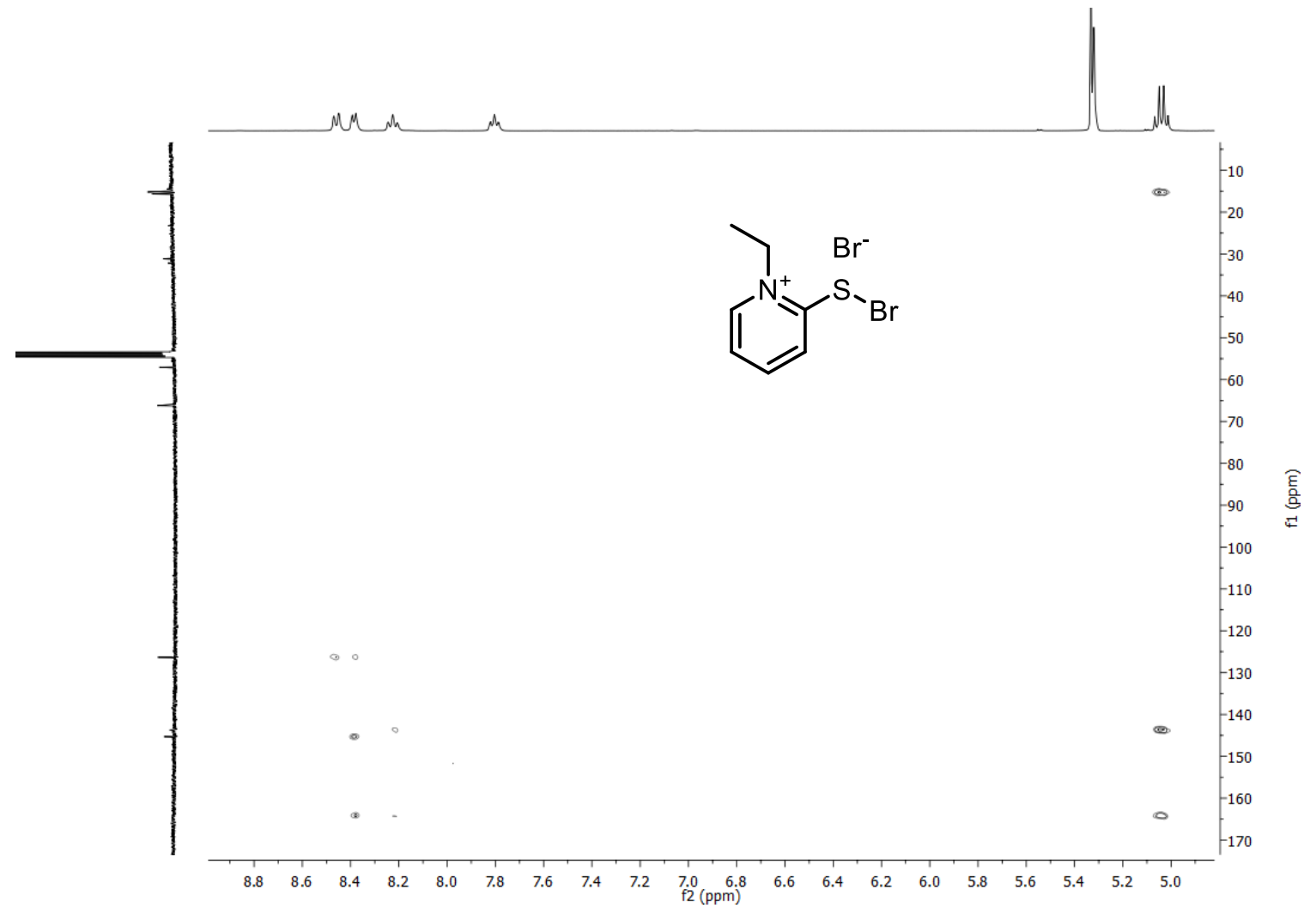

${ }^{1} \mathrm{H}$ NMR of compound 154bb, 2-(chlorothio)-1-ethylpyridin-1-ium chloride, $300 \mathrm{MHz}, \mathrm{CDCl}_{3}$, $303 \mathrm{~K}$

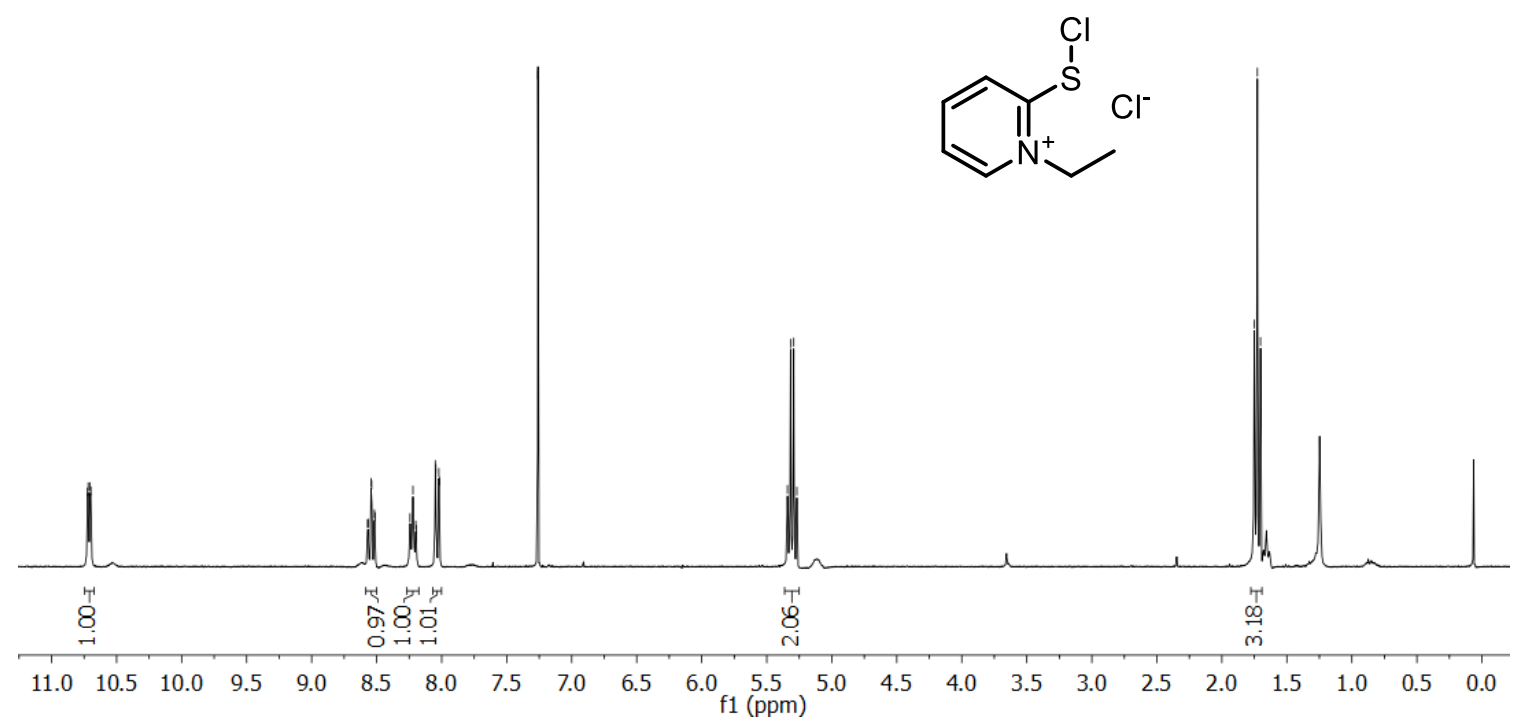


${ }^{1} \mathrm{H}$ NMR of compound 154c, 2-(bromothio)-1-ethyl-3,5-dimethylpyridin-1-ium bromide, 400 $\mathrm{MHz}, \mathrm{CD}_{2} \mathrm{Cl}_{2}, 298 \mathrm{~K}$
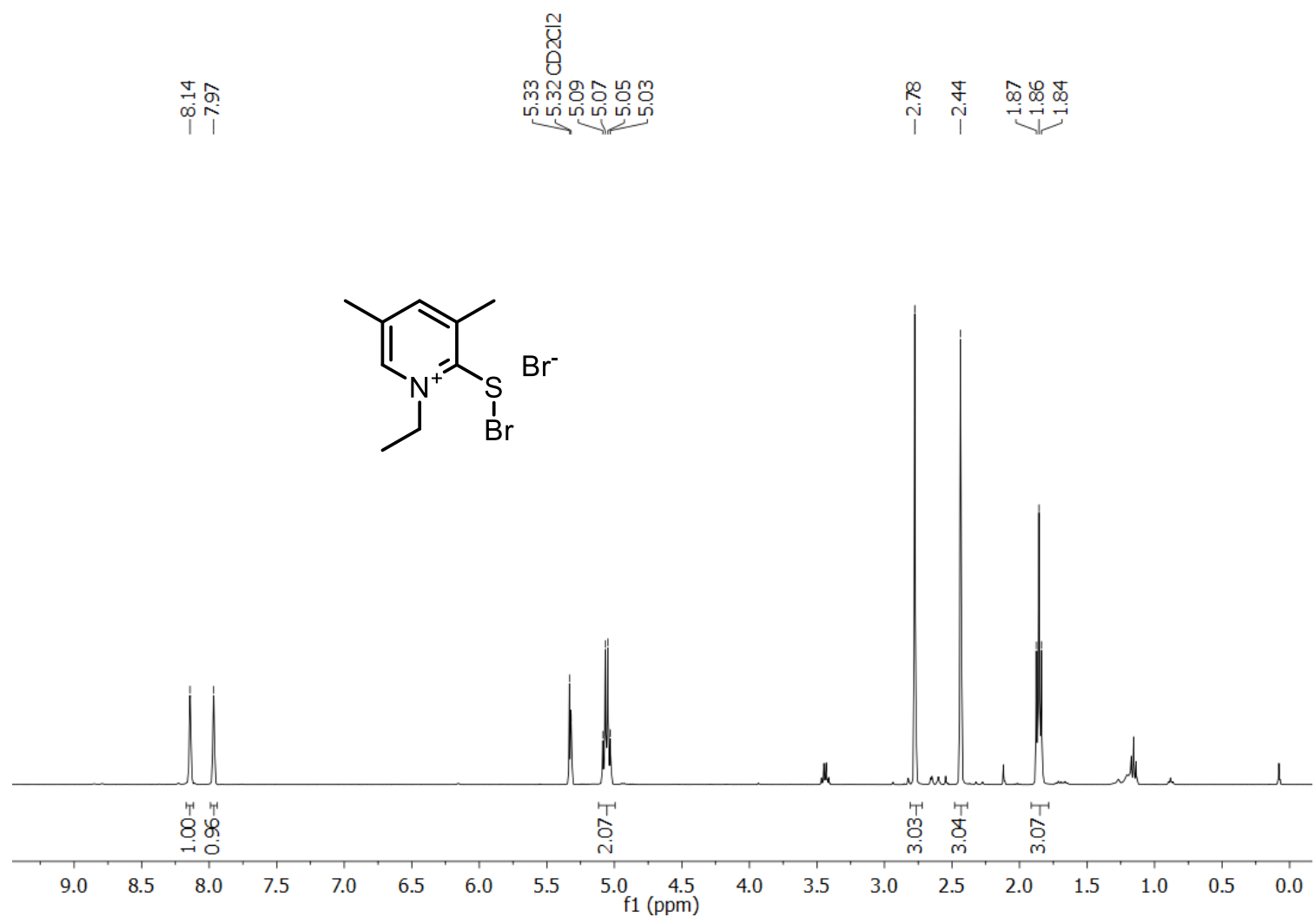

${ }^{13} \mathrm{C}$ NMR of compound 154c, 2-(bromothio)-1-ethyl-3,5-dimethylpyridin-1-ium bromide, $101 \mathrm{MHz}, \mathrm{CD}_{2} \mathrm{Cl}_{2}, 298 \mathrm{~K}$

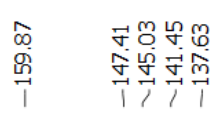

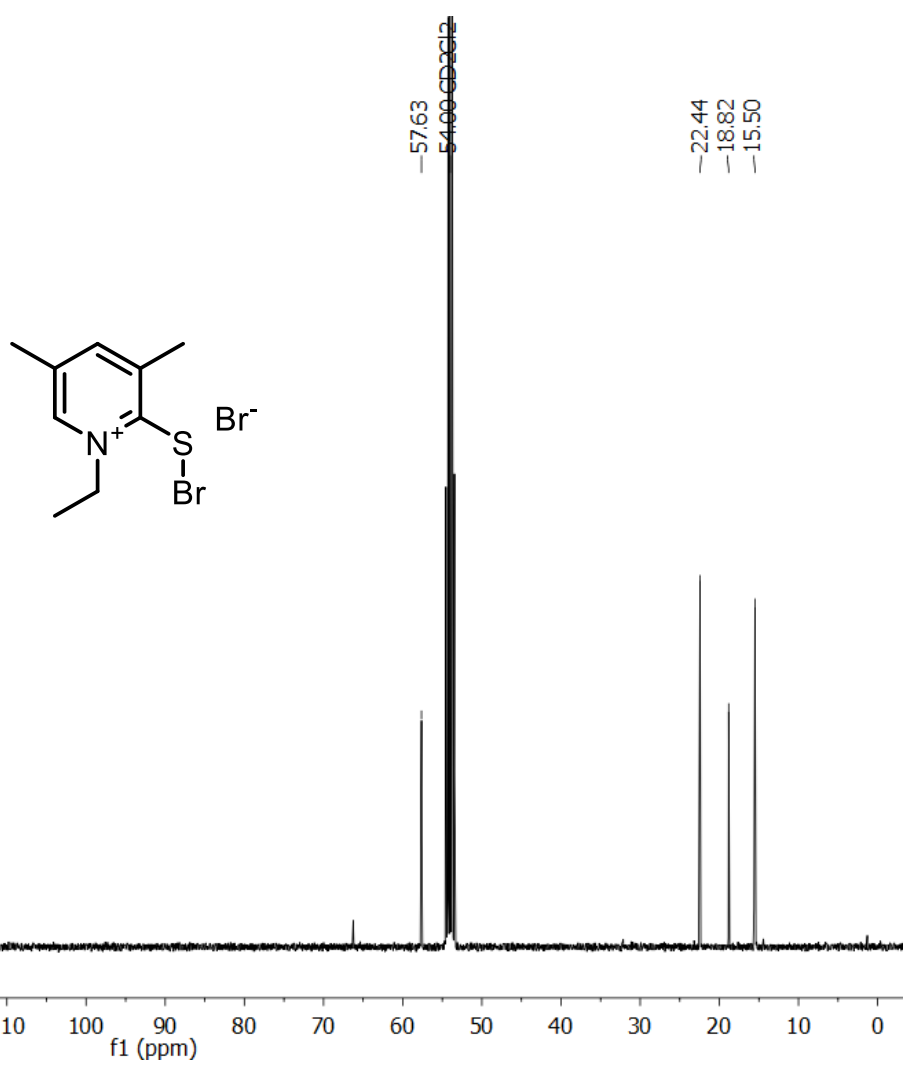


${ }^{1} \mathrm{H}$ NMR of compound 155a, 1-ethyl-3,5-dimethyl-2-thiocyanatopyridin-1-ium bromide, 400 $\mathrm{MHz}, \mathrm{CD}_{3} \mathrm{CN}, 300 \mathrm{~K}$

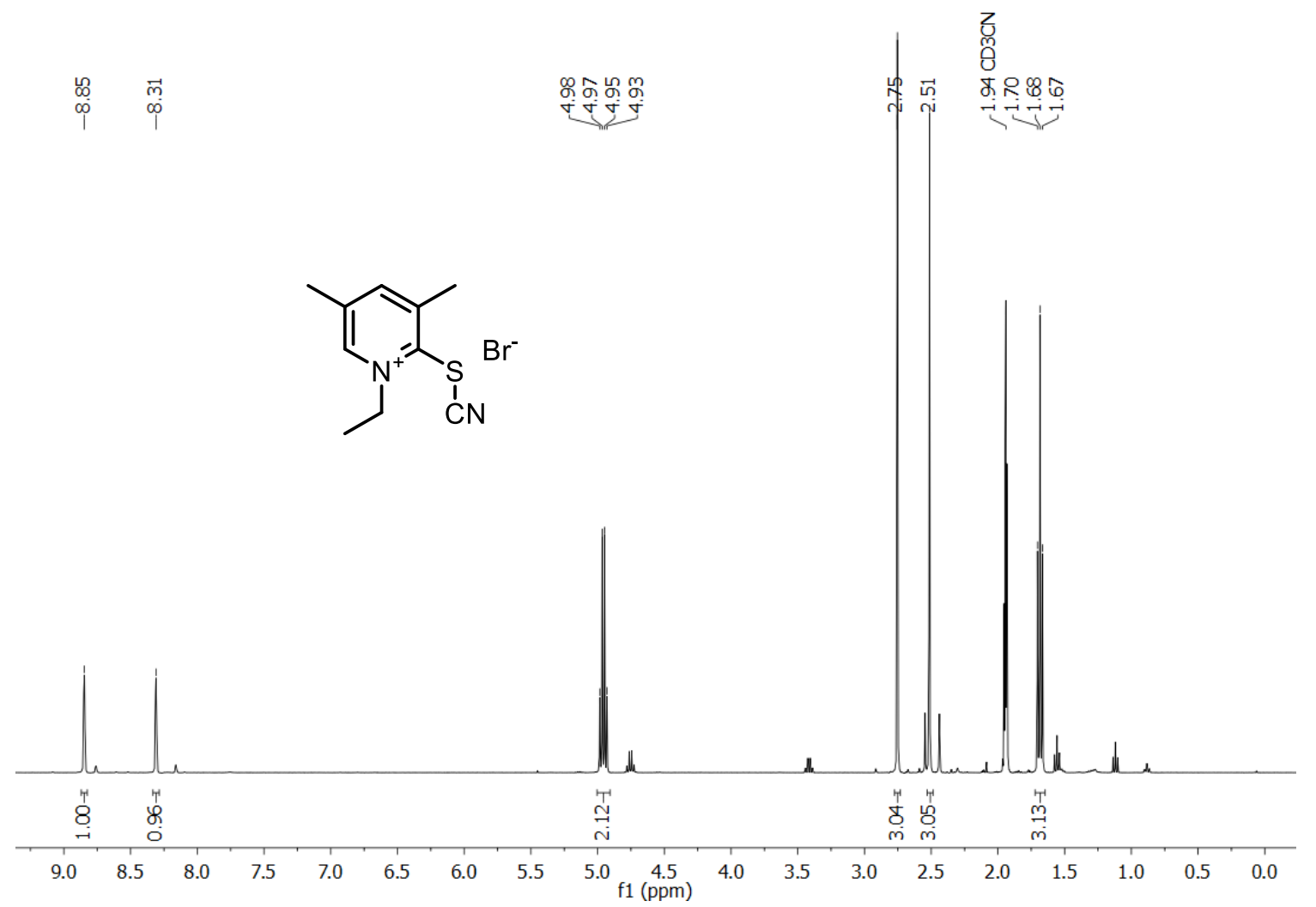

${ }^{1} \mathrm{H} \quad \mathrm{NMR}$ of compound 155b, 1-ethyl-3,5-dimethyl-2-thiocyanatopyridin-1-ium hexafluoroantimonate, $400 \mathrm{MHz}, \mathrm{CD}_{3} \mathrm{CN}, 300 \mathrm{~K}$

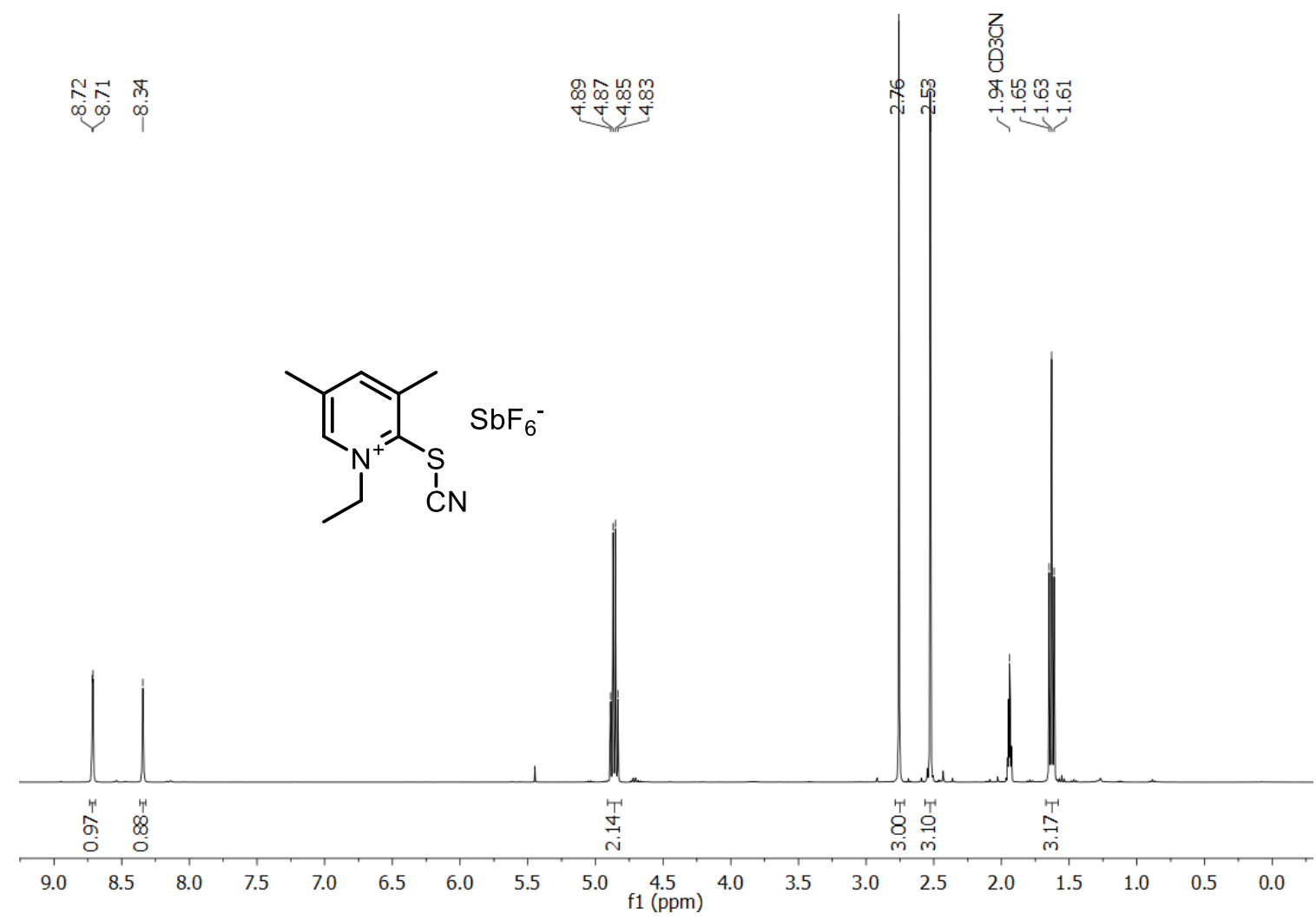


${ }^{13} \mathrm{C}$ NMR of compound 155b, 1-ethyl-3,5-dimethyl-2-thiocyanatopyridin-1-ium hexafluoroantimonate, $101 \mathrm{MHz}, \mathrm{CD}_{3} \mathrm{CN}, 300 \mathrm{~K}$

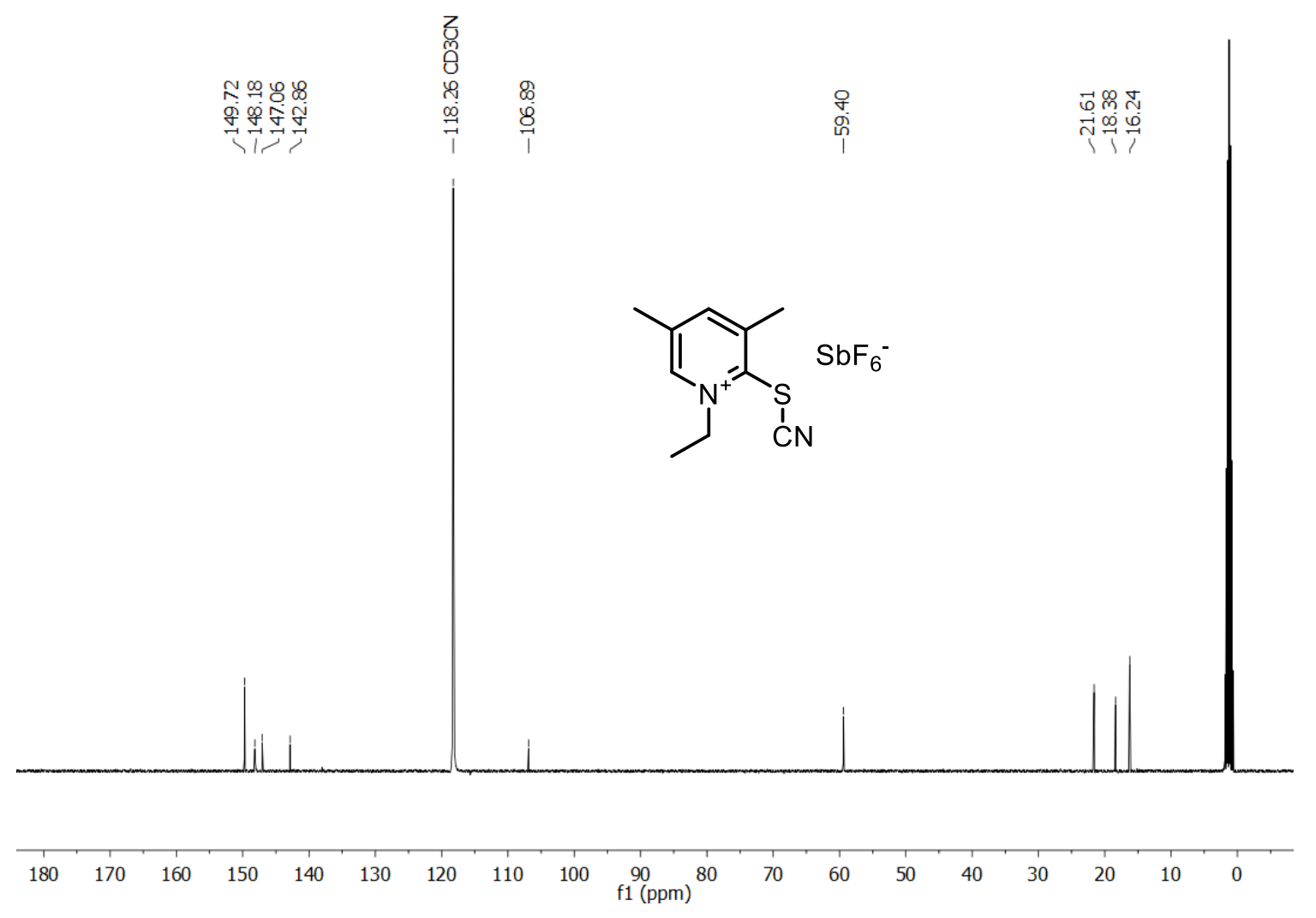

${ }^{19} \mathrm{~F} \quad \mathrm{NMR}$ of compound 155b, 1-ethyl-3,5-dimethyl-2-thiocyanatopyridin-1-ium hexafluoroantimonate, $282 \mathrm{MHz}, \mathrm{CD}_{3} \mathrm{CN}, 303 \mathrm{~K}$

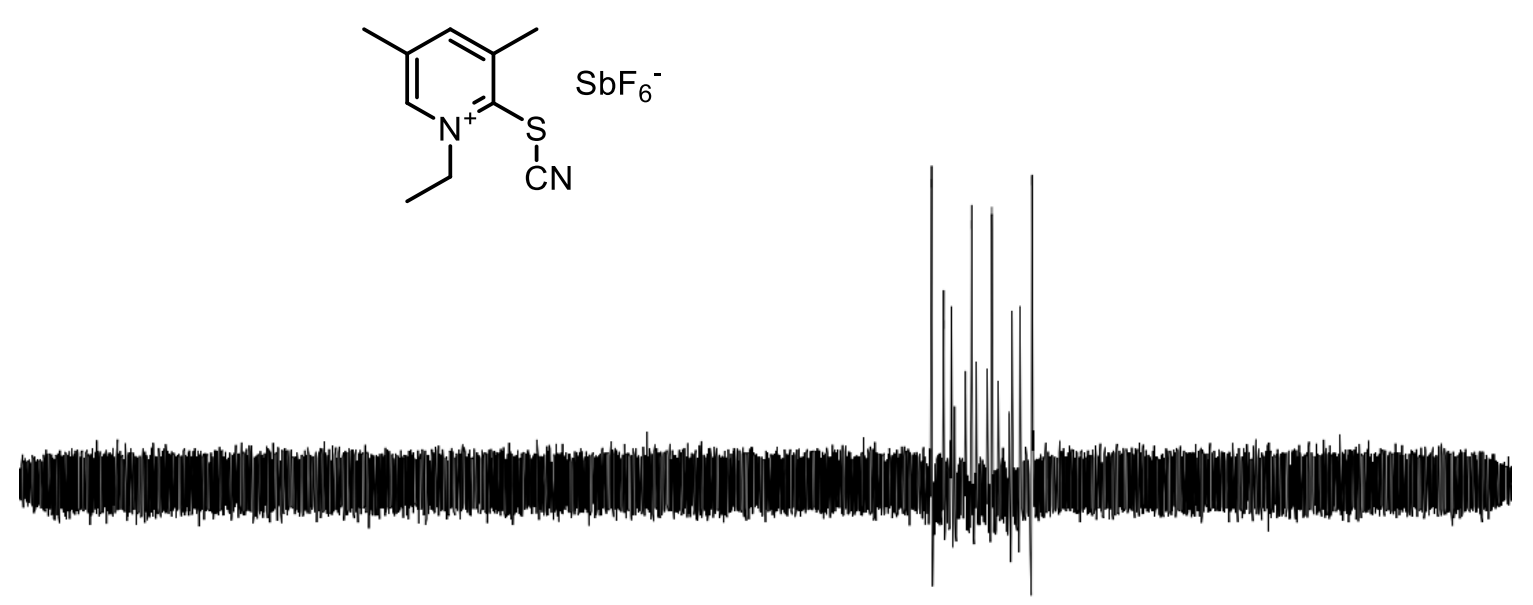

$\begin{array}{llllllllllllllllllllllllll}200 & 180 & 160 & 140 & 120 & 100 & 80 & 60 & 40 & 20 & 0 & -20 & -40 & -60 & -80 & -100 & -120 & -140 & -160 & -180 & -200 & -220 & -240 & -260 & -280 & -300\end{array}$ 
${ }^{1} \mathrm{H}$ NMR of compound 155c, 1-ethyl-2-thiocyanatopyridin-1-ium tetrafluoroborate, 300 $\mathrm{MHz}, \mathrm{CD}_{3} \mathrm{CN}, 303 \mathrm{~K}$

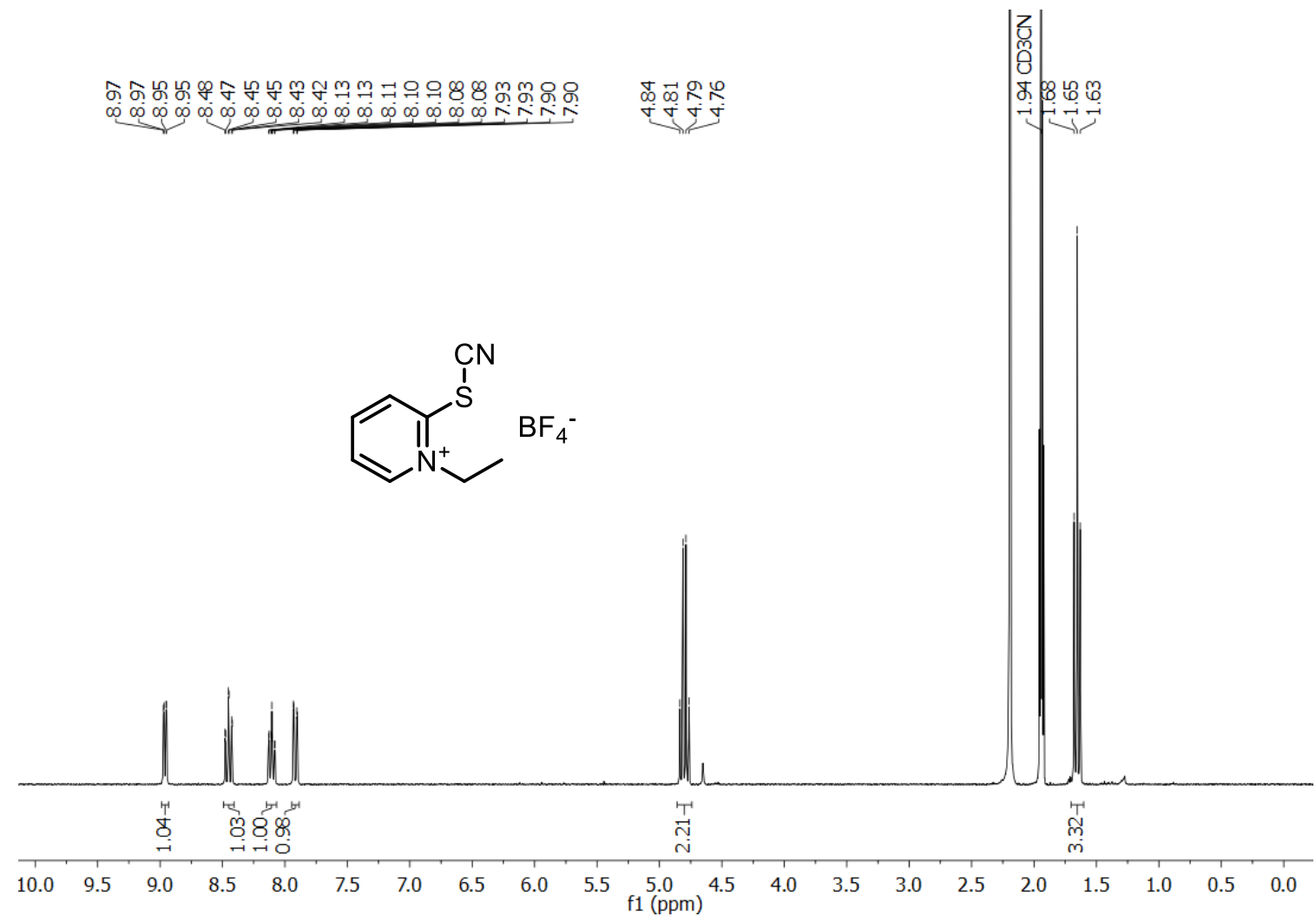

${ }^{19} \mathrm{~F}$ NMR of compound 155c, 1-ethyl-2-thiocyanatopyridin-1-ium tetrafluoroborate, 282 $\mathrm{MHz}, \mathrm{CD}_{3} \mathrm{CN}, 303 \mathrm{~K}$

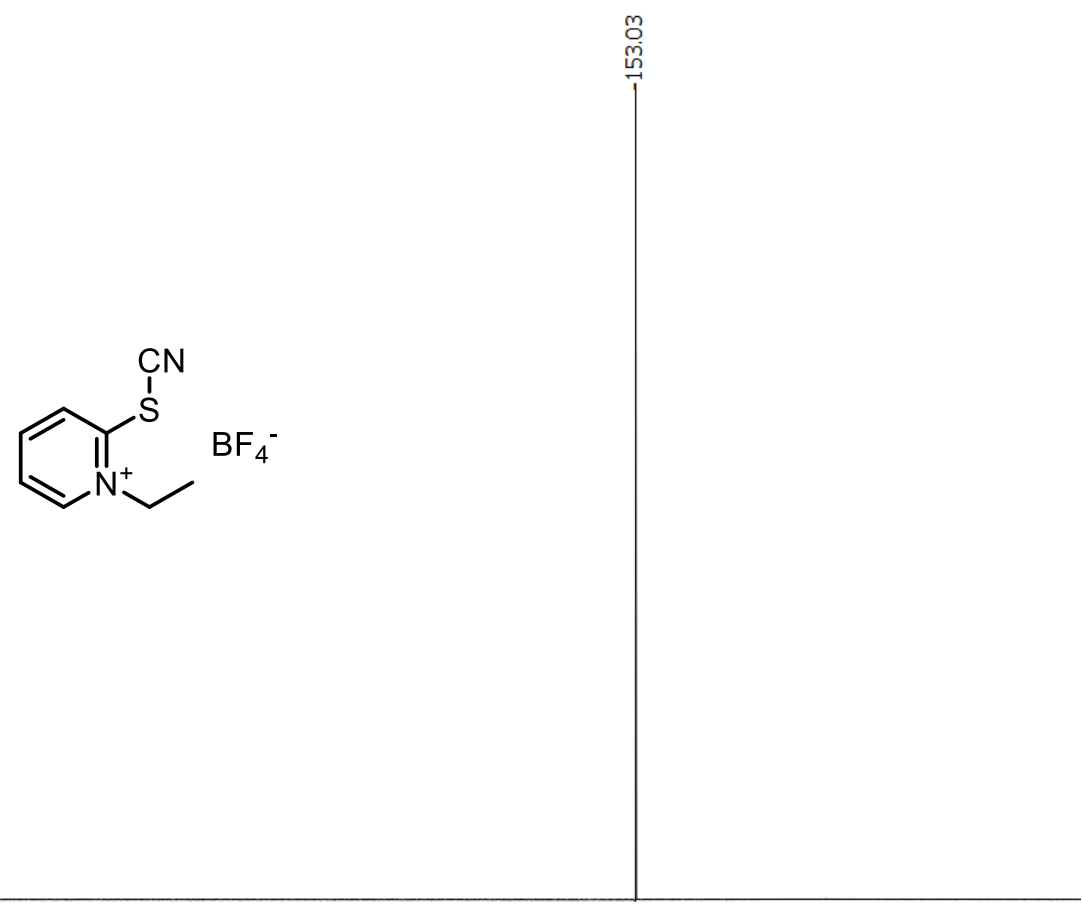

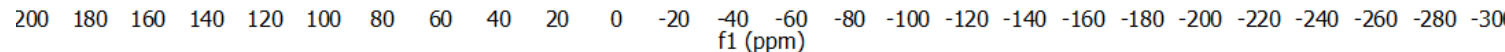


${ }^{1} \mathrm{H}$ NMR of compound 156a, 2-chloro-1-methylpyridin-1-ium tetrafluoroborate, $400 \mathrm{MHz}$, $\mathrm{CD}_{3} \mathrm{CN}, 298 \mathrm{~K}$

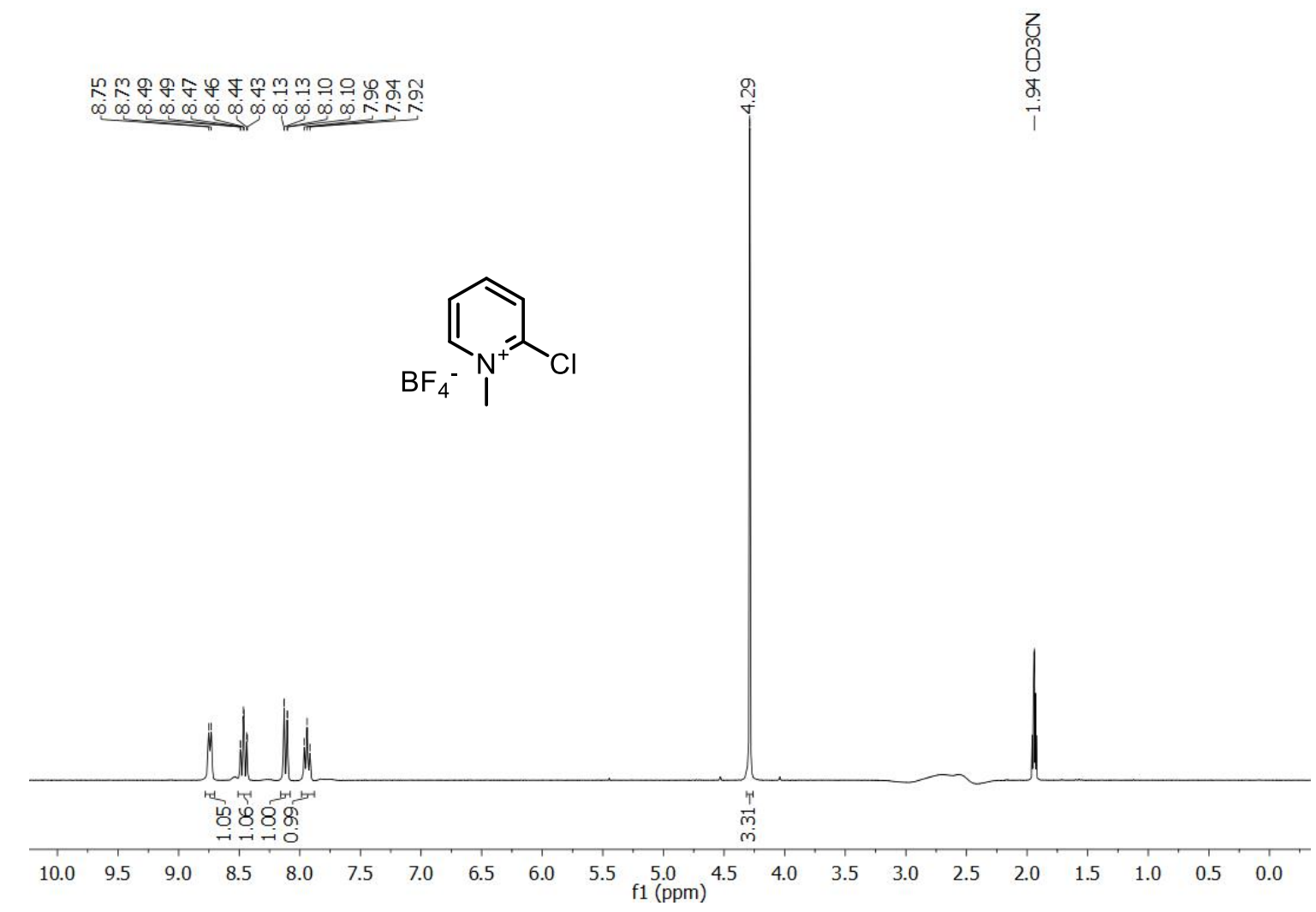

${ }^{19} \mathrm{~F}$ NMR of compound 156a, 2-chloro-1-methylpyridin-1-ium tetrafluoroborate, $282 \mathrm{MHz}$, $\mathrm{CD}_{3} \mathrm{CN}, 298 \mathrm{~K}$<smiles>C[n+]1cccc(Cl)c1</smiles> 
${ }^{1} \mathrm{H}$ NMR of compound 156b, 2-chloro-1-ethylpyridin-1-ium tetrafluoroborate, $400 \mathrm{MHz}$, $\mathrm{CD}_{3} \mathrm{CN}, 298 \mathrm{~K}$

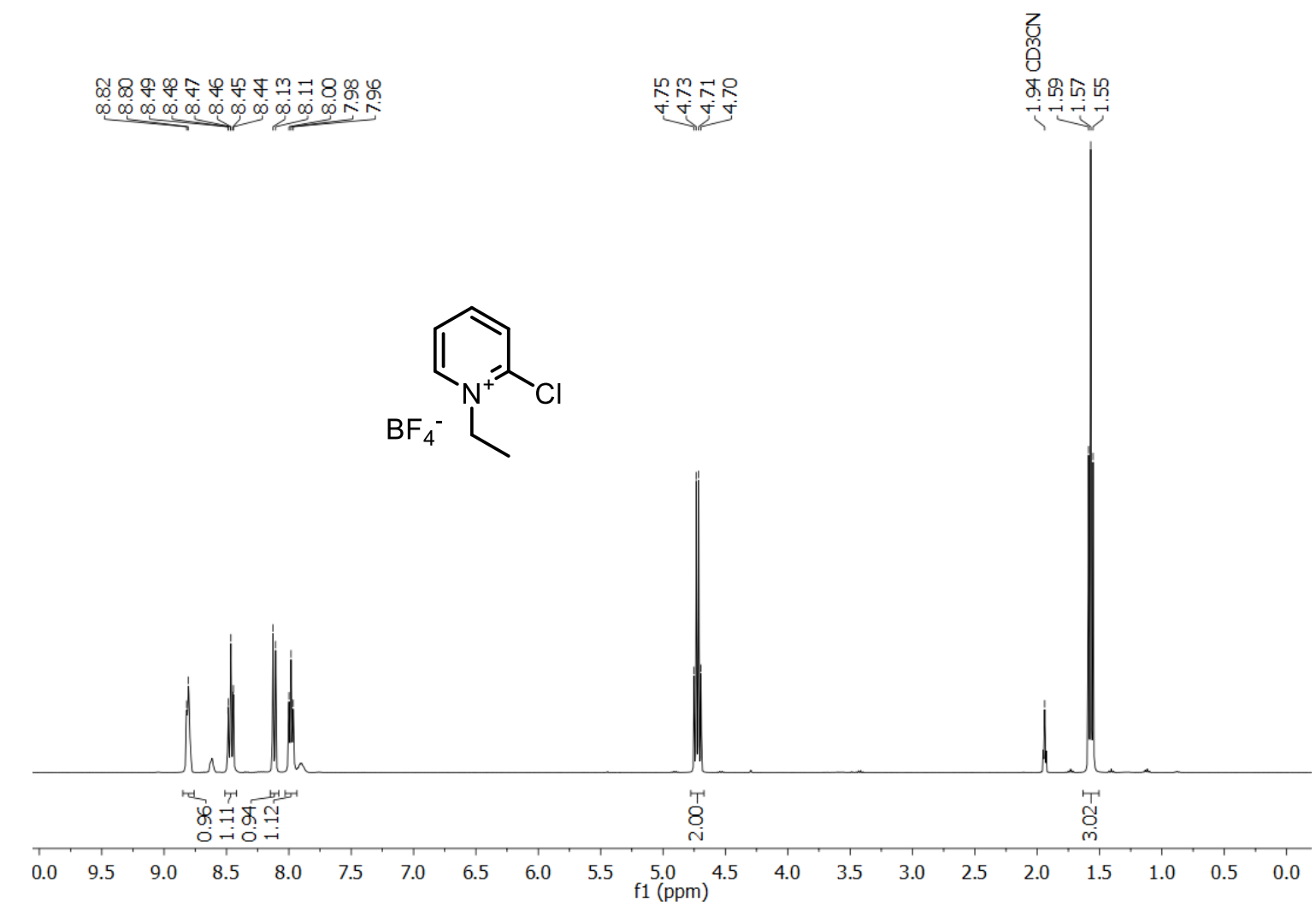

${ }^{13} \mathrm{C}$ NMR of compound 156b, 2-chloro-1-ethylpyridin-1-ium tetrafluoroborate, $101 \mathrm{MHz}$, $\mathrm{CD}_{3} \mathrm{CN}, 298 \mathrm{~K}$

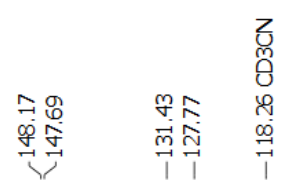
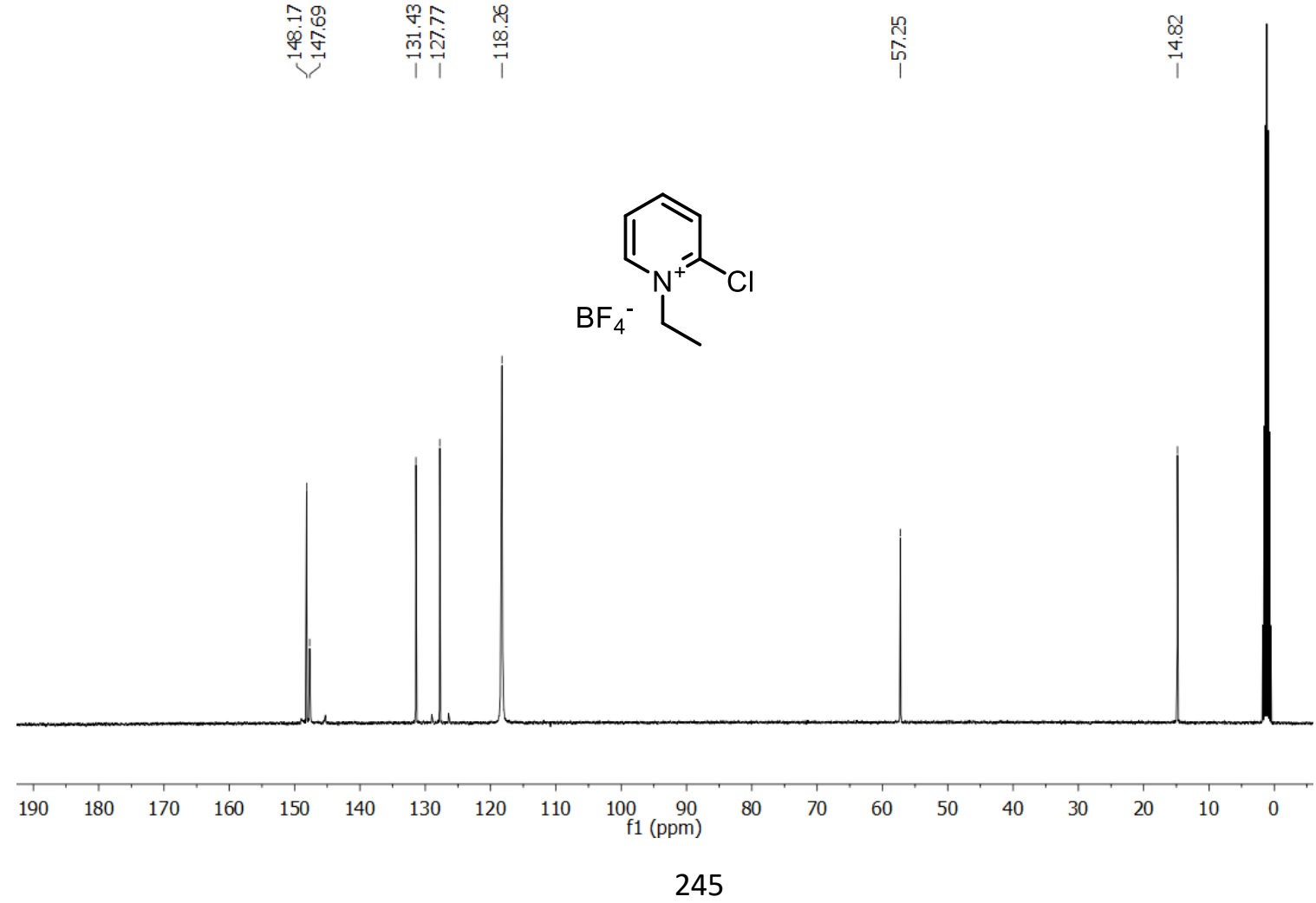
${ }^{1} \mathrm{H}$ NMR of compound 156c, 1-ethyl-3,5-dimethylpyridin-1-ium tetrafluoroborate, 400 $\mathrm{MHz}, \mathrm{CD}_{3} \mathrm{CN}, 298 \mathrm{~K}$

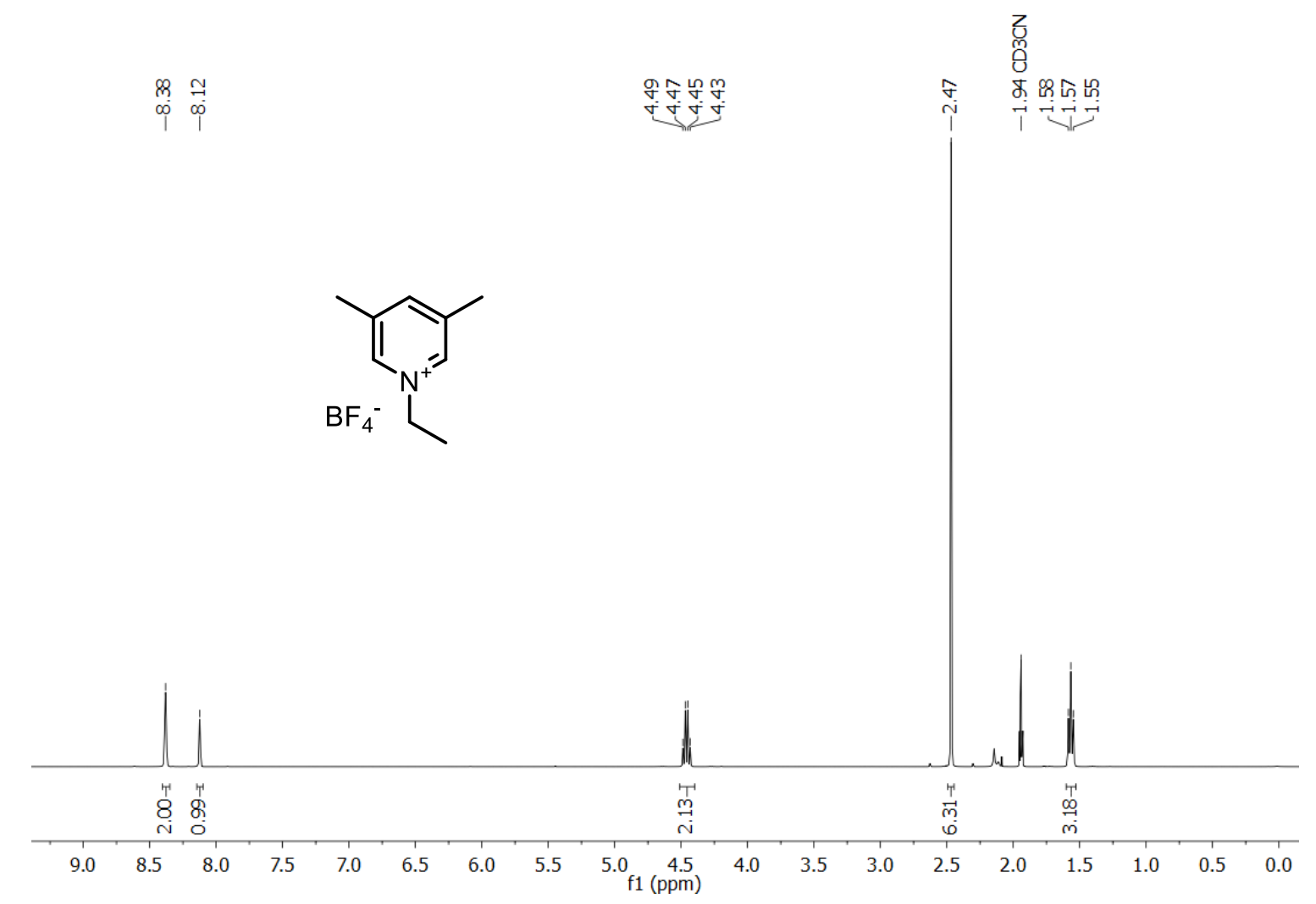

${ }^{13} \mathrm{C}$ NMR of compound 156c, 1-ethyl-3,5-dimethylpyridin-1-ium tetrafluoroborate, 101 $\mathrm{MHz}, \mathrm{CD}_{3} \mathrm{CN}, 298 \mathrm{~K}$

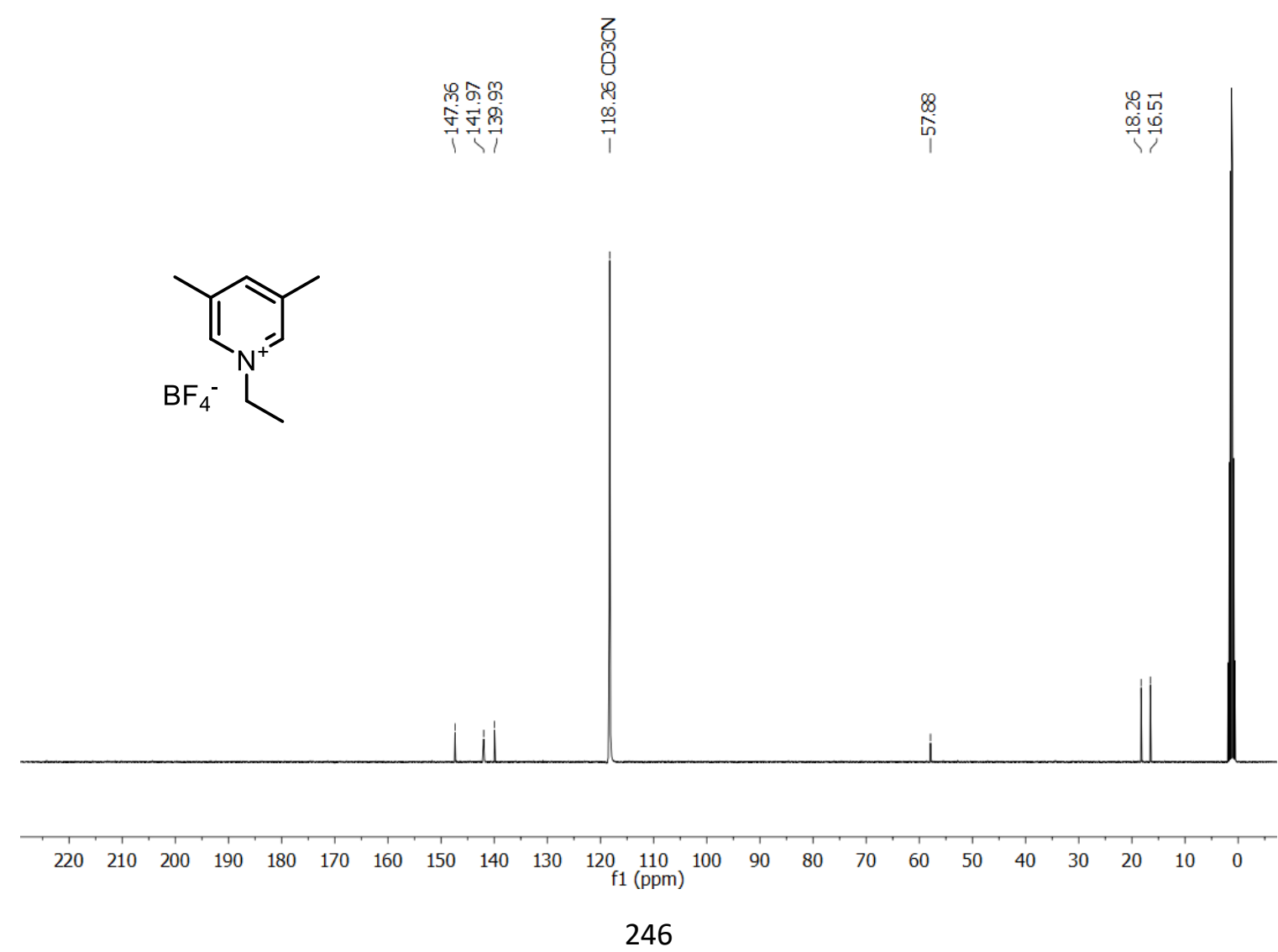


${ }^{19} \mathrm{~F}$ NMR of compound 156c, 1-ethyl-3,5-dimethylpyridin-1-ium tetrafluoroborate, 377 $\mathrm{MHz}, \mathrm{CD}_{3} \mathrm{CN}, 298 \mathrm{~K}$

$$
\stackrel{\circ}{\stackrel{9}{i}}
$$<smiles>CC[n+]1cc(C)cc(C)c1</smiles>

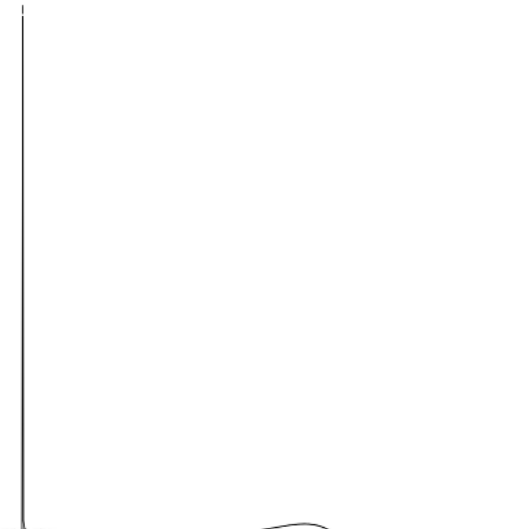

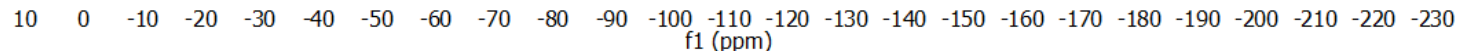

${ }^{1} \mathrm{H}$ NMR of compound 157, 2,2'-thiobis(1-ethylpyridin-1-ium) bishexafluoroantimonate, $400 \mathrm{MHz}, \mathrm{DMSO}-\mathrm{d}_{6}, 300 \mathrm{~K}$

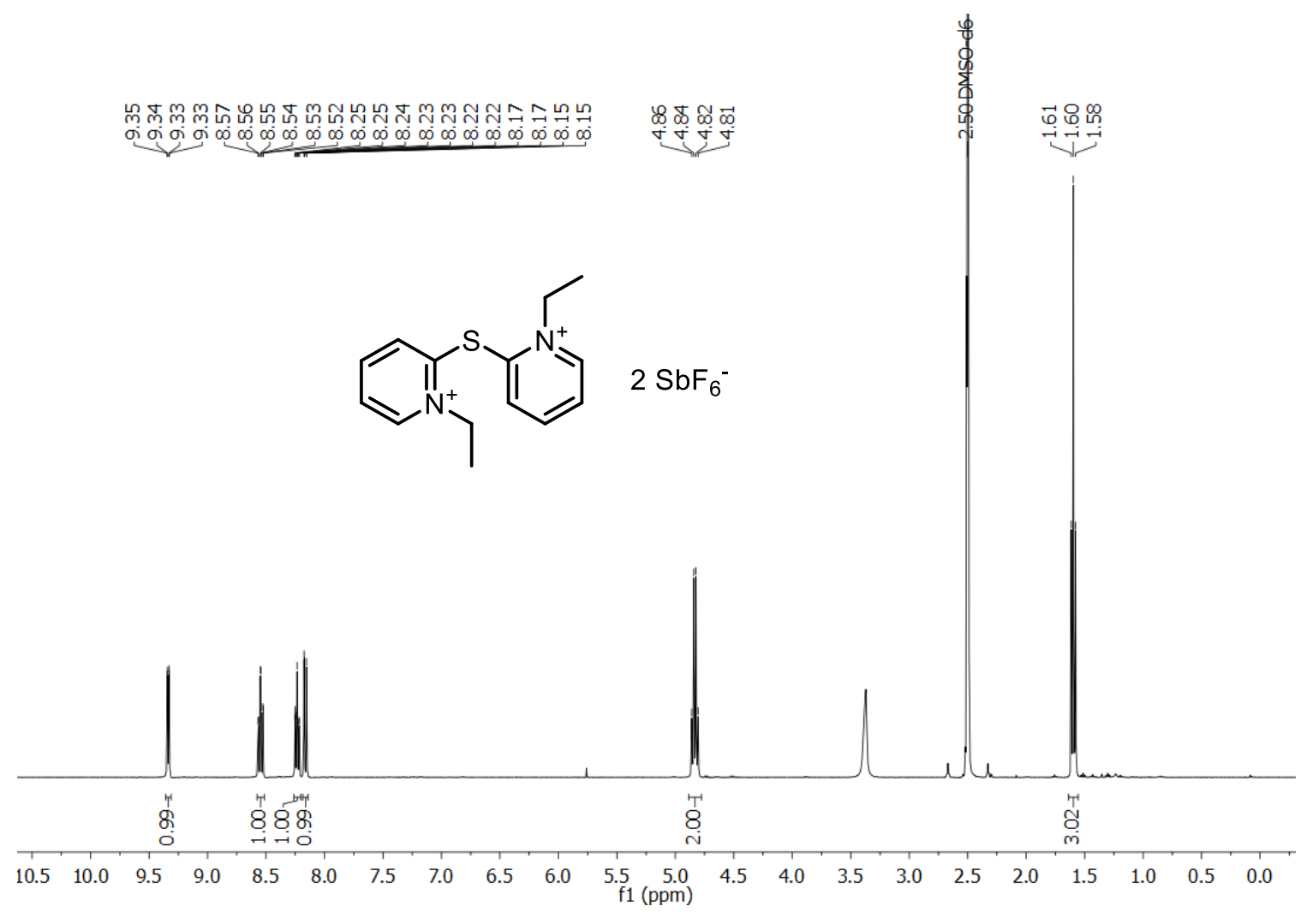


${ }^{13} \mathrm{C}$ NMR of compound 157, 2,2'-thiobis(1-ethylpyridin-1-ium) bishexafluoroantimonate, $101 \mathrm{MHz}$, DMSO-d $6,300 \mathrm{~K}$

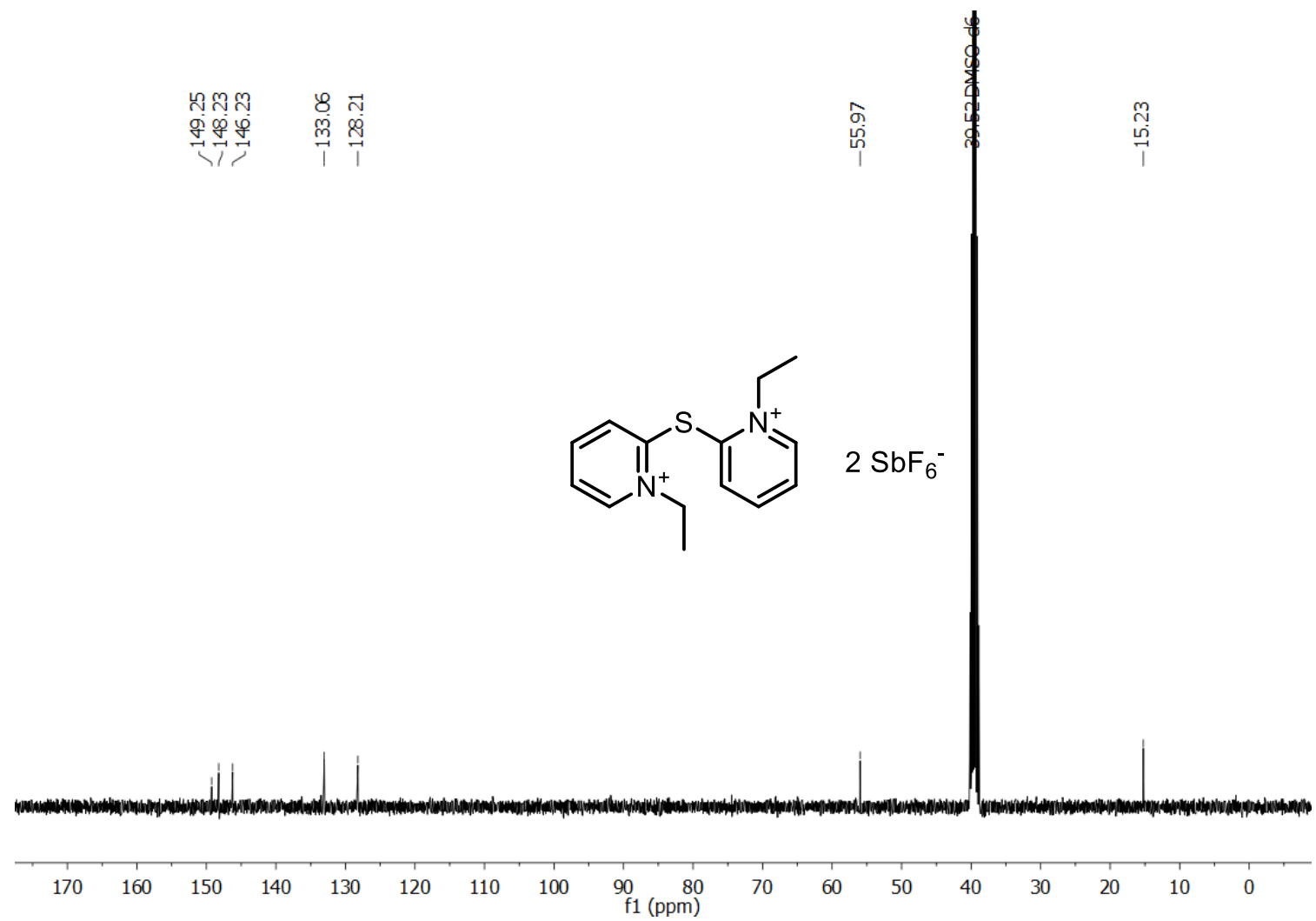

${ }^{19} \mathrm{~F}$ NMR of compound 157, 2,2'-thiobis(1-ethylpyridin-1-ium) bishexafluoroantimonate, $282 \mathrm{MHz}$, DMSO-d $6,303 \mathrm{~K}$<smiles>CC[n+]1ccccc1Sc1cccc[n+]1CC</smiles> 
${ }^{1} \mathrm{H} \quad \mathrm{NMR}$ of compound 160a, 1-methyl-2-[(phenylethynyl)thio]pyridin-1-ium tetrabromozincate, $400 \mathrm{MHz}, \mathrm{CD}_{3} \mathrm{CN}, 298 \mathrm{~K}$

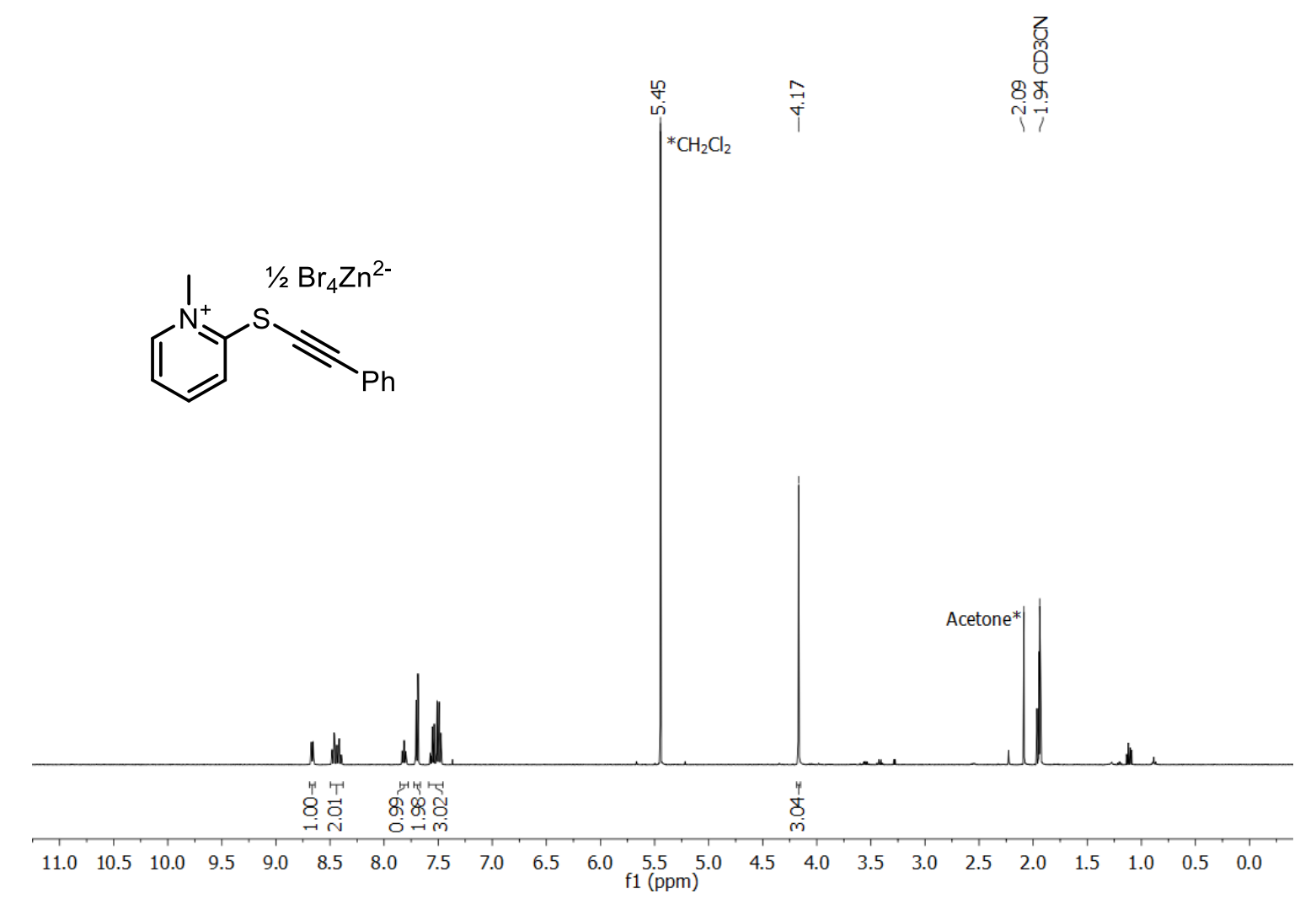

${ }^{13} \mathrm{C}$ NMR of compound 160a, 1-methyl-2-[(phenylethynyl)thio]pyridin-1-ium tetrabromozincate, $101, \mathrm{CD}_{3} \mathrm{CN}, 298 \mathrm{~K}$

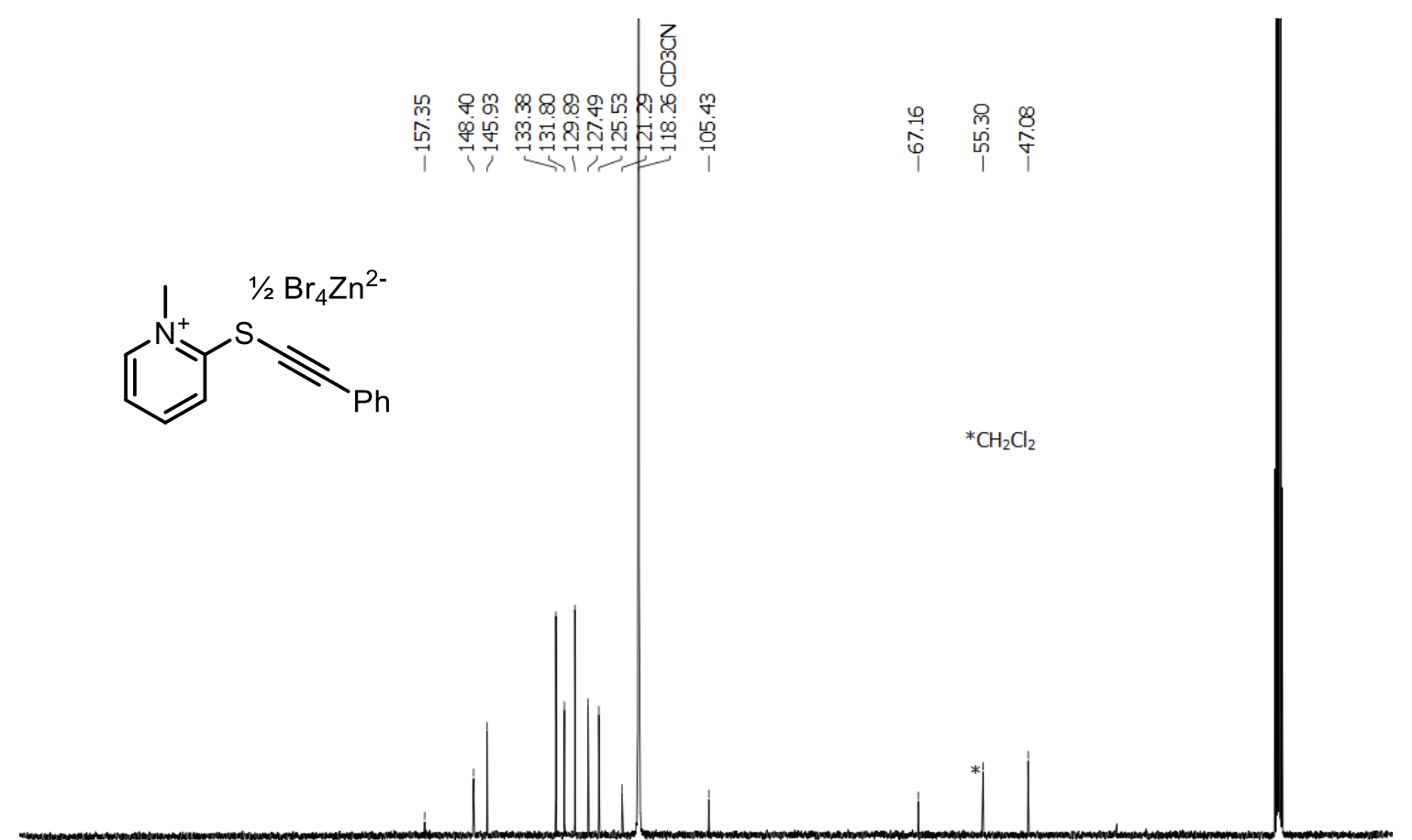

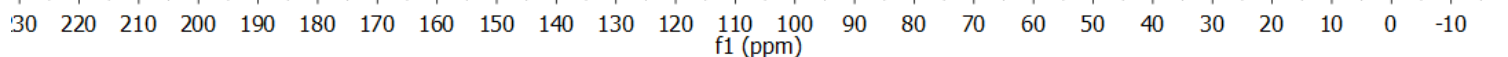


${ }^{1} \mathrm{H} \quad \mathrm{NMR}$ of compound 160b, 1-ethyl-2-[(phenylethynyl)thio]pyridin-1-ium tetrabromozincate, $400 \mathrm{MHz}, \mathrm{CD}_{3} \mathrm{CN}, 300 \mathrm{~K}$<smiles>[Y20][R17](Br)(Br)Br</smiles>

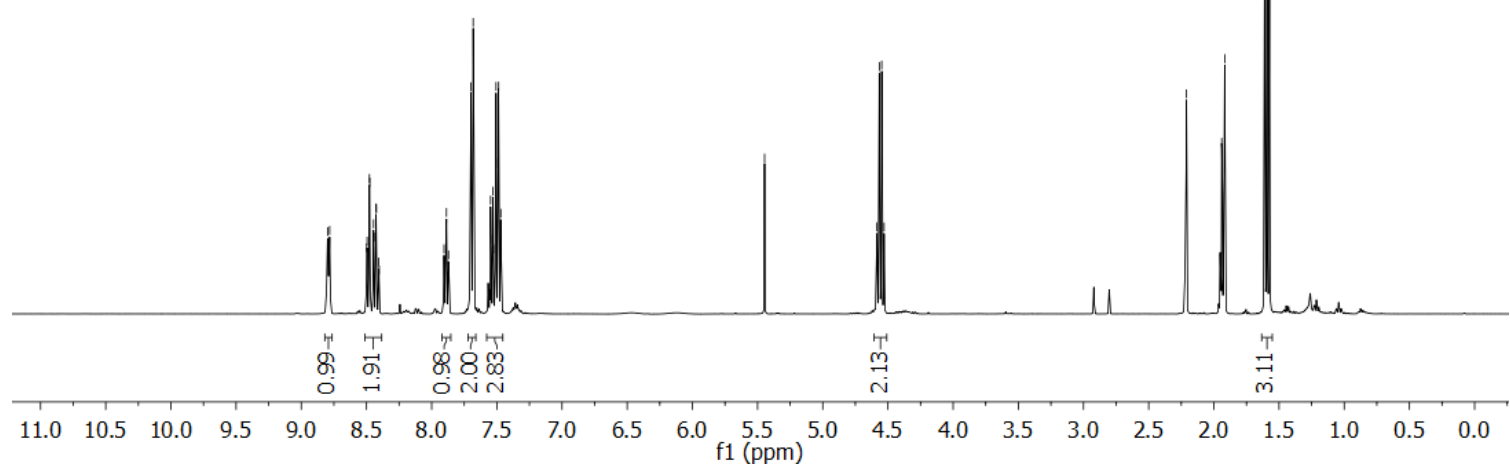

${ }^{13} \mathrm{C}$ NMR of compound 160b, 1-ethyl-2-[(phenylethynyl)thio]pyridin-1-ium tetrabromozincate, $101 \mathrm{MHz}, \mathrm{CD}_{3} \mathrm{CN}, 300 \mathrm{~K}$

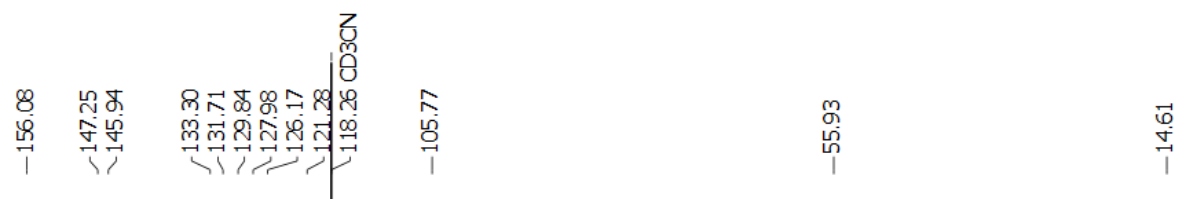

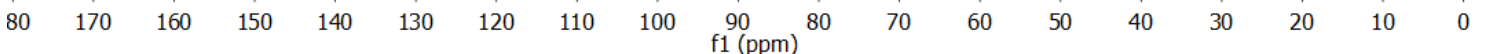


${ }^{1} \mathrm{H}$ NMR of compound 160c, 1-ethyl-3,5-dimethyl-2-[(phenylethynyl)thio]pyridin-1-ium tetrabromozincate, $400 \mathrm{MHz}, \mathrm{CD}_{3} \mathrm{CN}, 300 \mathrm{~K}$

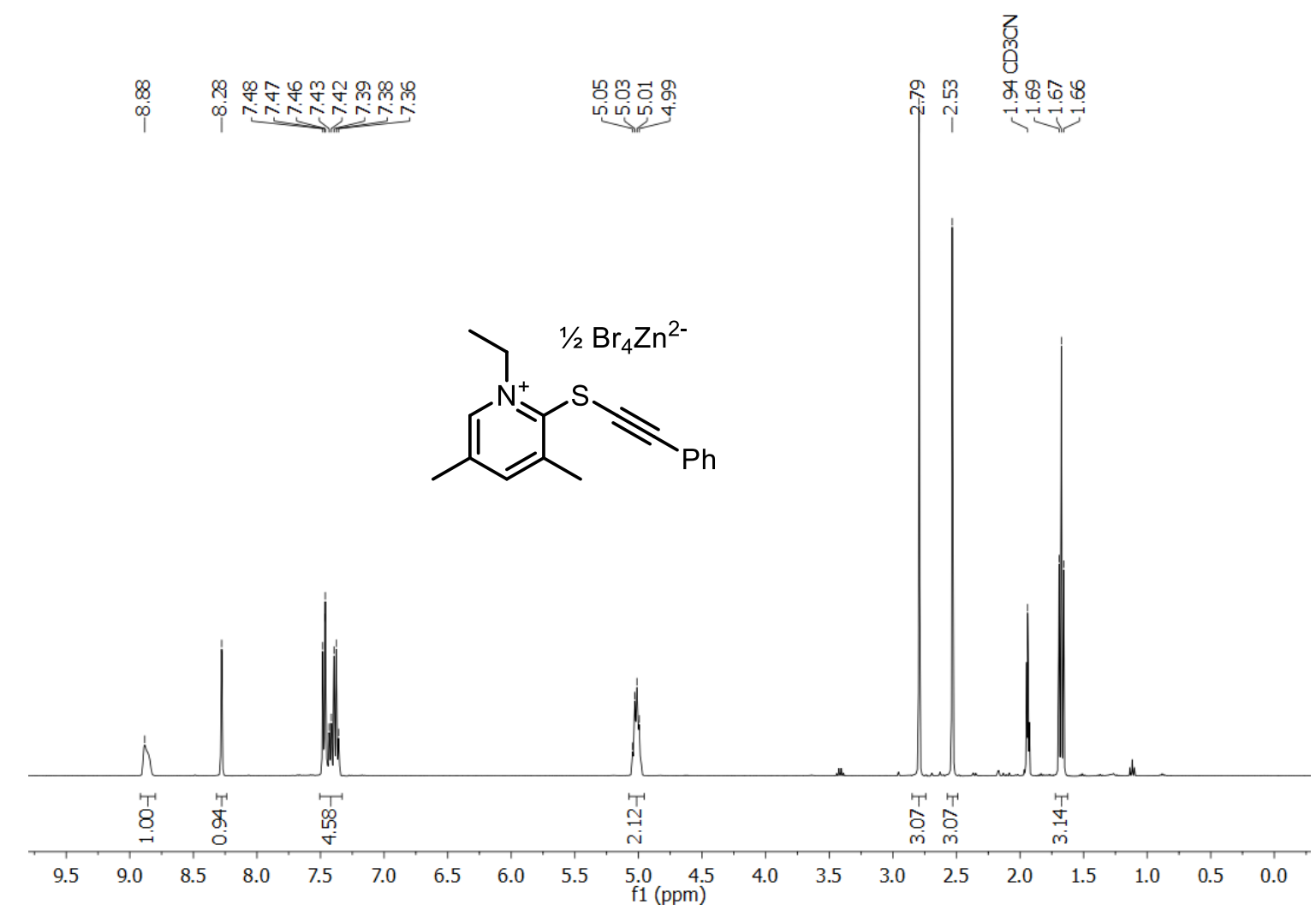

${ }^{13} \mathrm{C}$ NMR of compound 160c, 1-ethyl-3,5-dimethyl-2-[(phenylethynyl)thio]pyridin-1-ium tetrabromozincate, $101 \mathrm{MHz}, \mathrm{CD}_{3} \mathrm{CN}, 300 \mathrm{~K}$

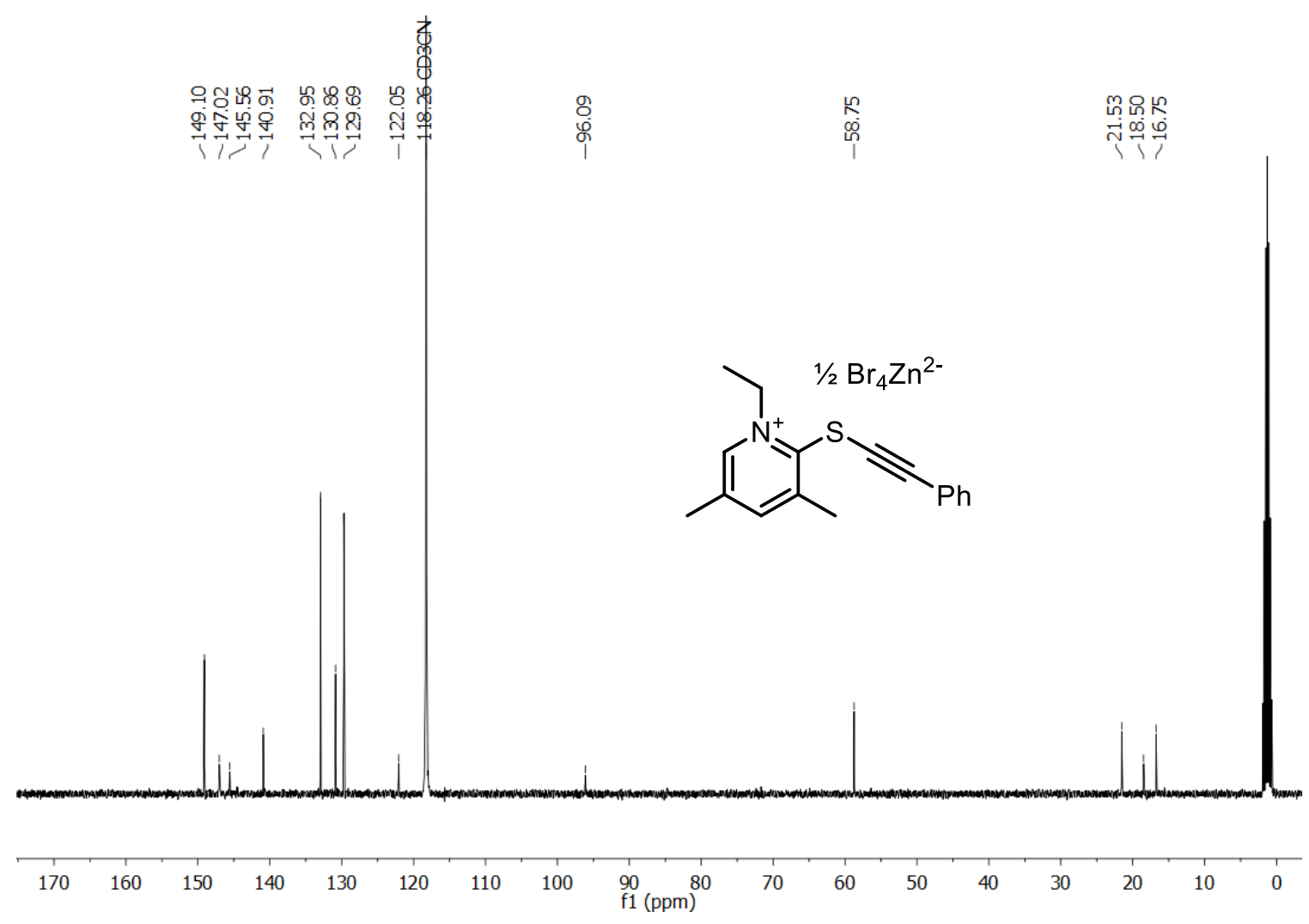


${ }^{1} \mathrm{H}$ NMR of compound 160d, 1-methyl-2-\{[(triisopropylsilyl)ethynyl]thio\}pyridin-1-ium hexafluoroantimonate, $300 \mathrm{MHz}, \mathrm{CD}_{3} \mathrm{CN}, 295 \mathrm{~K}$

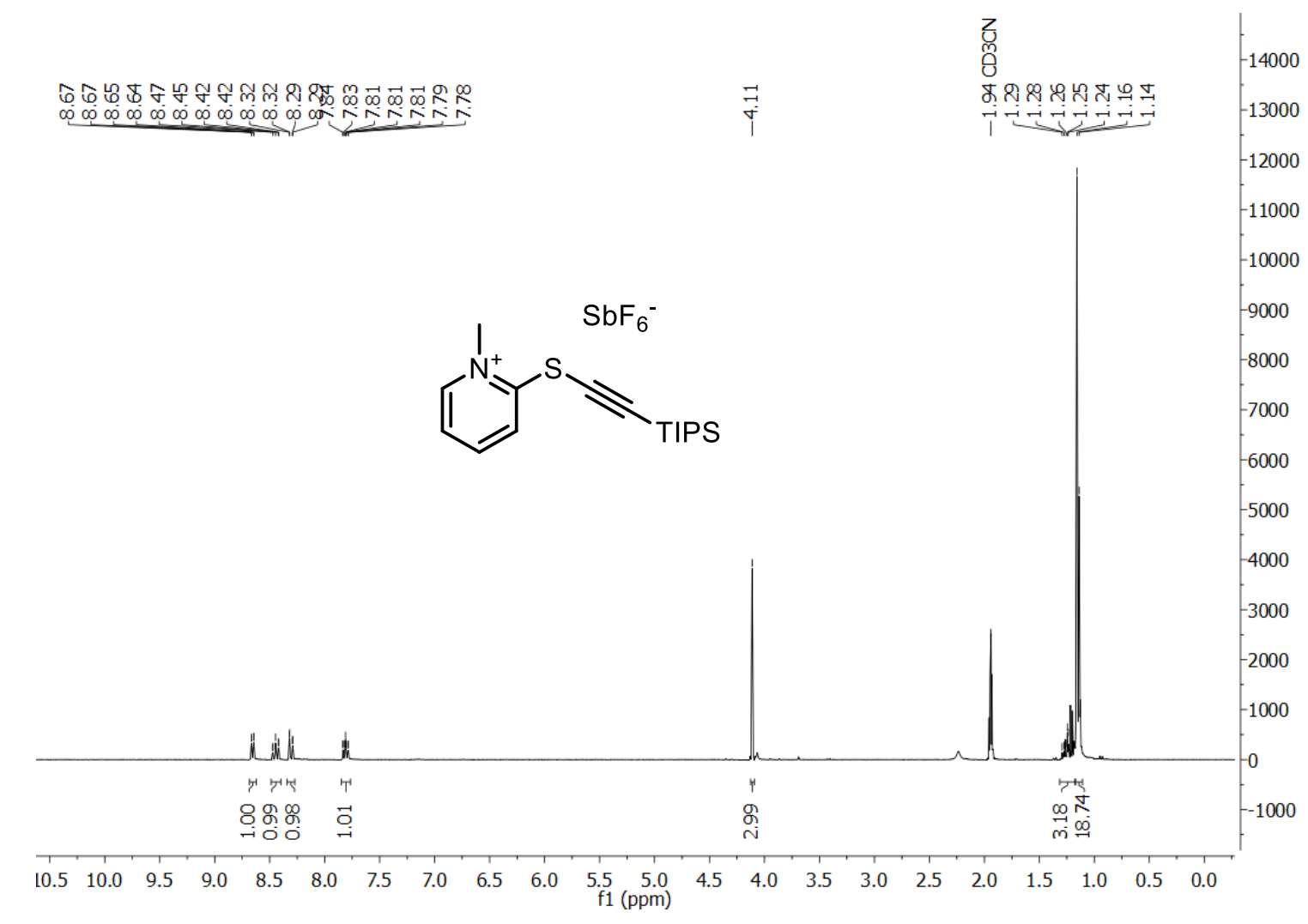

${ }^{13} \mathrm{C}$ NMR of compound $160 \mathrm{~d}, 1$-methyl-2-\{[(triisopropylsilyl)ethynyl]thio\}pyridin-1-ium hexafluoroantimonate, $400 \mathrm{MHz}, \mathrm{CD}_{3} \mathrm{CN}, 295 \mathrm{~K}$
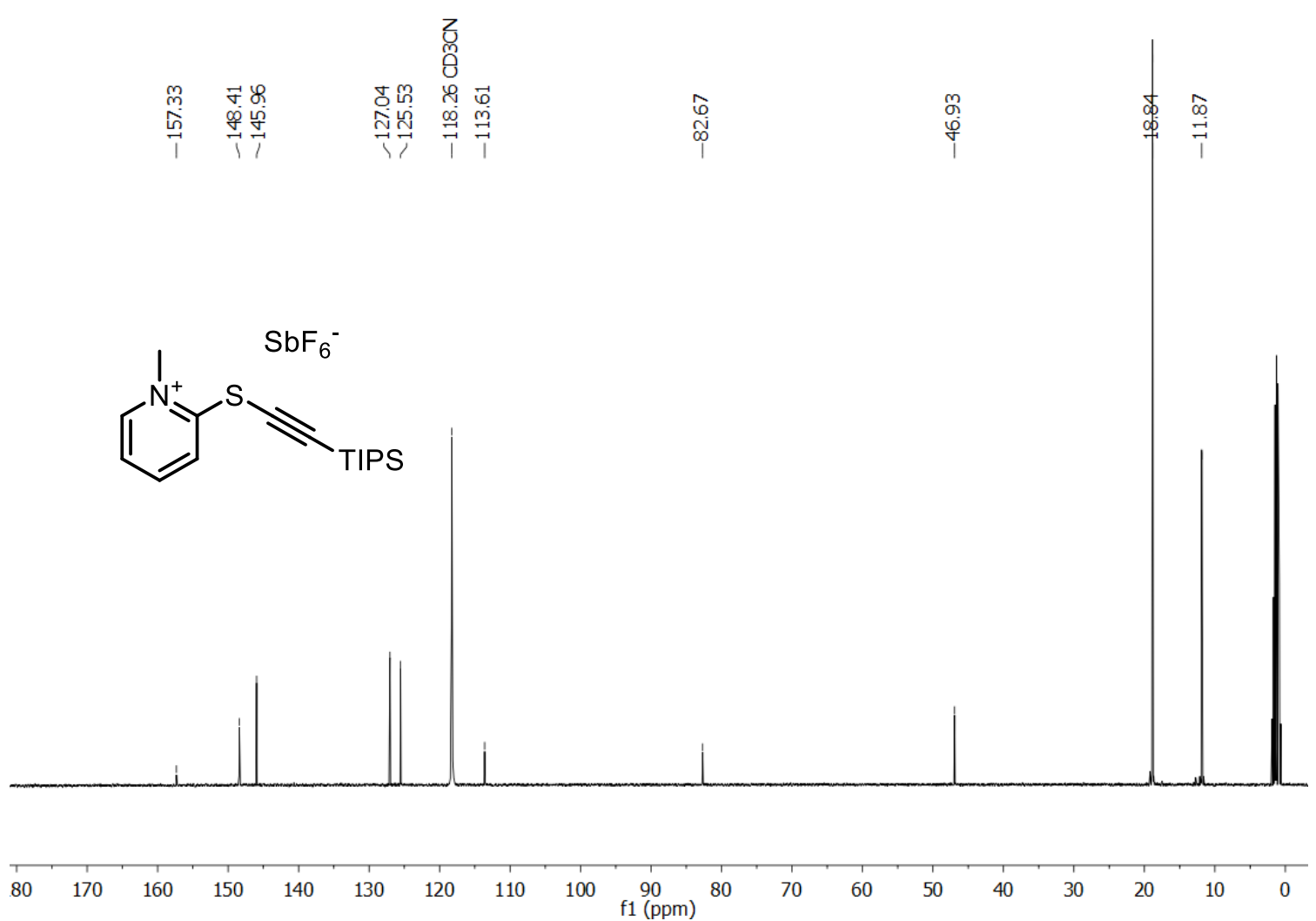
${ }^{19} \mathrm{~F}$ NMR of compound 160d, 1-methyl-2-\{[(triisopropylsilyl)ethynyl]thio\}pyridin-1-ium hexafluoroantimonate, $282 \mathrm{MHz}, \mathrm{CD}_{3} \mathrm{CN}, 295 \mathrm{~K}$

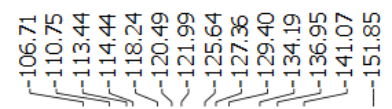<smiles>C[n+]1ccccc1SC#C[In]</smiles>

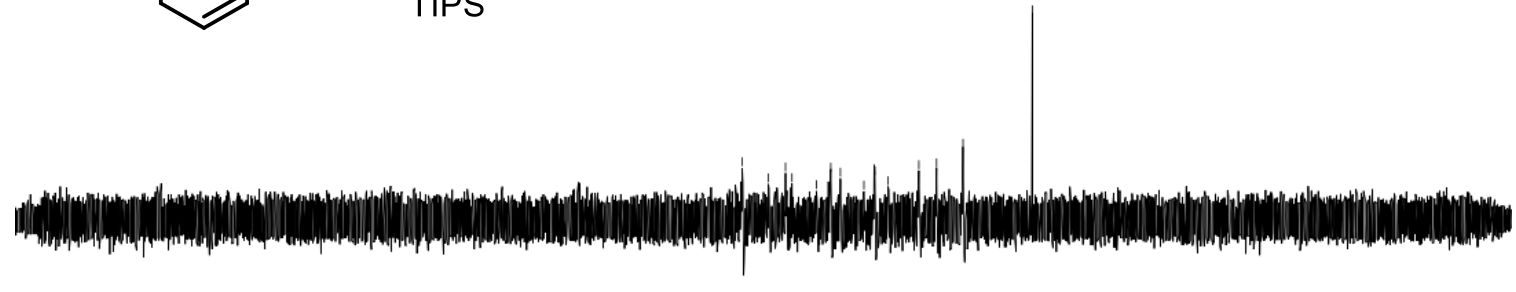

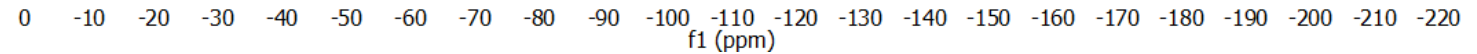

${ }^{1} \mathrm{H}$ NMR of compound 161a, 2-cyclohexylidene-2-phenyl-1-(pyrrolidin-1-yl)ethane-1thione, $500 \mathrm{MHz}, \mathrm{CD}_{2} \mathrm{Cl}_{2}, 298 \mathrm{~K}$

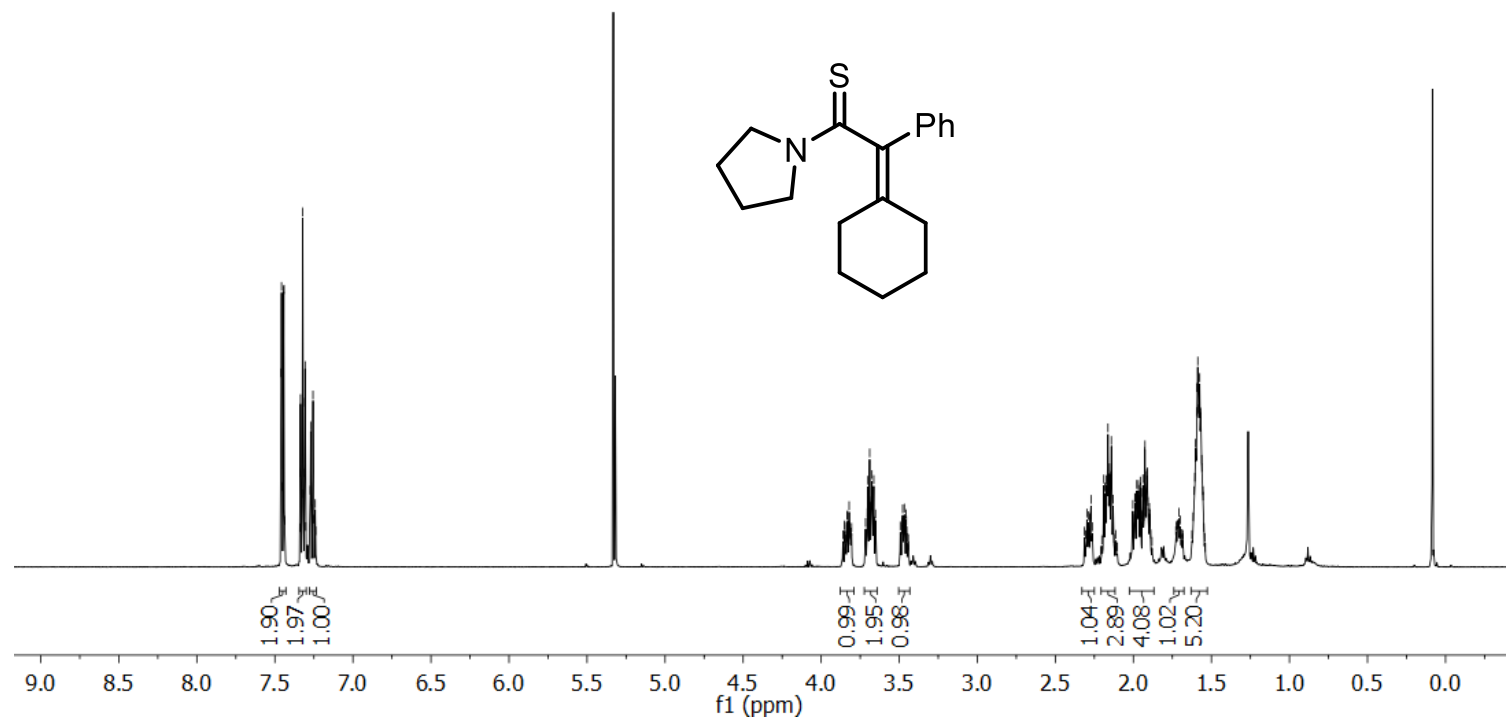


${ }^{13} \mathrm{C}$ NMR of compound 161a, 2-cyclohexylidene-2-phenyl-1-(pyrrolidin-1-yl)ethane-1thione, $126 \mathrm{MHz}, \mathrm{CD}_{2} \mathrm{Cl}_{2}, 300 \mathrm{~K}$

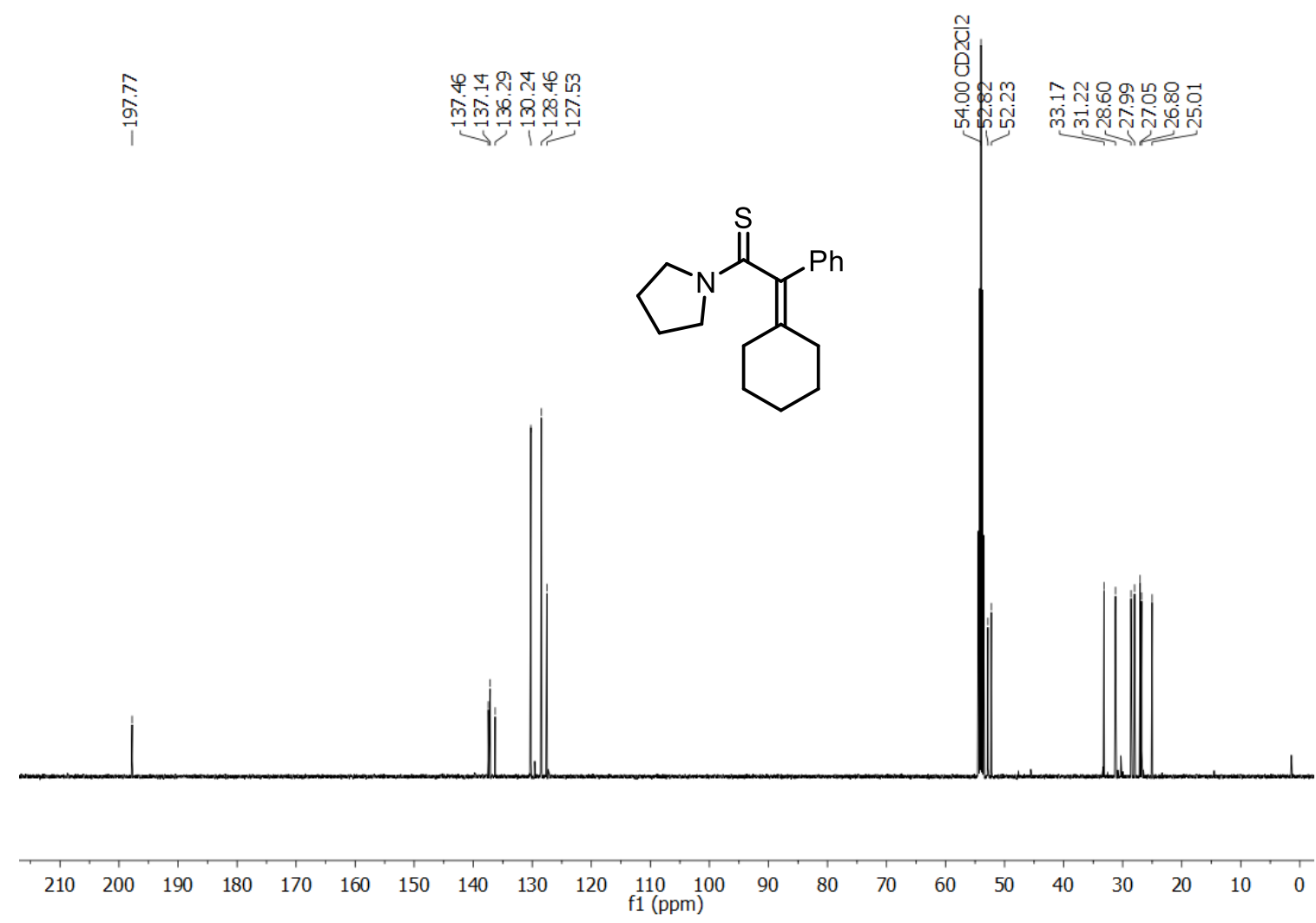

${ }^{1} \mathrm{H}$ NMR of compound 161b, 2-phenyl-1-(pyrrolidin-1-yl)ethane-1-thione, $400 \mathrm{MHz}, \mathrm{CD}_{2} \mathrm{Cl}_{2}$, $298 \mathrm{~K}$

$\frac{\bar{Q}}{8}$

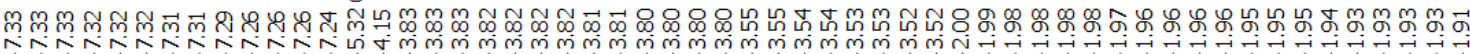

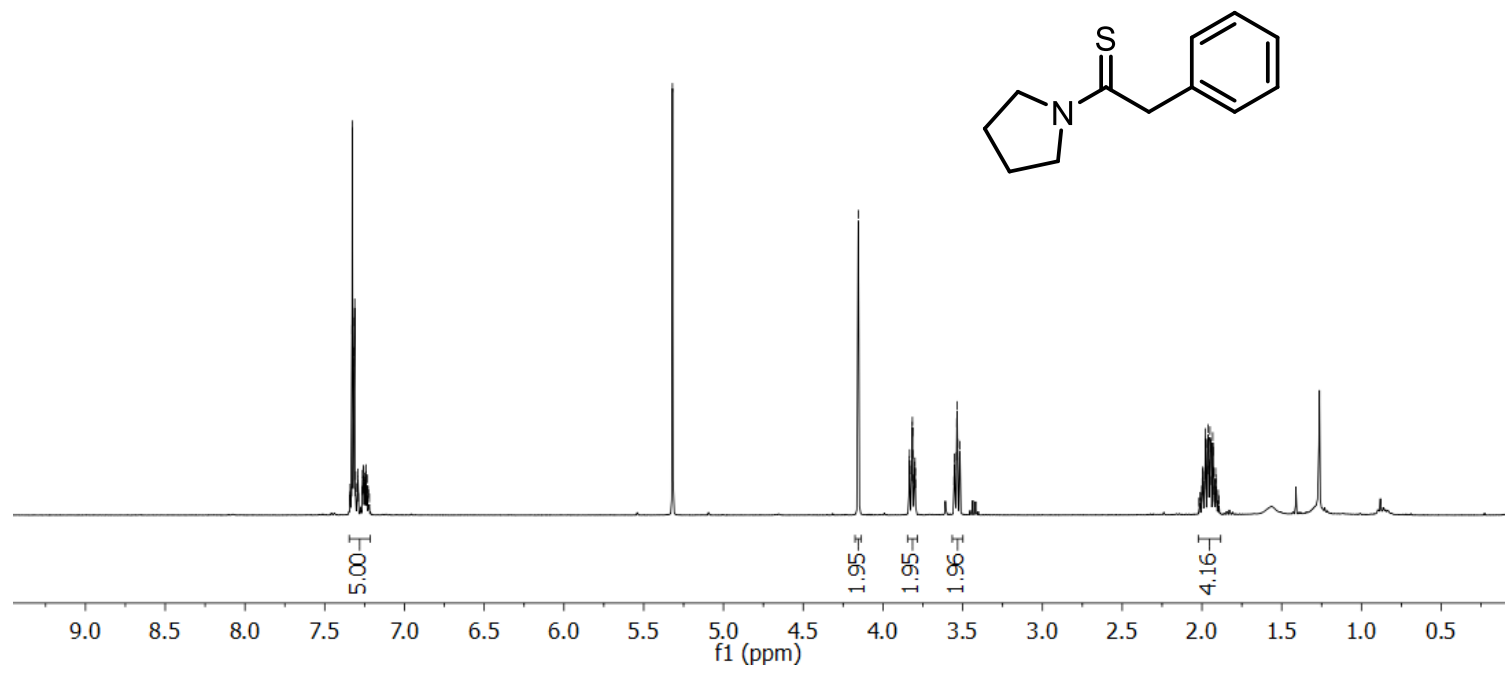


${ }^{13} \mathrm{C}$ NMR of compound 161b, 2-phenyl-1-(pyrrolidin-1-yl)ethane-1-thione, $126 \mathrm{MHz}, \mathrm{CD}_{2} \mathrm{Cl}_{2}$, $298 \mathrm{~K}$

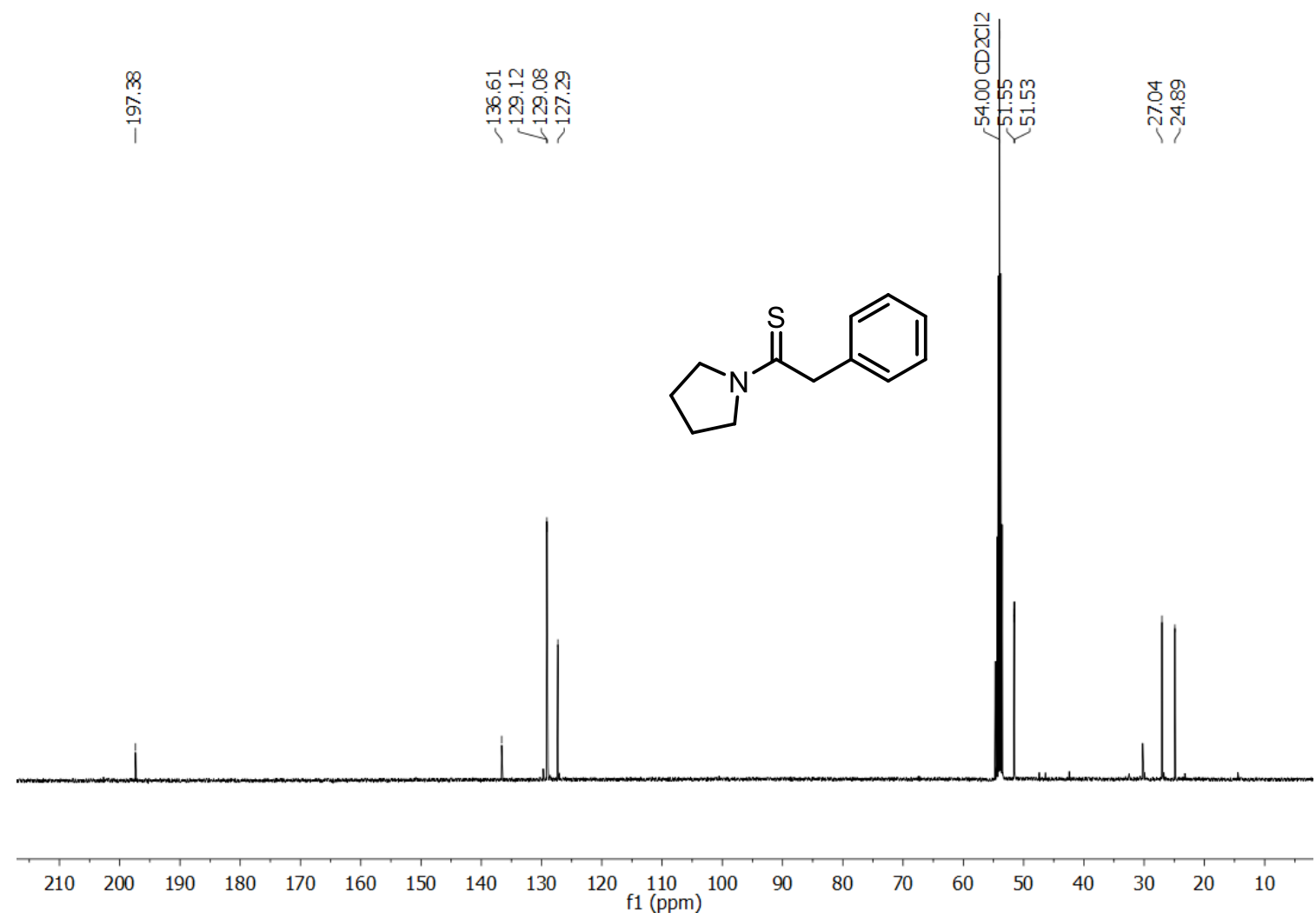

${ }^{1} \mathrm{H}$ NMR of compound 161c, $\mathrm{N}$, $\mathrm{N}$-diethyl-2-phenylethanethioamide, $600 \mathrm{MHz}, \mathrm{CD}_{2} \mathrm{Cl}_{2}, 298 \mathrm{~K}$
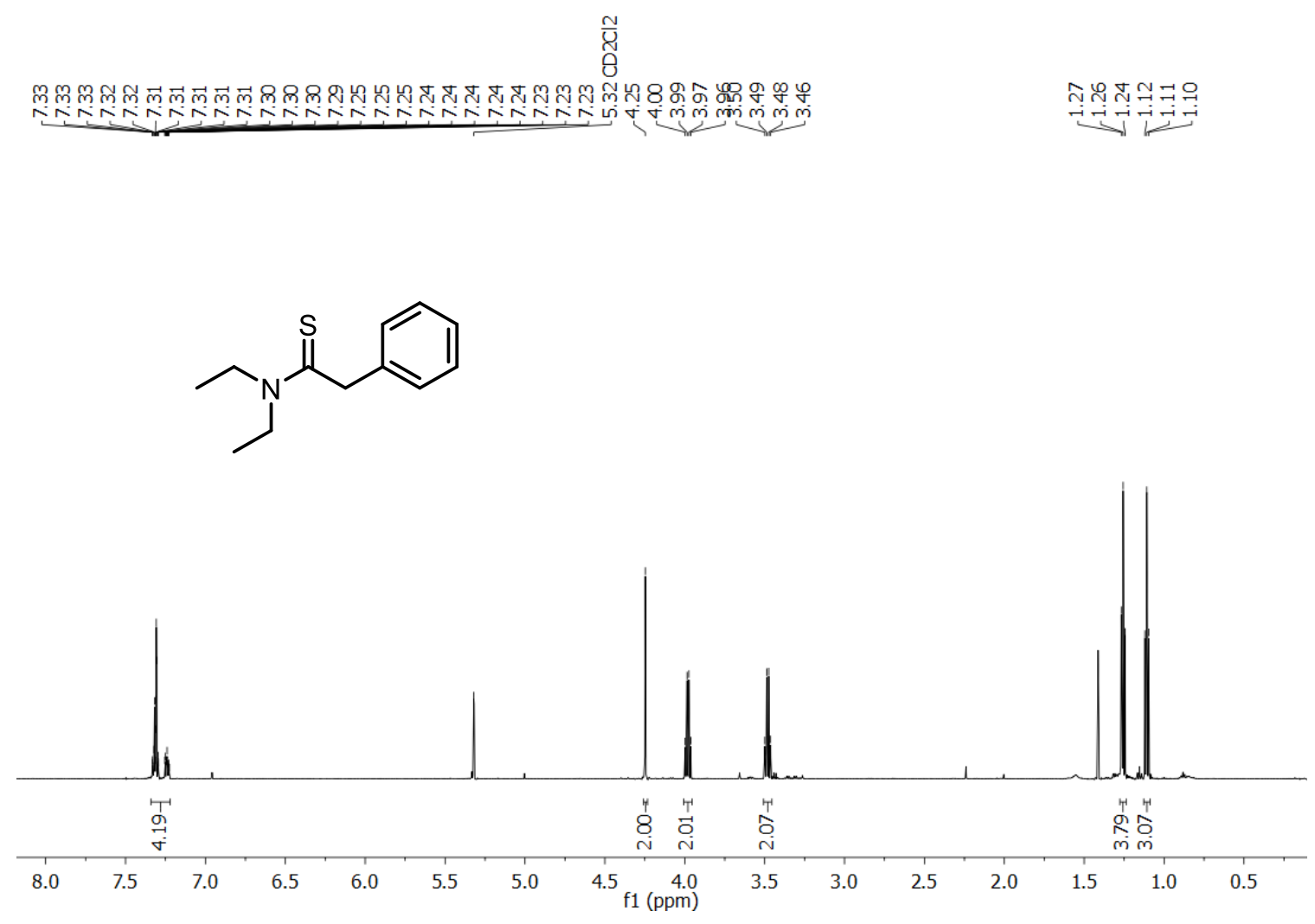
${ }^{13} \mathrm{C}$ NMR of compound 161c, $\mathrm{N}, \mathrm{N}$-diethyl-2-phenylethanethioamide, $126 \mathrm{MHz}, \mathrm{CD}_{2} \mathrm{Cl}_{2}, 300$ K

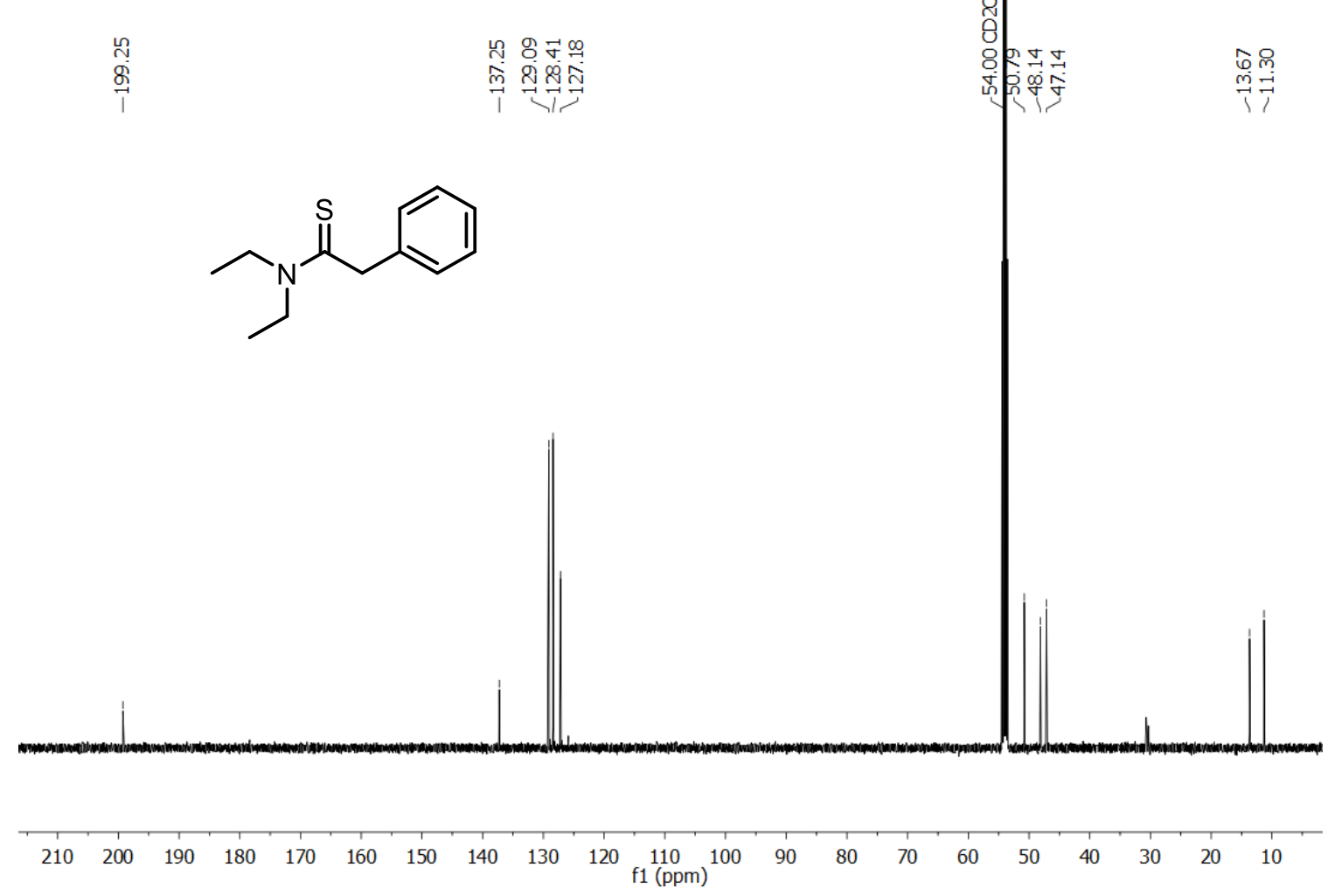

${ }^{1} \mathrm{H}$ NMR of compound 168a, 4-methoxybenzyl 2-(triisopropylsilyl)ethanedithioate, 300 $\mathrm{MHz}, \mathrm{CD}_{3} \mathrm{CN}, 298 \mathrm{~K}$

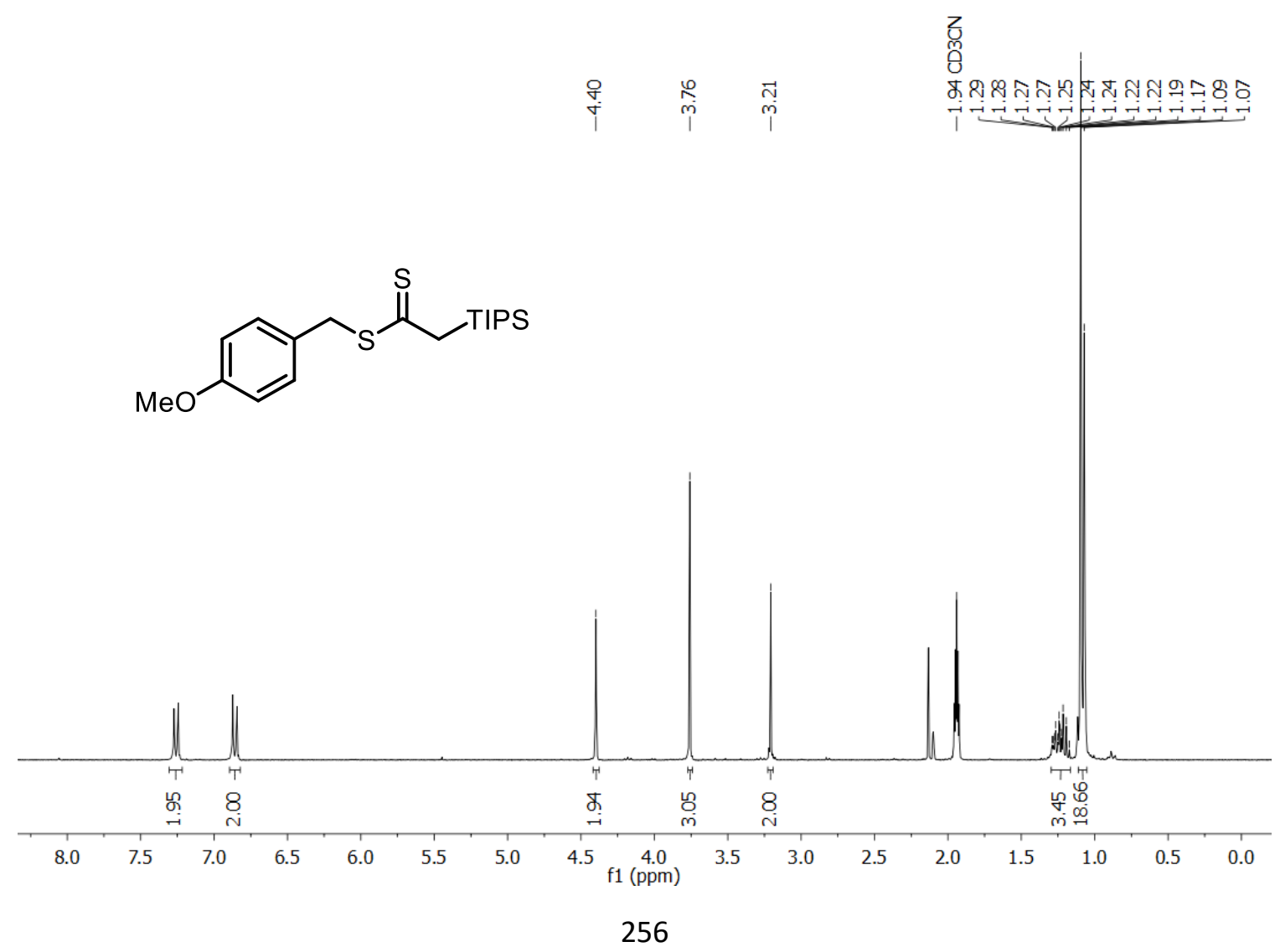


${ }^{13} \mathrm{C}$ NMR of compound 168a, 4-methoxybenzyl 2-(triisopropylsilyl)ethanedithioate, 126 $\mathrm{MHz}, \mathrm{CD}_{3} \mathrm{Cl}, 298 \mathrm{~K}$

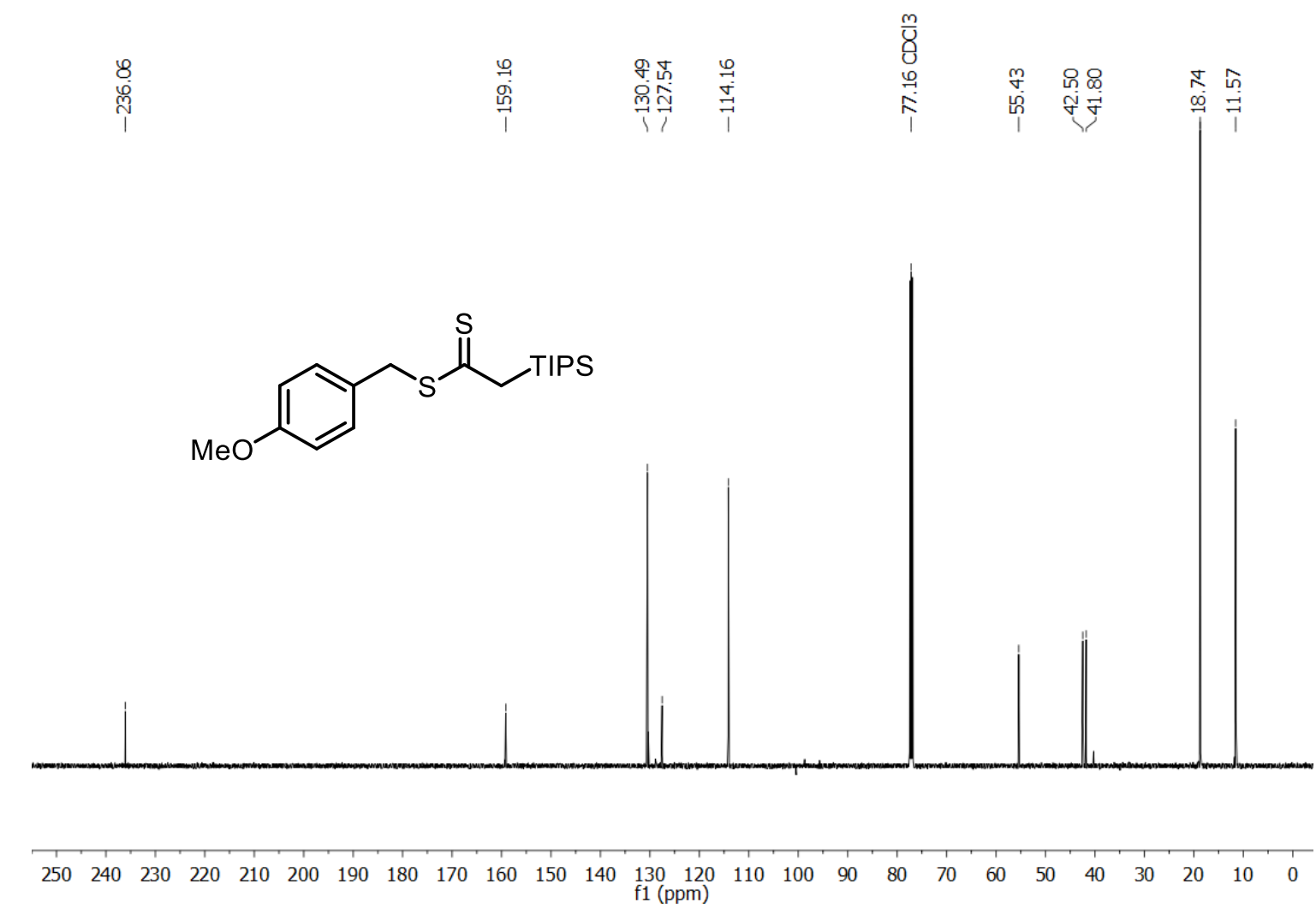

${ }^{1} \mathrm{H}$ NMR of compound 168b, 2-[(4-methoxybenzyl)thio]-1-methylpyridin-1-ium hexafluoroantimonate, $600 \mathrm{MHz}, \mathrm{CD}_{3} \mathrm{CN}, 298 \mathrm{~K}$<smiles>COc1ccc(CSc2cccc[n+]2C)cc1</smiles>

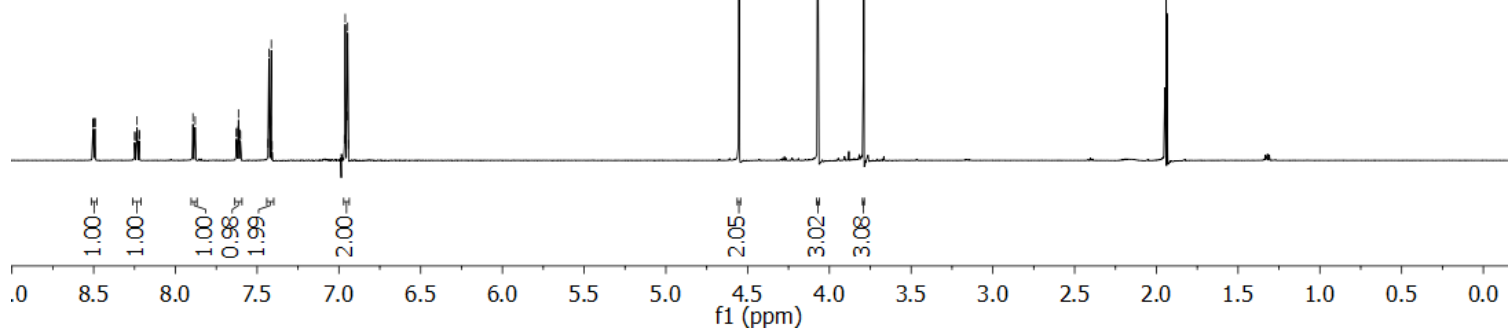


${ }^{13} \mathrm{C}$ NMR of compound 168b, 2-[(4-methoxybenzyl)thio]-1-methylpyridin-1-ium hexafluoroantimonate, $126 \mathrm{MHz}, \mathrm{CD}_{3} \mathrm{CN}, 298 \mathrm{~K}$

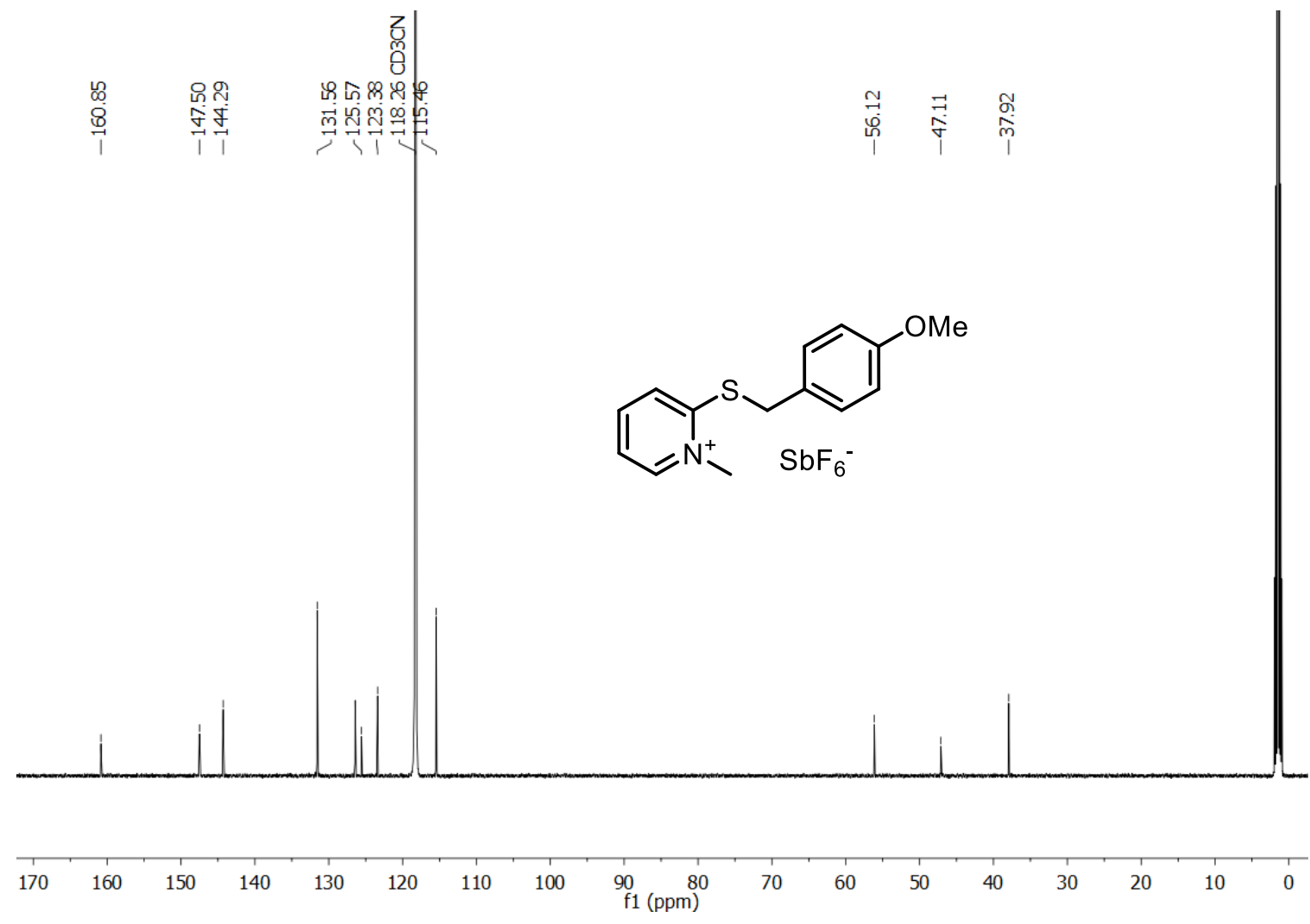

${ }^{19} \mathrm{~F}$ NMR of compound 168b, 2-[(4-methoxybenzyl)thio]-1-methylpyridin-1-ium hexafluoroantimonate, $283 \mathrm{MHz}, \mathrm{CD}_{3} \mathrm{CN}, 299 \mathrm{~K}$
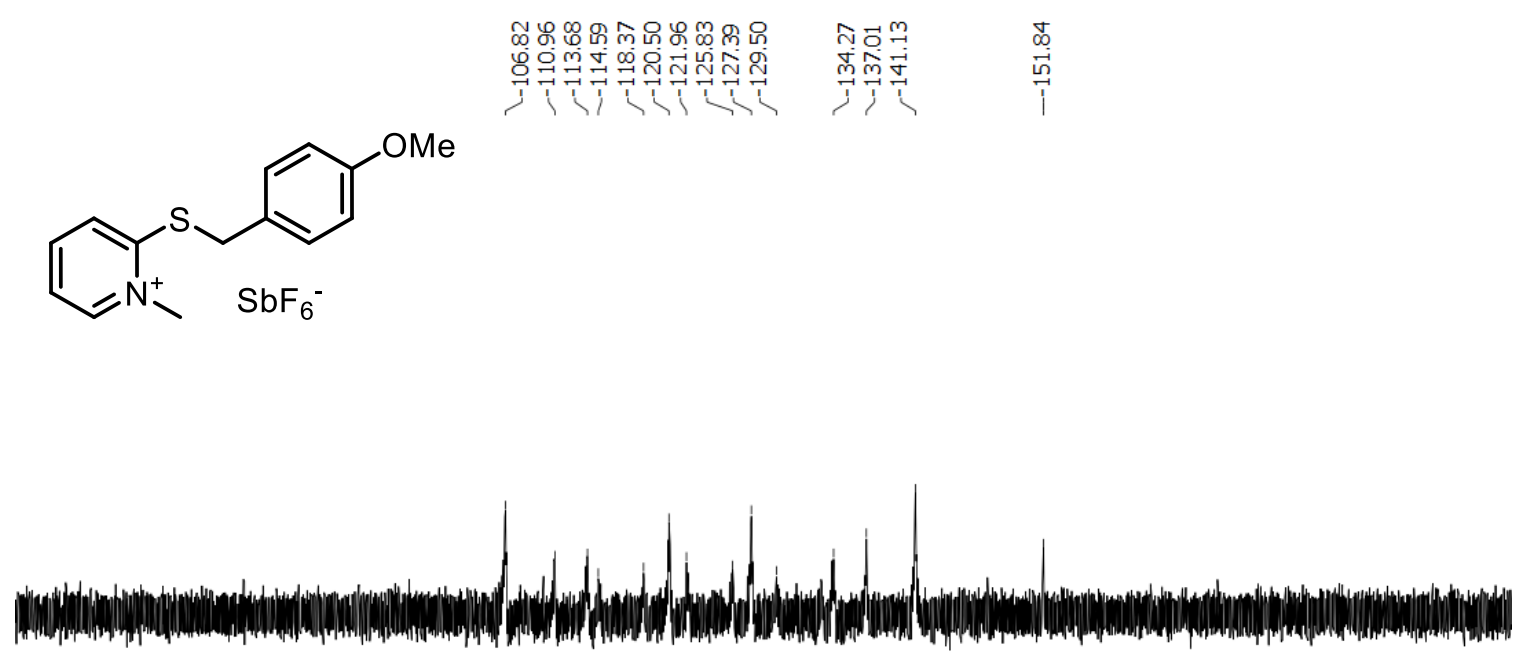

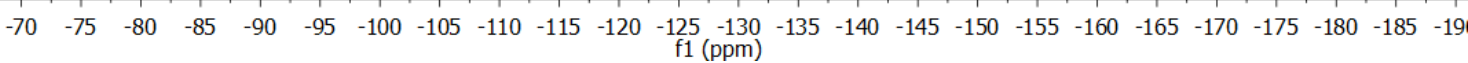


${ }^{1} \mathrm{H}$ NMR of compound 169, methyl 2-\{[(2-(triisopropylsilyl)ethanethioyl]thio\}acetate, 300 $\mathrm{Mhz}, \mathrm{CDCl}_{3}, 302 \mathrm{~K}$

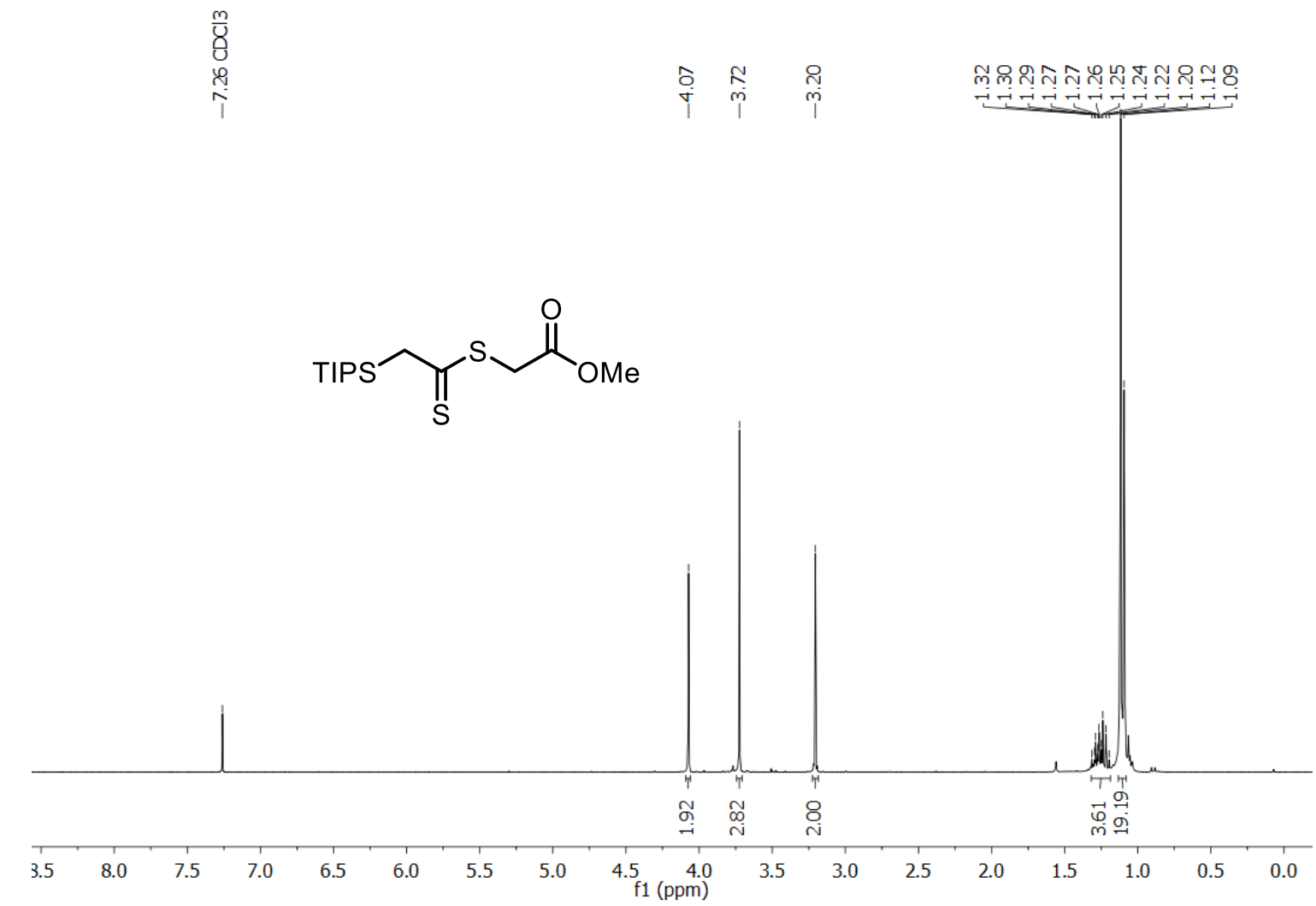

${ }^{13} \mathrm{C}$ NMR of compound 169, methyl 2-\{[(2-(triisopropylsilyl)ethanethioyl]thio\}acetate, 126 $\mathrm{Mhz}, \mathrm{CDCl}_{3}, 302 \mathrm{~K}$

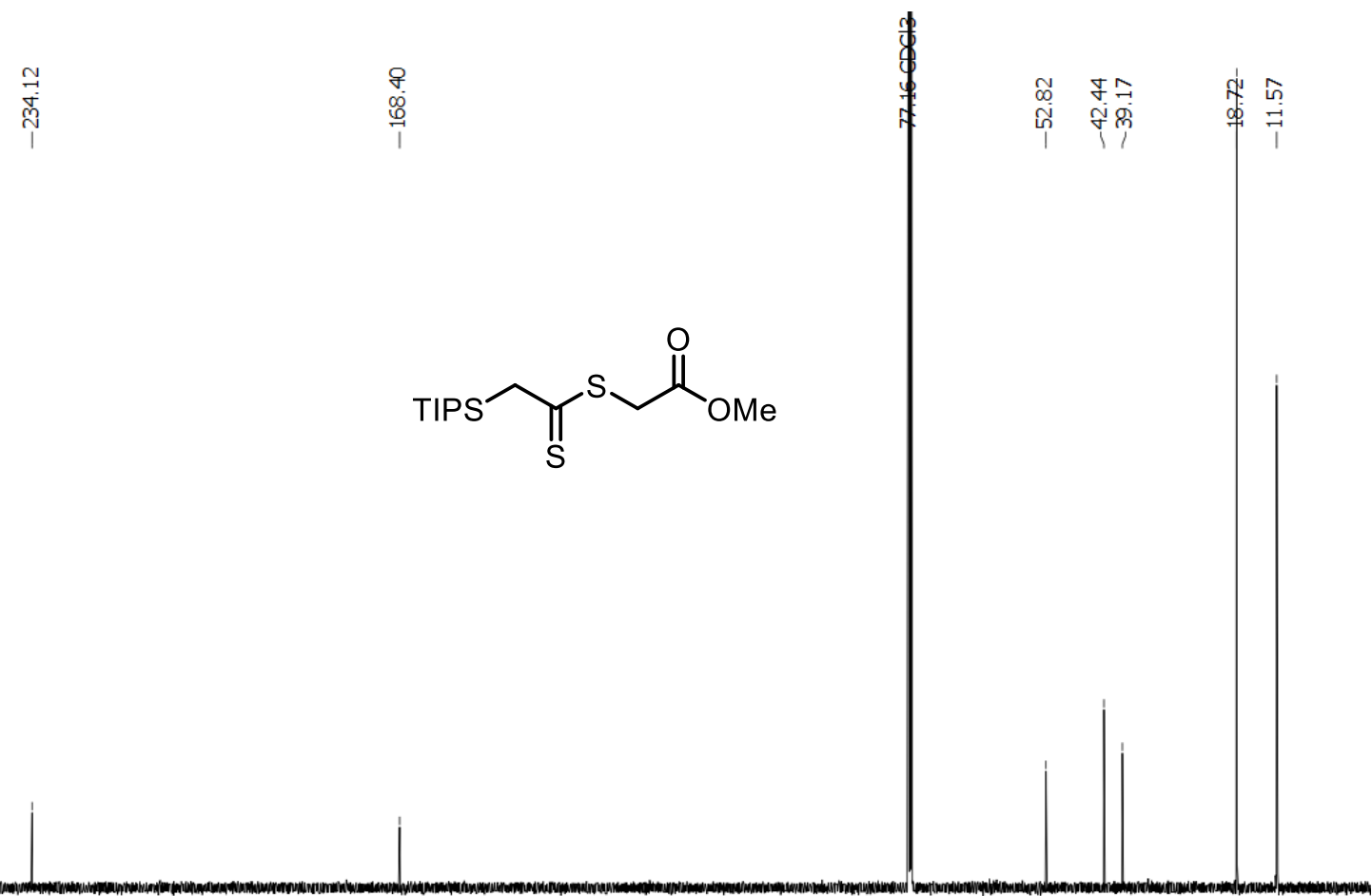

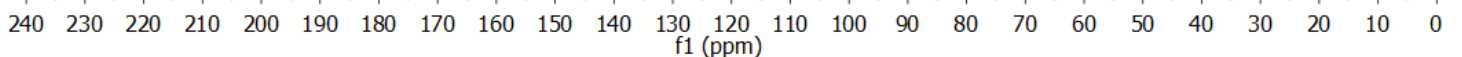


${ }^{1} \mathrm{H} \quad \mathrm{NMR}$ of compound 170, methyl $\mathbf{N}$-(tert-butoxycarbonyl)-S-[2(triisopropylsilyl)ethanethioyl]-L-cysteinate, $300 \mathrm{MHz}^{\mathrm{CDCl}} 3,302 \mathrm{~K}$

$\frac{m}{8}$
8
0
$i$
$i$<smiles>COC(=O)C(N)CSC(=S)C[I+]#P</smiles>

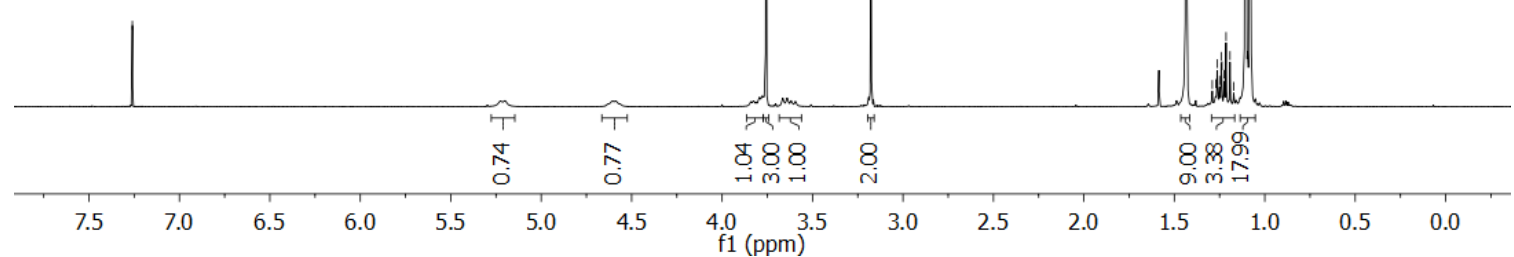

${ }^{13} \mathrm{C} \quad \mathrm{NMR}$ of compound 170, methyl N-(tert-butoxycarbonyl)-S-[2(triisopropylsilyl)ethanethioyl]-L-cysteinate, $126 \mathrm{MHz}^{\mathrm{CDCl}}, 298 \mathrm{~K}$ $\stackrel{\vec{b}}{\stackrel{m}{n}}$<smiles></smiles> 
${ }^{1} \mathrm{H}$ NMR of compound 171, ethane-1,2-diyl bis[2-(triisopropylsilyl)ethanedithioate], 400 $\mathrm{MHz}, \mathrm{CDCl}_{3}, 298 \mathrm{~K}$

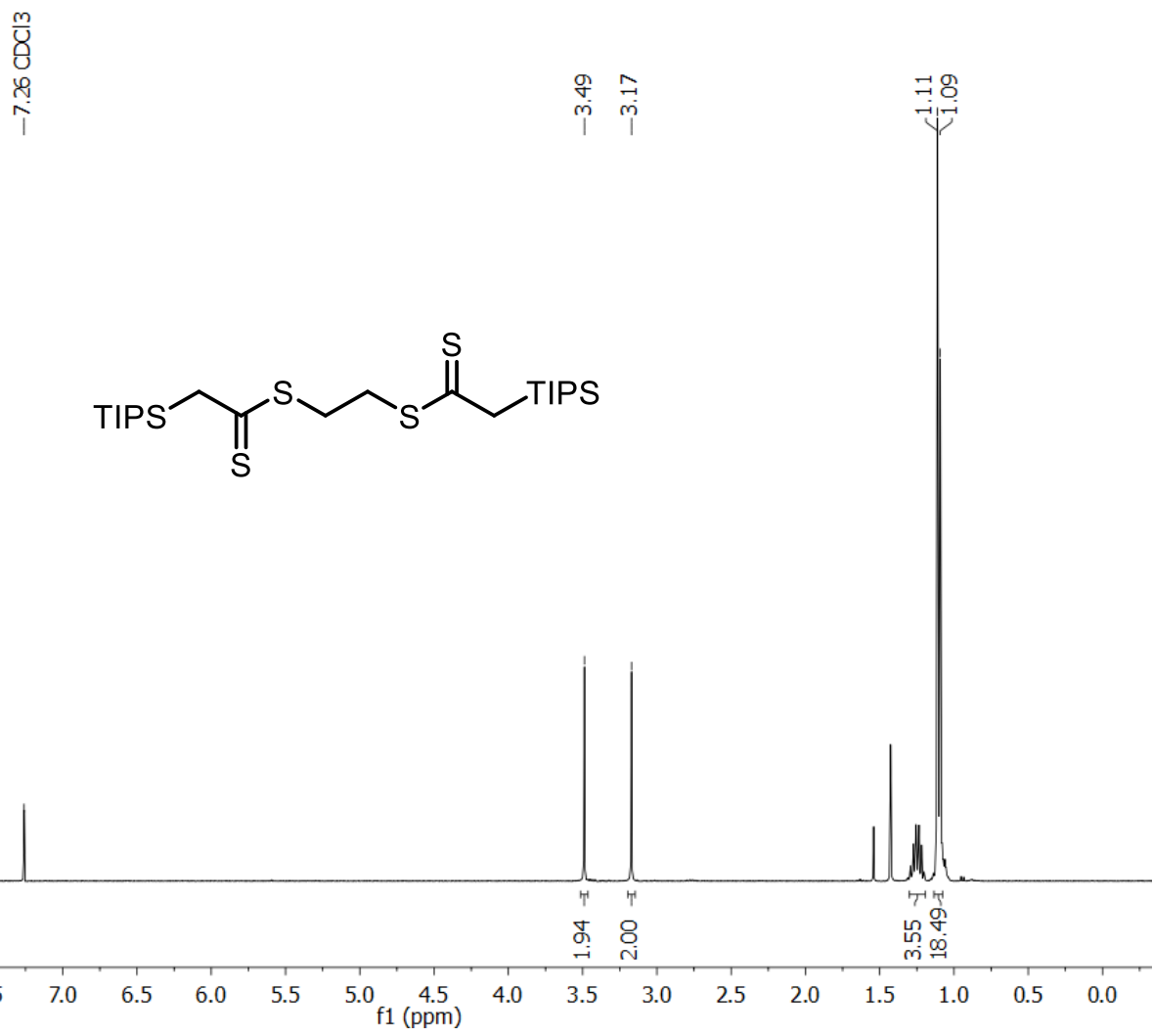

${ }^{13} \mathrm{C}$ NMR of compound 171, ethane-1,2-diyl bis[2-(triisopropylsilyl)ethanedithioate], 100 $\mathrm{MHz}, \mathrm{CDCl}_{3}, 298 \mathrm{~K}$

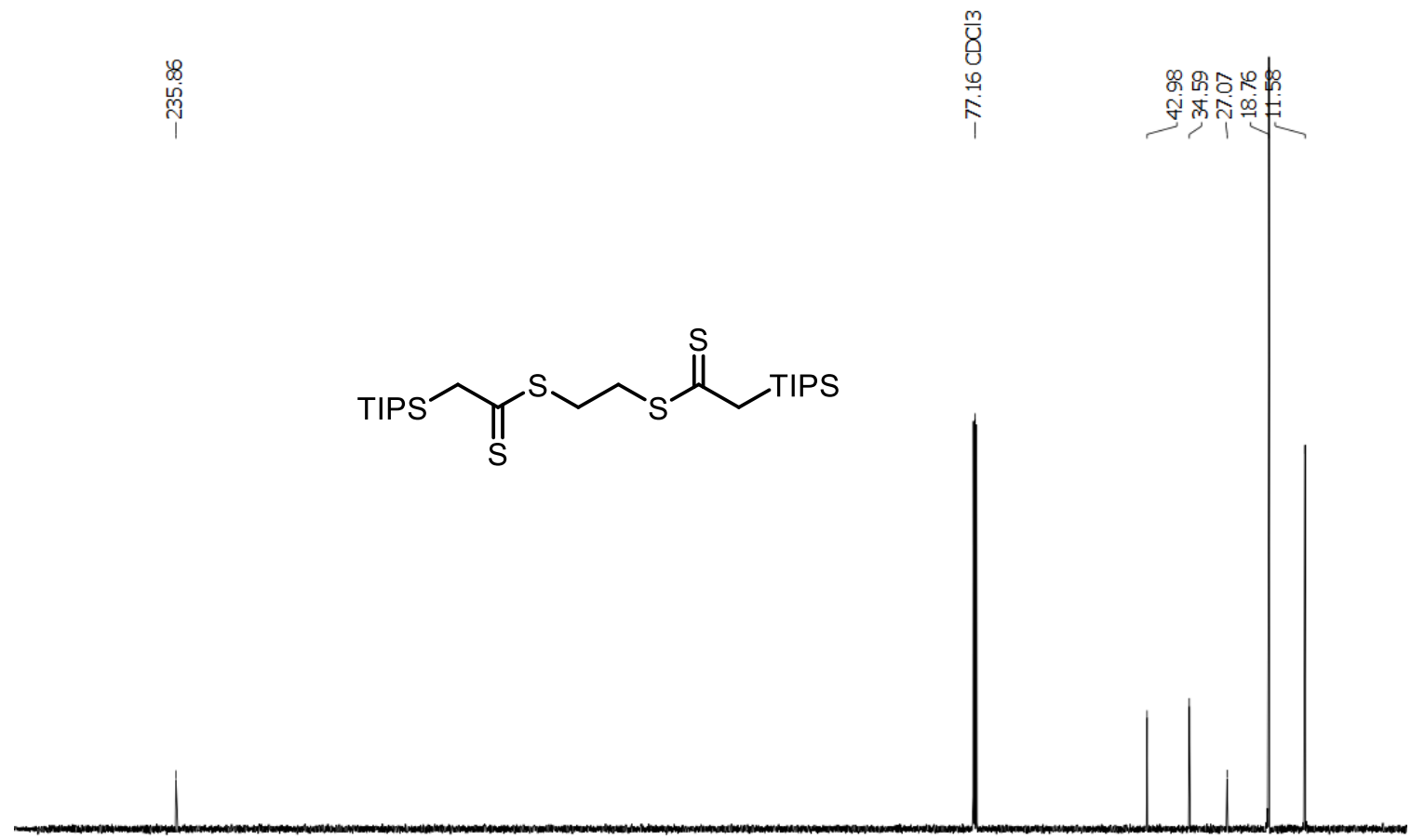

$\begin{array}{lllllllllllllllllllllllllll}260 & 250 & 240 & 230 & 220 & 210 & 200 & 190 & 180 & 170 & 160 & 150 & 140 & 130 & 120 & 110 & 100 & 90 & 80 & 70 & 60 & 50 & 40 & 30 & 20 & 10 & 0\end{array}$ 
${ }^{1} \mathrm{H}$ NMR of compound 172, propyl 2-(triisopropylsilyl)ethanedithioate, $300 \mathrm{MHz}, \mathrm{CDCl}_{3}, 302$ K

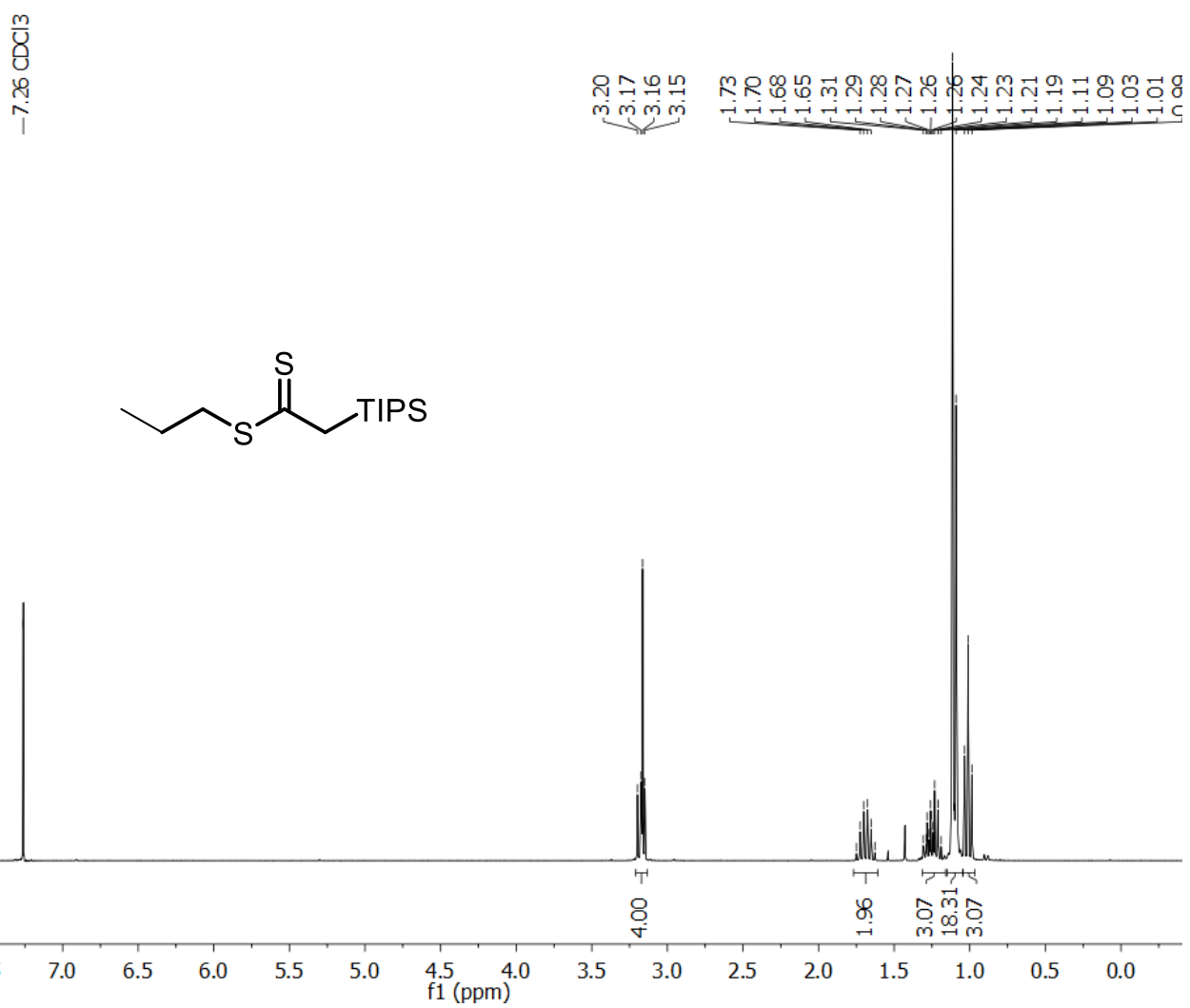

${ }^{13} \mathrm{C}$ NMR of compound 172, propyl 2-(triisopropylsilyl)ethanedithioate, $126 \mathrm{MHz}, \mathrm{CDCl}_{3}$, $298 \mathrm{~K}$

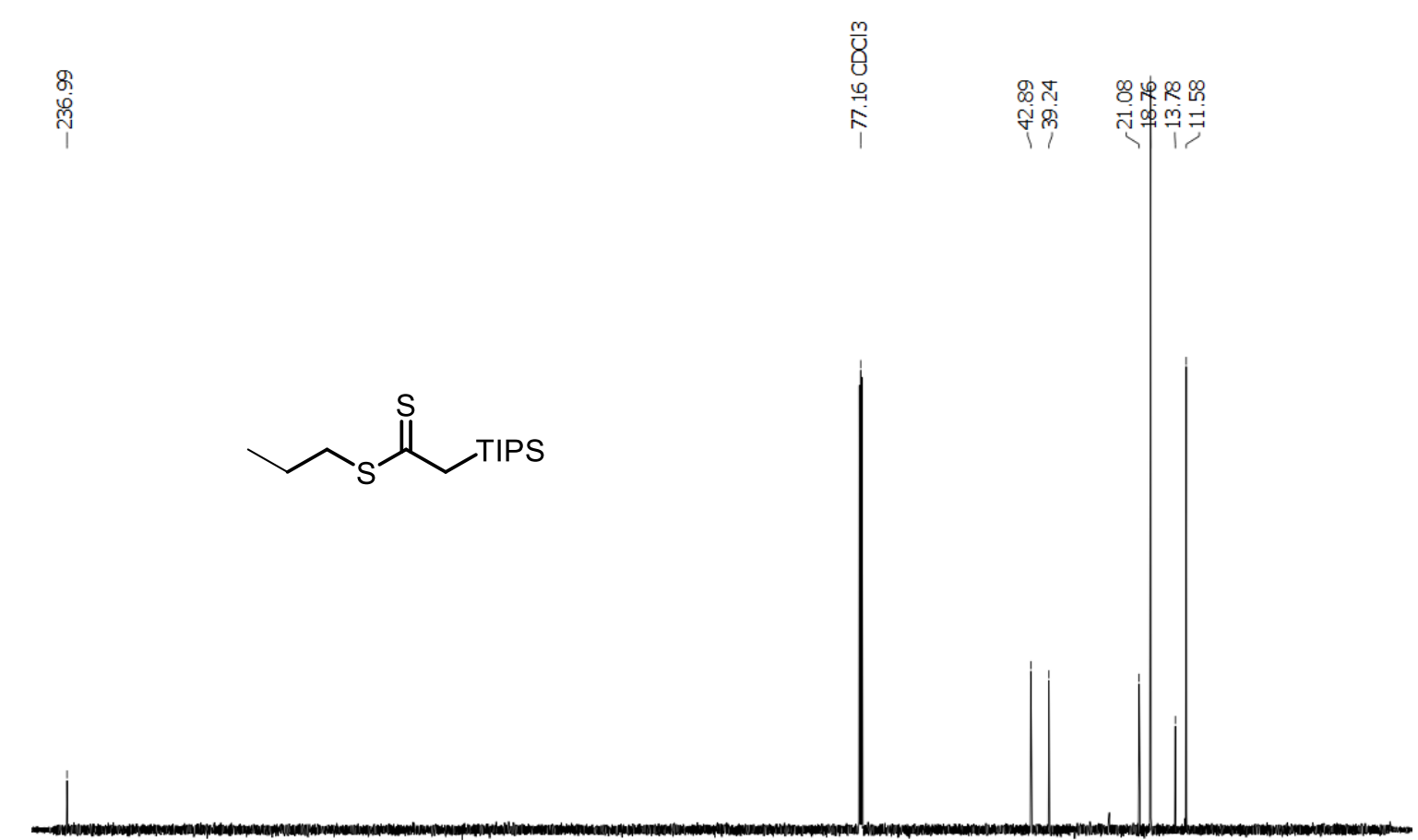

$\begin{array}{llllllllllllllllllllllllllll}240 & 230 & 220 & 210 & 200 & 190 & 180 & 170 & 160 & 150 & 140 & 130 & 120 & 110 & 100 & 90 & 80 & 70 & 60 & 50 & 40 & 30 & 20 & 10 & 0 & -10 & -20 & -30\end{array}$ 
${ }^{1} \mathrm{H}$ NMR of compound 173, 4-methoxyphenyl 2-(triisopropylsilyl)ethanedithioate, $300 \mathrm{MHz}$, $\mathrm{CDCl}_{3}, 302 \mathrm{~K}$

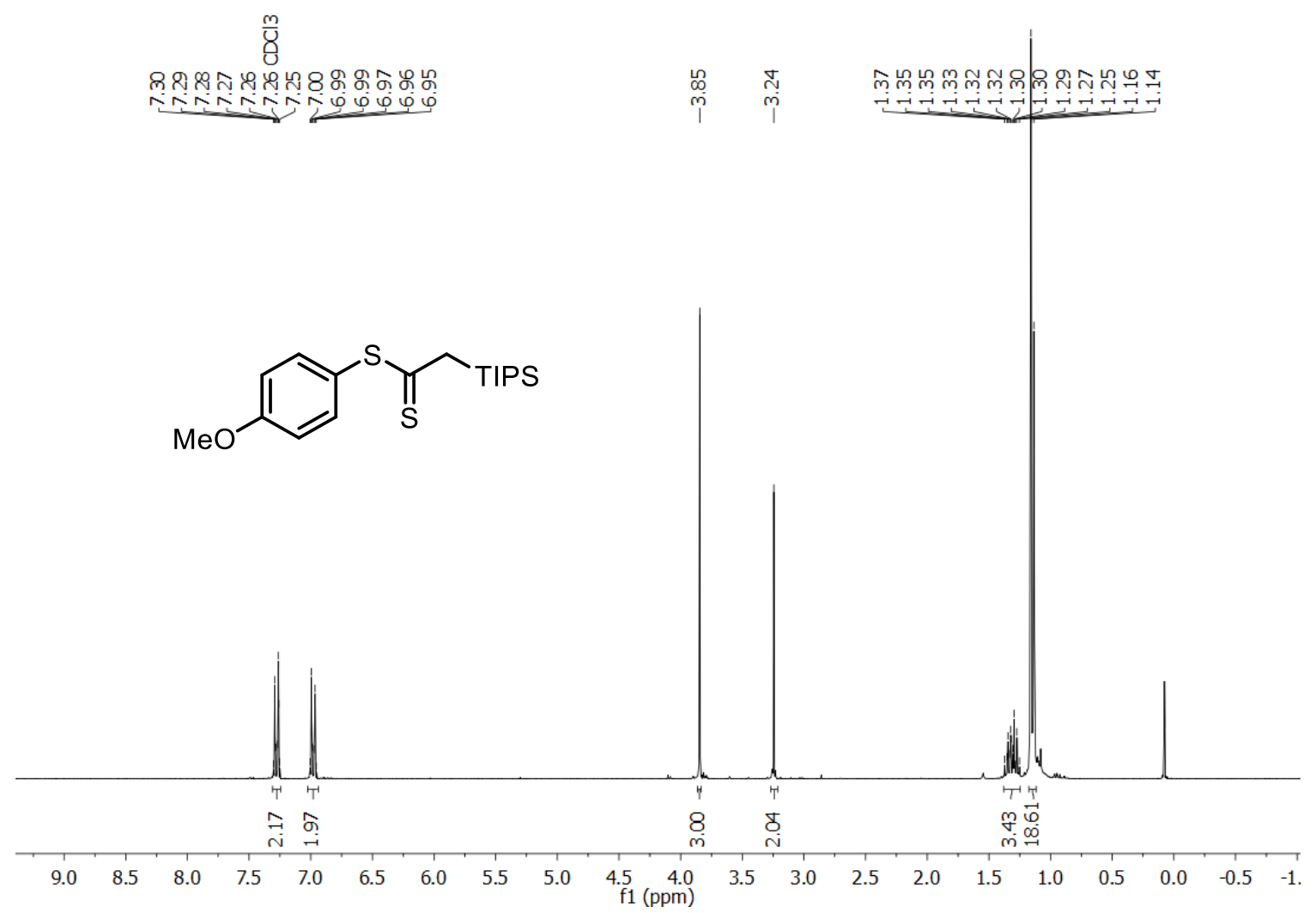

${ }^{13} \mathrm{C}$ NMR of compound 173, 4-methoxyphenyl 2-(triisopropylsilyl)ethanedithioate, $126 \mathrm{MHz}$, $\mathrm{CDCl}_{3}, 298 \mathrm{~K}$

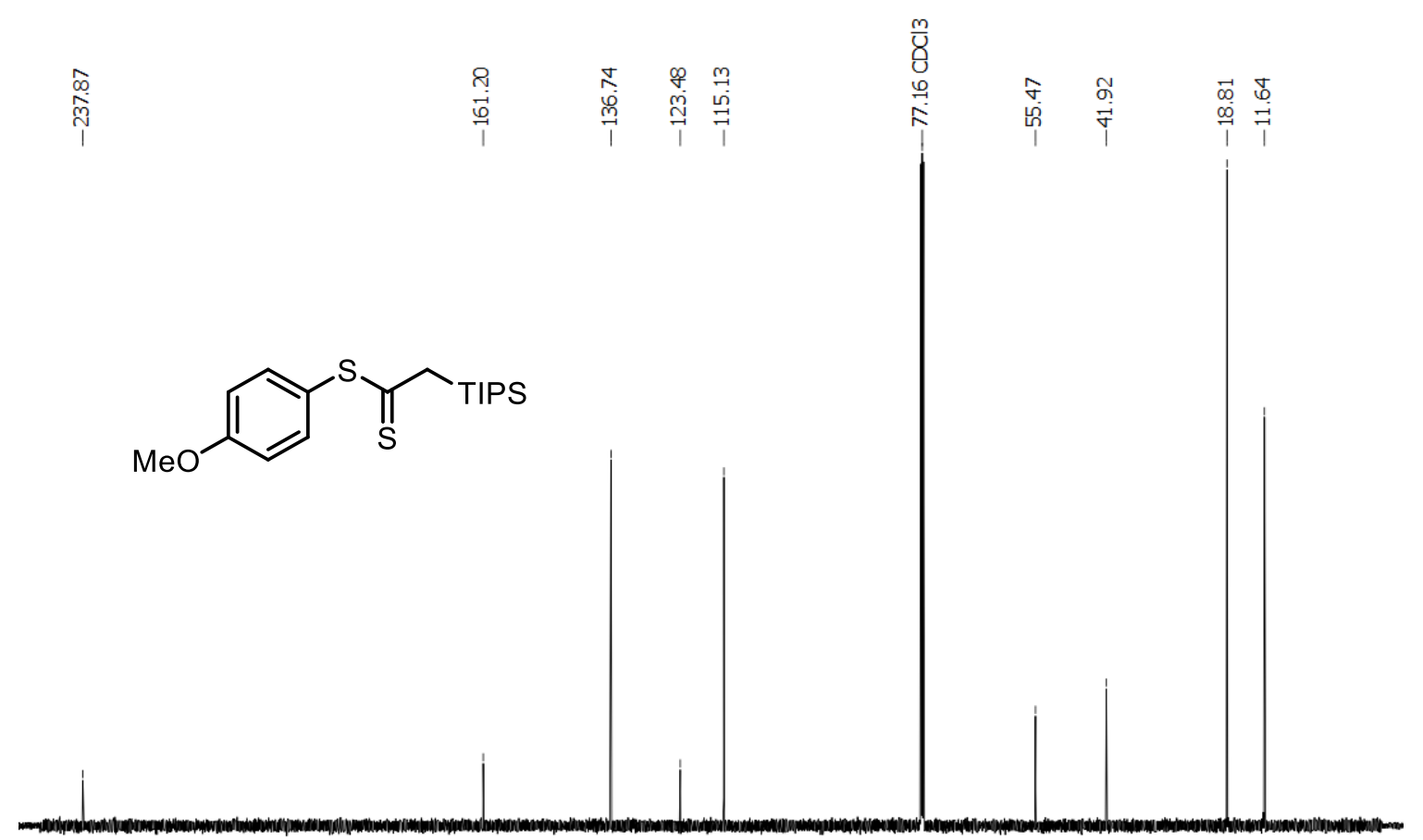

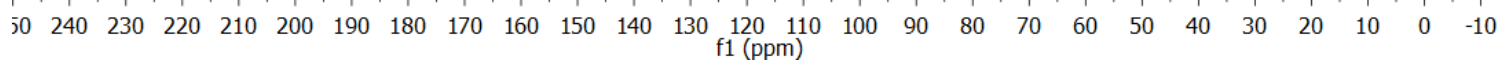


${ }^{1} \mathrm{H}$ NMR of compound 174, 4-nitrophenyl 2-(triisopropylsilyl)ethanedithioate, $300 \mathrm{MHz}$, $\mathrm{CDCl}_{3}, 302 \mathrm{~K}$<smiles></smiles>

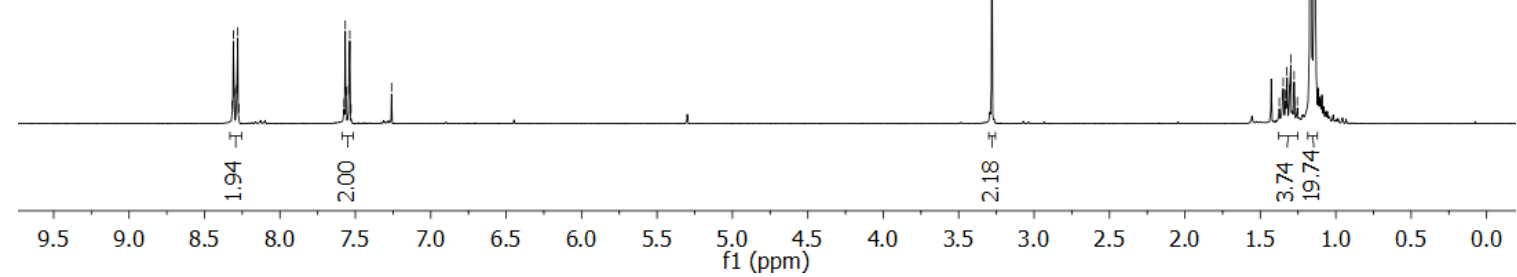

${ }^{13} \mathrm{C}$ NMR of compound 174, 4-nitrophenyl 2-(triisopropylsilyl)ethanedithioate, $126 \mathrm{MHz}$, $\mathrm{CDCl}_{3}, 298 \mathrm{~K}$

$\underset{\substack{m \\ i}}{\stackrel{m}{i}}$<smiles>O=[N+]([O-])c1ccc(SC(=S)C[In])cc1</smiles>

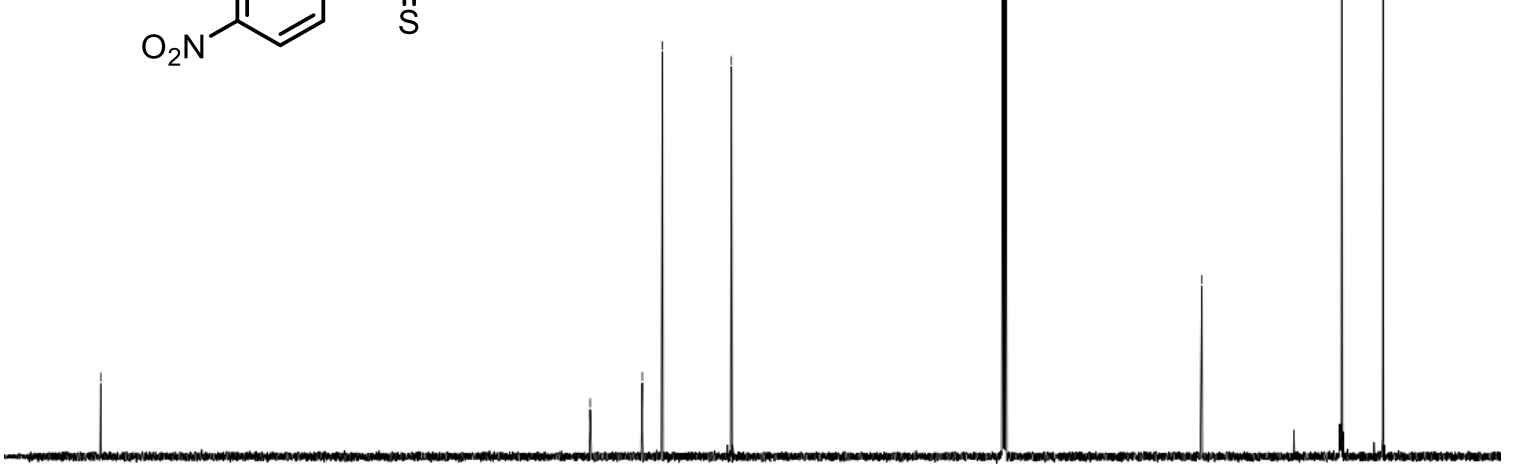

$\begin{array}{lllllllllllllllllllllllllll}30 & 240 & 230 & 220 & 210 & 200 & 190 & 180 & 170 & 160 & 150 & 140 & 130 & 120 & 110 & 100 & 90 & 80 & 70 & 60 & 50 & 40 & 30 & 20 & 10 & 0\end{array}$ 
${ }^{1} \mathrm{H}$ NMR of compound 175, 2-\{[2-(triisopropylsilyl)ethanethioyl]thio\}benzoic acid, $400 \mathrm{MHz}$, $\mathrm{CDCl}_{3}, 298 \mathrm{~K}$

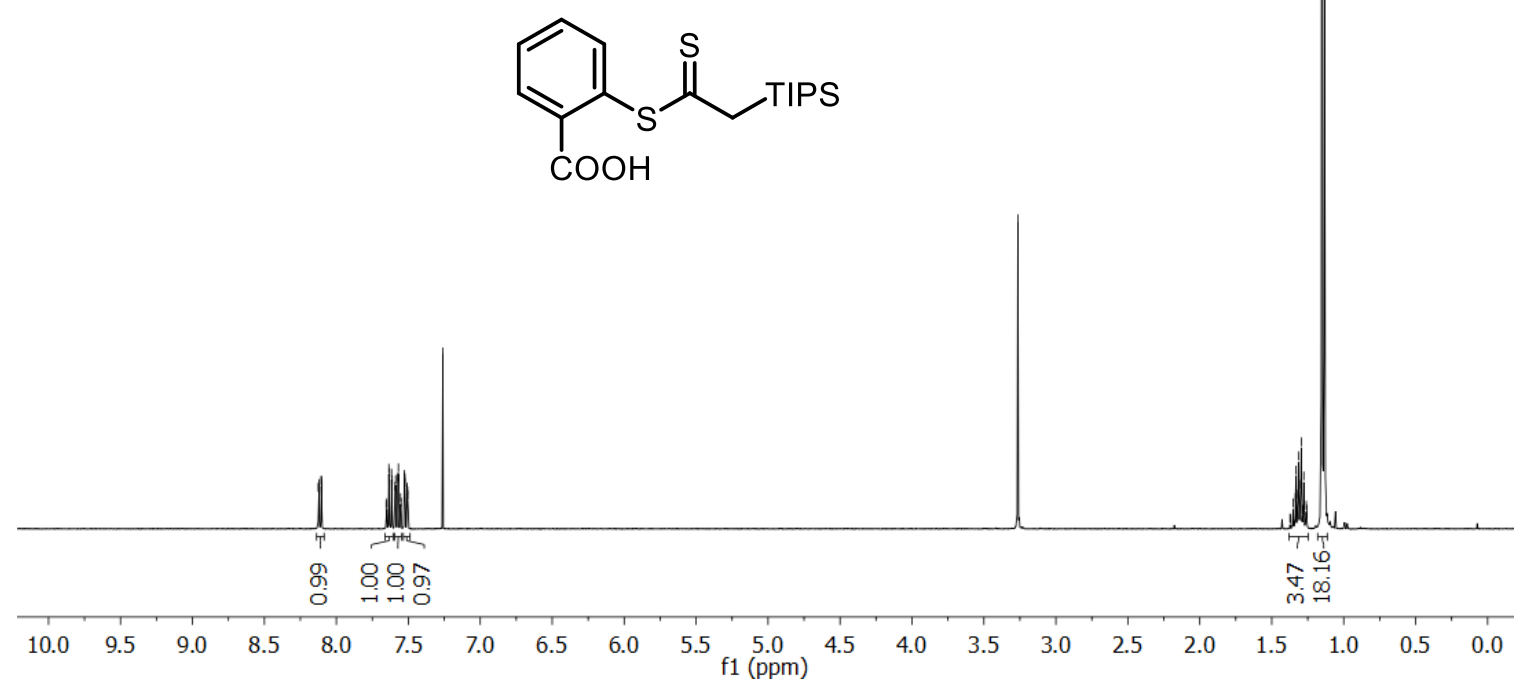

${ }^{13} \mathrm{C}$ NMR of compound 175, 2-\{[2-(triisopropylsilyl)ethanethioyl]thio\}benzoic acid, 100 $\mathrm{MHz}, \mathrm{CDCl}_{3}, 298 \mathrm{~K}$

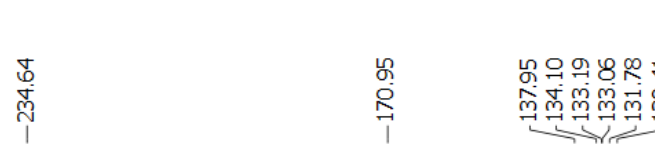<smiles></smiles> 
${ }^{1} \mathrm{H}$ NMR of compound $176, \mathrm{~N}$-ethyl-2-(triisopropylsilyl)ethanethioamide, $300 \mathrm{MHz}, \mathrm{CDCl}_{3}$, $298 \mathrm{~K}$

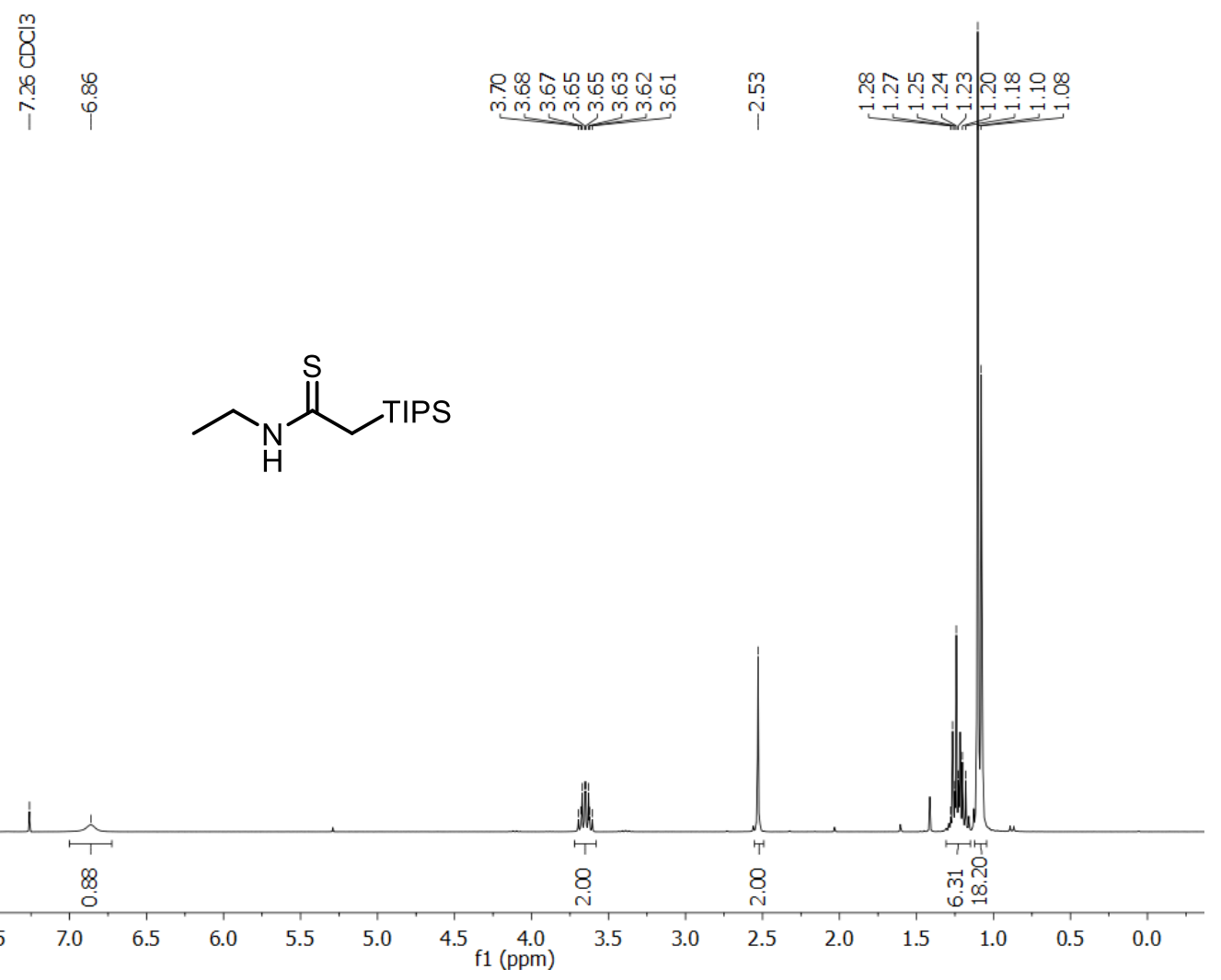

${ }^{13} \mathrm{C} \mathrm{NMR}$ of compound $176, \mathrm{~N}$-ethyl-2-(triisopropylsilyl)ethanethioamide, $76 \mathrm{MHz}^{\mathrm{CDCl}} \mathrm{C}_{3}$, $298 \mathrm{~K}$

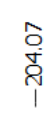

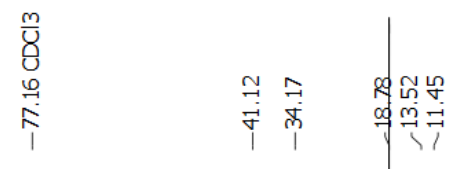<smiles>CCNC(=S)C[In]S</smiles>

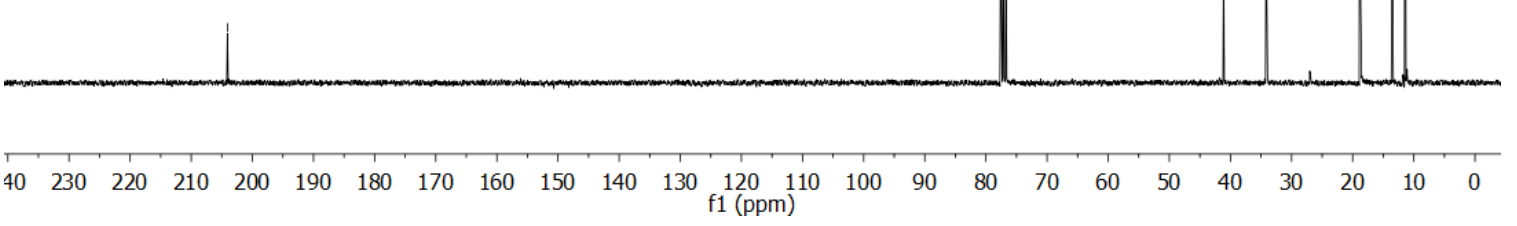


${ }^{1} \mathrm{H}$ NMR of compound $177, \mathrm{~N}, \mathrm{~N}$-diethyl-2-(triisopropylsilyl)ethanethioamide, $400 \mathrm{MHz}$, CDCl3, $299 \mathrm{~K}$

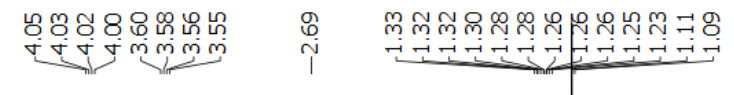<smiles>CCN(CC)C(=S)C[In]S</smiles>

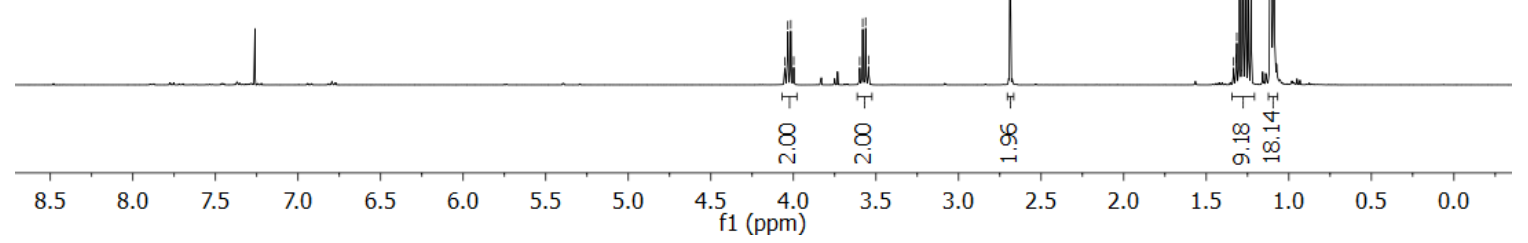

${ }^{13} \mathrm{C}$ NMR of compound 177, N,N-diethyl-2-(triisopropylsilyl)ethanethioamide, $101 \mathrm{MHz}$, $\mathrm{CDCl}_{3}, 300 \mathrm{~K}$
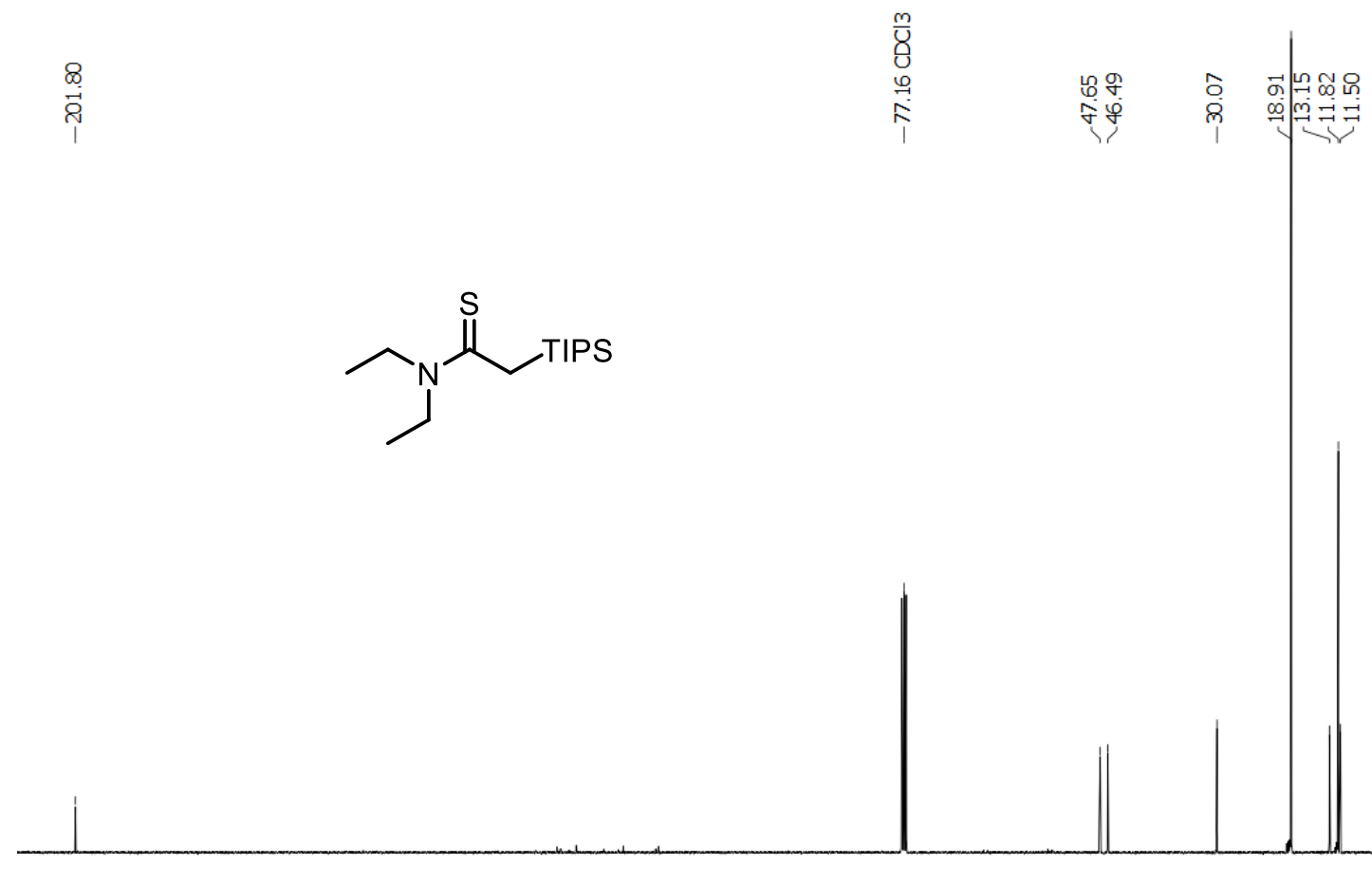

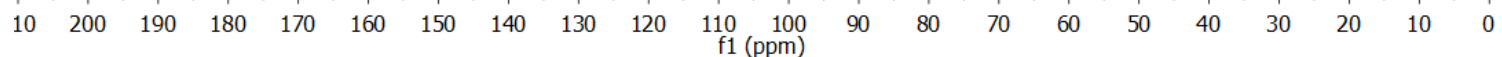


${ }^{1} \mathrm{H}$ NMR of compound 178, 1-morpholino-2-(triisopropylsilyl)ethane-1-thione, $300 \mathrm{MHz}$, $\mathrm{CDCl}_{3}, 302 \mathrm{~K}$

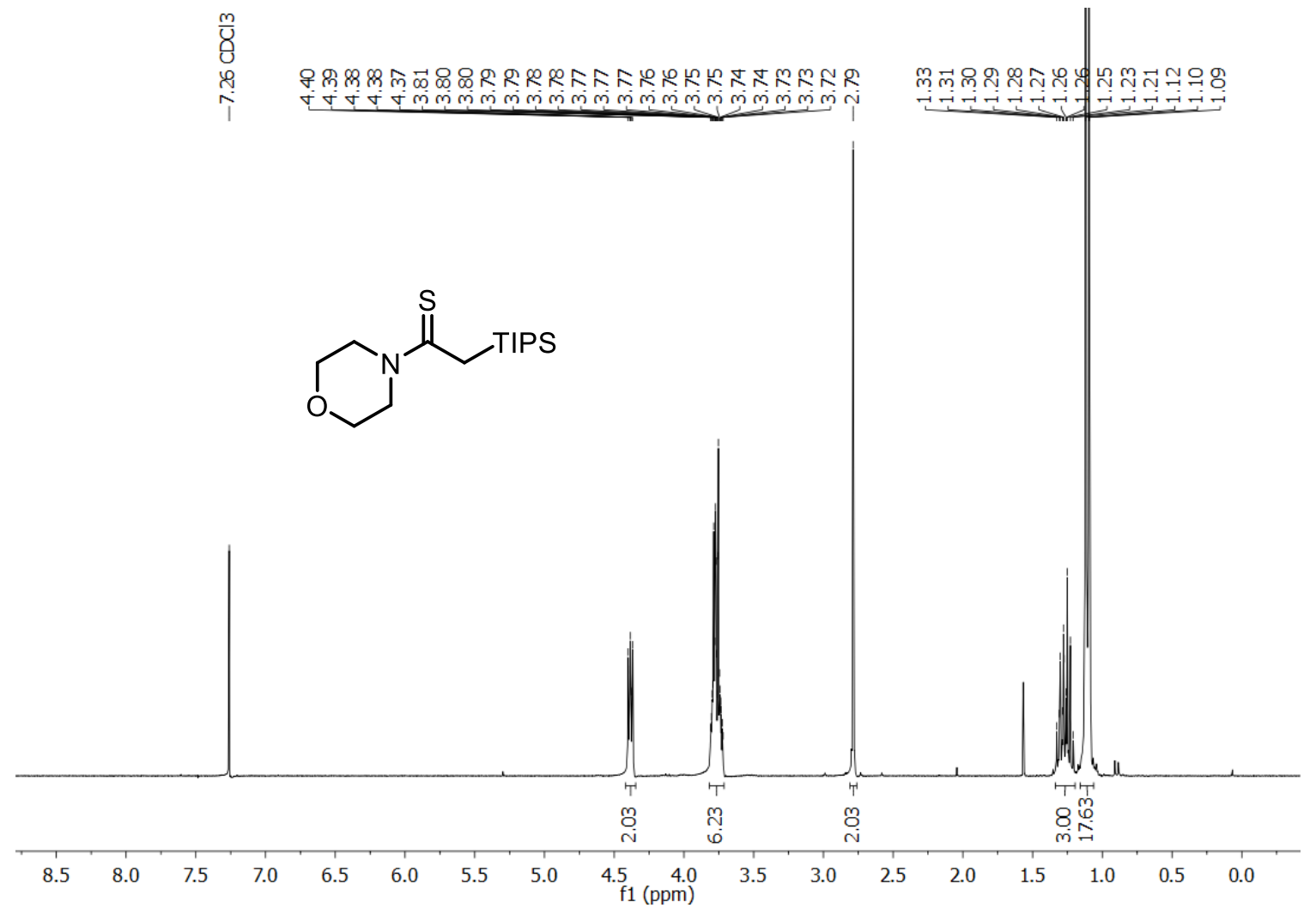

${ }^{13} \mathrm{C}$ NMR of compound 178, 1-morpholino-2-(triisopropylsilyl)ethane-1-thione, $126 \mathrm{MHz}$, $\mathrm{CDCl}_{3}, 298 \mathrm{~K}$

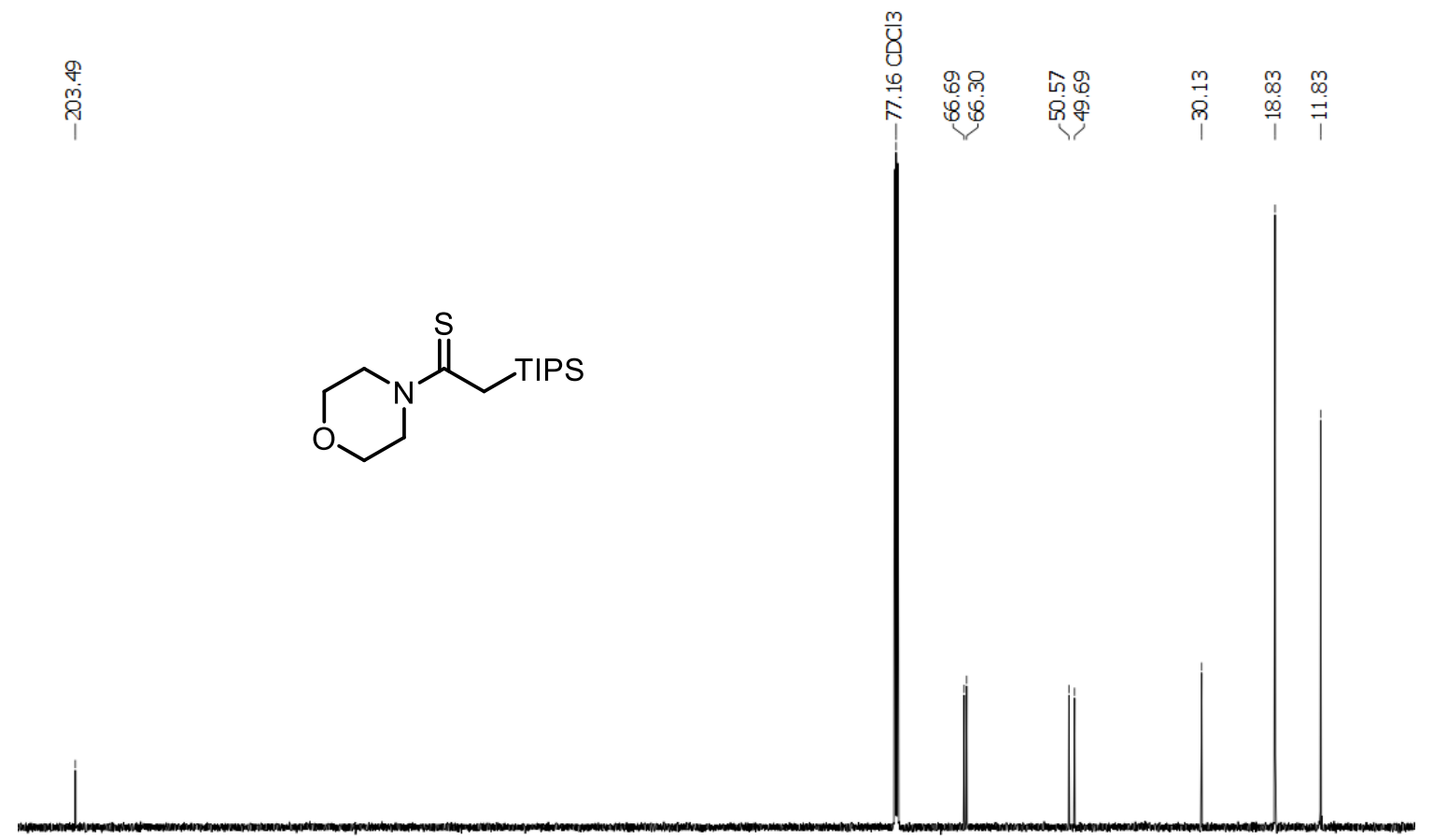

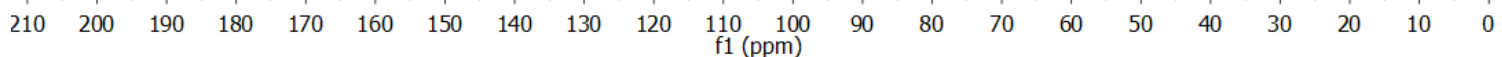


${ }^{1} \mathrm{H}$ NMR of compound $179, \mathrm{~N}$-cyclopropyl-2-(triisopropylsilyl)ethanethioamide, $300 \mathrm{MHz}$, $\mathrm{CDCl}_{3}, 302 \mathrm{~K}$

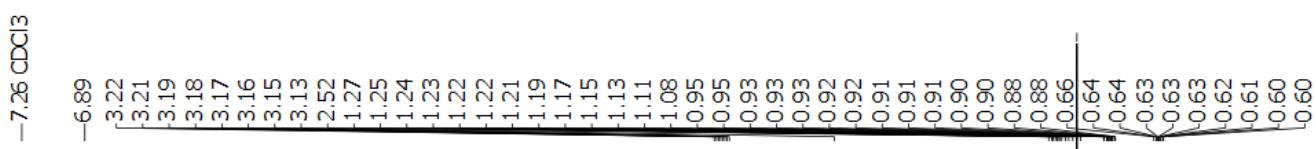<smiles>S=C(C[In])NC1CC1</smiles>

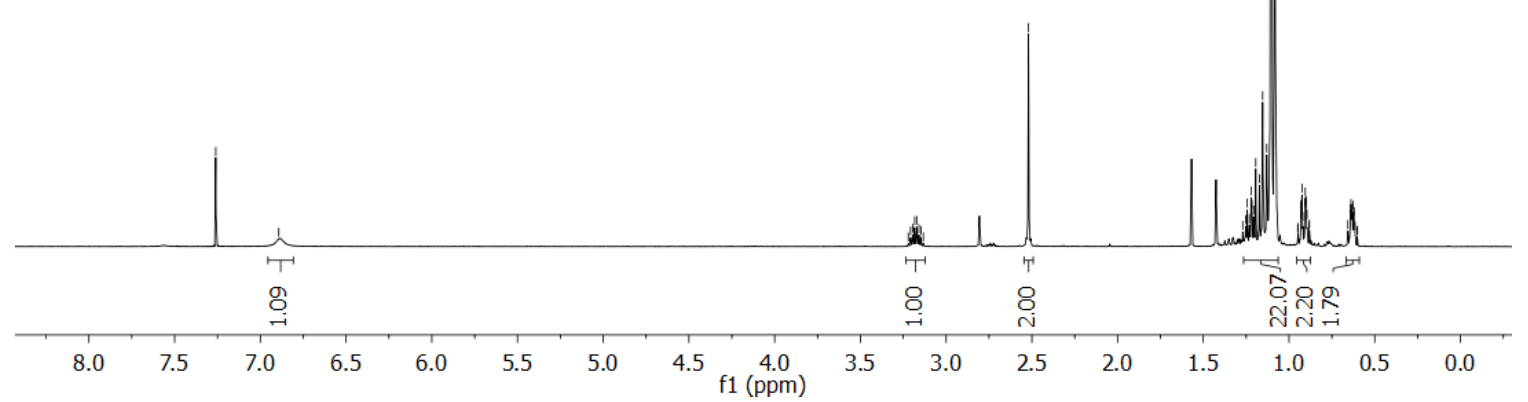

${ }^{13} \mathrm{C}$ NMR of compound 179, $\mathrm{N}$-cyclopropyl-2-(triisopropylsilyl)ethanethioamide, $101 \mathrm{MHz}$, $\mathrm{CDCl}_{3} ; 298 \mathrm{~K}$

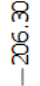<smiles>S=C(C[Te]S)NC1CC1</smiles>

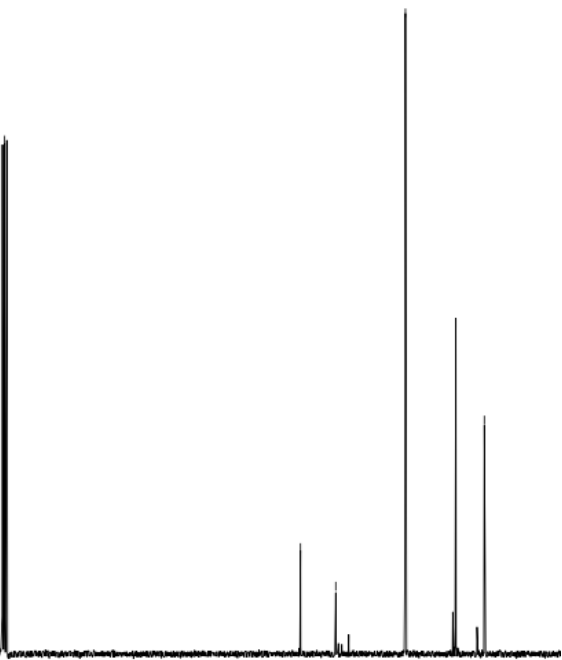

$\begin{array}{lllllllllll}210 & 200 & 190 & 180 & 170 & 160 & 150 & 140 & 130 & 120 & 110 \\ \mathrm{f} 1(\mathrm{ppm})\end{array}$ 
${ }^{1} \mathrm{H}$ NMR of compound 180, $\mathrm{N}$-[2-(1H-indol-3-yl)ethyl]-2-(triisopropylsilyl)ethanethioamide, $500 \mathrm{MHz}, \mathrm{CDCl}_{3} ; 298 \mathrm{~K}$

$\frac{m}{8}$

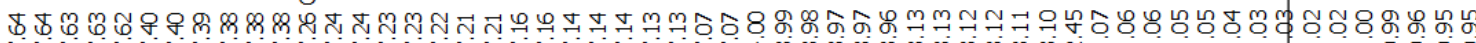

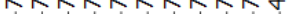<smiles>[AlH]CC(=S)NCCc1c[nH]c2ccccc12</smiles>

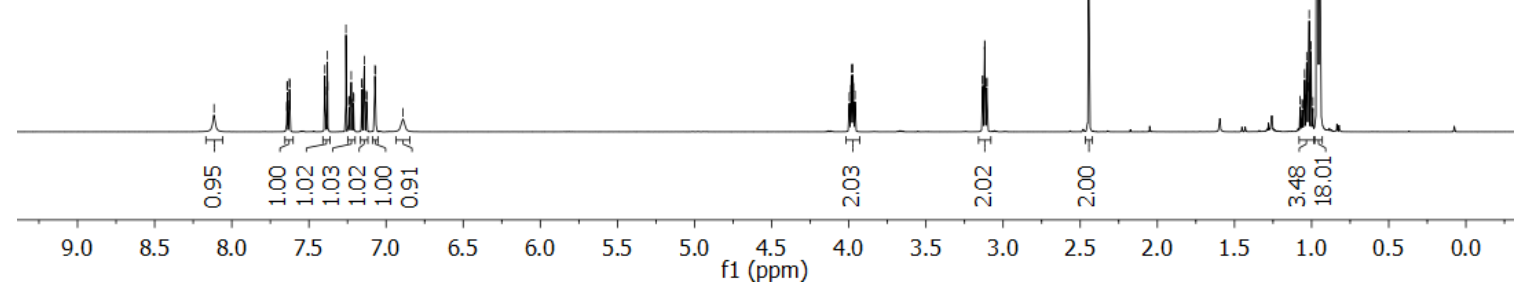

${ }^{13} \mathrm{C}$ NMR of compound 180, $\mathrm{N}$-[2-(1H-indol-3-yl)ethyl]-2-(triisopropylsilyl)ethanethioamide, $126 \mathrm{MHz}, \mathrm{CDCl}_{3} ; 298 \mathrm{~K}$<smiles>F[AlH]CC(=S)NCCc1c[nH]c2ccccc12</smiles>

$\begin{array}{llllllllllll}20 & 210 & 200 & 190 & 180 & 170 & 160 & 150 & 140 & 130 & 120 & \begin{array}{l}110 \\ \mathrm{f} 1(\mathrm{ppm})\end{array}\end{array}$ 
${ }^{1} \mathrm{H}$ NMR of compound 181, ethyl $N, S$-bis[2-(triisopropylsilyl)ethanethioyl]-L-cysteinate, 600 $\mathrm{MHz}, \mathrm{CDCl}_{3}, 298 \mathrm{~K}$

$\frac{m}{8}$

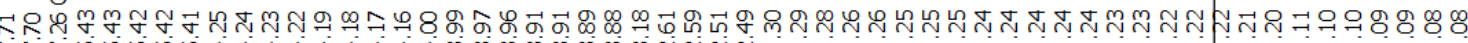
섯ำ<smiles></smiles>

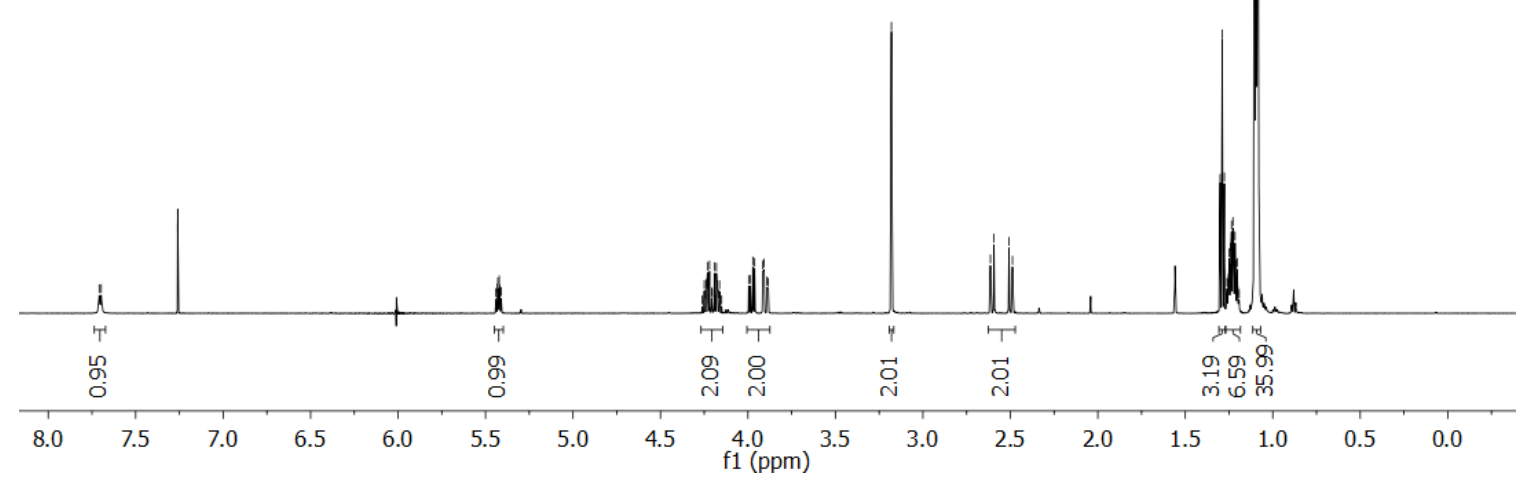

${ }^{13} \mathrm{C}$ NMR of compound 181, ethyl N,S-bis[2-(triisopropylsilyl)ethanethioyl]-L-cysteinate, $126 \mathrm{MHz}, \mathrm{CDCl}_{3}, 298 \mathrm{~K}$

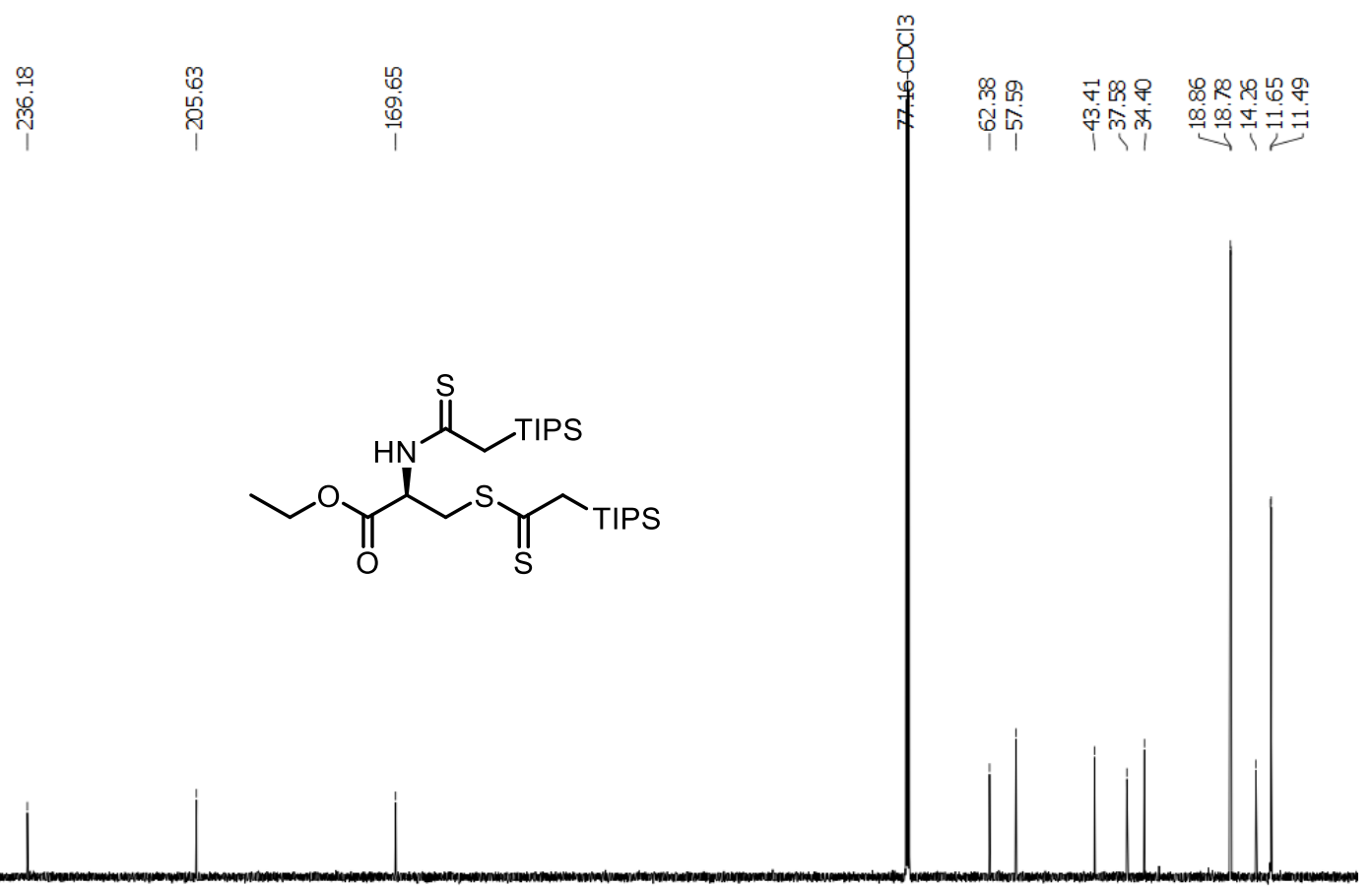

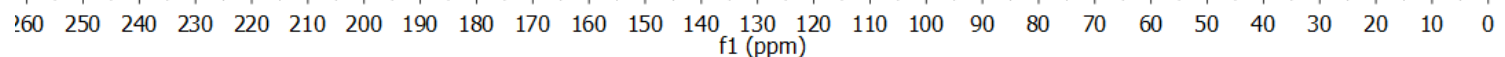


Selected region of NH-HSCQ for compound 181, methyl $\mathrm{N}$-(tert-butoxycarbonyl)-S-[2(triisopropylsilyl)ethanethioyl]-L-cysteinate, $600 \mathrm{MHz} / 126 \mathrm{MHz}, \mathrm{CDCl}_{3}, 302 \mathrm{~K}$

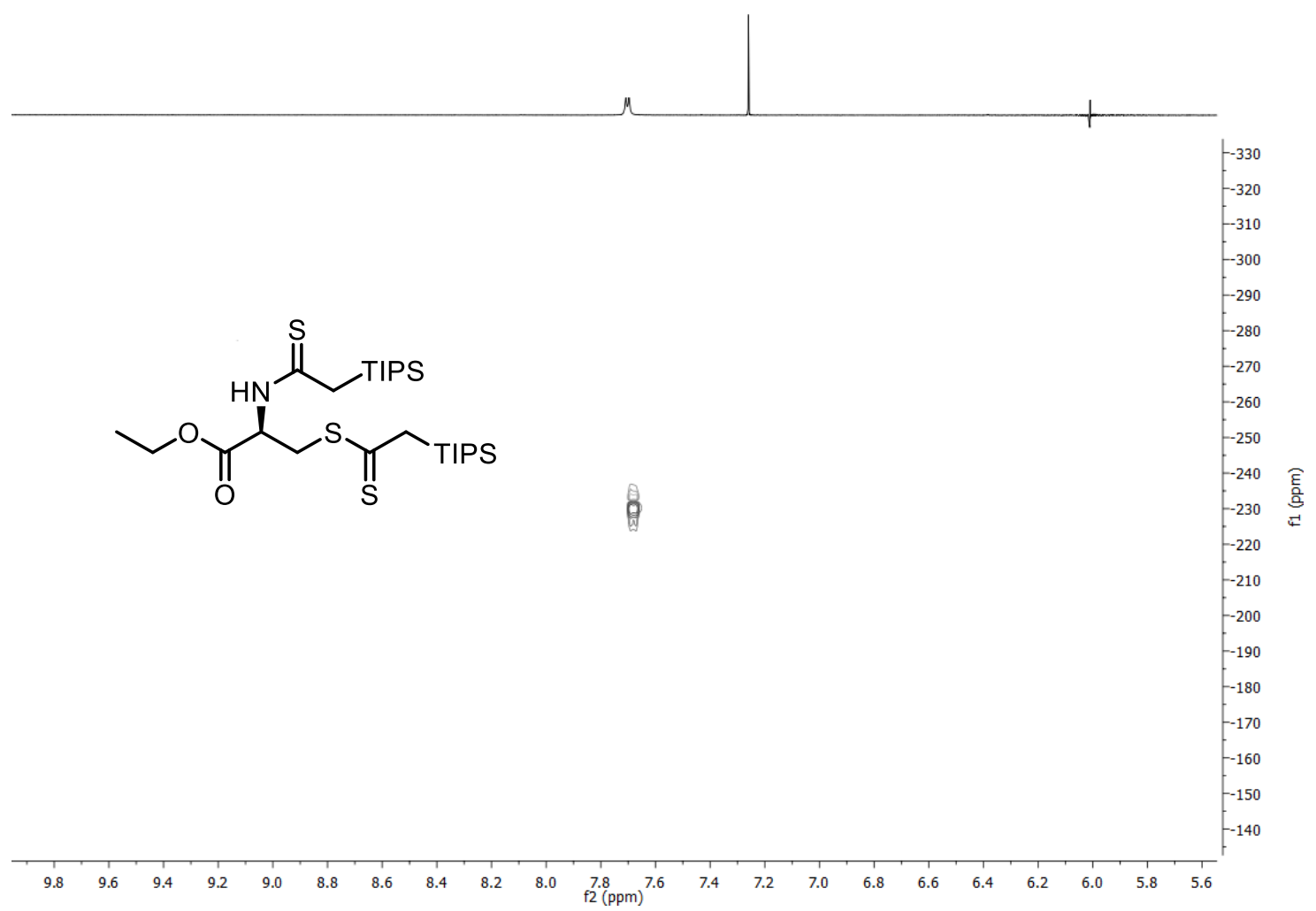

${ }^{1} \mathrm{H}$ NMR of compound $182, O-[(8 R, 9 S, 13 S, 14 S, 17 R)$-17-Ethynyl-17-hydroxy-13-methyl7,8,9,11,12,13,14,15,16,17-decahydro-6H-cyclopenta[a]phenanthren-3-yl] 2-(triisopropylsilyl)ethanethioate, $600 \mathrm{MHz}, \mathrm{CD}_{3} \mathrm{Cl}, 298 \mathrm{~K}$

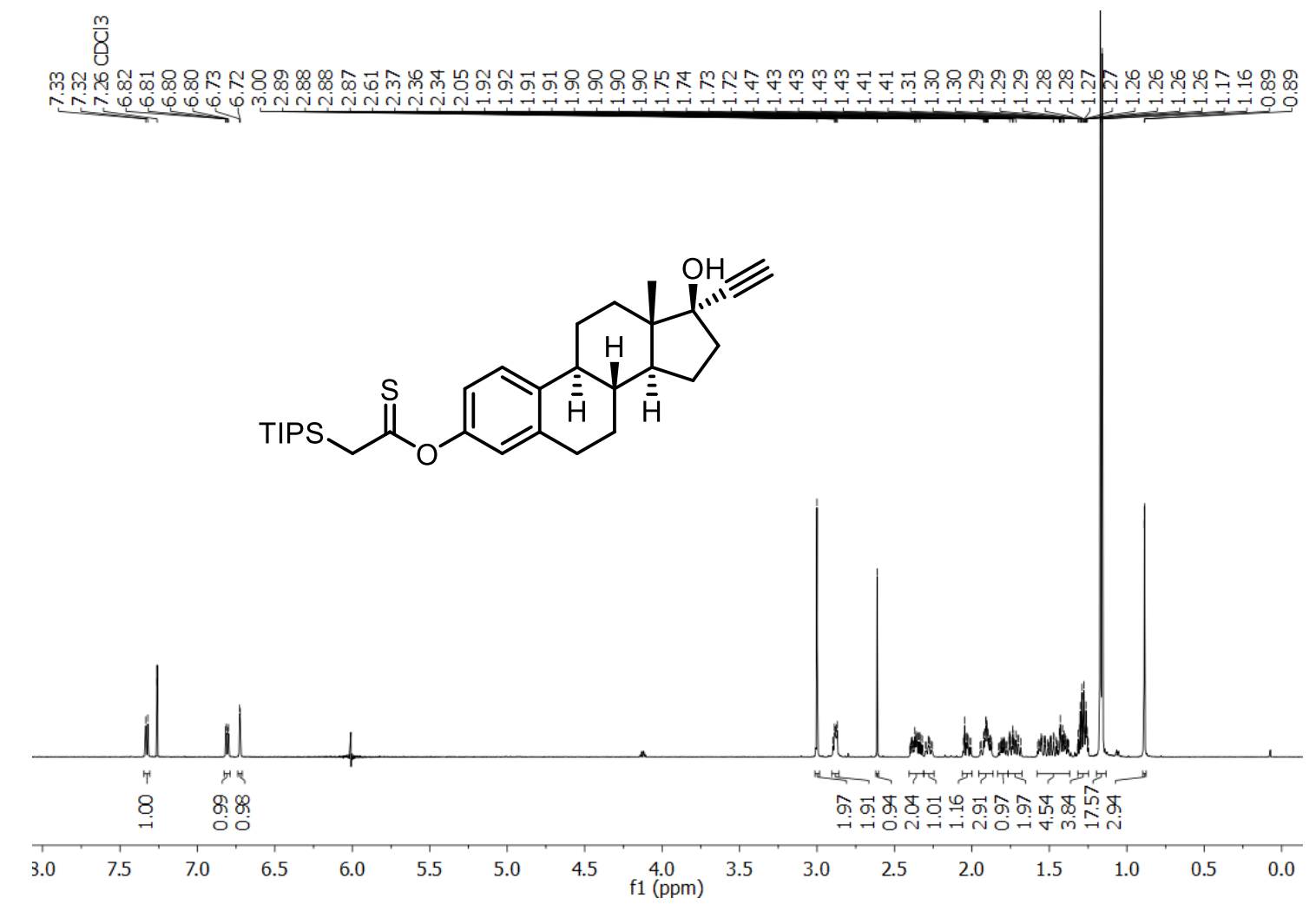


${ }^{13} \mathrm{C}$ NMR of compound 182, $O-[(8 R, 9 S, 13 S, 14 S, 17 R)$-17-Ethynyl-17-hydroxy-13-methyl7,8,9,11,12,13,14,15,16,17-decahydro-6H-cyclopenta[a]phenanthren-3-yl] 2-(triisopropylsilyl)ethanethioate, $126 \mathrm{MHz}, \mathrm{CD}_{3} \mathrm{Cl}, 298 \mathrm{~K}$

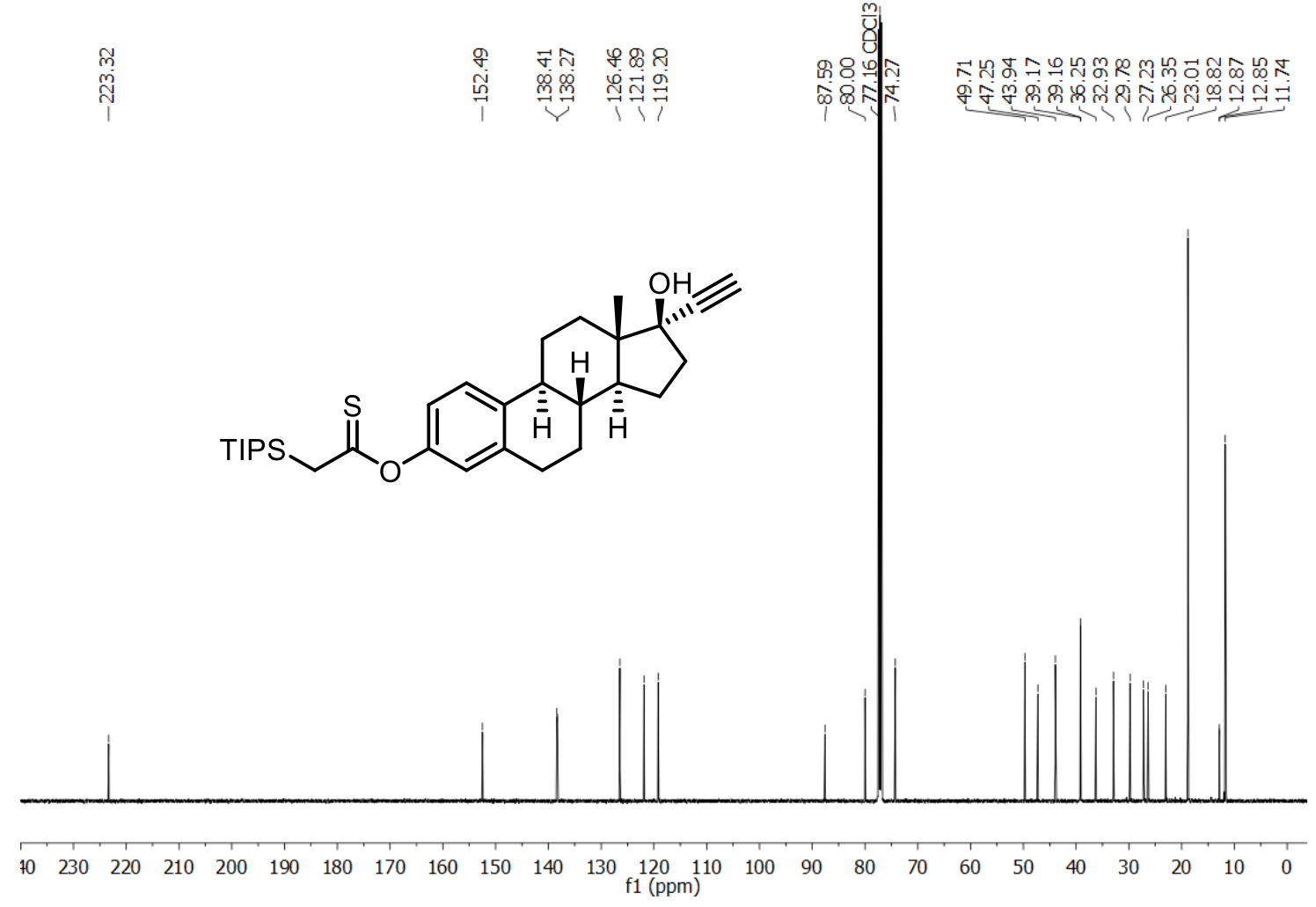

${ }^{1} \mathrm{H}$ NMR of compound 183, O-(naphthalen-2-yl) 2-(triisopropylsilyl)ethanethioate, 300 $\mathrm{MHz}, \mathrm{CDCl}_{3}, 302 \mathrm{~K}$<smiles>S=C(C[In]S)Oc1ccc2ccccc2c1</smiles>

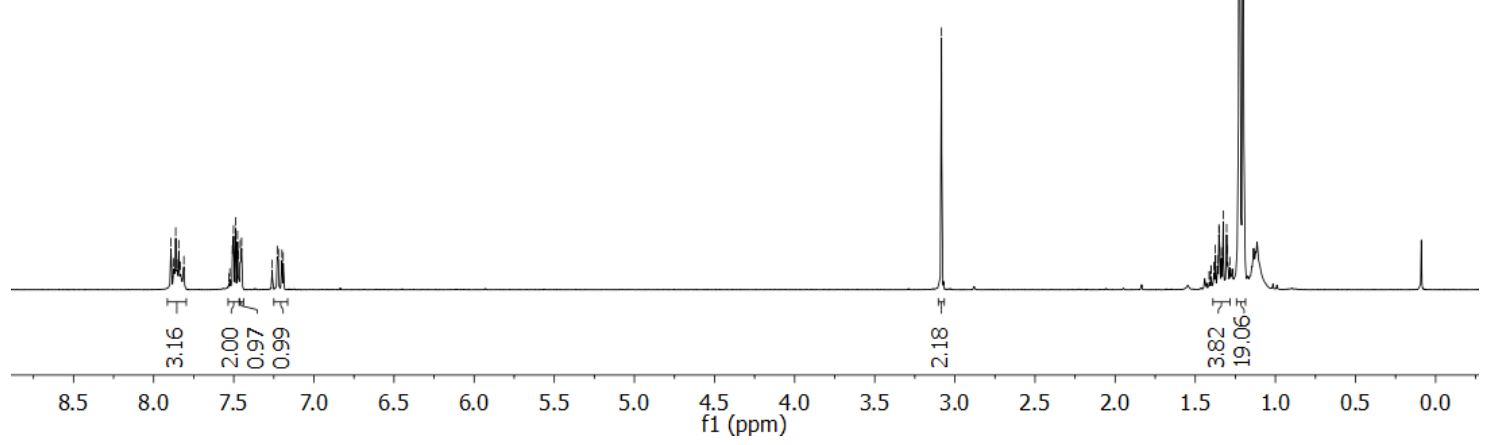


${ }^{13} \mathrm{C}$ NMR of compound 183, O-(naphthalen-2-yl) 2-(triisopropylsilyl)ethanethioate, 126 $\mathrm{MHz}, \mathrm{CDCl}_{3}, 298 \mathrm{~K}$

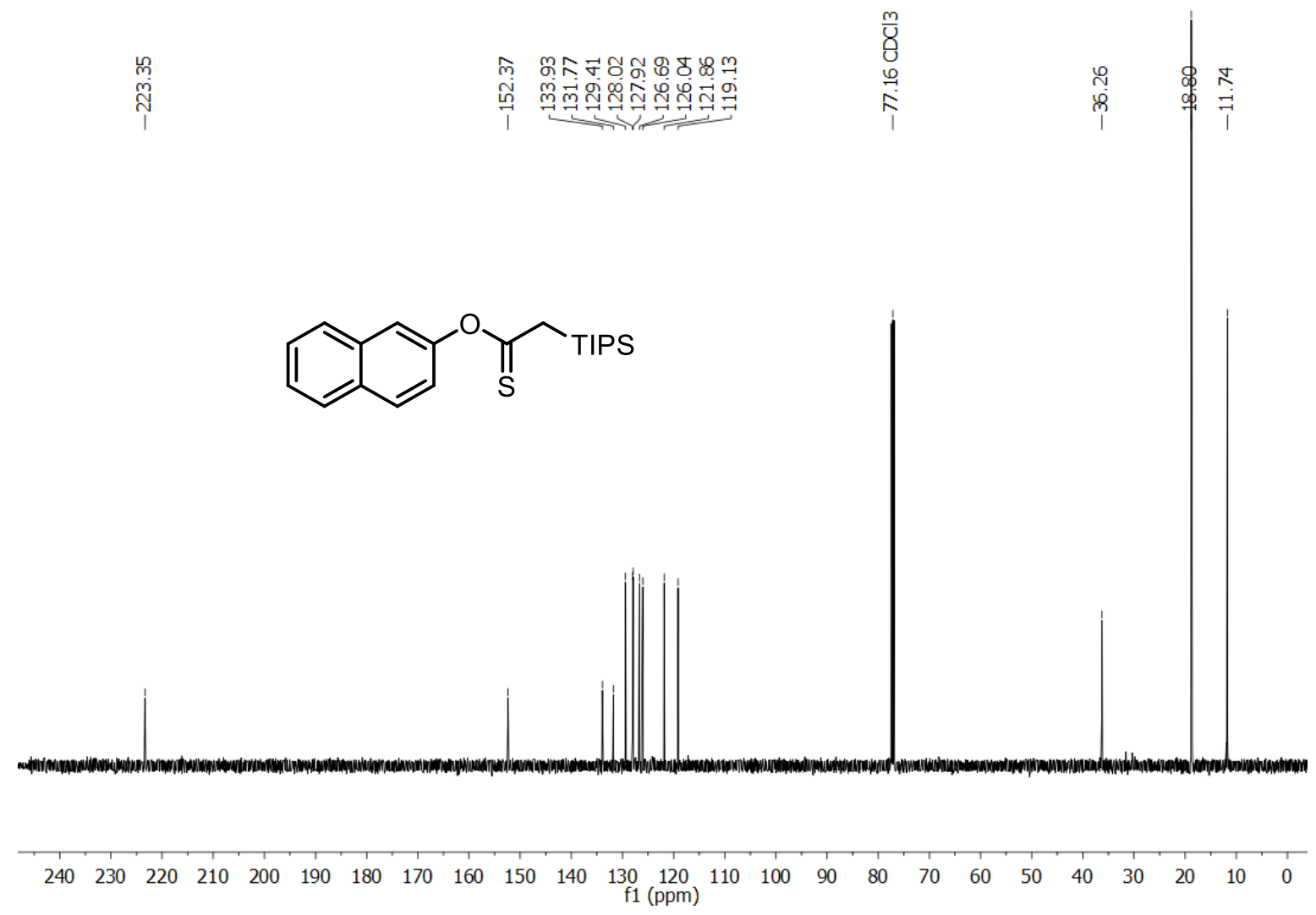

${ }^{1} \mathrm{H}$ NMR of compound $184, N, N^{\prime}$-diphenyl-2-(triisopropylsilyl)acetimidamide, $400 \mathrm{MHz}$, $\mathrm{CDCl}_{3}, 298 \mathrm{~K}$

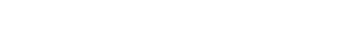<smiles>S=[SH][In](CS)CC(=Nc1ccccc1)Nc1ccccc1</smiles>

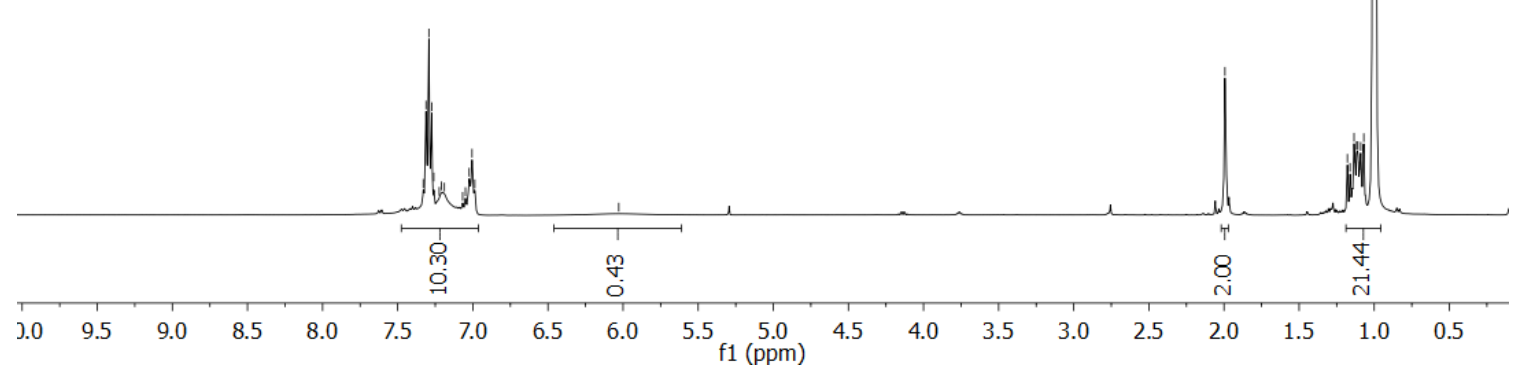


${ }^{13} \mathrm{C}$ NMR of compound 184, $N, N^{\prime}$-diphenyl-2-(triisopropylsilyl)acetimidamide, $101 \mathrm{MHz}$, $\mathrm{CDCl}_{3}, 298 \mathrm{~K}$

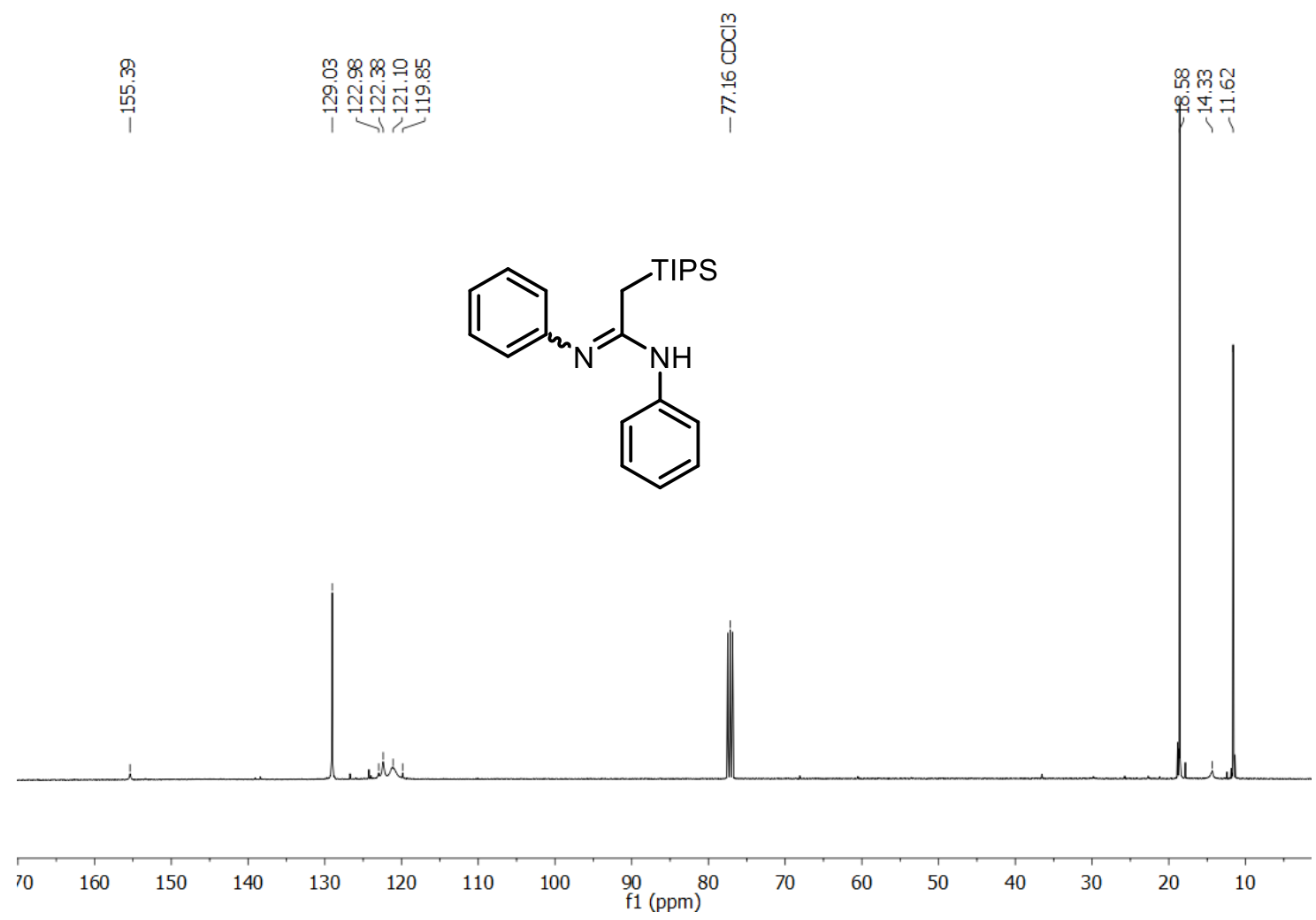

${ }^{1} \mathrm{H}$ NMR of compound 185, S-(triisopropylsilyl)ethynyl benzothioate, $400 \mathrm{MHz}, \mathrm{CDCl}_{3}, 298 \mathrm{~K}$

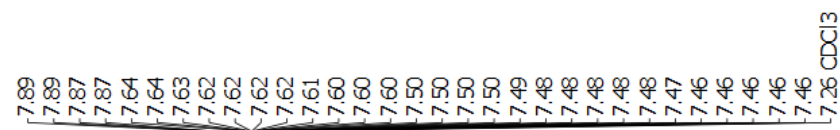

\section{学}<smiles>O=C(SC#C[In]S)c1ccccc1</smiles>

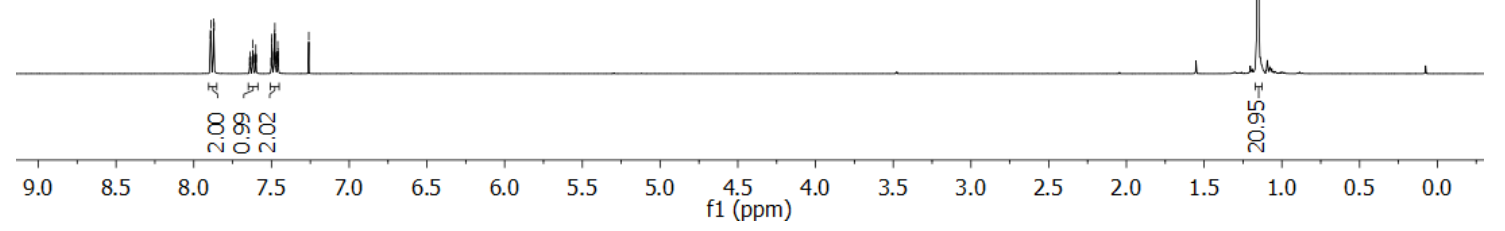


${ }^{1} \mathrm{H}$ NMR of compound 185, S-(triisopropylsilyl)ethynyl benzothioate, $100 \mathrm{MHz}, \mathrm{CDCl}_{3}, 299 \mathrm{~K}$

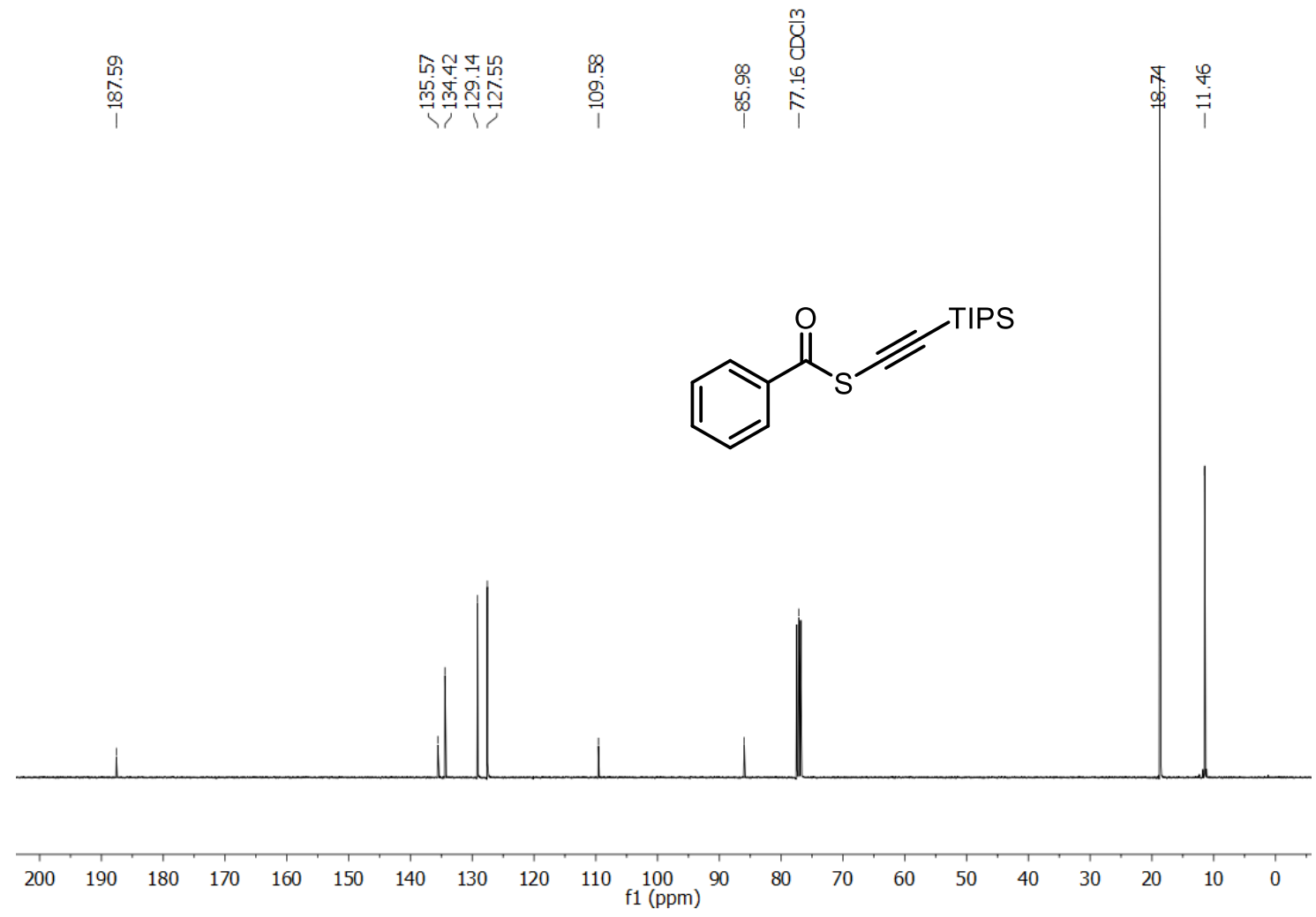

${ }^{1} \mathrm{H}$ NMR of compound 186, triisopropyl[(tritylthio)ethynyl]silane, $300 \mathrm{MHz}, \mathrm{CD}_{2} \mathrm{Cl}_{2}, 302 \mathrm{~K}$

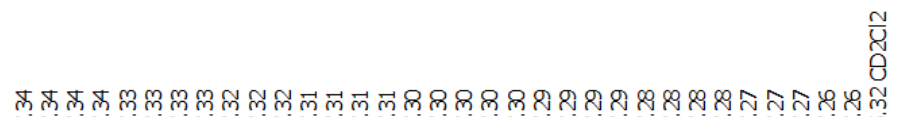
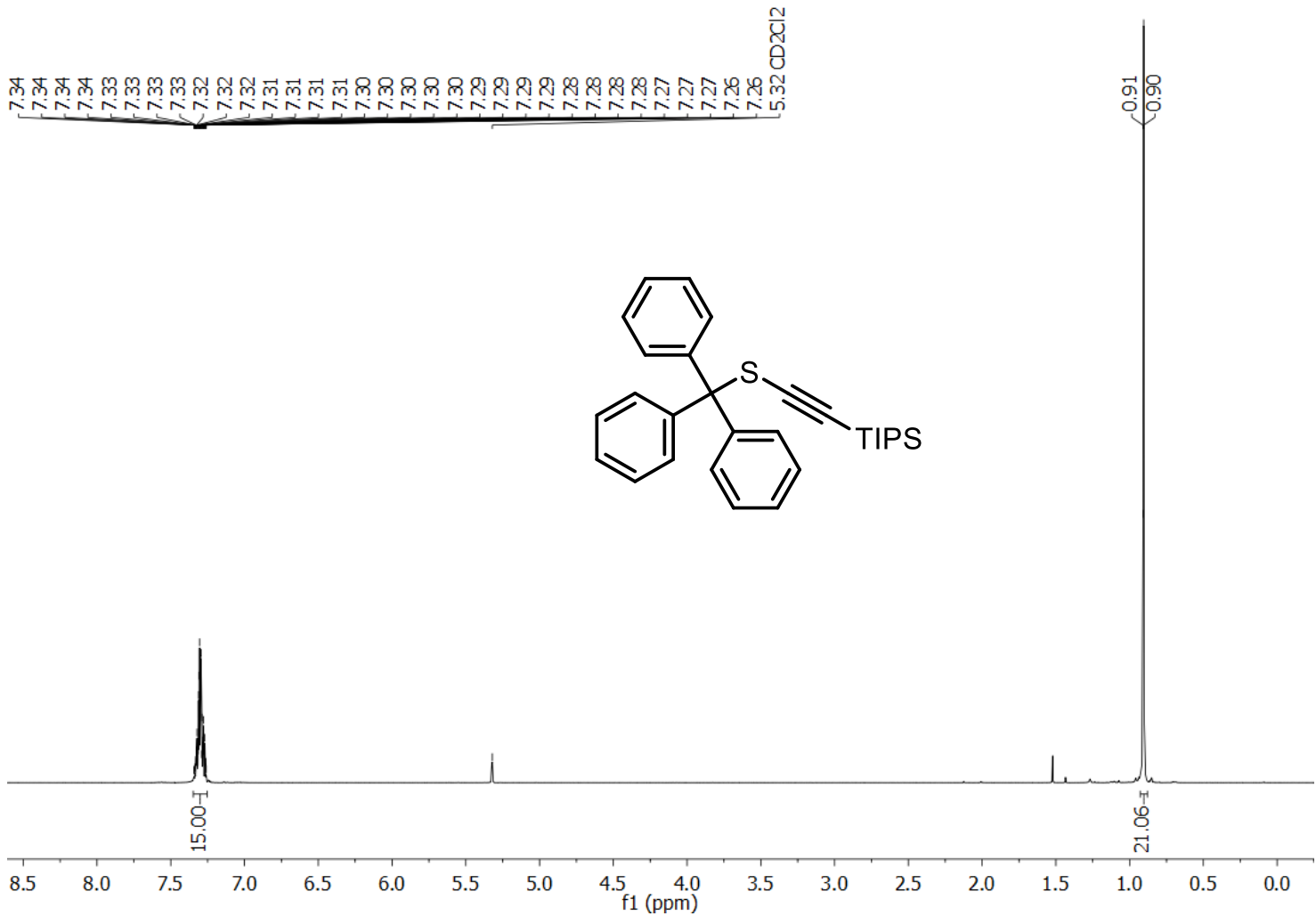
${ }^{13} \mathrm{C}$ NMR of compound 186, triisopropyl[(tritylthio)ethynyl]silane, $300 \mathrm{MHz}, \mathrm{CD}_{2} \mathrm{Cl}_{2}, 298 \mathrm{~K}$

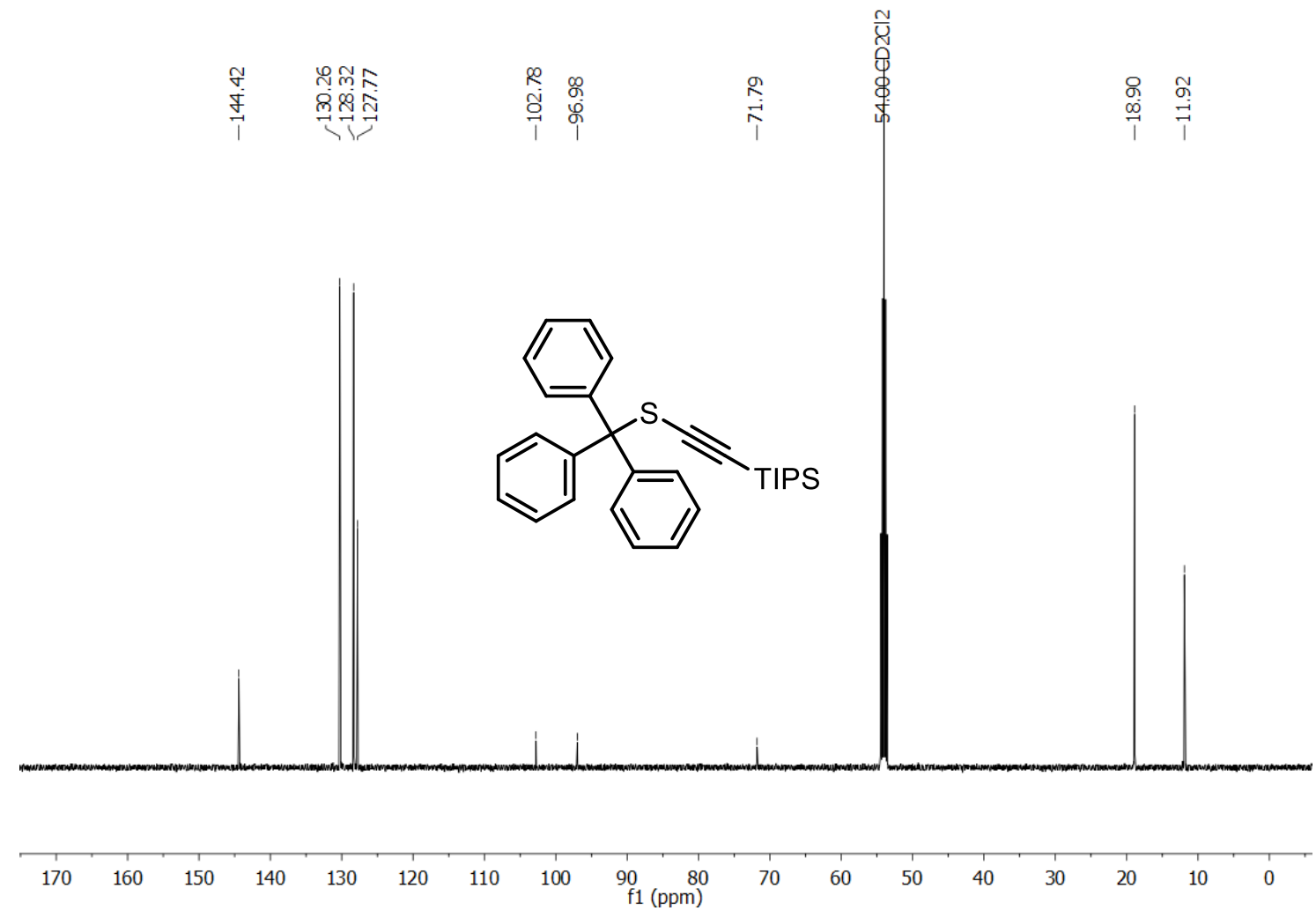

${ }^{1} \mathrm{H}$ NMR of compound 187, 2-(triisopropylsilyl)benzo[d]thiazolo[2,3-b]thiazol-4-ium-3thiolate, $600 \mathrm{MHz}, \mathrm{CD}_{3} \mathrm{Cl}, 298 \mathrm{~K}$

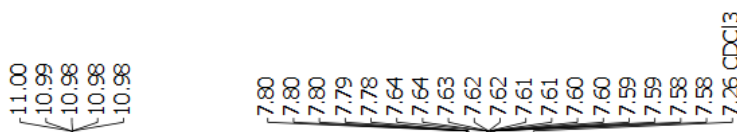

$\frac{m}{8}$<smiles>[S-][I+]c1sc2sc3ccccc3[n+]2c1[S-]</smiles>

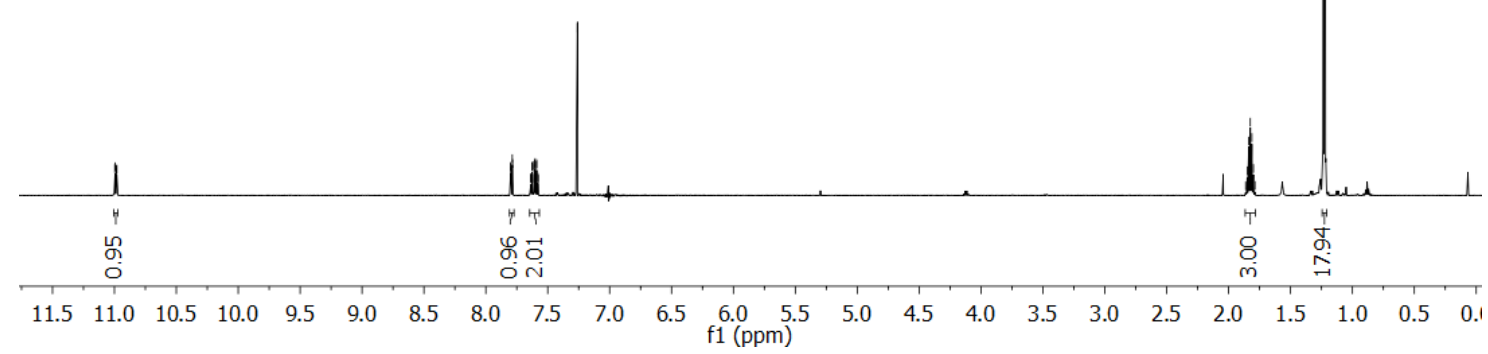


${ }^{13} \mathrm{C}$ NMR of compound 187, 2-(triisopropylsilyl)benzo[d]thiazolo[2,3-b]thiazol-4-ium-3thiolate, $126 \mathrm{MHz}, \mathrm{CD}_{3} \mathrm{Cl}, 298 \mathrm{~K}$

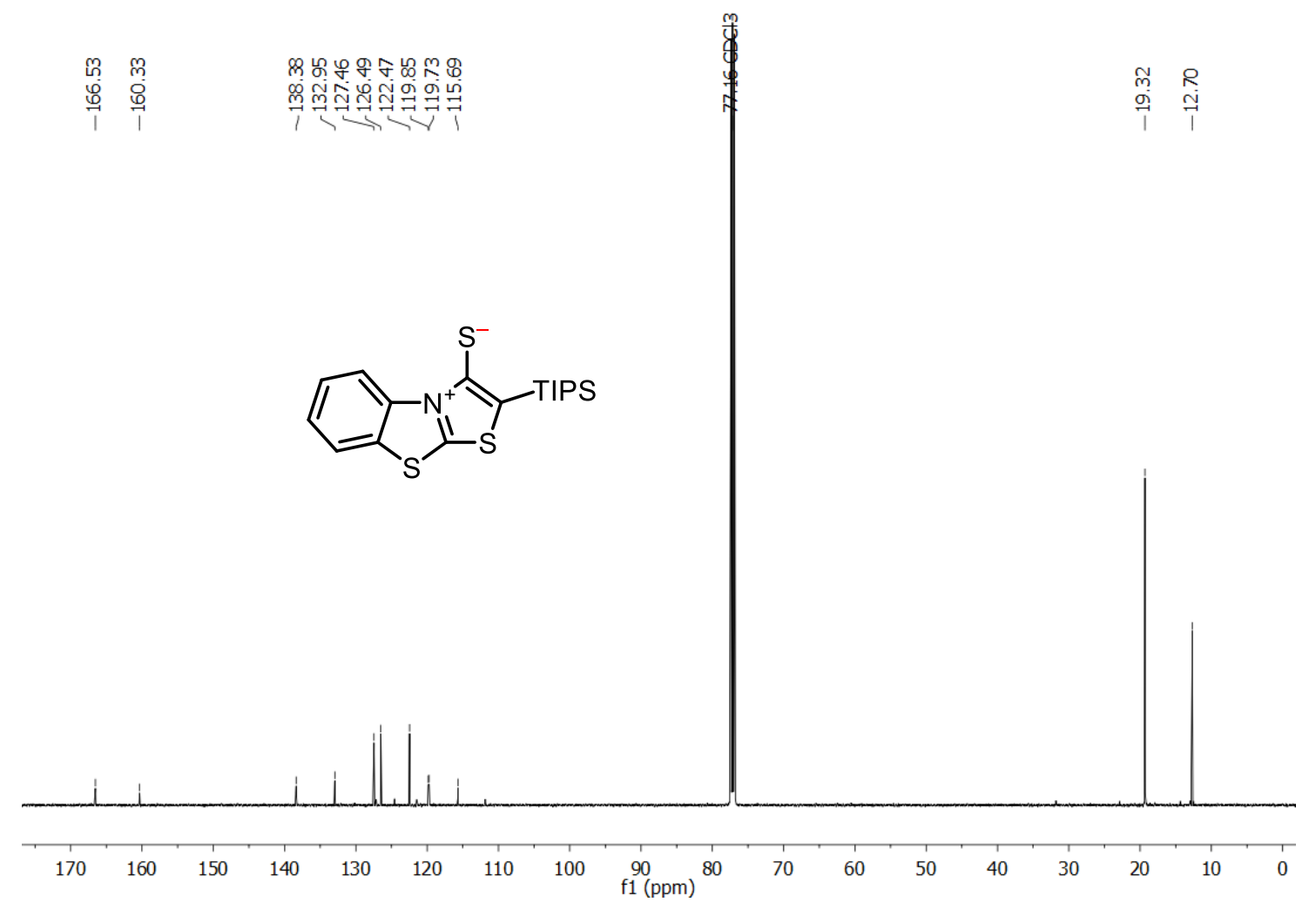

${ }^{1} \mathrm{H} \quad$ NMR of compound $188, \quad(Z)-1$-(pyrimidin-2-ylthio)-2-(triisopropylsilyl)vinyl 2(triisopropylsilyl)ethanedithioate, $600 \mathrm{MHz}, \mathrm{CD}_{3} \mathrm{Cl}, 298 \mathrm{~K}$

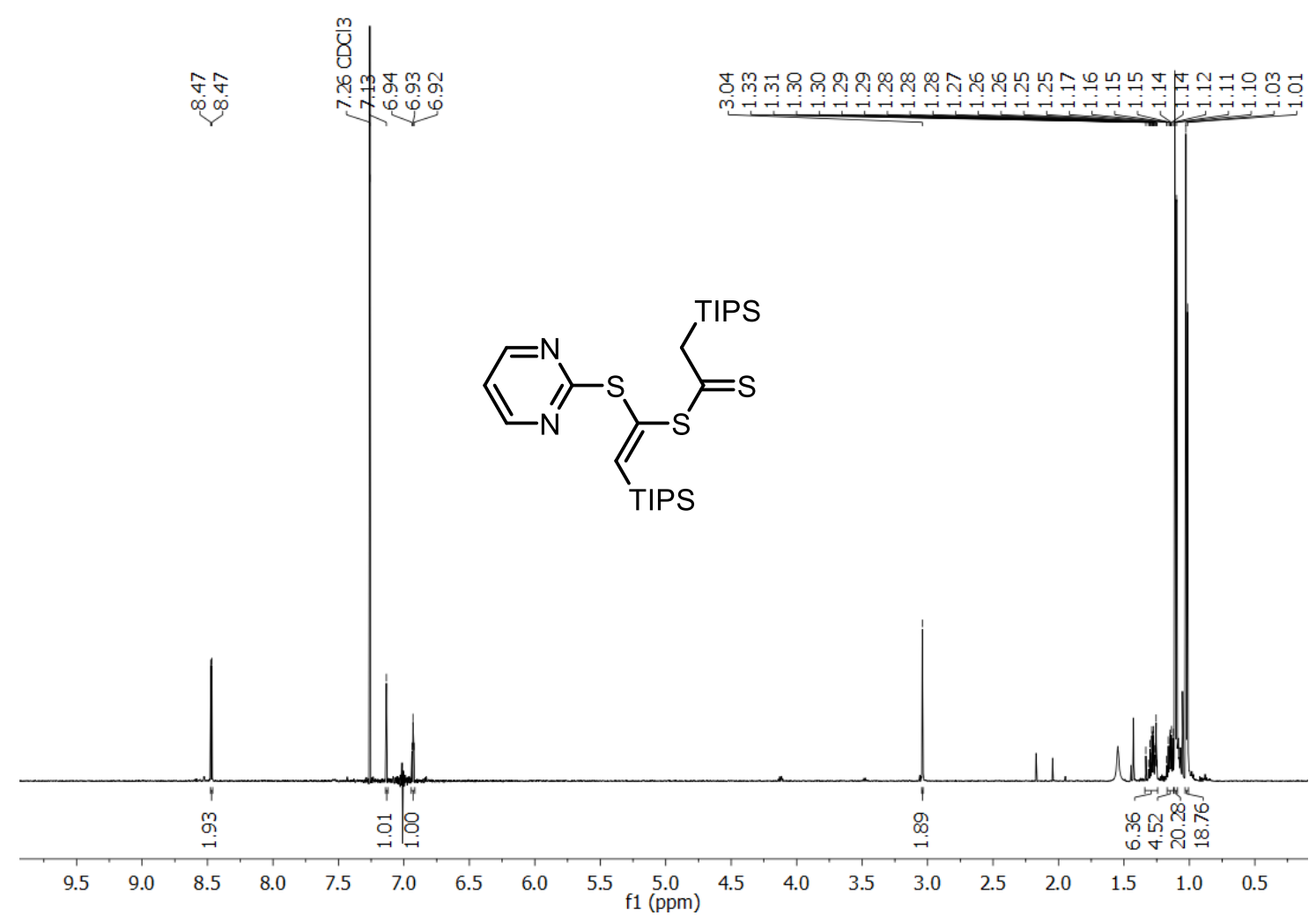


${ }^{13} \mathrm{C}$ NMR of compound 188, (Z)-1-(pyrimidin-2-ylthio)-2-(triisopropylsilyl)vinyl 2(triisopropylsilyl)ethanedithioate, $126 \mathrm{MHz}, \mathrm{CD}_{3} \mathrm{Cl}, 298 \mathrm{~K}$

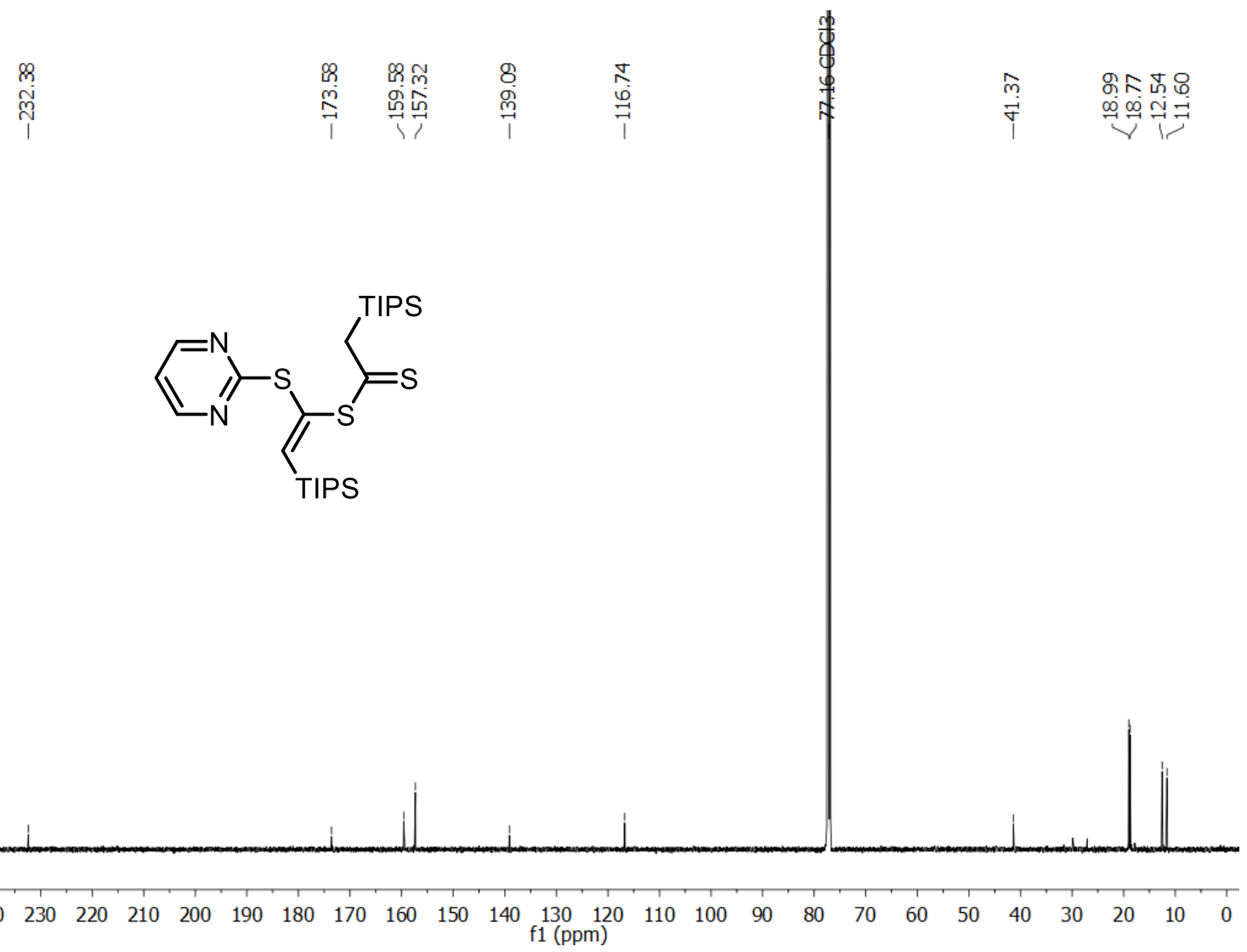

${ }^{1} \mathrm{H}$ NMR of compound 189, 2,4,6-trimethylbenzoic anhydride, $300 \mathrm{MHz}, \mathrm{CDCl}_{3}, 298 \mathrm{~K}$

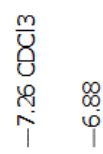<smiles>Cc1cc(C)c(C(=O)OC(=O)c2c(C)cc(C)cc2C)c(C)c1</smiles>

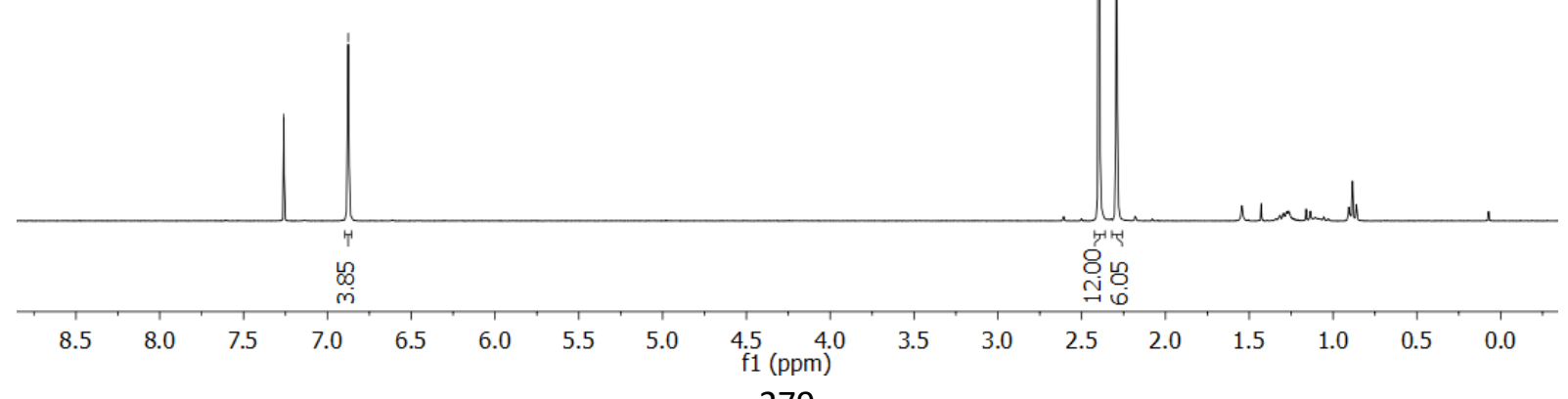


${ }^{1} \mathrm{H}$ NMR of compound 190a, (Z)-2-[\{1-[2-(tert-butoxycarbonyl)-1-oxo-2,3-dihydro-1H-inden2-yl]-2-phenylvinyl\}thio]-1-ethylpyridin-1-ium tetrabromizincate, $500 \mathrm{MHz}, \mathrm{CD}_{2} \mathrm{Cl}_{2}, 298 \mathrm{~K}$

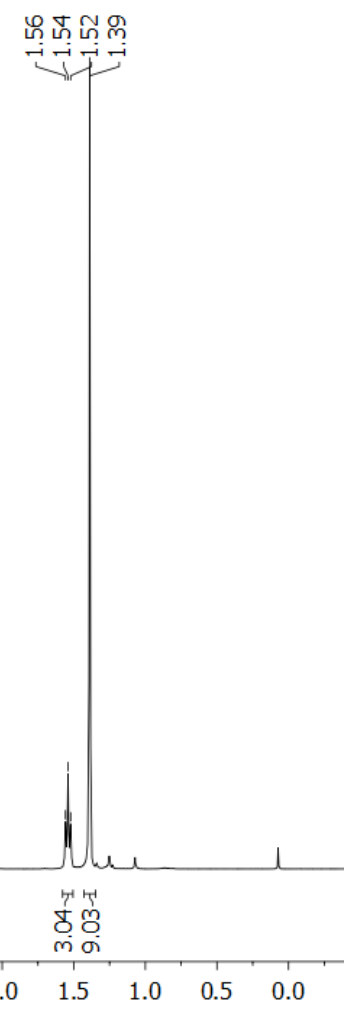<smiles>CC[n+]1ccccc1S/C(=C\c1ccccc1)C1(C(=O)OC(C)(C)C)Cc2ccccc2C1=O</smiles>

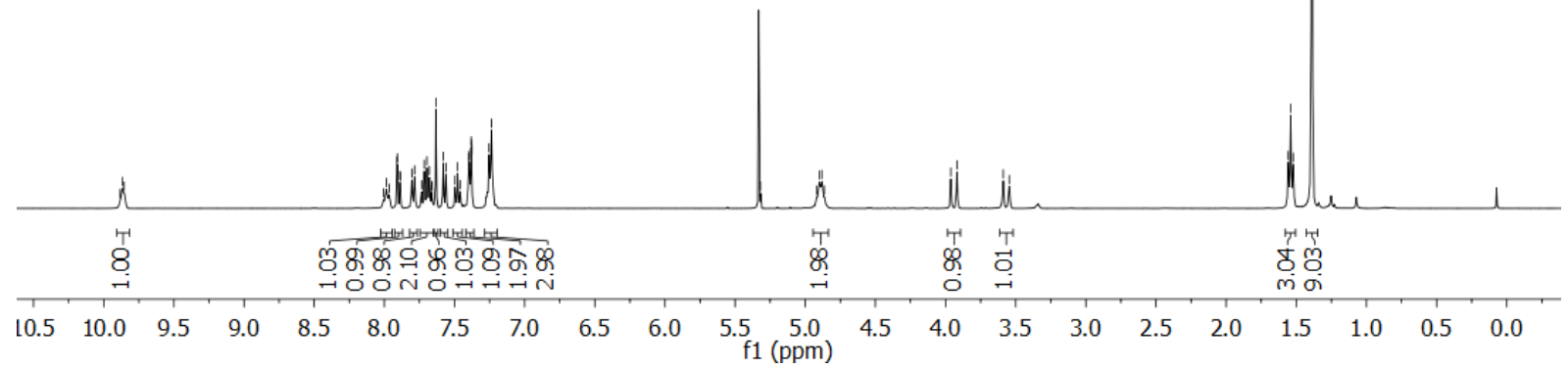

${ }^{13} \mathrm{C}$ NMR of compound 190, (Z)-2-[\{1-[2-(tert-butoxycarbonyl)-1-oxo-2,3-dihydro-1H-inden2-yl]-2-phenylvinyl\}thio]-1-ethylpyridin-1-ium tetrabromozincate, $126 \mathrm{MHz}, \mathrm{CD}_{2} \mathrm{Cl}_{2}, 298 \mathrm{~K}$

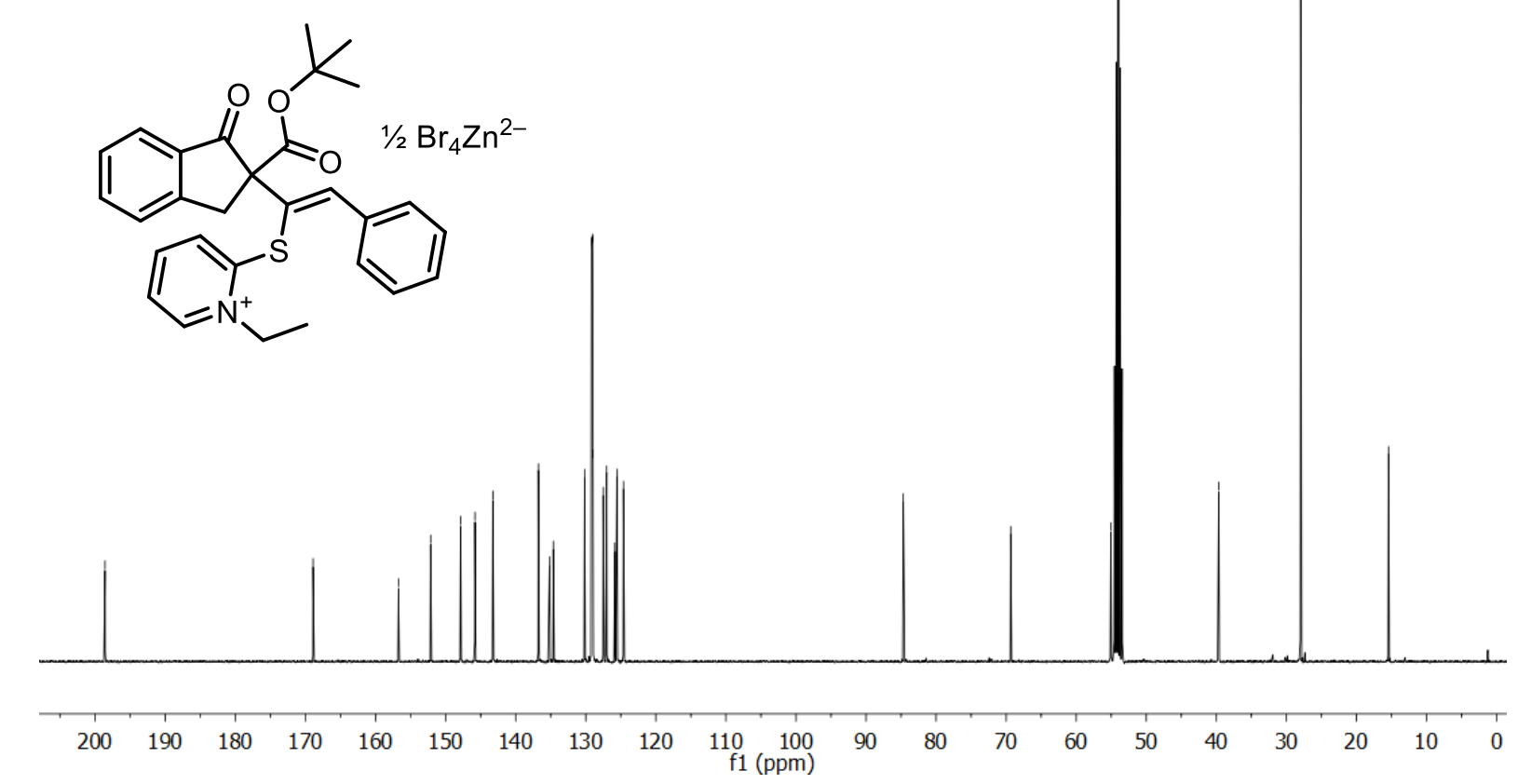


${ }^{1} \mathrm{H}$ NMR of compound 190b, (Z)-1-ethyl-2-[\{1-[2-(methoxycarbonyl)-1-oxo-2,3-dihydro-1Hinden-2-yl]-2-phenylvinyl\}thio]pyridin-1-ium tetrabromozincate, $500 \mathrm{MHz}, \mathrm{CDCl}_{3}, 298 \mathrm{~K}$

$\frac{\mathrm{m}}{\mathrm{Z}}$

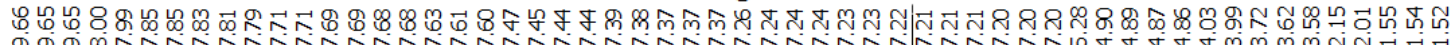<smiles>CC[n+]1ccccc1S/C(=C\c1ccccc1)C1(C(=O)OC)Cc2ccccc2C1=O</smiles>

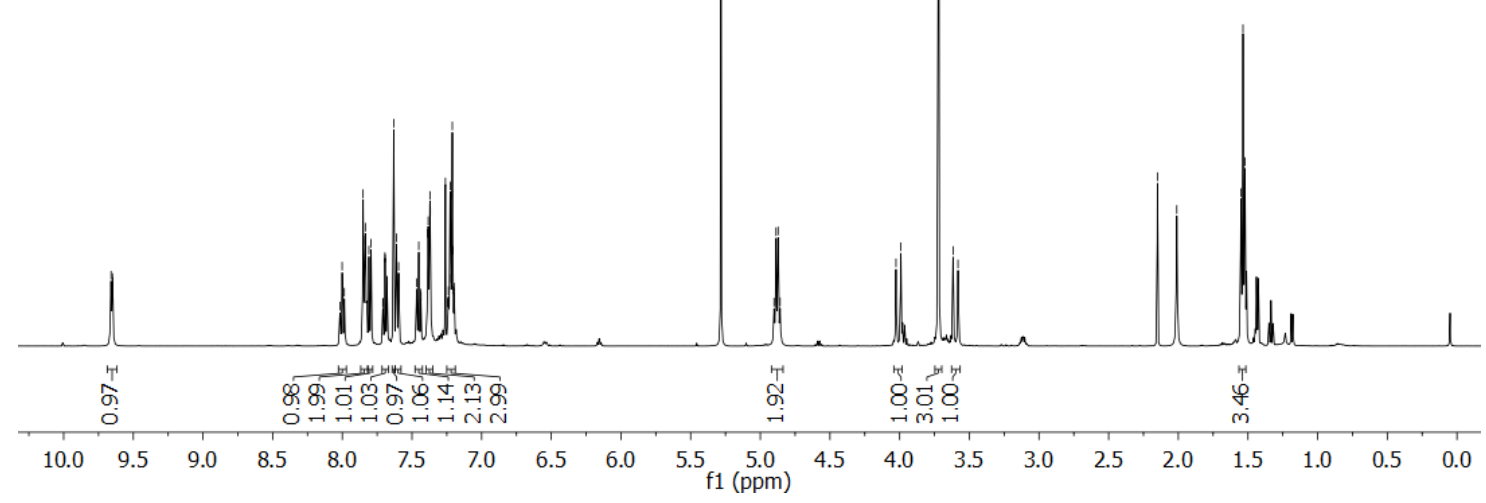

${ }^{13} \mathrm{C}$ NMR of compound 190b, (Z)-1-ethyl-2-[\{1-[2-(methoxycarbonyl)-1-oxo-2,3-dihydro-1Hinden-2-yl]-2-phenylvinyl\}thio]pyridin-1-ium tetrabromozincate, $126 \mathrm{MHz}, \mathrm{CDCl}_{3}, 298 \mathrm{~K}$

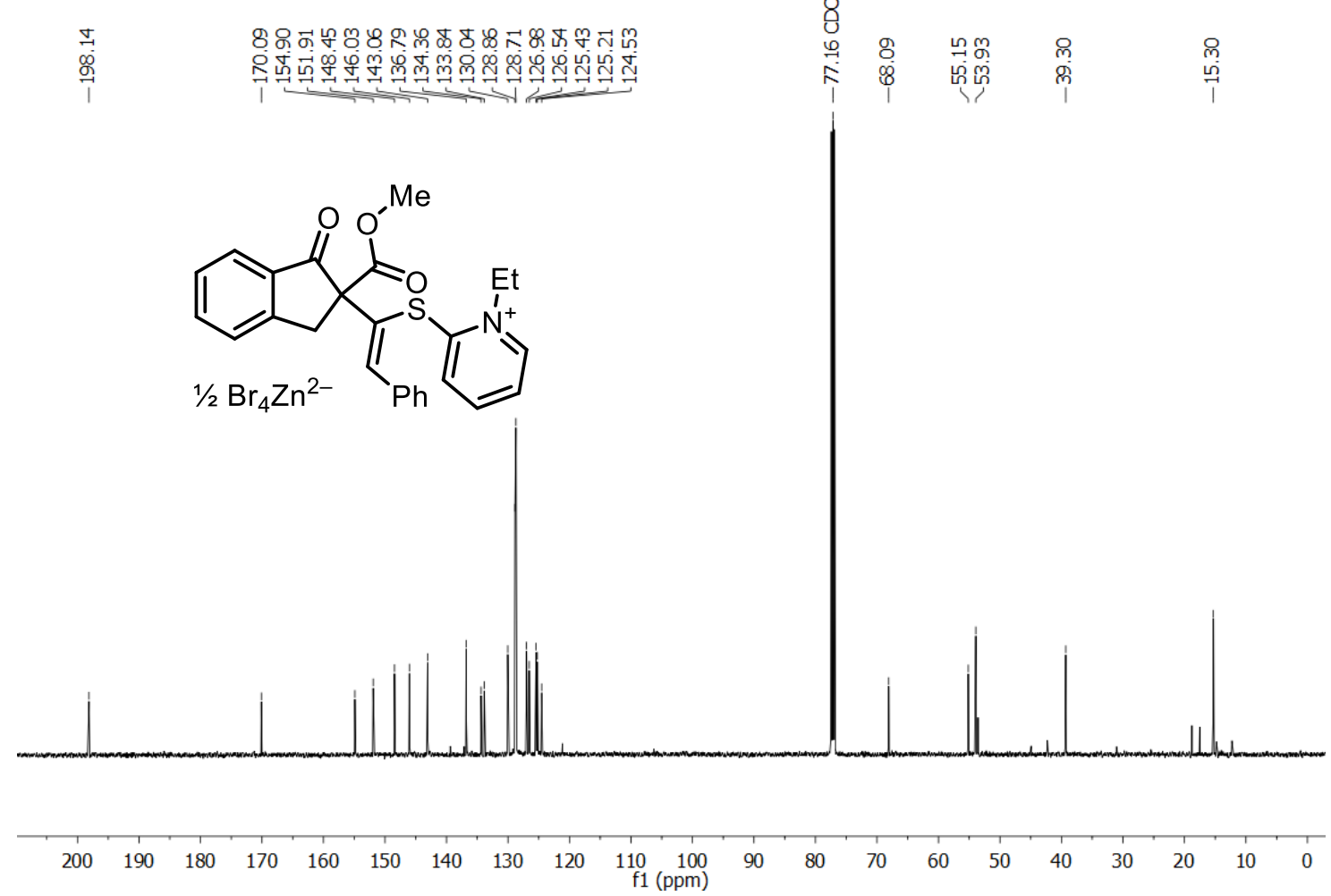


${ }^{1} \mathrm{H}$ NMR of compound 191, (E/Z)-2-benzylidene-4-phenyl-1,3-dithiole, $300 \mathrm{MHz}, \mathrm{CDCl}_{3}, 298$ K

$\frac{m}{8}$

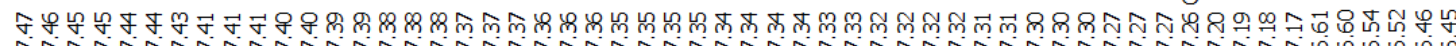

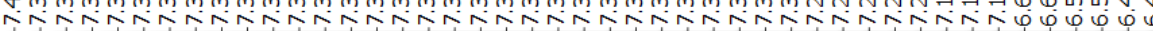

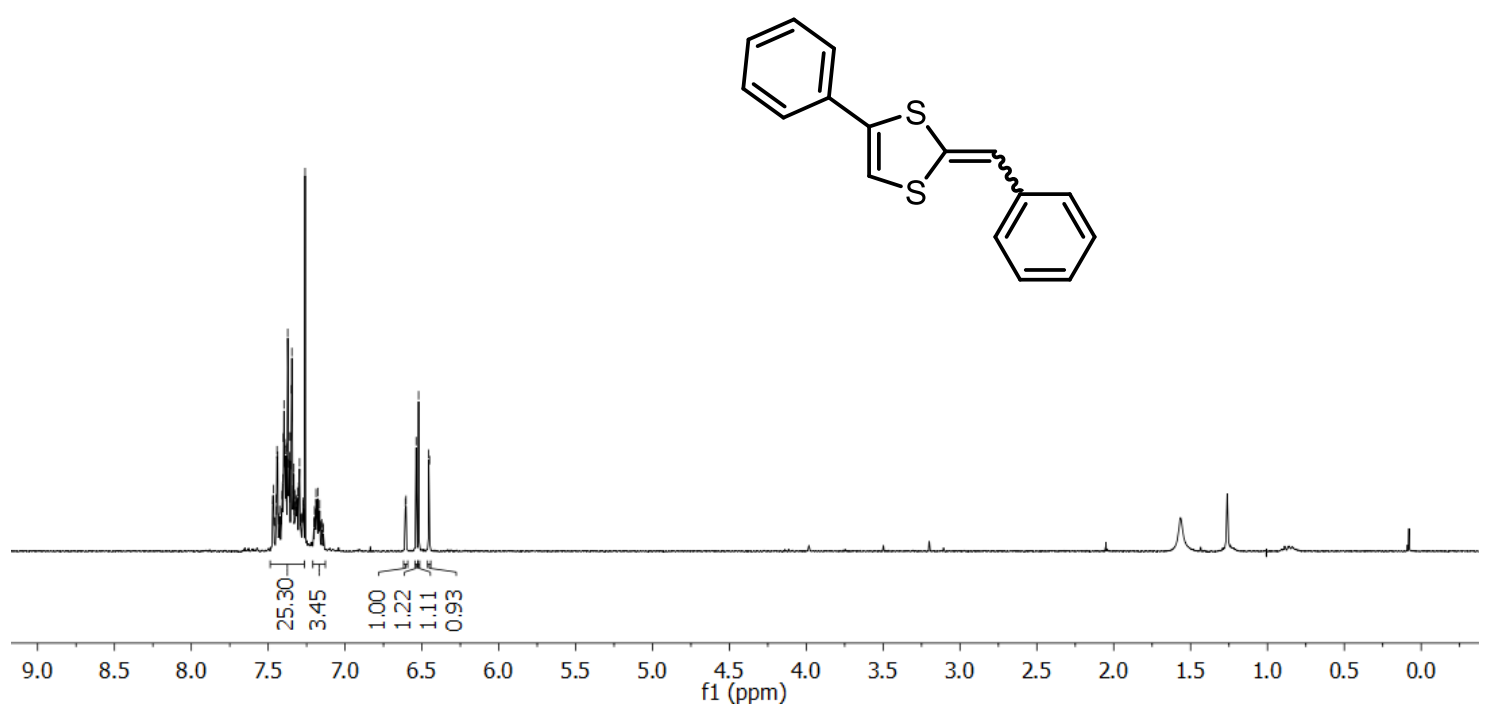

${ }^{13} \mathrm{C}$ NMR of compound 191, (E/Z)-2-benzylidene-4-phenyl-1,3-dithiole, $101 \mathrm{MHz}, \mathrm{CDCl} 3,298$ K
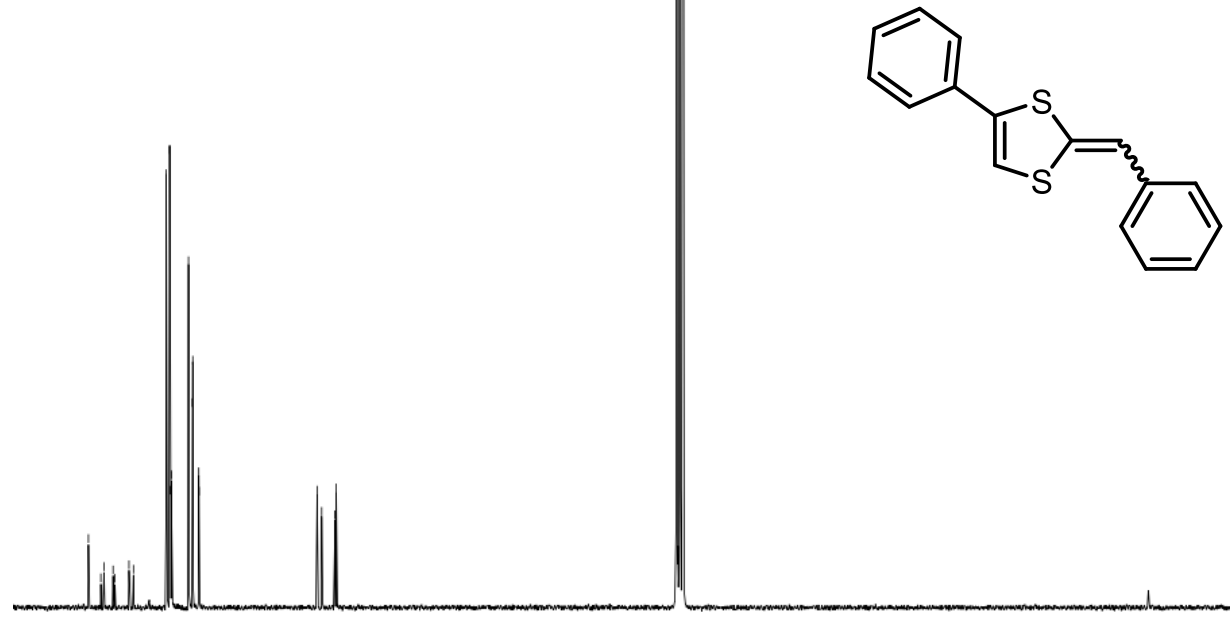

$\begin{array}{lllllllllllllllllllllllllllllllll}140 & 135 & 130 & 125 & 120 & 115 & 110 & 105 & 100 & 95 & 90 & 85 & 80 & 75 & 70 & 65 & 60 & 55 & 50 & 45 & 40 & 35 & 30 & 25 & 20 & 15 & 10 & 5 & 0\end{array}$ 
${ }^{1} \mathrm{H} \quad \mathrm{NMR}$ of compound 192, 4-methoxyphenyl 2,2-dideuterio-2-

(triisopropylsilyl)ethanedithioate, $300 \mathrm{MHz}^{\mathrm{CDCl}} 3,302 \mathrm{~K}$

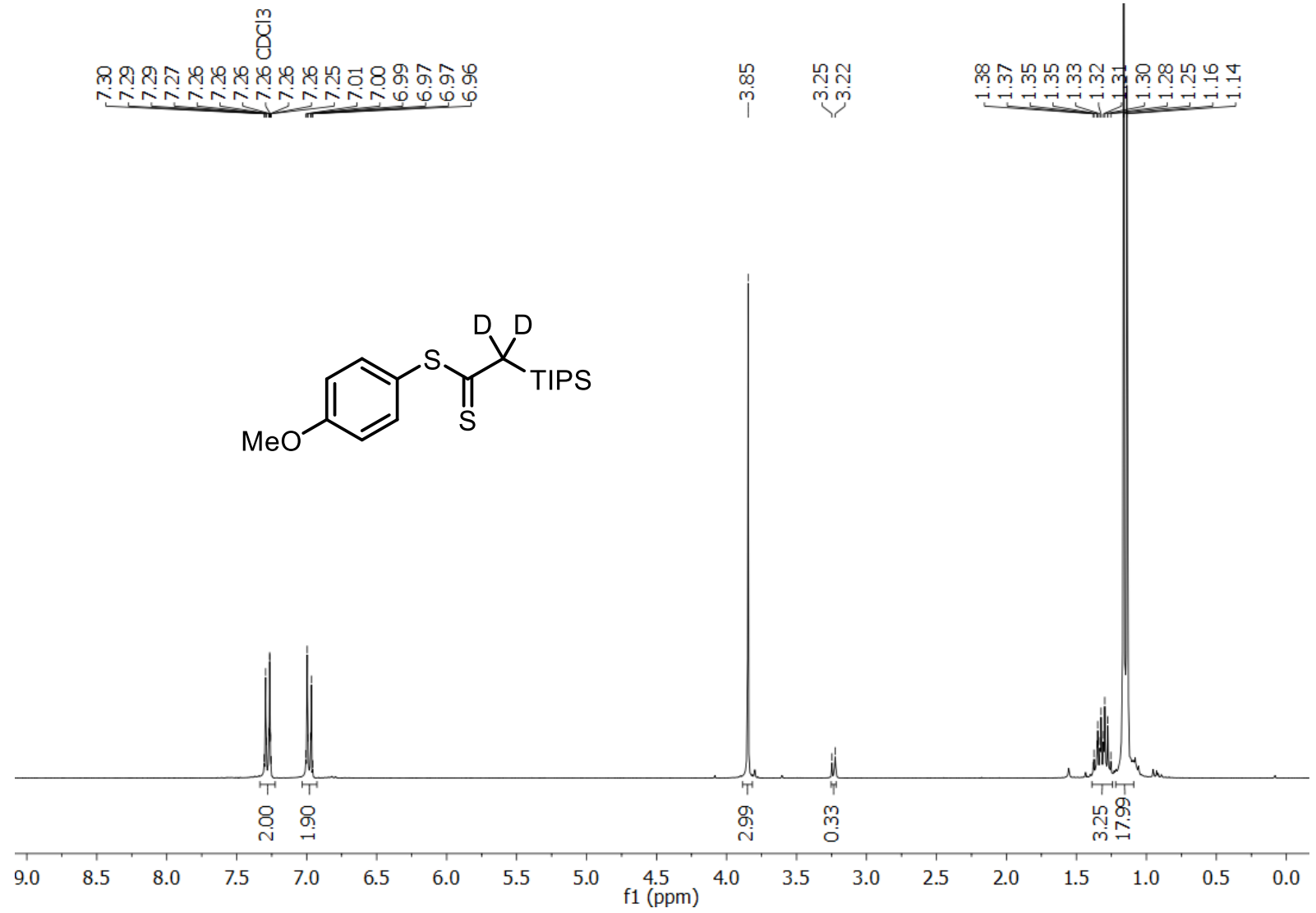

${ }^{13} \mathrm{C} \quad \mathrm{NMR}$ of compound 192, 4-methoxyphenyl 2,2-dideuterio-2-

(triisopropylsilyl)ethanedithioate, $101 \mathrm{MHz}, \mathrm{CDCl}_{3}, 298 \mathrm{~K}$

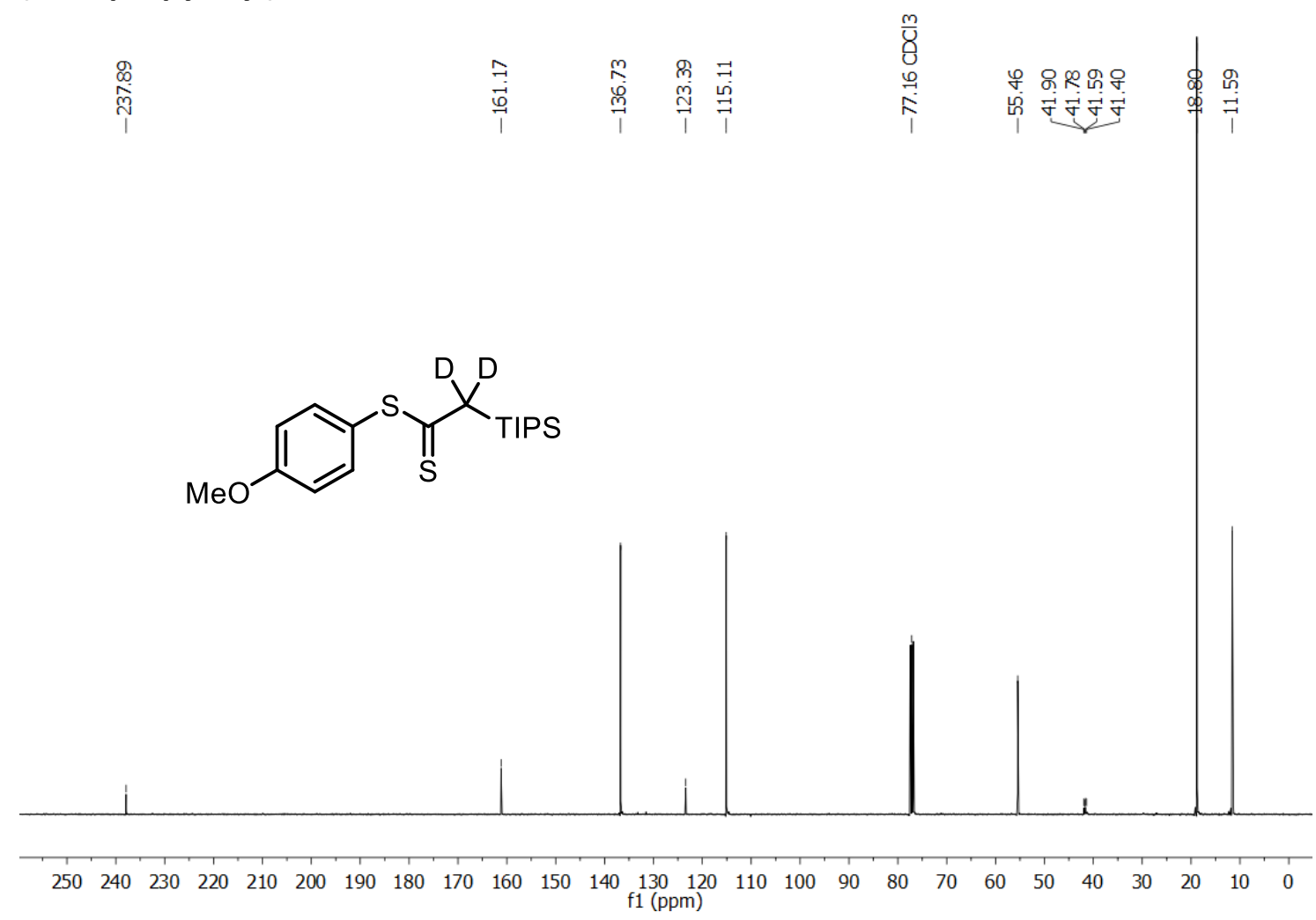


${ }^{1} \mathrm{H}$ NMR of compound $\left\{{ }^{13} \mathrm{C}\right\}-197,1,3-$ diisopropyl-4,5-dimethyl-2-[(phenylethynyl-2${ }^{13} \mathrm{C}$ )thio]-1H-imidazol-3-ium hexafluoroantimonate, $600 \mathrm{MHz}, \mathrm{CDCl}_{3}, 298 \mathrm{~K}$
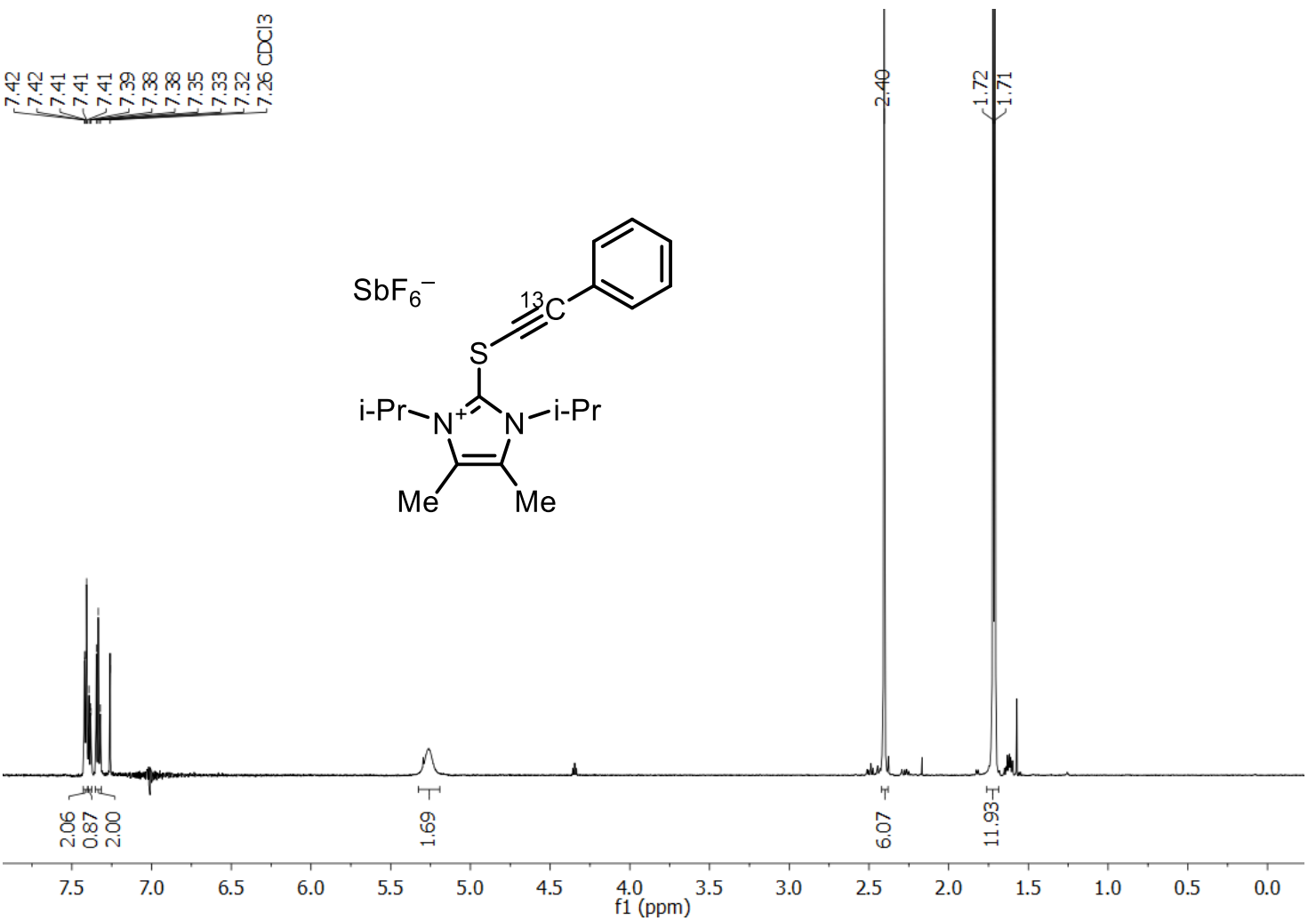

${ }^{13} \mathrm{C}$ NMR of compound $\left\{{ }^{13} \mathrm{C}\right\}-197,1,3-$ diisopropyl-4,5-dimethyl-2-[(phenylethynyl-2${ }^{13} \mathrm{C}$ )thio]-1H-imidazol-3-ium hexafluoroantimonate, $126 \mathrm{MHz}, \mathrm{CDCl}_{3}, 298 \mathrm{~K}$

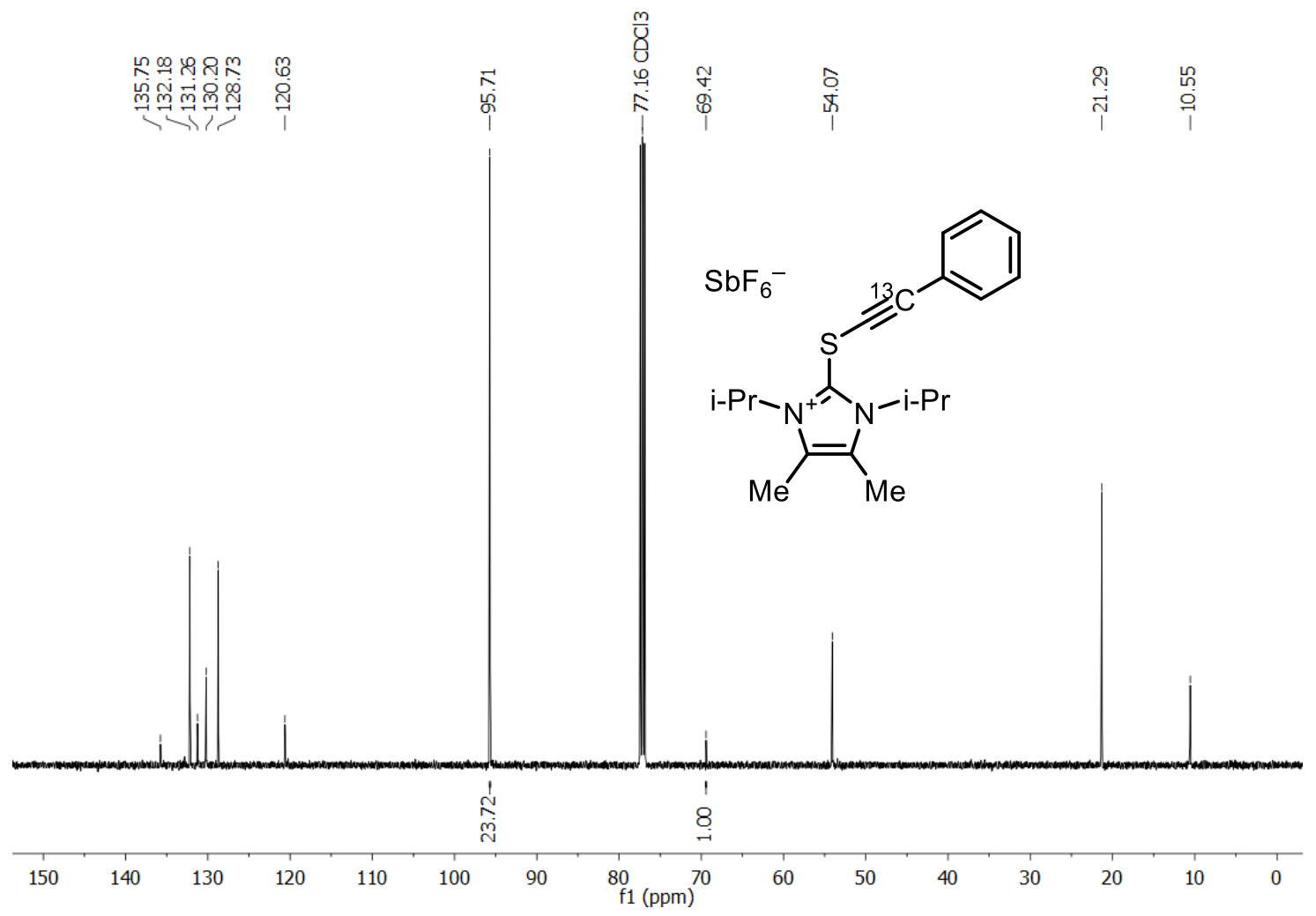


${ }^{1} \mathrm{H}$ NMR of compound 202, 1,3-diisopropyl-4,5-dimethyl-2-\{[(triisopropylsilyl)ethynyl]thio $\}-$ $1 \mathrm{H}$-imidazol-3-ium hexafluoroantimonate, $500 \mathrm{MHz} \mathrm{CDCl}_{3}, 298 \mathrm{~K}$

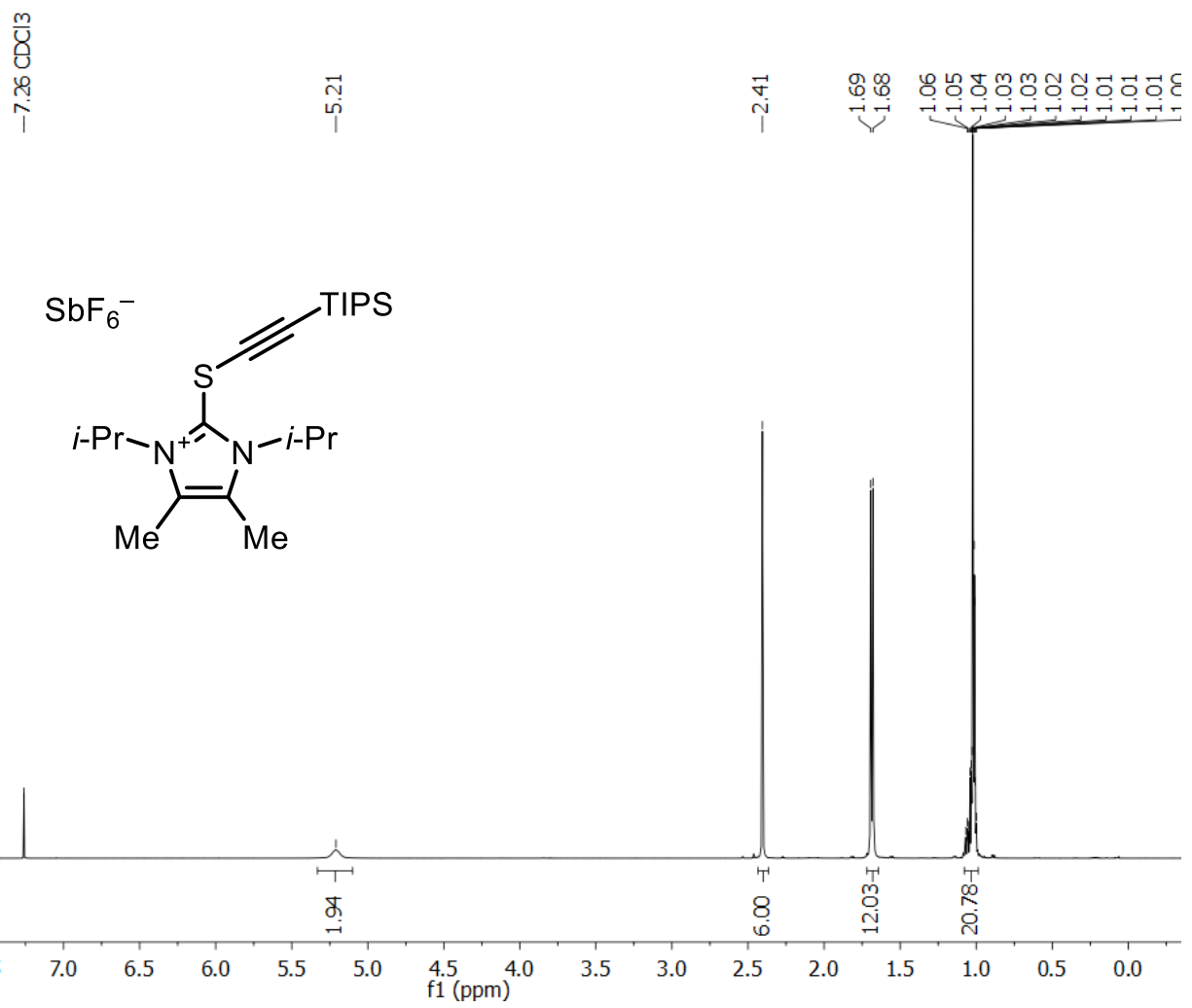

${ }^{13} \mathrm{C}$ NMR of compound 202, 1,3-diisopropyl-4,5-dimethyl-2-\{[(triisopropylsilyl)ethynyl]thio\}$1 \mathrm{H}$-imidazol-3-ium hexafluoroantimonate, $126 \mathrm{MHz}^{\mathrm{CDCl}} \mathrm{CD}_{3}, 298 \mathrm{~K}$

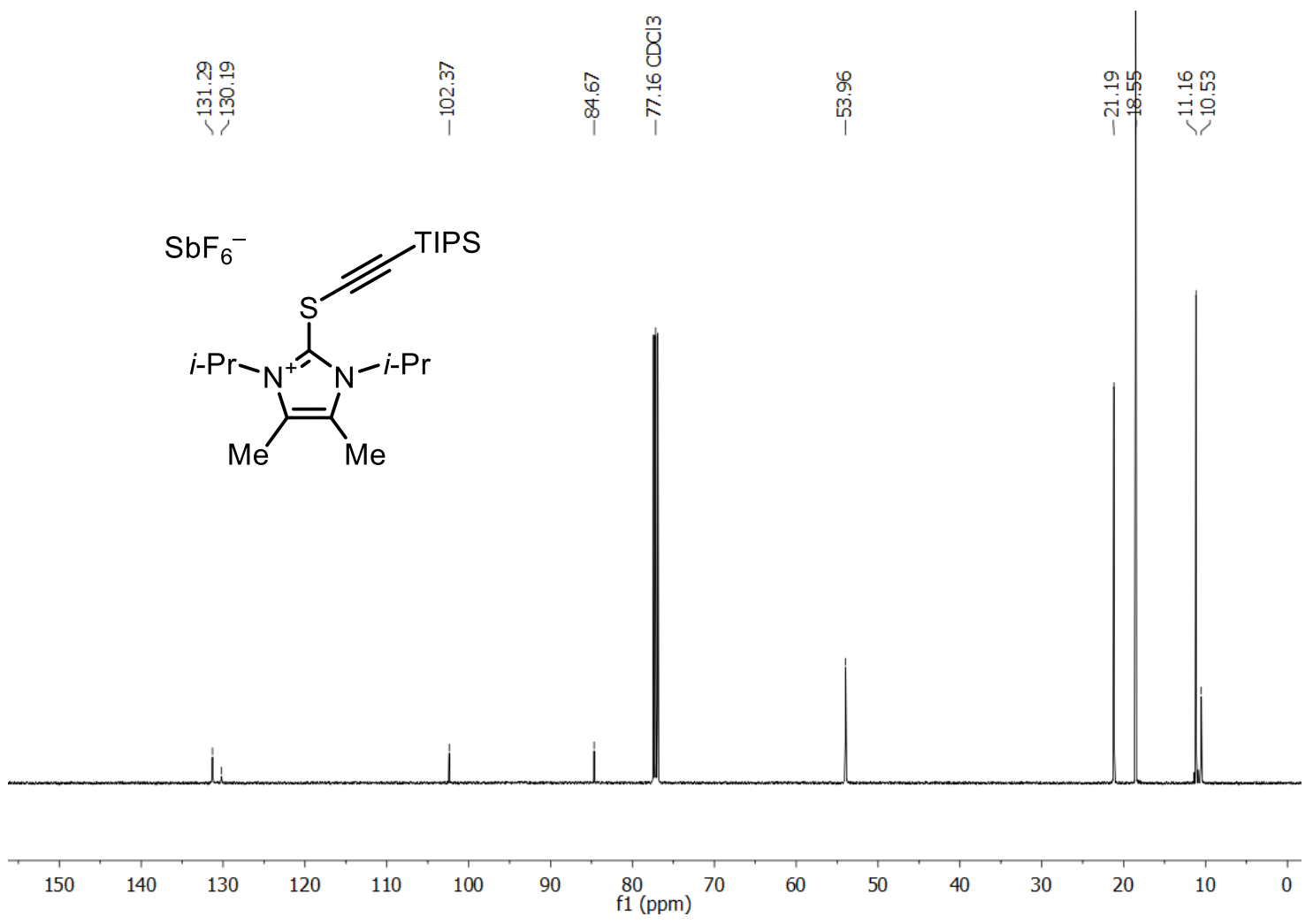


${ }^{1} \mathrm{H}$ NMR of compound 203, 5-phenylpyrazolo[1,2-a]cinnolin-4-ium hexafluoroantimonate, $400 \mathrm{MHz}, \mathrm{CD}_{3} \mathrm{CN}, 298 \mathrm{~K}$

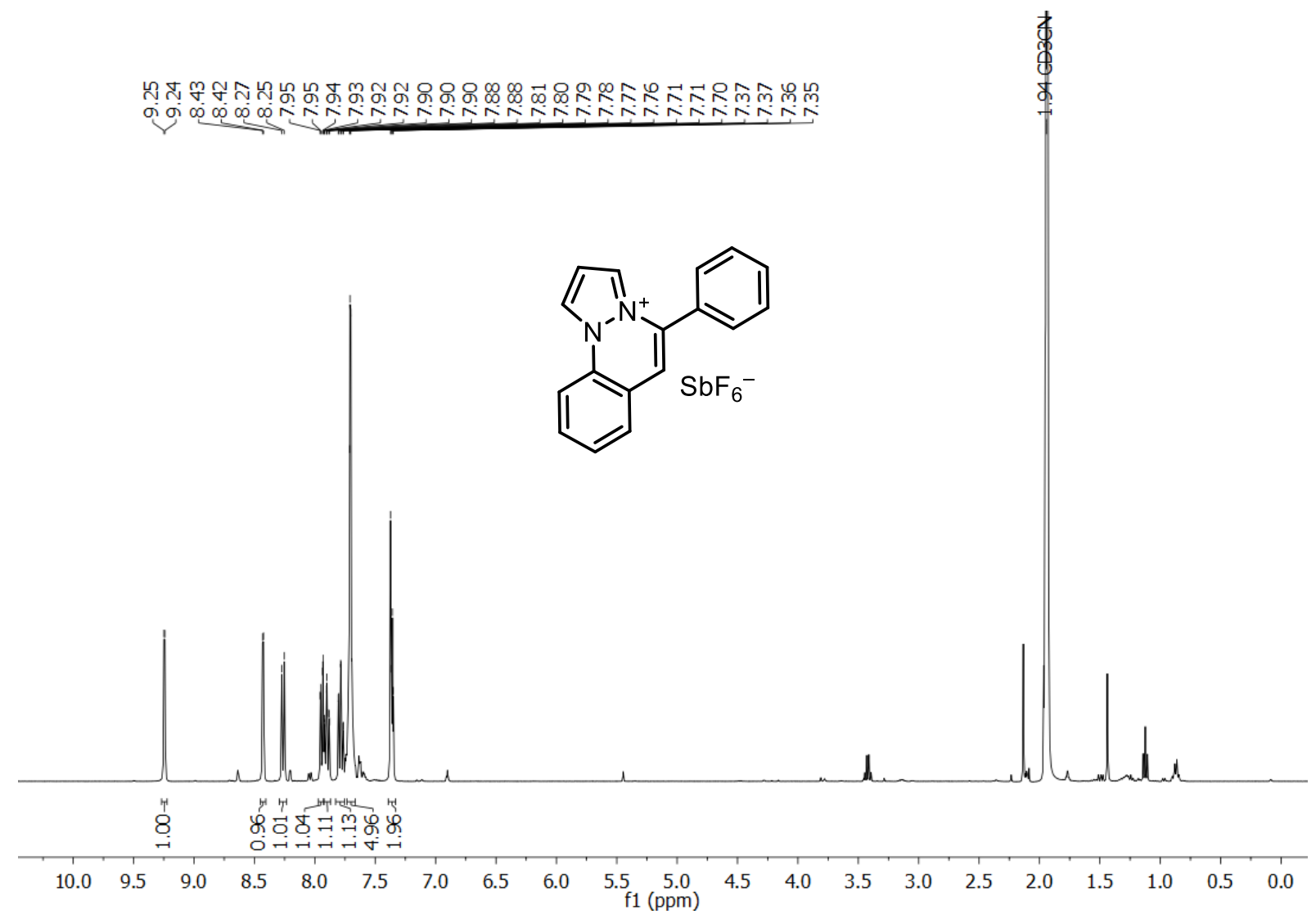

${ }^{13} \mathrm{C}$ NMR of compound 203, 5-phenylpyrazolo[1,2-a]cinnolin-4-ium hexafluoroantimonate, $151 \mathrm{MHz}$, DMSO-d $6,303 \mathrm{~K}$
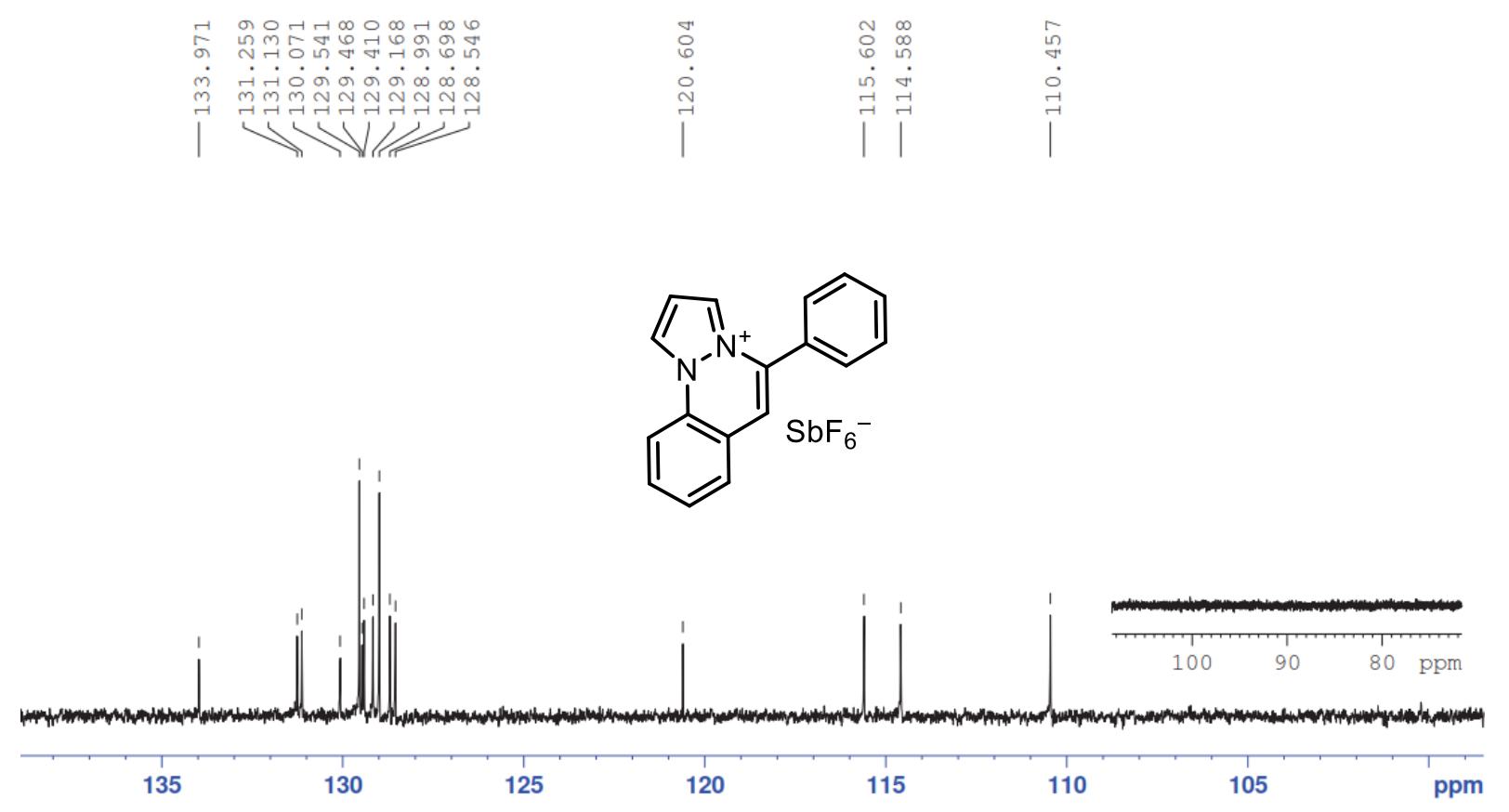
${ }^{19} \mathrm{~F}$ NMR of compound 203, 5-phenylpyrazolo[1,2-a]cinnolin-4-ium hexafluoroantimonate, $377 \mathrm{MHz}, \mathrm{CD}_{3} \mathrm{CN}, 298 \mathrm{~K}$<smiles>Oc1cc2ccccc2n2ccc[n+]12</smiles>

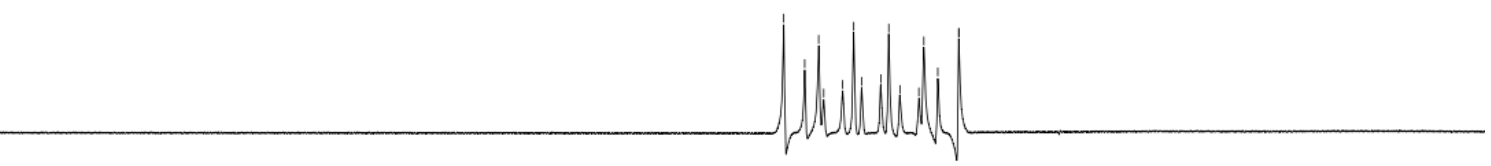

$\begin{array}{llllllllllllllllllllllll}0 & -10 & -20 & -30 & -40 & -50 & -60 & -70 & -80 & -90 & -100 & -110 & -120 & -130 & -140 & -150 & -160 & -170 & -180 & -190 & -200 & -211\end{array}$

${ }^{1} \mathrm{H}$ NMR of compound 204, 2-(2-Benzoylphenyl)-1-phenylethan-1-one, $400 \mathrm{Mhz}$, DMSO-d6, $300 \mathrm{~K}$

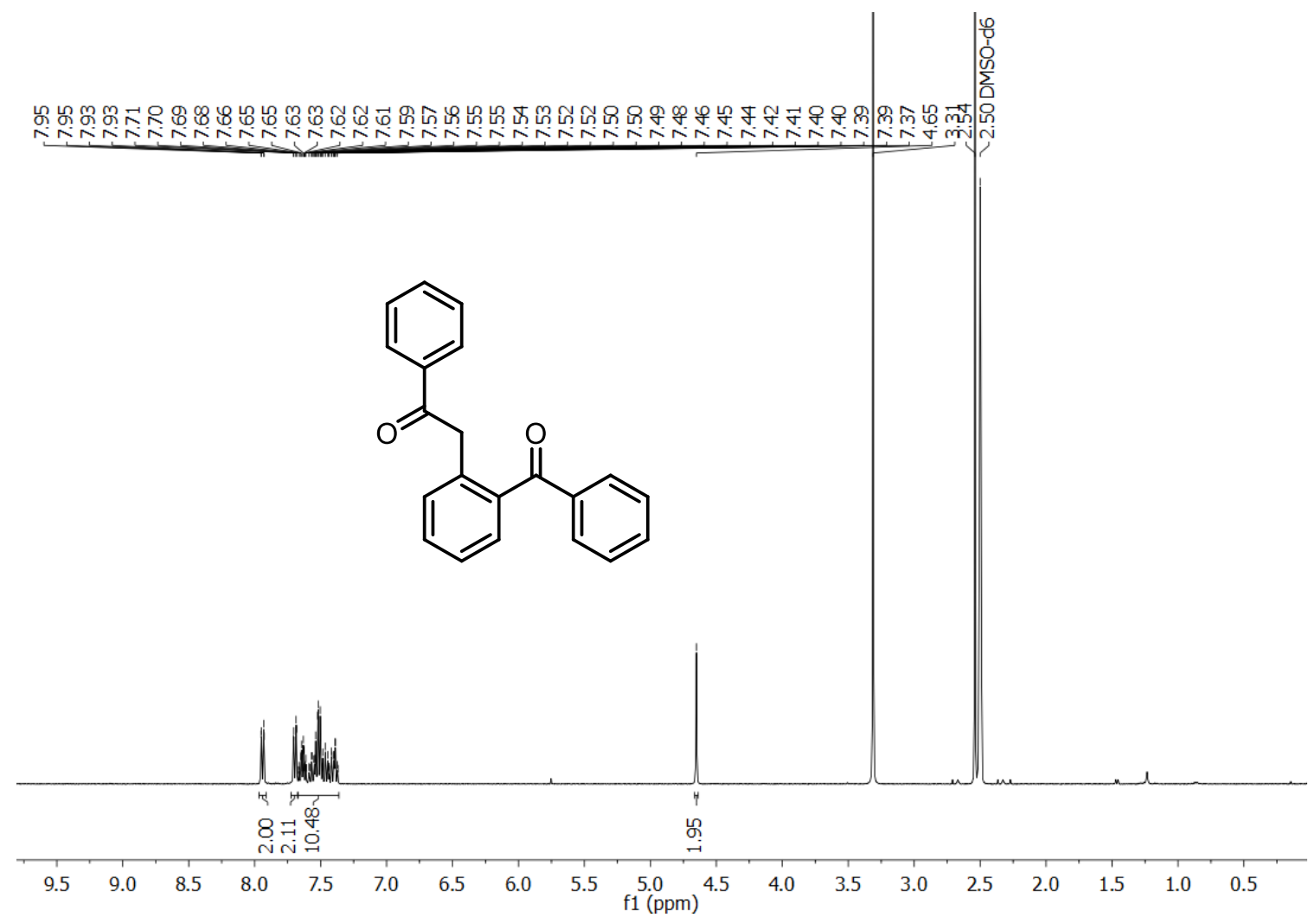


${ }^{13} \mathrm{C}$ NMR of compound 204, 2-(2-Benzoylphenyl)-1-phenylethan-1-one, $126 \mathrm{Mhz}$, DMSO- $d_{6}$, $303 \mathrm{~K}$

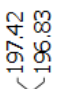

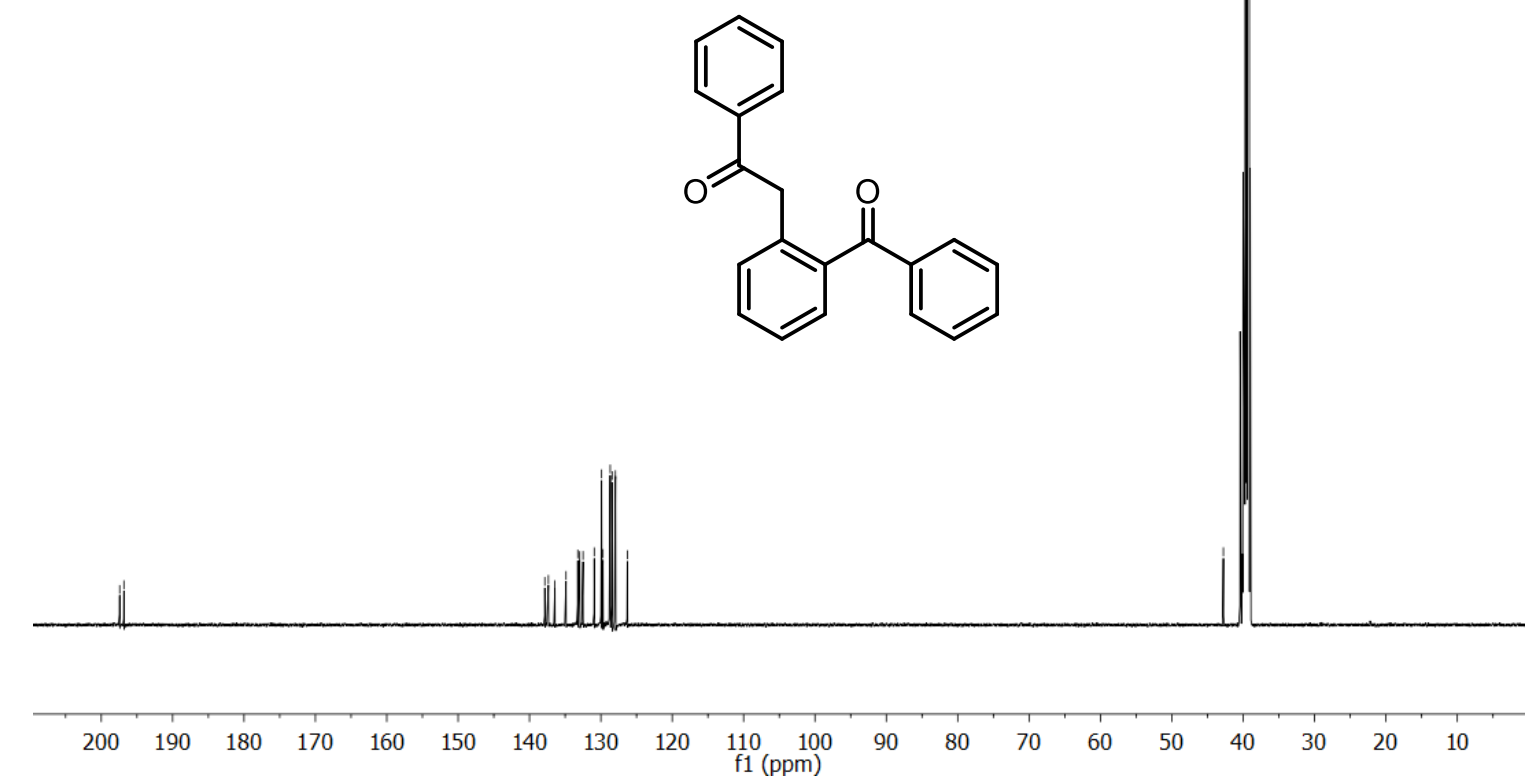

${ }^{1} \mathrm{H}$ NMR of compound 207, 3-phenyl-1H-isochromen-1-one (Homalicine), $300 \mathrm{MHz}^{\mathrm{CDCl}}{ }_{3}$, $300 \mathrm{~K}$

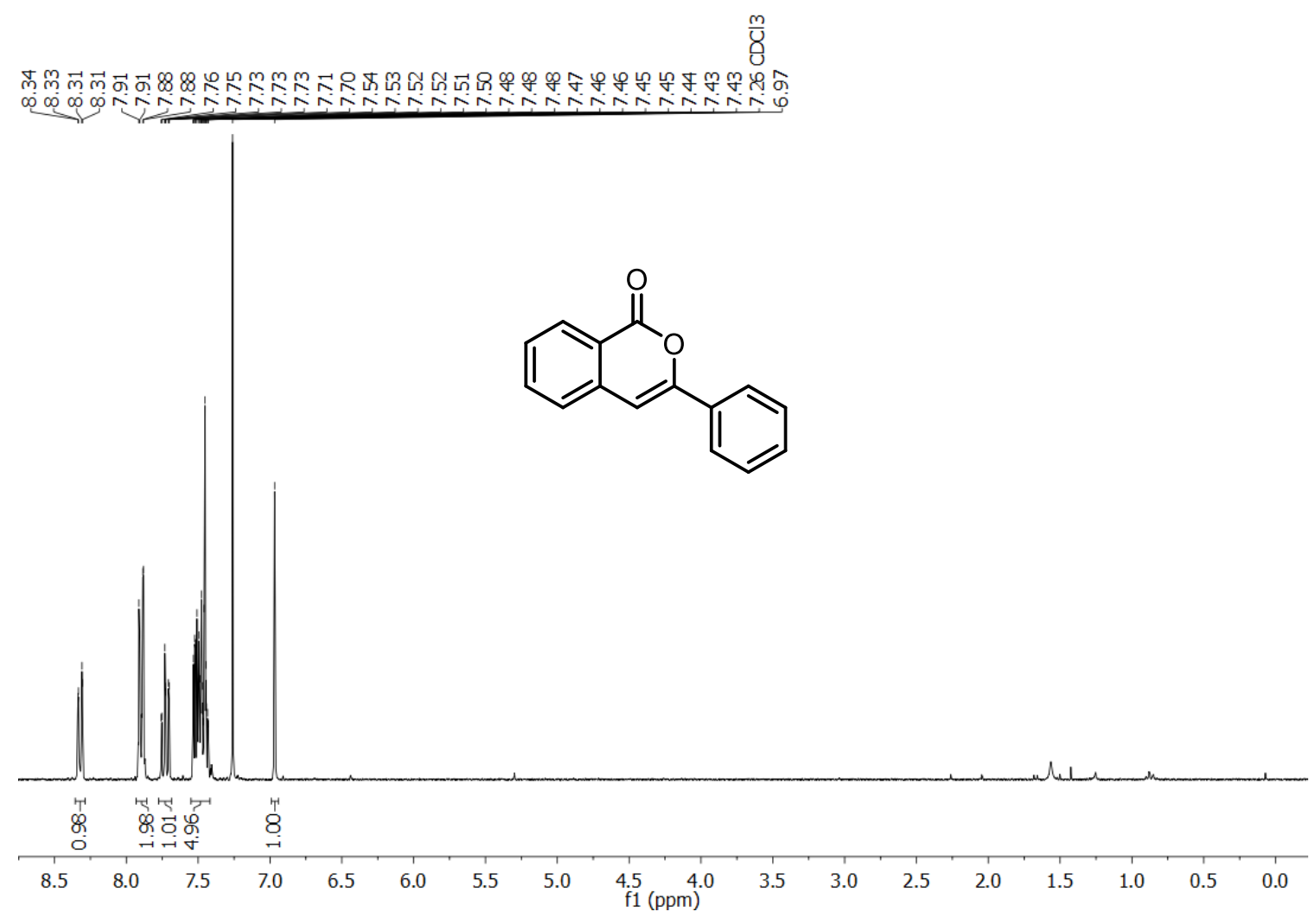


${ }^{13} \mathrm{C}$ NMR of compound 207, 3-phenyl-1H-isochromen-1-one (Homalicine), $126 \mathrm{MHz}, \mathrm{CDCl}_{3}$, $300 \mathrm{~K}$
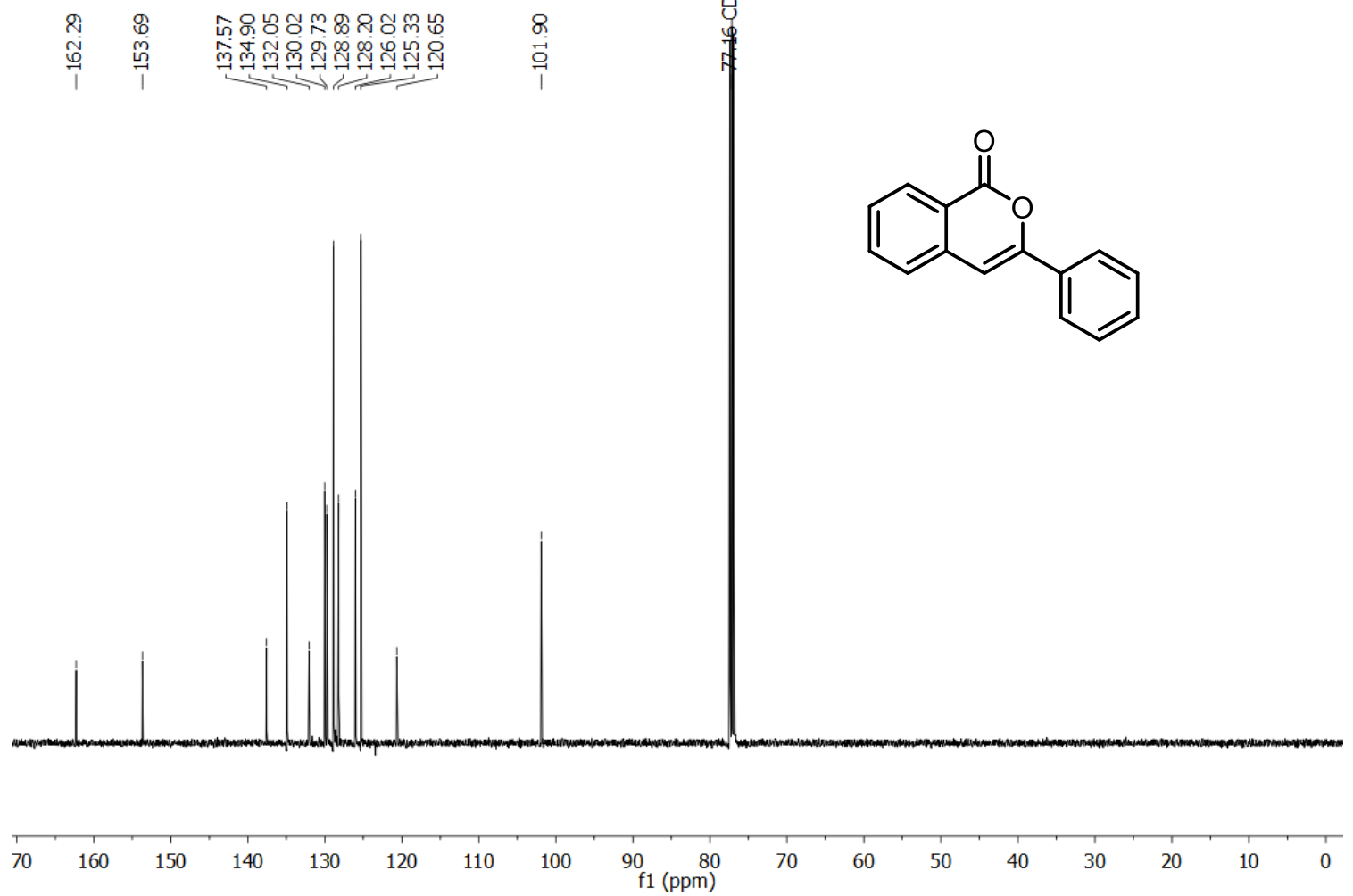

${ }^{1} \mathrm{H} \quad$ NMR of compound $\quad\left\{{ }^{13} \mathrm{C}\right\}-207, \quad 3-\left\{{ }^{13} \mathrm{C}\right\}$-3-phenyl-1H-isochromen-1-one $\quad\left(3-\left\{{ }^{13} \mathrm{C}\right\}\right.$ Homalicine), $300 \mathrm{MHz}, \mathrm{CDCl}_{3}, 298 \mathrm{~K}$<smiles>O=c1oc(-c2ccccc2)cc2ccccc12</smiles>

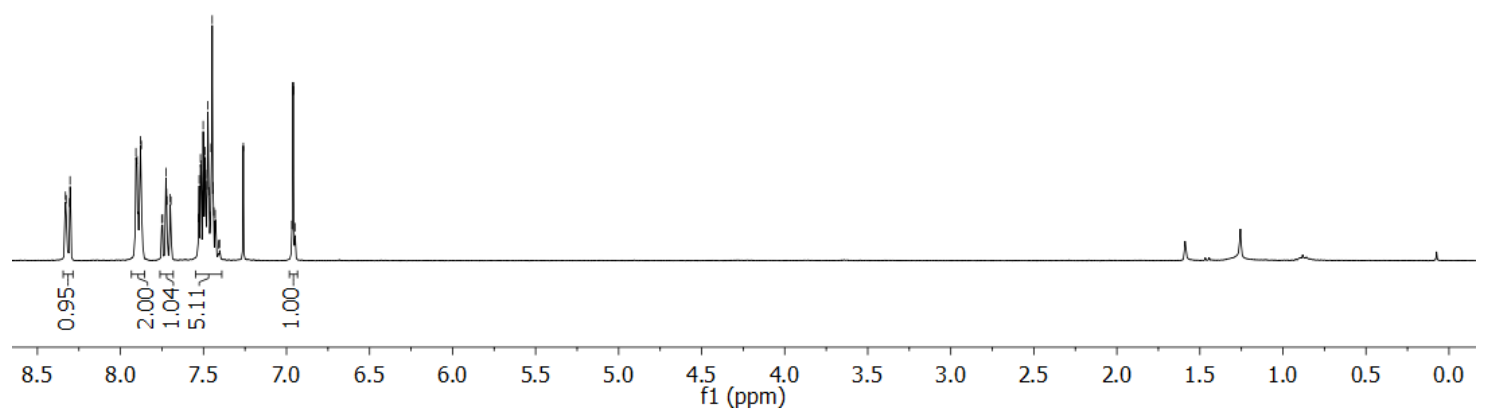

289 
${ }^{13} \mathrm{C}$ NMR of compound $\left\{{ }^{13} C\right\}-207,3-\left\{{ }^{13} C\right\}$-3-phenyl-1H-isochromen-1-one $\left(3-\left\{{ }^{13} C\right\}\right.$ -

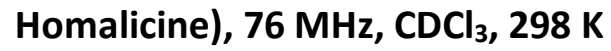

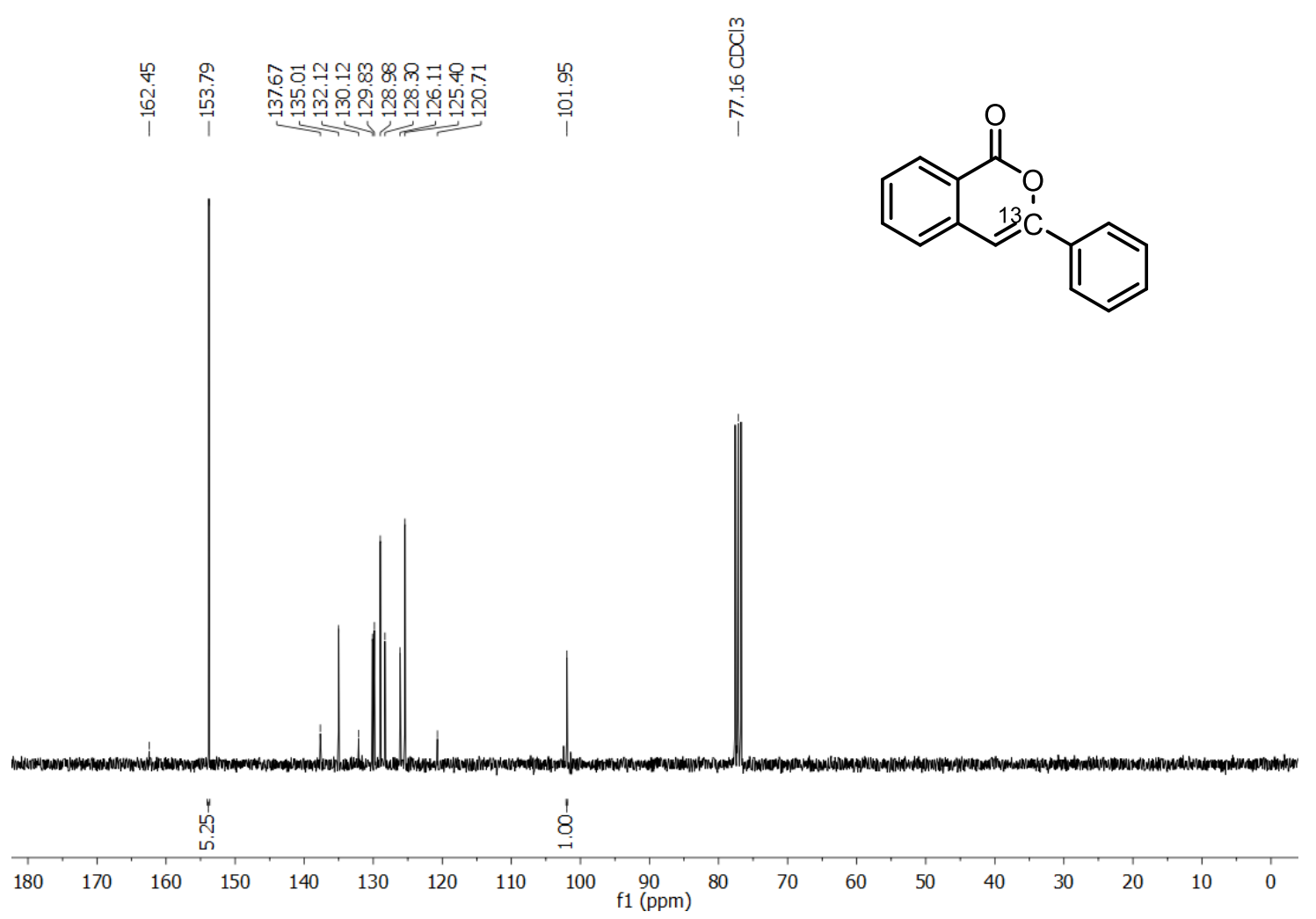

${ }^{1} \mathrm{H}$ NMR of compound 208, 6-methyl-3-phenyl-1H-isochromen-1-one, $400 \mathrm{MHz}, \mathrm{CDCl}_{3}, 299 \mathrm{~K}$
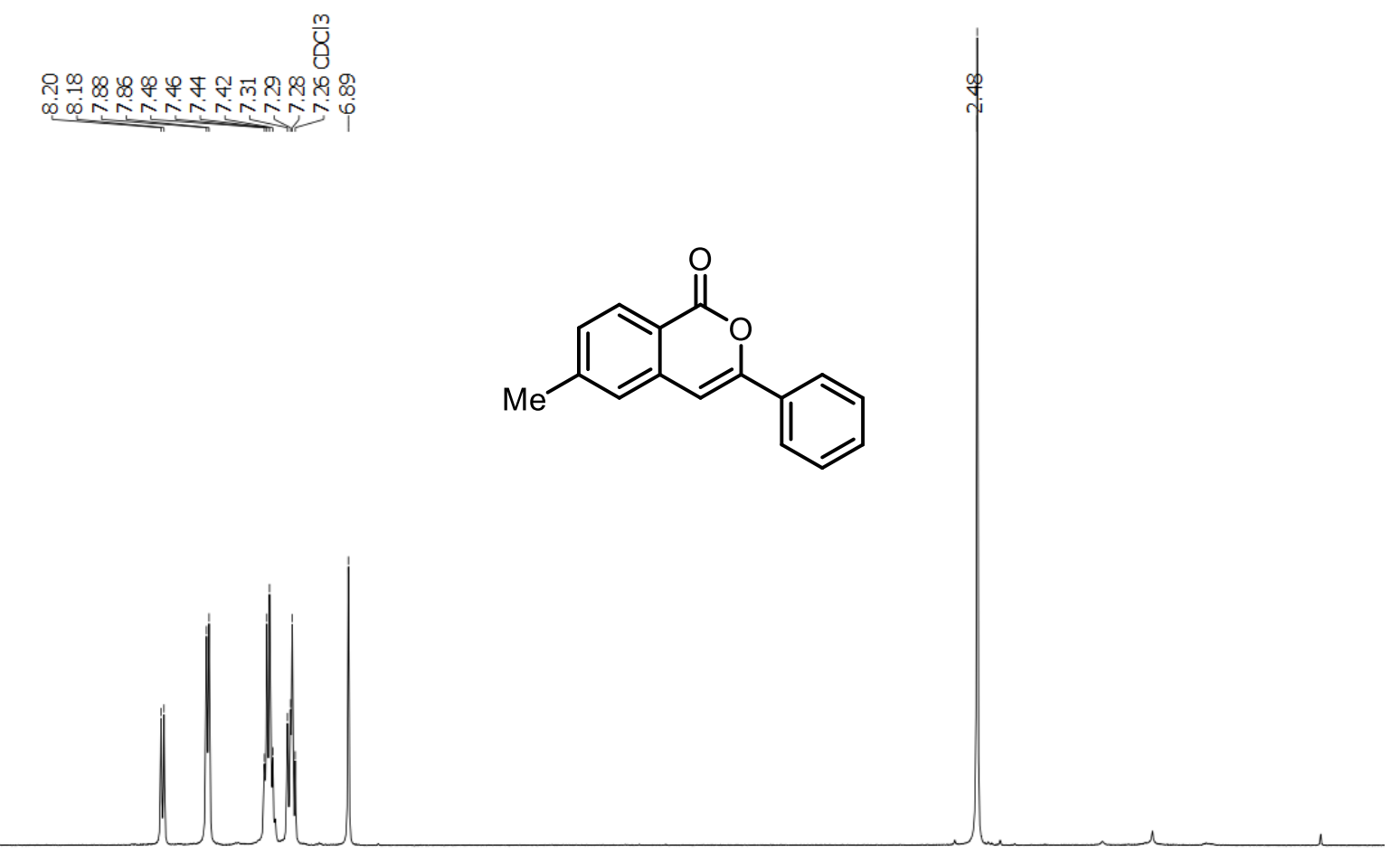

$9.0 \quad 8.5$

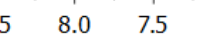

6.5

$\begin{array}{lll}6.0 & 5.5 & 5.0 \\ & & \begin{array}{c}4.5 \\ \mathrm{f} 1(\mathrm{ppm})\end{array}\end{array}$ 
${ }^{13} \mathrm{C}$ NMR of compound 208, 6-methyl-3-phenyl-1H-isochromen-1-one, $300 \mathrm{MHz}, \mathrm{CDCl}_{3}, 300 \mathrm{~K}$

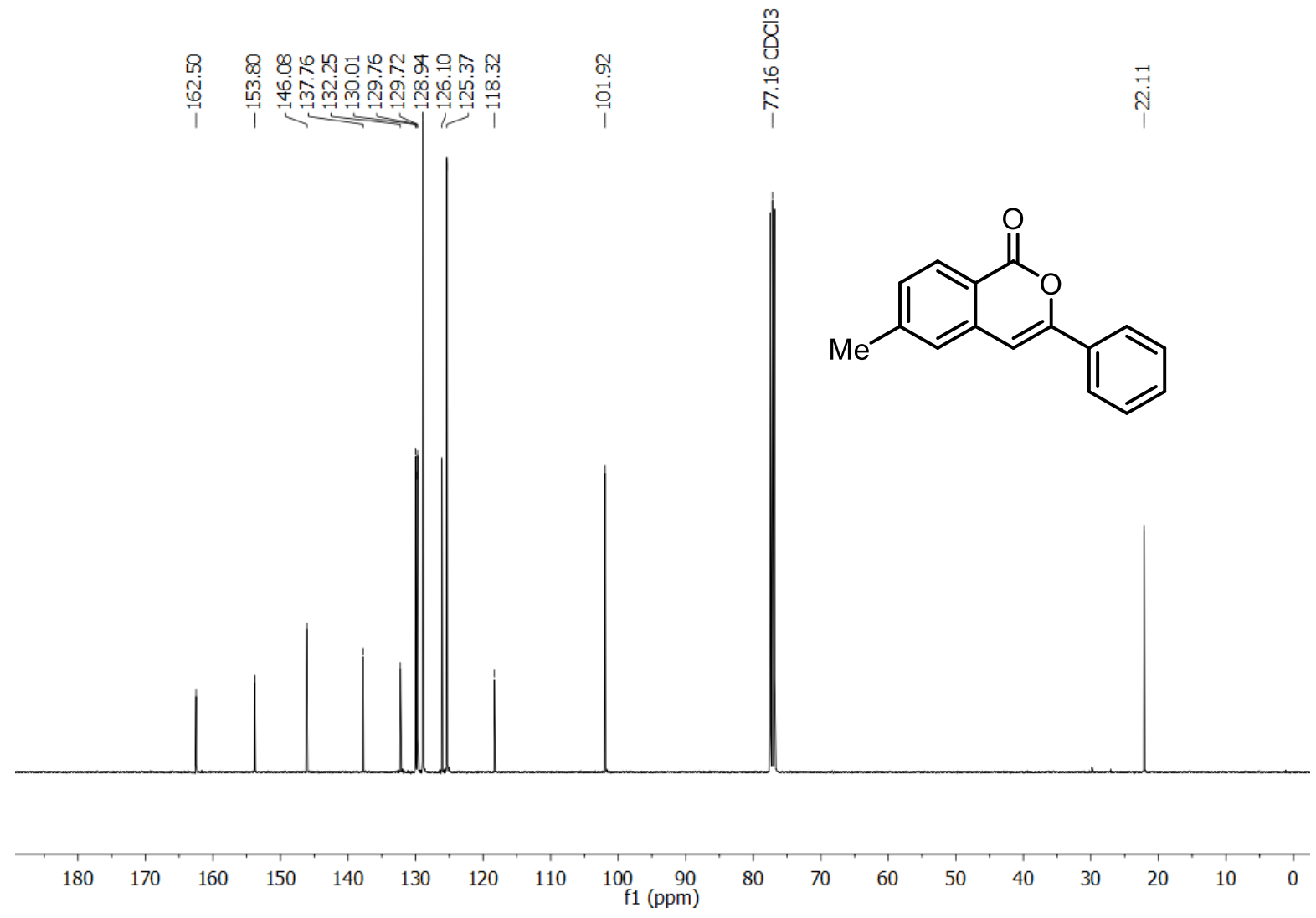

${ }^{1} \mathrm{H}$ NMR of compound 209, 6-methoxy-3-phenyl-1H-isochromen-1-one, $400 \mathrm{MHz}, \mathrm{CDCl}_{3}$, $298 \mathrm{~K}$

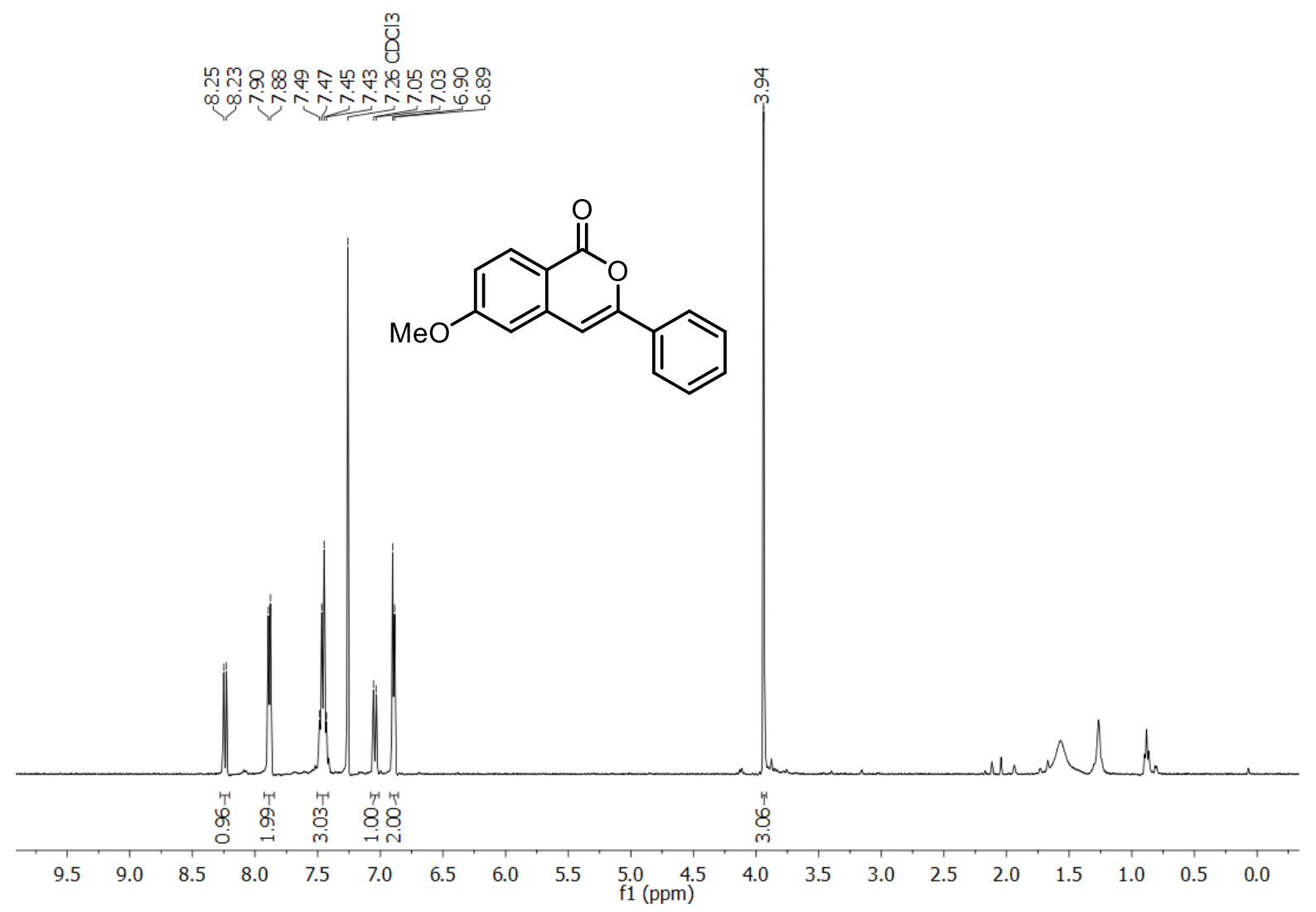


${ }^{13} \mathrm{C}$ NMR of compound 209, 6-methoxy-3-phenyl-1H-isochromen-1-one, $101 \mathrm{MHz}, \mathrm{CDCl}_{3}, 298$ $\mathrm{K}$
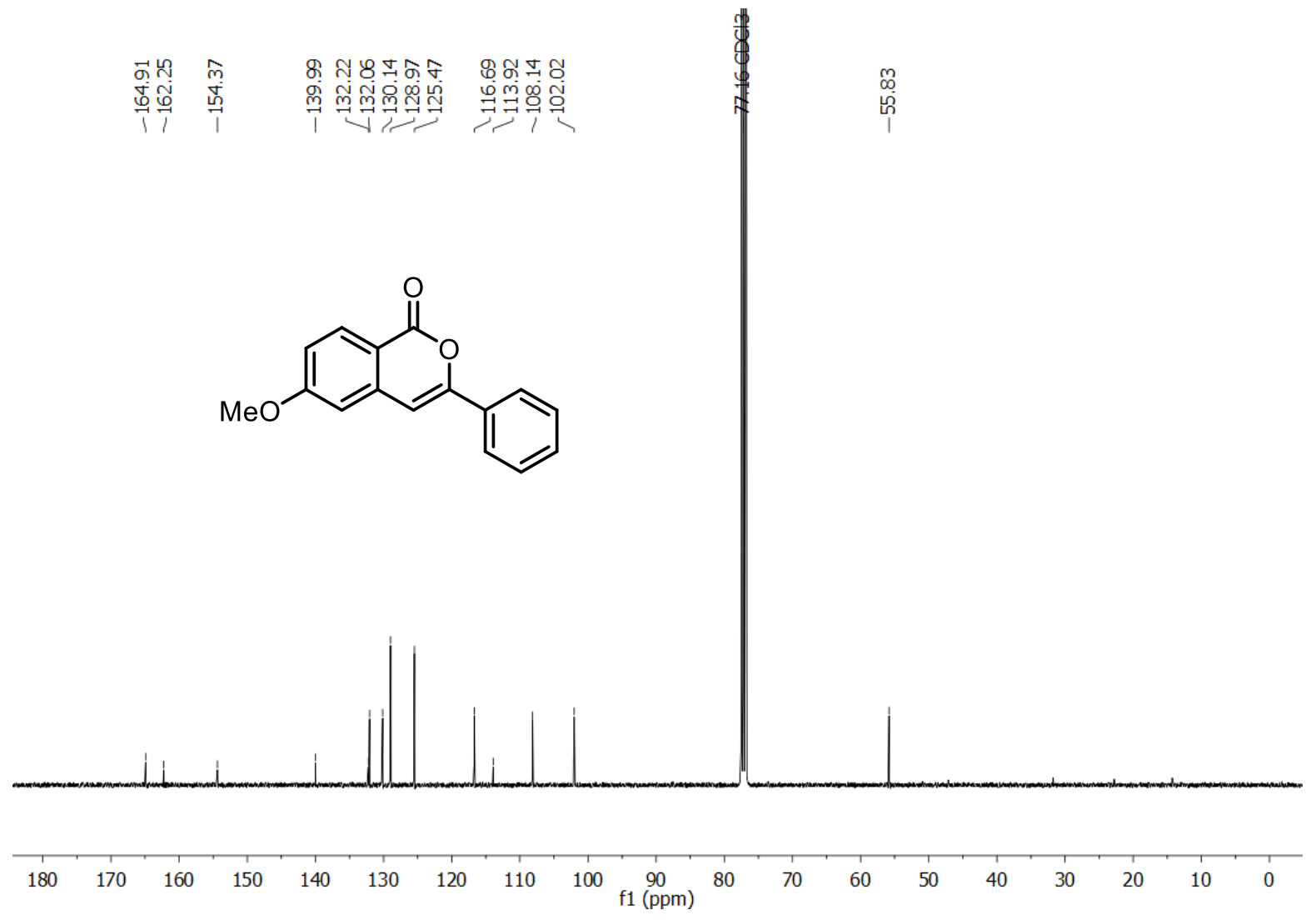

${ }^{1} \mathrm{H}$ NMR of compound 210, 6-fluoro-3-phenyl-1H-isochromen-1-one, $400 \mathrm{MHz}, \mathrm{CDCl}_{3}, 299 \mathrm{~K}$

$\frac{m}{8}$

品

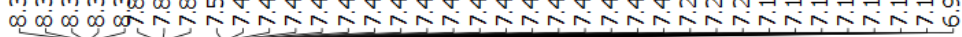

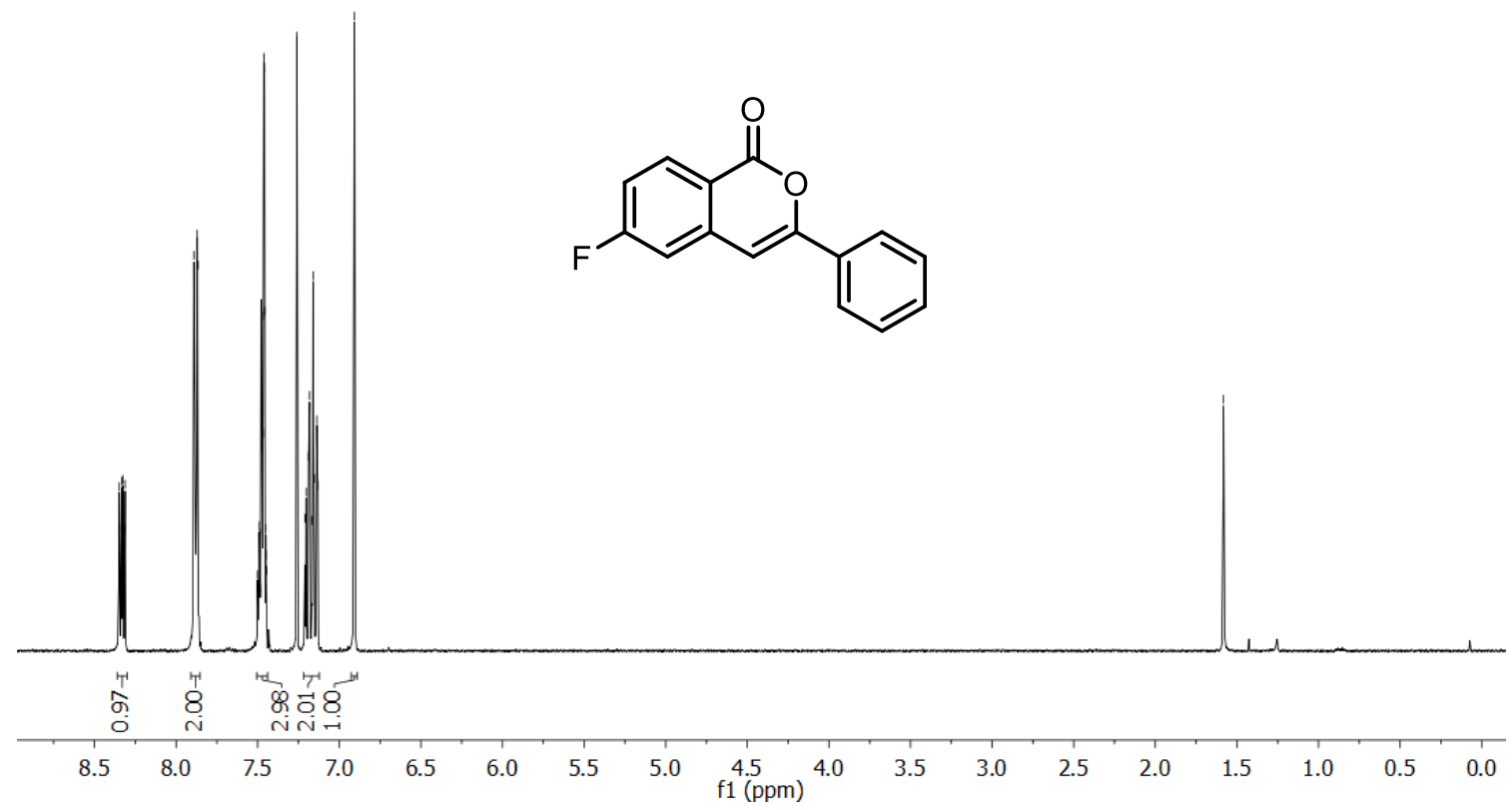


${ }^{13} \mathrm{C}$ NMR of compound 210, 6-fluoro-3-phenyl-1H-isochromen-1-one, $126 \mathrm{MHz}, \mathrm{CDCl}_{3}, 300$ K

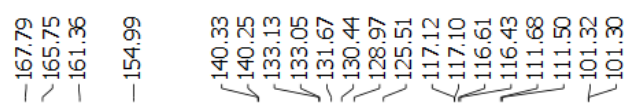

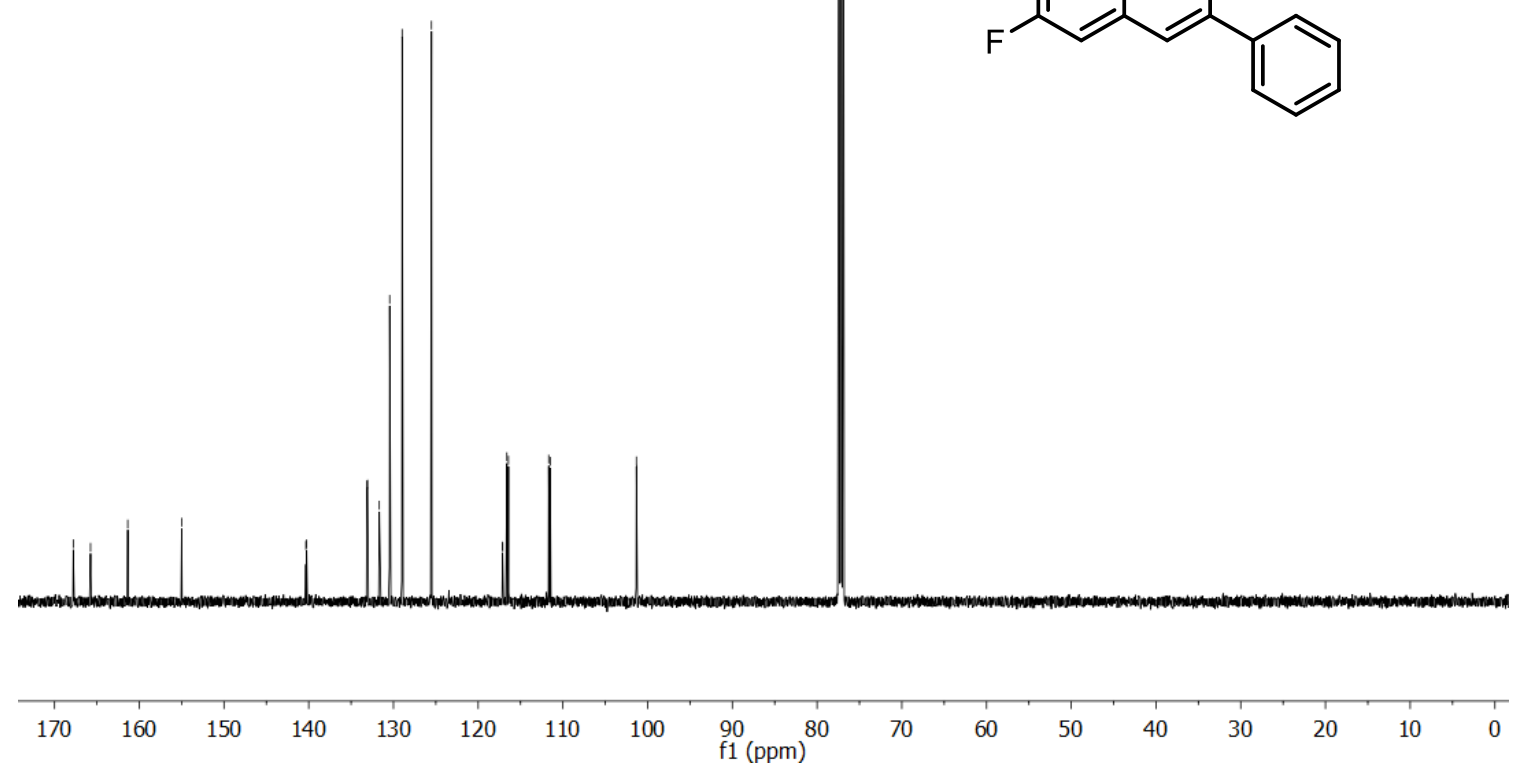

${ }^{19} \mathrm{~F}$ NMR of compound 210, 6-fluoro-3-phenyl-1H-isochromen-1-one, $377 \mathrm{MHz}, \mathrm{CDCl}_{3}, 299 \mathrm{~K}$<smiles>O=c1oc(-c2ccccc2)cc2cc(F)ccc12</smiles> 
${ }^{1} \mathrm{H}$ NMR of compound 211, 6-chloro-3-phenyl-1H-isochromen-1-one, $300 \mathrm{MHz}, \mathrm{CDCl}_{3}, 298 \mathrm{~K}$

นัก

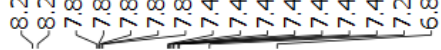<smiles>O=c1oc(-c2ccccc2)cc2cc(Cl)ccc12</smiles>

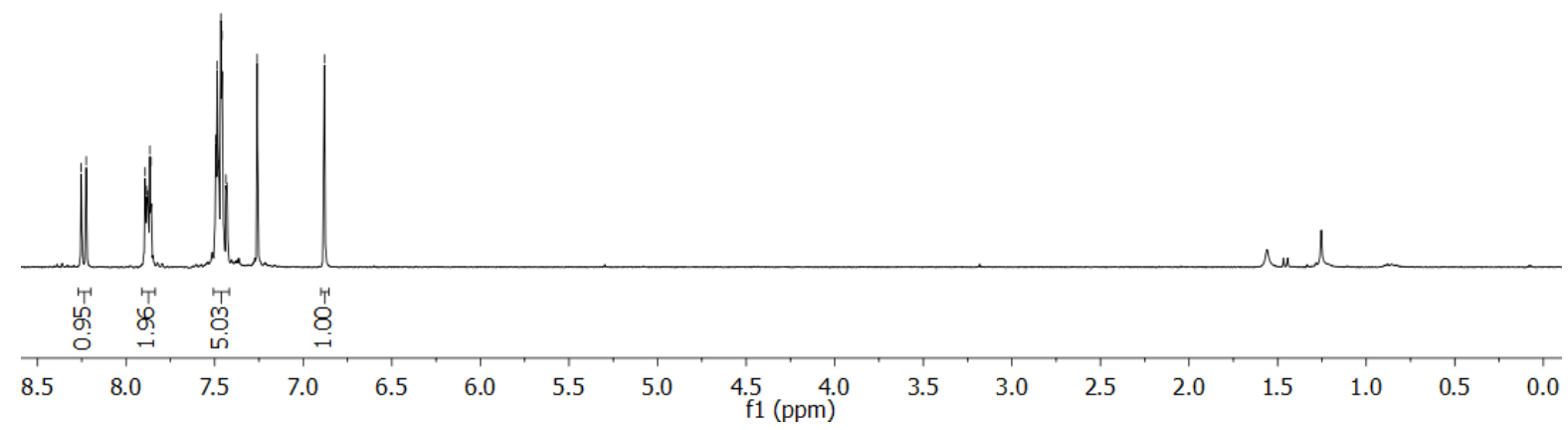

${ }^{13} \mathrm{C}$ NMR of compound 211, 6-chloro-3-phenyl-1H-isochromen-1-one, $126 \mathrm{MHz}, \mathrm{CDCl}_{3}, 298$ $\mathrm{K}$

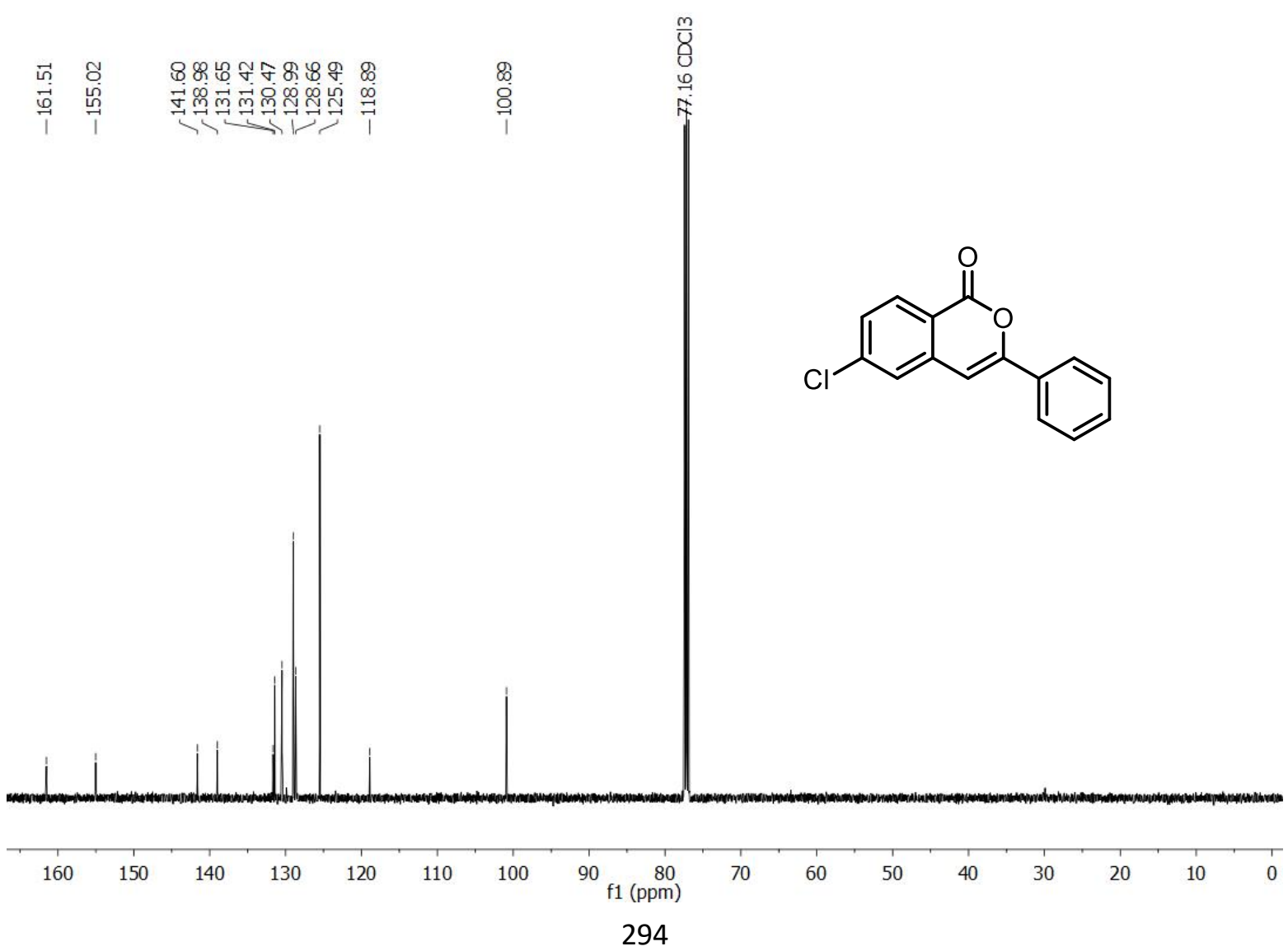


${ }^{1} \mathrm{H}$ NMR of compound 212, 6-bromo-3-phenyl-1H-isochromen-1-one, $400 \mathrm{MHz}, \mathrm{CDCl}_{3}, 298$ K

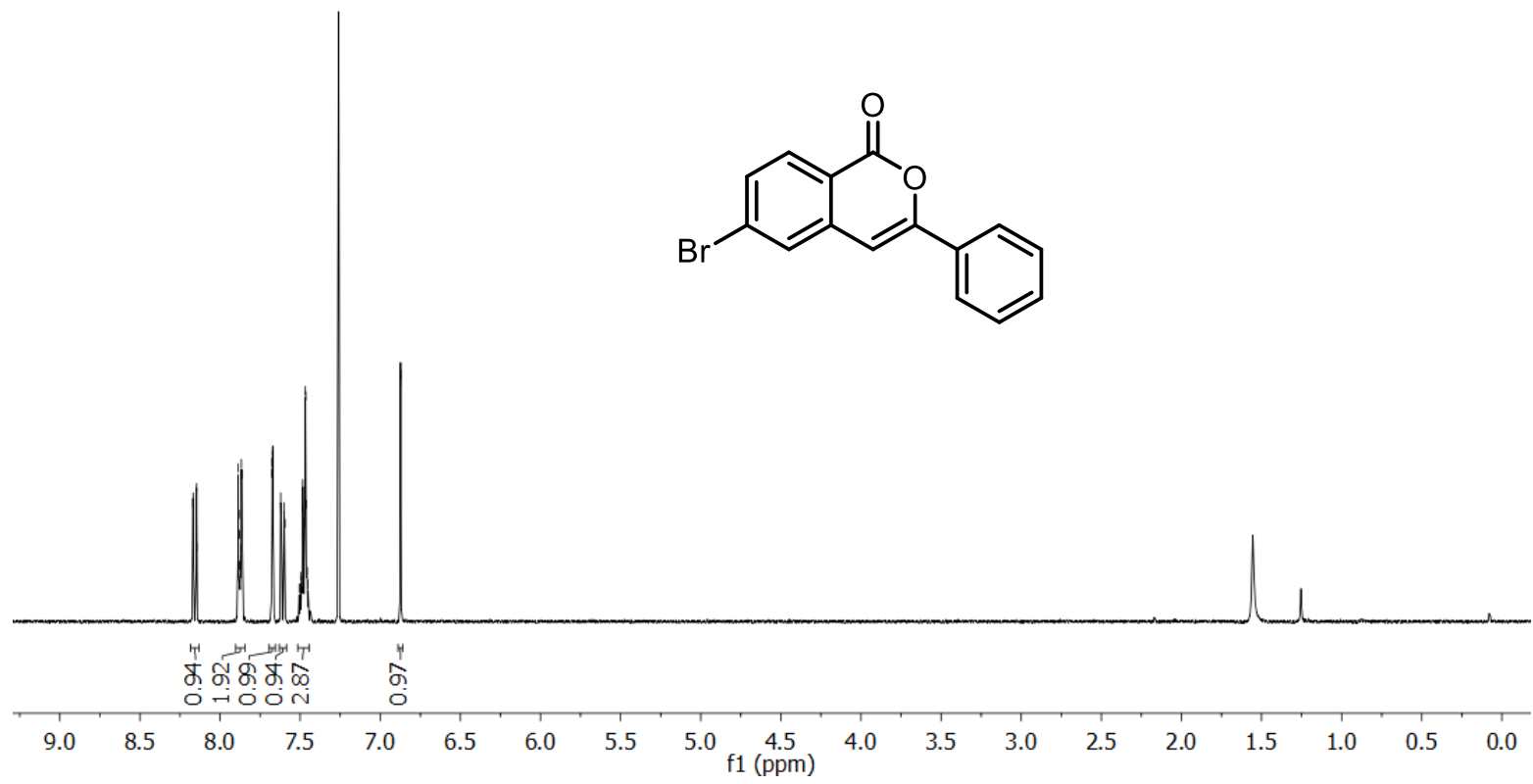

${ }^{13} \mathrm{C}$ NMR of compound 212, 6-bromo-3-phenyl-1H-isochromen-1-one, $101 \mathrm{MHz}, \mathrm{CDCl}_{3}, 299$ K

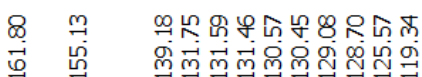

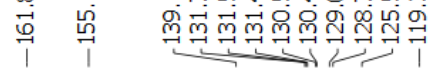
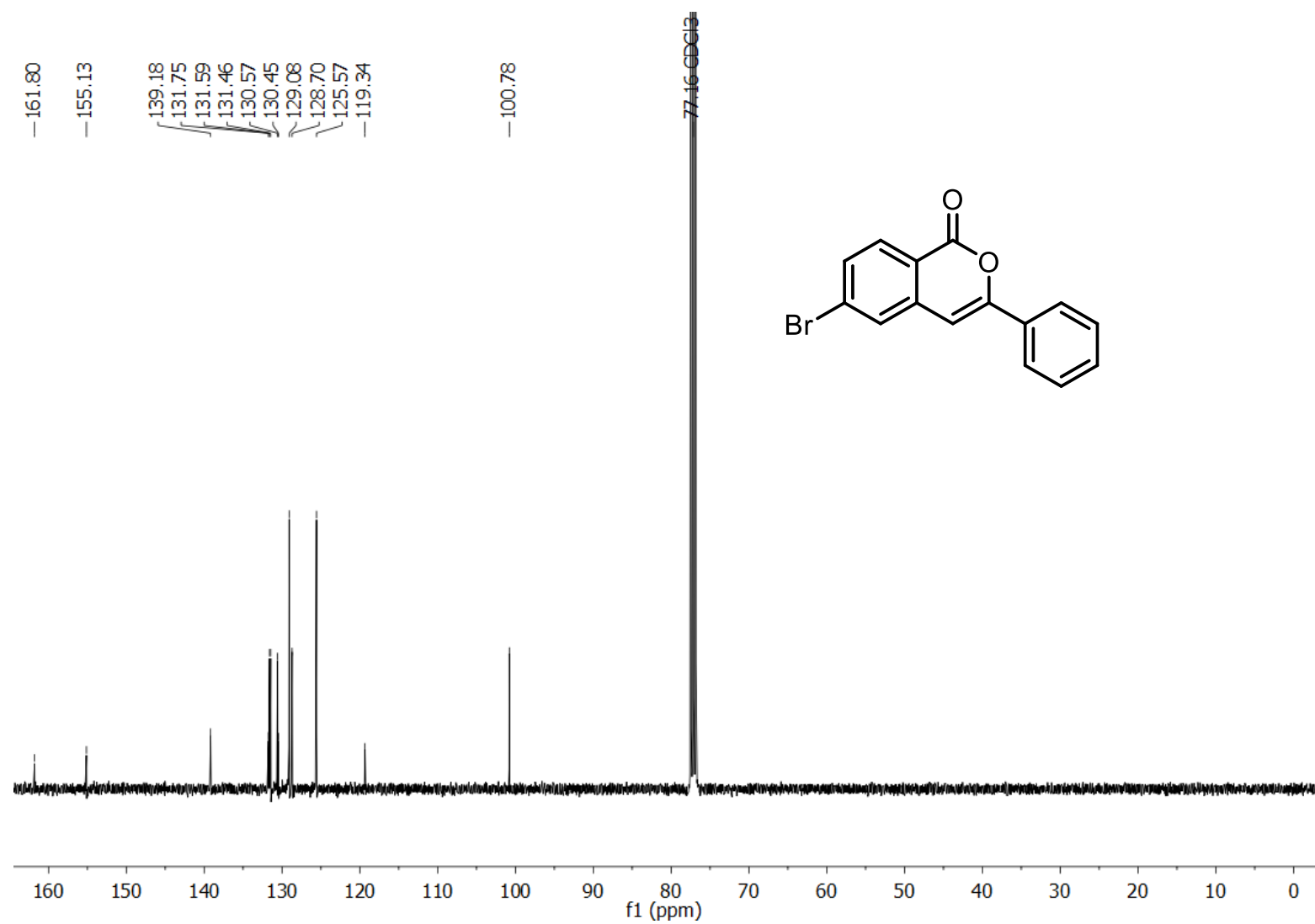
${ }^{1} \mathrm{H}$ NMR of compound 213, 6-iodo-3-phenyl-1H-isochromen-1-one, $300 \mathrm{MHz}, \mathrm{CDCl}_{3}, 298 \mathrm{~K}$

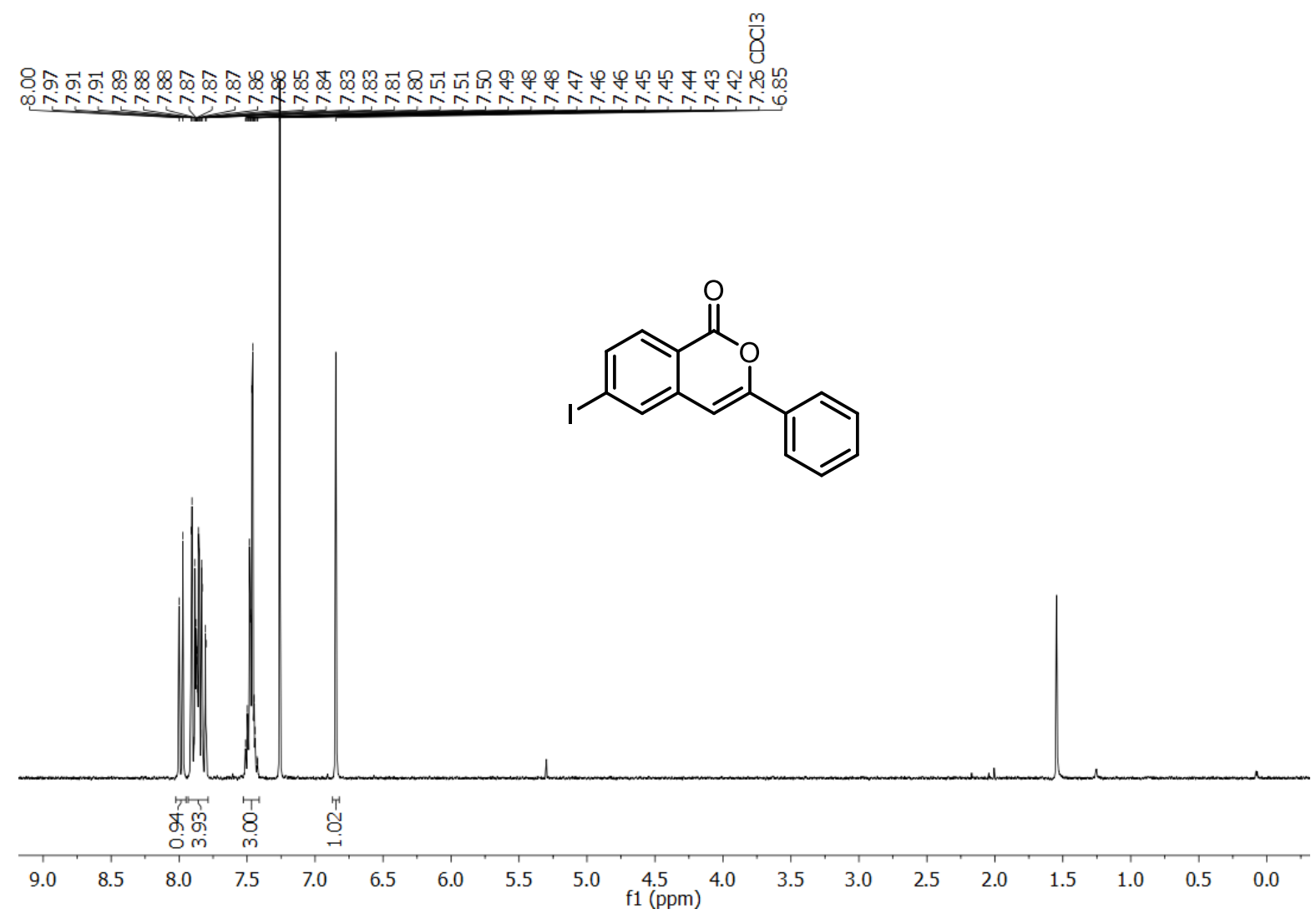

${ }^{13} \mathrm{C}$ NMR of compound 213, 6-iodo-3-phenyl-1H-isochromen-1-one, $126 \mathrm{MHz}, \mathrm{CDCl}_{3}, 298 \mathrm{~K}$

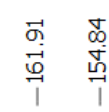

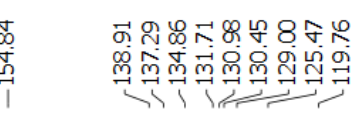

岕禺
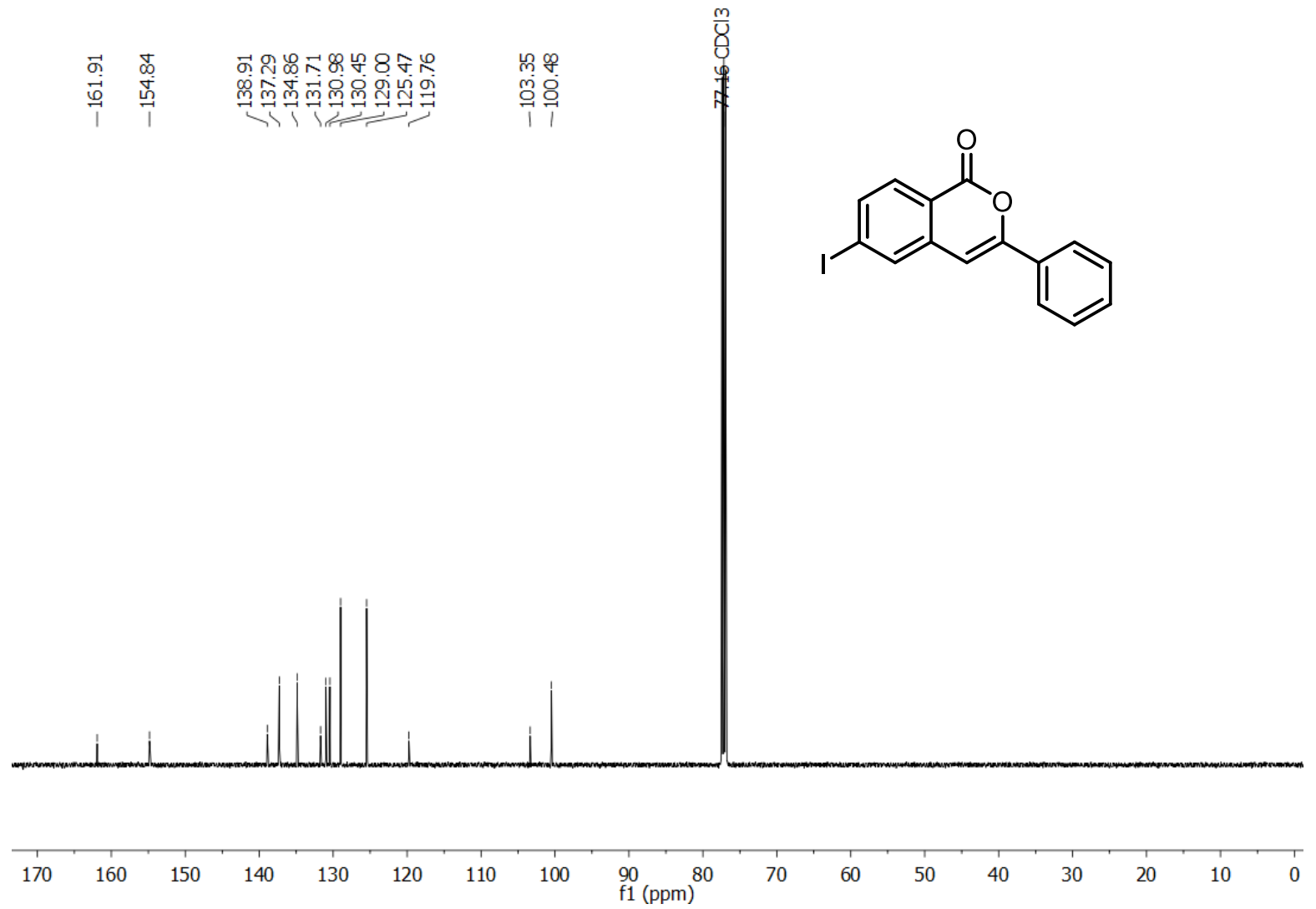

296 
${ }^{1} \mathrm{H}$ NMR of compound 214, 3-phenyl-6-(trifluoromethyl)-1H-isochromen-1-one, $300 \mathrm{MHz}$, $\mathrm{CDCl}_{3}, 300 \mathrm{~K}$

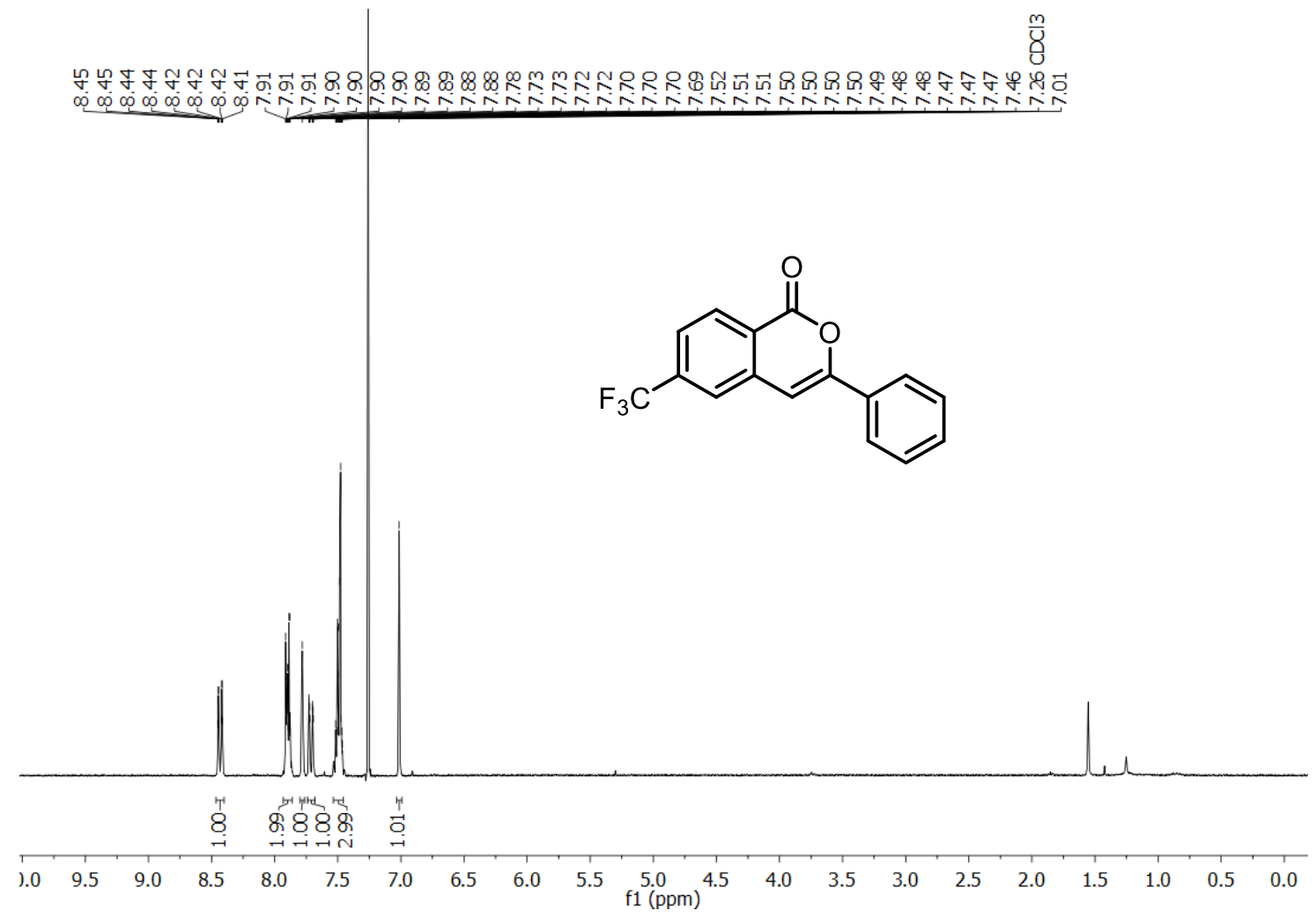

${ }^{13} \mathrm{C}$ NMR of compound 214, 3-phenyl-6-(trifluoromethyl)-1H-isochromen-1-one, $101 \mathrm{MHz}$, $\mathrm{CDCl}_{3}, 298 \mathrm{~K}$

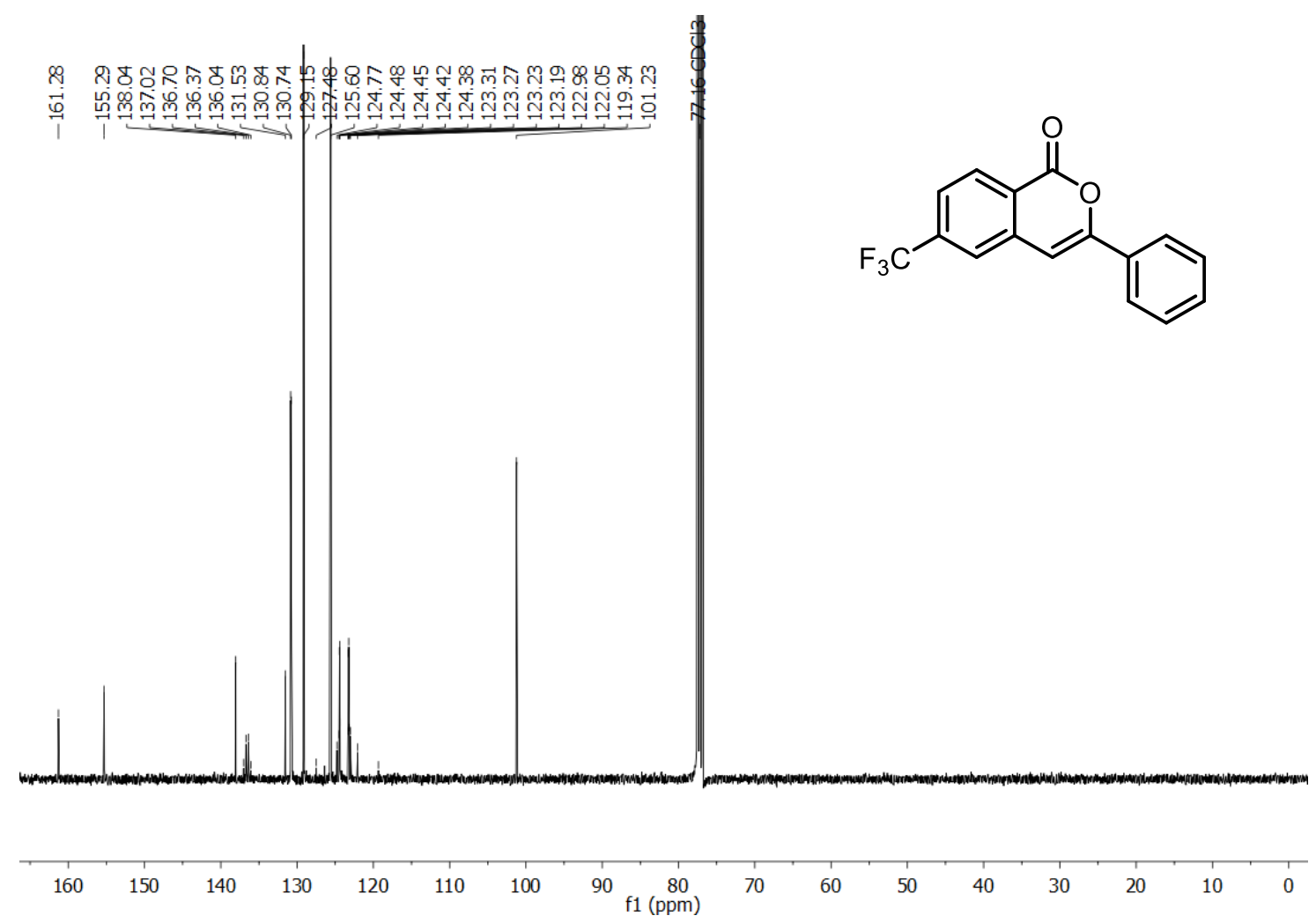


${ }^{19} \mathrm{~F}$ NMR of compound 214, 3-phenyl-6-(trifluoromethyl)-1H-isochromen-1-one, $282 \mathrm{MHz}$, $\mathrm{CDCl}_{3}, 300 \mathrm{~K}$<smiles>O=c1oc(-c2ccccc2)cc2cc(C(F)(F)F)ccc12</smiles>

${ }^{1} \mathrm{H}$ NMR of compound 215, methyl 1-oxo-3-phenyl-1H-isochromene-6-carboxylate, 400 $\mathrm{MHz}, \mathrm{CDCl}_{3}, 299 \mathrm{~K}$

씼

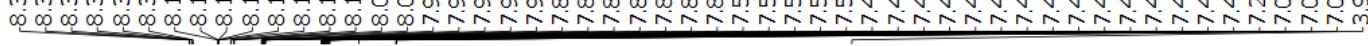<smiles>COC(=O)c1ccc2c(=O)oc(-c3ccccc3)cc2c1</smiles>

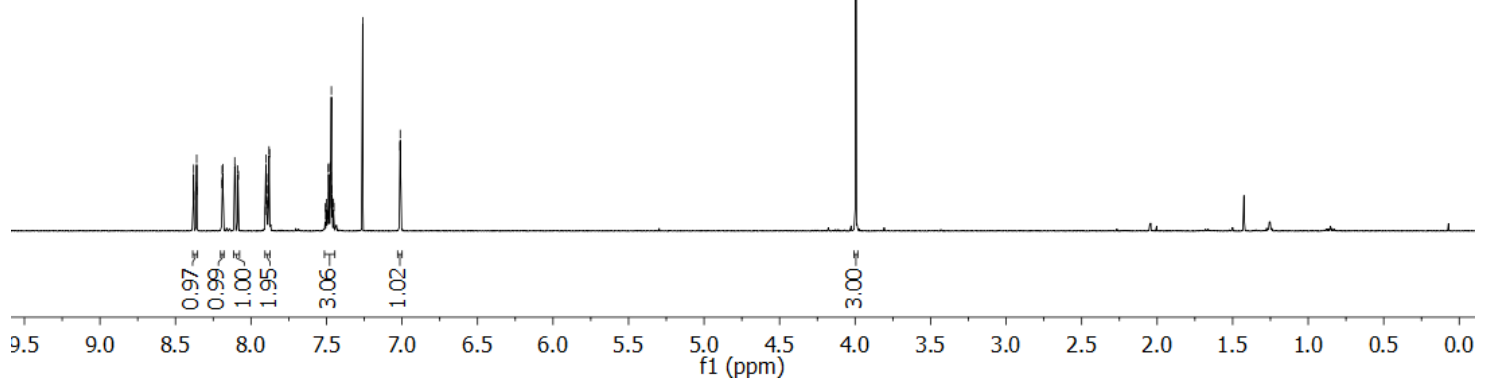

298 
${ }^{13} \mathrm{C}$ NMR of compound 215, methyl 1-oxo-3-phenyl-1H-isochromene-6-carboxylate, 101 $\mathrm{MHz}, \mathrm{CDCl}_{3}, 300 \mathrm{~K}$

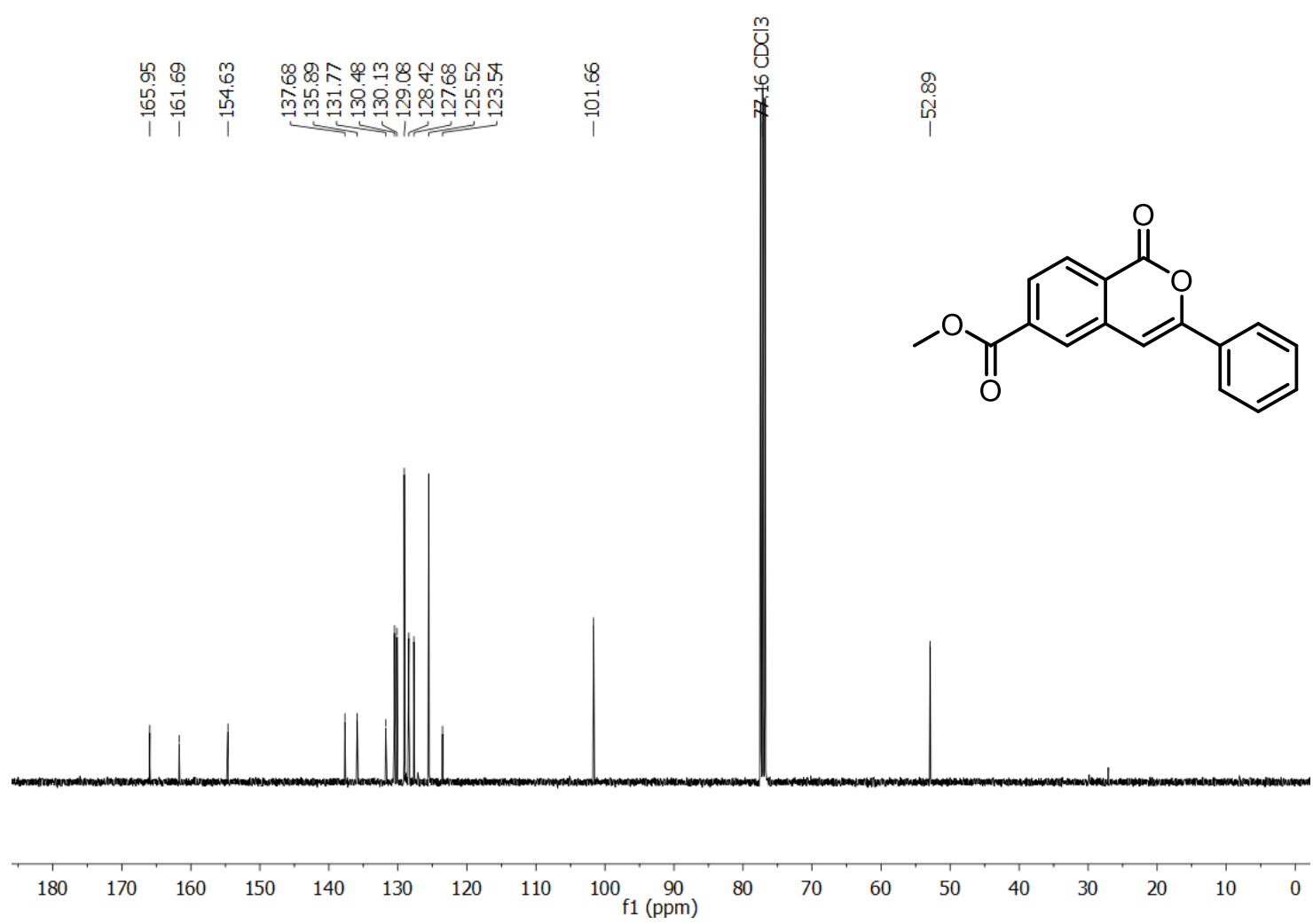

${ }^{1} \mathrm{H}$ NMR of compound 216, 8-phenyl-6H-[1,3]dioxolo[4,5-f] isochromen-6-one, $300 \mathrm{MHz}$, $\mathrm{CDCl}_{3}, 298 \mathrm{~K}$

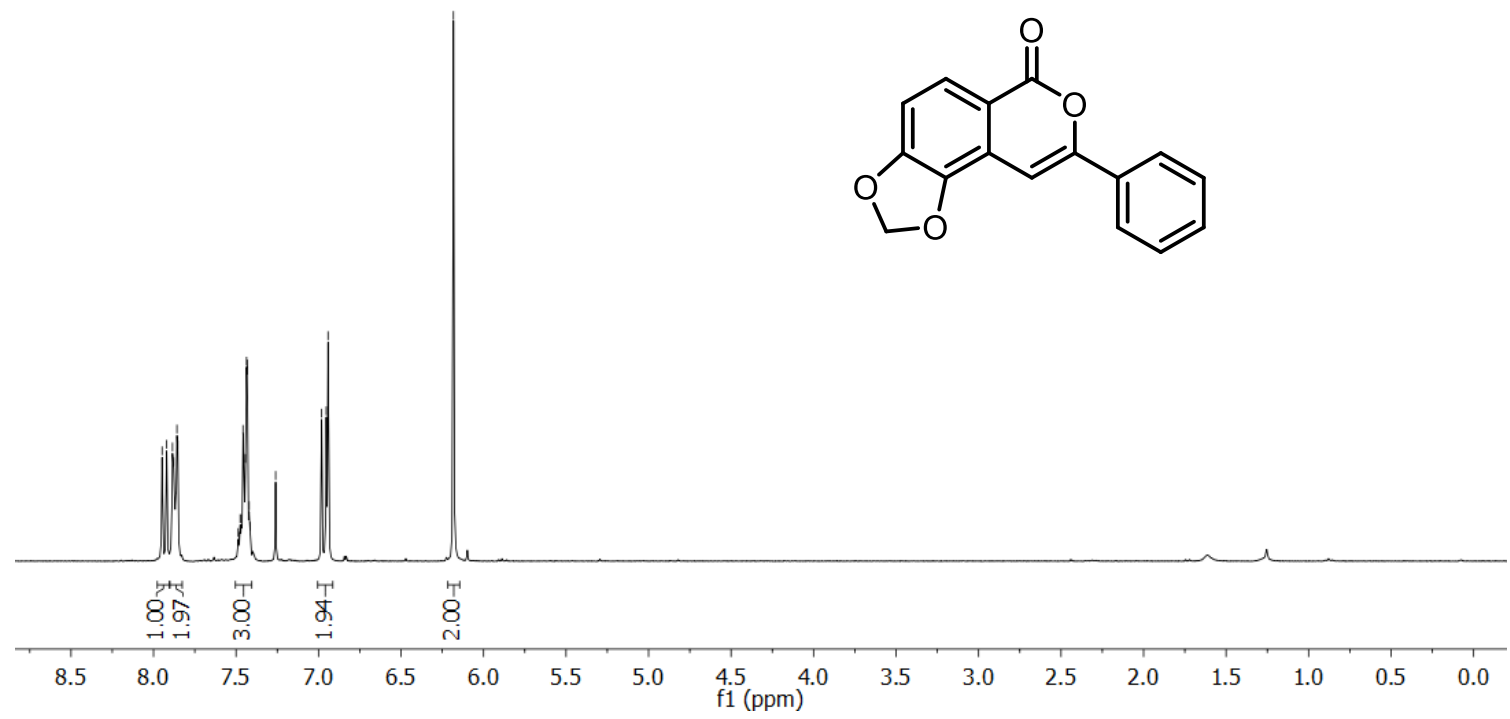


${ }^{13} \mathrm{C}$ NMR of compound 216, 8-phenyl-6H-[1,3]dioxolo[4,5-f] isochromen-6-one, $100 \mathrm{MHz}$, $\mathrm{CDCl}_{3}, 298 \mathrm{~K}$
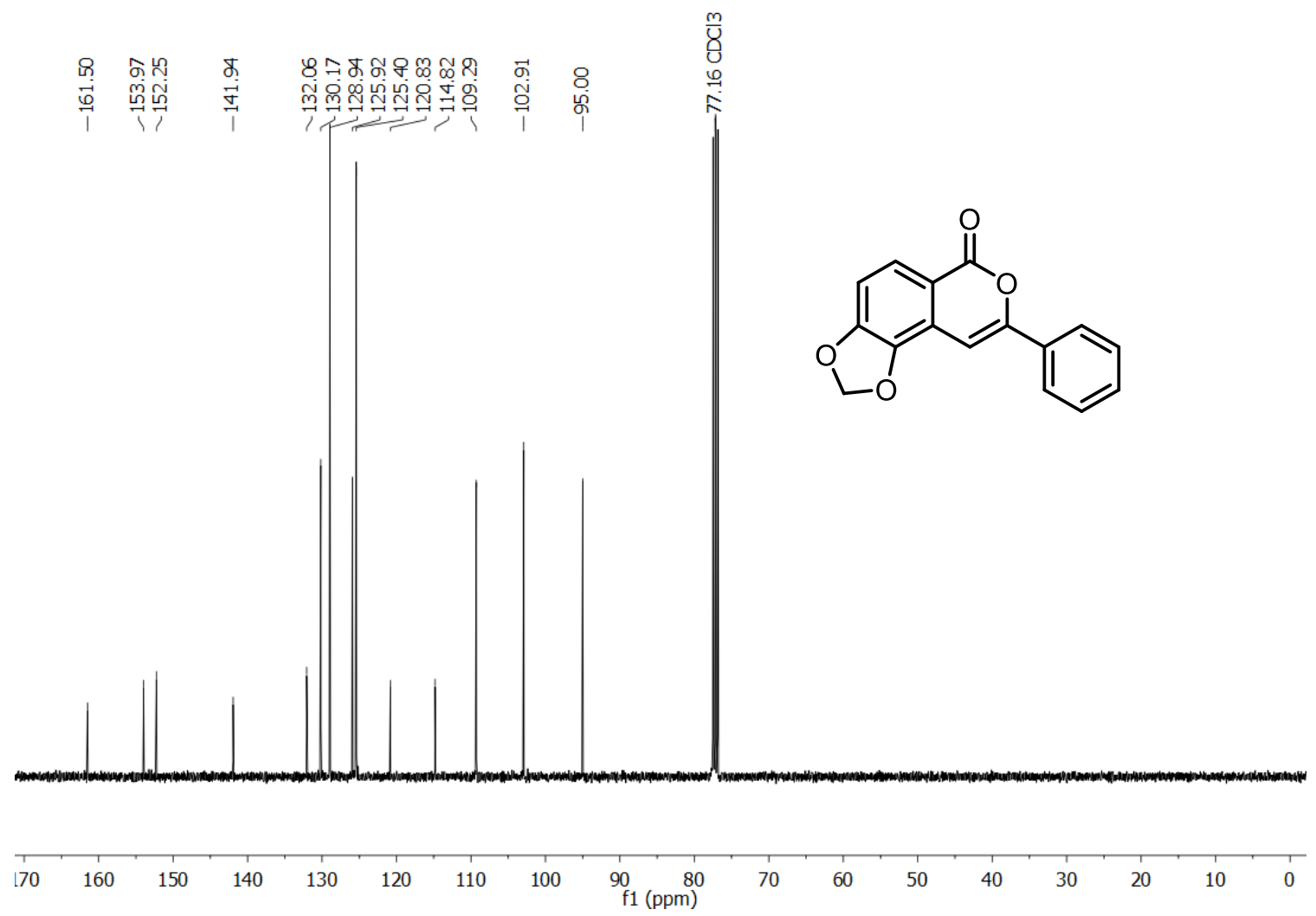

${ }^{1} \mathrm{H}$ NMR of compound 217, 7-methyl-3-phenyl-1H-isochromen-1-one, $300 \mathrm{MHz}, \mathrm{CDCl}_{3}, 298$ K<smiles>Cc1ccc2cc(-c3ccccc3)oc(=O)c2c1</smiles>

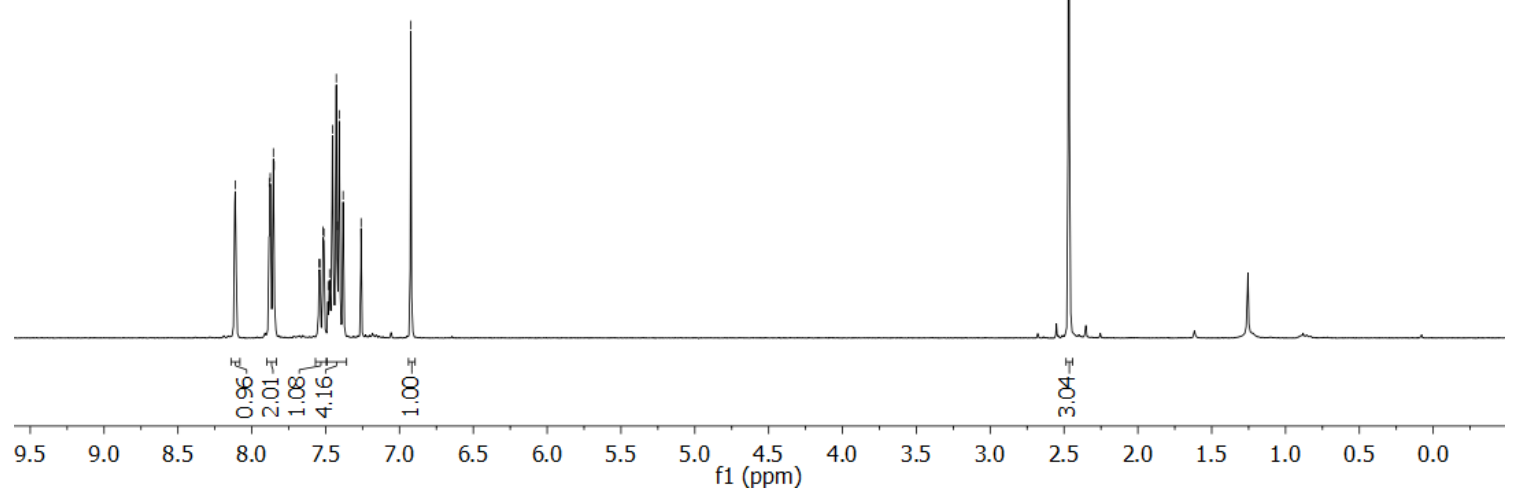

300 
${ }^{13} \mathrm{C}$ NMR of compound 217, 7-methyl-3-phenyl-1H-isochromen-1-one, $300 \mathrm{MHz}, \mathrm{CDCl}_{3}, 298$ K

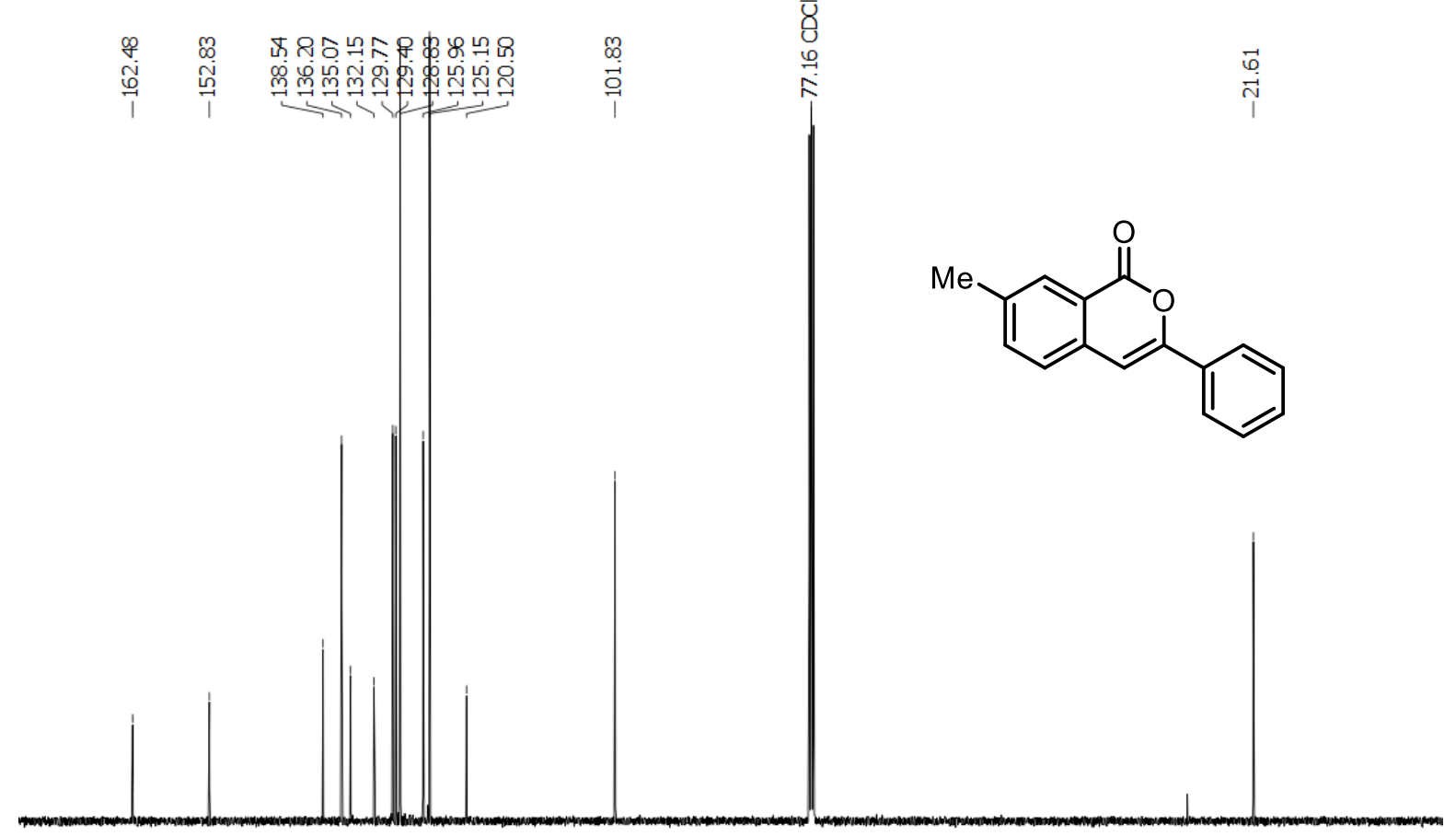

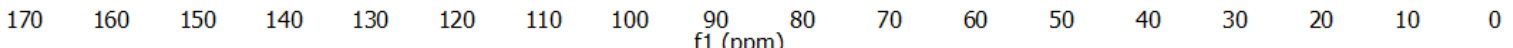

${ }^{1} \mathrm{H}$ NMR of compound 218a, 7-methoxy-3-phenyl-1H-isochromen-1-one, $500 \mathrm{MHz}, \mathrm{CD}_{3} \mathrm{CN}$, $298 \mathrm{~K}$

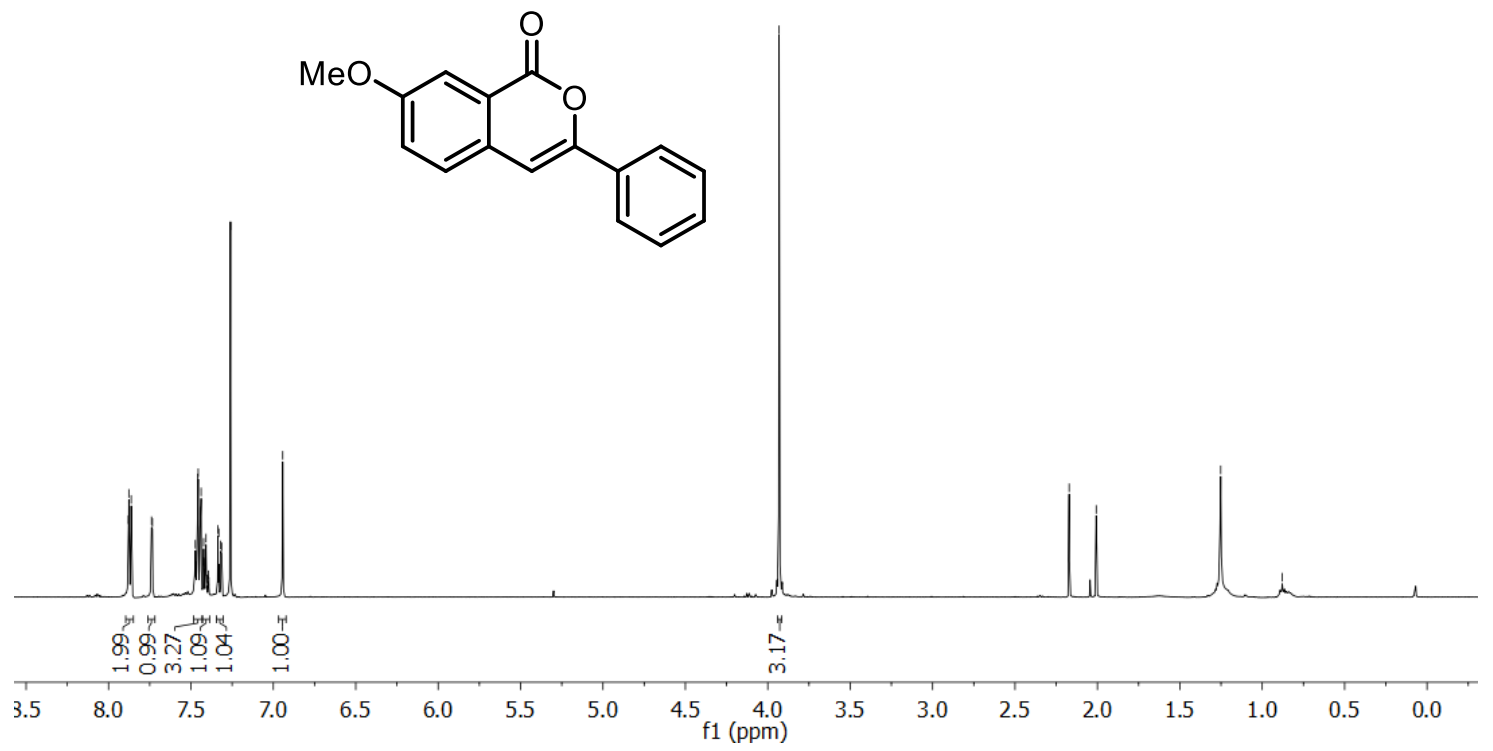


${ }^{13} \mathrm{C}$ NMR of compound 218a, 7-methoxy-3-phenyl-1H-isochromen-1-one, $126 \mathrm{MHz}, \mathrm{CD}_{3} \mathrm{CN}$, $298 \mathrm{~K}$

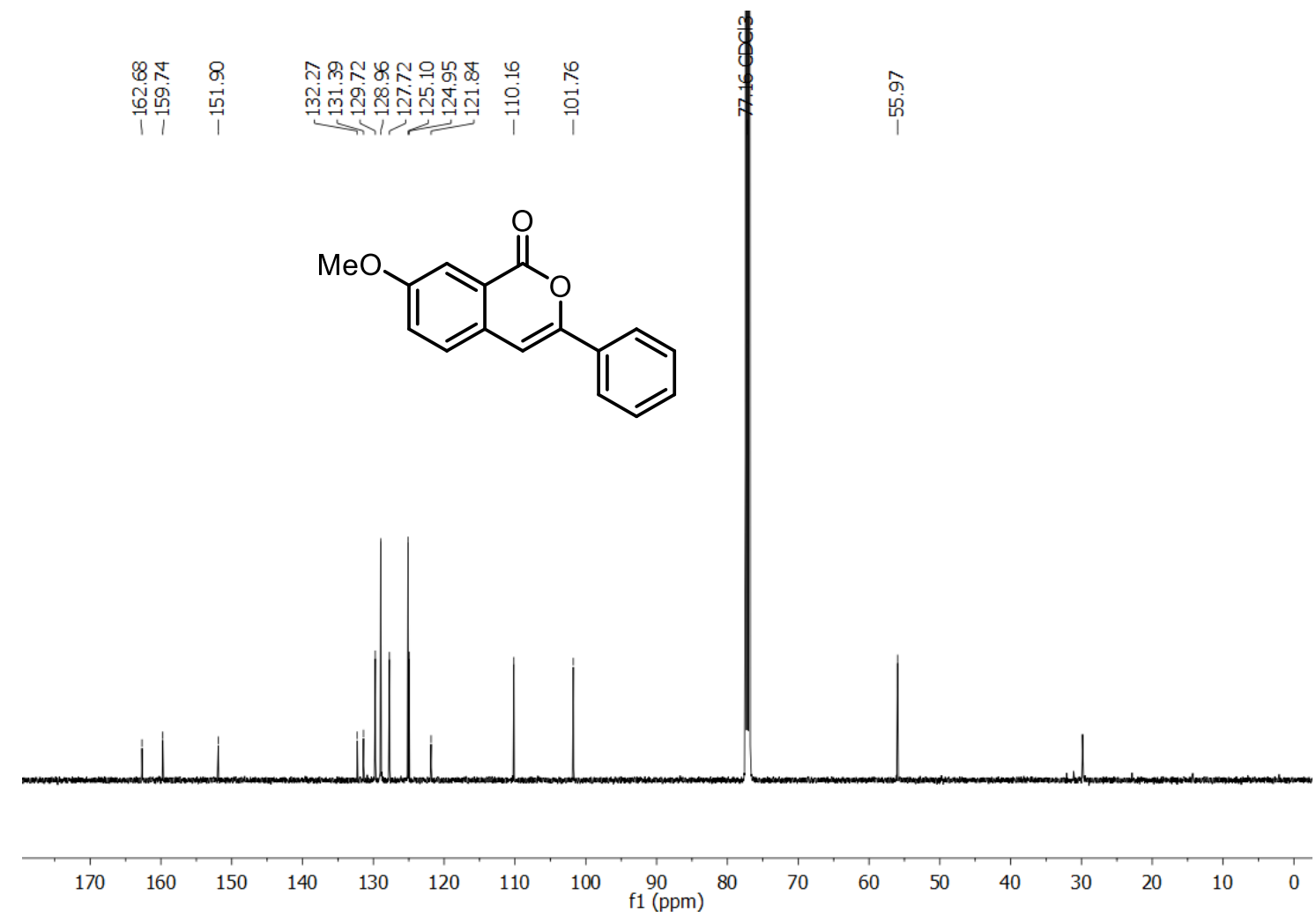

${ }^{1} \mathrm{H}$ NMR of compound 218b, 5-methoxy-3-phenyl-1H-isochromen-1-one, $500 \mathrm{MHz}^{\mathrm{CDCl}}{ }_{3}$, $298 \mathrm{~K}$
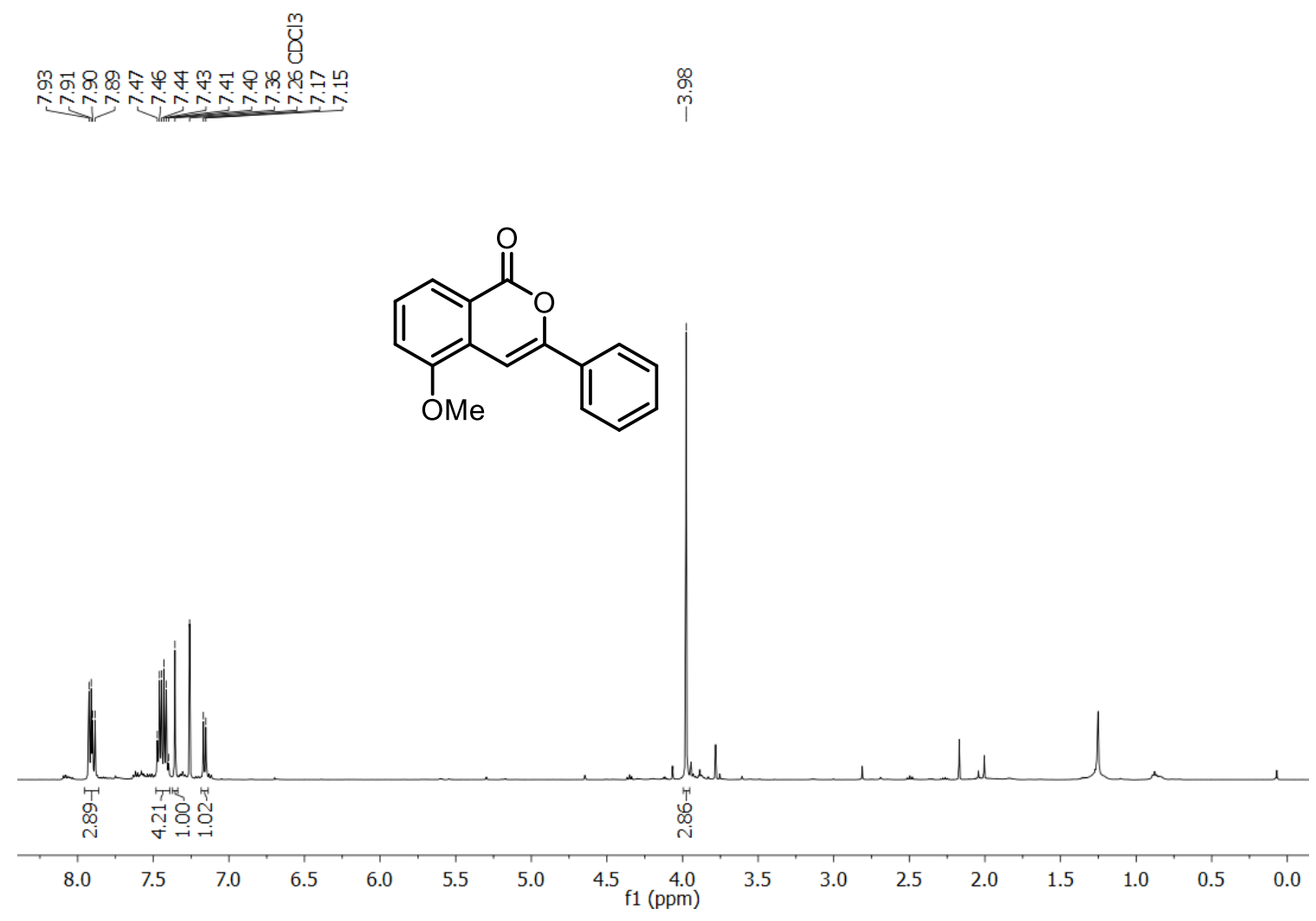
${ }^{13} \mathrm{C}$ NMR of compound 218b, 5-methoxy-3-phenyl-1H-isochromen-1-one, $126 \mathrm{MHz}, \mathrm{CDCl}_{3}$, $298 \mathrm{~K}$

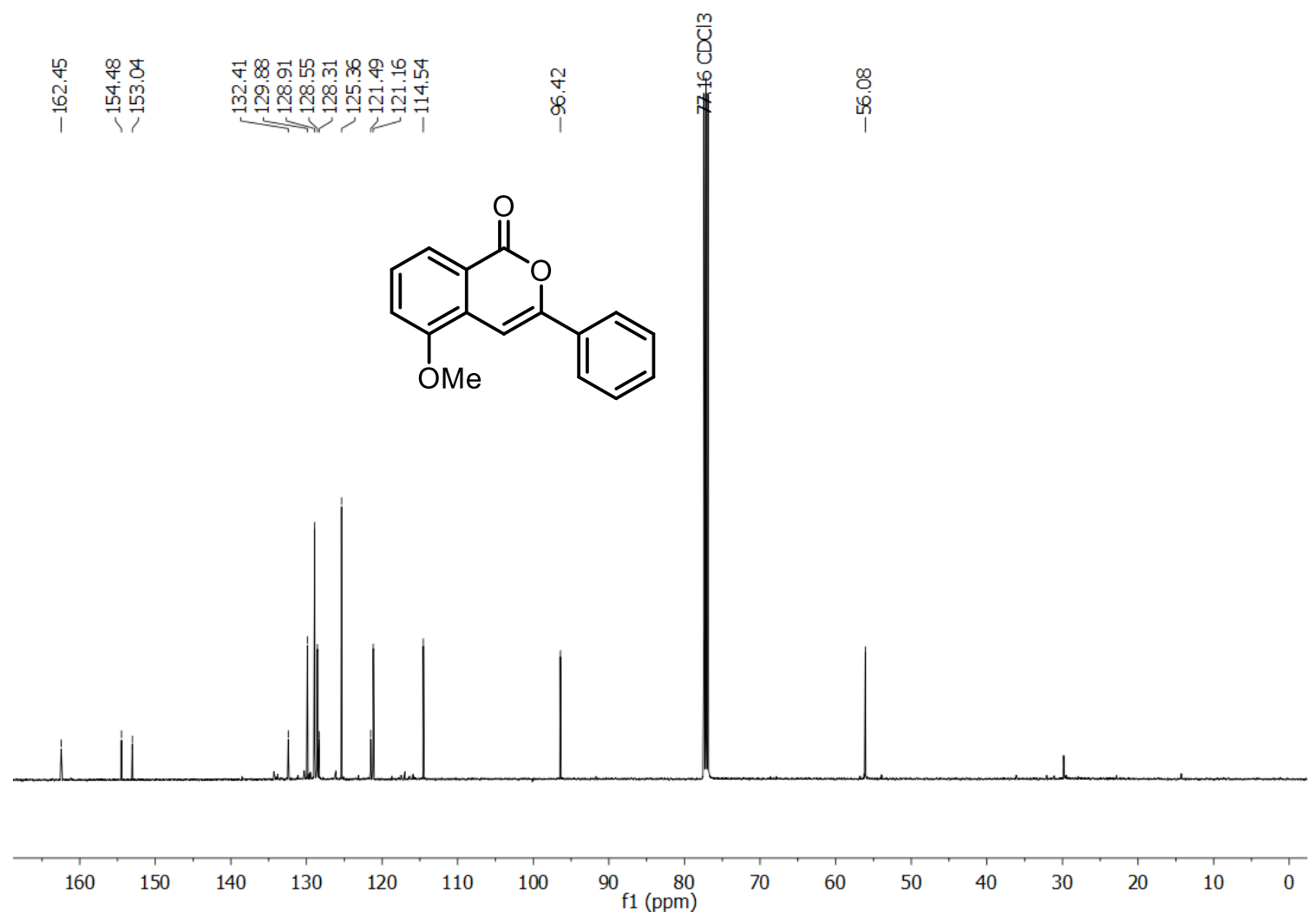

${ }^{1} \mathrm{H}$ NMR of compound 219a and 219b, 8-methyl-3-phenyl-1H-isochromen-1-one and (Z)-3-

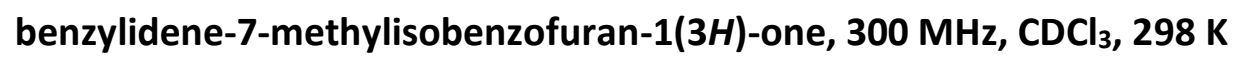

$\frac{m}{8}$

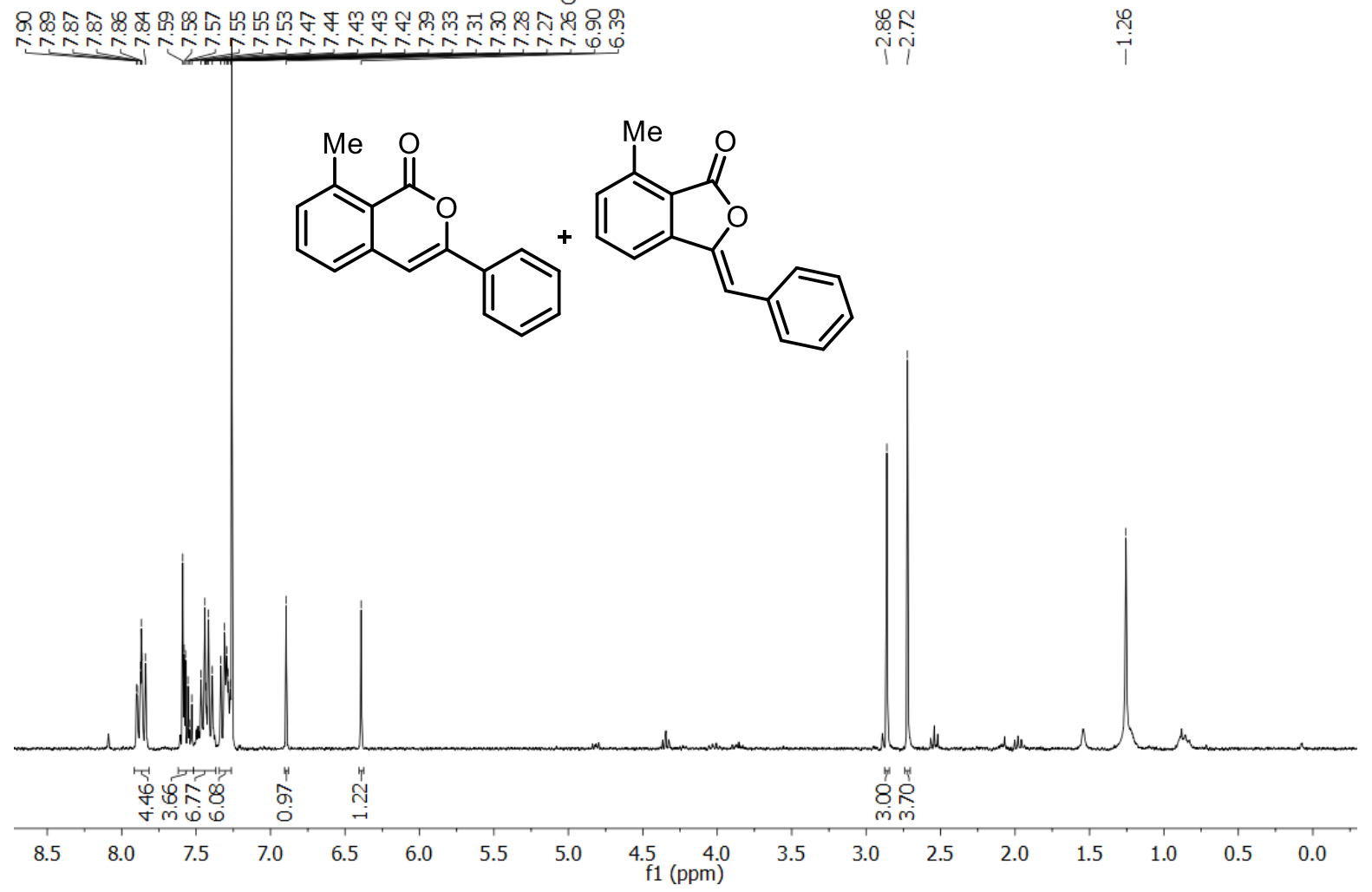


${ }^{1} \mathrm{H}$ NMR of compound 220a, 8-methoxy-3-phenyl-1H-isochromen-1-one, $600 \mathrm{MHz}, \mathrm{CDCl}_{3}$, $298 \mathrm{~K}$

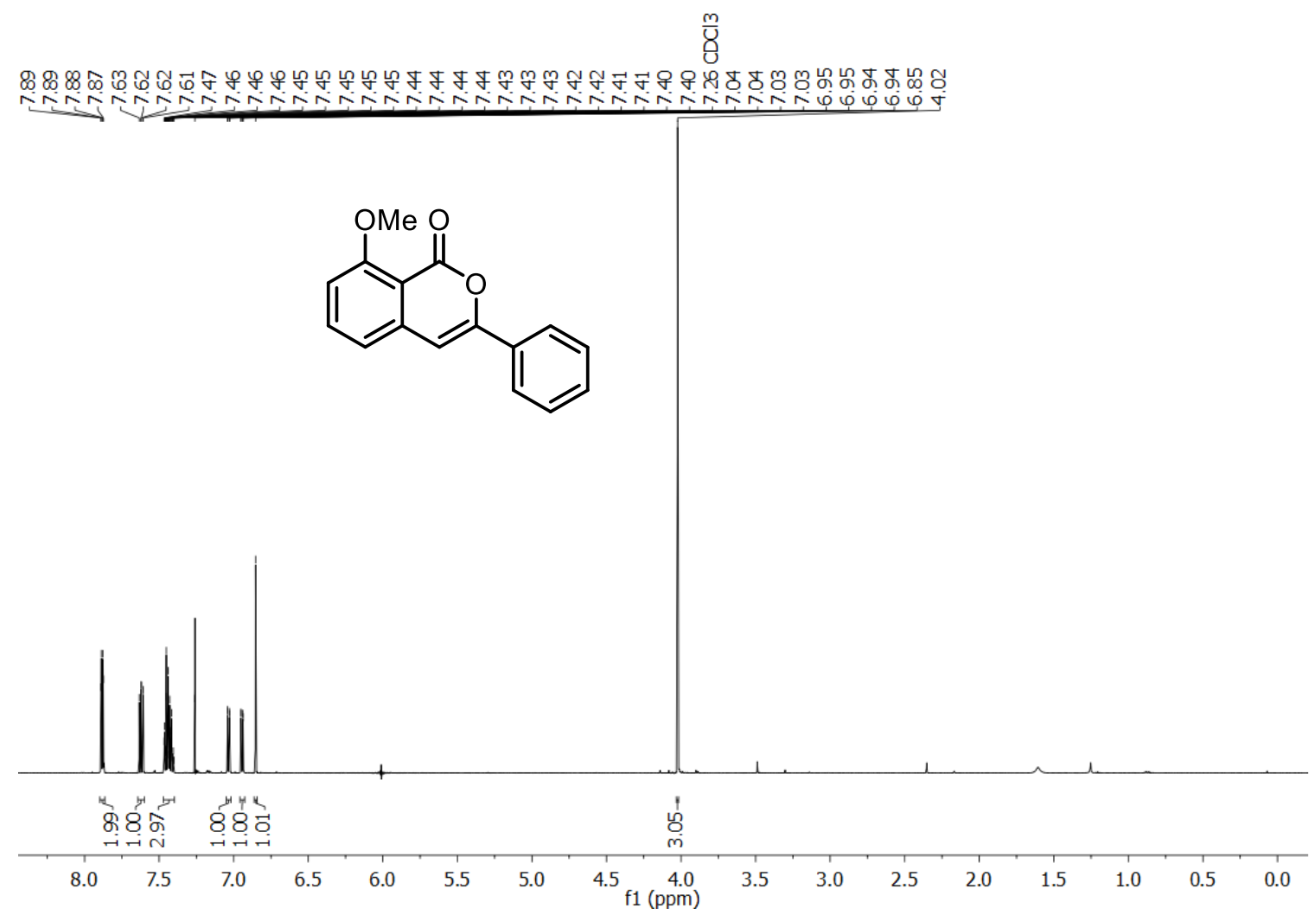

${ }^{13} \mathrm{C}$ NMR of compound 220a, 8-methoxy-3-phenyl-1H-isochromen-1-one, $126 \mathrm{MHz}, \mathrm{CDCl}_{3}$, $298 \mathrm{~K}$

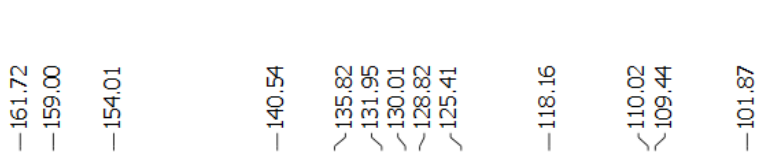
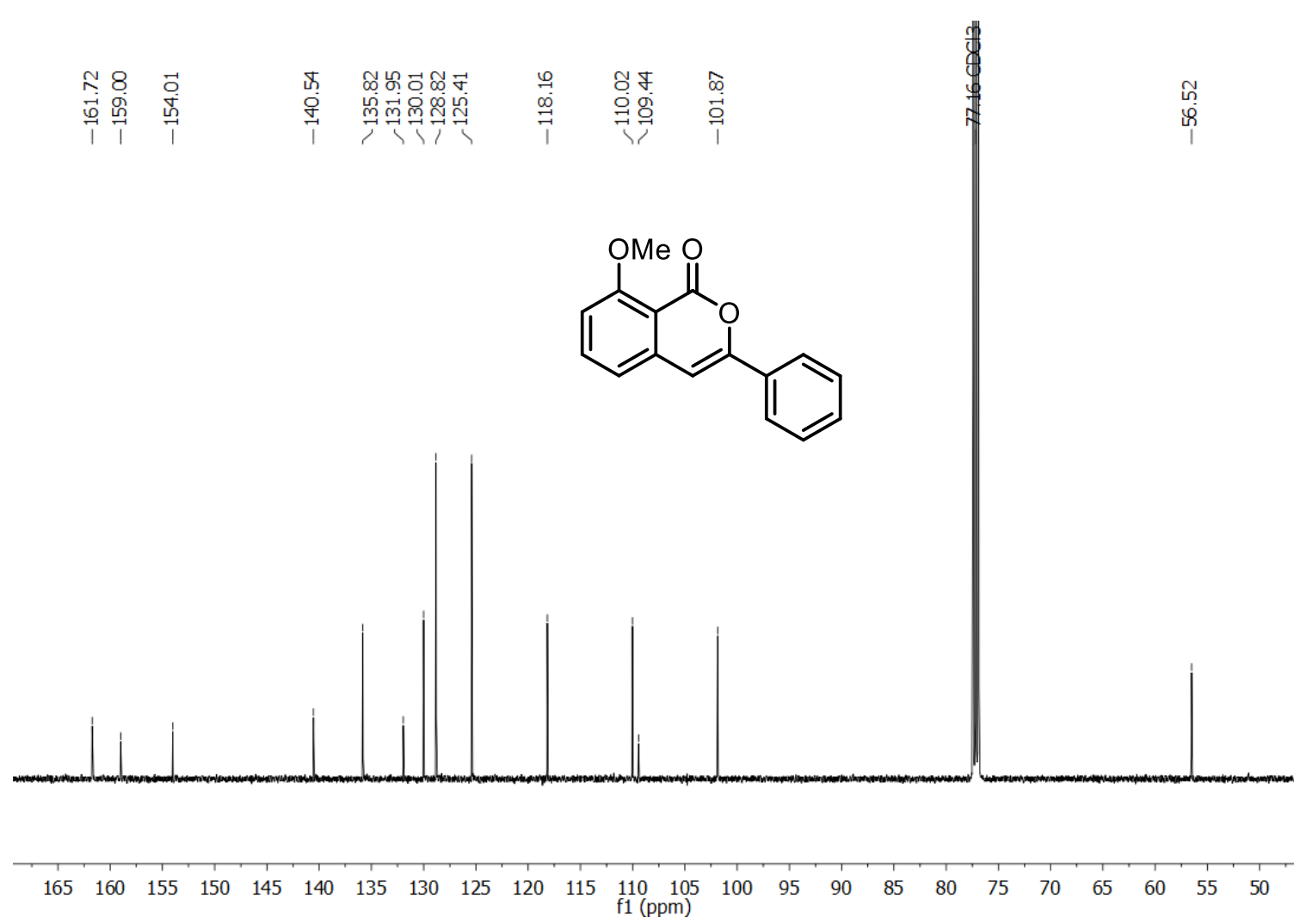
${ }^{1} \mathrm{H}$ NMR of compound 220b, (Z)-3-benzylidene-7-methoxyisobenzofuran-1(3H)-one, 600 $\mathrm{MHz}, \mathrm{CDCl}_{3}, 298 \mathrm{~K}$

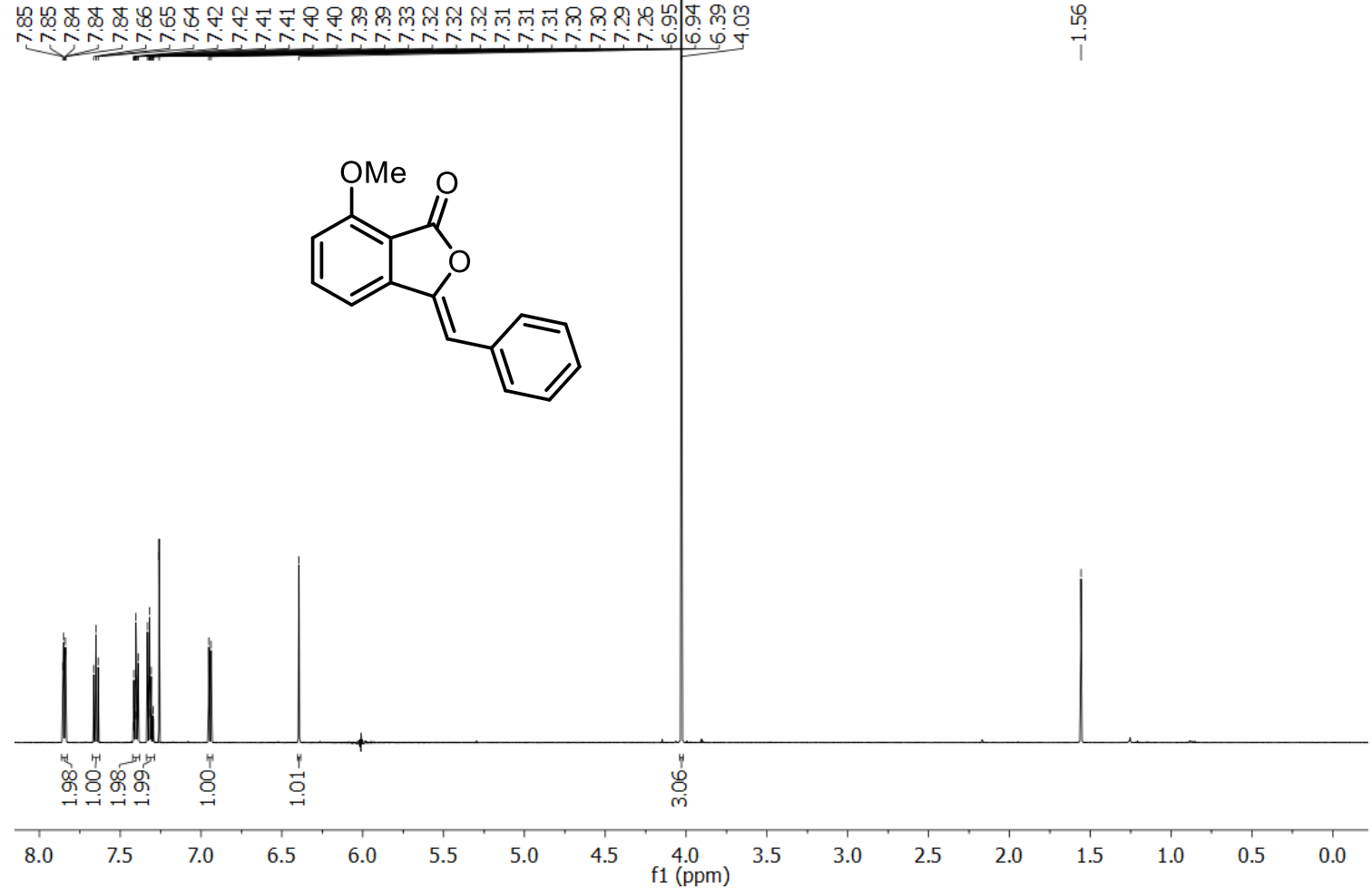

${ }^{13} \mathrm{C}$ NMR of compound 220b, (Z)-3-benzylidene-7-methoxyisobenzofuran-1(3H)-one, 126 $\mathrm{MHz}, \mathrm{CDCl}_{3}, 298 \mathrm{~K}$

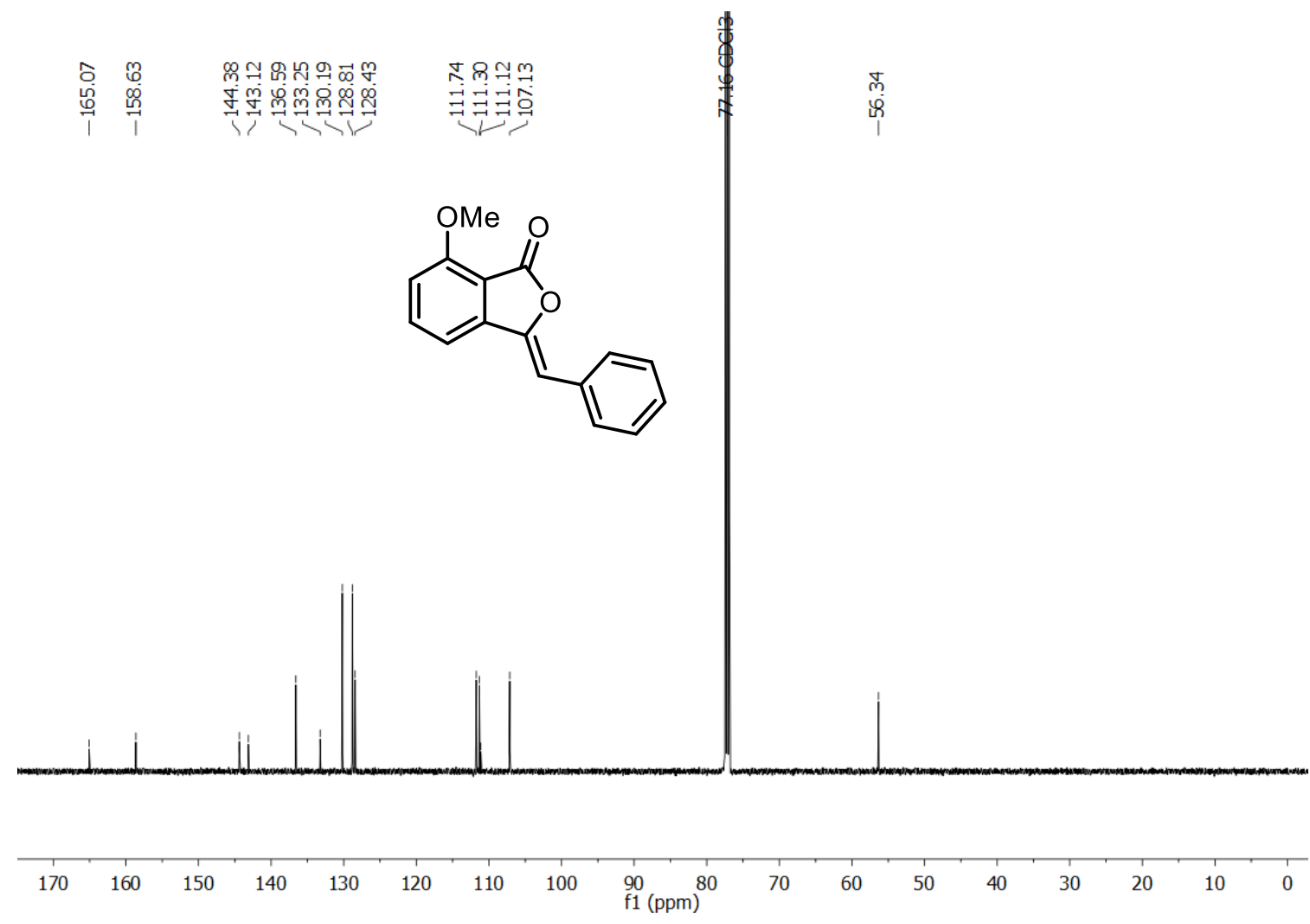


${ }^{1} \mathrm{H}$ NMR of compound 221a, 3-phenyl-1H-benzo[g]isochromen-1-one, $300 \mathrm{MHz}, \mathrm{CDCl}_{3}, 298$ K

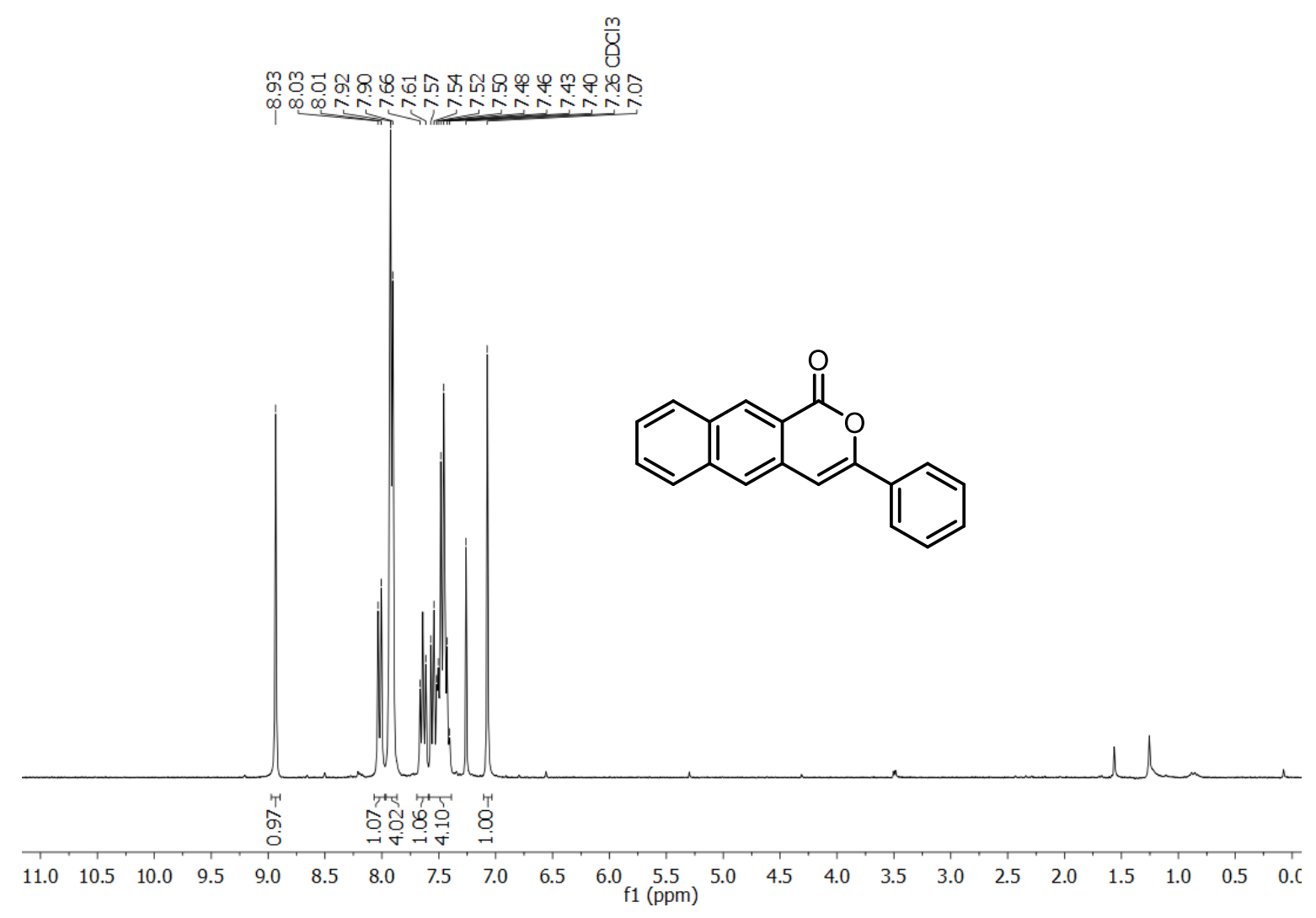

${ }^{13} \mathrm{C}$ NMR of compound 221a, 3-phenyl-1H-benzo[g]isochromen-1-one,101 $\mathrm{MHz}, \mathrm{CDCl}_{3}, 298$ K

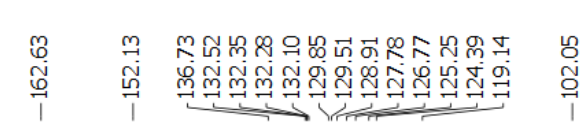<smiles>O=c1oc(-c2ccccc2)cc2cc3ccccc3cc12</smiles>

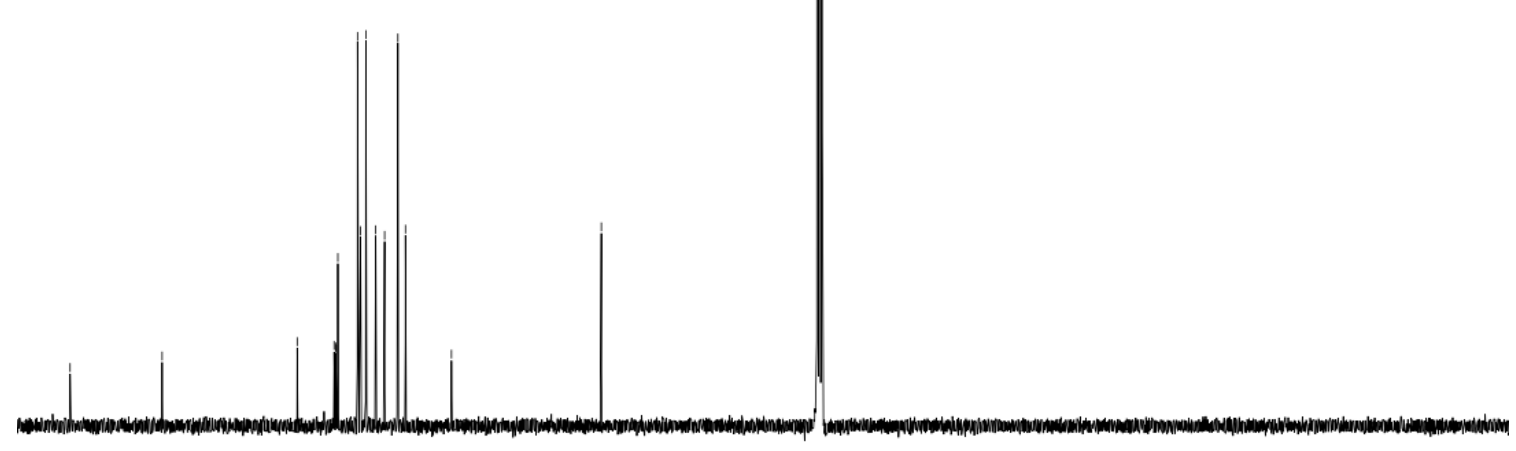

$\begin{array}{llllllllllllllll}160 & 150 & 140 & 130 & 120 & 110 & 100 & 90 \begin{array}{r}80 \\ \mathrm{f} 1(\mathrm{ppm})\end{array} & 70 & 60 & 50 & 40 & 30 & 20 & 10 & 0\end{array}$ 
${ }^{1} \mathrm{H}$ NMR of compound 221b, 2-phenyl-4H-benzo[f] isochromen-4-one, $300 \mathrm{MHz}, \mathrm{CDCl}_{3}, 302$ K

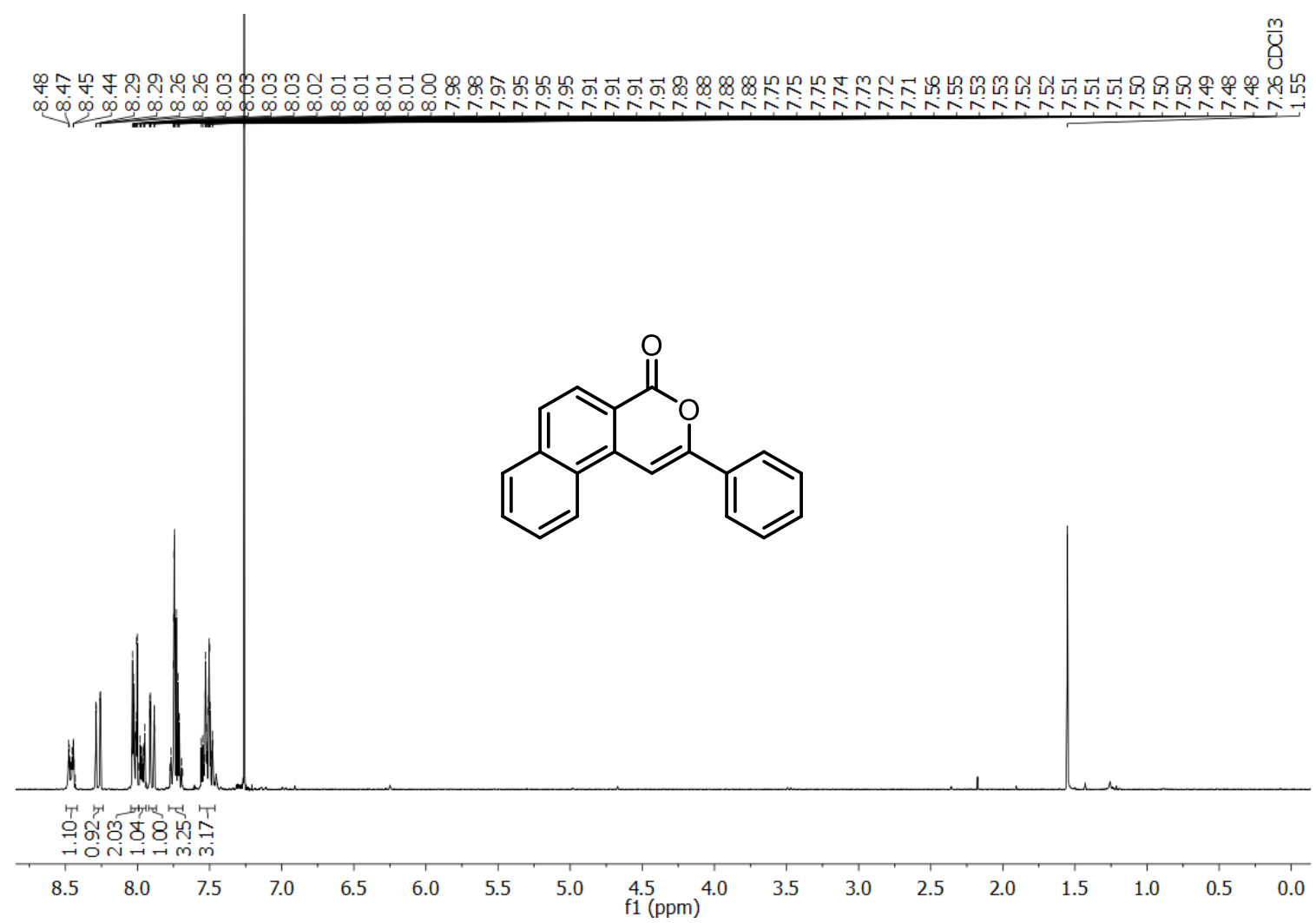

${ }^{13} \mathrm{C}$ NMR of compound 221b, 2-phenyl-4H-benzo[f] isochromen-4-one, $126 \mathrm{MHz}, \mathrm{CDCl}_{3}, 298$ K

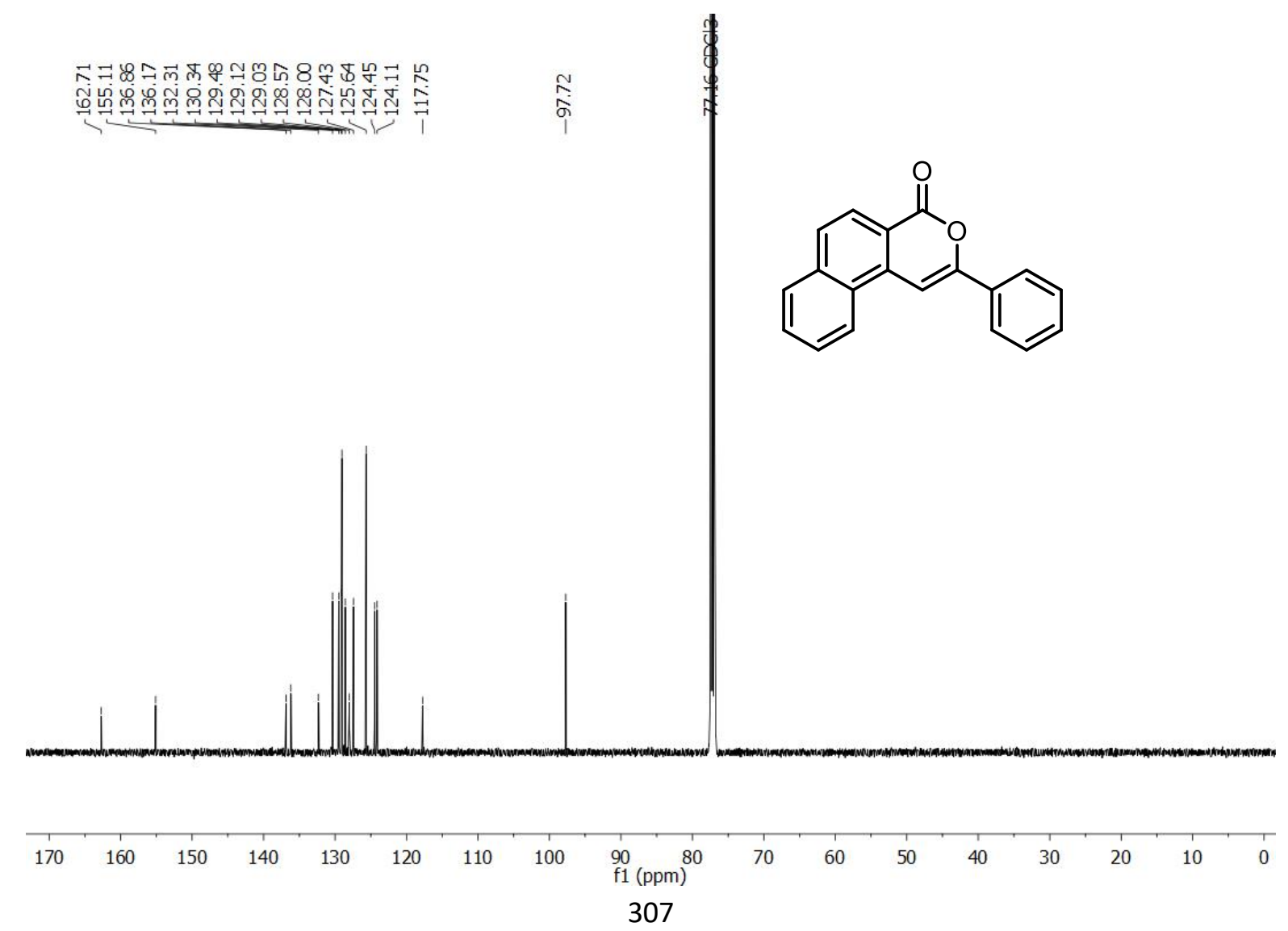


${ }^{1} \mathrm{H}$ NMR of compound 222, 3-(4-methoxyphenyl)-1H-isochromen-1-one, $600 \mathrm{MHz}, \mathrm{CDCl}_{3}$, $298 \mathrm{~K}$

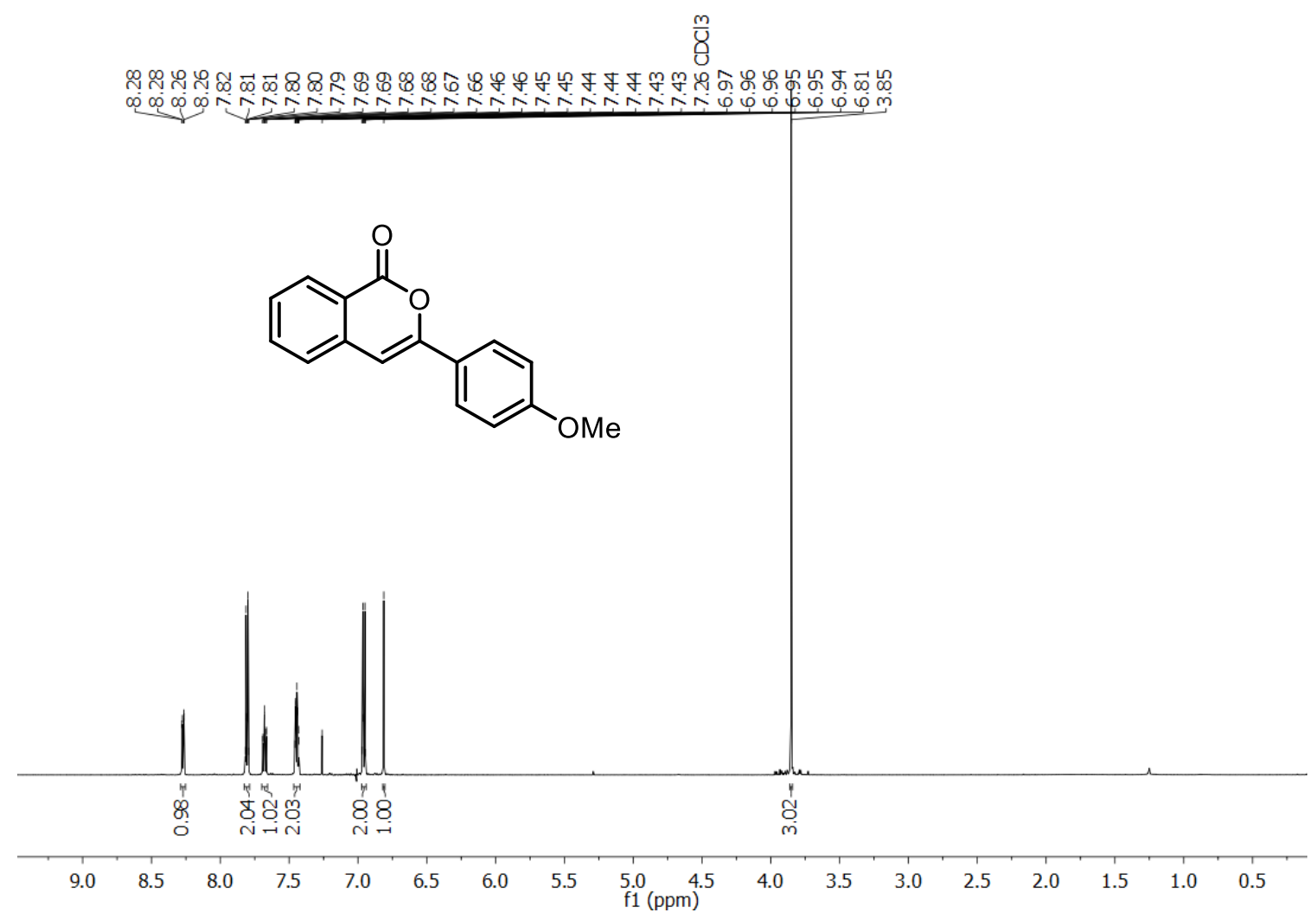

${ }^{13} \mathrm{C}$ NMR of compound 222, 3-(4-methoxyphenyl)-1H-isochromen-1-one, $126 \mathrm{MHz}, \mathrm{CDCl}_{3}$, $298 \mathrm{~K}$

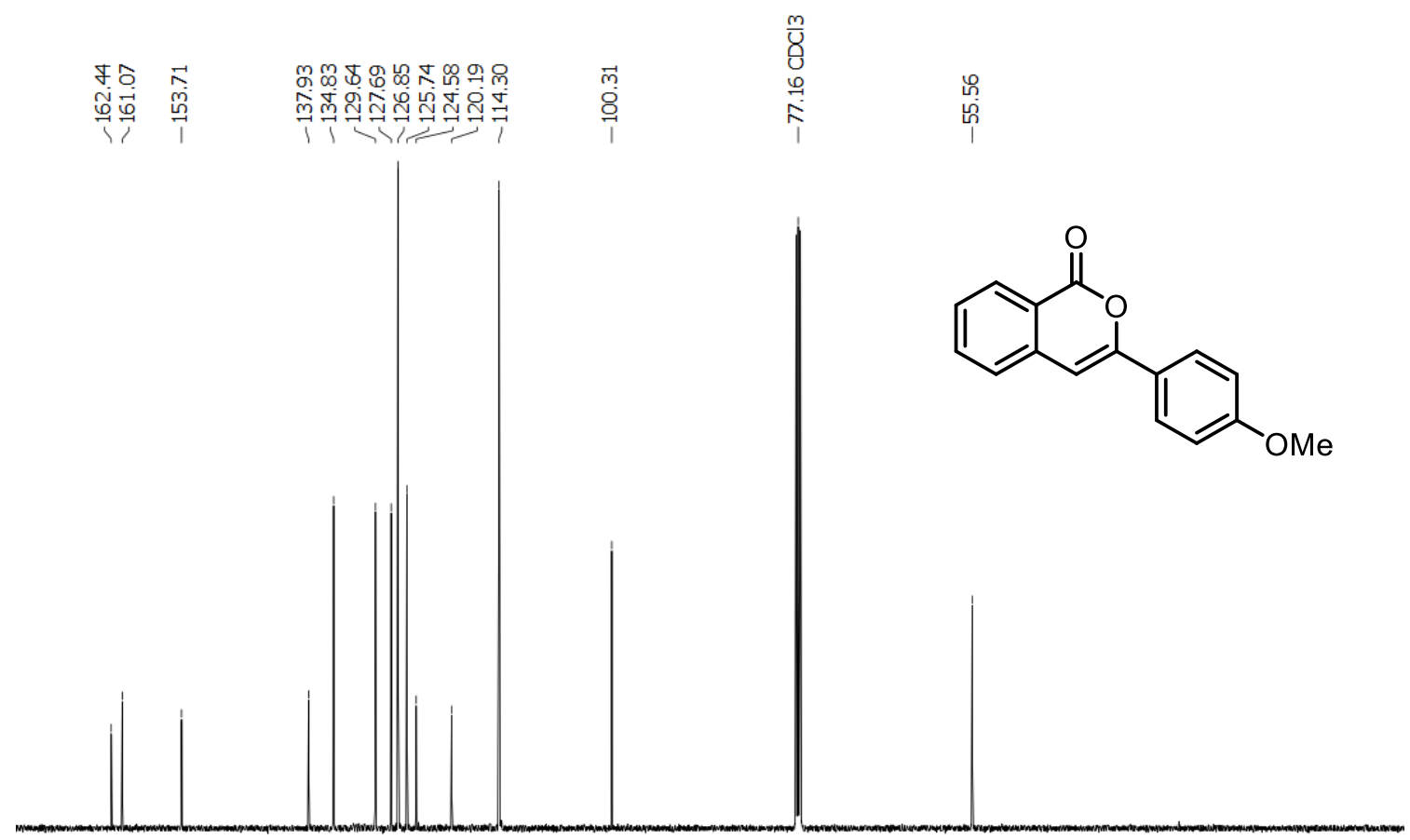

$\begin{array}{lllllllll}170 & 160 & 150 & 140 & 130 & 120 & 110 & 100 & \underset{\mathrm{f} 1(\mathrm{ppm})}{90}\end{array}$ 
${ }^{1} \mathrm{H}$ NMR of compound 223, 3,6-diphenyl-1H-isochromen-1-one, $300 \mathrm{MHz}^{\mathrm{CDCl}}{ }_{3}, 302 \mathrm{~K}$

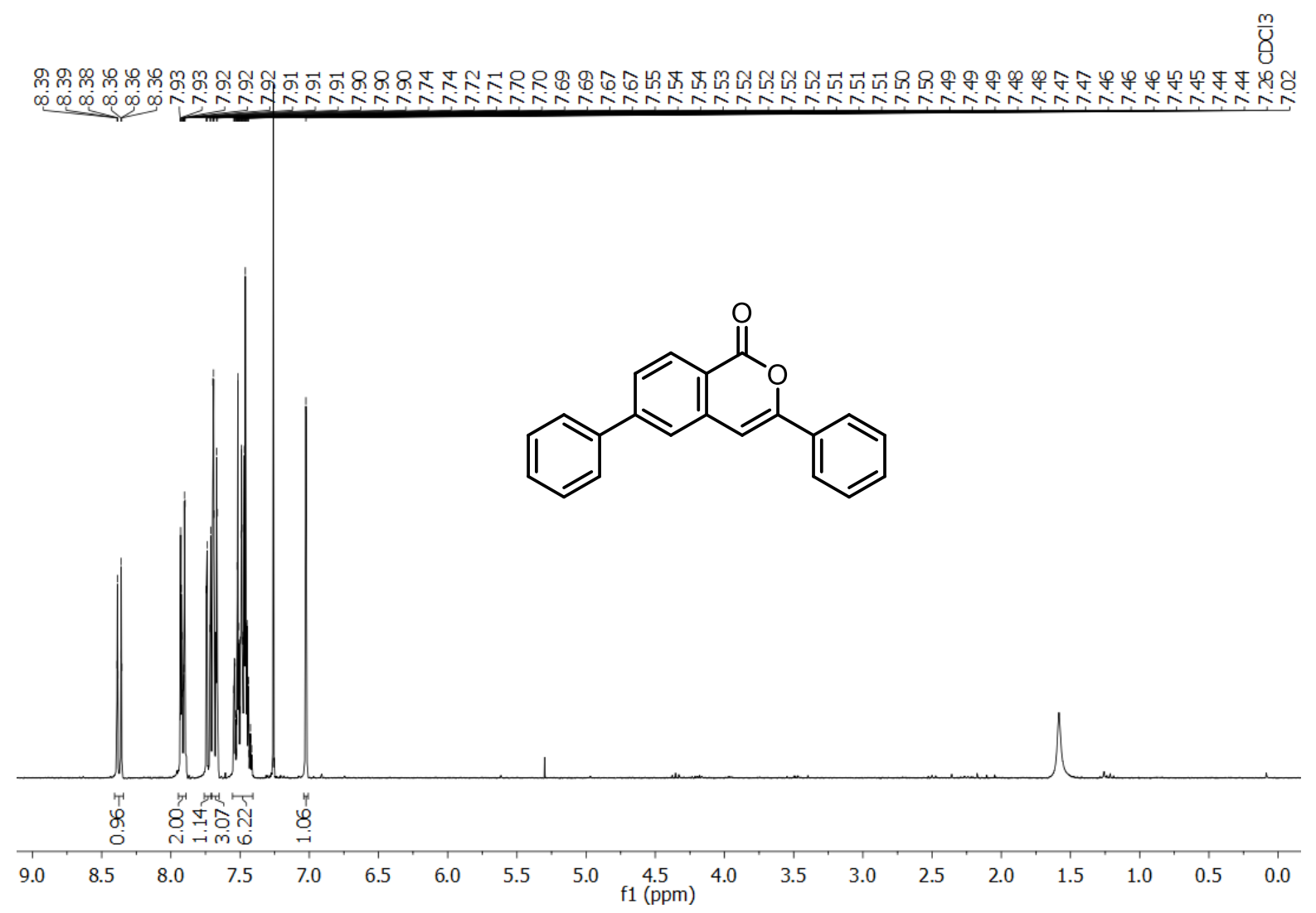

${ }^{13} \mathrm{C}$ NMR of compound 223, 3,6-diphenyl-1H-isochromen-1-one, $126 \mathrm{MHz}, \mathrm{CDCl}_{3}, 298 \mathrm{~K}$
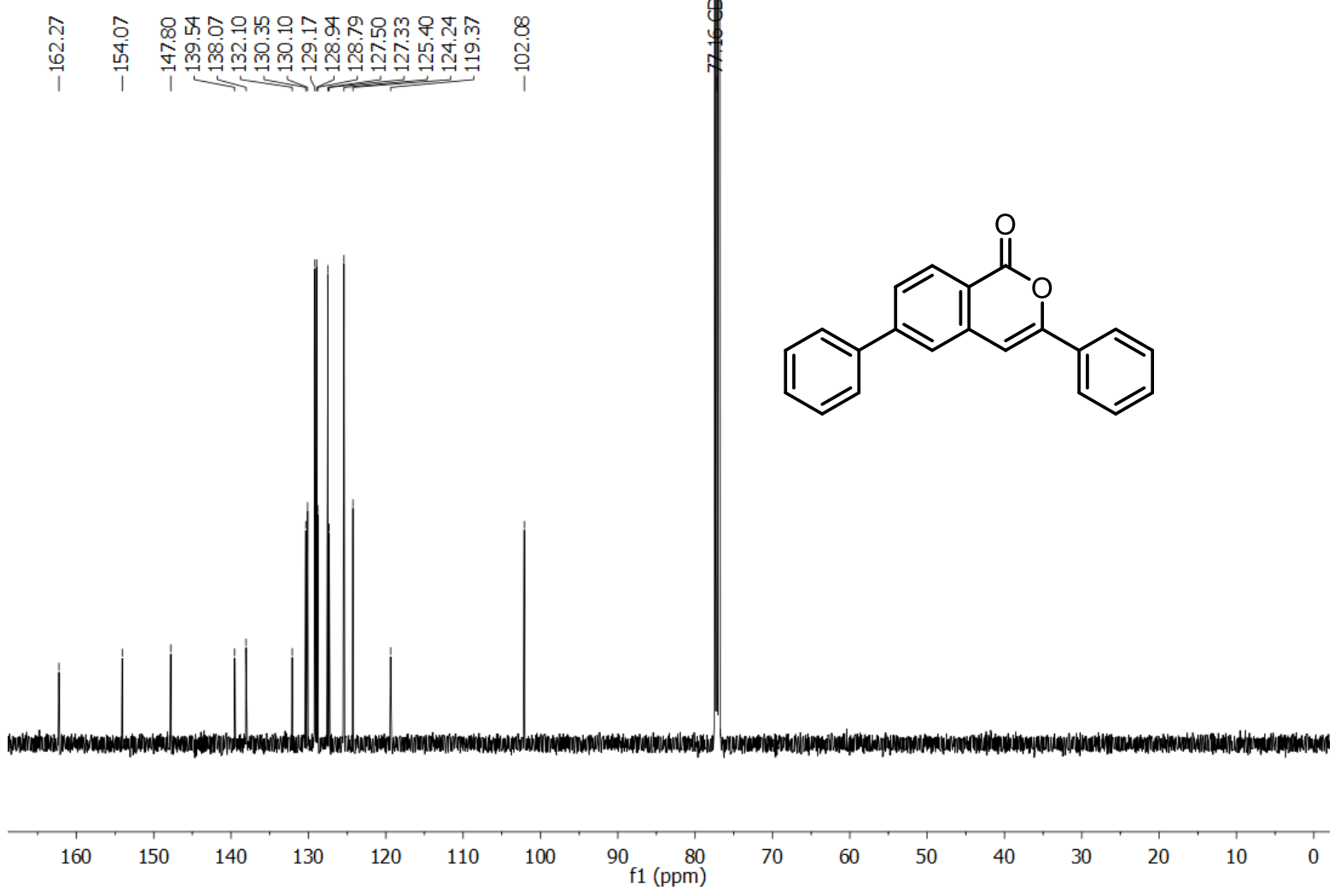

309 
${ }^{1} \mathrm{H}$ NMR of compound 224, 8-fluoro-3-phenyl-1H-isochromen-1-one, $400 \mathrm{MHz}, \mathrm{CDCl}_{3}, 298 \mathrm{~K}$

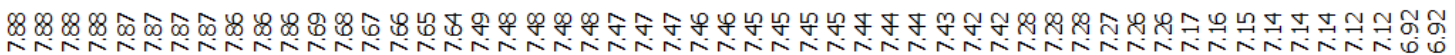

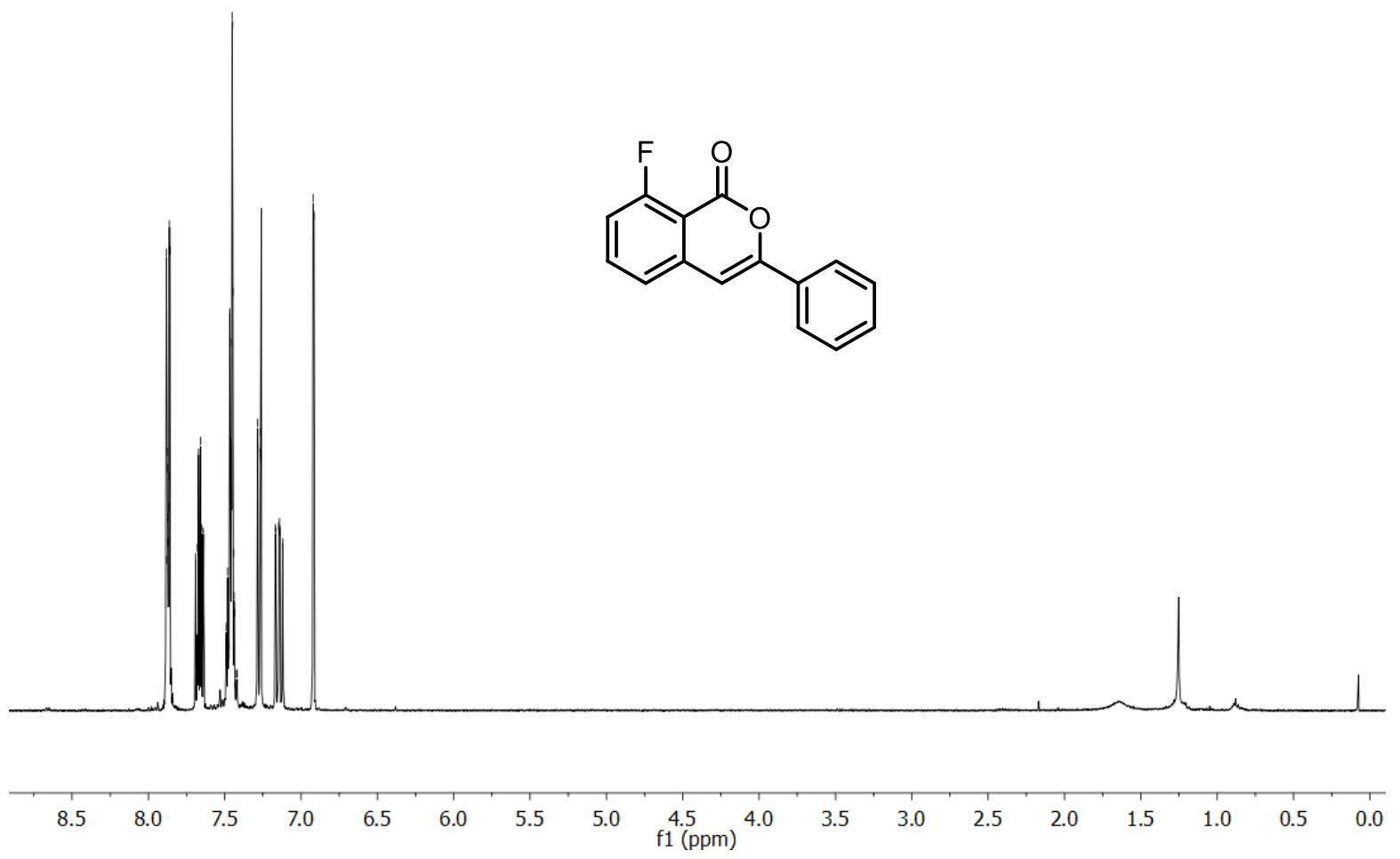

${ }^{13} \mathrm{C}$ NMR of compound 224, 8-fluoro-3-phenyl-1H-isochromen-1-one, $101 \mathrm{MHz}, \mathrm{CDCl}_{3}, 300$ $\mathrm{K}$
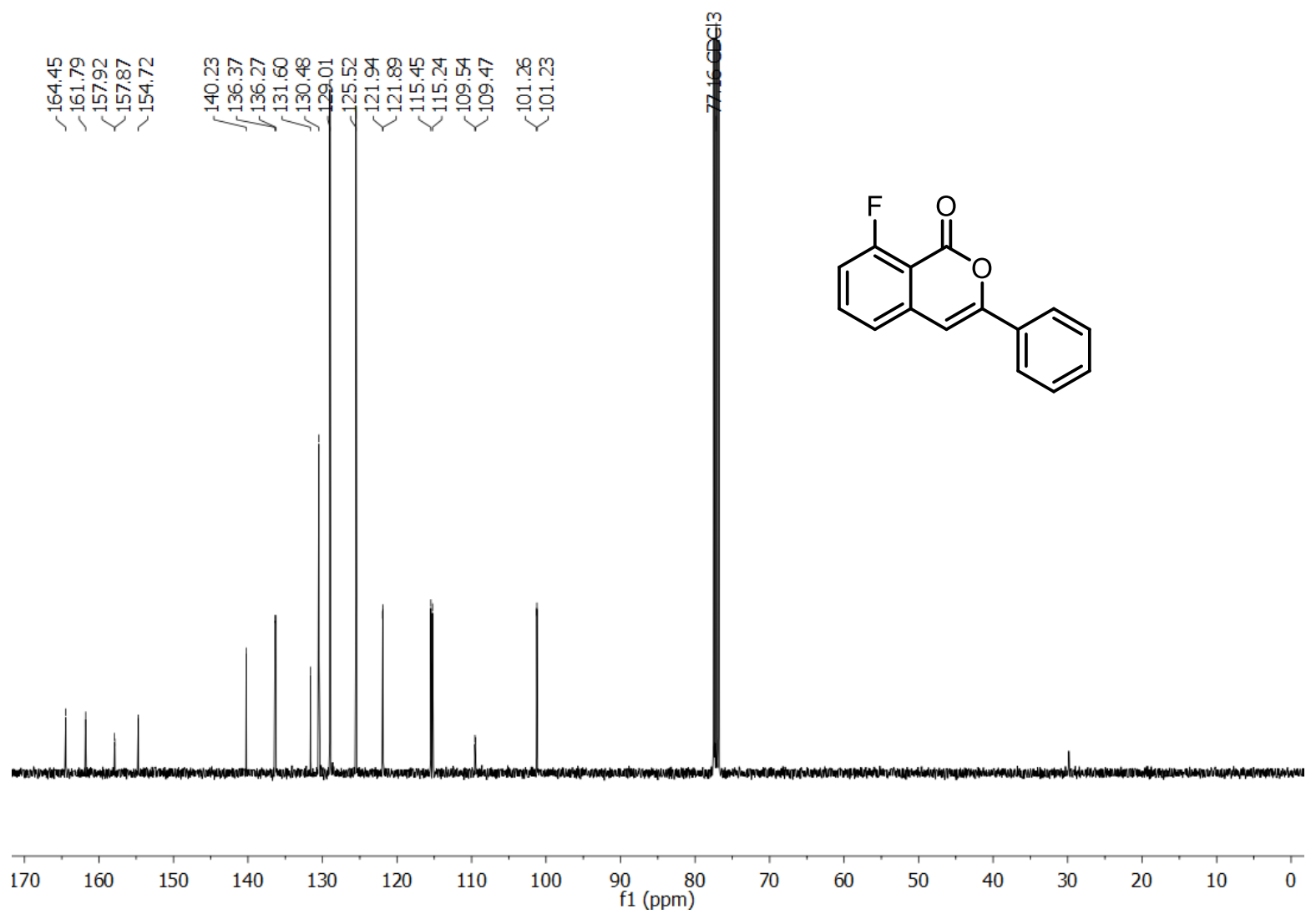

310 
${ }^{19} \mathrm{~F}$ NMR of compound 224, 8-fluoro-3-phenyl-1H-isochromen-1-one, $377 \mathrm{MHz}, \mathrm{CDCl}_{3}, 299 \mathrm{~K}$

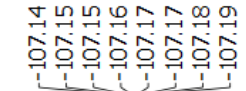<smiles>O=c1oc(-c2ccccc2)cc2cccc(F)c12</smiles>

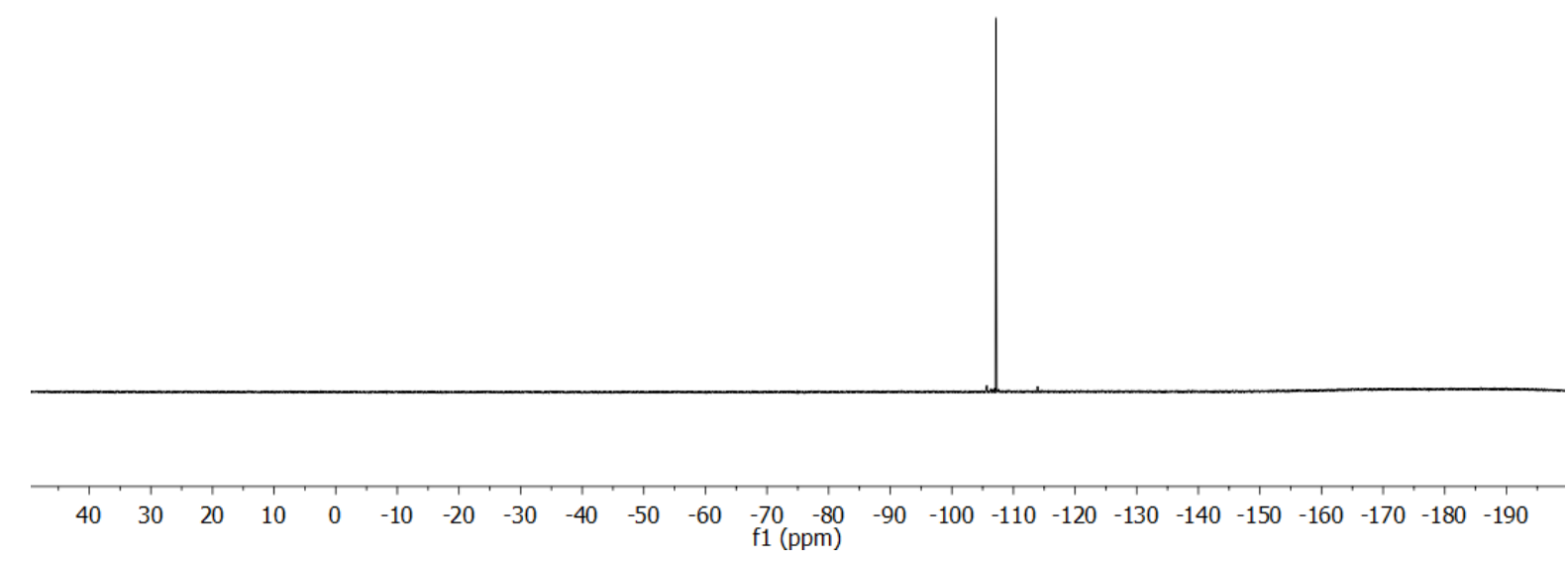

${ }^{1} \mathrm{H}$ NMR of compound 225, 3-phenyl-1H-pyrano[3,4-b]benzofuran-1-one, $300 \mathrm{MHz}, \mathrm{CDCl}_{3}$, $298 \mathrm{~K}$

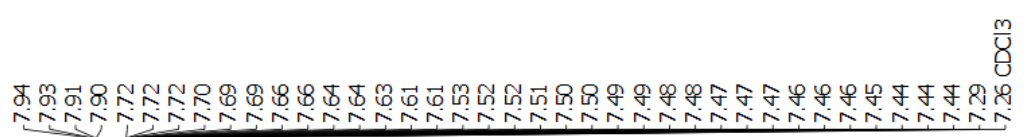<smiles>O=c1oc(-c2ccccc2)cc2c1oc1ccccc12</smiles>

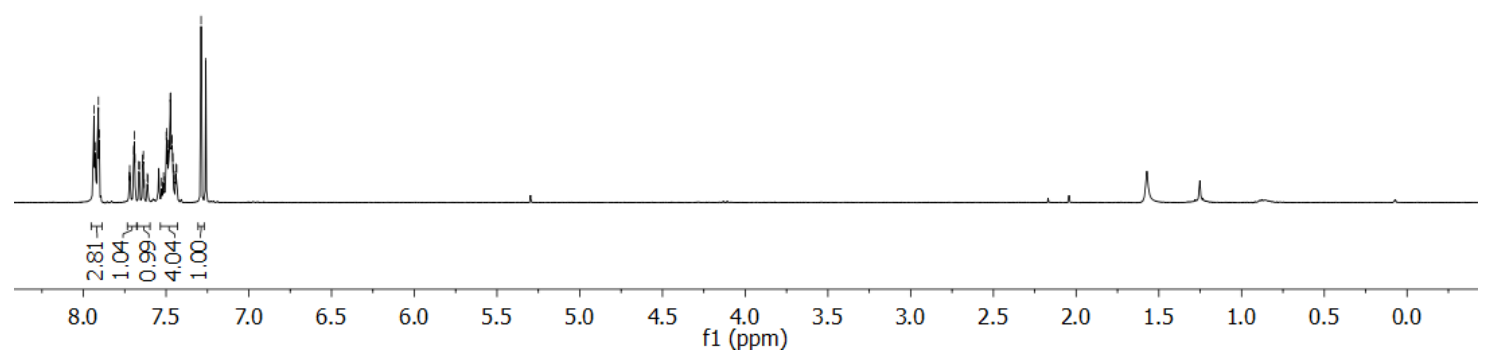


${ }^{13} \mathrm{C}$ NMR of compound 225, 3-phenyl-1H-pyrano[3,4-b]benzofuran-1-one, $101 \mathrm{MHz}, \mathrm{CDCl}_{3}$, $299 \mathrm{~K}$

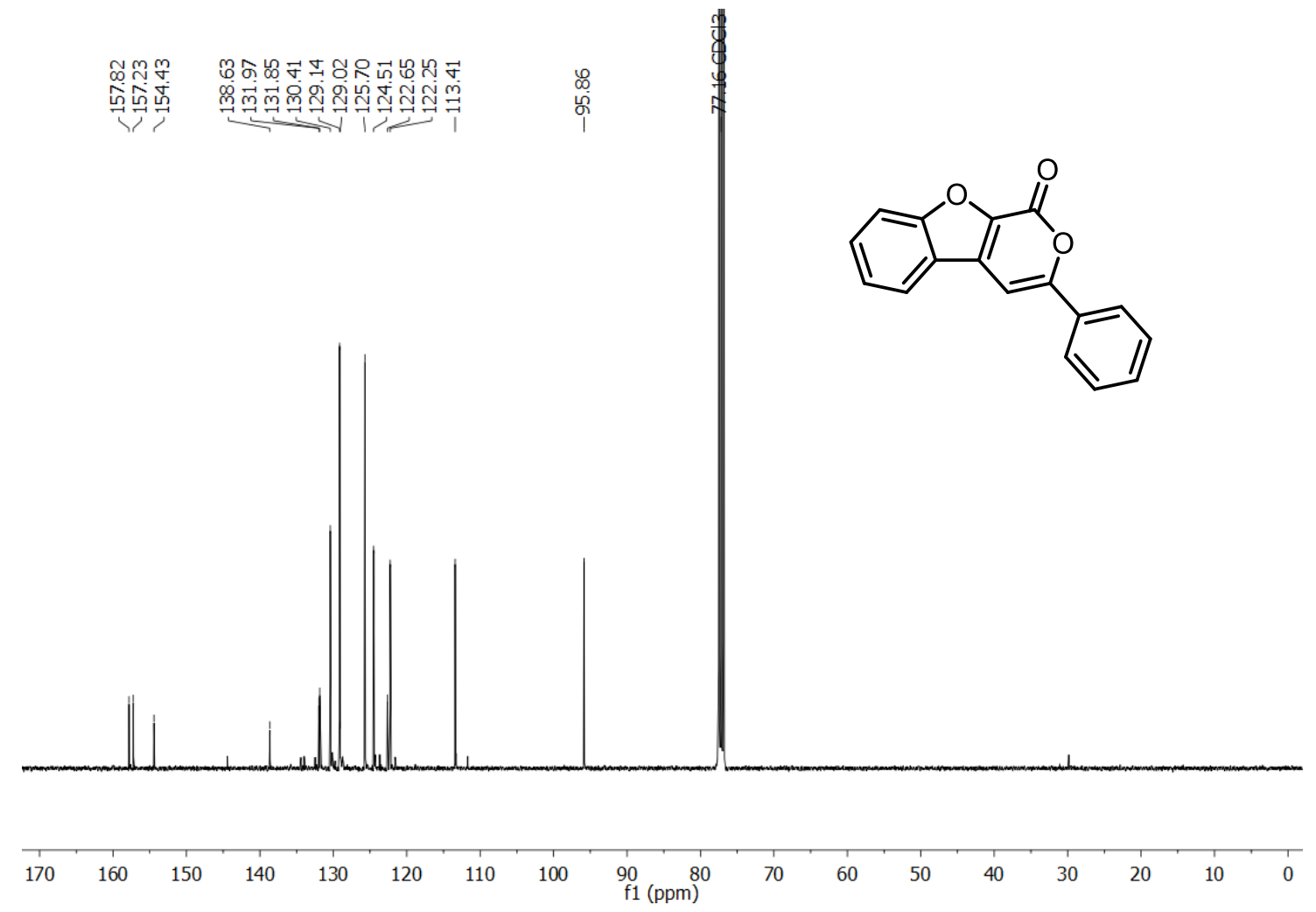

${ }^{1} \mathrm{H}$ NMR of compound 226, 6-fluoro-3-(4-fluorophenyl)-1H-isochromen-1-one, $300 \mathrm{MHz}$, $\mathrm{CDCl}_{3}, 300 \mathrm{~K}$

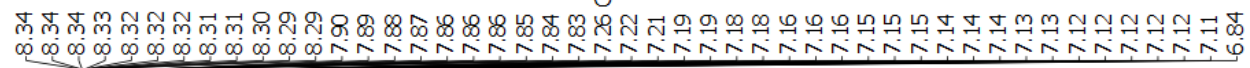

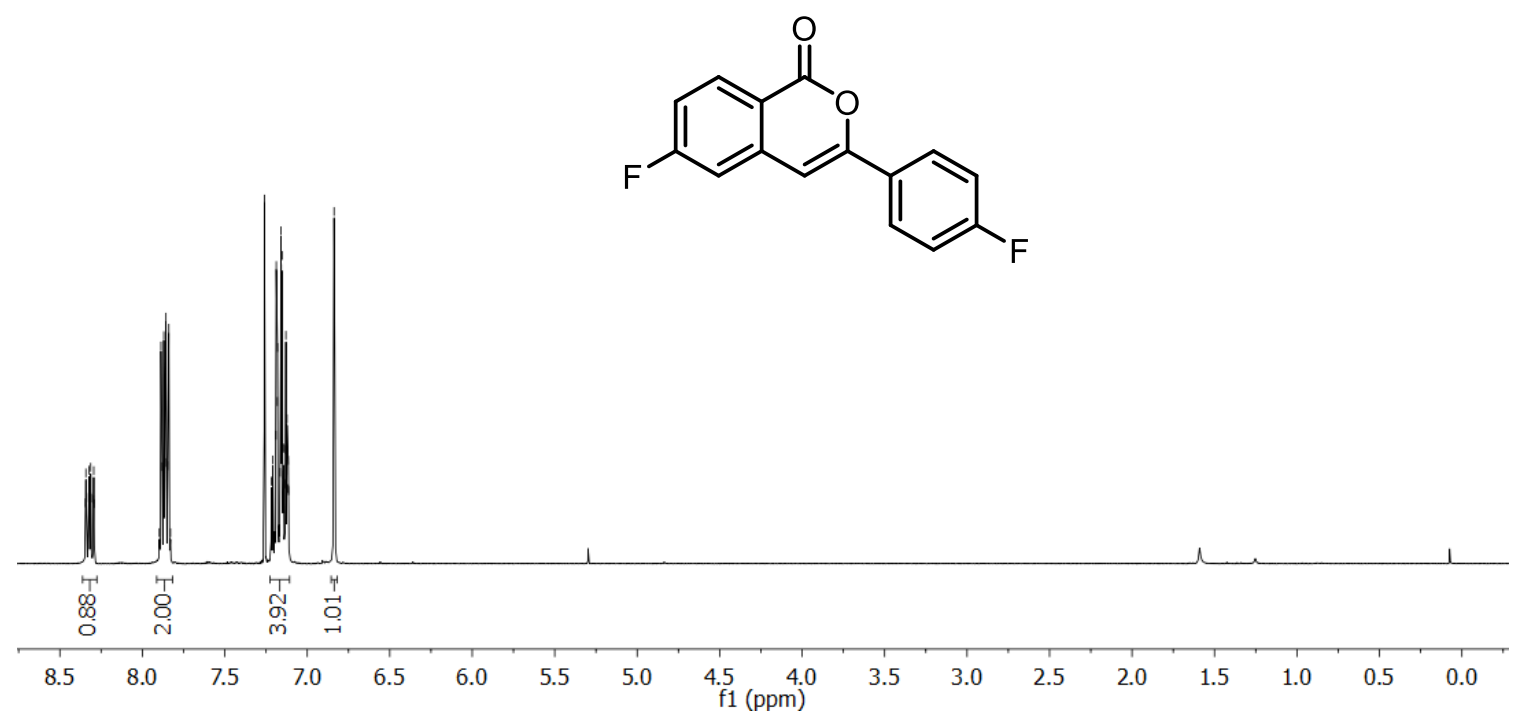


${ }^{13} \mathrm{C}$ NMR of compound 226, 6-fluoro-3-(4-fluorophenyl)-1H-isochromen-1-one, $126 \mathrm{MHz}$, $\mathrm{CDCl}_{3}, 300 \mathrm{~K}$

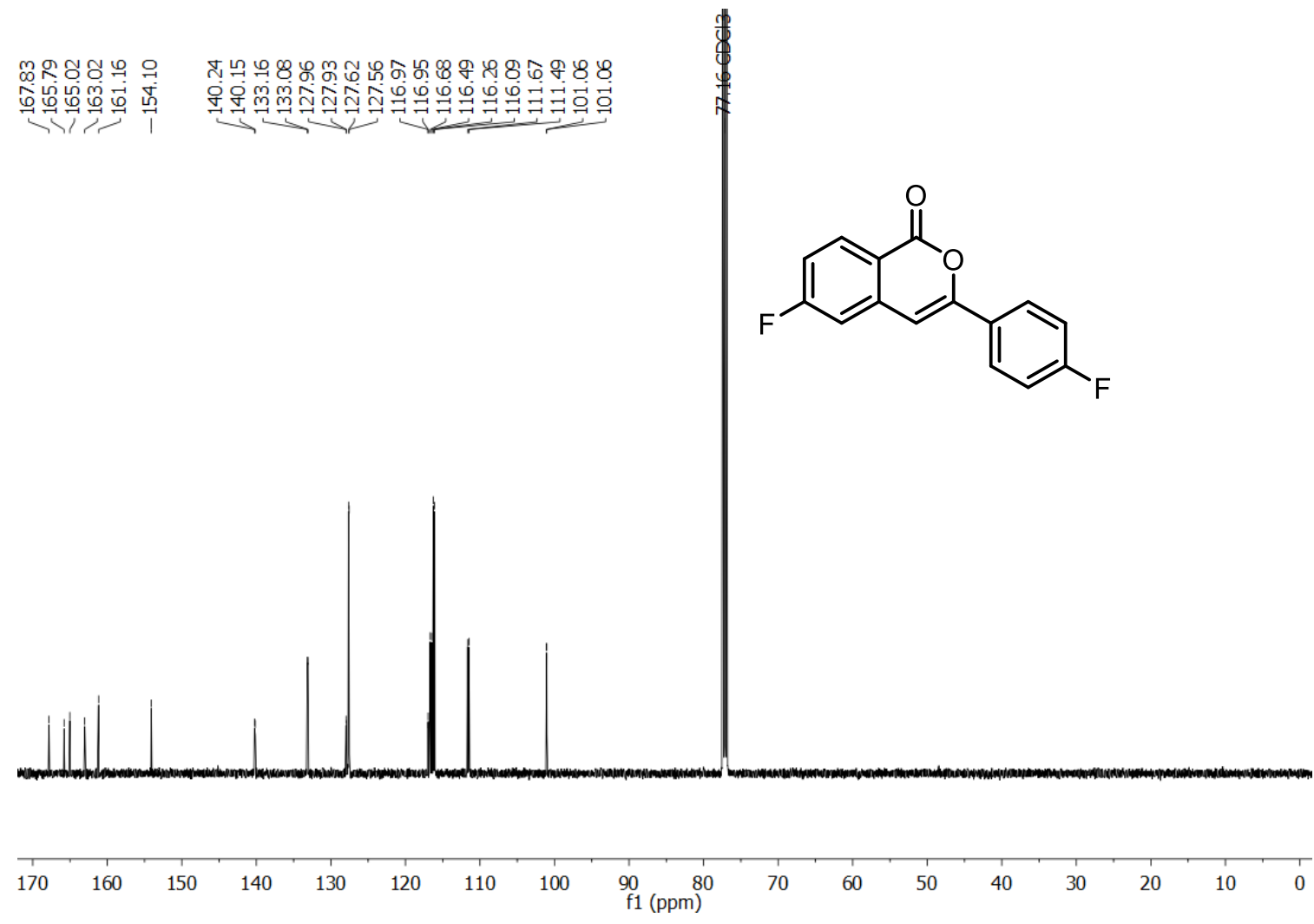

${ }^{19} \mathrm{~F}$ NMR of compound 226, 6-fluoro-3-(4-fluorophenyl)-1H-isochromen-1-one, $282 \mathrm{MHz}$, $\mathrm{CDCl}_{3}, 308 \mathrm{~K}$

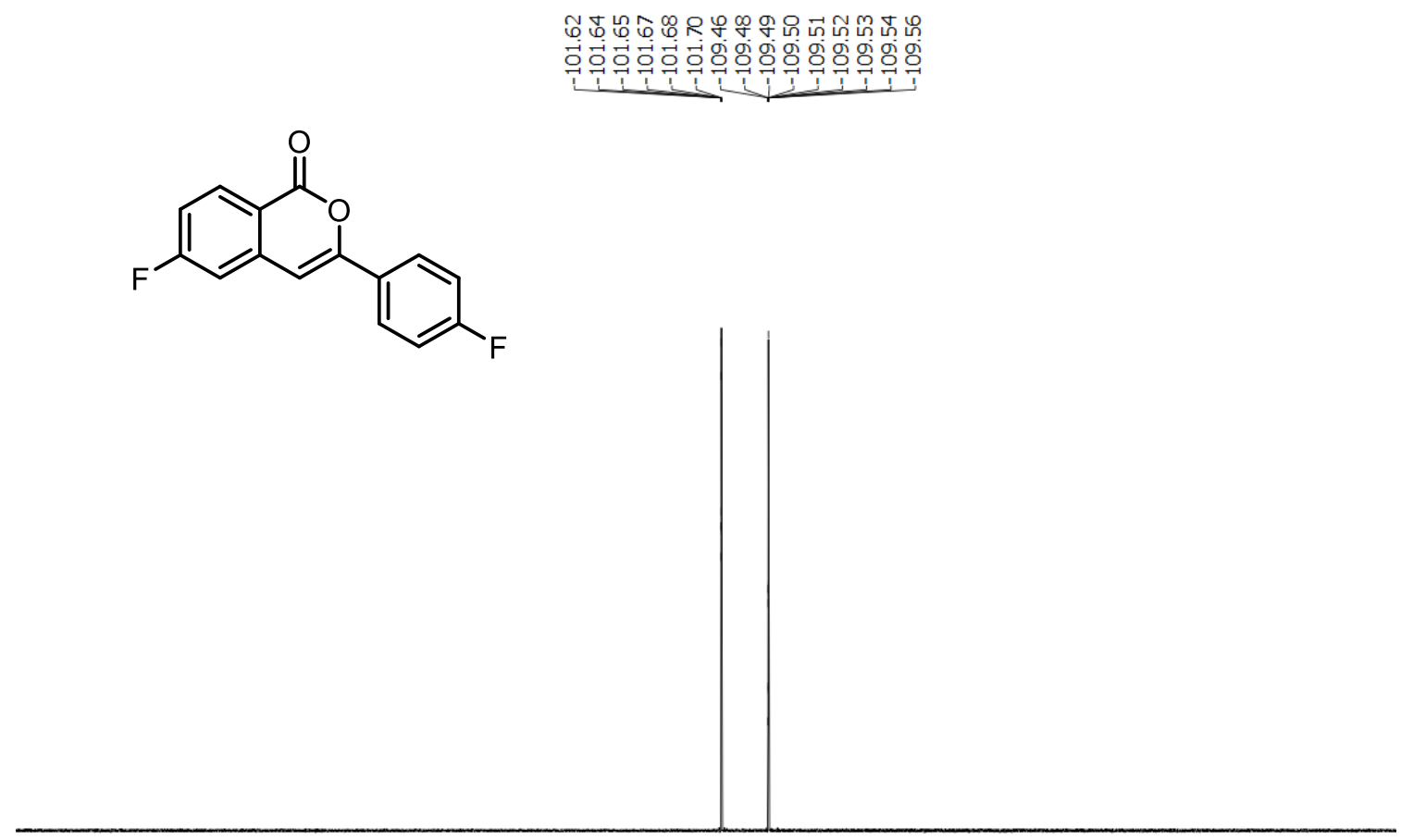

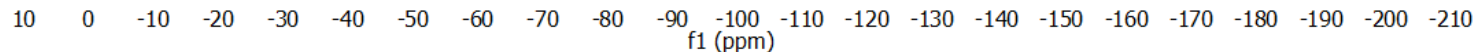


${ }^{1} \mathrm{H}$ NMR of compound 227, $\mathrm{N}$-methyl-1-oxo-3-phenyl-1H-isochromene-7-carboxamide, 300 $\mathrm{MHz}_{2} \mathrm{CDCl}_{3}, 298 \mathrm{~K}$

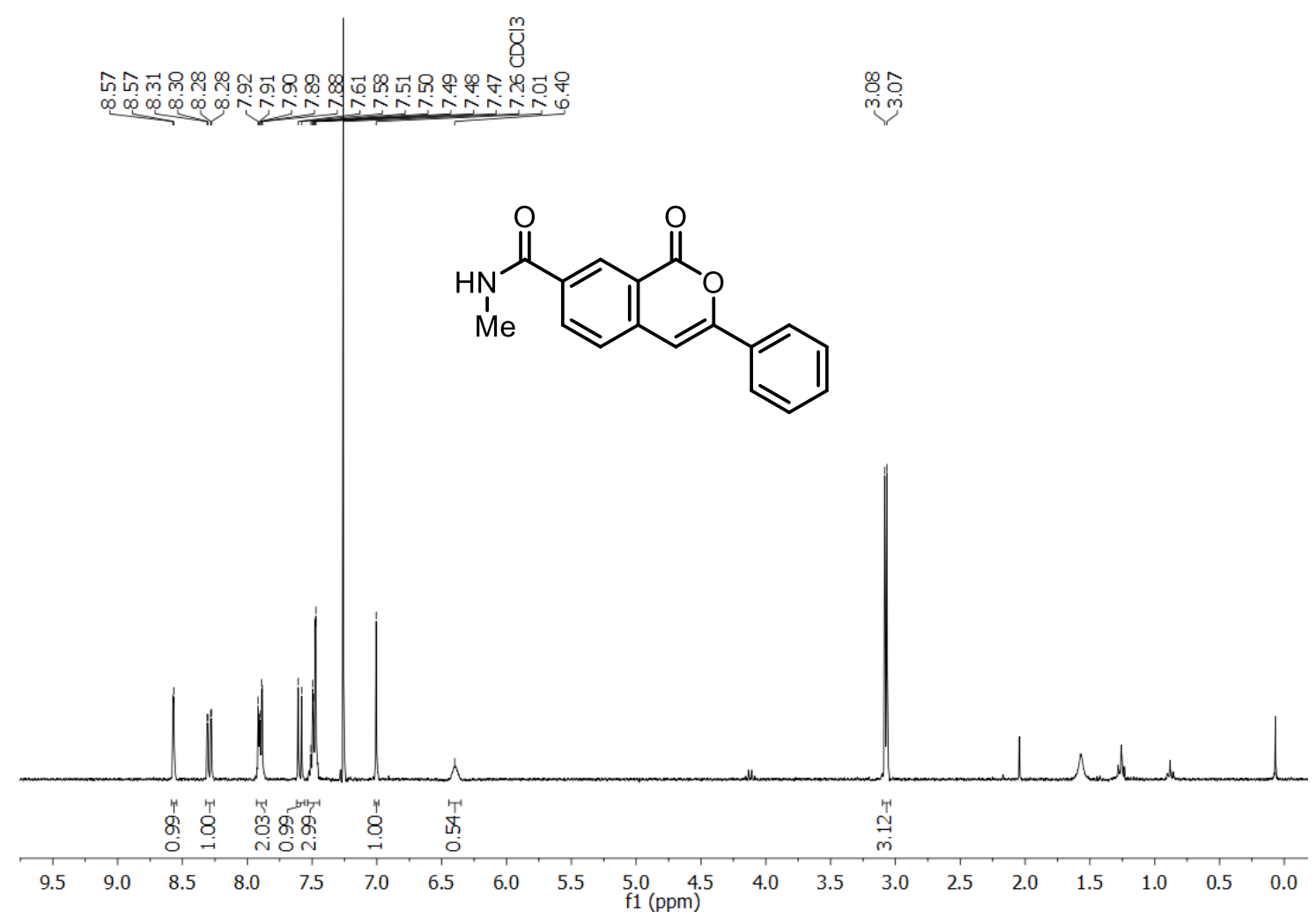

${ }^{13} \mathrm{C}$ NMR of compound 227, $\mathrm{N}$-methyl-1-oxo-3-phenyl-1H-isochromene-7-carboxamide, 126 $\mathrm{MHz}, \mathrm{CDCl}_{3}, 298 \mathrm{~K}$

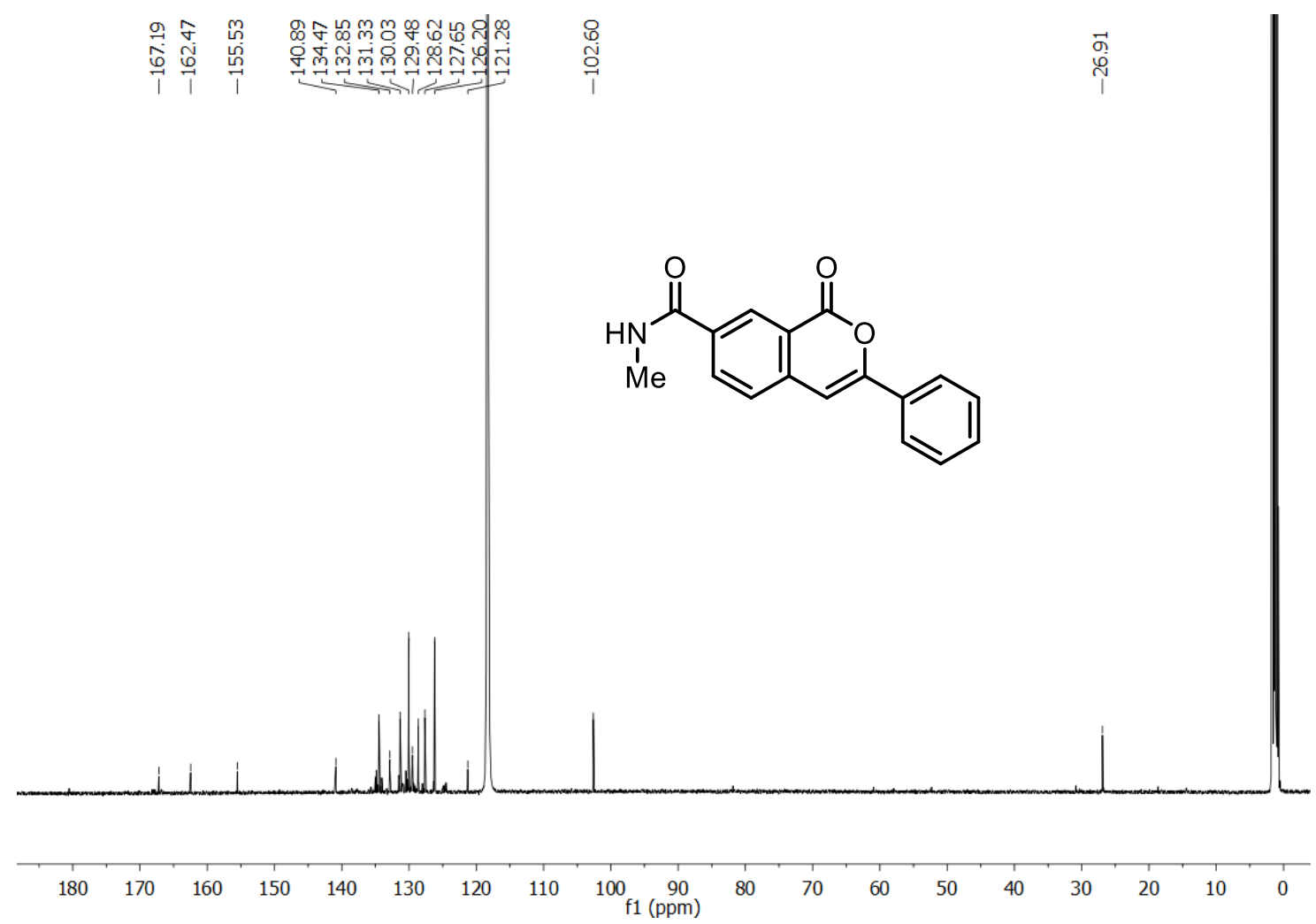


${ }^{1} \mathrm{H}$ NMR of compound 228, 3-phenyl-7-(trifluoromethyl)-1H-isochromen-1-one, $300 \mathrm{MHz}$, $\mathrm{CDCl}_{3}, 298 \mathrm{~K}$

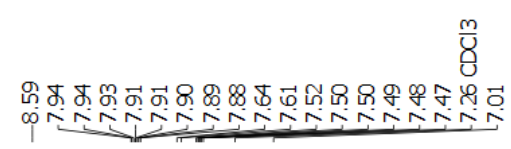<smiles>O=c1oc(-c2ccccc2)cc2ccc(C(F)(F)F)cc12</smiles>

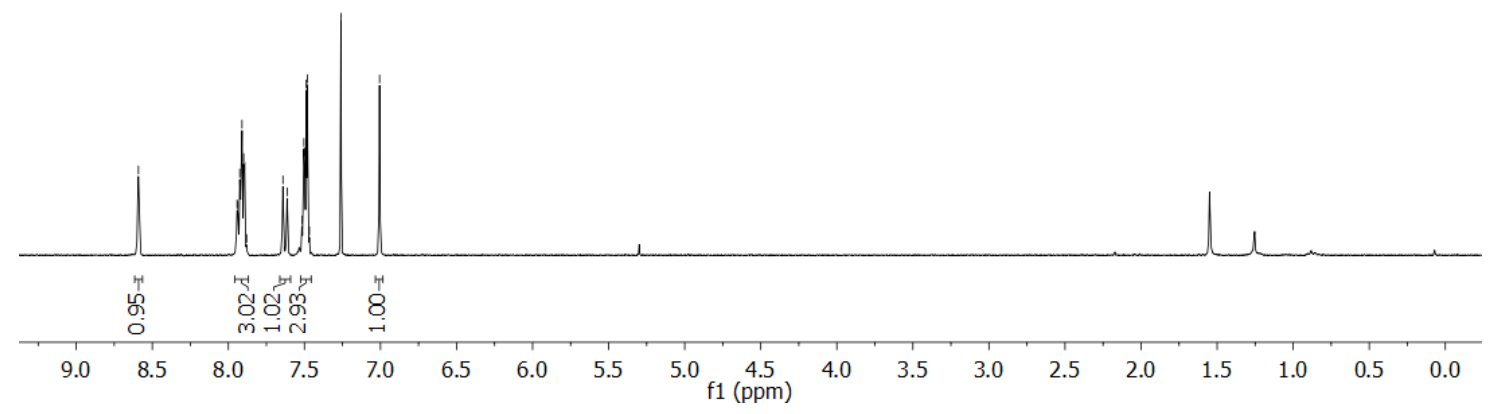

${ }^{13} \mathrm{C}$ NMR of compound 228, 3-phenyl-7-(trifluoromethyl)-1H-isochromen-1-one, $101 \mathrm{MHz}$, $\mathrm{CDCl}_{3}, 299 \mathrm{~K}$

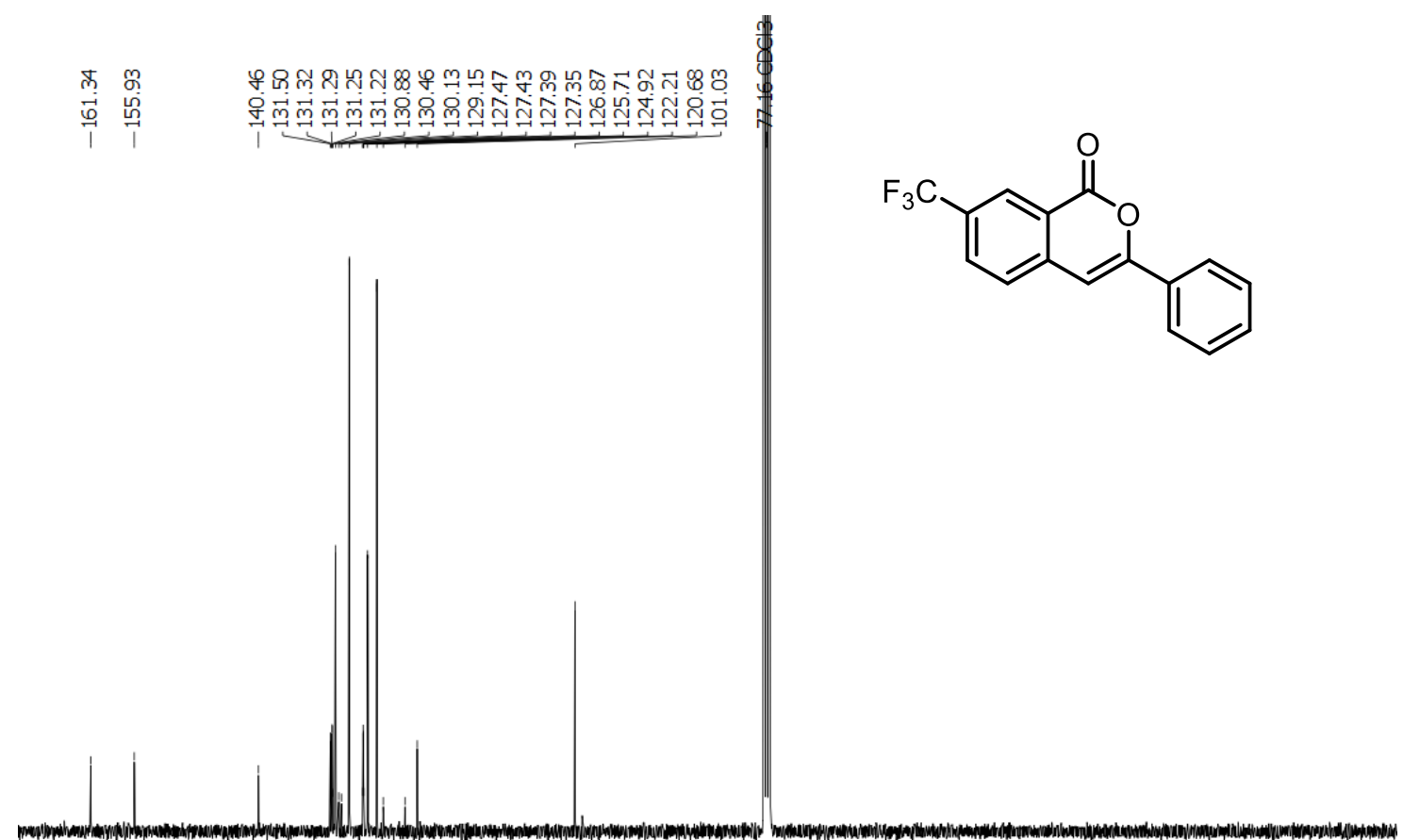

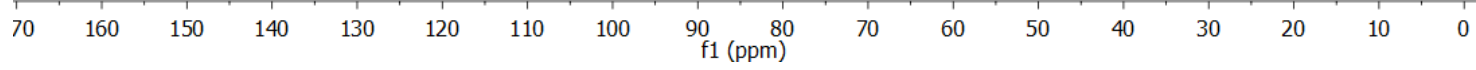


${ }^{19} \mathrm{~F}$ NMR of compound 228, 3-phenyl-7-(trifluoromethyl)-1H-isochromen-1-one, $282 \mathrm{MHz}$, $\mathrm{CDCl}_{3}, 298 \mathrm{~K}$

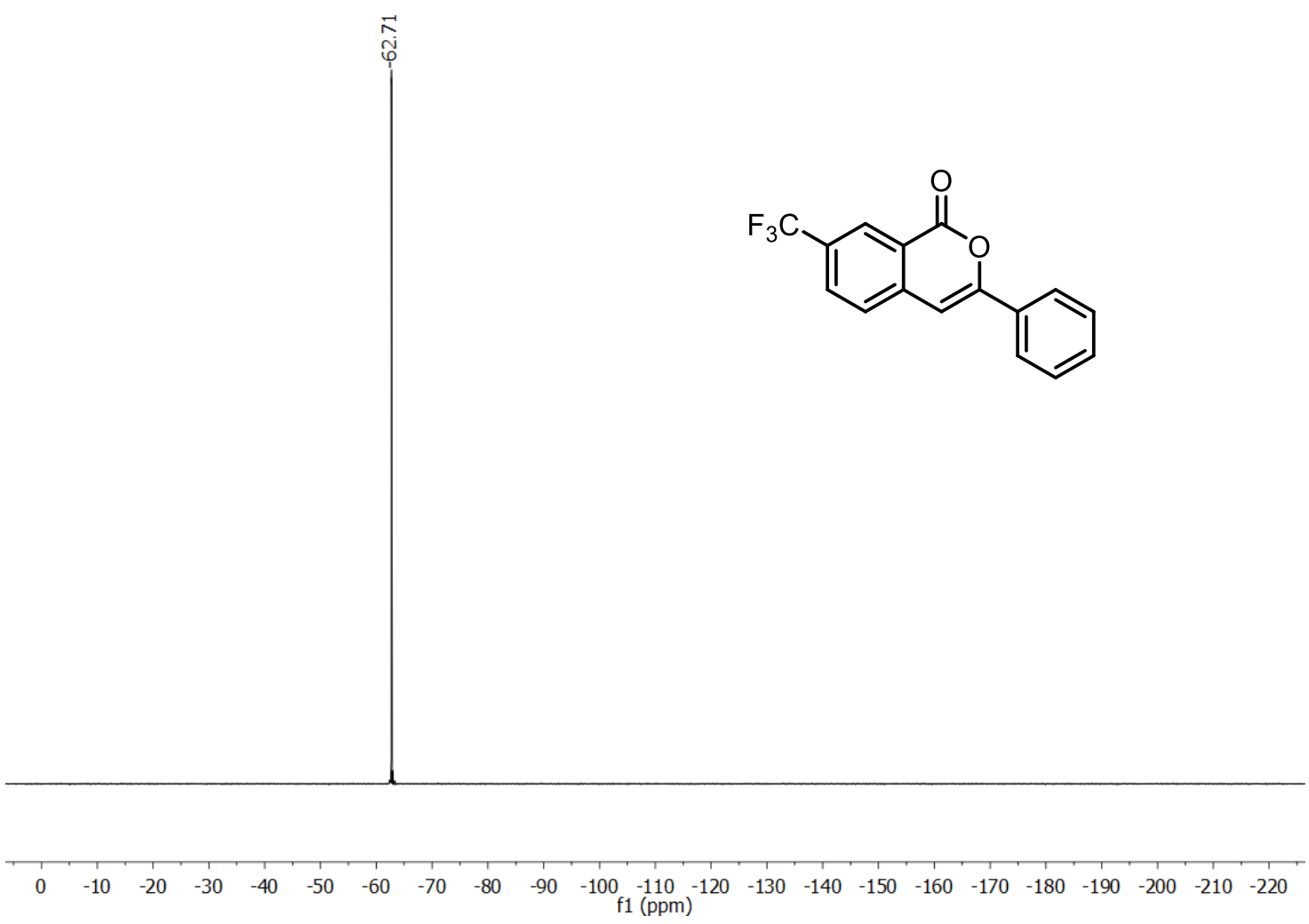

${ }^{1} \mathrm{H}$ NMR of compound 229, (Z)-N-(6-methoxy-3-phenyl-1H-isochromen-1-ylidene)methanaminium hexafluoroantimonate, $500 \mathrm{MHz}, \mathrm{CD}_{3} \mathrm{CN}, 298 \mathrm{~K}$

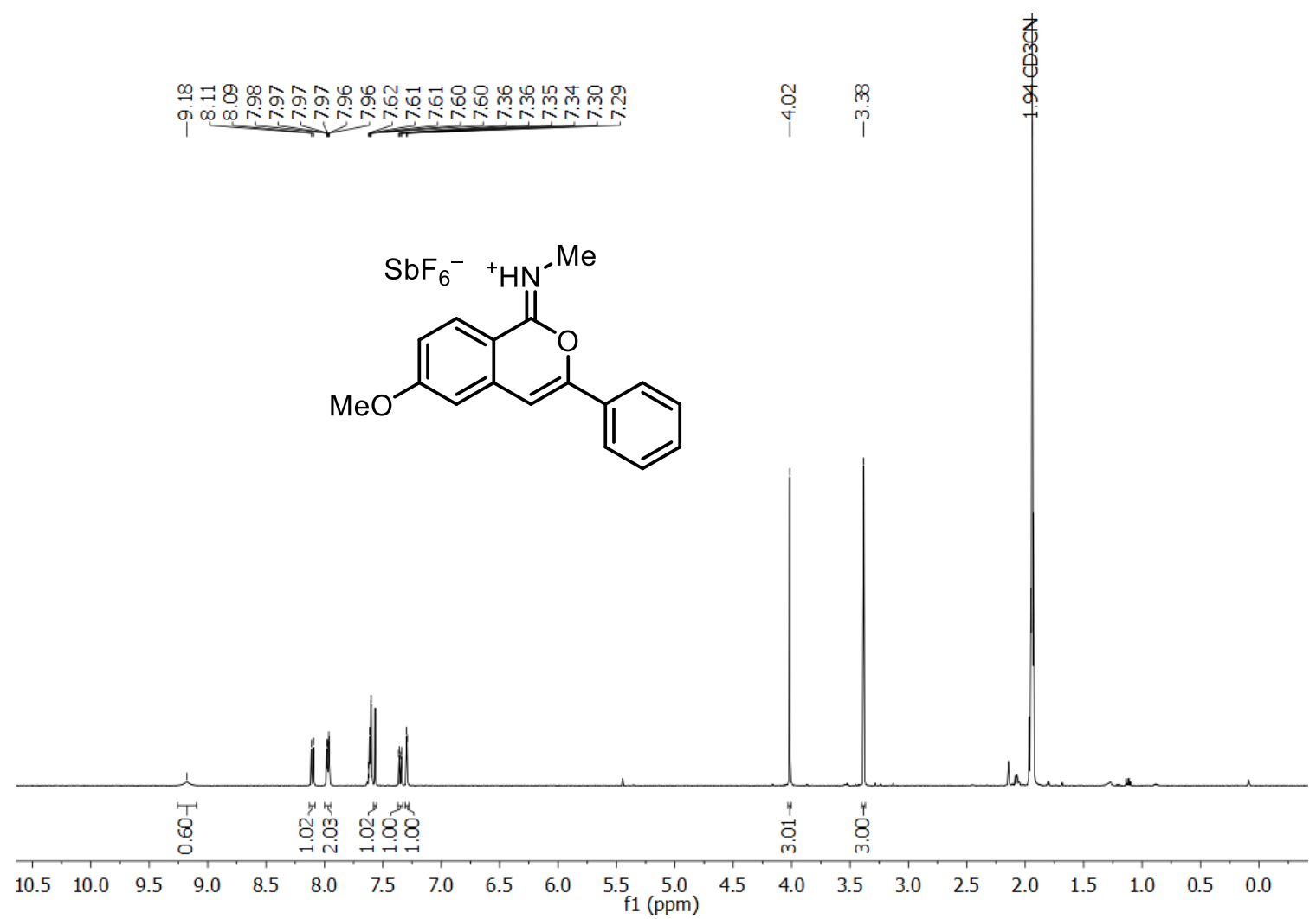


${ }^{13} \mathrm{C}$ NMR of compound 229, (Z)-N-(6-methoxy-3-phenyl-1H-isochromen-1-ylidene)methanaminium hexafluoroantimonate, $126 \mathrm{MHz}, \mathrm{CD}_{3} \mathrm{CN}, 298 \mathrm{~K}$

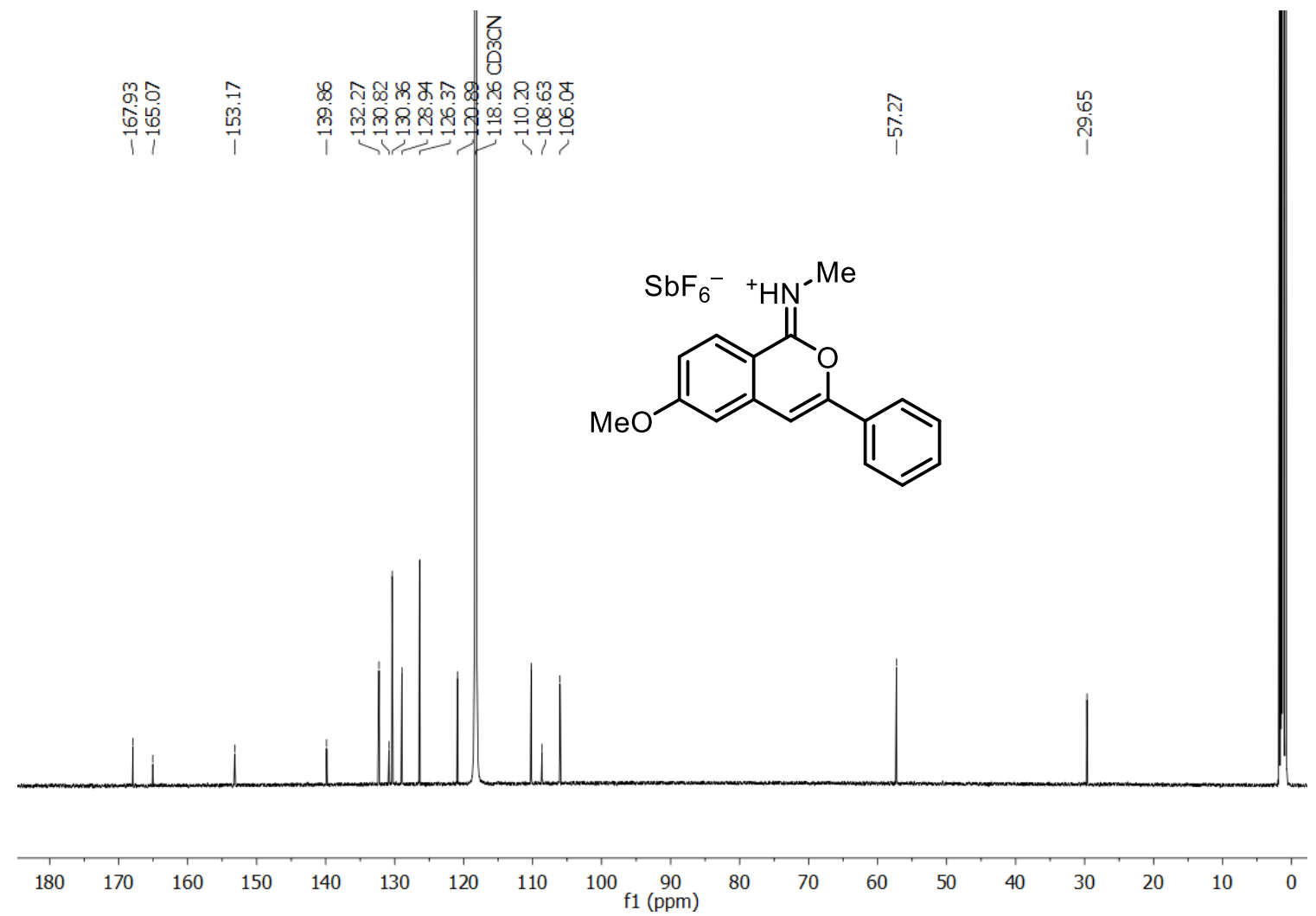

${ }^{19} \mathrm{~F}$ NMR of compound 229, (Z)-N-(6-methoxy-3-phenyl-1H-isochromen-1-ylidene)methanaminium hexafluoroantimonate, $471 \mathrm{MHz}, \mathrm{CD}_{3} \mathrm{CN}, 298 \mathrm{~K}$<smiles>CN=c1oc(-c2ccccc2)cc2cc(OC)ccc12</smiles>

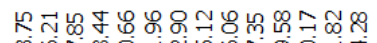

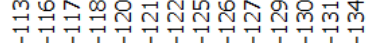

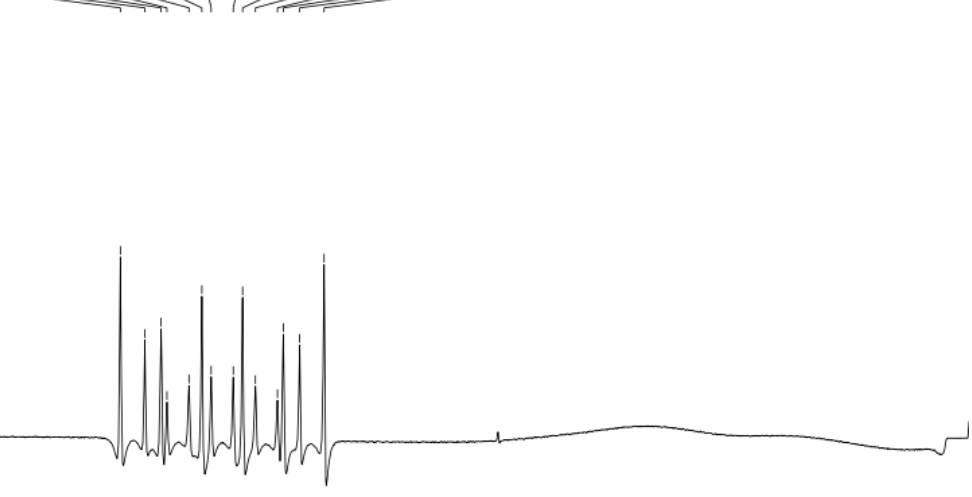


${ }^{1} \mathrm{H}$ NMR of compound 236, bis(acetonitrile)\{[(1,3-diisopropyl-4,5-dimethyl-1H-imidazol-3ium-2-yl]thio\}(pentamethylcyclopentadienyl)rhodium(I) bis(hexafluoroantimonate), 500 $\mathrm{MHz}, \mathrm{CDCl}_{3}, 298 \mathrm{~K}$
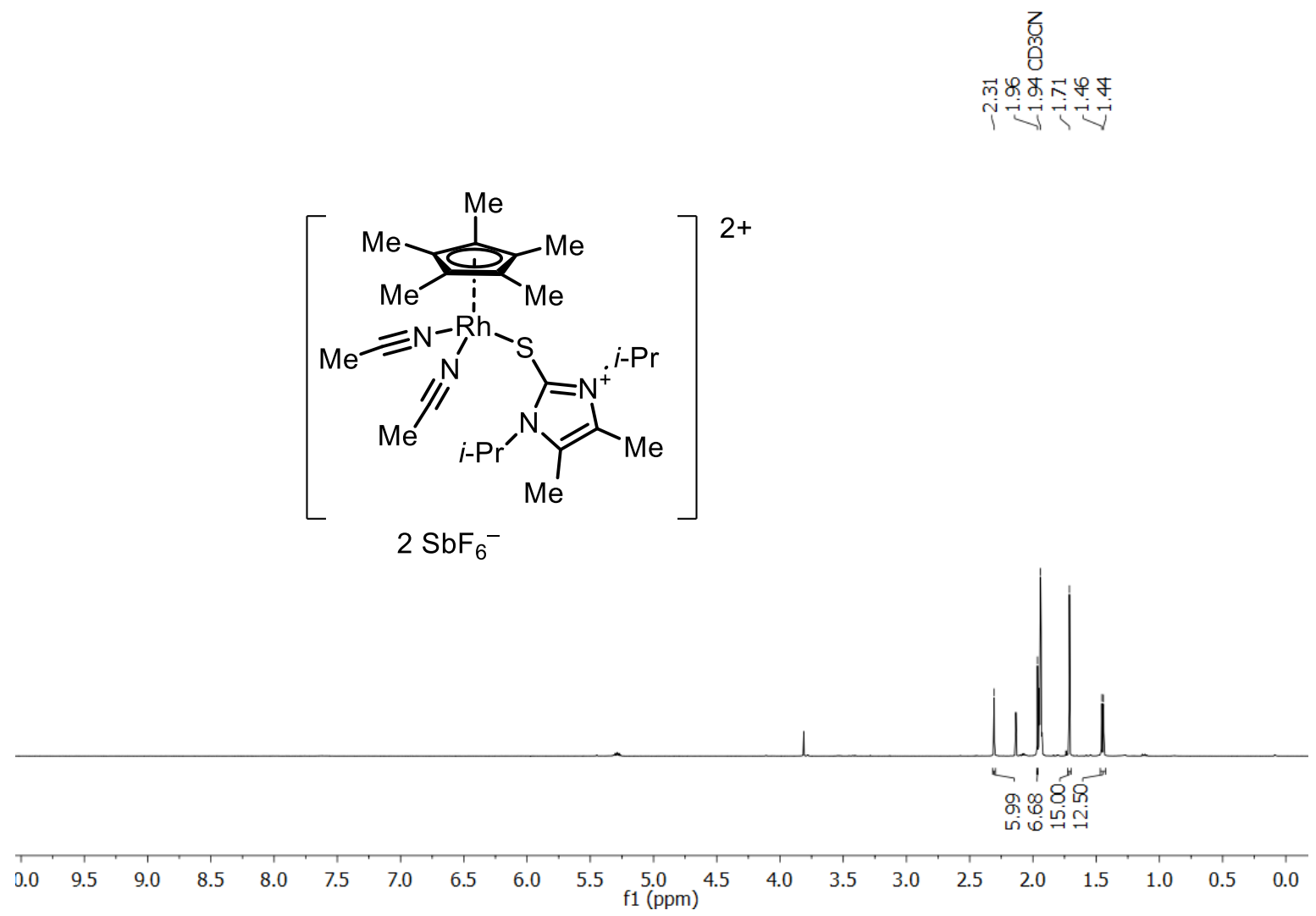

${ }^{13} \mathrm{C}$ NMR of compound 236, bis(acetonitrile)\{[(1,3-diisopropyl-4,5-dimethyl-1H-imidazol-3ium-2-yl]thio\}(pentamethylcyclopentadienyl)rhodium(I) bis(hexafluoroantimonate), 126 $\mathrm{MHz}, \mathrm{CDCl}_{3}, 298 \mathrm{~K}$

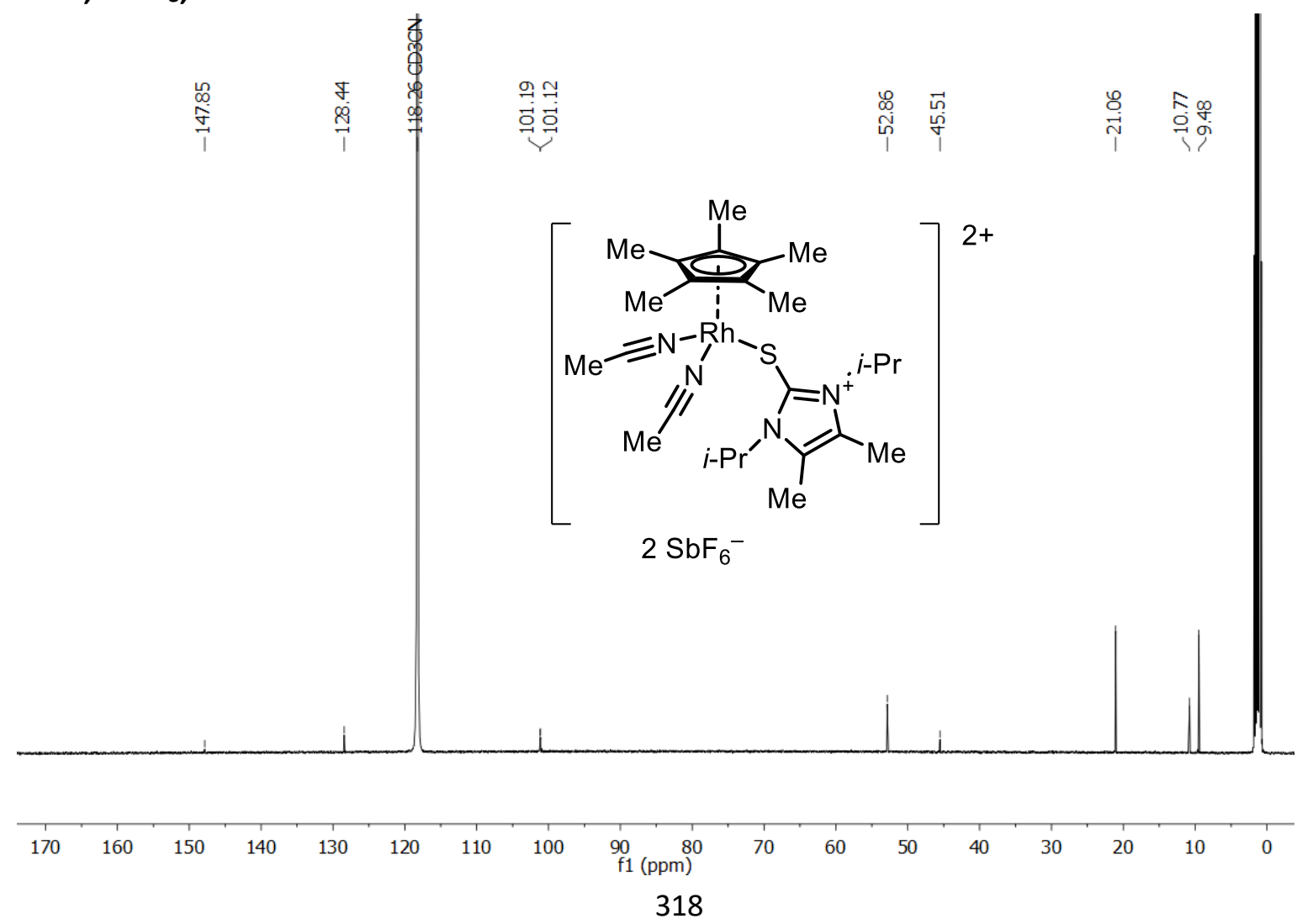


${ }^{19} \mathrm{~F}$ NMR of compound 236, bis(acetonitrile)\{[(1,3-diisopropyl-4,5-dimethyl-1H-imidazol-3ium-2-yl]thio\}(pentamethylcyclopentadienyl)rhodium(I) bis(hexafluoroantimonate), 471 $\mathrm{MHz}, \mathrm{CDCl}_{3}, 298 \mathrm{~K}$

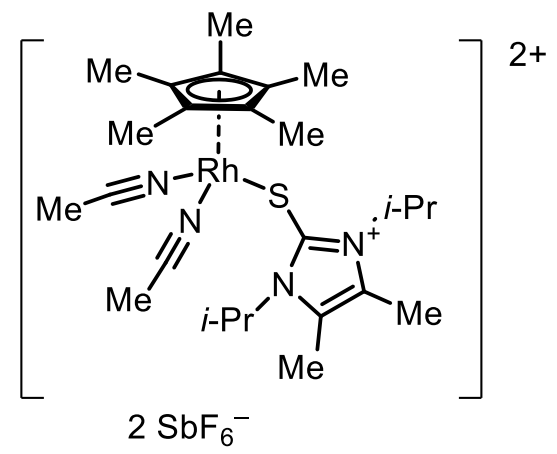

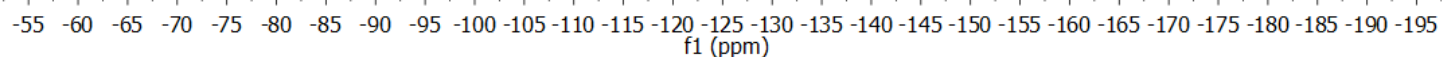

${ }^{1} \mathrm{H}$ NMR of compound 238, 3-[(1,3-diisopropyl-4,5-dimethyl-1H-imidazol-3-ium-2-yl)thio]2,4-diphenylcinnolin-2-ium, $300 \mathrm{MHz}^{\mathrm{CDCl}} 3,300 \mathrm{~K}$

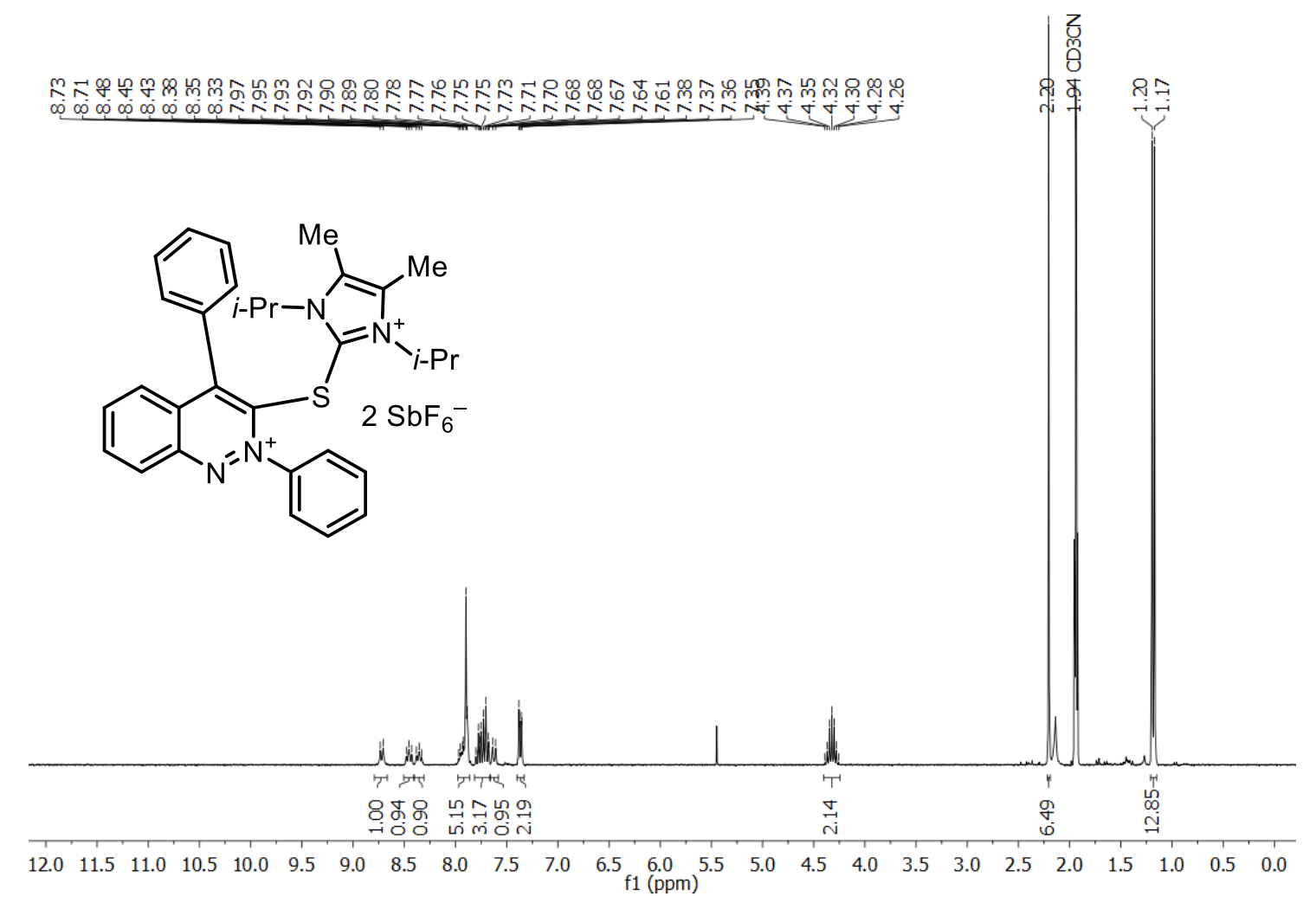


${ }^{13} \mathrm{C}$ NMR of compound 238, 3-[(1,3-diisopropyl-4,5-dimethyl-1H-imidazol-3-ium-2-yl)thio]2,4-diphenylcinnolin-2-ium, $101 \mathrm{MHz}, \mathrm{CDCl}_{3}, 298 \mathrm{~K}$

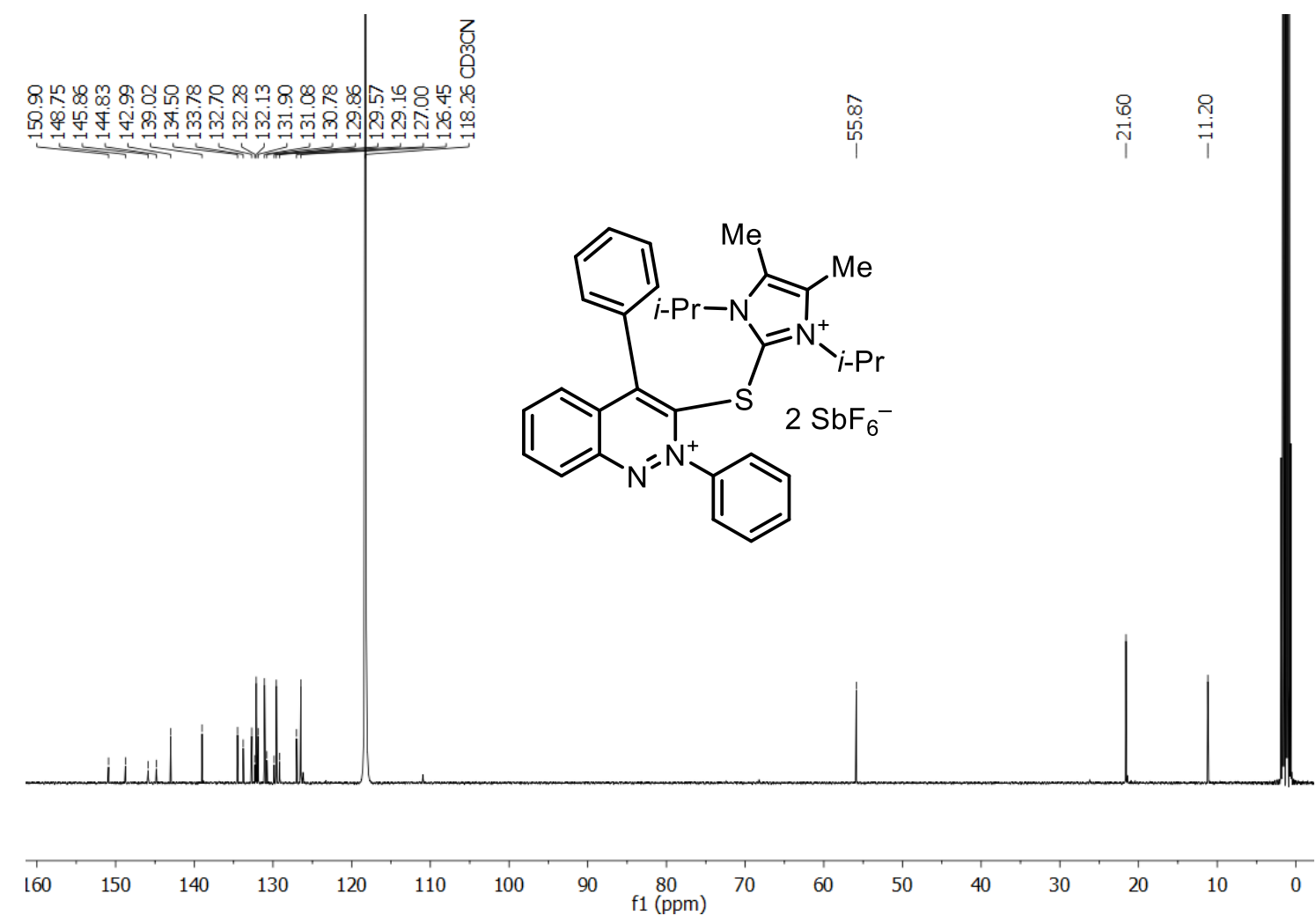

${ }^{19} \mathrm{~F}$ NMR of compound 238, 3-[(1,3-diisopropyl-4,5-dimethyl-1H-imidazol-3-ium-2-yl)thio]2,4-diphenylcinnolin-2-ium, $101 \mathrm{MHz}, \mathrm{CDCl}_{3}, 298 \mathrm{~K}$<smiles>CCCn1c(C)c(C)[n+](C(C)C)c1Sc1c(-c2ccccc2)c2ccccc2n[n+]1-c1ccccc1</smiles>

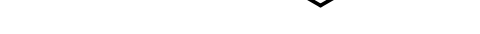


${ }^{1} \mathrm{H}$ NMR of compound $\left\{{ }^{13} \mathrm{C}\right\}-238,4-\left\{{ }^{13} \mathrm{C}\right\}-3-[(1,3-d i i s o p r o p y l-4,5-d i m e t h y l-1 H$-imidazol-3ium-2-yl)thio]-2,4-diphenylcinnolin-2-ium hexafluoroantimonate, $500 \mathrm{MHz}, \mathrm{CDCl}_{3}, 298 \mathrm{~K}$

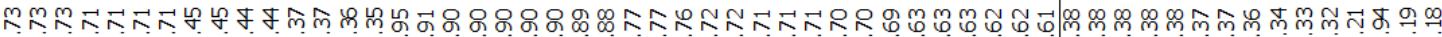

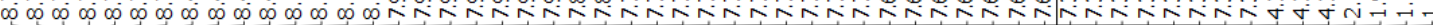<smiles></smiles>

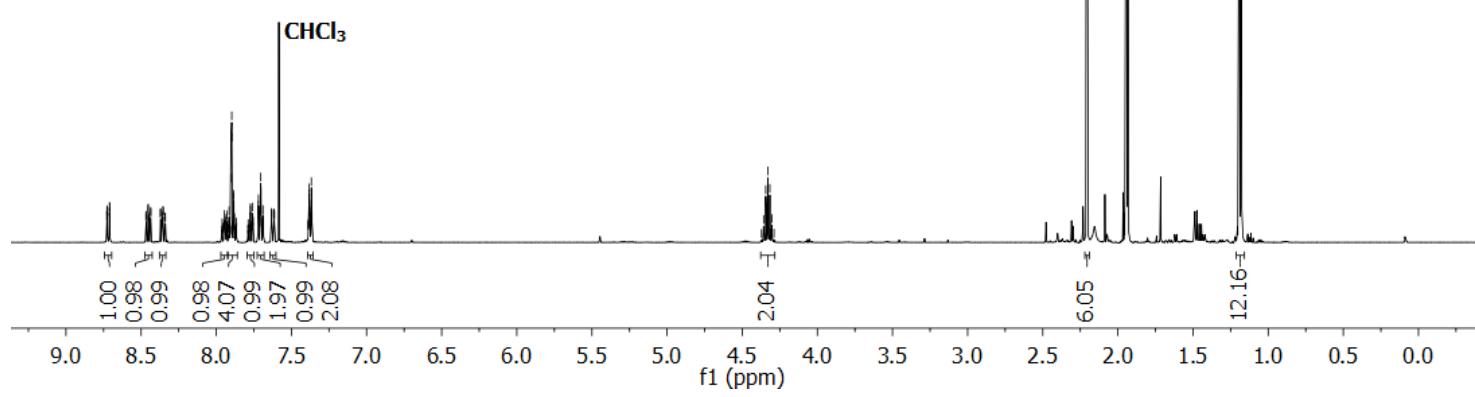

${ }^{13} \mathrm{C}$ NMR of compound $\left\{{ }^{13} \mathrm{C}\right\}-238,4-\left\{{ }^{13} \mathrm{C}\right\}-3-[(1,3-$ diisopropyl-4,5-dimethyl-1H-imidazol-3ium-2-yl)thio]-2,4-diphenylcinnolin-2-ium hexafluoroantimonate, $126 \mathrm{MHz}, \mathrm{CDCl}_{3}, 298 \mathrm{~K}$

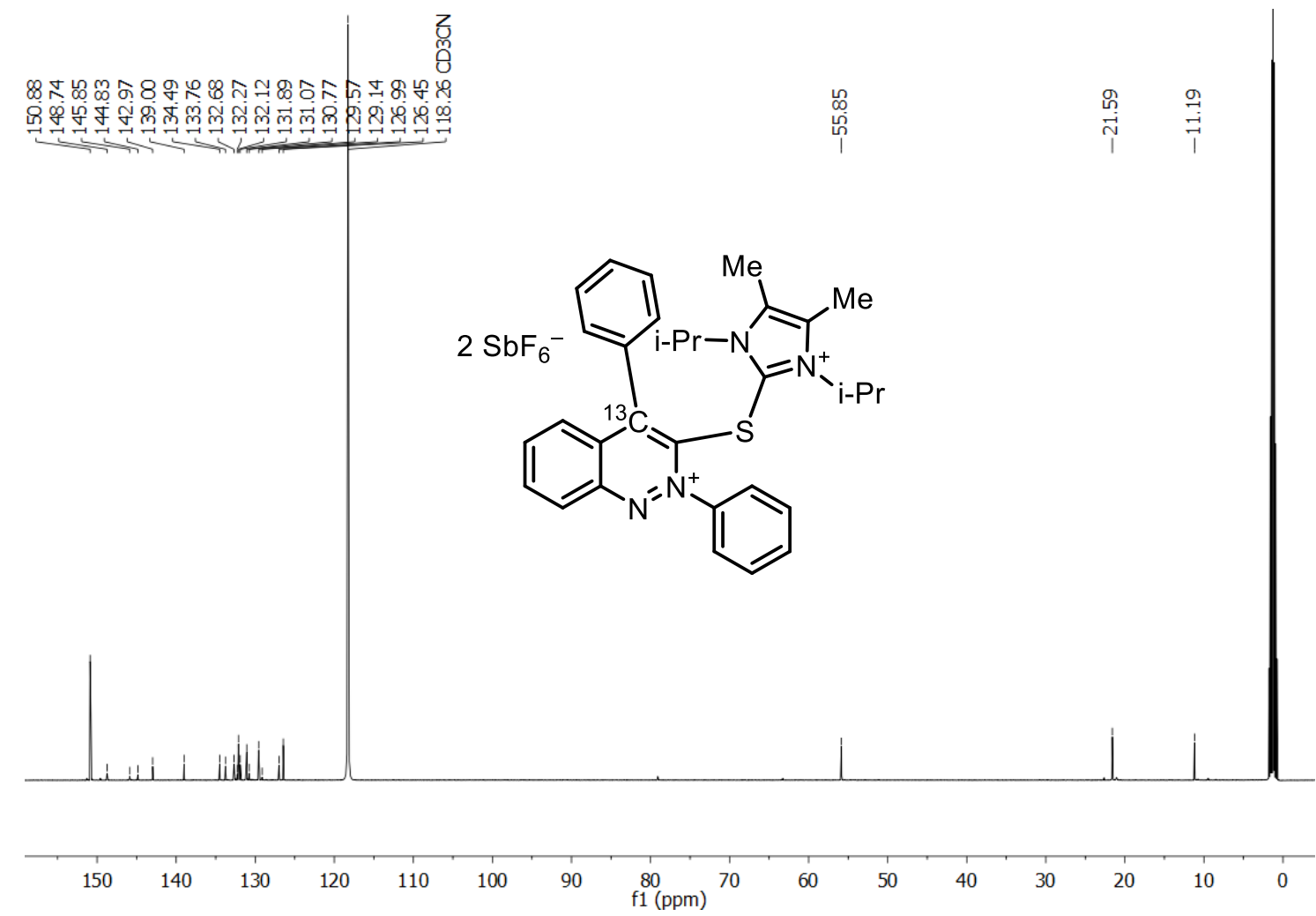


${ }^{19} \mathrm{~F}$ NMR of compound $\left\{{ }^{13} \mathrm{C}\right\}-238,4-\left\{{ }^{13} \mathrm{C}\right\}-3-[(1,3-$ diisopropyl-4,5-dimethyl-1H-imidazol-3ium-2-yl)thio]-2,4-diphenylcinnolin-2-ium hexafluoroantimonate, $471 \mathrm{MHz}, \mathrm{CDCl}_{3}, 298 \mathrm{~K}$

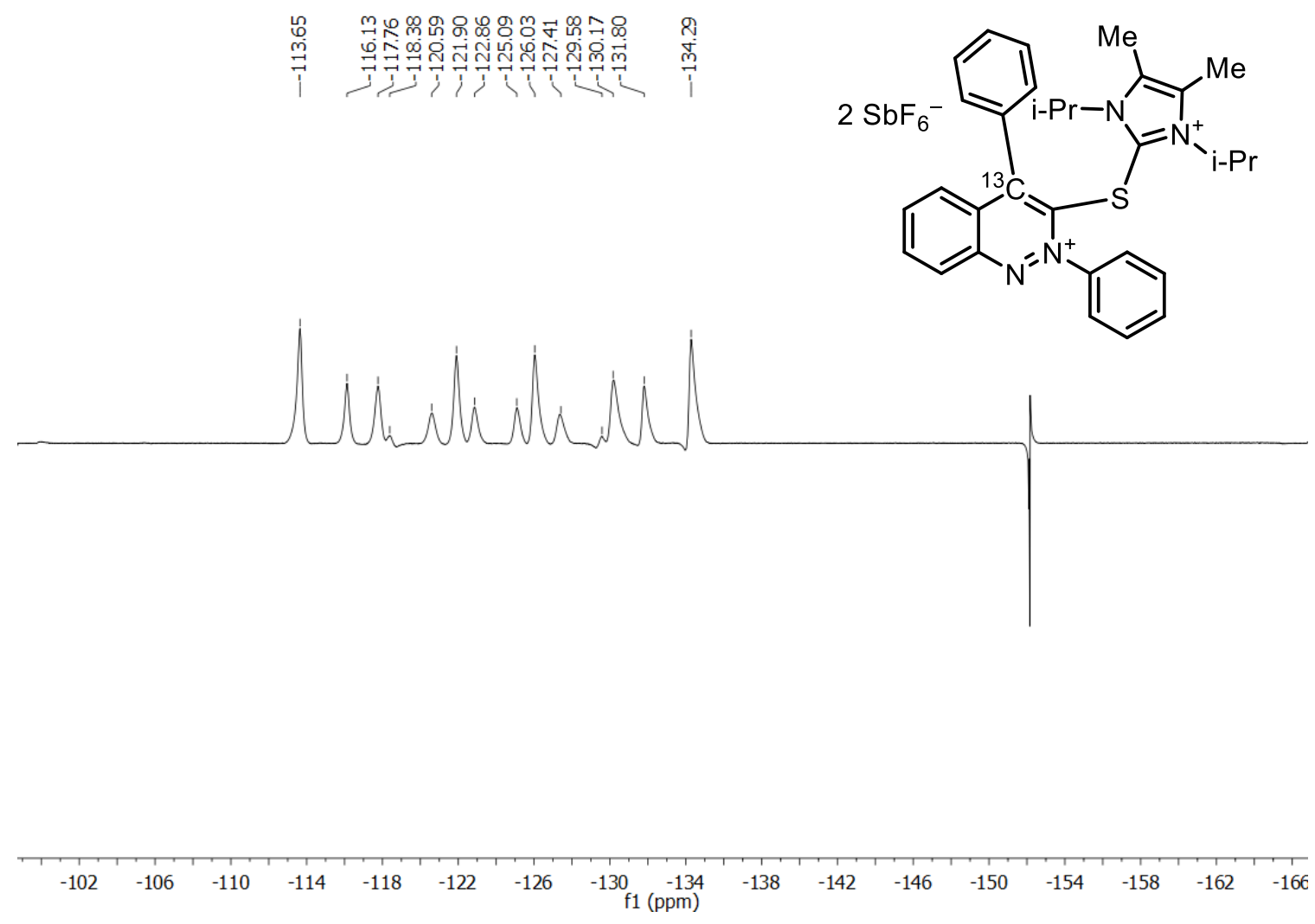

${ }^{1} \mathrm{H}$ NMR of compound 240, (1- $\left\{{ }^{13} \mathrm{C}\right\}$-2,2-dibromovinyl)benzene, $300 \mathrm{MHz}, \mathrm{CDCl}_{3}, 298 \mathrm{~K}$

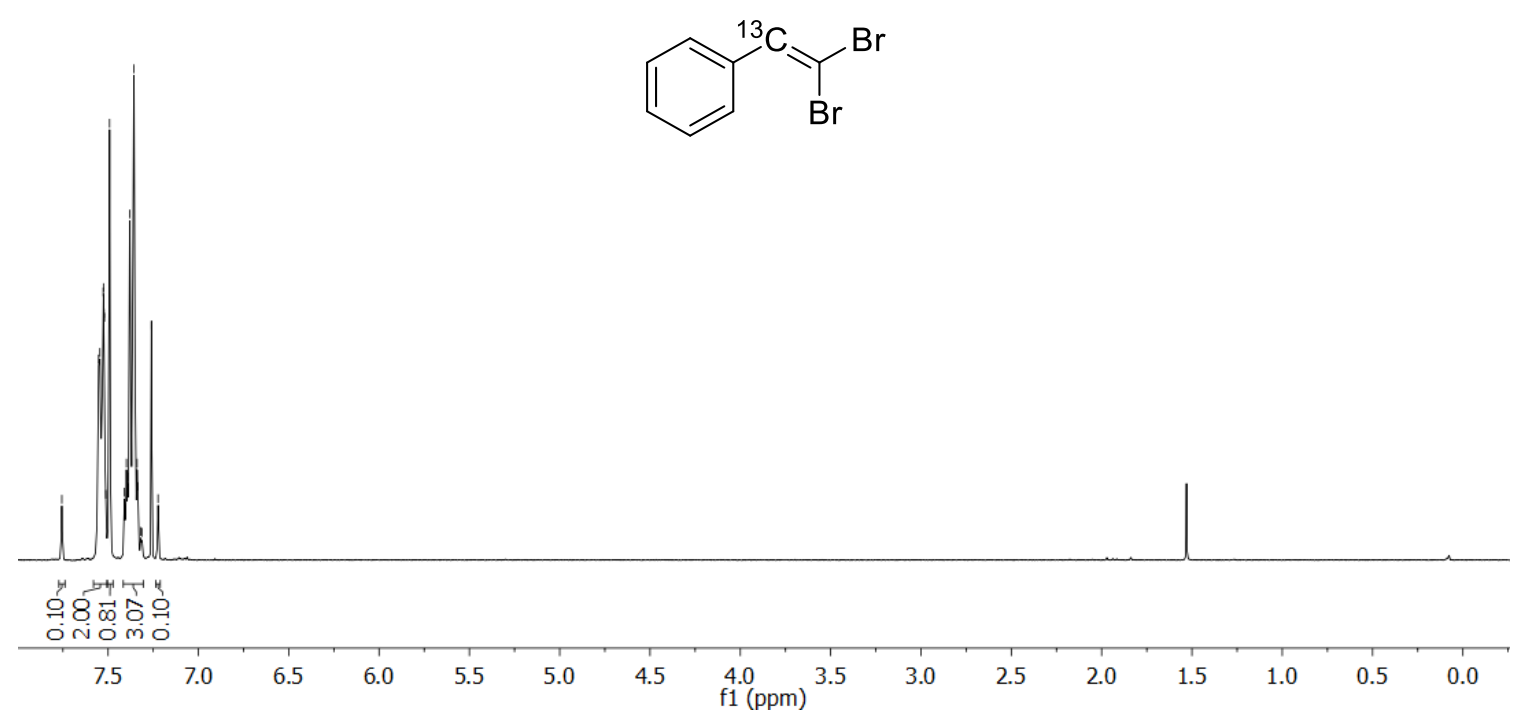


${ }^{13} \mathrm{C}$ NMR of compound 240, (1- $\left\{{ }^{13} \mathrm{C}\right\}$-2,2-dibromovinyl)benzene, $101 \mathrm{MHz}, \mathrm{CDCl}_{3}, 300 \mathrm{~K}$

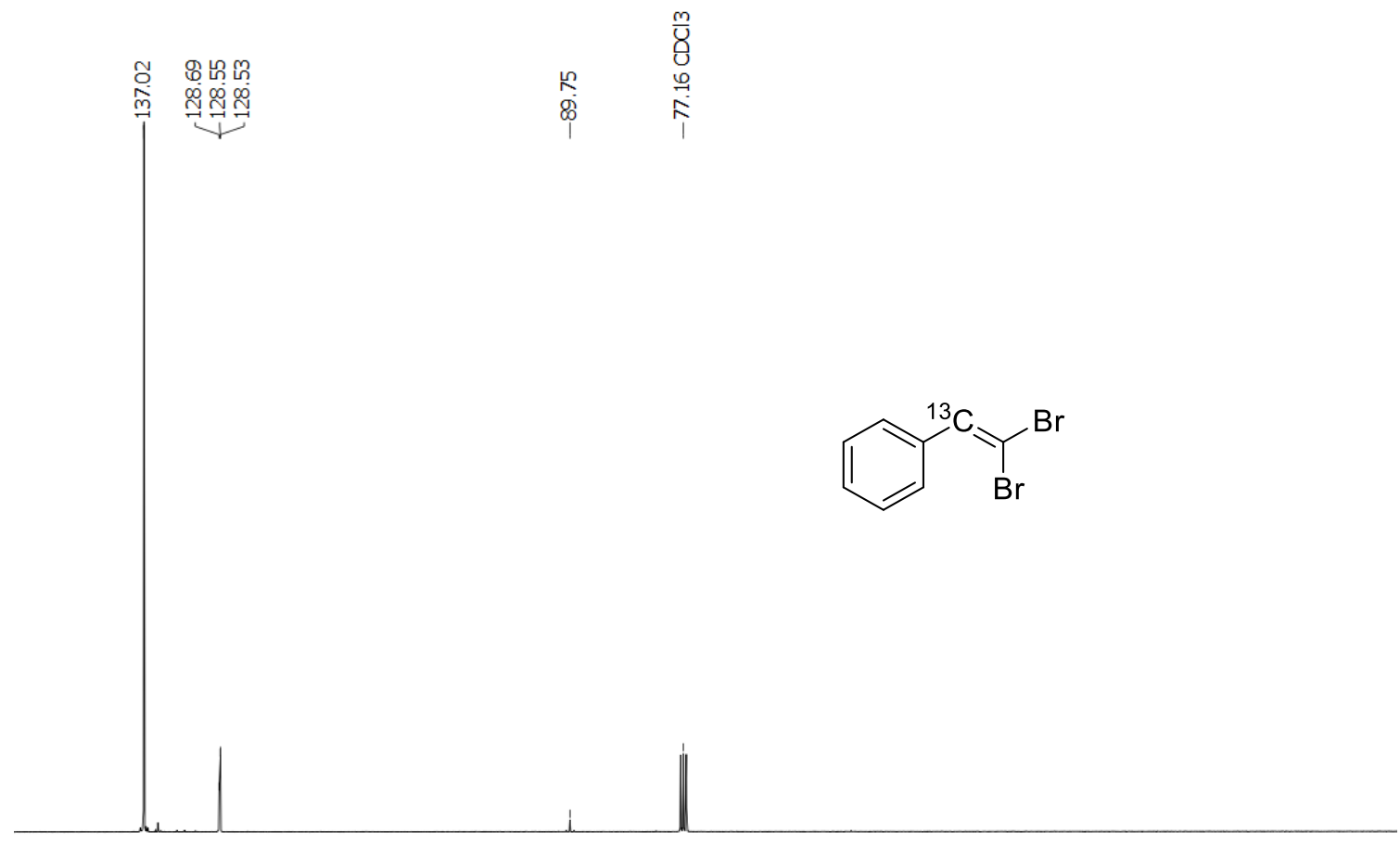

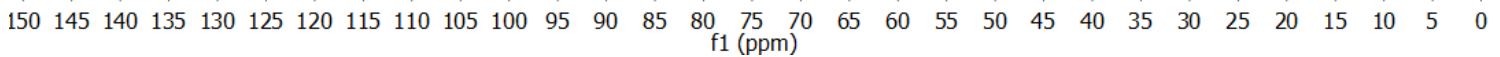

${ }^{1} \mathrm{H}$ NMR of compound 241, 2,4-diphenylcinnolin-3(2H)-one, $300 \mathrm{MHz}, \mathrm{CDCl}_{3}, 298 \mathrm{~K}$

$\frac{m}{8}$

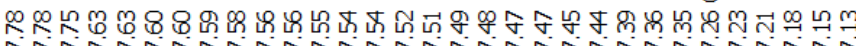

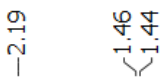<smiles>O=c1c(-c2ccccc2)c2ccccc2nn1-c1ccccc1</smiles>

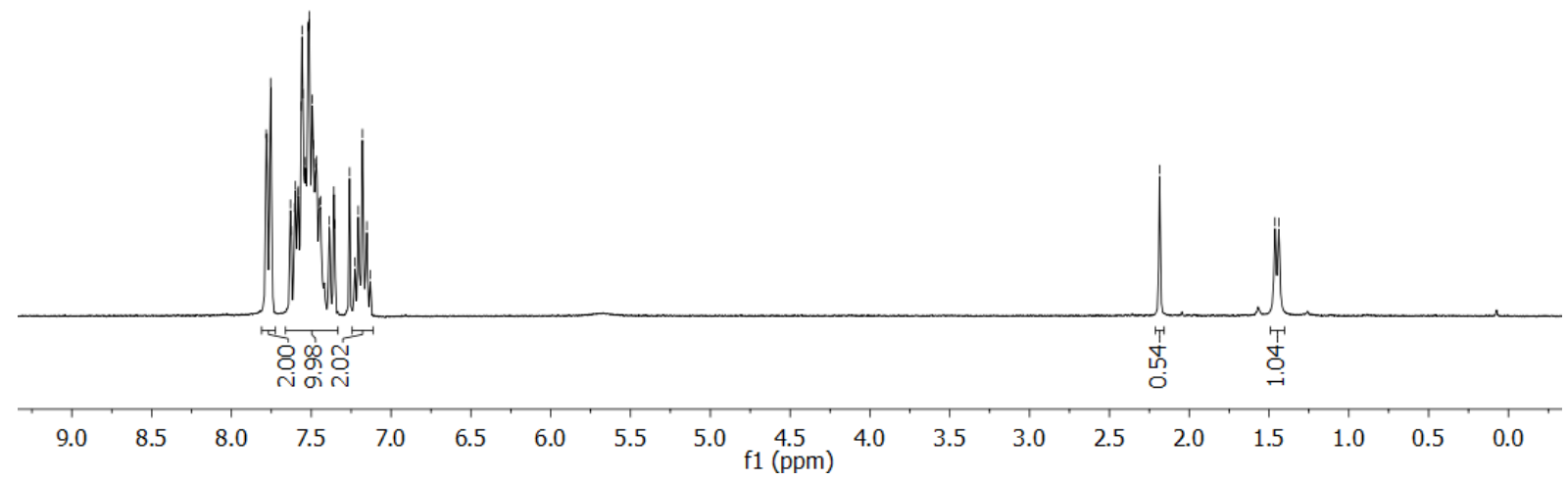


${ }^{13} \mathrm{C}$ NMR of compound 241, 2,4-diphenylcinnolin-3(2H)-one, $101 \mathrm{MHz}, \mathrm{CDCl}_{3}, 298 \mathrm{~K}$
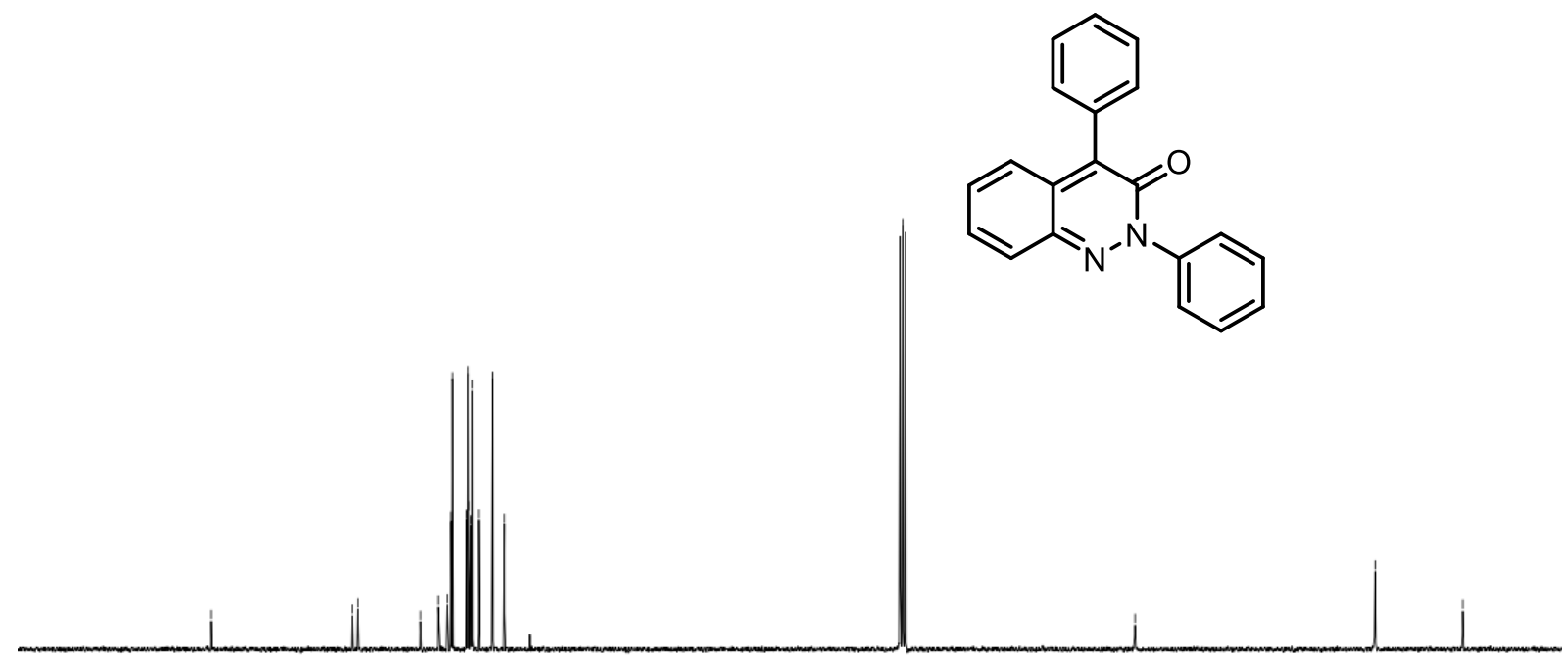

${ }^{1} \mathrm{H}$ NMR of compound 242, 2,3-diphenyl-2H-indazole, $400 \mathrm{MHz}, \mathrm{CDCl}_{3}, 298 \mathrm{~K}$

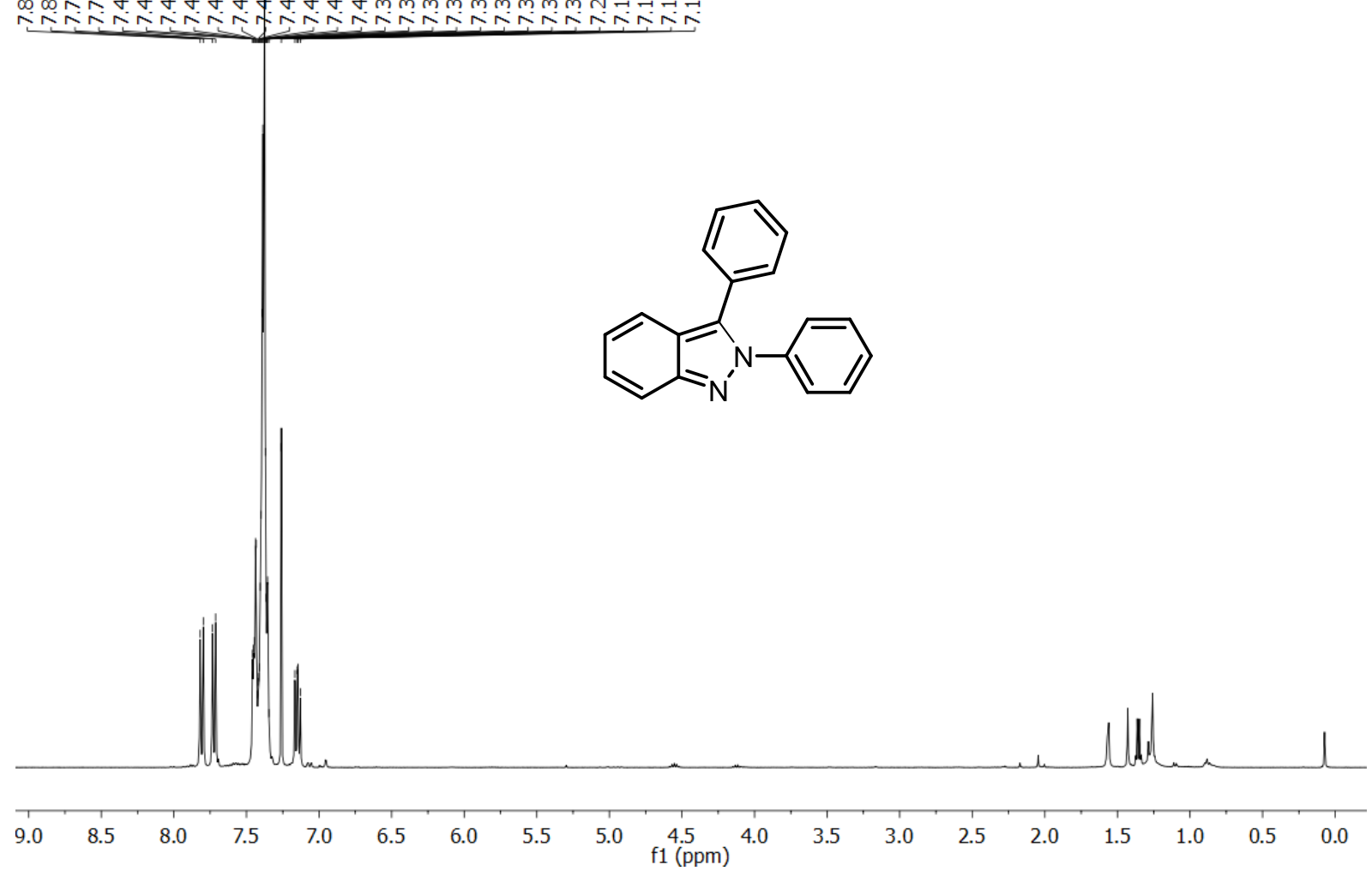


${ }^{13} \mathrm{C}$ NMR of compound 242, 2,3-diphenyl-2H-indazole, $101 \mathrm{MHz}, \mathrm{CDCl}_{3}, 298 \mathrm{~K}$

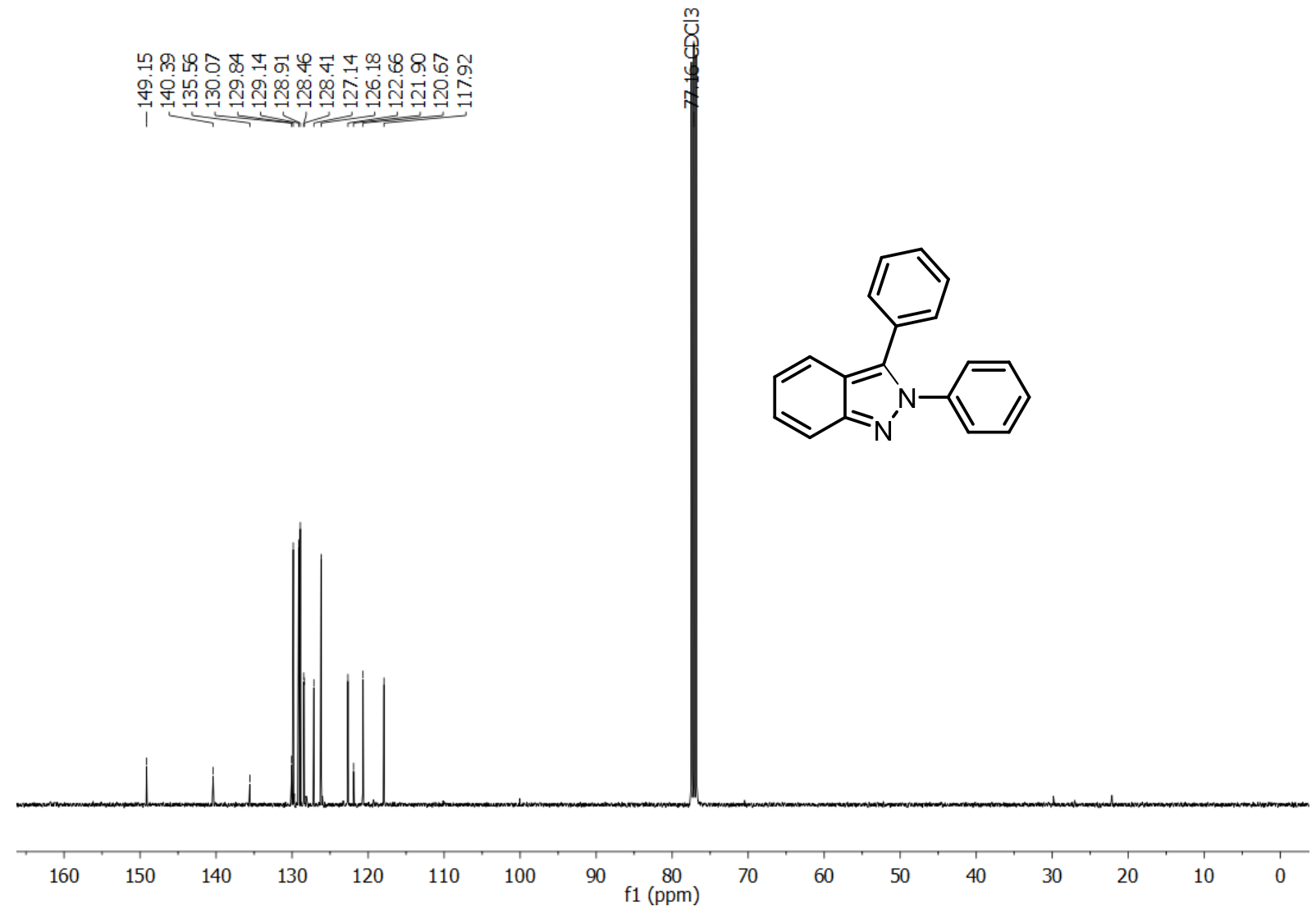




\section{Curriculum VitaE}

Personal Data:

Vogtei 20 B

Kai Florian Gustav Averesch

45239 Essen

Born on the 3rd of July, 1988 in Essen

Germany

Family status: Single

Ph.: +4915758506898

Nationality: German

kai.averesch@tu-dortmund.de

\section{Employment and Research History}

2016-2019

2016

2016

2015

2014-2015

2014

2013

2011

2011
PhD studies (continuation) at Georg August Universität Göttingen

Internship at Bayer Research Center, Wuppertal

Relocation with the research group to Georg August Universität Göttingen

Commencement of PhD studies at the Max-Planck-Institut für

Kohlenforschung with Prof. Dr. Manuel Alcarazo. On "Novel cationic Sulfur Reagents and their Application in Electrophilic Group Transfer Reactions " Editorial work on supporting material and self-education of the book "Technische Biochemie - Die Biochemie und industrielle Nutzung von Naturstoffen" (eds. O. Kayser and N. Averesch), ISBN: 978-3-658-05547-9 Degree in Chemistry (Master of Science) from the TU Dortmund University, Germany (Prof. Dr. Arno Behr) on the "Ruthenium-catalyzed Tandemreaction for the Synthesis of branched, primary higher Alcohols from 1Alkenes"

Internship at the Centre for Microbial Synthesis (CEMES) within the Advanced Water Management Centre (AWMC) at the University of Queensland, Australia

Degree in Chemistry (Bachelor of Science) from the TU Dortmund University, Germany (Prof. Dr. Arno Behr) on the "Cooligomerization of Isobutene with Dienes"

Student assistant at the chair for organic chemistry; supervision and assistance for undergraduate students in the degree-program chemistry or chemical biology at the TU Dortmund University 
2019 K. F. G. Averesch, H. Pesch, C. Golz. M. Alcarazo, "Synthesis of Alkynylthiopyridinium Salts and Their Use as Thioketene Equivalents", Chem. Eur.J .2019, 25, 10472-10477.

2013 Attended 4th International Microbial Fuel Cell Conference (MFC4) in Cairns, Australia with a poster presentation

Language Skills: German (native), English (excellent command) 